\title{
Modular Fragment Synthesis and Bioinformatic Analysis Propose a Revised Vancoresmycin Stereoconfiguration
}

Stefanie Spindler, ${ }^{\dagger, \dagger}$ Lukas M. Wingen, ${ }^{\dagger, \dagger}$ Max Schönenbroicher, ${ }^{\dagger}$ Maximilian Seul, ${ }^{\dagger}$ Martina Adamek, ${ }^{\S}$ Sebastian Essig, ${ }^{\|}$Michael Kurz, ${ }^{\dagger \dagger}$ Nadine Ziemert, ${ }^{\S}$ and Dirk Menche ${ }^{\dagger, *}$

${ }^{\dagger}$ Kekulé-Institut für Organische Chemie und Biochemie, Universität Bonn, Gerhard-Domagk-Str. 1, D-53121 Bonn, Germany,

${ }^{\S}$ Interfaculty Institute of Microbiology and Infection Medicine, , German Centre for Infection Research (DZIF), Partnersite Tuebingen, Auf der Morgenstelle 28, D-72076 Tübingen, Germany,

"Bayer AG, Aprather Weg 18a, D-42113 Wuppertal, Germany, and

${ }^{\dagger}$ Sanofi-Aventis Deutschland GmbH, Industriepark Höchst, D-65926 Frankfurt am Main, Germany.

*Correspondence: dirk.menche@uni-bonn.de

\section{Contents}

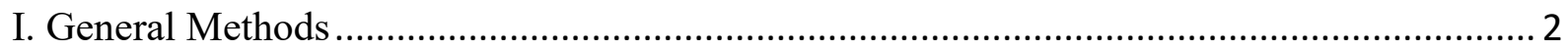

II. Experimental Details and Characterization Data............................................................. 4

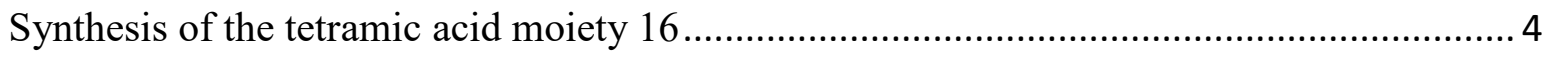

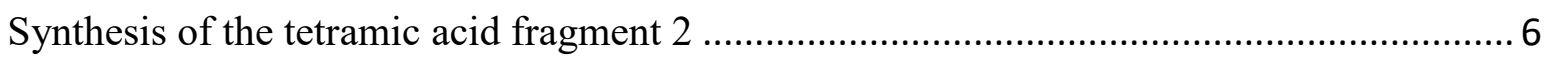

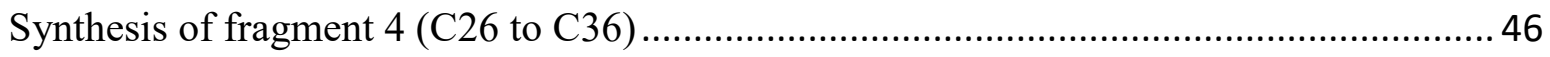

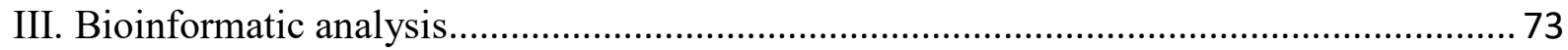

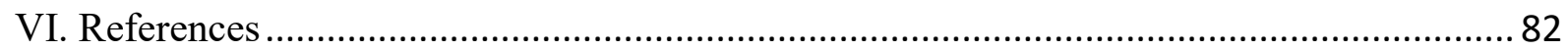

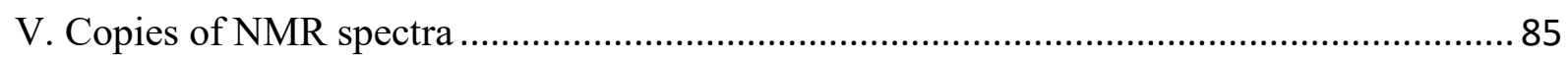




\section{General Methods}

Reaction conditions All reagents were purchased from commercial suppliers (Sigma-Aldrich, TCI, Acros, AlfaAesar, Merck, Carbolution) in the highest purity grade available and used without further purification. Anhydrous solvents (THF, DCM, MeCN, $\mathrm{Et}_{2} \mathrm{O}$, toluene) were obtained from a solvent drying system MB SPS-800 (MBraun) and stored over molecular sieves (4 $\AA$ ). All reactions with dry solvents were performed under an argon atmosphere. The glassware was flame dried and cooled under argon. All flasks were equipped with rubber septa and reactants were handled using standard Schlenk techniques. Reaction temperatures above r.t. $\left(23^{\circ} \mathrm{C}\right)$ refer to oil bath temperatures, which were controlled by a temperature modulator. For cooling, the following baths were used: acetone/dry ice $\left(-78^{\circ} \mathrm{C}\right), \mathrm{ACN} / \mathrm{dry}$ ice $\left(-40^{\circ} \mathrm{C}\right)$ and water/ice $\left(0^{\circ} \mathrm{C}\right)$. For other temperatures below $0{ }^{\circ} \mathrm{C}$ a HuBER TC100E-F-NR was used.

TLC monitoring was performed with silica gel $60 \mathrm{~F}_{254}$ pre-coated polyester sheets $(0.2 \mathrm{~mm}$ silica gel, Macherey-Nagel) and TLC silica gel $60 \mathrm{RP}-18 \mathrm{~F}_{254} \mathrm{~S}$ (MERCK). They were visualized using UV light and/or staining with a solution of CAM $\left(1.0 \mathrm{~g} \mathrm{Ce}\left(\mathrm{SO}_{4}\right)_{2}\right), 2.5 \mathrm{~g}\left(\mathrm{NH}_{4}\right)_{6} \mathrm{Mo}_{7} \mathrm{O}_{24}, 8 \mathrm{~mL}$ conc. $\mathrm{H}_{2} \mathrm{SO}_{4}$ in $\left.100 \mathrm{~mL} \mathrm{H}_{2} \mathrm{O}\right)$ or $\mathrm{KMnO}_{4}$ solution $\left(1.3 \mathrm{~g} \mathrm{KMnO}_{4}, 10 \mathrm{~g} \mathrm{~K}_{2} \mathrm{CO}_{3}, 1.25 \mathrm{~mL} 10 \% \mathrm{NaOH}\right.$ in $\left.200 \mathrm{~mL} \mathrm{H}_{2} \mathrm{O}\right)$ and subsequent heating. For column chromatography, silica gel (pore size $60 \AA$, 40-63 $\mu \mathrm{m}$ ) obtained from Merck or Macherey-Nagel was used. The elution was performed under a positive pressure of air using the stated solvent mixtures. The solvents were distilled prior use.

Analytical methods All NMR spectra were recorded on Bruker spectrometers at the University of Bonn under supervision of Dr. Senada Nozinovic with operating frequencies of $125\left({ }^{13} \mathrm{C}\right), 150\left({ }^{13} \mathrm{C}\right)$, $175\left({ }^{13} \mathrm{C}\right), 400\left({ }^{1} \mathrm{H}\right), 500\left({ }^{1} \mathrm{H}\right), 600\left({ }^{1} \mathrm{H}\right)$ and $700 \mathrm{MHz}\left({ }^{1} \mathrm{H}\right)$ in deuterated solvents obtained from Deutero and Carl Roth. Spectra were measured at room temperature unless stated otherwise and chemical shifts are reported in ppm relative to $(\mathrm{Me})_{4} \mathrm{Si}(\delta=0.00 \mathrm{ppm})$ and were calibrated to the residual signal of undeuterated solvents. ${ }^{[1]}$ Data for ${ }^{1} \mathrm{H}-\mathrm{NMR}$ spectra are reported as follows: chemical shift (multiplicity, coupling constants in $\mathrm{Hz}$, number of hydrogens, assignment). The following abbreviations are used: s (singlet), d (doublet), $\mathrm{t}$ (triplet), q (quartet), quint (quintet), hept (heptet), $\mathrm{m}$ (multiplet), br (broad).

Mass spectra (MS) and High-resolution-mass spectra (HRMS) were recorded on the documented systems in Table $S 1$ at the University of Bonn under supervision of PD Dr. Marianne Engeser. 
Table S1: Used MS systems for MS and HRMS.

$\begin{array}{lll}\text { System } & \text { Manufacturer } & \text { Measurements } \\ \text { MAT 95 XL } & \text { Thermo Finnigan (Bremen) } & \text { EI } \\ \text { MAT 90 } & \text { Thermo Finnigan (Bremen) } & \text { EI, LIFDI, CI, FAB } \\ \text { micrOTOF-Q } & \text { Bruker Daltonik (Bremen) } & \begin{array}{l}\text { ESI, APCI, nano-ESI, MS/MS, LC- } \\ \text { MS, DC-MS }\end{array} \\ \text { Orbitrap XL } & \begin{array}{l}\text { Thermo Fisher Scientific } \\ \text { (Bremen) }\end{array} & \text { ESI, APCI, APPI, nano-ESI } \\ \text { Apex IV FT-ICR } & \text { Bruker Daltonik (Bremen) } & \text { ESI, nano-ESI, MALDI, EI, CI }\end{array}$

The HPLC analyses (preparative and analytical) were performed by Andreas J. Schneider at the Kekulé-Institute of Organic Chemistry and Biochemistry at the University of Bonn. Systems of the company Knauer Wissenschaftliche Geräte GmbH were used (See Table XX). The solvents were purchased in HPLC grade from commercial suppliers. HPLC-water (type I) was supplied by Elga/Veolia PureLab Flex 4 with microendfilter $(0.2 \mu \mathrm{m})$, conductivity $\leq 18.2 \mathrm{MOhm}$; TOC $\leq 2 \mathrm{ppb}$.

Table S2: HPLC configuration for analytical and preparative HPLC.

$\begin{array}{lll}\text { System } & \text { A (analytical) } & \text { B (preparative) } \\ \text { series } & \text { PLATINblue } & \text { Smartline } \\ \text { pumps } & \text { binary, HPG 6.1L, 10 mL } & \text { binary, HPG 6.1L, 50 ml } \\ \text { pressure } & \text { 700 bar } & \text { 200-300 bar } \\ \text { autosampler } & \text { AS1 with } 10 \mu \mathrm{L} \text { injection loop } & 100 \\ & & \text { integrated static mixing } \\ \text { mixing chamber } & \text { integrated static mixing } & - \\ \text { column heater } & \text { CT 6.1 } & \text { MWL 2.1L, 190-700 nm } \\ \text { detection type } & \text { PDA UV/VIS detection DAD } & \\ & \text { 6.1L, } \mathrm{D}_{2} / \mathrm{Hg} \text { halogen lamps, 190-1020 } & \\ \text { nm } & \text { 2-channel-online-degasser }\end{array}$

Optical rotations were measured with an Anton Paar modular compact polarimeter MCP 150 at $20.0^{\circ} \mathrm{C}$ unless stated otherwise, with either a $10 \mathrm{~mm}$ cuvette $(0.2 \mathrm{ml})$ or $100 \mathrm{~mm}$ cuvette $(0.7 \mathrm{ml})$, using a LED (589 nm) as a light source. The optical rotation was measured 3 times and the specific rotation was calculated. $[\alpha]_{\lambda}^{T}=\alpha_{\lambda}^{T} /(l \cdot c)$

$[\alpha]$ : specific rotation $\left[\mathrm{deg} \cdot \mathrm{ml} \cdot \mathrm{dm}^{-1} \cdot \mathrm{g}^{-1}\right], \lambda$ : wave length of the light source $[\mathrm{nm}], \mathrm{D}$ refers to the sodium D line (589.0 and $589.6 \mathrm{~nm}), \mathrm{T}$ : temperature $\left[{ }^{\circ} \mathrm{C}\right], \alpha$ : optical rotation, 1 : path length of the cuvette $[\mathrm{dm}]$, c: concentration $[\mathrm{g} / 100 \mathrm{ml}]$. 


\section{Experimental Details and Characterization Data}

\section{Synthesis of the tetramic acid moiety 16}

(Z)-4-Methoxy-5-(2-methylpropylidene)-1,5-dihydro-2H-pyrrol-2-one (16a)

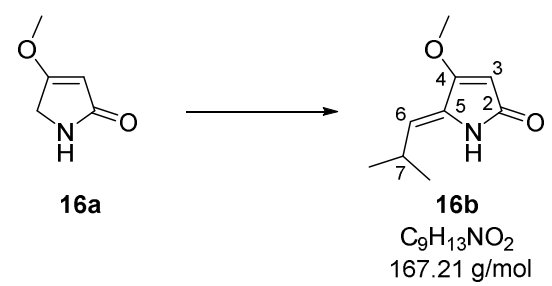

To a solution of compound 16a $(3.00 \mathrm{~g}, 26.5 \mathrm{mmol}, 1.0$ eq. $)$ in aq. $\mathrm{NaOH}(30 \mathrm{~mL}, 1 \mathrm{M})$ was added isobutyraldehyde $\left(2.42 \mathrm{~mL}, 26.5 \mathrm{mmol}, 1.0\right.$ eq.). The reaction mixture was stirred for $7 \mathrm{~h}$ at $60{ }^{\circ} \mathrm{C}$. The formed precipitate was filtered, washed with cold water, redissolved in DCM, dried over $\mathrm{Na}_{2} \mathrm{SO}_{4}$ and the solvent was removed under reduced pressure to yield $\mathbf{1 6 b}$ as a colorless solid (3.70 g, $22.1 \mathrm{mmol}$, $83 \%)$.

$\mathbf{R}_{f}=0.14(50 \% \mathrm{EtOAc} / \mathrm{CyH}) ;{ }^{1} \mathbf{H}$ NMR $\left(700 \mathrm{MHz}, \mathrm{CD}_{2} \mathrm{Cl}_{2}\right) \delta=7.35(\mathrm{~s}, 1 \mathrm{H}, \mathrm{NH}), 5.25(\mathrm{~d}, J=9.9 \mathrm{~Hz}$, 1H, H-6), 5.07 (d, $J=1.7 \mathrm{~Hz}, 1 \mathrm{H}, \mathrm{H}-3), 3.82$ (s, 3H, O-CH $\mathrm{CH}_{3}, 2.56-2.50$ (m, 1H, H-7), 1.08 (d, $J=$ $\left.6.7 \mathrm{~Hz}, 6 \mathrm{H}, 7-\left(\mathrm{CH}_{3}\right)_{2}\right) \mathrm{ppm} ;{ }^{13} \mathrm{C}$ NMR $\left(175 \mathrm{MHz}, \mathrm{CD}_{2} \mathrm{Cl}_{2}\right) \delta=171.5(\mathrm{C}-2), 167.2(\mathrm{C}-4), 131.7(\mathrm{C}-5)$, 116.9 (C-6), $93.2(\mathrm{C}-3), 58.6\left(\mathrm{OCH}_{3}\right), 27.7(\mathrm{C}-7), 23.0\left(\mathrm{CH}\left(\underline{\mathrm{CH}}_{3}\right)_{2}\right) \mathrm{ppm}$; HRMS (ESI+) $\mathrm{m} / z$ : calcd for $\mathrm{C}_{9} \mathrm{H}_{13} \mathrm{NO}_{2} \mathrm{H}^{+}[\mathrm{M}+\mathrm{H}]^{+}: 168.1019$, found: 168.1017 .

The spectroscopic data were in agreement with those previously reported. ${ }^{[2]}$

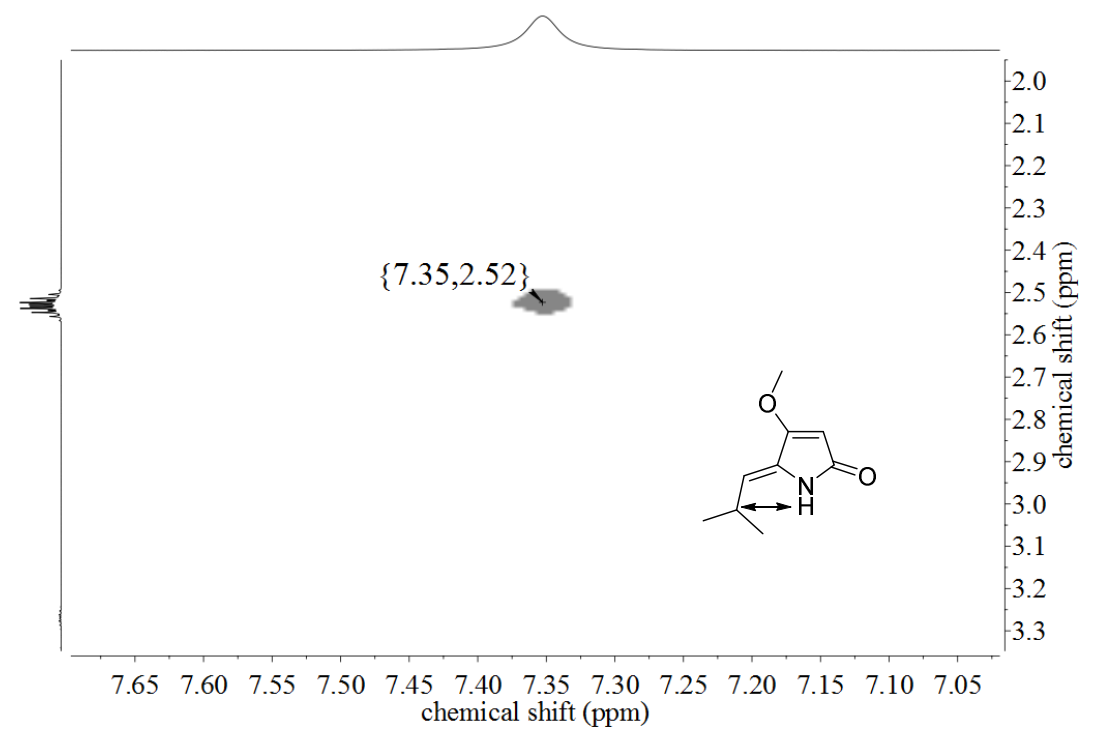

Figure 1. Selected NOESY signal of compound $\mathbf{1 6 b}$, which was used for the determination of the Z-configuration 


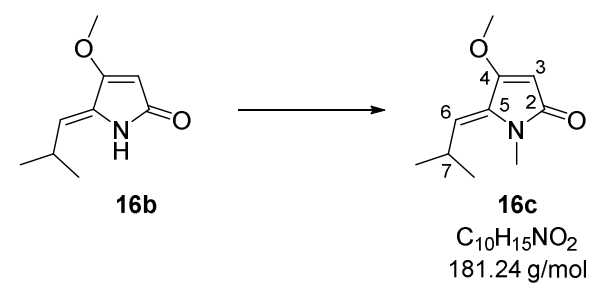

A solution of compound $\mathbf{1 6 b}\left(3.50 \mathrm{~g}, 20.9 \mathrm{mmol}, 1.0 \mathrm{eq}\right.$.) in DMF $(30 \mathrm{~mL})$ was cooled to $0{ }^{\circ} \mathrm{C}$ and $\mathrm{NaH}$ (921 mg, $23.0 \mathrm{mmol}, 1.1$ eq. $60 \%$ dispersion in mineral oil) was added. The reaction mixture was stirred $15 \mathrm{~min}$ at $\mathrm{rt}$ before cooling again to $0{ }^{\circ} \mathrm{C}$. Iodomethane $(1.43 \mathrm{~mL}, 23.0 \mathrm{mmol}, 1.1 \mathrm{eq}$.) was added and the mixture was stirred for $3 \mathrm{~h}$ at $\mathrm{rt}$. Water $(20 \mathrm{~mL})$ and aq. sat. $\mathrm{NH}_{4} \mathrm{Cl}$ solution $(10 \mathrm{~mL})$ were added. After separation of the organic phase, the aqueous phase was extracted with DCM $(3 \times 30 \mathrm{~mL})$. The combined organic layers were washed with water $(30 \mathrm{~mL})$ and brine $(30 \mathrm{~mL})$, dried over $\mathrm{Na}_{2} \mathrm{SO}_{4}$ and the solvent was removed under reduced pressure. The crude product was purified by flash chromatography $(50 \%$ EtOAc/CyH) to yield the title compound $\mathbf{1 6 c}$ as a colorless solid $(3.66 \mathrm{~g}$, $20.2 \mathrm{mmol}, 97 \%)$.

$\mathbf{R}_{f}=0.24(50 \% \mathrm{EtOAc} / \mathrm{CyH}) .{ }^{1} \mathbf{H}$ NMR $\left(500 \mathrm{MHz}, \mathrm{CD}_{2} \mathrm{Cl}_{2}\right) \delta=5.23(\mathrm{~d}, J=10.7 \mathrm{~Hz}, 1 \mathrm{H}, \mathrm{H}-6), 5.02$ (s, 1H, H-3), 3.79 (s, 3H, OCH $), 3.22$ (s, 3H, N-CH $), 3.15-2.99$ (m, 1H, H-7), 1.09 (d, J = 6.6 Hz, 6H, 7-( $\left.\left(\mathrm{CH}_{3}\right)_{2}\right)$ ppm; ${ }^{13} \mathrm{C}$ NMR $\left(125 \mathrm{MHz}, \mathrm{CD}_{2} \mathrm{Cl}_{2}\right) \delta=170.7$ (C-2), 166.8 (C-4), $132.9(\mathrm{C}-5), 117.7$ (C-

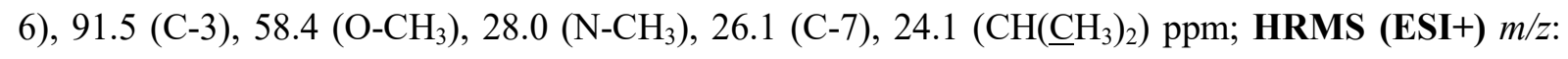
calcd for $\mathrm{C}_{10} \mathrm{H}_{15} \mathrm{NO}_{2} \mathrm{H}^{+}[\mathrm{M}+\mathrm{H}]^{+}$: 182.1176, found: 182.1175 .

\section{(Z)-1-Methyl-5-(2-methylpropylidene)pyrrolidine-2,4-dione (16) ${ }^{[2]}$}

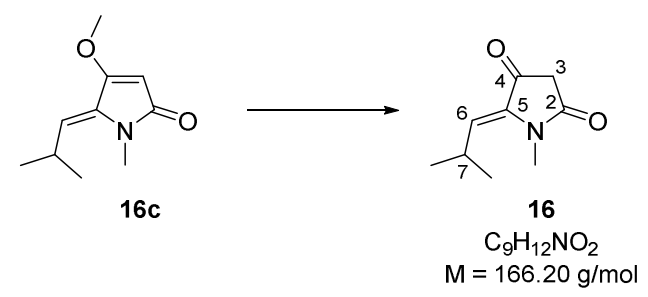

A solution of compound $\mathbf{1 6 c}(50.0 \mathrm{mg}, 276 \mu \mathrm{mol}, 1.0$ eq. $)$ in conc. $\mathrm{HCl}(1.5 \mathrm{~mL})$ was stirred at $\mathrm{rt}$ for $8 \mathrm{~h}$. The reaction mixture was cooled to $0{ }^{\circ} \mathrm{C}$ and diluted with water $(20 \mathrm{~mL})$. The aqueous layer was extracted with DCM $(3 \times 20 \mathrm{~mL})$, dried over $\mathrm{Na}_{2} \mathrm{SO}_{4}$ and the solvent was removed under reduced pressure to yield $\mathbf{1 6}$ as a yellow solid (45.8 mg, $274 \mu \mathrm{mol}, 99 \%)$. If necessary, the product can be further purified by flash chromatography $(50 \% \mathrm{EtOAc} / \mathrm{CyH})$.

$\mathbf{R}_{f}=0.32(50 \% \mathrm{EtOAc} / \mathrm{CyH}) .{ }^{1} \mathbf{H} \mathbf{N M R}\left(700 \mathrm{MHz}, \mathrm{CD}_{2} \mathrm{Cl}_{2}\right) \delta=5.01(\mathrm{~d}, J=9.9 \mathrm{~Hz}, 1 \mathrm{H}, \mathrm{H}-6), 3.67-$ 3.57 (m, 1H, H-7), 3.01 (s, 3H, N-CH $), 3.01$ (s, 2H, H-3), 1.04 (d, J=6.7 Hz, 6H, 7- $\left.\left(\mathrm{CH}_{3}\right)_{2}\right) \mathrm{ppm} ;{ }^{13} \mathbf{C}$ NMR $\left(175 \mathrm{MHz}, \mathrm{CD}_{2} \mathrm{Cl}_{2}\right.$ ) $\delta=194.9$ (C-4), 168.0 (C-2), 135.7 (C-5), 123.7 (C-6), 41.5 (C-3), 26.2 
$\left(\mathrm{NCH}_{3}\right), 25.7(\mathrm{C}-7), 23.6\left(\mathrm{CH}\left(\underline{\mathrm{CH}}_{3}\right)_{2}\right)$ ppm; HRMS (ESI+) $\mathrm{m} / z$ : calcd for $\mathrm{C}_{9} \mathrm{H}_{13} \mathrm{NO}_{2} \mathrm{H}^{+}[\mathrm{M}+\mathrm{H}]^{+}$: 168.1019, found: 168.1017 .

\section{Synthesis of the tetramic acid fragment 2}

3-((tert-Butyldimethylsilyl)oxy)propan-1-ol (43)

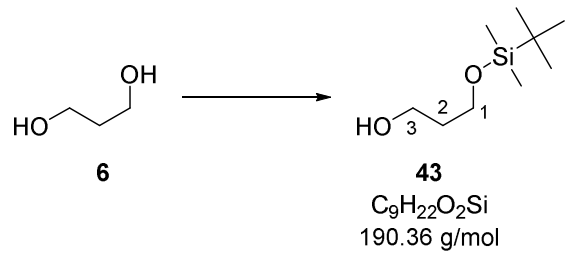

A solution of compound $6(7.21 \mathrm{~mL}, 99.5 \mathrm{mmol}, 5.0$ eq.) and $1 H$-imidazole $(1.49 \mathrm{~g}, 21.9 \mathrm{mmol}$, 1.1 eq.) in THF $(40 \mathrm{~mL})$ was cooled to $0{ }^{\circ} \mathrm{C}$. tert-Butyldimethylsilyl chloride $(3.00 \mathrm{~g}, 19.9 \mathrm{mmol}$, 1.0 eq.) was added and the reaction mixture was stirred $30 \mathrm{~min}$ at $0{ }^{\circ} \mathrm{C}$ and on at rt. sat. aq. $\mathrm{NH}_{4} \mathrm{Cl}$ solution $(20 \mathrm{~mL})$ was added and the aqueous phase was extracted with $\mathrm{Et}_{2} \mathrm{O}(3 \times 60 \mathrm{~mL})$. The combined organic layers were washed with water $(30 \mathrm{~mL})$, dried over $\mathrm{MgSO}_{4}$ and the solvent was removed under reduced pressure. The crude product was purified by flash chromatography $(30 \% \mathrm{EtOAc} / \mathrm{CyH})$ to yield the title compound $\mathbf{4 3}$ as a colorless oil (3.40 g, $17.9 \mathrm{mmol}, 90 \%)$.

$\mathbf{R}_{f}=0.34(30 \% \mathrm{EtOAc} / \mathrm{CyH}) .{ }^{1} \mathbf{H}$ NMR $\left(500 \mathrm{MHz}, \mathrm{CDCl}_{3}\right) \delta=3.83(\mathrm{t}, J=5.7 \mathrm{~Hz}, 2 \mathrm{H}, \mathrm{H}-1), 3.80(\mathrm{t}, J$ $=5.7 \mathrm{~Hz}, 2 \mathrm{H}, \mathrm{H}-3), 2.07(\mathrm{~s}, 1 \mathrm{H}, \mathrm{OH}), 1.78(\mathrm{p}, 2 \mathrm{H}, \mathrm{H}-2), 0.90(\mathrm{~s}, 9 \mathrm{H}, \mathrm{TBS}), 0.08$ (s, 6H, TBS) ppm; ${ }^{13} \mathrm{C}$ NMR $\left(125 \mathrm{MHz}, \mathrm{CDCl}_{3}\right.$ ) $\delta=63.1$ (C-1), 62.6 (C-3), 34.3 (C-2), 26.0 (TBS), 18.3 (TBS), -5.4 (TBS) ppm; MS (EI) $m / z$ : calcd for $\mathrm{C}_{5} \mathrm{H}_{13} \mathrm{O}_{2} \mathrm{Si}^{+\bullet}\left[\mathrm{M}-{ }^{t} \mathrm{Bu}\right]^{+\bullet}:$ 133.0, found: 133.0. The spectroscopic data were in agreement with those previously reported. ${ }^{[3]}$

\section{3-((tert-Butyldimethylsilyl)oxy)propanal (7)}

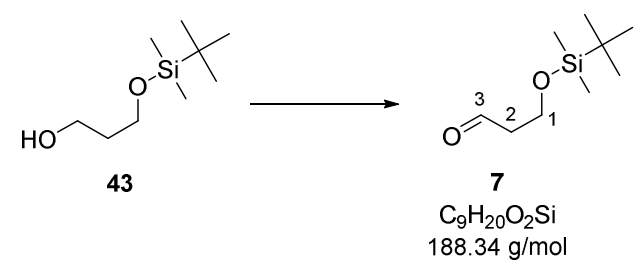

DCM (45 mL) was added to sulfur trioxide pyridine complex $(8.78 \mathrm{~g}, 55.2 \mathrm{mmol}, 3.0$ eq.) followed by the addition of $N, N$-diisopropylethylamine $(12.5 \mathrm{~mL}, 73.6 \mathrm{mmol}, 4.0$ eq.) and DMSO (13.1 mL, $164 \mathrm{mmol}, 10.0$ eq.). The reaction mixture was cooled to $0{ }^{\circ} \mathrm{C}$ and alcohol 43 (3.50 g, $18.4 \mathrm{mmol}$, 1.0 eq.) was added. After stirring at $\mathrm{rt}$ for $1.5 \mathrm{~h}$, sat. aq. $\mathrm{NaHCO}_{3}$ solution $(150 \mathrm{~mL})$ was added and the aqueous phase was extracted with DCM $(3 \times 150 \mathrm{~mL})$. The combined organic layers were washed with $\mathrm{CuSO}_{4}, \mathrm{NH}_{4} \mathrm{Cl}$ and brine solution (each aq. sat. $2 \times 150 \mathrm{~mL}$ ). The organic phase was dried over $\mathrm{MgSO}_{4}$ and the solvent was removed under reduced pressure to yield the title compound 7 as a colorless oil (3.40 g, $18.0 \mathrm{mmol}, 98 \%$ ), which was used without further purification. 
$\mathbf{R}_{f}=0.41(20 \% \mathrm{EtOAc} / \mathrm{CyH}) .{ }^{1} \mathbf{H} \mathbf{N M R}\left(500 \mathrm{MHz}, \mathrm{CDCl}_{3}\right) \delta=9.80(\mathrm{t}, J=2.1 \mathrm{~Hz}, 1 \mathrm{H}, \mathrm{H}-3), 3.98(\mathrm{t}, J$ $=6.0 \mathrm{~Hz}, 2 \mathrm{H}, \mathrm{H}-1), 2.59$ (td, $J=6.0,2.1 \mathrm{~Hz}, 2 \mathrm{H}, \mathrm{H}-2), 0.88$ (s, 9H, TBS), 0.06 (s, 6H, TBS) ppm; ${ }^{13}$ C NMR (125 MHz, $\mathrm{CDCl}_{3}$ ) $\delta=202.2$ (C-3), 57.6 (C-1), 46.7 (C-2), 26.0 (TBS), 18.4 (TBS), -5.3 (TBS) ppm; MS (ESI+) $\mathrm{m} / \mathrm{z}$ : calcd for $\mathrm{C}_{9} \mathrm{H}_{20} \mathrm{O}_{2} \mathrm{SiH}^{+}[\mathrm{M}+\mathrm{H}]^{+}: 189.131$, found: 189.130. The spectroscopic data were in agreement with those previously reported. ${ }^{[4]}$

Ethyl (E)-5-((tert-butyldimethylsilyl)oxy)-2-methylpent-2-enoate (44E) and ethyl (Z)-5-((tertbutyldimethylsilyl)oxy)-2-methylpent-2-enoate (44Z)

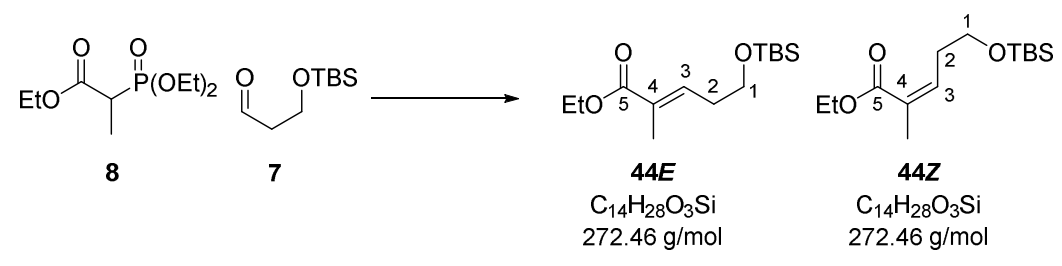

$\mathrm{Ba}(\mathrm{OH})_{2}\left(1.37 \mathrm{~g}, 7.99 \mathrm{mmol}, 3.5 \mathrm{eq}\right.$.) was dried for $5 \mathrm{~h}$ at $120^{\circ} \mathrm{C}$ using high vacuum. After cooling to rt, THF (14 mL) was added followed by phosphonate $8(686 \mu \mathrm{L}, 3.20 \mathrm{mmol}, 1.4$ eq. $)$. The suspension was stirred for $30 \mathrm{~min}$ at $\mathrm{rt}$. Freshly prepared aldehyde $7(430 \mathrm{mg}, 2.28 \mathrm{mmol}, 1.0$ eq. in 40:1 THF/water $(7 \mathrm{~mL})$ ) was added and the reaction was stirred overnight at $\mathrm{rt}$. The suspension was filtered over Celite ${ }^{\circledR}$ and washed with $\mathrm{Et}_{2} \mathrm{O}$. The solvent was removed under reduced pressure and the crude product was purified by flash chromatography $\left(3 \% \mathrm{Et}_{2} \mathrm{O} / \mathrm{CyH}\right)$ to yield the title compounds $\mathbf{4 4 E}$ (472 mg, $1.73 \mathrm{mmol}, 76 \%, \underline{E} / Z=16: 1)$ and $\mathbf{4 4 Z}(29.5 \mathrm{mg}, 108 \mu \mathrm{mol}, 5 \%, E / \underline{Z}=16: 1)$ as colorless oils.

Analytical data for $44 E$ :

$\mathbf{R}_{f}=0.22\left(5 \% \mathrm{Et}_{2} \mathrm{O} / \mathrm{CyH}\right) .{ }^{1} \mathbf{H}$ NMR $\left(700 \mathrm{MHz}, \mathrm{CDCl}_{3}\right) \delta=6.78(\mathrm{tq}, J=6.8,1.3 \mathrm{~Hz}, 1 \mathrm{H}, \mathrm{H}-3), 4.19$ (q, $J=7.1 \mathrm{~Hz}, 2 \mathrm{H}, \mathrm{OEt}), 3.70(\mathrm{t}, J=6.7 \mathrm{~Hz}, 2 \mathrm{H}, \mathrm{H}-1), 2.40$ (dt, $J=7.2,6.8 \mathrm{~Hz}, 2 \mathrm{H}, \mathrm{H}-2), 1.85(\mathrm{~s}, 3 \mathrm{H}$, 4- $\mathrm{CH}_{3}$ ), 1.29 (t, $\left.J=7.1 \mathrm{~Hz}, 3 \mathrm{H}, \mathrm{OEt}\right), 0.89$ (s, 9H, TBS), 0.05 (s, 6H, TBS) ppm; ${ }^{13} \mathbf{C}$ NMR $\left(175 \mathrm{MHz}, \mathrm{CDCl}_{3}\right) \delta=168.2(\mathrm{C}-5), 138.6$ (C-3), 129.4 (C-4), 61.9 (C-1), 60.6 (OEt), 32.5 (C-2), 26.0 (TBS), 18.5 (TBS), 14.4 (OEt), $12.7\left(4-\mathrm{CH}_{3}\right),-5.2$ (TBS) ppm; HRMS (ESI+) $m / z$ : calcd for $\mathrm{C}_{14} \mathrm{H}_{28} \mathrm{O}_{3} \mathrm{SiH}^{+}[\mathrm{M}+\mathrm{H}]^{+}: 273.1880$, found: 273.1880 . The spectroscopic data were in agreement with those previously reported. ${ }^{[5]}$ 


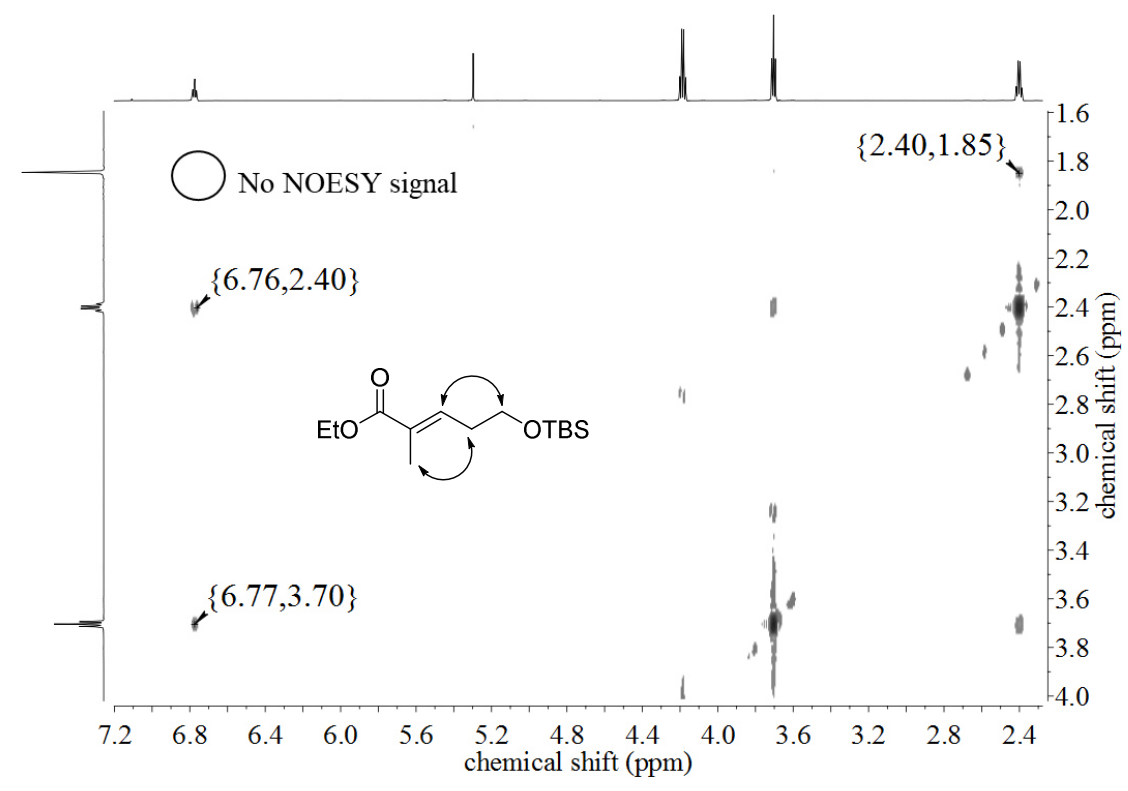

Figure 2. Selected NOESY signals of compound $\mathbf{4 4 E}$, which were used for the determination of the $E$-configuration. No NOESY signal could be detected between $\mathrm{H}-3$ and $4-\mathrm{CH}_{3}$.

Analytical data for $44 Z$ :

$\mathbf{R}_{f}=0.27\left(5 \% \mathrm{Et}_{2} \mathrm{O} / \mathrm{CyH}\right) .{ }^{1} \mathbf{H}$ NMR $\left(500 \mathrm{MHz}, \mathrm{CDCl}_{3}\right) \delta=6.01(\mathrm{tq}, J=7.3,1.6 \mathrm{~Hz}, 1 \mathrm{H}, \mathrm{H}-3), 4.19$ (q, $J=7.1 \mathrm{~Hz}, 2 \mathrm{H}, \mathrm{OEt}), 3.68(\mathrm{t}, J=6.5 \mathrm{~Hz}, 2 \mathrm{H}, \mathrm{H}-1), 2.71-2.64(\mathrm{~m}, 2 \mathrm{H}, \mathrm{H}-2), 1.91$ (q, $J=1.6 \mathrm{~Hz}$, $\left.3 \mathrm{H}, 4-\mathrm{CH}_{3}\right), 1.30$ (t, $J=7.1 \mathrm{~Hz}, 3 \mathrm{H}, \mathrm{OEt}$ ), 0.89 (s, 9H, TBS), 0.05 (s, 6H, TBS) ppm; ${ }^{13} \mathbf{C}$ NMR $\left(125 \mathrm{MHz}, \mathrm{CDCl}_{3}\right) \delta=168.2(\mathrm{C}-5), 139.4$ (C-3), 128.7 (C-4), 62.7 (C-1), 60.2 (OEt), 33.3 (C-2), 26.1 (TBS), $20.8\left(4-\mathrm{CH}_{3}\right), 18.5$ (TBS), 14.4 (OEt), -5.1 (TBS) ppm; HRMS (ESI+) m/z: calcd for $\mathrm{C}_{14} \mathrm{H}_{28} \mathrm{O}_{3} \mathrm{SiH}^{+}[\mathrm{M}+\mathrm{H}]^{+}: 273.1880$, found: 273.1883 . The spectroscopic data were in agreement with those previously reported. ${ }^{5}$

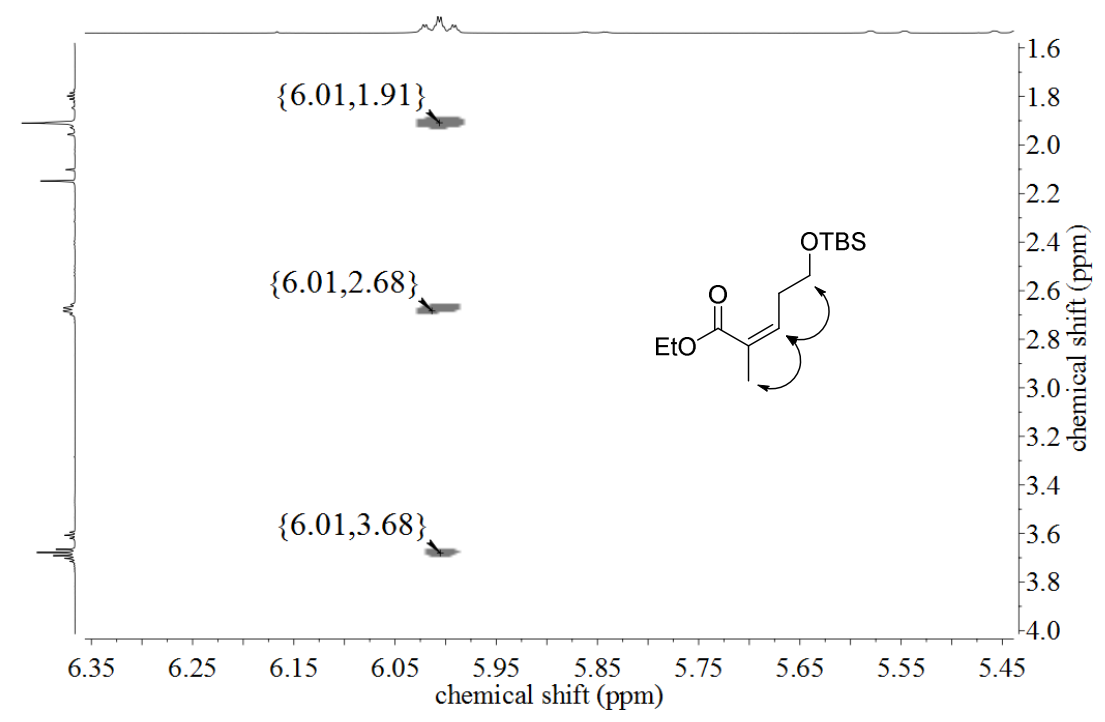

Figure 3. Selected NOESY signals of compound $44 Z$, which were used for the determination of the $Z$-configuration. 


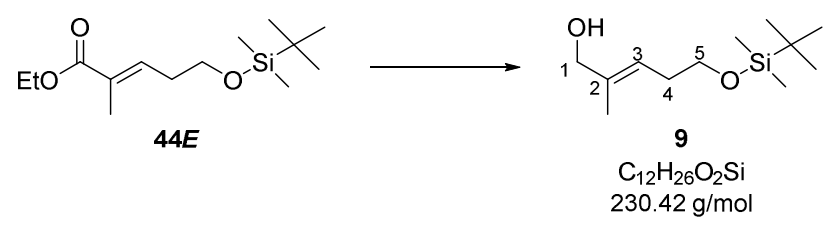

Ester $44 \boldsymbol{E}(1.00 \mathrm{~g}, 3.67 \mathrm{mmol}, 1.00$ eq.) was dissolved in THF $(6 \mathrm{~mL})$, the solution was cooled to $-78^{\circ} \mathrm{C}$ and DIBAL-H $(8.07 \mathrm{~mL}, 8.07 \mathrm{mmol}, 2.20$ eq. $1 \mathrm{M}$ in $\mathrm{CyH})$ was added slowly. After stirring $1 \mathrm{~h}$ at $-78^{\circ} \mathrm{C}$, methanol $(1.5 \mathrm{~mL})$ and sat. aq. Rochelle salt solution $(5.8 \mathrm{~mL})$ were added. The mixture was allowed to warm to $\mathrm{rt}$ and stirred until clearly two phases appeared $(2 \mathrm{~h})$. The phases were separated, the aqueous layer was extracted with $\mathrm{Et}_{2} \mathrm{O}(3 \times 30 \mathrm{~mL})$, the combined organic phases were dried over $\mathrm{MgSO}_{4}$ and the solvent was removed under reduced pressure. Purification of the crude product was achieved by flash chromatography $(30 \% \mathrm{EtOAc} / \mathrm{CyH})$ to yield the title compound 9 as a colorless oil (787 mg, $3.42 \mathrm{mmol}$, 93\%).

$\mathbf{R}_{f}=0.25(20 \% \mathrm{EtOAc} / \mathrm{CyH}) .{ }^{1} \mathbf{H}$ NMR $\left(700 \mathrm{MHz}, \mathrm{CDCl}_{3}\right) \delta=5.42(\mathrm{tq}, J=7.1,1.3 \mathrm{~Hz}, 1 \mathrm{H}, \mathrm{H}-3)$, 4.01 (s, 2H, H-1), 3.62 (t, $J=7.0 \mathrm{~Hz}, 2 \mathrm{H}, \mathrm{H}-5), 2.29$ (dt, $J=7.1,7.0 \mathrm{~Hz}, 2 \mathrm{H}, \mathrm{H}-4), 1.68$ (s, 3H, 2$\mathrm{CH}_{3}$ ), 1.30 (brs, $\left.1 \mathrm{H}, \mathrm{OH}\right), 0.89$ (s, 9H, TBS), 0.05 (s, 6H, TBS) ppm; ${ }^{13} \mathbf{C} \mathbf{N M R}\left(175 \mathrm{MHz}, \mathrm{CDCl}_{3}\right) \delta=$ 136.8 (C-2), 122.3 (C-3), 69.0 (C-1), 62.9 (C-5), 31.6 (C-4), 26.1 (TBS), 18.5 (TBS), 13.9 (2- $\mathrm{CH}_{3}$ ), 5.1 (TBS) ppm; HRMS (ESI+) $m / z$ : calcd for $\mathrm{C}_{12} \mathrm{H}_{26} \mathrm{O}_{2} \mathrm{SiH}^{+}[\mathrm{M}+\mathrm{H}]^{+}: 231.1775$, found: 231.1772. The spectroscopic data were in agreement with those previously reported. ${ }^{[6]}$

\section{(E)-tert-Butyl((5-((4-methoxybenzyl)oxy)-4-methylpent-3-en-1-yl)oxy)dimethylsilane (45)}

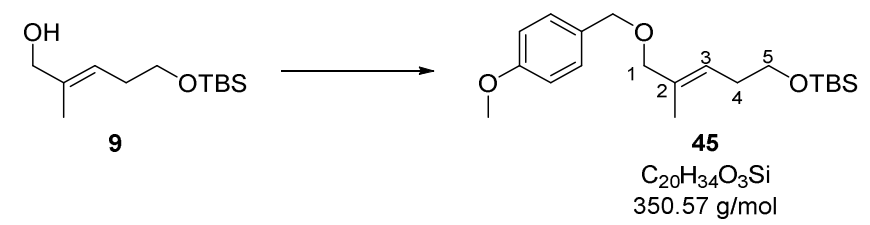

Alcohol 9 (600 mg, $2.60 \mathrm{mmol}, 1.00$ eq.) was dissolved in DMF $(3 \mathrm{~mL})$, cooled to $0{ }^{\circ} \mathrm{C}$ and 4-methoxybenzyl chloride ( $388 \mu \mathrm{L}, 2.86 \mathrm{mmol}, 1.10$ eq.) was added. $\mathrm{NaH}$ (115 mg, $2.86 \mathrm{mmol}$, 1.10 eq. $60 \%$ dispersion in mineral oil) was added over the period of $20 \mathrm{~min}$ and the reaction mixture was stirred for $3 \mathrm{~h}$ at $0{ }^{\circ} \mathrm{C}$ followed by the addition of sat. aq. $\mathrm{NH}_{4} \mathrm{Cl}$ solution $(5 \mathrm{~mL}), \mathrm{DCM}(15 \mathrm{~mL})$ and water $(10 \mathrm{~mL})$. The phases were separated, the aqueous phase extracted with DCM $(3 \times 15 \mathrm{~mL})$, the combined organic phases were dried over $\mathrm{MgSO}_{4}$ and the solvent was removed under reduced pressure. Purification of the crude product was achieved by flash chromatography ( $5 \% \mathrm{EtOAc} / \mathrm{CyH})$ to yield the title compound $\mathbf{4 5}$ as a colorless oil (792 mg, $2.26 \mathrm{mmol}, 87 \%$ ).

$\mathbf{R}_{f}=0.18\left(5 \%\right.$ EtOAc/CyH). ${ }^{1} \mathbf{H}$ NMR $\left(700 \mathrm{MHz}, \mathrm{CDCl}_{3}\right) \delta=7.26\left(\mathrm{~d}, J=8.7 \mathrm{~Hz}, 2 \mathrm{H}, \mathrm{H}_{\text {arom }}\right), 6.87(\mathrm{~d}$, $J=8.7 \mathrm{~Hz}, 2 \mathrm{H}, \mathrm{H}_{\text {arom. }}$ ), 5.43 (tq, $\left.J=7.1,1.3 \mathrm{~Hz}, 1 \mathrm{H}, \mathrm{H}-3\right), 4.38$ (s, 2H, $\left.\operatorname{Ar}-\mathrm{CH}_{2}\right), 3.88$ (s, 2H, H-1), 3.80 (s, 3H, O-CH ${ }_{3}$ ), 3.62 (t, $\left.J=7.0 \mathrm{~Hz}, 2 \mathrm{H}, \mathrm{H}-5\right), 2.29$ (dt, $\left.J=7.1,7.0 \mathrm{~Hz}, 2 \mathrm{H}, \mathrm{H}-4\right), 1.68$ (s, 3H, 2- 
$\left.\mathrm{CH}_{3}\right), 0.89$ (s, 9H, TBS), 0.05 (s, 6H, TBS) ppm; ${ }^{13} \mathbf{C ~ N M R}\left(175 \mathrm{MHz}, \mathrm{CDCl}_{3}\right) \delta=159.2\left(\mathrm{C}_{\text {arom. }} \mathrm{O}\right)$, $134.2(\mathrm{C}-2), 130.9\left(\mathrm{C}_{\text {arom. }}\right), 129.5\left(\mathrm{C}_{\text {arom. }} \mathrm{H}\right), 124.4(\mathrm{C}-3), 113.9\left(\mathrm{C}_{\text {arom. }} \mathrm{H}\right), 76.0(\mathrm{C}-1), 71.3\left(\mathrm{Ar}_{-} \mathrm{CH}_{2}\right)$, 62.9 (C-5), $55.4\left(\mathrm{O}-\mathrm{CH}_{3}\right), 31.7$ (C-4), 26.1 (TBS), 18.5 (TBS), $14.2\left(2-\mathrm{CH}_{3}\right),-5.1$ (TBS) ppm; HRMS (ESI+) $m / z$ : calcd for $\mathrm{C}_{20} \mathrm{H}_{34} \mathrm{O}_{3} \mathrm{SiH}^{+}[\mathrm{M}+\mathrm{H}]^{+}: 351.2350$, found: 351.2348 .

\section{(E)-5-((4-Methoxybenzyl)oxy)-4-methylpent-3-en-1-ol (46)}

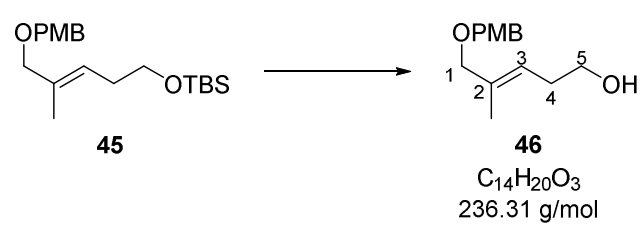

Compound 45 (630 mg, $1.80 \mathrm{mmol}, 1.00$ eq.) was dissolved in methanol $(20 \mathrm{~mL})$, cooled to $0{ }^{\circ} \mathrm{C}$ and $1 \mathrm{M}$ aq. $\mathrm{HCl}\left(3.05 \mathrm{~mL}, 3.05 \mathrm{mmol}, 1.70\right.$ eq.) was added. The reaction mixture was stirred $1 \mathrm{~h}$ at $0{ }^{\circ} \mathrm{C}$ followed by the addition of DCM $(10 \mathrm{~mL})$ and water $(10 \mathrm{~mL})$. The organic layer was separated, the aqueous layer was extracted with DCM $(3 \times 15 \mathrm{~mL})$, the combined organic phases were dried over $\mathrm{MgSO}_{4}$ and the solvent was removed under reduced pressure. Purification of the crude product was achieved by flash chromatography $(40 \% \mathrm{EtOAc} / \mathrm{CyH})$ to yield the title compound $\mathbf{4 6}$ as a colorless oil (383 mg, $1.62 \mathrm{mmol}, 90 \%$ ).

$\mathbf{R}_{f}=0.18(40 \% \mathrm{EtOAc} / \mathrm{CyH}) .{ }^{1} \mathbf{H}$ NMR $\left(700 \mathrm{MHz}, \mathrm{CDCl}_{3}\right) \delta=7.26\left(\mathrm{~d}, J=8.7 \mathrm{~Hz}, 2 \mathrm{H}, \mathrm{H}_{\text {arom. }}\right), 6.88$ (d, $J=8.7 \mathrm{~Hz}, 2 \mathrm{H}, \mathrm{H}_{\text {arom }}$ ), 5.45 (tq, $\left.J=7.1,1.3 \mathrm{~Hz}, 1 \mathrm{H}, \mathrm{H}-3\right), 4.40$ (s, $\left.2 \mathrm{H}, \mathrm{Ar}_{-} \mathrm{CH}_{2}\right), 3.89$ (s, 2H, H-1), 3.80 (s, 3H, O-CH $\left.{ }_{3}\right), 3.66$ (t, $\left.J=6.5 \mathrm{~Hz}, 2 \mathrm{H}, \mathrm{H}-5\right), 2.34$ (dt, $\left.J=7.1,6.5 \mathrm{~Hz}, 2 \mathrm{H}, \mathrm{H}-4\right), 1.70$ (s, 3H, 2$\left.\mathrm{CH}_{3}\right), 1.53$ (brs, $\left.1 \mathrm{H}, \mathrm{OH}\right) \mathrm{ppm} ;{ }^{13} \mathbf{C}$ NMR $\left(175 \mathrm{MHz}, \mathrm{CDCl}_{3}\right) \delta=159.3\left(\mathrm{C}_{\text {arom. }} \mathrm{O}\right), 135.6(\mathrm{C}-2), 130.7$ $\left(\mathrm{C}_{\text {arom. }}\right), 129.5\left(\mathrm{C}_{\text {arom. }} \mathrm{H}\right), 123.6(\mathrm{C}-3), 113.9\left(\mathrm{C}_{\text {arom. }} \mathrm{H}\right), 75.8(\mathrm{C}-1), 71.6\left(\mathrm{Ar}_{-} \mathrm{CH}_{2}\right), 62.4(\mathrm{C}-5), 55.4(\mathrm{O}-$ $\left.\mathrm{CH}_{3}\right), 31.4(\mathrm{C}-4), 14.3\left(2-\mathrm{CH}_{3}\right)$ ppm; HRMS (ESI+) $m / z$ : calcd for $\mathrm{C}_{14} \mathrm{H}_{20} \mathrm{O}_{3} \mathrm{H}^{+}[\mathrm{M}+\mathrm{H}]^{+}: 237.1485$, found: 237.1484 . The spectroscopic data were in agreement with those previously reported. ${ }^{[7]}$

\section{(E)-5-((4-Methoxybenzyl)oxy)-4-methylpent-3-enal (10)}

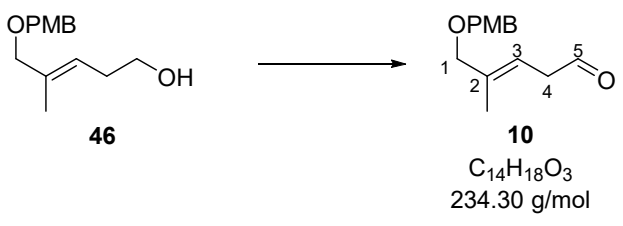

\section{Parikh-Doering oxidation:}

DCM (1.5 mL) was added to sulfur trioxide pyridine complex (303 mg, $1.90 \mathrm{mmol}, 3.00$ eq.) followed by the addition of $N, N$-diisopropylethylamine $(432 \mu \mathrm{L}, 2.54 \mathrm{mmol}, 4.00$ eq.) and DMSO (451 $\mu \mathrm{L}$, $6.35 \mathrm{mmol}, 10.0$ eq.). The reaction mixture was cooled to $0{ }^{\circ} \mathrm{C}$ and alcohol $46(150 \mathrm{mg}, 635 \mu \mathrm{mol}$, 1.00 eq.) was added. After stirring at $\mathrm{rt}$ for $1.5 \mathrm{~h}$, sat. aq. $\mathrm{NaHCO}_{3}$ solution $(4 \mathrm{~mL})$ was added and the aqueous phase was extracted with DCM $(3 \times 10 \mathrm{~mL})$. The combined organic layers were washed with 
$\mathrm{CuSO}_{4}, \mathrm{NH}_{4} \mathrm{Cl}$ and brine solution (each aq. sat. $2 \times 10 \mathrm{~mL}$ ). The organic phase was dried over $\mathrm{MgSO}_{4}$ and the solvent was removed under reduced pressure. Purification of the crude product was achieved by flash chromatography $(20 \% \mathrm{EtOAc} / \mathrm{CyH})$ yielding the title compound $\mathbf{1 0}$ as a yellowish oil (47.3 mg, $202 \mu \mathrm{mol}, 32 \%)$.

\section{IBX oxidation:}

Alcohol 46 (138 mg, $584 \mu \mathrm{mol}, 1.00$ eq.) was dissolved in DMSO (1 mL) and 2-iodoxybenzoic acid (IBX, $245 \mathrm{mg}, 876 \mu \mathrm{mol}, 1.50$ eq.) was added. After stirring $1 \mathrm{~h}$ at rt, DCM (5 mL) and water $(5 \mathrm{~mL})$ were added and the mixture was filtered over Celite ${ }^{\circledR}$. The organic layer was separated, the aqueous layer was extracted with DCM $(3 \times 10 \mathrm{~mL})$, the combined organic phases were dried over $\mathrm{MgSO}_{4}$ and the solvent was removed under reduced pressure. Purification of the crude product was achieved by flash chromatography (35\% EtOAc/CyH) giving back start material 46 (48.8 mg, $207 \mu \mathrm{mol}, 35 \%)$ and to yield the title compound $\mathbf{1 0}$ as a yellowish oil (137 mg, $340 \mu \mathrm{mol}, 58 \%, 90 \% \mathrm{brsm}$.).

\section{TEMPO/BAIB oxidation:}

Alcohol 46 (147 mg, $622 \mu \mathrm{mol}, 1.0$ eq.) was dissolved in DCM (1.0 mL) and TEMPO (11.0 mg, $70.0 \mu \mathrm{mol}, 0.1$ eq.) and BAIB (232 mg, $720 \mu \mathrm{mol}, 1.2$ eq.) were added in one portion. The reaction mixture was stirred for $45 \mathrm{~min}$ at rt. Afterwards, DCM (2 mL) and $\mathrm{Na}_{2} \mathrm{~S}_{2} \mathrm{O}_{3}$-solution (aq., sat., $2 \mathrm{~mL}$ ) were added and the different phases were separated. The aqueous layer was extracted with DCM ( $3 \mathrm{x}$ $20 \mathrm{~mL}$ ). The organic phases were combined and washed with $\mathrm{NaHCO}_{3}$-solution (aq., sat., $50 \mathrm{~mL}$ ) and brine $(50 \mathrm{~mL})$, dried over $\mathrm{MgSO} 4$ and the solvent was removed under reduced pressure. The crude product was purified by column chromatography (cyclohexane/ethyl acetate 6:4) and product $\mathbf{1 0}$ was obtained as yellow oil (109 mg, $465 \mu \mathrm{mol}, 75 \%)$.

$\mathbf{R}_{\boldsymbol{f}}=0.16(20 \% \mathrm{EtOAc} / \mathrm{CyH}) .{ }^{1} \mathbf{H}$ NMR $\left(300 \mathrm{MHz}, \mathrm{CDCl}_{3}\right) \delta=9.68(\mathrm{t}, J=1.9 \mathrm{~Hz}, 1 \mathrm{H}, \mathrm{H}-5), 7.27$ (d, $\left.J=8.7 \mathrm{~Hz}, 2 \mathrm{H}, \mathrm{H}_{\text {arom. }}\right), 6.88$ (d, $J=8.7 \mathrm{~Hz}, 2 \mathrm{H}, \mathrm{H}_{\text {arom. }}$ ), 5.65 (tq, $\left.J=7.2,1.4 \mathrm{~Hz}, 1 \mathrm{H}, \mathrm{H}-3\right), 4.42$ (s, 2H, $\mathrm{Ar}-\mathrm{CH}_{2}$ ), 3.93 (s, 2H, H-1), 3.81 (s, 3H, O-CH 3 ), 3.23 - 3.19 (m, 2H, H-4), 1.69 (s, 3H, 2- $\mathrm{CH}_{3}$ ) ppm;

${ }^{13} \mathbf{C}$ NMR $\left(75 \mathrm{MHz}, \mathrm{CDCl}_{3}\right) \delta=199.6(\mathrm{C}-5), 159.4\left(\mathrm{C}_{\text {arom. }} \mathrm{O}\right), 138.1(\mathrm{C}-2), 130.5\left(\mathrm{C}_{\text {arom. }}\right), 129.5$ $\left(\mathrm{C}_{\text {arom. }} \mathrm{H}\right), 116.2(\mathrm{C}-3), 113.9\left(\mathrm{C}_{\text {arom. }} \mathrm{H}\right), 75.2(\mathrm{C}-1), 71.7\left(\mathrm{Ar}-\mathrm{CH}_{2}\right), 55.4\left(\mathrm{O}^{-} \mathrm{CH}_{3}\right), 43.1(\mathrm{C}-4), 14.5$ (2$\mathrm{CH}_{3}$ ) ppm; HRMS (ESI+) $\mathrm{m} / z$ : calcd for $\mathrm{C}_{14} \mathrm{H}_{18} \mathrm{O}_{3} \mathrm{H}^{+}[\mathrm{M}+\mathrm{H}]^{+}: 235.1329$, found: 235.1329 . 


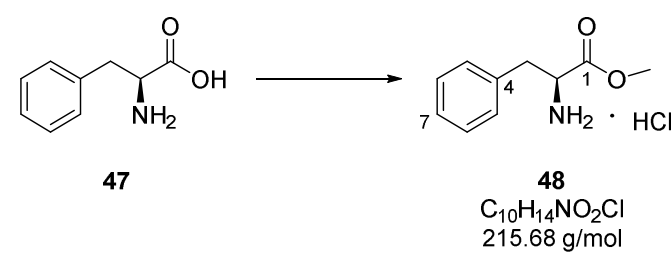

$\mathrm{MeOH}(70 \mathrm{~mL})$ was cooled down to $-5^{\circ} \mathrm{C}$. Thionyl chloride $(14.0 \mathrm{~mL}, 182 \mathrm{mmol}, 3.0$ eq.) was added dropwise within $10 \mathrm{~min}$. L-Phenylalanine $(47,10.0 \mathrm{~g}, 60.6 \mathrm{mmol})$ was added. The mixture was stirred for $20 \mathrm{~h}$ at room temperature and afterwards concentrated in vacuo. The precipitate was dissolved in $\mathrm{MeOH}(50 \mathrm{~mL})$. The solvent was removed and the procedure was repeated once to remove the excess of $\mathrm{SOCl}_{2}$. The solid was dried to yield the hydrochloride 48 (13.1 g, $60.6 \mathrm{mmol}$, quant.) as a colorless solid.

$\mathbf{R}_{f}=0.35$ (DCM/MeOH 10:1); ${ }^{1} \mathbf{H}$ NMR (500 MHz, MeOD): $\delta=7.41-7.38$ (m, 2H, H-6, H-8), 7.36 -7.33 (m, 1H, H-7), $7.29-7.27$ (m, 2H, H-5, H-9), 4.34 (t, J=6.8 Hz, 1H, H-2), 3.82 (s, 3H, - $\mathrm{OCH}_{3}$ ), $3.31-3.18$ (m, 2H, H-3) ppm; ${ }^{13} \mathbf{C}$ NMR (126 MHz, MeOD): $\delta=170.5$ (C-1), 135.3 (C-4), 130.4 (C-5, C-9), 130.2 (C-6, C-8), 129.0 (C-7), 55.2 (C-2), $53.6\left(\mathrm{OCH}_{3}\right), 37.4$ (C-3) ppm; HRMS (ESI+) $m / z:[\mathrm{M}+\mathrm{H}]^{+}$Calcd for $\mathrm{C}_{10} \mathrm{H}_{14} \mathrm{NO}_{2}^{+}$180.1019, found 180.1017. The spectroscopic data were consistent with those previously reported. ${ }^{[8]}$

(S)-3-Benzyl-1-methyl-1,4-diazaspiro[4.4]nonan-2-one $(49)^{[9]}$

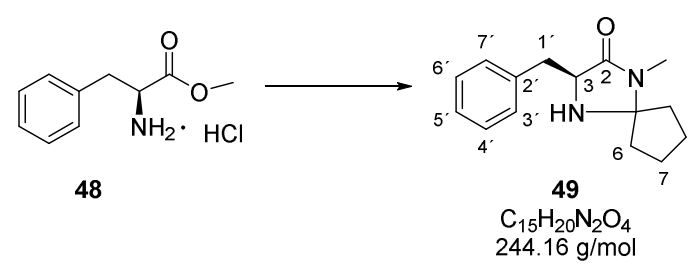

To a solution of ethanolic $\mathrm{MeNH}_{2}$ (33\%weight, $38 \mathrm{~mL}, 400 \mathrm{mmol}, 6.6$ eq.) and $\mathrm{EtOH}(20 \mathrm{~mL})$ was added $(S)$-phenylalanine methyl ester hydrochloride $48(13.1 \mathrm{~g}, 60.6 \mathrm{mmol})$ and the resulting solution was stirred at room temperature for $20 \mathrm{~h}$. The solvent was removed in vacuo. The residue was suspended in $\mathrm{Et}_{2} \mathrm{O}$ and then concentrated again. This procedure was repeated two times until $(S)$ phenylalanine- $N$-methyl amide hydrochloride was obtained as a white solid. The solid was treated with aqueous saturated $\mathrm{NaHCO}_{3}$ solution $(50 \mathrm{~mL})$ and the aqueous phase was extracted with $\mathrm{CHCl}_{3}(3$ x $50 \mathrm{~mL}$ ). The combined organic phases were dried over $\mathrm{MgSO}_{4}$ and the solvent was removed under reduced pressure. The residue was dissolved in $\mathrm{MeOH}(120 \mathrm{~mL})$ and cyclopentanone $(26.8 \mathrm{~mL}$, $303 \mathrm{mmol}, 5.0$ eq.) and $p$ TSA (116 mg, $0.61 \mathrm{mmol}, 0.01$ eq.) were added and the reaction mixture was heated to reflux for $18 \mathrm{~h}$. The mixture was cooled down to room temperature and concentrated in 
vacuo. The residue was taken up in $\mathrm{Et}_{2} \mathrm{O}$ and a solution of $\mathrm{HCl}$ in dioxane $(4.0 \mathrm{M}, 12.0 \mathrm{~mL})$ was added to precipitate the catalyst. The precipitate was recrystallized by solving it in a minimum of hot isopropanol. Then, $n$-hexane was added until the cloudiness persisted and then, isopropanol was added until the solution became homogeneous again. After cooling to room temperature, the formed precipitate was filtered off and dried to yield the hydrochloride $(9.2 \mathrm{~g}, 32.9 \mathrm{mmol}, 54 \%)$ as a colorless solid. It was stored at $-20^{\circ} \mathrm{C}$. For the organo catalyst reaction, the catalyst was treated with aq. sat. $\mathrm{NaHCO}_{3}$ solution until no further gas evolution was observed. The aqueous phase was extracted with $\mathrm{CHCl}_{3}(3 \times 50 \mathrm{~mL})$ and the solvent was removed in vacuo to yield catalyst 49 ( $8.0 \mathrm{~g}, 32.9 \mathrm{mmol}$, quant.) as colorless oil.

$\mathbf{R}_{f}=0.11$ (1:1 cyclohexane/ethyl acetate); $[\alpha]_{\mathbf{D}}{ }^{\mathbf{2 0}}=-70.3\left(c 1.32, \mathrm{CHCl}_{3}\right) ;{ }^{1} \mathbf{H}$ NMR $\left(500 \mathrm{MHz}, \mathrm{d}_{6}\right.$ DMSO): $\delta=10.70\left(\mathrm{~s}_{\mathrm{br}}, 1 \mathrm{H}, \mathrm{NH}\right), 7.46-7.44$ (m, 2H, H-3', H-7'), $7.37-7.34$ (m, J=7.3 Hz, 2H, H-4', H-6'), 7.28 (t, $J=7.3 \mathrm{~Hz}, 1 \mathrm{H}, \mathrm{H}-5^{\prime}$ ), 4.49 (s $\mathrm{s}_{\mathrm{b}}, 1 \mathrm{H}, \mathrm{H}-3$ ), 3.25 (d, J=3.7 Hz, 2H, H-1'), 2.80 (s, $3 \mathrm{H}, \mathrm{N}-\mathrm{CH}_{3}$ ), $2.28-2.09$ (m, 3H, H-6, H-9), 1.83 (dt, $J=14.3,8.4$ Hz, 1H, H-6, H-9), 1.76 - 1.69 (m, 4H, H-7, H-8) ppm; ${ }^{13}$ C NMR (125 MHz, d $\mathrm{d}_{6}$-DMSO): $\delta=166.7$ (C-2), 136.3 (C $\mathrm{C}_{\mathrm{q}}$-benzyl), 129.4 (C3', C-6'), 128.4 (C-4', C-6'), 126.9 (C-5'), 86.1 (C-5), 57.5 (C-3), 33.2 (C-1'), 31.9 (C-6, C-9), 25.1 $\left(\mathrm{NCH}_{3}\right), 23.6(\mathrm{C}-7 / \mathrm{C}-8), 23.3(\mathrm{C}-8 / \mathrm{C}-7)$ ppm; HRMS (ESI+) $\mathrm{m} / z$ : $[\mathrm{M}+\mathrm{H}]^{+}$Calcd for $\mathrm{C}_{15} \mathrm{H}_{21} \mathrm{~N}_{2} \mathrm{O}^{+}$ 245.1648 , found 245.1651 .

The spectroscopic data were consistent with those previously reported. ${ }^{[9,10]}$

Ethyl-3,4,-dihydroxybenzoate (51) ${ }^{[11]}$

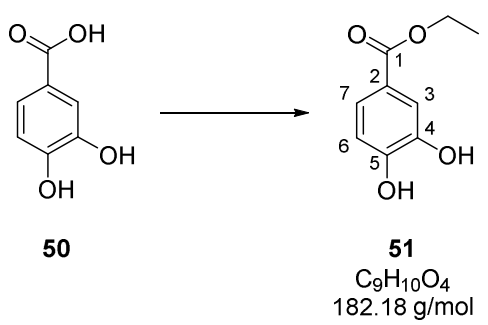

Concentrated sulfuric acid ( $2 \mathrm{~mL}, 37.5 \mathrm{mmol}, 98 \%, 0.6$ eq.) was added dropwise to a solution of 3,4-dihydroxybenzoic acid $(\mathbf{5 0}, 10.0 \mathrm{~g}, 64.9 \mathrm{mmol})$ in ethanol $(100 \mathrm{~mL})$ at $0{ }^{\circ} \mathrm{C}$. The mixture was allowed to warm to room temperature, then, heated to $60^{\circ} \mathrm{C}$ and stirred overnight. The solvent was removed and the precipitate was dissolved in EtOAc. The organic phase was washed with aq. $\mathrm{NaHCO}_{3}$ solution $(10 \%, 100 \mathrm{~mL})$ and brine $(100 \mathrm{~mL})$. The organic phase was dried over $\mathrm{MgSO}_{4}$ and the solvent was removed under reduced pressure to yield cocatalyst $\mathbf{5 1}$ as a colorless solid ( $8.60 \mathrm{~g}, 47.2 \mathrm{mmol}$, $73 \%)$.

$\mathbf{R}_{f}=0.18$ (3:1 $n$-pentane/ethyl acetate); ${ }^{1} \mathbf{H}$ NMR (500 MHz, DMSO): $\delta=9.46\left(\mathrm{~s}_{\mathrm{br}}, 2 \mathrm{H},-\mathrm{OH}\right), 7.35$ (d, $J=2.1 \mathrm{~Hz}, 1 \mathrm{H}, \mathrm{H}-3), 7.30$ (dd, $J=8.3,2.1 \mathrm{~Hz}, 1 \mathrm{H}, \mathrm{H}-7), 6.80$ (d, $J=8.3 \mathrm{~Hz}, 1 \mathrm{H}, \mathrm{H}-6), 4.22$ (q, $J=$ $7.1 \mathrm{~Hz}, 2 \mathrm{H}, \mathrm{OEt}), 1.27$ (t, $J=7.1 \mathrm{~Hz}, 3 \mathrm{H}, \mathrm{OEt}) \mathrm{ppm} ;{ }^{13} \mathbf{C}$ NMR (125 MHz, DMSO): $\delta=165.7$ (C-1), 
150.4 (C-5), 145.1 (C-4), 121.8 (C-7), 120.8 (C-2), 116.3 (C-3), 115.3 (C-6), 60.1 (OEt), 14.3 (OEt) ppm; HRMS (ESI+) $m / z$ : [M - H] $]^{-}$Calcd for $\mathrm{C}_{9} \mathrm{H}_{9} \mathrm{O}_{4}{ }^{-}$181.0506, found 181.0509.

The spectroscopic data were consistent with those previously reported. ${ }^{[11,12]}$

\section{(R)-2-Methyl-5-oxohexanal (12)}

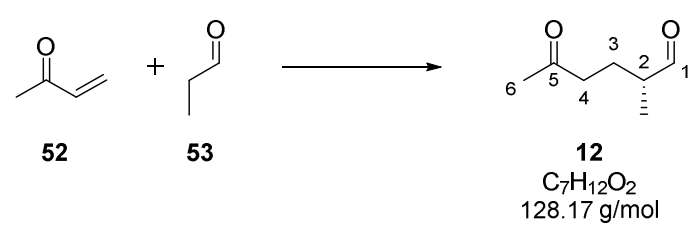

To organo catalyst 49 (840 mg, $3.44 \mathrm{mmol}, 0.2$ eq.), ethyl-3,4,-dihydroxybenzoate (51, $626 \mathrm{mg}$, $3.44 \mathrm{mmol}, 0.2$ eq.), methyl vinyl ketone (52, $4.8 \mathrm{~mL}, 51.6 \mathrm{mmol}, 90 \%, 3.0$ eq.) and propionaldehyde $(\mathbf{5 3}, 1.23 \mathrm{~mL}, 17.2 \mathrm{mmol}, 1.0$ eq.) were added. The mixture was stirred for $16 \mathrm{~h}$ at room temperature. The mixture was purified by silica gel flash column chromatography ( $n$-pentane/diethyl ether 80:20 $\rightarrow$ 70:30) to yield aldehyde $\mathbf{1 2}(1.24 \mathrm{~g}, 9.6 \mathrm{mmol}, 56 \%)$ as a colorless oil.

$\mathbf{R}_{f}=0.17$ (7:3 n-pentane/diethyl ether); $[\alpha]_{\mathbf{D}}{ }^{20}=+1.9\left(c 1.04, \mathrm{CHCl}_{3}\right) ;{ }^{1} \mathbf{H} \mathbf{N M R}\left(500 \mathrm{MHz}, \mathrm{CD}_{2} \mathrm{Cl}_{2}\right): \delta$ $=9.58(\mathrm{~d}, J=1.8 \mathrm{~Hz}, 1 \mathrm{H}, \mathrm{H}-1), 2.47$ (t, $J=7.5 \mathrm{~Hz} ; 2 \mathrm{H}, \mathrm{H}-4), 2.33$ (qd, $J=7.0,1.7 \mathrm{~Hz}, 1 \mathrm{H}, \mathrm{H}-2), 2.10$ (s, 3H, H-6), $1.96-1.89$ (m, 1H, H-3a), $1.64-1.57$ (m, 1H, H-3b), 1.08 (d, $J=7.1 \mathrm{~Hz}, 3 \mathrm{H}, 2-\mathrm{CH}_{3}$ ) ppm; ${ }^{13} \mathrm{C}$ NMR (125 MHz, $\mathrm{CD}_{2} \mathrm{Cl}_{2}$ ): $\delta=208.2$ (C-5), 204.9 (C-1), 46.0 (C-2), 41.0 (C-4), 30.3 (C-6), $24.6(\mathrm{C}-3), 13.7\left(2-\mathrm{CH}_{3}\right)$ ppm; HRMS (ESI+) $\mathrm{m} / \mathrm{z}$ : $[\mathrm{M}+\mathrm{H}]^{+}$Calcd for $\mathrm{C}_{7} \mathrm{H}_{13} \mathrm{O}_{2}{ }^{+}$129.0910, found 129.0911.

The spectroscopic data were consistent with those previously reported. ${ }^{[13,14]}$

\section{(2R)-2-Methylhexane-1,5-diol (54)}

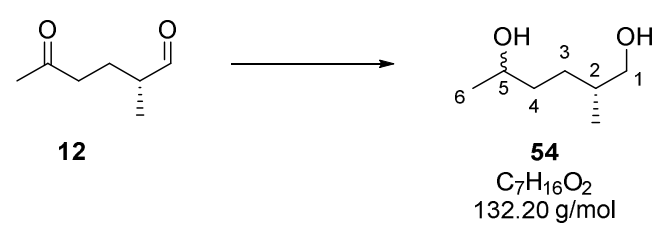

A solution of aldehyde $12(100 \mathrm{mg}, 0.78 \mathrm{mmol})$ in $\mathrm{DCM}(3 \mathrm{~mL})$ and $\mathrm{MeOH}(0.5 \mathrm{~mL})$ was cooled down to $0{ }^{\circ} \mathrm{C}$ and then, $\mathrm{NaBH}_{4}(59 \mathrm{mg}, 1.56 \mathrm{mmol}, 2.0$ eq.) was added. The reaction was quenched by addition of $\mathrm{H}_{2} \mathrm{O}(2 \mathrm{~mL})$ as TLC indicated full conversion (approx. $5 \mathrm{~min}$ ). The mixture was stirred for 30 min. Then, the phases were separated and the aqueous phase was extracted with DCM and diethyl 
ether (each 3 x $5 \mathrm{~mL}$ ), successively. The solvents were removed and the crude product $54(120 \mathrm{mg})$, a mixture of diastereomers, was directly used for the next reaction step.

${ }^{1} \mathbf{H}$ NMR (500 MHz, $\mathrm{CD}_{2} \mathrm{Cl}_{2}$ ): $\delta=3.78-3.70(\mathrm{~m}, 1 \mathrm{H}, \mathrm{H}-6), 3.47-3.39(\mathrm{~m}, 2 \mathrm{H}, \mathrm{H}-1), 1.64-1.55$ (m, 1H, H-2), $1.56-1.07$ (m, 4H, H-3, H-4), 1.16/1.15 (d, J=6.2 Hz, 3H, C-6), 0.91/0.90 (d, J=6.7 Hz, $\left.3 \mathrm{H}, 2-\mathrm{CH}_{3}\right) \mathrm{ppm} ;{ }^{13} \mathrm{C}$ NMR (125 MHz, $\mathrm{CD}_{2} \mathrm{Cl}_{2}$ ): $\delta=68.9 / 68.6$ (C-5), 68.5/68.4 (C-1), 37.2/37.0 (C4), 36.4/36.2 (C-2), 29.7/29.6 (C-3), 24.0/23.9 (C-6), 17.0/16.9 (2- $\left.\mathrm{CH}_{3}\right) \mathrm{ppm}$; HRMS (ESI+) $\mathrm{m} / \mathrm{z}$ : [M $+\mathrm{H}]^{+}$Calcd for $\mathrm{C}_{7} \mathrm{H}_{17} \mathrm{O}_{2}{ }^{+}$133.1223, found 133.1224.

The spectroscopic data were consistent with those previously reported. ${ }^{[15]}$ 


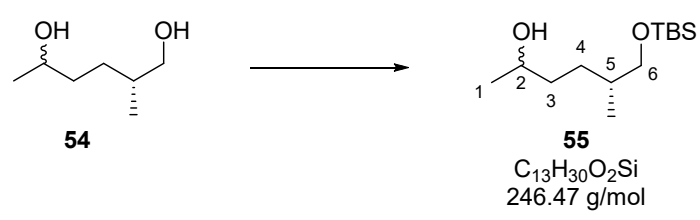

The crude diol 54 (120 mg, max. $0.78 \mathrm{mmol})$ was dissolved in dry DCM (2.5 mL). Imidazole $(80 \mathrm{mg}$, $1.17 \mathrm{mmol}, 1.5$ eq.) was added. Then, a solution of TBSCl (105 mg, $0.70 \mathrm{mmol}, 0.9$ eq.) and DCM $(1.5 \mathrm{~mL})$ was added dropwise at $0{ }^{\circ} \mathrm{C}$. The mixture was stirred for $25 \mathrm{~min}$ at $0{ }^{\circ} \mathrm{C}$. The reaction was quenched by addition of aq. sat. $\mathrm{NH}_{4} \mathrm{Cl}$ solution $(4 \mathrm{~mL})$. The phases were separated and the aqueous phase was extracted with diethyl ether and DCM (3 x $4 \mathrm{~mL})$, successively. The combined organic phases were dried over $\mathrm{MgSO}_{4}$ and the solvent was removed under reduced pressure. The crude product was purified by silica gel flash column chromatography ( $n$-pentane/diethyl ether 100:0 $\rightarrow$ 70:30) to yield TBS protected alcohol $\mathbf{5 5}(106 \mathrm{mg}, 0.43 \mathrm{mmol}, 55 \%)$ as a mixture of diastereomers as a colorless oil.

$\mathbf{R}_{f}=0.56\left(1: 1\right.$ cyclohexane/ethyl acetate); ${ }^{1} \mathbf{H}$ NMR $\left(500 \mathrm{MHz}, \mathrm{CD}_{2} \mathrm{Cl}_{2}\right): \delta=3.76-3.69(\mathrm{~m}, 1 \mathrm{H}, \mathrm{H}-2)$, 3.46 - 3.37 (m, 2H, H-6), 1.51 - 1.35 (m, 4H, H-3, H-4a, H-5), 1.20 - 1.04 (m, 1H, H-4b), 1.15 (2d, J $=6.2 \mathrm{~Hz}, 3 \mathrm{H}, \mathrm{H}-1), 0.91-0.87\left(\mathrm{~m}, 12 \mathrm{H}\right.$, OTBS, 5- $\left.\mathrm{CH}_{3}\right), 0.04(\mathrm{~s}, 6 \mathrm{H}$, OTBS $) \mathrm{ppm} ;{ }^{13} \mathbf{C}$ NMR (125 MHz, $\mathrm{CD}_{2} \mathrm{Cl}_{2}$ ): $\delta=68.76 / 68.73$ (C-6), 68.71 (C-2), 37.4/37.3 (C-3), 36.4/36.3 (C-5), 29.8 (C-4), 26.3 (OTBS), 23.9/23.8 (C-1), 18.8 (OTBS), 17.1 (5-- $\mathrm{CH}_{3}$ ), -5.1 (OTBS) ppm; HRMS (ESI+) $m / z:[\mathrm{M}+\mathrm{H}]^{+}$Calcd for $\mathrm{C}_{13} \mathrm{H}_{31} \mathrm{O}_{2} \mathrm{Si}^{+}$247.2088, found 247.2085.

\section{(R)-6-((tert-Butyldimethylsilyl)oxy)-5-methylhexan-2-one (11)}

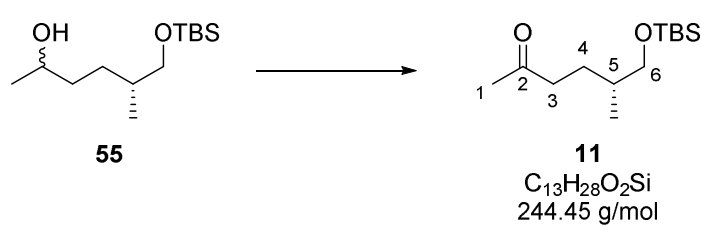

Sulfur trioxide pyridine complex $(2.40 \mathrm{~g}, 15.1 \mathrm{mmol}, 3.0 \mathrm{eq}$.) was dried in HV for an hour. Then, DCM (40 mL), DIPEA (3.4 mL, $20.1 \mathrm{mmol}, 4.0$ eq.) and DMSO (3.6 mL, $50.3 \mathrm{mmol}, 10$ eq.) were added. The mixture was cooled down to $0{ }^{\circ} \mathrm{C}$ and the alcohol $55(1.24 \mathrm{~g}, 5.03 \mathrm{mmol})$ was added dropwise as a solution in DCM $(30 \mathrm{~mL})$. After $10 \mathrm{~min}$, the reaction was quenched by addition of aq. sat. $\mathrm{NaHCO}_{3}$ solution $(20 \mathrm{~mL})$. The phases were separated and the aqueous phase was extracted with DCM $(3 \times 20 \mathrm{~mL})$. The combined organic phases were dried over $\mathrm{Na}_{2} \mathrm{SO}_{4}$ and the solvent was removed under reduced pressure. The crude product was purified by silica gel flash column 
chromatography ( $n$-pentane/diethyl ether 70:30) to yield $\mathbf{1 1}(1.05 \mathrm{~g}, 4.30 \mathrm{mmol}, 86 \%)$ as a colorless oil.

$\mathbf{R}_{f}=0.56$ (2:1 cyclohexane/ethyl acetate); $[\alpha]_{\mathbf{D}}{ }^{20}=+44.1\left(c 1.36, \mathrm{CH}_{2} \mathrm{Cl}_{2}\right) ;{ }^{1} \mathbf{H} \mathbf{~ N M R}(500 \mathrm{MHz}$, $\mathrm{CD}_{2} \mathrm{Cl}_{2}$ ): $\delta=3.45-3.39$ (m, 2H, H-6), $2.49-2.34$ (m, 2H, H-3), 2.10 (s, 3H, H-1), $1.70-1.63$ (m, 1H, H-4a), $1.58-1.52$ (m, 1H, H-5), $1.37-1.30$ (m, 1H, H-4b), 0.89 (s, 9H, OTBS), 0.86 (d, $J=$ $\left.6.7 \mathrm{~Hz}, 3 \mathrm{H}, 5-\mathrm{CH}_{3}\right), 0.04$ (s, 6H, OTBS) ppm; ${ }^{13} \mathbf{C} \mathbf{~ N M R}\left(125 \mathrm{MHz}, \mathrm{CD}_{2} \mathrm{Cl}_{2}\right): \delta=209.3\left(\mathrm{C}_{\mathrm{q}}-2\right), 68.5$ (C-6), 41.9 (C-3), 35.9 (C-5), 30.1 (C-1), 27.8 (C-4), 26.2 (OTBS), 18.8 (OTBS), 16.9 (5-CH ${ }_{3}$ ), -5.2 (OTBS) ppm; HRMS (ESI+) m/z: [M+ H] ${ }^{+}$Calcd for $\mathrm{C}_{13} \mathrm{H}_{29} \mathrm{O}_{2} \mathrm{Si}^{+} 245.1931$, found 245.1928 .

The spectroscopic data were consistent with those previously reported. ${ }^{[16]}$

\section{(2R,7R,E)-1-((tert-Butyldimethylsilyl)oxy)-7-hydroxy-11-((4-methoxybenzyl)oxy)-2,10-} dimethylundec-9-en-5-one (56)

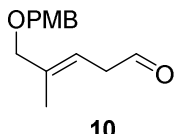

10

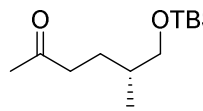

11

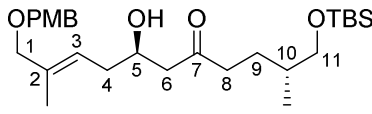

56

$\mathrm{C}_{27} \mathrm{H}_{46} \mathrm{O}_{5} \mathrm{Si}$

$(+)-[\mathrm{Ipc}]_{2} \mathrm{BCl}(102 \mathrm{mg}, 316 \mu \mathrm{mol}, 1.10$ eq. $)$ was dissolved in DCM $(1 \mathrm{~mL})$. After cooling to $-78{ }^{\circ} \mathrm{C}$, DIPEA (97.8 $\mu \mathrm{L}, 575 \mu \mathrm{mol}, 2.00$ eq.) and ketone 11 (70.3 mg, $288 \mu \mathrm{mol}, 1.00$ eq. stored over $3 \AA \mathrm{MS}$ in $2 \mathrm{~mL} \mathrm{DCM}$ ) were added dropwise. The reaction mixture was stirred $3 \mathrm{~h}$ at $-78^{\circ} \mathrm{C}$ and freshly prepared aldehyde 10 (70.3 mg, $288 \mu \mathrm{mol}, 1.15$ eq. stored $14 \mathrm{~h}$ over $3 \AA \mathrm{MS}$ in $2 \mathrm{~mL}$ DCM) was added dropwise. After stirring $1 \mathrm{~h}$ at $-78{ }^{\circ} \mathrm{C}$, the reaction vessel was placed in a deep freezer $\left(-15^{\circ} \mathrm{C}\right)$ overnight. Methanol (3.3 mL), pH 7 buffer $(1.8 \mathrm{~mL})$ and $\mathrm{H}_{2} \mathrm{O}_{2}(1.8 \mathrm{~mL}, 35 \%)$ were added to quench the reaction. After stirring $1 \mathrm{~h}$ at $\mathrm{rt}$, brine $(8 \mathrm{~mL})$ was added, the phases were separated, the aqueous layer was extracted with DCM $(3 \times 10 \mathrm{~mL})$, the combined organic phases were dried over $\mathrm{MgSO}_{4}$ and the solvent was removed under reduced pressure. Purification of the crude product was achieved by flash chromatography (20\% EtOAc/CyH) giving back starting material 11 (24.1 mg, $98.7 \mu$ mol, 34\%) and yielding the title compound $\mathbf{5 6}$ as a colorless oil (50.7 mg, $106 \mu \mathrm{mol}, 37 \%, 56 \% \mathrm{brsm}$., $d r=4: 1$ ). Separation of the diastereomers was not possible.

$\mathbf{R}_{f}=0.15(40 \% \mathrm{EtOAc} / \mathrm{CyH}) \cdot[\boldsymbol{\alpha}]_{\boldsymbol{D}}^{\mathbf{2 0}}=-3.6^{\circ}\left(c=1.40\right.$ in $\left.\mathrm{CH}_{2} \mathrm{Cl}_{2}\right) .{ }^{1} \mathbf{H} \mathbf{N M R}\left(700 \mathrm{MHz}, \mathrm{CDCl}_{3}\right) \delta=$ $7.26\left(\mathrm{~d}, J=8.7 \mathrm{~Hz}, 2 \mathrm{H}, \mathrm{H}_{\text {arom. }}\right), 6.87$ (d, $\left.J=8.7 \mathrm{~Hz}, 2 \mathrm{H}, \mathrm{H}_{\text {arom. }}\right), 5.47$ (tq, $\left.J=7.4,1.1 \mathrm{~Hz}, 1 \mathrm{H}, \mathrm{H}-3\right)$, 4.39 (s, 2H, Ar-CH $), 4.12-4.08$ (m, 1H, H-5), 3.89 (s, 2H, H-1), 3.80 (s, 3H, O-CH $)_{3}, 3.41$ (d, $J=$ $6.0 \mathrm{~Hz}, 2 \mathrm{H}, \mathrm{H}-11$ ), 3.03 (d, J = 3.2 Hz, 1H, OH), $2.63-2.60$ (m, 1H, H-6), $2.54-2.41$ (m, 3H, H-6', H-8), $2.32-2.28$ (m, 1H, H-4), $2.24-2.20$ (m, 1H, H-4'), $1.72-1.68$ (m, 1H, H-9), 1.68 (s, 3H, 2$\mathrm{CH}_{3}$ ), $1.59-1.54$ (m, 1H, H-10), $1.41-1.35$ (m, 1H, H-9'), 0.89 (s, 9H, TBS), 0.86 (d, $J=6.7 \mathrm{~Hz}$, $\left.3 \mathrm{H}, 10-\mathrm{CH}_{3}\right), 0.03$ (s, 6H, TBS) ppm; ${ }^{13} \mathbf{C}$ NMR $\left(175 \mathrm{MHz}, \mathrm{CDCl}_{3}\right) \delta=212.4(\mathrm{C}-7), 159.3\left(\mathrm{C}_{\text {arom. }} \mathrm{O}\right)$, 
$135.5(\mathrm{C}-2), 130.7\left(\mathrm{C}_{\text {arom. }}\right), 129.5\left(\mathrm{C}_{\text {arom. }} \mathrm{H}\right), 123.1(\mathrm{C}-3), 113.9\left(\mathrm{C}_{\text {arom. }} \mathrm{H}\right), 75.9(\mathrm{C}-1), 71.6\left(\mathrm{Ar}-\mathrm{CH}_{2}\right)$,

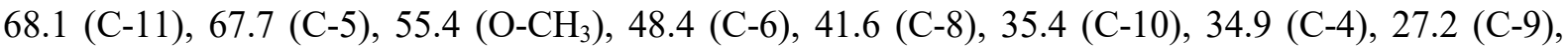
26.1 (TBS), 18.5 (TBS), $16.7\left(10-\mathrm{CH}_{3}\right), 14.4\left(2-\mathrm{CH}_{3}\right),-5.2$ (TBS) ppm; HRMS (ESI+) $\mathrm{m} / z$ : calcd for $\mathrm{C}_{27} \mathrm{H}_{46} \mathrm{O}_{5} \mathrm{SiH}^{+}[\mathrm{M}+\mathrm{H}]^{+}: 479.3187$, found: 479.3193, calcd for $\mathrm{C}_{27} \mathrm{H}_{46} \mathrm{O}_{5} \mathrm{SiNH}_{4}\left[\mathrm{M}+\mathrm{NH}_{4}\right]^{+}: 496.3453$, found: 496.3458, calcd for $\mathrm{C}_{27} \mathrm{H}_{46} \mathrm{O}_{5} \mathrm{SiNa}^{+}[\mathrm{M}+\mathrm{Na}]^{+}$: 501.3007, found: 501.3009.

(5R,10R,E)-11-((tert-Butyldimethylsilyl)oxy)-1-((4-methoxybenzyl)oxy)-2,10-dimethyl-7oxoundec-2-en-5-yl (S)-3,3,3-trifluoro-2-methoxy-2-phenylpropanoate (57)

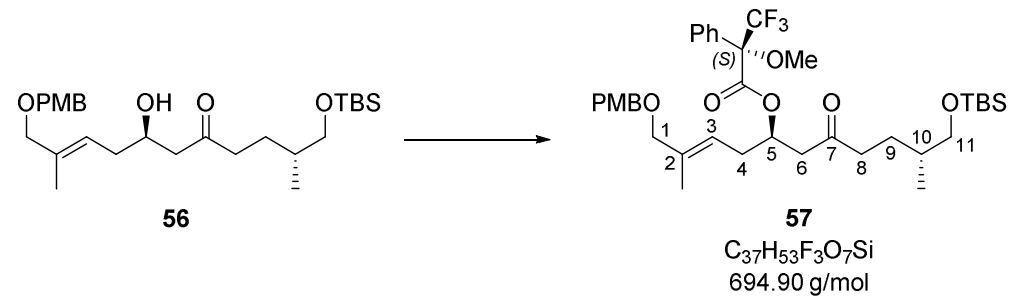

Alcohol $56(5.00 \mathrm{mg}, 10.4 \mu \mathrm{mol}, 1.00$ eq. $)$ was dissolved in pyridine $(120 \mu \mathrm{L})$ and $(-)-(R)-\mathrm{MTPA}-\mathrm{Cl}$ $\left(11.7 \mu \mathrm{L}, 62.7 \mu \mathrm{mol}, 6.00\right.$ eq.) was added at $0{ }^{\circ} \mathrm{C}$. The reaction mixture was stirred $2 \mathrm{~h}$ at $\mathrm{rt}$ followed by the addition of sat. aq. $\mathrm{NH}_{4} \mathrm{Cl}$ solution $(1 \mathrm{~mL})$ and EtOAc $(2 \mathrm{~mL})$. After separation of the organic layer, the aqueous phase was extracted with EtOAc $(3 \times 2 \mathrm{~mL})$, the combined organic phases were dried over $\mathrm{Na}_{2} \mathrm{SO}_{4}$ and the solvent was removed under reduced pressure. Purification of the crude product was achieved by preparative TLC $(20 \% \mathrm{EtOAc} / \mathrm{CyH})$ and HPLC $(90 \% \mathrm{MeCN} /$ water, retention time 20.4 min, using a MACHEREY-NAGEL Nucleodur 110-5 Gravity C18; $5 \mu \mathrm{m} ; 250$ x $10 \mathrm{~mm}$ ) to yield the title compound 57 as a colorless oil $(6.60 \mathrm{mg}, 9.50 \mu \mathrm{mol}, 91 \%, d r=4: 1$ resulting out of the aldol reaction to compound $\mathbf{5 6}$ ).

$\mathbf{R}_{f}=0.37(20 \% \mathrm{EtOAc} / \mathrm{CyH}) \cdot[\boldsymbol{\alpha}]_{\boldsymbol{D}}^{\mathbf{2 0}}=-15.9^{\circ}\left(c=0.44\right.$ in $\left.\mathrm{CH}_{2} \mathrm{Cl}_{2}\right) .{ }^{1} \mathbf{H} \mathbf{N M R}\left(700 \mathrm{MHz}, \mathrm{CDCl}_{3}\right) \delta=$ $7.49-7.47$ (m, 2H, $\left.\mathrm{H}_{\text {arom., MTPA }}\right), 7.37-7.35$ (m, 3H, $\left.\mathrm{H}_{\text {arom., MTPA }}\right), 7.24$ (d, J = 8.7 Hz, 2H, $\mathrm{H}_{\text {arom. }}$ ), 6.87 (d, $J=8.7 \mathrm{~Hz}, 2 \mathrm{H}, \mathrm{H}_{\text {arom. }}$ ), $5.60-5.56$ (m, 1H, H-5), 5.42 (t, $\left.J=7.3 \mathrm{~Hz}, 1 \mathrm{H}, \mathrm{H}-3\right), 4.37$ (s, 2H, Ar$\mathrm{CH}_{2}$ ), 3.85 (s, 2H, H-1), 3.80 (s, 3H, O-CH $), 3.51$ (s, 3H, O-CH $\left.\mathrm{CH}_{3, \mathrm{MTPA}}\right), 3.38-3.37$ (m, 2H, H-11), 2.79 (dd, $J=17.1,7.9 \mathrm{~Hz}, 1 \mathrm{H}, \mathrm{H}-6), 2.57$ (dd, $J=17.1,4.8 \mathrm{~Hz}, 1 \mathrm{H}, \mathrm{H}-6$ '), $2.53-2.49$ (m, 1H, H-4), $2.47-2.43$ (m, 1H, H-4'), $2.41-2.35$ (m, 1H, H-8), 2.28 - 2.24 (m, 1H, H-8'), 1.66 (s, 3H, 2-CH ( $_{3}$, $1.61-1.57$ (m, 1H, H-9), $1.53-1.48$ (m, 1H, H-10), 1.30 - 1.26 (m, 1H, H-9'), 0.88 (s, 9H, TBS), $0.82\left(\mathrm{~d}, J=6.7 \mathrm{~Hz}, 3 \mathrm{H}, 10-\mathrm{CH}_{3}\right), 0.03(\mathrm{~s}, 6 \mathrm{H}, \mathrm{TBS}) \mathrm{ppm} ;{ }^{13} \mathbf{C} \mathbf{N M R}\left(175 \mathrm{MHz}, \mathrm{CDCl}_{3}\right) \delta=207.2(\mathrm{C}-$ 7), $165.9\left(\mathrm{C}=\mathrm{O}_{\mathrm{MTPA}}\right), 159.3\left(\mathrm{C}_{\text {arom. }} \mathrm{O}\right), 136.8(\mathrm{C}-2), 132.4\left(\mathrm{C}_{\text {arom.MTPA }}\right), 130.5\left(\mathrm{C}_{\text {arom. }}\right), 129.6$ $\left(\mathrm{C}_{\text {arom. }} \mathrm{H}_{\mathrm{MTPA}}\right), 129.4\left(\mathrm{C}_{\text {arom. }} \mathrm{H}\right), 128.5\left(\mathrm{C}_{\text {arom. }} \mathrm{H}_{\mathrm{MTPA}}\right), 127.5\left(\mathrm{C}_{\text {arom. }} \mathrm{H}_{\mathrm{MTPA}}\right), 123.4\left(\mathrm{q}, J=283.7 \mathrm{~Hz}, \mathrm{CF}_{3}\right)$, 120.8 (C-3), $113.9\left(\mathrm{C}_{\text {arom. }} \mathrm{H}\right), 75.4(\mathrm{C}-1), 72.7$ (C-5), $71.7\left(\mathrm{Ar}^{-} \mathrm{CH}_{2}\right), 68.1(\mathrm{C}-11), 55.4\left(\mathrm{O}^{-} \mathrm{CH}_{3}\right), 45.5$ (C-6), 41.4 (C-8), 35.3 (C-10), 32.1 (C-4), 27.0 (C-9), 26.1 (TBS), 18.5 (TBS), 16.6 (10- $\mathrm{CH}_{3}$ ), 14.3 (2$\mathrm{CH}_{3}$ ), -5.3 (TBS) ppm; HRMS (ESI+) $m / z$ : calcd for $\mathrm{C}_{37} \mathrm{H}_{53} \mathrm{~F}_{3} \mathrm{O}_{7} \mathrm{SiH}^{+}[\mathrm{M}+\mathrm{H}]^{+}: 695.3585$, found: 695.3591. 
(5R,10R,E)-11-((tert-Butyldimethylsilyl)oxy)-1-((4-methoxybenzyl)oxy)-2,10-dimethyl-7oxoundec-2-en-5-yl ( $R$ )-3,3,3-trifluoro-2-methoxy-2-phenylpropanoate (58)

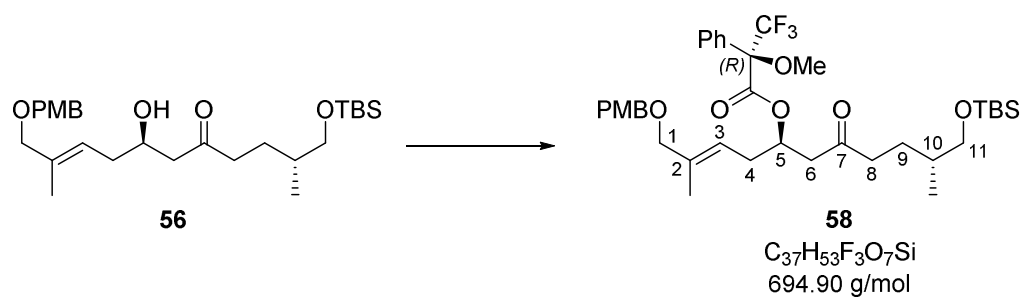

Alcohol 56 (5.00 mg, $10.4 \mu \mathrm{mol}, 1.00$ eq.) was dissolved in pyridine (120 $\mu \mathrm{L})$ and (+)-(S)-MTPA-Cl $\left(11.7 \mu \mathrm{L}, 62.7 \mu \mathrm{mol}, 6.00\right.$ eq.) was added at $0{ }^{\circ} \mathrm{C}$. The reaction mixture was stirred $2 \mathrm{~h}$ at $\mathrm{rt}$ followed by the addition of sat. aq. $\mathrm{NH}_{4} \mathrm{Cl}$ solution $(1 \mathrm{~mL})$ and EtOAc $(2 \mathrm{~mL})$. After separation of the organic layer, the aqueous phase was extracted with EtOAc $(3 \times 2 \mathrm{~mL})$, the combined organic phases were dried over $\mathrm{Na}_{2} \mathrm{SO}_{4}$ and the solvent was removed under reduced pressure. Purification of the crude product was achieved by preparative TLC (15\% EtOAc/CyH) and HPLC $(90 \% \mathrm{MeCN} /$ water, retention time $20.4 \mathrm{~min}$, using a MACHEREY-NAGEL Nucleodur 110-5 Gravity C18; $5 \mu \mathrm{m} ; 250$ x $10 \mathrm{~mm}$ ) to yield the title compound $\mathbf{5 8}$ as a colorless oil $(6.80 \mathrm{mg}, 9.79 \mu \mathrm{mol}, 94 \%, d r=4: 1$ resulting out of the aldol reaction to compound $\mathbf{5 6}$ ).

$\mathbf{R}_{\boldsymbol{f}}=0.26(15 \% \mathrm{EtOAc} / \mathrm{CyH}) \cdot[\boldsymbol{\alpha}]_{\boldsymbol{D}}^{\mathbf{2 0}}=+11.8^{\circ}\left(c=0.68\right.$ in $\left.\mathrm{CH}_{2} \mathrm{Cl}_{2}\right) .{ }^{1} \mathbf{H} \mathbf{N M R}\left(700 \mathrm{MHz}, \mathrm{CDCl}_{3}\right) \delta=$ $7.49-7.47$ (m, 2H, $\left.\mathrm{H}_{\text {arom., MTPA }}\right), 7.37-7.35$ (m, 3H, $\left.\mathrm{H}_{\text {arom., MTPA }}\right), 7.24$ (d, J=8.6 Hz, 2H, $\mathrm{H}_{\text {arom. }}$ ), 6.87 (d, $J=8.6 \mathrm{~Hz}, 2 \mathrm{H}, \mathrm{H}_{\text {arom }}$ ), $5.57-5.53$ (m, 1H, H-5), 5.30 (t, $\left.J=6.9 \mathrm{~Hz}, 1 \mathrm{H}, \mathrm{H}-3\right), 4.35$ (s, 2H, Ar$\mathrm{CH}_{2}$ ), 3.81 (s, 5H, H-1, O-CH CH $_{3} 3.48$ (s, 3H, O-CH $\mathrm{CH}_{3, \mathrm{MTPA}}$ ), 3.38 (d, $\left.J=6.0 \mathrm{~Hz}, 2 \mathrm{H}, \mathrm{H}-11\right), 2.82$ (dd, $J$ $=17.2,8.4 \mathrm{~Hz}, 1 \mathrm{H}, \mathrm{H}-6), 2.62$ (dd, $J=17.2,4.3 \mathrm{~Hz}, 1 \mathrm{H}, \mathrm{H}-6$ '), 2.47 - 2.42 (m, 2H; H-4, H-8), 2.39 2.33 (m, 2H, H-4', H-8'), 1.68 - 1.63 (m, 1H, H-9), 1.63 (s, 3H, 2- $\mathrm{CH}_{3}$ ), 1.56 - 1.49 (m, 1H, H-10), $1.36-1.32$ (m, 1H, H-9'), 0.88 (s, 9H, TBS), 0.83 (d, $J=6.7 \mathrm{~Hz}, 3 \mathrm{H}, 10-\mathrm{CH}_{3}$ ), 0.02 (s, 6H, TBS) ppm; ${ }^{13} \mathrm{C}$ NMR $\left(175 \mathrm{MHz}, \mathrm{CDCl}_{3}\right) \delta=207.4(\mathrm{C}-7), 166.0\left(\mathrm{C}=\mathrm{O}_{\text {MTPA }}\right), 159.3\left(\mathrm{C}_{\text {arom. }} \mathrm{O}\right), 136.7(\mathrm{C}-2), 132.4$ $\left(\mathrm{C}_{\text {arom.,MTPA }}\right), \quad 130.6 \quad\left(\mathrm{C}_{\text {arom. }}\right), 129.7\left(\mathrm{C}_{\text {arom. }} \mathrm{H}_{\mathrm{MTPA}}\right), 129.4\left(\mathrm{C}_{\text {arom. }} \mathrm{H}\right), 128.5 \quad\left(\mathrm{C}_{\text {arom. }} \mathrm{H}_{\mathrm{MTPA}}\right), 127.6$ $\left(\mathrm{C}_{\text {arom. }} \mathrm{H}_{\mathrm{MTPA}}\right), 123.4\left(\mathrm{q}, J=285.9 \mathrm{~Hz}, \mathrm{CF}_{3}\right), 120.7(\mathrm{C}-3), 113.9\left(\mathrm{C}_{\text {arom. }} \mathrm{H}\right), 75.5$ (C-1), 72.5 (C-5), 71.6 (Ar- $\mathrm{CH}_{2}$ ), 68.1 (C-11), $55.4\left(\mathrm{O}-\mathrm{CH}_{3}\right), 45.5$ (C-6), 41.4 (C-8), 35.3 (C-10), 31.8 (C-4), 27.1 (C-9), 26.1 (TBS), 18.5 (TBS), $16.6\left(10-\mathrm{CH}_{3}\right), 14.3\left(2-\mathrm{CH}_{3}\right),-5.3$ (TBS) ppm; HRMS (ESI+) $\mathrm{m} / z$ : calcd for $\mathrm{C}_{37} \mathrm{H}_{53} \mathrm{~F}_{3} \mathrm{O}_{7} \mathrm{SiH}^{+}[\mathrm{M}+\mathrm{H}]^{+}: 695.3585$, found: 695.3585 .

\section{Mosher Ester Analysis for Compound 56}

Determination of the absolute configuration was accomplished using the Mosher ester analysis. For this purpose, the two Mosher ester derivatives $\mathbf{5 7}$ and $\mathbf{5 8}$ have been synthesized and the respective 
NMR data have been compared as shown in Table 1 . The resulting difference between the ${ }^{1} \mathrm{H}$ NMR data of the $(S)$ - and $(R)$-MTPA ester led to the determination of the absolute configuration as shown below. ${ }^{[17]}$

Main diastereomer:

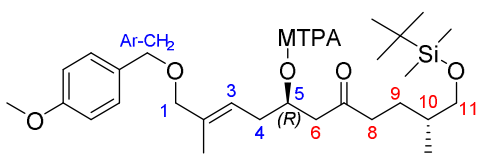

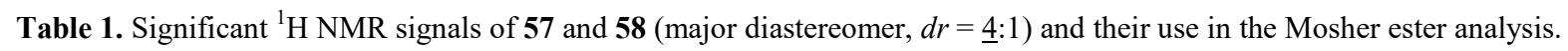

$\begin{array}{cccc}\text { position } & \begin{array}{c}\delta_{H}[\mathrm{ppm}] \\ (\mathbf{5 7})(S)\end{array} & \begin{array}{c}\delta_{H}[\mathrm{ppm}] \\ (\mathbf{5 8})(R)\end{array} & \Delta \delta^{S-R} \\ 8 & 2.26 & 2.37 & -0.11 \\ 8^{\circ} & 2.38 & 2.45 & -0.07 \\ 9 & 1.59 & 1.66 & -0.07 \\ 9^{\circ} & 1.28 & 1.34 & -0.06 \\ 6 & 2.57 & 2.62 & -0.05 \\ 6^{6} & 2.79 & 2.82 & -0.03 \\ 10 & 1.51 & 1.53 & -0.02 \\ 10-\mathrm{CH}_{3} & 0.82 & 0.83 & -0.01 \\ 11 & 3.37 & 3.38 & -0.01 \\ \mathrm{Ar}^{\circ} \mathrm{CH}_{2} & 4.37 & 4.35 & +0.02 \\ 2-\mathrm{CH}_{3} & 1.66 & 1.63 & +0.03 \\ 5 & 5.58 & 5.55 & +0.03 \\ 4 & 2.50 & 2.45 & +0.05 \\ 4^{6} & 2.46 & 2.37 & +0.09 \\ 3 & 5.42 & 5.30 & +0.12\end{array}$




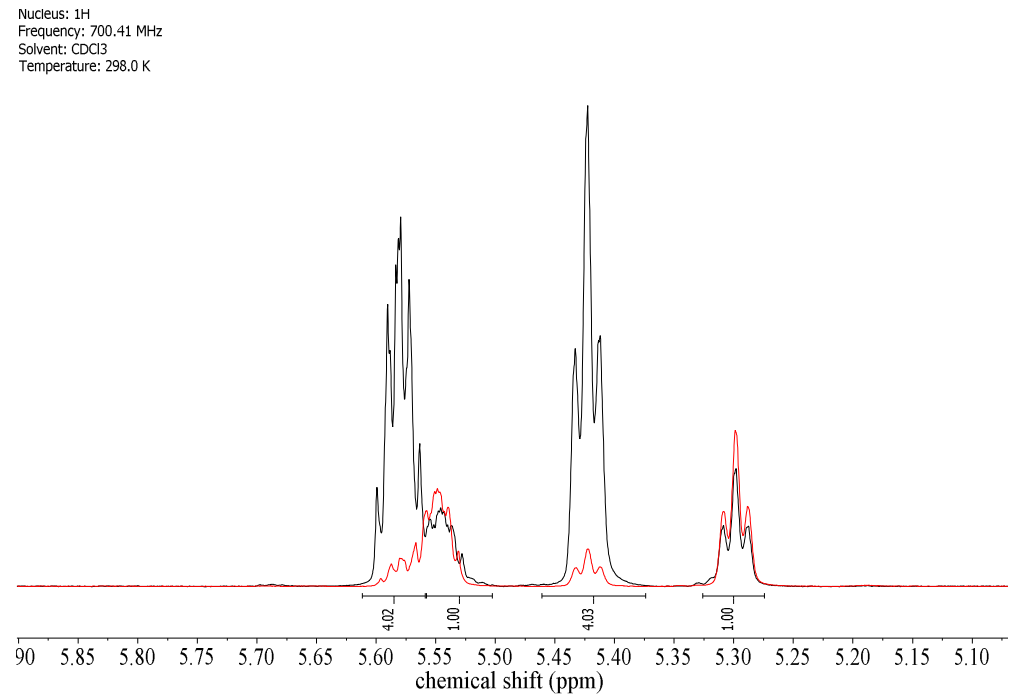

Figure 4. Superimposed ${ }^{1} \mathrm{H}$ NMR spectra of the two Mosher esters showing the $d r$ of 4:1, resulting out of the aldol reaction to compound 56.

$(5 R, 7 S, 10 R, E)-11-(($ tert-Butyldimethylsilyl)oxy)-1-((4-methoxybenzyl)oxy)-2,10-dimethylundec-2ene-5,7-diol (59)

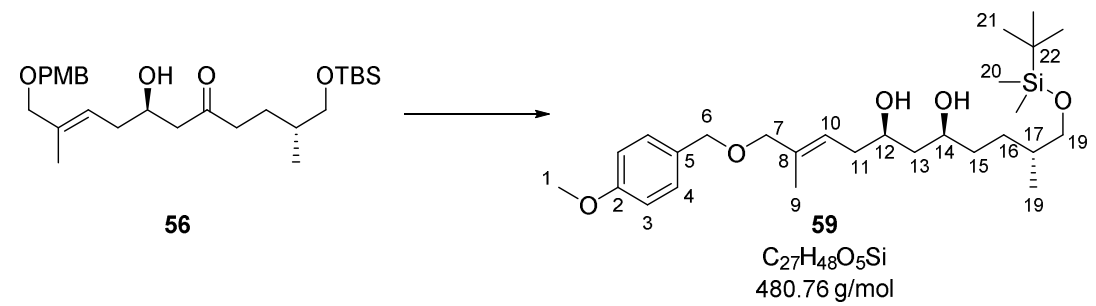

Alcohol 56 (29.0 mg, $60.6 \mu \mathrm{mol}, 1.00$ eq.) was dissolved in THF/methanol (4:1, $0.75 \mathrm{~mL})$ and cooled to $-78{ }^{\circ} \mathrm{C}$. $\mathrm{Et}_{2} \mathrm{BOMe}(66.6 \mu \mathrm{L}, 66.6 \mu \mathrm{mol}, 1.10$ eq. $1 \mathrm{M}$ in THF) was added slowly and the mixture was stirred $15 \mathrm{~min}$ at $-78{ }^{\circ} \mathrm{C}$ followed by the addition of $\mathrm{NaBH}_{4}(2.52 \mathrm{mg}, 66.6 \mu \mathrm{mol}, 1.10$ eq. $)$ and further stirring for $4 \mathrm{~h}$ at $-78^{\circ} \mathrm{C}$. The reaction mixture was allowed to warm to $0{ }^{\circ} \mathrm{C}$ and quenched with aq. $\mathrm{NaOH}(0.6 \mathrm{~mL}, 3 \mathrm{M})$ and $\mathrm{H}_{2} \mathrm{O}_{2}(0.8 \mathrm{~mL}, 35 \%)$. After stirring $3 \mathrm{~h}$ at $0{ }^{\circ} \mathrm{C}$ water $(1.5 \mathrm{~mL})$ and $\mathrm{Et}_{2} \mathrm{O}(4 \mathrm{~mL})$ were added the phases separated, the aqueous phase was extracted with $\mathrm{Et}_{2} \mathrm{O}(3 \times 5 \mathrm{~mL})$, the combined organic phases were washed with water $(10 \mathrm{~mL})$, dried over $\mathrm{MgSO}_{4}$ and the solvent was removed under reduced pressure. Purification of the crude product was achieved by flash chromatography $(40 \% \mathrm{EtOAc} / \mathrm{CyH})$ to yield the title compound $\mathbf{5 9}$ as a colorless oil $(28.5 \mathrm{mg}$, $59.3 \mu \mathrm{mol}, 98 \%, d r=4: 1$ resulting out of the aldol reaction to compound $\mathbf{5 6}$ ).

$\mathbf{R}_{f}=0.20(40 \% \mathrm{EtOAc} / \mathrm{CyH}) \cdot[\boldsymbol{\alpha}]_{\boldsymbol{D}}^{\mathbf{2 0}}=+2.9^{\circ}\left(c=1.02\right.$ in $\left.\mathrm{CH}_{2} \mathrm{Cl}_{2}\right) .{ }^{1} \mathbf{H} \mathbf{N M R}\left(500 \mathrm{MHz}, \mathrm{CD}_{2} \mathrm{Cl}_{2}\right) \delta=$ 7.25 (d, 2H, $J=8.8 \mathrm{~Hz}, \mathrm{H}-4), 6.87$ (d, 2H, $J=8.6 \mathrm{~Hz}, \mathrm{H}-3$ ), 5.47 (tq, $1 \mathrm{H}, J=7.3 \mathrm{~Hz}, 1.3 \mathrm{~Hz}, \mathrm{H}-10$ ), 4.36 (s, 2H, H-6), 3.89 (s, 2H, H-7), $3.88-3.84$ (m, 1H, H-12), $3.83-3.80$ (m, 1H, H-14), 3.79 (s, 3H, H-1), $3.48-3.37$ (m, 2H, H-19), 2.91 (br s, 2H, 2 x OH), 2.25 - 2.20 (m, 2H, H-11), 1.67 (s, 3H, H-9), 1.65 - 1.60 (m, 1H, H-13a), 1.59 - 1.56 (m, 1H, H-17), 1.52 - 1.37 (m, 4H, H-13b, H-15, H-16a), 1.14 - 1.05 (m, 1H, H-16b), 0.89 (s, 9H, H-21), 0.87 (d, 3H, $J=6.7$ Hz, H-18), 0.04 (s, 6H, H-20); ${ }^{13} \mathbf{C}-$ 
NMR (126 MHz, $\left.\mathrm{CD}_{2} \mathrm{Cl}_{2}, 298 \mathrm{~K}\right): \delta$ [ppm] = 159.7 (C-2), 136.0 (C-8), 131.4 (C-5), 129.8 (2C, C-4), 123.6 (C-10), 114.2 (2C, C-3), 76.3 (C-7), 73.9 (C-14), 73.3 (C-12), 71.9 (C-6), 68.7 (C-19), 55.8 (C1), 43.1 (C-13), 37.0 (C-11), 36.4 (C-17), 36.1 (C-15), 29.4 (C-16), 26.3 (3C, C-21), 18.8 (C-22), 17.1 (C-18), 14.5 (C-9), -5.1 (2C, C-20) ppm; HRMS (ESI+) $m / z$ : calcd for $\mathrm{C}_{27} \mathrm{H}_{48} \mathrm{O}_{5} \mathrm{SiH}^{+}[\mathrm{M}+\mathrm{H}]^{+}$: 481.3344, found: 481.3342 .

${ }^{1}$ H-NMR (500 MHz, MeOD): $\delta=7.25$ (d, 2H, $\left.J=8.5 \mathrm{~Hz}, \mathrm{H}-4\right), 6.89$ (d, 2H, $J=8.6 \mathrm{~Hz}, \mathrm{H}-3$ ), 5.54 (t, 1H, $J=7.3 \mathrm{~Hz}, \mathrm{H}-10), 4.37$ (s, 2H, H-6), 3.91 (s, 2H, H-7), $3.88-3.81$ (m, 1H, H-12), 3.78 (s, 3H, H-1), $3.76-3.70$ (m, 1H, H-14), $3.50-3.43$ (m, 2H, H-19), 2.26 (t, 2H, $J=7.0 \mathrm{~Hz}, 5.8 \mathrm{~Hz}$ ), 1.69 (s, 3H, H-9), 1.65 - 1.60 (m, 1H, H-13a), 1.58 - 1.51 (m, 4H, H-13b, H-15a, H-16a, H-17), 1.48 - 1.38 (m, 1H, H-13b), 1.15 - 1.07 (m, 1H, H-16b), $0.90-0.88$ (m, 12H, H-18, H-21), 0.04 (s, 6H, H-20) ppm; ${ }^{13}$ C-NMR (126 MHz, MeOD): $\delta=160.85$ (C-2), 135.30 (C-8), 131.60 (C-5), 130.68 (2C, C-4), 125.65 (C-10), 114.67 (2C, C-3), 76.97 (C-7), 72.21 (C-6), 71.92 (C-14), 71.55 (C-12), 69.29 (C-19), 55.68 (C-1), 44.12 (C-13), 37.14 (C-17), 36.82 (C-11), 36.05 (C-15), 29.97 (C-16), 26.43 (3C, C-21), 19.18 (C-22), 17.24 (C-18), 14.38 (C-9), -5.23 (2C, C-20) ppm.

tert-Butyl((R)-4-((4S,6R)-6-((E)-4-((4-methoxybenzyl)oxy)-3-methylbut-2-en-1-yl)-2,2-dimethyl1,3-dioxan-4-yl)-2-methylbutoxy)dimethylsilane (13)

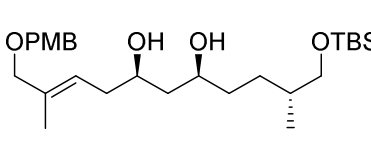

59

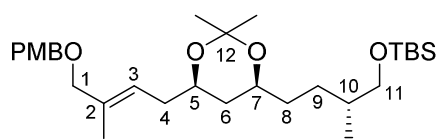

13

$\mathrm{C}_{30} \mathrm{H}_{52} \mathrm{O}_{5} \mathrm{Si}$

$520.83 \mathrm{~g} / \mathrm{mol}$

2,2-Dimethoxypropane ( $1 \mathrm{~mL}$ ) was added to diol 59 (28.5 $\mathrm{mg}, 59.3 \mu \mathrm{mol}, 1.00$ eq.) followed by the addition of CSA $(12.8 \mu \mathrm{L}, 0.59 \mu \mathrm{mol}, 0.01$ eq. $43 \mathrm{mM}$ in DCM). The reaction mixture was stirred $1.5 \mathrm{~h}$ at $\mathrm{rt}$ and quenched with the addition of $\mathrm{NEt}_{3}(20 \mu \mathrm{L})$. All volatiles were removed under reduced pressure. Purification of the crude product was achieved by flash chromatography $(10 \% \mathrm{EtOAc} / \mathrm{CyH})$ to yield the title compound $\mathbf{1 3}$ as a colorless oil $(29.5 \mathrm{mg}, 56.6 \mu \mathrm{mol}, 96 \%, d r=4: 1$ resulting out of the aldol reaction to compound 56).

$\mathbf{R}_{\boldsymbol{f}}=0.24(10 \% \mathrm{EtOAc} / \mathrm{CyH}) .[\boldsymbol{\alpha}]_{\boldsymbol{D}}^{\mathbf{2 0}}=+1.1^{\circ}\left(c=0.94\right.$ in $\left.\mathrm{CH}_{2} \mathrm{Cl}_{2}\right) .{ }^{1} \mathbf{H} \mathbf{N M R}\left(700 \mathrm{MHz}, \mathrm{CDCl}_{3}\right) \delta=$ $7.26\left(\mathrm{~d}, J=8.7 \mathrm{~Hz}, 2 \mathrm{H}, \mathrm{H}_{\text {arom }}\right), 6.87$ (d, $J=8.7 \mathrm{~Hz}, 2 \mathrm{H}, \mathrm{H}_{\text {arom. }}$ ), 5.45 (tq, $\left.J=7.4,1.1 \mathrm{~Hz}, 1 \mathrm{H}, \mathrm{H}-3\right)$, 4.38 (s, 2H, Ar- $\mathrm{CH}_{2}$ ), 3.88 (s, 2H, H-1), $3.84-3.82$ (m, 1H, H-5), 3.80 (s, 3H, O-CH - $_{3}, 3.77-3.73$ (m, 1H, H-7), 3.43 (dd, $J=9.8,6.0 \mathrm{~Hz}, 1 \mathrm{H}, \mathrm{H}-11$ ), 3.35 (dd, $J=9.8,6.6 \mathrm{~Hz}, 1 \mathrm{H}, \mathrm{H}-11$ '), 2.31 - 2.27 (m, 1H, H-4), $2.21-2.16$ (m, 1H, H-4'), 1.67 (s, 3H, 2-CH CH $_{3} 1.57$ - 1.43 (m, 5H, H-6, H-8, H-9, H10), 1.42 (s, 3H, 12- $\mathrm{CH}_{3}$ ), 1.39 (s, 3H, 12- $\mathrm{CH}_{3}$ ), 1.13 - 1.02 (m, 2H, H-6', H-9'), 0.89 (s, 9H, TBS), $0.87\left(\mathrm{~d}, J=6.7 \mathrm{~Hz}, 3 \mathrm{H}, 10-\mathrm{CH}_{3}\right), 0.03$ (s, 6H, TBS) ppm; ${ }^{13} \mathbf{C} \mathbf{N M R}\left(175 \mathrm{MHz}, \mathrm{CDCl}_{3}\right) \delta=159.3$ $\left(\mathrm{C}_{\text {arom. }} \mathrm{O}\right), 134.6(\mathrm{C}-2), 130.8\left(\mathrm{C}_{\text {arom }}\right), 129.55\left(\mathrm{C}_{\text {arom. }} \mathrm{H}\right), 123.4(\mathrm{C}-3), 113.9\left(\mathrm{C}_{\text {arom. }} \mathrm{H}\right), 98.6(\mathrm{C}-12), 76.0$ 
(C-1), $71.3\left(\mathrm{Ar}^{-} \mathrm{CH}_{2}\right), 69.6$ (C-7), 69.1 (C-5), 68.4 (C-11), $55.4\left(\mathrm{O}-\mathrm{CH}_{3}\right), 36.7$ (C-6), 35.9 (C-10), 35.1 (C-4), 34.0 (C-8), 30.4 (12- $\left.\mathrm{CH}_{3}\right), 28.5$ (C-9), 26.1 (TBS), 20.0 (12- $\left.\mathrm{CH}_{3}\right), 18.5$ (TBS), $16.8\left(10-\mathrm{CH}_{3}\right)$, $14.4\left(2-\mathrm{CH}_{3}\right),-5.2$ (TBS) ppm; HRMS (ESI+) $\mathrm{m} / z$ : calcd for $\mathrm{C}_{30} \mathrm{H}_{52} \mathrm{O}_{5} \mathrm{SiNH}_{4}{ }^{+}\left[\mathrm{M}+\mathrm{NH}_{4}\right]^{+}: 538.3922$, found: 538.3923 .

\section{Acetonide Analysis for Compound 59}

The determination of the correct relative stereochemistry of diol 59 (stereocenter at position 7) was accomplished by the accepted model for the analysis of the corresponding acetonide 13 . Thus, the ${ }^{13} \mathrm{C}$ NMR was used and analyzed showing different chemical shifts for the two methyl groups of the acetonide, which indicates a chair conformation, resulting out of the $s y n-1,3$-diol acetonide $\mathbf{1 3} .^{[18-21]}$

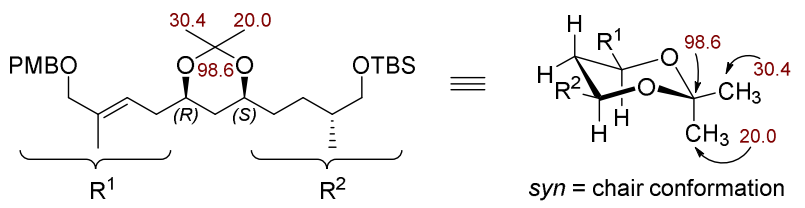

Figure 5. Relevant ${ }^{13} \mathrm{C}$ NMR signals used for the determination of the 1,3-syn-diol acetonide $\mathbf{1 3}$.

\section{(R)-4-((4S,6R)-6-((E)-4-((4-Methoxybenzyl)oxy)-3-methylbut-2-en-1-yl)-2,2-dimethyl-1,3-dioxan-} 4-yl)-2-methylbutan-1-ol (60)

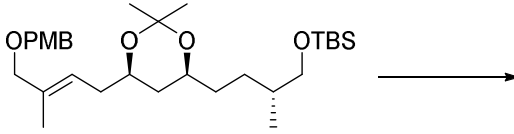

13

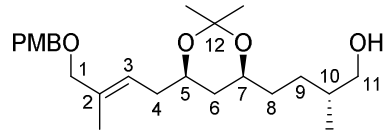

60

$\mathrm{C}_{24} \mathrm{H}_{38} \mathrm{O}_{5}$

$406.56 \mathrm{~g} / \mathrm{mo}$

TBS-Ether 13 (95.0 mg, $0.182 \mathrm{mmol}, 1.0$ eq.) was dissolved in THF $(12.0 \mathrm{~mL})$ and cooled to $0{ }^{\circ} \mathrm{C}$. Then, TBAF ( $1 \mathrm{M}$ in THF, $290 \mu \mathrm{L}, 0.29 \mathrm{mmol}, 1.6$ eq.) was added dropwise. The reaction mixture was stirred for $20 \mathrm{~min}$ at $0{ }^{\circ} \mathrm{C}$ and then $4.5 \mathrm{~h}$ at rt. Water $(20 \mathrm{~mL})$ and $\mathrm{Et}_{2} \mathrm{O}(20 \mathrm{~mL})$ were added and the phases were separated. The aqueous phase was extracted with $\mathrm{Et}_{2} \mathrm{O}(3 \times 30 \mathrm{~mL})$. The combined organic layers were dried over $\mathrm{Na}_{2} \mathrm{SO}_{4}$ and the solvent was removed under reduced pressure. The crude product was purified by column chromatography $(\mathrm{CyH} / \mathrm{EtOAc} 2: 1)$ and the product $\mathbf{6 0}$ was obtained as yellow oil $(73.8 \mathrm{mg}, 0.182 \mathrm{mmol}$, quant. $d r=4: 1$ resulting out of the aldol reaction to compound 56).

$\mathbf{R}_{\boldsymbol{f}}=0.27(50 \% \mathrm{EtOAc} / \mathrm{CyH}) \cdot[\boldsymbol{\alpha}]_{\boldsymbol{D}}^{\mathbf{2 0}}=+1.0^{\circ}\left(c=1.02\right.$ in $\left.\mathrm{CH}_{2} \mathrm{Cl}_{2}\right) .{ }^{1} \mathbf{H} \mathbf{N M R}\left(500 \mathrm{MHz}, \mathrm{CDCl}_{3}\right) \delta=$ $7.26\left(\mathrm{~d}, J=8.6 \mathrm{~Hz}, 2 \mathrm{H}, \mathrm{H}_{\text {arom. }}\right), 6.88\left(\mathrm{~d}, J=8.6 \mathrm{~Hz}, 2 \mathrm{H}, \mathrm{H}_{\text {arom. }}\right), 5.44$ (t, $\left.J=7.4 \mathrm{~Hz}, 1 \mathrm{H}, \mathrm{H}-3\right), 4.38$ (s, $\left.2 \mathrm{H}, \mathrm{Ar}-\mathrm{CH}_{2}\right), 3.88$ (s, 2H, H-1), $3.86-3.82(\mathrm{~m}, 1 \mathrm{H}, \mathrm{H}-5), 3.80$ (s, 3H, O-CH $\mathrm{CH}_{3}, 3.78-3.75(\mathrm{~m}, 1 \mathrm{H}, \mathrm{H}-$ 7), $3.50-3.42$ (m, 2H, H-11), $2.32-2.27$ (m, 1H, H-4), $2.21-2.15$ (m, 1H, H-4'), 1.67 (s, 3H, 2$\mathrm{CH}_{3}$ ), 1.63 - 1.45 (m, 5H, H-6, H-8, H-9, H-10), 1.43 (s, 3H, 12- $\mathrm{CH}_{3}$ ), 1.39 (s, 3H, 12- $\left.\mathrm{CH}_{3}\right), 1.16$ $1.11\left(\mathrm{~m}, 2 \mathrm{H}, \mathrm{H}-6\right.$ ', H-9'), $0.91\left(\mathrm{~d}, J=6.7 \mathrm{~Hz}, 3 \mathrm{H}, 10-\mathrm{CH}_{3}\right) \mathrm{ppm} ;{ }^{13} \mathbf{C} \mathbf{N M R}\left(125 \mathrm{MHz}, \mathrm{CDCl}_{3}\right) \delta=$ $159.3\left(\mathrm{C}_{\text {arom. }} \mathrm{O}\right), 134.6(\mathrm{C}-2), 130.8\left(\mathrm{C}_{\text {arom. }}\right), 129.5\left(\mathrm{C}_{\text {arom. }} \mathrm{H}\right), 123.3(\mathrm{C}-3), 113.9\left(\mathrm{C}_{\text {arom. }} \mathrm{H}\right), 98.6(\mathrm{C}-12)$, 
75.9 (C-1), $71.3\left(\mathrm{Ar}^{-} \mathrm{CH}_{2}\right), 69.6$ (C-7), 69.1 (C-5), $68.2(\mathrm{C}-11), 55.4\left(\mathrm{O}-\mathrm{CH}_{3}\right), 36.7$ (C-6), 35.9 (C-10), 35.0 (C-4), 33.9 (C-8), $30.4\left(12-\mathrm{CH}_{3}\right), 28.5(\mathrm{C}-9), 20.0\left(12-\mathrm{CH}_{3}\right), 16.8\left(10-\mathrm{CH}_{3}\right), 14.4\left(2-\mathrm{CH}_{3}\right)$ ppm; HRMS (ESI+) $m / z$ : calcd for $\mathrm{C}_{24} \mathrm{H}_{38} \mathrm{O}_{5} \mathrm{NH}_{4}{ }^{+}\left[\mathrm{M}+\mathrm{NH}_{4}\right]^{+}: 424.3057$, found: 424.3068 . 


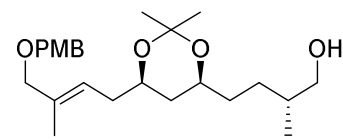

60

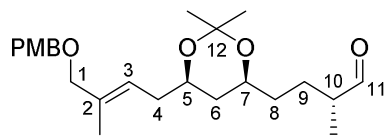

61

$\mathrm{C}_{24} \mathrm{H}_{36} \mathrm{O}_{5}$

$404.55 \mathrm{~g} / \mathrm{mol}$

Alcohol 60 (16.0 mg, $39.4 \mathrm{mmol}, 1.0$ eq.) was dissolved in dry DMSO (0.5 mL). IBX (24.0 mg, $85.7 \mu \mathrm{mol}, 2.2 \mathrm{eq}$.) was added at $\mathrm{rt}$ and the reaction mixture was stirred for $2 \mathrm{~h}$ at r.t. Afterwards, DCM $(2 \mathrm{~mL})$ was added and the suspension was filtrated over Celite ${ }^{\circledR}$. Water $(10 \mathrm{~mL})$ was added and the phases were separated. The aqueous phase was extracted with DCM $(3 \times 10 \mathrm{~mL})$. The combined organic layers were dried over $\mathrm{MgSO}_{4}$ and the solvent was removed under reduced pressure. The crude product $\mathbf{6 1}$ was directly used in the next reaction.

$\mathbf{R}_{\boldsymbol{f}}=0.36(30 \% \mathrm{EtOAc} / \mathrm{CyH}) .{ }^{1} \mathbf{H} \mathbf{N M R}\left(500 \mathrm{MHz}, \mathrm{CDCl}_{3}\right) \delta=9.62(\mathrm{t}, J=1.9 \mathrm{~Hz}, 1 \mathrm{H}, \mathrm{H}-11), 7.26(\mathrm{~d}$, $\left.J=8.6 \mathrm{~Hz}, 2 \mathrm{H}, \mathrm{H}_{\text {arom. }}\right), 6.88$ (d, $\left.J=8.6 \mathrm{~Hz}, 2 \mathrm{H}, \mathrm{H}_{\text {arom }}\right), 5.44$ (t, $\left.J=7.4 \mathrm{~Hz}, 1 \mathrm{H}, \mathrm{H}-3\right), 4.39$ (s, 2H, Ar$\mathrm{CH}_{2}$ ), 3.88 (s, 2H, H-1), 3.86 - 3.82 (m, 1H, H-5), 3.81 (s, 3H, O-CH $\mathrm{CH}_{3}, 3.79-3.76$ (m, 1H, H-7), 2.37 -2.20 (m, 1H, H-4), $1.87-1.20$ (m, 7H, H-6, H-8, H-9, H-10) 1.67 (s, 3H, 2-CH CH $_{3} 1.42$ (s, 3H, 12$\left.\mathrm{CH}_{3}\right), 1.39\left(\mathrm{~s}, 3 \mathrm{H}, 12-\mathrm{CH}_{3}\right), 1.10\left(\mathrm{~d}, J=7.0 \mathrm{~Hz}, 3 \mathrm{H}, 10-\mathrm{CH}_{3}\right) \mathrm{ppm}$; HRMS (ESI+) $\mathrm{m} / \mathrm{z}$ : calcd for $\mathrm{C}_{24} \mathrm{H}_{36} \mathrm{O}_{5} \mathrm{NH}_{4}^{+}\left[\mathrm{M}+\mathrm{NH}_{4}\right]^{+}: 422.2901$, found: 422.2903 . Due to instability, the compound was freshly prepared and used for the next reaction without further analysis.

(4R)-6-((4S,6R)-6-((E)-4-((4-Methoxybenzyl)oxy)-3-methylbut-2-en-1-yl)-2,2-dimethyl-1,3dioxan-4-yl)-4-methylhexan-3-ol (62)

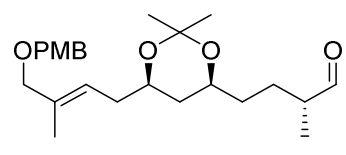

61

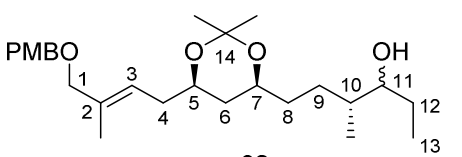

62

$\mathrm{C}_{26} \mathrm{H}_{42} \mathrm{O}_{5}$

$434.62 \mathrm{~g} / \mathrm{mol}$

Aldehyde 61 (max. $15.9 \mathrm{mg}, 39.4 \mu \mathrm{mol}, 1.0$ eq.) was dissolved in THF $(1 \mathrm{~mL})$ and cooled to $0{ }^{\circ} \mathrm{C}$. EtMgBr $(0.9 \mathrm{M}$ in THF, $0.100 \mathrm{~mL}, 90.0 \mu \mathrm{mol}, 2.3$ eq. $)$ was added dropwise and the reaction was stirred for $1 \mathrm{~h}$ at $0{ }^{\circ} \mathrm{C}$. Afterwards, EtMgBr $(0.9 \mathrm{~m}$ in THF, $0.100 \mathrm{~mL}, 90.0 \mu \mathrm{mol}, 2.3$ eq. $)$ was added again. After further stirring for $2 \mathrm{~h}$ at $0{ }^{\circ} \mathrm{C}, \mathrm{NH}_{4} \mathrm{Cl}$-solution (aq., sat., $\left.10 \mathrm{~mL}\right)$ and EtOAc $(10 \mathrm{~mL})$ were added. The phases were separated and the aqueous phase was extracted with DCM $(3 \times 10 \mathrm{~mL})$. The combined organic phases were dried over $\mathrm{MgSO}_{4}$ and the solvent was removed under reduced pressure. The crude product was purified by column chromatography (CyH/EtOAc7:3) and the product $\mathbf{6 2}$ was obtained as yellow oil (9.08 $\mathrm{mg}, 20.9 \mathrm{mmol}, 53 \%$ over 2 steps). 
$\mathbf{R}_{\boldsymbol{f}}=0.21(30 \% \mathrm{EtOAc} / \mathrm{CyH}) \cdot[\boldsymbol{\alpha}]_{\boldsymbol{D}}^{\mathbf{2 0}}=+3.8^{\circ}\left(c=0.78\right.$ in $\left.\mathrm{CH}_{2} \mathrm{Cl}_{2}\right) .{ }^{\mathbf{1}} \mathbf{H} \mathbf{N M R}\left(700 \mathrm{MHz}, \mathrm{CDCl}_{3}\right) \delta=$ $7.26\left(\mathrm{~d}, J=8.6 \mathrm{~Hz}, 2 \mathrm{H}, \mathrm{H}_{\text {arom. }}\right), 6.88$ (d, $J=8.6 \mathrm{~Hz}, 2 \mathrm{H}, \mathrm{H}_{\text {arom. }}$ ), 5.45 (t, $\left.J=7.4 \mathrm{~Hz}, 1 \mathrm{H}, \mathrm{H}-3\right), 4.39$ (s, $\left.2 \mathrm{H}, \mathrm{Ar}-\mathrm{CH}_{2}\right), 3.88$ (s, 2H, H-1), $3.84-3.83$ (m, 1H, H-5), 3.80 (s, 3H, O-CH CH $_{3} 3.78-3.75$ (m, 1H, H7), $3.45-3.33$ (m, 1H, H-11), $2.31-2.28$ (m, 1H, H-4), $2.22-2.16$ (m, 1H, H-4'), 1.67 (s, 3H, 2$\mathrm{CH}_{3}$ ), $1.58-1.44$ (m, 7H, H-6, H-8, H-9, H-10, H-12), 1.43 (s, 3H, 14-CH $\mathrm{CH}_{3}$, 1.39 (s, 3H, 14-CH ( $_{3}$, $1.17-1.10$ (m, 2H, H-6', H-9'), $0.97-0.93$ (m, 3H, H-13), $0.89-0.86$ (m, 3H, 10-CH ${ }_{3}$ ppm; ${ }^{13} \mathbf{C}$ NMR $\left(175 \mathrm{MHz}, \mathrm{CDCl}_{3}\right) \delta=159.3,134.6,130.8,129.5,123.3,113.9,98.6,76.5,76.4,75.9,71.3$, 69.7, 69.4, 69.1, 55.4, 38.7, 38.4, 38.0, 37.8, 36.8, 36.7, 35.0, 34.4, 34.3, 34.2, 30.4, 28.9, 28.7, 27.4, $27.2,27.1,26.5,20.0,20.0,15.7,14.4,13.7,13.5,10.8,10.7,10.5 \mathrm{ppm}$; Due to the extended stereoisomerism, the carbon atoms were not assigned at this stage. HRMS (ESI+) $\mathrm{m} / \mathrm{z}$ : calcd for $\mathrm{C}_{26} \mathrm{H}_{42} \mathrm{O}_{5} \mathrm{H}^{+}[\mathrm{M}+\mathrm{H}]^{+}$: 435.3105, found: 435.3106.

(R)-6-((4S,6R)-6-((E)-4-((4-Methoxybenzyl)oxy)-3-methylbut-2-en-1-yl)-2,2-dimethyl-1,3-dioxan4-yl)-4-methylhexan-3-one (14)

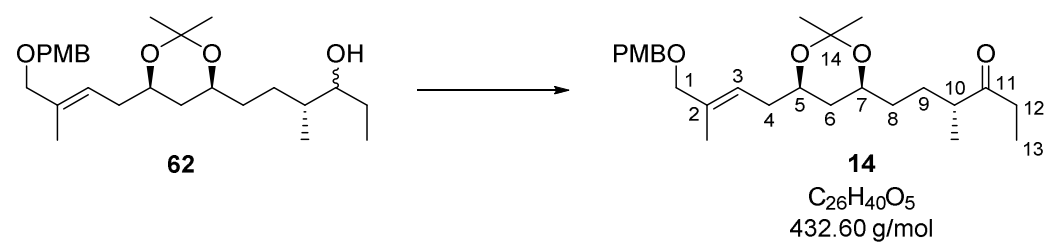

$\mathrm{Py} \cdot \mathrm{SO}_{3}$ (31.4 mg, $197 \mu \mathrm{mol}, 4.0$ eq.) was dried for $1 \mathrm{~h}$ at high vacuum. Afterwards, dry DCM (0.5 mL), DIPEA ( $40.0 \mu \mathrm{L}, 235 \mu \mathrm{mol}, 4.8$ eq.) and DMSO (40.0 $\mu \mathrm{L}, 563 \mathrm{mmol}, 11.4$ eq.) were added. The reaction mixture was cooled to $0{ }^{\circ} \mathrm{C}$ and alcohol $62(21.5 \mathrm{mg}, 49.5 \mu \mathrm{mol}, 1.0 \mathrm{eq}$.) was added. The reaction mixture was stirred for $30 \mathrm{~min}$ at $0{ }^{\circ} \mathrm{C}$. Afterwards, water $(15 \mathrm{~mL})$ and DCM $(15 \mathrm{~mL})$ were added. The phases were separated and the aqueous phase was extracted with DCM $(3 \times 20 \mathrm{~mL})$. The combined organic layers were dried over $\mathrm{MgSO}_{4}$ and the solvent was removed under reduced pressure. The crude product was purified by column chromatography $\left(\mathrm{SiO}_{2}\right.$, cyclohexane/ethyl acetate $\left.4: 1\right)$ and the product 14 was obtained as yellow oil (16.5 mg, $38.1 \mathrm{mmol}, 77 \%)$.

$\mathbf{R}_{f}=0.20(20 \% \mathrm{EtOAc} / \mathrm{CyH}) \cdot[\boldsymbol{\alpha}]_{\boldsymbol{D}}^{\mathbf{2 0}}=-6.9^{\circ}\left(c=0.58\right.$ in $\left.\mathrm{CH}_{2} \mathrm{Cl}_{2}\right) .{ }^{\mathbf{1}} \mathbf{H} \mathbf{N M R}\left(700 \mathrm{MHz}, \mathrm{CDCl}_{3}\right) \delta=$ $7.26\left(\mathrm{~d}, J=8.6 \mathrm{~Hz}, 2 \mathrm{H}, \mathrm{H}_{\text {arom }}\right), 6.88$ (d, $\left.J=8.6 \mathrm{~Hz}, 2 \mathrm{H}, \mathrm{H}_{\text {arom }}\right), 5.44$ (t, $\left.J=7.4 \mathrm{~Hz}, 1 \mathrm{H}, \mathrm{H}-3\right), 4.38$ (s, $\left.2 \mathrm{H}, \mathrm{Ar}-\mathrm{CH}_{2}\right), 3.88$ (s, 2H, H-1), $3.85-3.82(\mathrm{~m}, 1 \mathrm{H}, \mathrm{H}-5), 3.81$ (s, 3H, O-CH $), 3.77-3.73(\mathrm{~m}, 1 \mathrm{H}, \mathrm{H}-$ 7), $2.54-2.41$ (m, 3H, H-10, H-12), $2.31-2.27$ (m, 1H, H-4), 2.20 - 2.16 (m, 1H, H-4'), 1.75 - 1.71 (m, 1H, H-9), $1.64-1.28$ (m, 5H, H-6, H-8, H-9'), 1.67 (s, 3H, 2- $\mathrm{CH}_{3}$ ), 1.41 (s, 3H, 14-CH CH $_{3}, 1.38$ (s, $\left.3 \mathrm{H}, 14-\mathrm{CH}_{3}\right), 1.07\left(\mathrm{~d}, J=6.9 \mathrm{~Hz}, 3 \mathrm{H}, 10-\mathrm{CH}_{3}\right), 1.04(\mathrm{t}, J=7.3 \mathrm{~Hz}, 3 \mathrm{H}, \mathrm{H}-13) \mathrm{ppm} ;{ }^{13} \mathbf{C} \mathbf{~ N M R}$ $\left(175 \mathrm{MHz}, \mathrm{CDCl}_{3}\right) \delta=215.4(\mathrm{C}-11), 159.3\left(\mathrm{C}_{\text {arom. }} \mathrm{O}\right), 134.7(\mathrm{C}-2), 130.8\left(\mathrm{C}_{\text {arom. }}\right), 129.5\left(\mathrm{C}_{\text {arom. }} \mathrm{H}\right)$, $123.2(\mathrm{C}-3), 113.9\left(\mathrm{C}_{\text {arom. }} \mathrm{H}\right), 98.6(\mathrm{C}-14), 75.9$ (C-1), $71.3\left(\mathrm{Ar}^{\left.-\mathrm{CH}_{2}\right)}, 69.1(\mathrm{C}-7), 69.0(\mathrm{C}-5), 55.4(\mathrm{O}-\right.$ $\mathrm{CH}_{3}$ ), 46.1 (C-10), 36.6 (C-6), 35.0 (C-4), 34.4 (C-12), 34.2 (C-8), 30.4 (14- $\mathrm{CH}_{3}$ ), 28.4 (C-9), 20.0 
(14- $\left.\mathrm{CH}_{3}\right), 16.7\left(10-\mathrm{CH}_{3}\right), 14.4\left(2-\mathrm{CH}_{3}\right), 7.9(\mathrm{C}-13)$ ppm; HRMS (ESI+) $\mathrm{m} / z$ : calcd for $\mathrm{C}_{26} \mathrm{H}_{40} \mathrm{O}_{5} \mathrm{NH}_{4}{ }^{+}$ $\left[\mathrm{M}+\mathrm{NH}_{4}\right]^{+}: 450.3214$, found: 450.3217 .

(E)-4-((4R,6S)-6-((R)-4-((tert-Butyldimethylsilyl)oxy)-3-methylbutyl)-2,2-dimethyl-1,3-dioxan-4yl)-2-methylbut-2-enal (63)

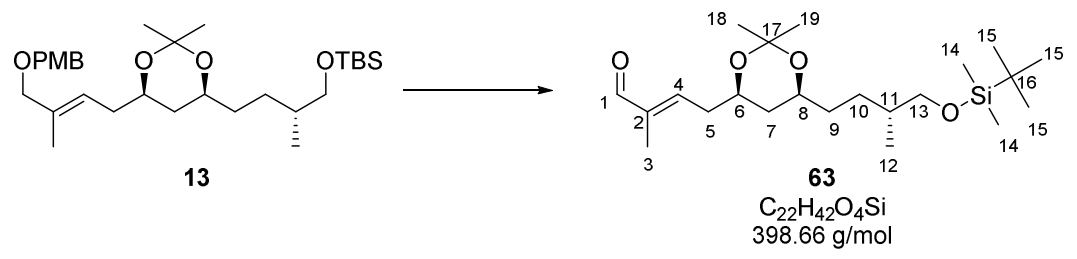

Compound 13 (29.0 mg, $55.7 \mu \mathrm{mol}, 1.0$ eq.) was dissolved in DCM (2 mL) and pH 7 buffer $(0.2 \mathrm{~mL})$. DDQ (40.4 mg, $178 \mu \mathrm{mol}, 3.2 \mathrm{eq}$.) was added in one portion and the reaction mixture was stirred for $4 \mathrm{~h}$ at r.t. Afterwards, water $(10 \mathrm{~mL})$ and DCM $(10 \mathrm{~mL})$ were added to the red suspension, the phases separated and the aqueous phase was extracted with DCM $(3 \times 20 \mathrm{~mL})$. The combined organic layers were dried over $\mathrm{MgSO}_{4}$ and the solvent was removed under reduced pressure. The crude product was purified by column chromatography (cyclohexane/ethyl acetate 85:15) and the product $\mathbf{6 3}$ was obtained as yellow oil (17.4 mg, $43.6 \mu \mathrm{mol}, 78 \%$ ).

$\mathbf{R}_{\mathbf{f}}=0.46$ (cyclohexane/ethyl acetate $\left.4: 1\right) ;[\boldsymbol{\alpha}]_{\mathbf{D}}{ }^{20}=+4.7^{\circ}\left(\mathrm{c}=1.07, \mathrm{CH}_{2} \mathrm{Cl}_{2}\right) ;{ }^{1} \mathbf{H}-\mathbf{N M R}(700 \mathrm{MHz}$, $\mathrm{CD}_{2} \mathrm{Cl}_{2}$ ): $\delta=9.41$ (s, 1H, H-1), 6.56 (tq, $\left.1 \mathrm{H}, J=7.3 \mathrm{~Hz}, 1.4 \mathrm{~Hz}, \mathrm{H}-4\right), 4.04-3.97$ (m, 1H, H-6), 3.82 3.77 (m, 1H, H-8), 3.46 - 3.36 (m, 2H, H-13), 2.51 - 2.46 (m, 2H, H-5), 1.72 (d, 3H, J=1.1 Hz H-3), $1.57-1.53$ (m, 2H, H-7a, H-11), $1.51-1.48$ (m, 1H, H-10a), $1.46-1.43$ (m, 2H, H-9), 1.42 (s, 3H, H-18), 1.33 (s, 3H, H-19), 1.19 - 1.10 (m, 1H, H-7b), 1.08 - 0.99 (m, 1H, H-10b), 0.89 (s, 9H, H-15), 0.86 (d, 3H, $J=6.6 \mathrm{~Hz}, \mathrm{H}-12), 0.04$ (s, 6H, H-14) ppm; ${ }^{13} \mathbf{C}-\mathbf{N M R}\left(175 \mathrm{MHz}, \mathrm{CD}_{2} \mathrm{Cl}_{2}\right): \delta=195.4$ (C-1), 150.4 (C-4), 141.2 (C-2), 99.1 (C-17), 69.7 (C-8), 68.7 (C-13), 68.5 (C-6), 37.2 (C-7), 36.5 (C-5), 36.4 (C-11), 34.4 (C-9), 30.5 (C-19), 28.9 (C-10), 26.3 (3C, C-15), 20.1 (C-18), 18.8 (C-16), 17.0 (C-12), 9.7 (C-3), -5.1 (2C, C-14) ppm; HRMS (ESI+) $\mathrm{m} / z:\left[\mathrm{M}+\mathrm{NH}_{4}\right]^{+}$Calcd. for $\mathrm{C}_{22} \mathrm{H}_{42} \mathrm{O}_{4} \mathrm{SiNH}_{4}^{+}$416.3191, found 416.3189. 


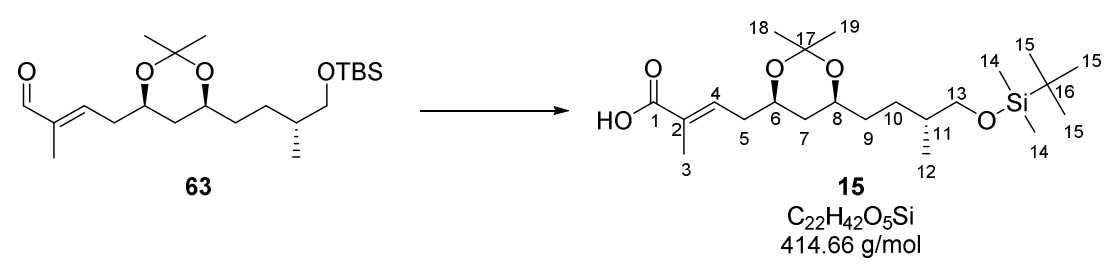

Aldehyde $63 \quad(16.2 \mathrm{mg}, \quad 40.6 \mu \mathrm{mol}, \quad 1.0$ eq. $)$ was dissolved in tert-butanol $(6 \mathrm{~mL})$ and 2-methyl-2-butene ( $2 \mathrm{M}$ in THF, $162 \mu \mathrm{L}, 0.325 \mathrm{mmol}, 8.0$ eq.) was added. $\mathrm{NaClO}_{2}(80 \%, 44.8 \mathrm{mg}$, 0.396 mmol, 9.8 eq.) and $\mathrm{NaH}_{2} \mathrm{PO}_{4}(49.7 \mathrm{mg}, 0.319$ mmol, 7.8 eq.) were dissolved in water $(0.5 \mathrm{~mL})$ and added dropwise to the dissolved aldehyde. The reaction mixture was stirred for $50 \mathrm{~min}$ at r.t. Afterwards, the reaction was quenched by the addition of $\mathrm{NH}_{4} \mathrm{Cl}$-solution $\left(1 \mathrm{M}\right.$ in $\left.\mathrm{H}_{2} \mathrm{O}, 0.8 \mathrm{~mL}\right)$. Water $(10 \mathrm{~mL})$ and DCM $(10 \mathrm{~mL})$ were added and the phases were separated. The aqueous phase was extracted with DCM $(5 \times 12 \mathrm{~mL})$. The combined organic layers were dried over $\mathrm{MgSO}_{4}$ and the solvent was removed under reduced pressure. The crude product was purified by column chromatography $\left(\mathrm{SiO}_{2}\right.$, cyclohexane/ethyl acetate 3:1) and the product 15 was obtained as yellow oil (16.1 mg, $38.8 \mu \mathrm{mol}, 96 \%)$.

$\mathbf{R}_{\mathbf{f}}=0.46$ (cyclohexane/ethyl acetate $\left.4: 1\right) ;[\boldsymbol{\alpha}]_{\mathbf{D}}{ }^{20}=+2.3^{\circ}\left(\mathrm{c}=1.36, \mathrm{CH}_{2} \mathrm{Cl}_{2}\right) ;{ }^{1} \mathbf{H}-\mathbf{N M R}(700 \mathrm{MHz}$, $\mathrm{CD}_{2} \mathrm{Cl}_{2}$ ): $\delta=6.89$ (tq, $\left.1 \mathrm{H}, J=7.3 \mathrm{~Hz}, 1.2 \mathrm{~Hz}, \mathrm{H}-4\right), 3.99-3.93(\mathrm{~m}, 1 \mathrm{H}, \mathrm{H}-6), 3.80-3.75(\mathrm{~m}, 1 \mathrm{H}$, $\mathrm{H}-8), 3.47-3.35$ (m, 2H, H-13), $2.41-2.30$ (m, 2H, H-5), 1.84 (d, 3H, J=1.2 Hz, H-3), $1.60-1.47$ (m, 3H, H-7a, H-10a, H-11) 1.45 - 1.42 (m, 2H, H-9), 1.42 (s, 3H, H-18), 1.33 (s, 3H, H-19), 1.15 1.08 (m, 1H, H-7b), $1.07-1.00$ (m, 1H, H-10b), 0.89 (s, 9H, H-15), 0.87 (d, 3H, $J=6.6 \mathrm{~Hz}, \mathrm{H}-12$ ), 0.04 (s, 6H, H-14) ppm; ${ }^{13} \mathrm{C}-\mathrm{NMR}\left(175 \mathrm{MHz}, \mathrm{CD}_{2} \mathrm{Cl}_{2}\right): \delta=170.9$ (C-1), 140.8 (C-4), 128.9 (C-2), 99.0 (C-17), 69.8 (C-8), 68.74 (C-13), 68.68 (C-6), 37.3 (C-7), 36.5 (C-5), 36.4 (C-11), 34.4 (C-9), 30.5 (C-19), 28.9 (C-10), 26.3 (3C, C-15), 20.1 (C-18), 18.8 (C-16), 17.0 (C-12), 12.8 (C-3), -5.1 (2C, C-14) ppm; HRMS (ESI+) $m / z$ : $[\mathrm{M}+\mathrm{H}]^{+}$Calcd. for $\mathrm{C}_{22} \mathrm{H}_{42} \mathrm{O}_{5} \mathrm{SiH}^{+} 415.2874$, found 415.2867. 

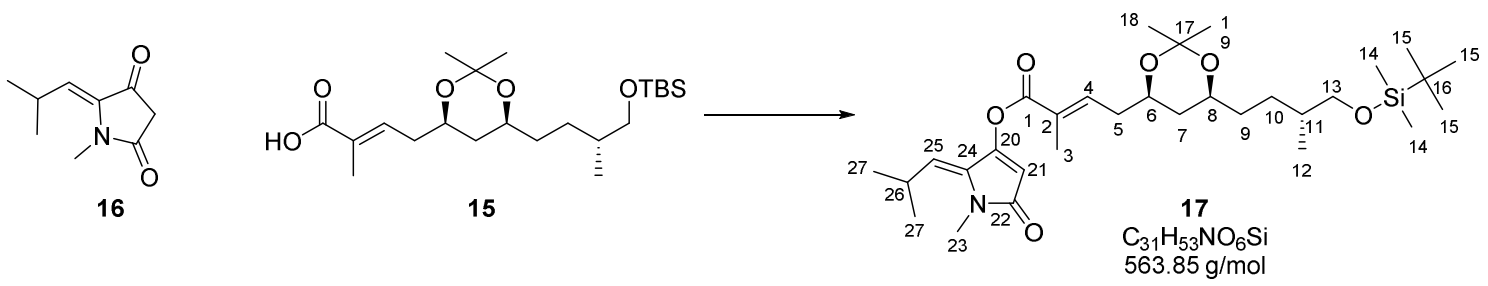

Carboxylic acid 15 (21.7 mg, $52.3 \mu \mathrm{mol}, 1.2$ eq.) was dissolved in dry DCM (0.5 mL) and cooled to $0{ }^{\circ} \mathrm{C}$. Then, DMAP $(2.2 \mathrm{mg}, 18.0 \mu \mathrm{mol}, 0.3$ eq. $)$ and DCC (1M in DCM, $\left.54.0 \mu \mathrm{L}, 54.0 \mu \mathrm{mol}, 1.2 \mathrm{eq}.\right)$ were added and the reaction mixture was stirred for $5 \mathrm{~min}$ at $0{ }^{\circ} \mathrm{C}$. The tetramic acid $16(7.29 \mathrm{mg}$, $43.6 \mu \mathrm{mol}, 1.0$ eq.) was added at $0{ }^{\circ} \mathrm{C}$. The reaction mixture was allowed to warm up to r.t. and stirring was continued overnight. Then, it was filtered over Celite ${ }^{\circledR}$ and washed with ethyl acetate. The product 17 was obtained after column chromatography $\left(\mathrm{SiO}_{2}\right.$, cyclohexane/ethyl acetate 85:15) as yellow oil (18.9 mg, $33.5 \mu \mathrm{mol}, 77 \%)$.

$\mathbf{R}_{\mathbf{f}}=0.34$ (cyclohexane/ethyl acetate 7:3); $[\boldsymbol{\alpha}]_{\mathbf{D}}{ }^{20}=+4.3^{\circ}\left(\mathrm{c}=0.93, \mathrm{CH}_{2} \mathrm{Cl}_{2}\right) ;{ }^{1} \mathbf{H}-\mathbf{N M R}(700 \mathrm{MHz}$, $\left.\mathrm{CD}_{2} \mathrm{Cl}_{2}\right): \delta=7.00(\mathrm{tq}, 1 \mathrm{H}, J=7.1 \mathrm{~Hz}, 1.5 \mathrm{~Hz}, \mathrm{H}-4), 6.14(\mathrm{~d}, 1 \mathrm{H}, J=1.4 \mathrm{~Hz}, \mathrm{H}-21), 5.23(\mathrm{dd}, 1 \mathrm{H}$, $J=10.0 \mathrm{~Hz}, 1.4 \mathrm{~Hz}, \mathrm{H}-25), 4.01-3.96$ (m, 1H, H-6), $3.81-3.77$ (m, 1H, H-8), 3.45 - 3.36 (m, 2H, H-13), $3.31-3.23$ (m, 1H, H-26), 3.02 (s, 3H, H-23), $2.48-2.37$ (m, 2H, H-5), 1.94 (d, 3H, $J=1.2 \mathrm{~Hz}, \mathrm{H}-3), 1.57-1.55$ (m, 2H, H-7a, H-11), $1.52-1.49$ (m, 1H, H-10a), $1.46-1.42$ (m, 2H, H-9), 1.42 (s, 3H, H-18), 1.33 (s, 3H, H-19), $1.18-1.13$ (m, 1H, H-7b), 1.12 (2d, 6H, $J=6.6 \mathrm{~Hz}$, H-27), $1.07-1.01$ (m, 1H, H-10b), 0.89 (s, 9H, H-15), 0.87 (d, 3H, $J=6.7$ Hz, H-12), 0.03 (s, 6H, H-14) ppm; ${ }^{13}$ C-NMR (175 MHz, $\mathrm{CD}_{2} \mathrm{Cl}_{2}$ ): $\delta=168.0$ (C-22), 164.3 (C-1), 155.1 (C-24), $142.6(\mathrm{C}-4)$, 133.9 (C-20), 128.8 (C-2), 122.7 (C-25), 108.5 (C-21), 99.1 (C-17), 69.7 (C-8), 68.7 (C-13), 68.6 (C-6), 37.3 (C-7), 36.7 (C-5), 36.4 (C-11), 34.4 (C-9), 30.5 (C-19), 28.9 (C-10), 26.7 (C-26), 26.3 (3C, C-15), 25.2 (C-23), 24.30/24.28 (C-27), 20.1 (C-18), 18.8 (C-16), 17.0 (C-12), 13.2 (C-3), -5.1 (2C, C-14) ppm; HRMS (ESI+) $m / z$ : [M+H] ${ }^{+}$Calcd. for $\mathrm{C}_{31} \mathrm{H}_{53} \mathrm{NO}_{6} \mathrm{SiH}^{+}$564.3715, found 564.3707. 
(Z)-3-((E)-4-((4R,6S)-6-((R)-4-((tert-Butyldimethylsilyl)oxy)-3-methylbutyl)-2,2-dimethyl-1,3dioxan-4-yl)-2-methylbut-2-enoyl)-4-hydroxy-1-methyl-5-(2-methylpropylidene)-1,5-dihydro$2 H$-pyrrol-2-one (2)

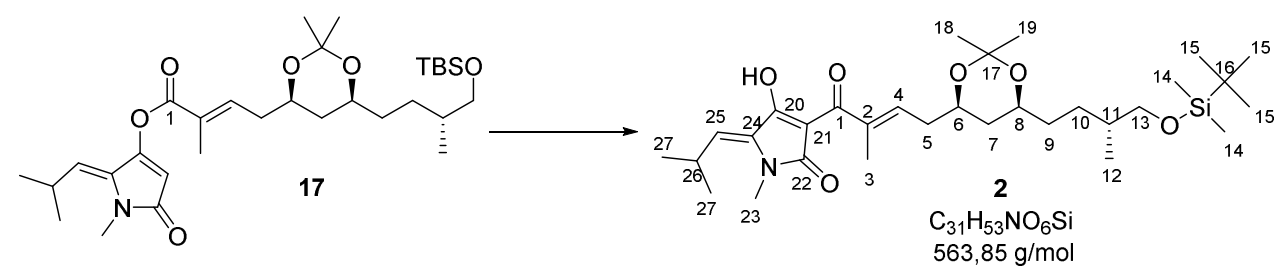

Ester 17 (13.5 mg, $23.9 \mu \mathrm{mol}, 1.0$ eq.) was dissolved in dry DCM (0.5 mL). NEt 3 (5.0 $\mu \mathrm{L}, 36.1 \mu \mathrm{mol}$, 1.5 eq.) was added and stirring at r.t. was continued for $5 \mathrm{~min}$. Afterwards, $\mathrm{CaCl}_{2}(5.0 \mathrm{mg}, 45.1 \mu \mathrm{mol}$, 1.9 eq.) and DMAP (1.2 mg, $9.8 \mu \mathrm{mol}, 0.4$ eq.) were added and the suspension was stirred for $24 \mathrm{~h}$ at r.t. DCM $(20 \mathrm{~mL})$ and $\mathrm{H}_{2} \mathrm{O}(20 \mathrm{~mL})$ were added and the aqueous phase was acidified with $1 \mathrm{M}$ aq. $\mathrm{HCl}$. The phases were separated and the aqueous phase was extracted with DCM $(3 \times 20 \mathrm{~mL})$. The combined organic phases were dried over $\mathrm{MgSO}_{4}$ and the solvent was removed under reduced pressure. The product 2 was obtained as yellow/orange oil (13.5 mg, $23.9 \mu \mathrm{mol}$, quant.).

$\mathbf{R}_{\mathbf{f}}=0.60$ (ethyl acetate); $[\boldsymbol{\alpha}]_{\mathbf{D}}{ }^{20}=+2.1^{\circ}\left(\mathrm{c}=0.97, \mathrm{CH}_{2} \mathrm{Cl}_{2}\right) ;{ }^{1} \mathbf{H}-\mathbf{N M R}\left(500 \mathrm{MHz}, \mathrm{CD}_{2} \mathrm{Cl}_{2}\right): \delta=$ 7.00/6.92 (2 x t, 1H, $J=7.1 \mathrm{~Hz}, \mathrm{H}-4), 5.23 / 5.13(2 \mathrm{x} \mathrm{d}, 1 \mathrm{H}, J=10.1 \mathrm{~Hz}, \mathrm{H}-25), 4.06-3.98$ (m, 1H, H-6), $3.86-3.76$ (m, 1H, H-8), $3.86-3.76 / 3.70-3.62$ (m, 1H, H-26), $3.47-3.33$ (m, 2H, H-13), 3.05/3.00 (s, 3H, H-23), $2.51-2.31$ (m, 2H, H-5), 1.93/1.92 (2x s, 3H, H-3), 1.66 (dt, 1H, $J=12.8 \mathrm{~Hz}, 2.4 \mathrm{~Hz}, \mathrm{H}-7 \mathrm{a}) 1.52-1.47$ (m, 2H, H-10a, H-11), $1.45-1.40$ (m, 2H, H-9), 1.43 (s, 3H, H-18), 1.33 (s, 3H, H-19), 1.18 - 1.13 (m, 1H, H-7b), 1.09/1.04 (2 x d, 6H, J=6.7 Hz, H-27), 1.07 1.01 (m, 1H, H-10b), 0.89 (s, 9H, H-15), 0.87 (d, 3H, J=6.7 Hz, H-12), 0.03 (s, 6H, H-14) ppm; ${ }^{13} \mathbf{C}-$ NMR $\left(125 \mathrm{MHz}, \mathrm{CD}_{2} \mathrm{Cl}_{2}\right): \delta=189.1 / 182.6$ (C-1), 188.3/180.9 (C-20), 173.0/163.9 (C-22), 142.7/140.6 (C-4), 134.5/132.7 (C-24), 132.1/130.6 (C-2), 125.3/124.8 (C-25), 103.4/100.4 (C-21), 99.1/98.9 (C-17), 69.9/69.7 (C-8), 68.85 (C-13), 68.75/68.70 (C-6), 37.4/37.3 (C-7), 36.6/36.4 (C-5), 36.5/36.3 (C-11), 34.5/34.4 (C-9), 30.5 (C-19), 28.9 (C-10), 26.3 (3C, C-15), 26.3/25.1 (C-26), 25.5/25.3 (C-23), 23.8/23.6 (2C, C-27), 20.2 (C-18), 18.8 (C-16), 17.03/17.00 (C-12), 13.3/13.1 (C-3), -5.1 (2C, C-20) ppm; HRMS (ESI+) $m / z$ : $[\mathrm{M}+\mathrm{H}]^{+}$Calcd. for $\mathrm{C}_{31} \mathrm{H}_{53} \mathrm{NO}_{6} \mathrm{SiH}^{+}$564.3715, found 564.3707. 


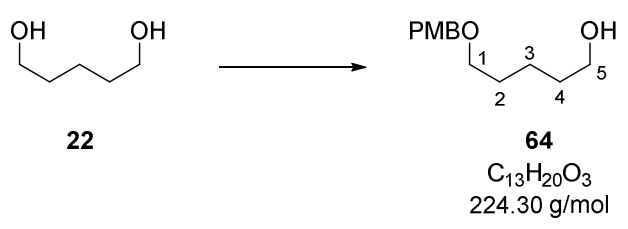

Diol 22 (3.02 mL, 28.9 mmol, 2.00 eq.) was dissolved in DMF (40 mL), cooled to $0{ }^{\circ} \mathrm{C}$ and $\mathrm{NaH}$ $(1.27 \mathrm{mg}, 31.8 \mathrm{mmol}, 2.20$ eq. $60 \%$ dispersion in mineral oil) was added. The reaction mixture was stirred for $1 \mathrm{~h}$ at $\mathrm{rt}$, cooled again to $0{ }^{\circ} \mathrm{C}$, 4-methoxybenzyl chloride $(1.95 \mathrm{~mL}, 14.4 \mathrm{mmol}, 1.00 \mathrm{eq}$.) was added and the reaction was stirred $2 \mathrm{~h}$ at $\mathrm{rt}$. Water $(30 \mathrm{~mL})$ was added slowly and the aqueous phase was extracted with EtOAc $(3 \times 40 \mathrm{~mL})$. The combined organic phases were washed with aq. sat. $\mathrm{NH}_{4} \mathrm{Cl}$ solution $(25 \mathrm{~mL})$, dried over $\mathrm{MgSO}_{4}$ and the solvent was removed under reduced pressure. Purification of the crude product was achieved by flash chromatography $(30-40 \% \mathrm{EtOAc} / \mathrm{CyH})$ to yield the title compound $\mathbf{6 4}$ as a colorless oil (2.50 g, $11.2 \mathrm{mmol}, 77 \%)$ and the double protected diol $\mathbf{6 5}$ as a side product (557 $\mathrm{mg}, 1.62 \mathrm{mmol}, 11 \%)$.

$\mathbf{R}_{\boldsymbol{f}}=0.10(30 \% \mathrm{EtOAc} / \mathrm{CyH}) .{ }^{1} \mathbf{H}$ NMR $\left(700 \mathrm{MHz}, \mathrm{CDCl}_{3}\right) \delta=7.26\left(\mathrm{~d}, J=8.6 \mathrm{~Hz}, 2 \mathrm{H}, \mathrm{H}_{\text {arom }}\right), 6.88$ (d, $J=8.6 \mathrm{~Hz}, 2 \mathrm{H}, \mathrm{H}_{\text {arom }}$ ), 4.43 (s, 2H, Ar- $\left.\mathrm{CH}_{2}\right), 3.80$ (s, 3H, $\left.\mathrm{OCH}_{3}\right), 3.63$ (t, $J=6.5 \mathrm{~Hz}, 2 \mathrm{H}, \mathrm{H}-5$ ), 3.45 (t, $J=6.5 \mathrm{~Hz}, 2 \mathrm{H}, \mathrm{H}-1), 1.63$ (dt, $J=14.7,6.5 \mathrm{~Hz}, 2 \mathrm{H}, \mathrm{H}-2), 1.58$ (dt, $J=14.7,6.5 \mathrm{~Hz}, 2 \mathrm{H}, \mathrm{H}-4$ ), $1.46-1.43$ (m, 2H, H-3), 1.37 (brs, $1 \mathrm{H}, \mathrm{OH}) \mathrm{ppm} ;{ }^{13} \mathbf{C ~ N M R}\left(175 \mathrm{MHz}, \mathrm{CDCl}_{3}\right) \delta=159.3\left(\mathrm{C}_{\text {arom. }} \mathrm{O}\right)$, $130.8\left(\mathrm{C}_{\text {arom. }}\right), 129.4\left(\mathrm{C}_{\text {arom. }} \mathrm{H}\right), 113.9\left(\mathrm{C}_{\text {arom. }} \mathrm{H}\right), 72.7\left(\mathrm{Ar}-\mathrm{CH}_{2}\right), 70.1(\mathrm{C}-1), 63.0(\mathrm{C}-5), 55.4\left(\mathrm{O}_{-} \mathrm{CH}_{3}\right)$, 32.7 (C-4), 29.6 (C-2), 22.6 (C-3) ppm; MS (EI) $m / z$ : calcd for $\mathrm{C}_{13} \mathrm{H}_{20} \mathrm{O}_{3}{ }^{+\bullet}[\mathrm{M}]^{+\bullet}: 224.2$, found: 224.2 . The spectroscopic data were in agreement with those previously reported. ${ }^{[22]}$

\section{1,5-Bis((4-methoxybenzyl)oxy)pentane (65)}

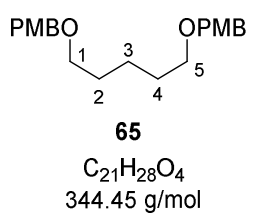

Compound 65 was isolated as side product.

$\mathbf{R}_{\boldsymbol{f}}=0.38(30 \% \mathrm{EtOAc} / \mathrm{CyH}) .{ }^{1} \mathbf{H}$ NMR $\left(700 \mathrm{MHz}, \mathrm{CDCl}_{3}\right) \delta=7.25\left(\mathrm{~d}, J=8.6 \mathrm{~Hz}, 4 \mathrm{H}, \mathrm{H}_{\text {arom. }}\right), 6.87$ (d, $J=8.6 \mathrm{~Hz}, 4 \mathrm{H}, \mathrm{H}_{\text {arom. }}$ ), 4.42 (s, 4H, 2xAr-CH 2$), 3.80$ (s, 6H, 2xO-CH $\left.\mathrm{CH}_{3}\right), 3.44$ (t, $J=6.6 \mathrm{~Hz}, 4 \mathrm{H}, \mathrm{H}-$ 1, H-5), 1.62 (dt, $J=14.5,6.7 \mathrm{~Hz}, 4 \mathrm{H}, \mathrm{H}-2, \mathrm{H}-4), 1.45-1.41$ (m, 2H, H-3) ppm; ${ }^{13} \mathbf{C}$ NMR $\left(175 \mathrm{MHz}, \mathrm{CDCl}_{3}\right) \delta=159.2\left(2 \mathrm{xC}_{\text {arom. }} \mathrm{O}\right), 130.9\left(2 \mathrm{xC}_{\text {arom. }}\right), 129.4\left(\mathrm{C}_{\text {arom. }} \mathrm{H}\right), 113.9\left(\mathrm{C}_{\text {arom. }} \mathrm{H}\right), 72.7$ (2xAr-CH 2$), 70.2$ (C-1, C-5), $55.4\left(2 \mathrm{xO}^{-\mathrm{CH}_{3}}\right), 29.7$ (C-2, C-4), 23.0 (C-3) ppm; HRMS (ESI+) $\mathrm{m} / z$ : calcd for $\mathrm{C}_{21} \mathrm{H}_{28} \mathrm{O}_{4} \mathrm{Na}^{+}[\mathrm{M}+\mathrm{Na}]^{+}: 367.1880$, found: 367.1880 . 


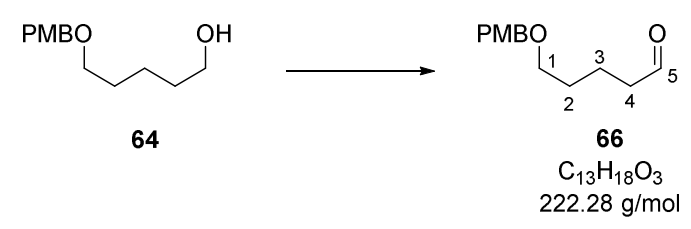

DCM (3.5 mL) was added to sulfur trioxide pyridine complex (532 mg, $3.34 \mathrm{mmol}, 3.00 \mathrm{eq}$.) and cooled to $0{ }^{\circ} \mathrm{C}$. $N, N$-Diisopropylethylamine $(758 \mu \mathrm{L}, 4.46 \mathrm{mmol}, 4.00$ eq.), DMSO $(792 \mu \mathrm{L}$, $11.2 \mathrm{mmol}, 10.0$ eq.) and alcohol 64 (250 mg, $1.11 \mu \mathrm{mol}, 1.00$ eq. in $1 \mathrm{~mL}$ DCM) were added. After stirring $1 \mathrm{~h}$ at $\mathrm{rt}$, sat. aq. $\mathrm{NaHCO}_{3}$ solution $(10 \mathrm{~mL})$ was added and the aqueous phase was extracted with DCM $(3 \times 10 \mathrm{~mL})$. The combined organic layers were washed with $\mathrm{CuSO}_{4}, \mathrm{NH}_{4} \mathrm{Cl}$ and brine solution (each aq. sat. $2 \times 70 \mathrm{~mL}$ ). The organic phase was dried over $\mathrm{MgSO}_{4}$ and the solvent was removed under reduced pressure to yield the title compound $\mathbf{6 6}$ as a colorless oil (241 mg, $1.08 \mathrm{mmol}$, $97 \%)$.

$\mathbf{R}_{f}=0.23(30 \% \mathrm{EtOAc} / \mathrm{CyH}) .{ }^{1} \mathbf{H}$ NMR $\left(300 \mathrm{MHz}, \mathrm{CDCl}_{3}\right) \delta=9.76-9.74(\mathrm{~m}, 1 \mathrm{H}, \mathrm{H}-5), 7.25(\mathrm{~d}, J=$ $8.5 \mathrm{~Hz}, 2 \mathrm{H}, \mathrm{H}_{\text {arom. }}$ ), 6.88 (d, J=8.5 Hz, 2H, $\mathrm{H}_{\text {arom. }}$ ), 4.42 (s, 2H, $\left.\mathrm{Ar}_{-} \mathrm{CH}_{2}\right), 3.80$ (s, 3H, O-CH $), 3.46$ (t, $J=6.0 \mathrm{~Hz}, 2 \mathrm{H}, \mathrm{H}-1), 2.44(\mathrm{t}, J=7.1 \mathrm{~Hz}, 2 \mathrm{H}, \mathrm{H}-4), 1.78-1.59$ (m, $1 \mathrm{H}, \mathrm{H}-2, \mathrm{H}-3)$ ppm; ${ }^{13} \mathbf{C} \mathbf{~ N M R}$ $\left(75 \mathrm{MHz}, \mathrm{CDCl}_{3}\right) \delta=202.7(\mathrm{C}-5), 159.3\left(\mathrm{C}_{\text {arom. }} \mathrm{O}\right), 130.7\left(\mathrm{C}_{\text {arom }}\right), 129.4\left(\mathrm{C}_{\text {arom. }} \mathrm{H}\right), 113.9\left(\mathrm{C}_{\text {arom. }} \mathrm{H}\right), 72.7$ $\left(\mathrm{Ar}-\mathrm{CH}_{2}\right), 69.6(\mathrm{C}-1), 55.4\left(\mathrm{O}-\mathrm{CH}_{3}\right), 43.7$ (C-4), 29.3 (C-2), 19.1 (C-3) ppm; HRMS (ESI+) m/z: calcd for $\mathrm{C}_{13} \mathrm{H}_{18} \mathrm{O}_{3} \mathrm{Na}^{+}[\mathrm{M}+\mathrm{Na}]^{+}: 245.1148$, found: 245.1148 . The spectroscopic data were in agreement with those previously reported. ${ }^{[23]}$

7-((4-Methoxybenzyl)oxy)heptan-3-ol (67)

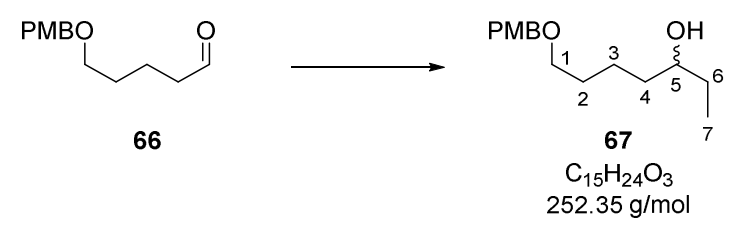

Aldehyde 66 ( $1.00 \mathrm{~g}, 4.50 \mathrm{mmol}, 1.00$ eq.) was dissolved in THF $(40 \mathrm{~mL})$, cooled to $0{ }^{\circ} \mathrm{C}$ and EtMgBr. (5.40 mL, $5.40 \mathrm{mmol}, 1.20$ eq. $1 \mathrm{M}$ in THF) was added. After stirring for $30 \mathrm{~min}$ at $0{ }^{\circ} \mathrm{C}$, EtOAc $(20 \mathrm{~mL})$ and aq. sat. $\mathrm{NH}_{4} \mathrm{Cl}(25 \mathrm{~mL})$ were added, the phases separated, the aqueous phase was extracted with EtOAc $(3 \times 25 \mathrm{~mL})$, the combined organic phases were dried over $\mathrm{MgSO}_{4}$ and the solvent was removed under reduced pressure. Purification of the crude product was achieved by flash chromatography $(30-40 \% \mathrm{EtOAc} / \mathrm{CyH})$ to yield the racemic title compound $\mathbf{6 7}$ as a colorless oil (946 mg, $3.75 \mathrm{mmol}, 83 \%$ ).

$\mathbf{R}_{f}=0.19(30 \% \mathrm{EtOAc} / \mathrm{CyH}) .{ }^{1} \mathbf{H}$ NMR $\left(500 \mathrm{MHz}, \mathrm{CDCl}_{3}\right) \delta=7.26\left(\mathrm{~d}, J=8.6 \mathrm{~Hz}, 2 \mathrm{H}, \mathrm{H}_{\text {arom }}\right), 6.88$ (d, $J=8.6 \mathrm{~Hz}, 2 \mathrm{H}, \mathrm{H}_{\text {arom. }}$ ), 4.43 (s, 2H, Ar- $\mathrm{CH}_{2}$ ), 3.80 (s, 3H, O-CH $\mathrm{CH}_{3}, 3.54-3.50$ (m, 1H, H-5), 3.45 (t, $J=6.5 \mathrm{~Hz}, 2 \mathrm{H}, \mathrm{H}-1), 1.66-1.39$ (m, 8H, H-2, H-3, H-4, H-6), 0.93 (t, J = 7.4 Hz, 3H, H-7) ppm; 
${ }^{13}$ C NMR $\left(125 \mathrm{MHz}, \mathrm{CDCl}_{3}\right) \delta=159.3\left(\mathrm{C}_{\text {arom. }} \mathrm{O}\right), 130.8\left(\mathrm{C}_{\text {arom. }}\right), 129.4\left(\mathrm{C}_{\text {arom. }} \mathrm{H}\right), 113.9\left(\mathrm{C}_{\text {arom. }} \mathrm{H}\right), 73.4$ (C-5), $72.7\left(\mathrm{Ar}^{-\mathrm{CH}_{2}}\right), 70.1$ (C-1), $55.4\left(\mathrm{O}-\mathrm{CH}_{3}\right), 36.8$ (C-4), 30.3 (C-6), 29.9 (C-2), 22.5 (C-3), 10.0 (C-7) ppm; HRMS (ESI+) $m / z$ : calcd for $\mathrm{C}_{15} \mathrm{H}_{24} \mathrm{O}_{3} \mathrm{H}^{+}[\mathrm{M}+\mathrm{H}]^{+}: 253.1798$, found: 253.1801 .

\section{7-((4-Methoxybenzyl)oxy)heptan-3-one (21)}

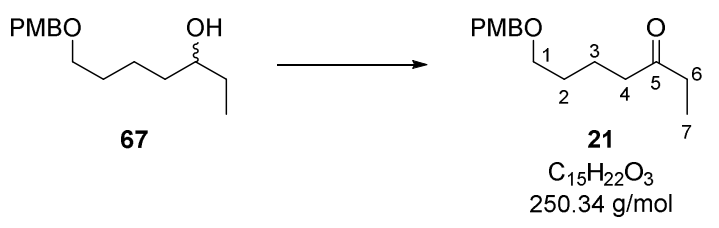

DCM (14 mL) was added to sulfur trioxide pyridine complex (1.78 g, $11.2 \mathrm{mmol}, 3.00$ eq. $)$ and cooled to $0{ }^{\circ} \mathrm{C}$. $N, N$-Diisopropylethylamine $(2.53 \mathrm{~mL}, 14.9 \mathrm{mmol}, 4.00$ eq.), DMSO (2.65 mL, $37.3 \mathrm{mmol}$, 10.0 eq.) and alcohol 67 (940 mg, $3.72 \mathrm{mmol}, 1.00$ eq. in $1 \mathrm{~mL} \mathrm{DCM})$ were added. After stirring $1 \mathrm{~h}$ at rt, sat. aq. $\mathrm{NaHCO}_{3}$ solution $(10 \mathrm{~mL})$ was added and the aqueous phase was extracted with DCM $(3 \times 20 \mathrm{~mL})$. The combined organic layers were washed with $\mathrm{CuSO}_{4}, \mathrm{NH}_{4} \mathrm{Cl}$ and brine solution (each aq. sat. $2 \times 70 \mathrm{~mL}$ ). The organic phase was dried over $\mathrm{MgSO}_{4}$ and the solvent was removed under reduced pressure. Purification of the crude product was achieved by flash chromatography $(20 \%$ EtOAc/CyH) to yield the title compound 21 as a colorless oil (874 mg, $3.49 \mathrm{mmol}, 94 \%$ ).

$\mathbf{R}_{f}=0.36(30 \% \mathrm{EtOAc} / \mathrm{CyH}) .{ }^{1} \mathbf{H}$ NMR $\left(500 \mathrm{MHz}, \mathrm{CDCl}_{3}\right) \delta=7.25\left(\mathrm{~d}, J=8.7 \mathrm{~Hz}, 2 \mathrm{H}, \mathrm{H}_{\text {arom. }}\right), 6.87$ (d, $\left.J=8.7 \mathrm{~Hz}, 2 \mathrm{H}, \mathrm{H}_{\text {arom. }}\right), 4.42\left(\mathrm{~s}, 2 \mathrm{H}, \mathrm{Ar}-\mathrm{CH}_{2}\right), 3.80\left(\mathrm{~s}, 3 \mathrm{H}, \mathrm{O}-\mathrm{CH}_{3}\right), 3.44$ (t, $\left.J=6.2 \mathrm{~Hz}, 3 \mathrm{H}, \mathrm{H}-1\right)$, $2.43-2.38$ (m, 4H, H-4, H-6), $1.69-1.58$ (m, 4H, H-2, H-3), 1.04 (t, J=7.4 Hz, 3H, H-7) ppm; ${ }^{13} \mathbf{C}$ NMR $\left(125 \mathrm{MHz}, \mathrm{CDCl}_{3}\right) \delta=211.7(\mathrm{C}-5), 159.3\left(\mathrm{C}_{\text {arom. }} \mathrm{O}\right), 130.8\left(\mathrm{C}_{\text {arom. }}\right), 129.4\left(\mathrm{C}_{\text {arom. }} \mathrm{H}\right), 113.9$ $\left(\mathrm{C}_{\text {arom. }} \mathrm{H}\right), 72.7\left(\mathrm{Ar}-\mathrm{CH}_{2}\right), 69.8(\mathrm{C}-1), 55.4\left(\mathrm{O}-\mathrm{CH}_{3}\right), 42.2$ (C-4), 36.0 (C-6), 29.4 (C-2), 20.8 (C-3), 8.0 (C-7) ppm; HRMS (ESI+) $m / z$ : calcd for $\mathrm{C}_{15} \mathrm{H}_{22} \mathrm{O}_{3} \mathrm{Na}^{+}[\mathrm{M}+\mathrm{Na}]^{+}: 273.1461$, found: 273.1459 .

\section{Methyl (R)-3-((tert-butyldimethylsilyl)oxy)-2-methylpropanoate (69)}

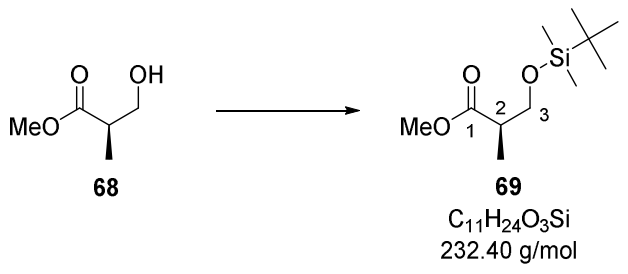

(R)-Roche ester 68 (467 mL, $4.23 \mathrm{mmol}, 1.00$ eq.) was dissolved in DCM (2.5 mL), imidazole (576 mg, $8.47 \mathrm{mmol}, 2.00$ eq. in $2 \mathrm{~mL} \mathrm{DCM}$ ) was added and the reaction mixture was cooled to $0{ }^{\circ} \mathrm{C}$ before the addition of $\mathrm{TBSCl}\left(766 \mathrm{mg}, 5.08 \mathrm{mmol}, 1.20\right.$ eq.). After stirring $4 \mathrm{~h}$ at rt, aq. sat. $\mathrm{NH}_{4} \mathrm{Cl}$ solution $(10 \mathrm{~mL})$ was added, the phases separated, the aqueous phase was extracted with DCM $(3 \times 15 \mathrm{~mL})$, the combined organic phases were dried over $\mathrm{MgSO}_{4}$ and the solvent was removed under 
reduced pressure. Purification of the crude product was achieved by flash chromatography (DCM) to yield the title compound $\mathbf{6 9}$ as a colorless oil ( $981 \mathrm{mg}, 4.22 \mathrm{mmol}$, quant.).

$\mathbf{R}_{f}=0.56(\mathrm{DCM}) \cdot[\boldsymbol{\alpha}]_{\boldsymbol{D}}^{20}=-19.8^{\circ}\left(c=0.86\right.$ in $\left.\mathrm{CH}_{2} \mathrm{Cl}_{2}\right) .{ }^{1} \mathbf{H} \mathbf{~ N M R}\left(500 \mathrm{MHz}, \mathrm{CD}_{2} \mathrm{Cl}_{2}\right) \delta=3.75(\mathrm{dd}, J=$ 9.7, 6.9 Hz, 1H, H-3), $3.69-3.61$ (dd, $J=9.7,6.9 \mathrm{~Hz}, 1 \mathrm{H}, \mathrm{H}-3$ '), 3.64 (s, 3H, O-CH $\mathrm{CH}_{3}$ ), $2.65-2.58$ (m, $1 \mathrm{H}, \mathrm{H}-2), 1.11$ (d, $\left.J=7.0 \mathrm{~Hz}, 3 \mathrm{H}, 2-\mathrm{CH}_{3}\right), 0.87$ (s, 9H, TBS), 0.04 (2xs, 6H, TBS) ppm; ${ }^{13} \mathbf{C}$ NMR

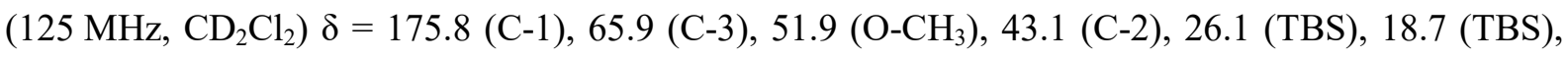
$13.8\left(2-\mathrm{CH}_{3}\right),-5.3($ TBS $)$ ppm; HRMS (ESI+) $m / z$ : calcd for $\mathrm{C}_{11} \mathrm{H}_{24} \mathrm{O}_{3} \mathrm{SiH}^{+}[\mathrm{M}+\mathrm{H}]^{+}: 233.1567$, found: 233.1567 . The spectroscopic data were in agreement with those previously reported. ${ }^{[2]}$

\section{(S)-3-((tert-Butyldimethylsilyl)oxy)-2-methylpropan-1-ol (70)}

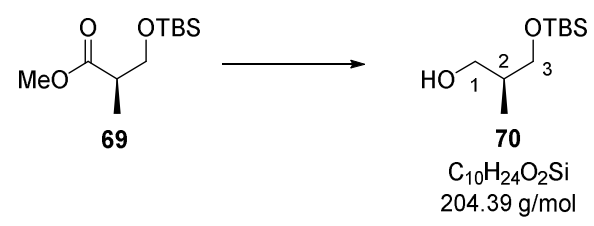

Ester $69(200 \mathrm{mg}, 861 \mu \mathrm{mol}, 1.00$ eq.) was dissolved in THF $(1 \mathrm{~mL})$, the solution was cooled to $-78^{\circ} \mathrm{C}$ and DIBAL-H (2.58 mL, $2.58 \mathrm{mmol}, 3.00$ eq. $1 \mathrm{M}$ in $\left.\mathrm{CyH}\right)$ was added slowly. After stirring $2 \mathrm{~h}$ at $-78^{\circ} \mathrm{C}$ and $30 \mathrm{~min}$ at $\mathrm{rt}$, the reaction mixture was cooled again to $-78^{\circ} \mathrm{C}$ before the addition of EtOAc $(1.5 \mathrm{~mL})$ and sat. aq. Rochelle salt solution $(3 \mathrm{~mL})$. The mixture was allowed to warm to $\mathrm{rt}$ and stirred until clearly two phases appeared $(2 \mathrm{~h})$. The phases were separated, the aqueous layer was extracted with EtOAc $(3 \times 10 \mathrm{~mL})$, the combined organic phases were dried over $\mathrm{MgSO}_{4}$ and the solvent was removed under reduced pressure. Purification of the crude product was achieved by flash chromatography (DCM) to yield the title compound 70 as a colorless oil (142 $\mathrm{mg}, 659 \mu \mathrm{mol}, 81 \%$ ).

$\mathbf{R}_{f}=0.15(\mathrm{DCM}) \cdot[\boldsymbol{\alpha}]_{\boldsymbol{D}}^{20}=-9.0^{\circ}\left(c=0.67\right.$ in $\left.\mathrm{CH}_{2} \mathrm{Cl}_{2}\right) .{ }^{1} \mathbf{H} \mathbf{N M R}\left(500 \mathrm{MHz}, \mathrm{CD}_{2} \mathrm{Cl}_{2}\right) \delta=3.70(\mathrm{dd}, J=$ 9.9, 4.8 Hz, 1H, H-3), 3.57 - 3.52 (m, 3H, H-1, H-3'), 2.45 (t, J=5.7 Hz, 1H, OH), $1.89-1.84$ (m, 1H, H-2), 0.90 (s, 9H, TBS), 0.83 (d, $J=6.9 \mathrm{~Hz}, 3 \mathrm{H}, 2-\mathrm{CH}_{3}$ ), 0.07 (s, 6H, TBS) ppm; ${ }^{13} \mathbf{C}$ NMR $\left(125 \mathrm{MHz}, \mathrm{CD}_{2} \mathrm{Cl}_{2}\right) \delta=68.7$ (C-3), $68.2(\mathrm{C}-1), 37.9$ (C-2), 26.2 (TBS), 18.6 (TBS), $13.5\left(2-\mathrm{CH}_{3}\right),-5.3$ (TBS) ppm; MS (EI) $m / z$ : calcd for $\mathrm{C}_{6} \mathrm{H}_{15} \mathrm{O}_{2} \mathrm{Si}^{+\bullet}\left[\mathrm{M}-{ }^{t} \mathrm{Bu}\right]^{+\bullet}: 147.1$, found: 147.1 . The spectroscopic data were in agreement with those previously reported. ${ }^{[24]}$

(R)-3-((tert-Butyldimethylsilyl)oxy)-2-methylpropanal (18)

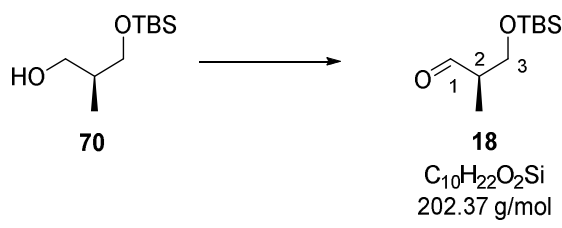

Dess-Martin-Periodinane oxidation: 
Alcohol 70 (70.0 mg, $343 \mu \mathrm{mol}$, 1.00 eq.) was dissolved in DCM (4 mL) and DMP (218 mg, $514 \mu$ mol, 1.50 eq.) was added. After stirring $2 \mathrm{~h}$ at $\mathrm{rt}$, the reaction mixture was concentrated, $\mathrm{Et}_{2} \mathrm{O}$ was added and the suspension was filtered over Celite ${ }^{\circledR}$. The solvent was removed under reduced pressure and the crude product was purified using flash chromatography (DCM) to yield the title compound 18 as a colorless oil (66.4 mg, $328 \mu \mathrm{mol}, 96 \%)$.

\section{Parikh-Doering oxidation:}

DCM (30 mL) was added to sulfur trioxide pyridine complex (4.67 g, $29.4 \mathrm{mmol}, 3.00$ eq.) and cooled to $0{ }^{\circ} \mathrm{C}$. $N, N$-Diisopropylethylamine $(6.66 \mathrm{~mL}, 39.1 \mathrm{mmol}, 4.00$ eq.), DMSO (6.95 mL, $97.9 \mathrm{mmol}$, 10.0 eq.) and alcohol 70 (2.00 g, 9.79 mmol, 1.00 eq.) were added. After stirring $1 \mathrm{~h}$ at rt, sat. aq. $\mathrm{NaHCO}_{3}$ solution $(20 \mathrm{~mL})$ was added and the aqueous phase was extracted with DCM $(3 \times 50 \mathrm{~mL})$. The combined organic layers were washed with $\mathrm{CuSO}_{4}, \mathrm{NH}_{4} \mathrm{Cl}$ and brine solution (each aq. sat. $2 \times 150 \mathrm{~mL}$ ). The organic phase was dried over $\mathrm{MgSO}_{4}$ and the solvent was removed under reduced pressure to yield the title compound $\mathbf{1 8}$ as a colorless oil (1.97 $\mathrm{g}, 9.73 \mathrm{mmol}$, quant.).

$\mathbf{R}_{\boldsymbol{f}}=0.51(\mathrm{DCM}) \cdot[\boldsymbol{\alpha}]_{\boldsymbol{D}}^{\mathbf{2 0}}=-34.4^{\circ}\left(c=0.84\right.$ in $\left.\mathrm{CH}_{2} \mathrm{Cl}_{2}\right) .{ }^{1} \mathbf{H} \mathbf{N M R}\left(500 \mathrm{MHz}, \mathrm{CD}_{2} \mathrm{Cl}_{2}\right) \delta=9.70(\mathrm{~d}, J=$ $1.6 \mathrm{~Hz}, 1 \mathrm{H}, \mathrm{H}-1), 3.87$ (dd, $J=10.2,5.0 \mathrm{~Hz}, 1 \mathrm{H}, \mathrm{H}-3$ ), 3.80 (dd, $J=10.2,6.3 \mathrm{~Hz}, 1 \mathrm{H}, \mathrm{H}-3$ '), 2.53 2.46 (m, 1H, H-2), 1.06 (d, $J=7.0 \mathrm{~Hz}, 3 \mathrm{H}, 2-\mathrm{CH} 3), 0.88$ (s, 9H, TBS), 0.06 (s, 6H, TBS) ppm; ${ }^{13} \mathbf{C}$ NMR (125 MHz, $\mathrm{CD}_{2} \mathrm{Cl}_{2}$ ) $\delta=205.0$ (C-1), 64.0 (C-3), 49.4 (C-2), 26.1 (TBS), 18.7 (TBS), 10.6 (2$\mathrm{CH}_{3}$ ), -5.3 (TBS) ppm; MS (EI) $m / z$ : calcd for $\mathrm{C}_{10} \mathrm{H}_{21} \mathrm{O}_{2} \mathrm{Si}^{+\bullet}[\mathrm{M}]^{+\bullet}: 201.2$, found: 201.2. The spectroscopic data were in agreement with those previously reported. ${ }^{[25]}$ 
Ethyl (S,E)-5-((tert-butyldimethylsilyl)oxy)-2,4-dimethylpent-2-enoate (19) and Ethyl (S,Z)-5((tert-butyldimethylsilyl)oxy)-2,4-dimethylpent-2-enoate (19Z)

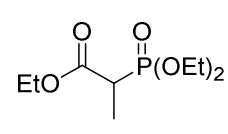<smiles>CC(C=O)C[18O][Mg]</smiles>

18<smiles>CCOC(=O)C(C)=CC(C)=C(C)CO[Mg]</smiles>

19

$\mathrm{C}_{15} \mathrm{H}_{30} \mathrm{O}_{3} \mathrm{Si}$ $286.49 \mathrm{~g} / \mathrm{mol}$<smiles>CCOC(=O)c1ccc(OCC)cc1</smiles>

$19 Z$

$\mathrm{C}_{15} \mathrm{H}_{30} \mathrm{O}_{3} \mathrm{Si}$ $286.49 \mathrm{~g} / \mathrm{mol}$

$\mathrm{Ba}(\mathrm{OH})_{2}(178 \mathrm{mg}, 1.04 \mathrm{mmol}, 3.50$ eq. $)$ was dried for $6 \mathrm{~h}$ at $120^{\circ} \mathrm{C}$ using high vacuum. After cooling to $\mathrm{rt}$, THF $(50 \mathrm{~mL})$ was added followed by phosphonate $8(2.82 \mathrm{~mL}, 13.1 \mathrm{mmol}, 1.40$ eq.). The suspension was stirred for $30 \mathrm{~min}$ at rt. Freshly prepared aldehyde 18 (1.90 g, 9.39 mmol, 1.00 eq. in 40:1 THF/water $(28 \mathrm{~mL}))$ was added and the reaction was stirred overnight at $\mathrm{rt}$. The suspension was filtered over Celite ${ }^{\circledR}$ and washed with $\mathrm{Et}_{2} \mathrm{O}$. The solvent was removed under reduced pressure and the crude product was purified by flash chromatography $\left(2 \% \mathrm{Et}_{2} \mathrm{O} / \mathrm{CyH}\right)$ to yield the title compounds 19 $(1.85 \mathrm{~g}, 6.46 \mathrm{mmol}, 69 \%, \underline{E} / Z=13: 1)$ and $19 Z(142 \mathrm{mg}, 496 \mu \mathrm{mol}, 5 \%, E / \underline{Z}=13: 1)$ as colorless oils. Analytical data for 20:

$\mathbf{R}_{f}=0.25\left(5 \% \mathrm{Et}_{2} \mathrm{O} / \mathrm{CyH}\right) \cdot[\boldsymbol{\alpha}]_{\boldsymbol{D}}^{\mathbf{2 0}}=-4.4^{\circ}\left(c=0.90\right.$ in $\left.\mathrm{CH}_{2} \mathrm{Cl}_{2}\right) .{ }^{1} \mathbf{H} \mathbf{N M R}\left(700 \mathrm{MHz}, \mathrm{CDCl}_{3}\right) \delta=6.55$ (dq, $J=9.9,1.4 \mathrm{~Hz}, 1 \mathrm{H}, \mathrm{H}-3), 4.22-4.15$ (m, $2 \mathrm{H}, \mathrm{OCH}_{2} \mathrm{CH}_{3}$ ), $3.52-3.47$ (m, 2H, H-5), $2.72-2.66$ (m, $1 \mathrm{H}, \mathrm{H}-4), 1.86\left(\mathrm{~d}, J=1.5 \mathrm{~Hz}, 3 \mathrm{H}, 2-\mathrm{CH}_{3}\right), 1.29$ (t, $\left.J=7.1 \mathrm{~Hz}, 3 \mathrm{H}, \mathrm{OCH}_{2} \underline{\mathrm{CH}}_{3}\right), 1.01$ (d, $J=6.7 \mathrm{~Hz}$, $\left.3 \mathrm{H}, 4-\mathrm{CH}_{3}\right), 0.88$ (s, 9H, TBS), 0.03 (2xs, 6H, TBS) ppm; ${ }^{13} \mathbf{C}$ NMR $\left(175 \mathrm{MHz}, \mathrm{CDCl}_{3}\right) \delta=168.4$ (C-1), 144.7 (C-3), 128.1 (C-2), 67.3 (C-5), $60.6\left(\mathrm{OCH}_{2} \mathrm{CH}_{3}\right), 36.4$ (C-4), 26.0 (TBS), 18.5 (TBS), $16.4\left(4-\mathrm{CH}_{3}\right), 14.4\left(\mathrm{OCH}_{2} \mathrm{CH}_{3}\right), 12.8\left(2-\mathrm{CH}_{3}\right),-5.2$ (TBS), -5.3 (TBS) ppm; HRMS (ESI+) $\mathrm{m} / z$ : calcd for $\mathrm{C}_{15} \mathrm{H}_{30} \mathrm{O}_{3} \mathrm{SiH}^{+}[\mathrm{M}+\mathrm{H}]^{+}: 287.2037$, found: 287.2035 . The spectroscopic data were in agreement with those previously reported. ${ }^{[26]}$

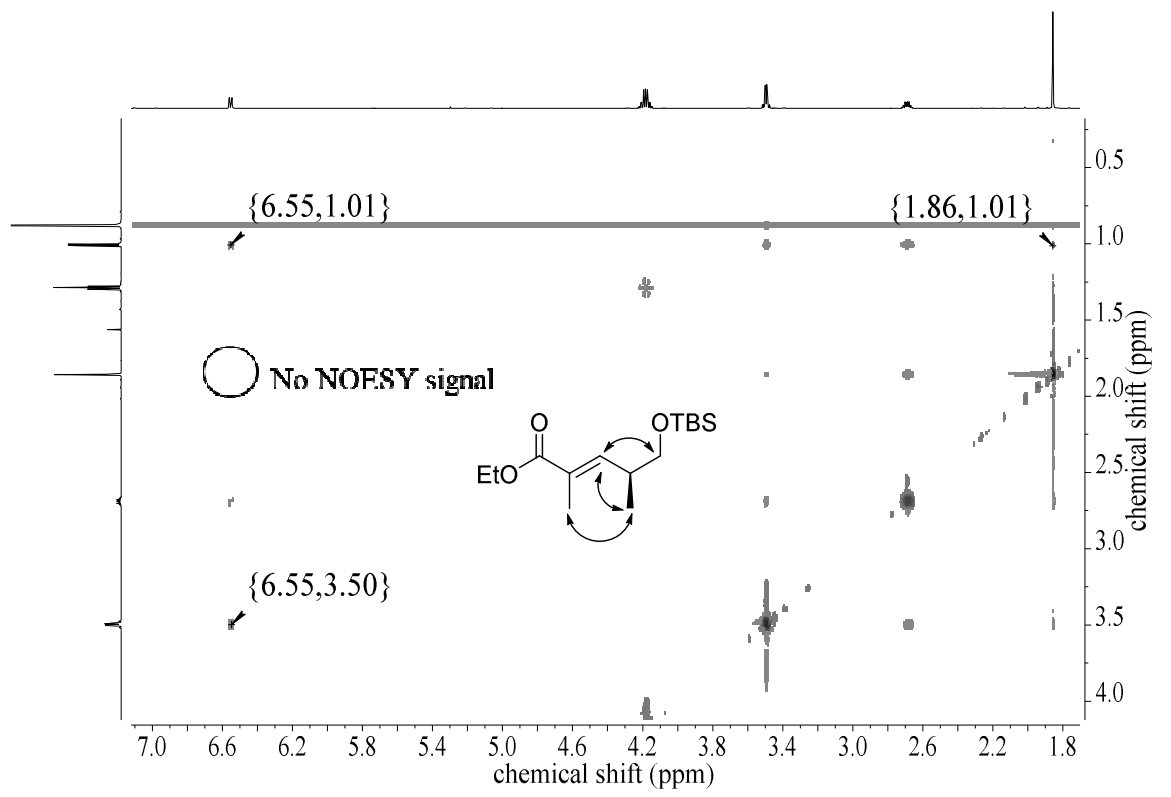

Figure 6. Selected NOESY signals of compound 19, which were used for the determination of the $E$-configuration. 
Analytical data for 19Z:

$\mathbf{R}_{\boldsymbol{f}}=0.33\left(5 \% \mathrm{Et}_{2} \mathrm{O} / \mathrm{CyH}\right) \cdot[\boldsymbol{\alpha}]_{\boldsymbol{D}}^{\mathbf{2 0}}=+27.8^{\circ}\left(c=0.72\right.$ in $\left.\mathrm{CH}_{2} \mathrm{Cl}_{2}\right) .{ }^{1} \mathbf{H} \mathbf{N M R}\left(700 \mathrm{MHz}, \mathrm{CDCl}_{3}\right) \delta=5.74$ (dq, $J=8.3,1.5 \mathrm{~Hz}, 1 \mathrm{H}, \mathrm{H}-3), 4.19$ (q, $\left.J=7.1 \mathrm{~Hz}, 2 \mathrm{H}, \mathrm{OCH}_{2} \mathrm{CH}_{3}\right), 3.51-3.44$ (m, 2H, H-5), $3.28-$ $3.23(\mathrm{~m}, 1 \mathrm{H}, \mathrm{H}-4), 1.89$ (d, $\left.J=1.4 \mathrm{~Hz}, 3 \mathrm{H}, 2-\mathrm{CH}_{3}\right), 1.30$ (t, $\left.J=7.1 \mathrm{~Hz}, 3 \mathrm{H}, \mathrm{OCH}_{2} \underline{\mathrm{CH}}_{3}\right), 0.99$ (d, $J=$

$\left.6.7 \mathrm{~Hz}, 3 \mathrm{H}, 4-\mathrm{CH}_{3}\right), 0.88$ (s, 9H, TBS), 0.03 (2xs, 6H, TBS) ppm ${ }^{13} \mathbf{C} \mathbf{N M R}\left(175 \mathrm{MHz}, \mathrm{CDCl}_{3}\right) \delta=$ 168.3 (C-1), 145.1 (C-3), 127.4 (C-2), 67.8 (C-5), $60.2\left(\mathrm{OCH}_{2} \mathrm{CH}_{3}\right), 36.4$ (C-4), 26.0 (TBS), 21.0 (2- $\left.\mathrm{CH}_{3}\right), 18.5$ (TBS), $17.0\left(4-\mathrm{CH}_{3}\right), 14.4\left(\mathrm{OCH}_{2} \mathrm{CH}_{3}\right),-5.2$ (TBS) ppm; HRMS (ESI+) m/z: calcd for $\mathrm{C}_{15} \mathrm{H}_{30} \mathrm{O}_{3} \mathrm{SiH}^{+}[\mathrm{M}+\mathrm{H}]^{+}: 287.2037$, found: 287.2036.

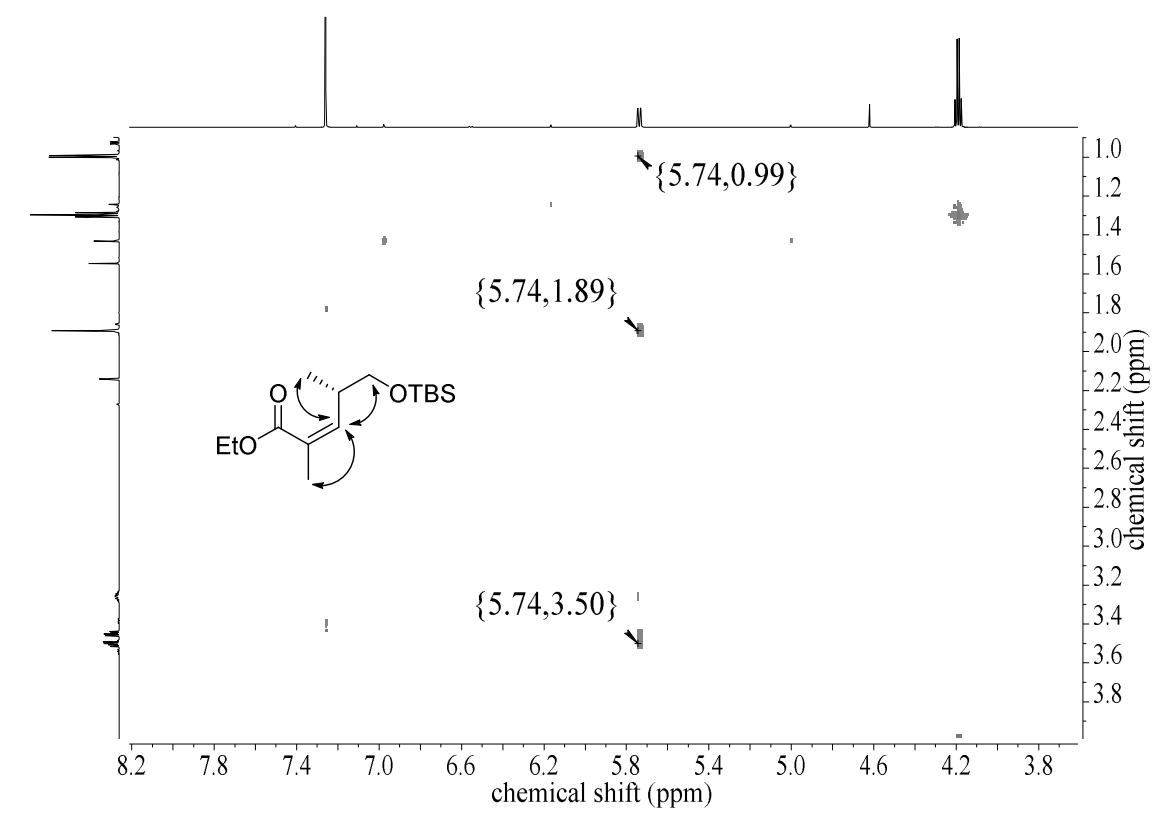

Figure 7. Selected NOESY signals of compound 19Z, which were used for the determination of the Z-configuration.

\section{(S,E)-5-((tert-Butyldimethylsilyl)oxy)-2,4-dimethylpent-2-en-1-ol (71)}

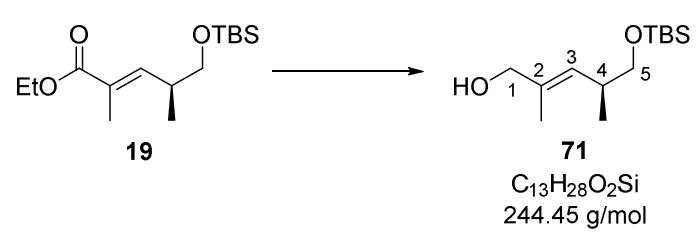

Ester 19 (1.86 g, $6.49 \mathrm{mmol}, 1.00$ eq.) was dissolved in THF $(27 \mathrm{~mL})$, the solution was cooled to $-78^{\circ} \mathrm{C}$ and DIBAL-H $(14.8 \mathrm{~mL}, 16.2 \mathrm{mmol}, 2.50$ eq. $1.1 \mathrm{M}$ in $\mathrm{CyH})$ was added slowly. After stirring $1 \mathrm{~h}$ at $-78{ }^{\circ} \mathrm{C}$, the reaction solution was poured into a sat. aq. Rochelle salt solution $(80 \mathrm{~mL})$. The mixture was stirred until clearly two phases appeared $(2 \mathrm{~h})$. The phases were separated, the aqueous layer was extracted with $\mathrm{Et}_{2} \mathrm{O}(3 \times 70 \mathrm{~mL})$, the combined organic phases were dried over $\mathrm{MgSO}_{4}$ and the solvent was removed under reduced pressure. Purification of the crude product was achieved by flash chromatography $(20 \% \mathrm{EtOAc} / \mathrm{CyH})$ to yield the title compound 71 as a colorless oil $(1.37 \mathrm{~g}$, $5.62 \mathrm{mmol}, 87 \%)$. 
$\mathbf{R}_{f}=0.24(20 \% \mathrm{EtOAc} / \mathrm{CyH}) \cdot[\boldsymbol{\alpha}]_{\boldsymbol{D}}^{\mathbf{2 0}}=+13.2^{\circ}\left(c=0.76\right.$ in $\left.\mathrm{CH}_{2} \mathrm{Cl}_{2}\right) .{ }^{1} \mathbf{H}$ NMR $\left(500 \mathrm{MHz}, \mathrm{CDCl}_{3}\right) \delta=$ 5.19 (d, $J=10.0 \mathrm{~Hz}, 1 \mathrm{H}, \mathrm{H}-3), 4.00$ (d, $J=5.6 \mathrm{~Hz}, 2 \mathrm{H}, \mathrm{H}-1), 3.47-3.35$ (m, 2H, H-5), $2.62-2.54$ (m, 1H, H-4), 1.69 (s, 3H, 2- $\left.\mathrm{CH}_{3}\right), 1.28(\mathrm{t}, J=5.6 \mathrm{~Hz}, 1 \mathrm{H}, \mathrm{OH}), 0.96\left(\mathrm{~d}, J=6.7 \mathrm{~Hz}, 3 \mathrm{H}, 4-\mathrm{CH}_{3}\right), 0.89$ (s, 9H, TBS), 0.04 (s, 6H, TBS) ppm; ${ }^{13} \mathbf{C}$ NMR (175 MHz, CDCl $\left.{ }_{3}\right) \delta=135.3$ (C-2), 129.1 (C-3), 69.1 (C-1), 68.0 (C-5), 35.3 (C-4), 26.1 (TBS), 18.5 (TBS), 17.3 (4- $\left.\mathrm{CH}_{3}\right), 14.1$ (2- $\left.\mathrm{CH}_{3}\right),-5.1$ (TBS) ppm; HRMS (ESI+) $m / z$ : calcd for $\mathrm{C}_{13} \mathrm{H}_{28} \mathrm{O}_{2} \mathrm{SiH}^{+}[\mathrm{M}+\mathrm{H}]^{+}: 245.1928$, found: 245.1928 . The spectroscopic data were in agreement with those previously reported. ${ }^{[26]}$

(S,E)-5-((tert-Butyldimethylsilyl)oxy)-2,4-dimethylpent-2-enal (20)

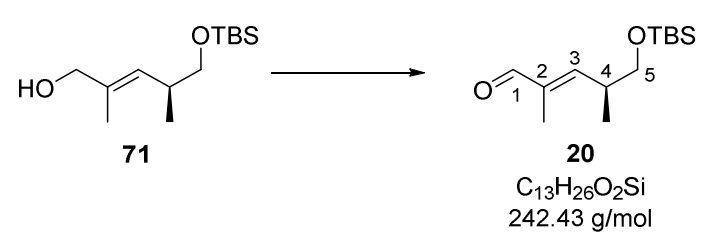

Alcohol 71 (260 mg, $1.06 \mathrm{mmol}$, 1.00 eq.) was dissolved in DMSO (1.3 mL) and 2-iodoxybenzoic acid (IBX, $596 \mathrm{mg}, 2.13 \mathrm{mmol}, 2.00$ eq.) was added. After stirring $1.5 \mathrm{~h}$ at rt, DCM (10 mL) and water $(10 \mathrm{~mL})$ were added and the mixture was filtered over Celite ${ }^{\circledR}$. The organic layer was separated, the aqueous layer was extracted with DCM $(3 \times 15 \mathrm{~mL})$, the combined organic phases were dried over $\mathrm{MgSO}_{4}$ and the solvent was removed under reduced pressure. Purification of the crude product was achieved by flash chromatography $(10 \% \mathrm{EtOAc} / \mathrm{CyH})$ to yield the title compound $\mathbf{2 0}$ as a colorless oil (257 mg, $1.06 \mathrm{mmol}$, quant.).

$\mathbf{R}_{f}=0.39(10 \% \mathrm{EtOAc} / \mathrm{CyH}) \cdot[\boldsymbol{\alpha}]_{\boldsymbol{D}}^{\mathbf{2 0}}=+8.3^{\circ}\left(c=0.84\right.$ in $\left.\mathrm{CH}_{2} \mathrm{Cl}_{2}\right) .{ }^{\mathbf{1}} \mathbf{H} \mathbf{N M R}\left(500 \mathrm{MHz}, \mathrm{CDCl}_{3}\right) \delta=$ 9.40 (s, 1H, H-1), 6.32 (dq, $J=9.7,1.3 \mathrm{~Hz}, 1 \mathrm{H}, \mathrm{H}-3), 3.61-3.53$ (m, 2H, H-5), $2.93-2.85$ (m, 1H, $\mathrm{H}-4), 1.77$ (d, $J=1.3 \mathrm{~Hz}, 3 \mathrm{H}, 2-\mathrm{CH}_{3}$ ), 1.06 (d, $J=6.8 \mathrm{~Hz}, 2 \mathrm{H}, 4-\mathrm{CH}_{3}$ ), 0.87 (s, 9H, TBS), 0.03 (2xs, 6H, TBS) ppm; ${ }^{13} \mathrm{C}$ NMR (125 MHz, $\left.\mathrm{CDCl}_{3}\right) \delta=195.7$ (C-1), 157.4 (C-3), 139.4 (C-2), 67.0 (C-5), 36.6 (C-4), 26.0 (TBS), 18.4 (TBS), $16.2\left(4-\mathrm{CH}_{3}\right), 9.6\left(2-\mathrm{CH}_{3}\right),-5.3$ (TBS) ppm; HRMS (ESI+) $m / z$ : calcd for $\mathrm{C}_{13} \mathrm{H}_{26} \mathrm{O}_{2} \mathrm{SiH}^{+}[\mathrm{M}+\mathrm{H}]^{+}: 243.1775$, found: 243.1775 . The spectroscopic data were in agreement with those previously reported. ${ }^{[26]}$

(6S,7S,10S,E)-11-((tert-Butyldimethylsilyl)oxy)-7-hydroxy-1-((4-methoxybenzyl)oxy)-6,8,10trimethylundec-8-en-5-one (23)

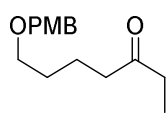

21

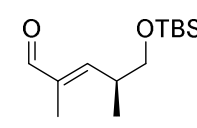

20

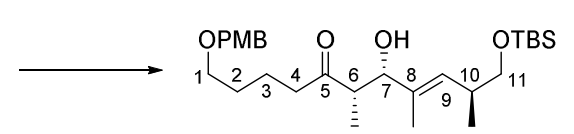

23

$\mathrm{C}_{28} \mathrm{H}_{48} \mathrm{O}_{5} \mathrm{Si}$

$492.77 \mathrm{~g} / \mathrm{mol}$ 
Preparation of (-)-[Ipc $]_{2}$ BOTf: First, (-)-[Ipc $]_{2} \mathrm{BH}$ was synthesized using the literature described hydroboration. ${ }^{27,28}$ Thus, $\mathrm{BH}_{3} \mathrm{xSMe} 2(3.87 \mathrm{~mL}, 40.8 \mathrm{mmol}, 1.00$ eq.) was added to THF (40 mL). This solution was cooled to $0{ }^{\circ} \mathrm{C}$ and $(\alpha)-(+)$-pinene $(13.0 \mathrm{~mL}, 81.6 \mathrm{mmol}, 2.00$ eq.) was added over the period of $20 \mathrm{~min}$ via syringe pump. Afterwards, the reaction vessel was stored $46 \mathrm{~h}$ at $0{ }^{\circ} \mathrm{C}$ under argon. The reaction mixture was allowed to warm to $\mathrm{rt}$ and the supernatant was removed from the formed precipitate using a syringe. Finally, the precipitate was washed with $\mathrm{Et}_{2} \mathrm{O}(3 \times 40 \mathrm{~mL})$ under argon atmosphere, dried at high vacuum and the yielded (-)-[Ipc $]_{2} \mathrm{BH}(6.99 \mathrm{~g}, 24.4 \mathrm{mmol}, 60 \%)$ was stored in the glove box fridge and was used over the period of two month. (-)-[Ipc $]_{2}$ BOTf was always prepared freshly and in situ before usage in the corresponding aldol reaction. For this purpose, hexane $(0.8 \mathrm{~mL})$ was added to $(-)$-[Ipc $]_{2} \mathrm{BH}(1.00 \mathrm{~g}, 3.49 \mathrm{mmol}, 1.00$ eq. $)$ and the mixture was cooled to $0{ }^{\circ} \mathrm{C}$. Triflic acid ( $308 \mu \mathrm{L}, 3.49 \mathrm{mmol}, 1.00$ eq.) was added carefully and the mixture was stirred at $\mathrm{rt}$ until complete disappearance of $(-)-[\mathrm{Ipc}]_{2} \mathrm{BH}$ crystals $(2-3 \mathrm{~h})$ resulting in a two phase system. Due to the literature described conversion of $60 \%{ }^{27}$, a $1.9 \mathrm{M}$ stock solution of (-)-[Ipc $]_{2} \mathrm{BOTf}$ in hexane (upper layer of the two phase system) was assumed.

(-)-[Ipc] ${ }_{2}$ BOTf ( $486 \mu \mathrm{L}, 923 \mu \mathrm{mol}, 1.10$ eq. 1.9M in hexane) was dissolved in DCM (3.5 mL). After cooling to $-78{ }^{\circ} \mathrm{C}$, DIPEA (300 $\mu \mathrm{L}, 1.76 \mathrm{mmol}, 2.10$ eq.) and ketone 21 (210 mg, $839 \mu \mathrm{mol}, 1.00 \mathrm{eq}$., stored over $3 \AA$ MS in $2 \mathrm{~mL}$ DCM) were added dropwise. The reaction mixture was stirred $2 \mathrm{~h}$ at $-78^{\circ} \mathrm{C}$ and freshly prepared aldehyde 20 (244 mg, $1.01 \mathrm{mmol}, 1.20$ eq., stored $14 \mathrm{~h}$ over $3 \AA \mathrm{MS}$ in $2 \mathrm{~mL}$ DCM) was added dropwise. After stirring $1 \mathrm{~h}$ at $-78^{\circ} \mathrm{C}$, the reaction vessel was placed in a deep freezer $\left(-15^{\circ} \mathrm{C}\right)$ overnight. Methanol $(1.8 \mathrm{~mL})$, pH 7 buffer $(2.7 \mathrm{~mL})$ and $\mathrm{H}_{2} \mathrm{O}_{2}(1.4 \mathrm{~mL}, 35 \%)$ were added to quench the reaction. After stirring $1 \mathrm{~h}$ at $\mathrm{rt}$, DCM $(15 \mathrm{~mL})$ and brine $(8 \mathrm{~mL})$ were added, the phases were separated, the aqueous layer was extracted with DCM $(3 \times 15 \mathrm{~mL})$, the combined organic phases were dried over $\mathrm{MgSO}_{4}$ and the solvent was removed under reduced pressure. Purification of the crude product was achieved by flash chromatography $(20 \% \mathrm{EtOAc} / \mathrm{CyH})$ and HPLC $(60 \%-100 \%$ $\mathrm{MeCN} /$ water, retention time $17.2 \mathrm{~min}$, using a KNAUER Eurospher II 100-5 C18; $5 \mu \mathrm{m} ; 250 \mathrm{x}$ $60 \mathrm{~mm})$ to give back ketone $21(93.6 \mathrm{mg}, 374 \mu \mathrm{mol}, 45 \%)$ and to yield the title compound $\mathbf{2 3}$ as a colorless oil (77.6 mg, $158 \mu \mathrm{mol}, 19 \%, 34 \%$ brsm., $d r=6: 1)$.

$\mathbf{R}_{\boldsymbol{f}}=0.18(20 \% \mathrm{EtOAc} / \mathrm{CyH}) .[\boldsymbol{\alpha}]_{\boldsymbol{D}}^{\mathbf{2 0}}=-2.1^{\circ}\left(c=0.94\right.$ in $\left.\mathrm{CH}_{2} \mathrm{Cl}_{2}\right) .{ }^{1} \mathbf{H} \mathbf{N M R}\left(500 \mathrm{MHz}, \mathrm{CDCl}_{3}\right) \delta=$ 7.25 (d, $J=8.7 \mathrm{~Hz}, 2 \mathrm{H}, \mathrm{H}_{\text {arom. }}$ ), 6.87 (d, $J=8.7 \mathrm{~Hz}, 2 \mathrm{H}, \mathrm{H}_{\text {arom. }}$ ), 5.26 (dq, $J=9.4,1.2 \mathrm{~Hz}, 1 \mathrm{H}, \mathrm{H}-9$ ), 4.42 (s, 2H, Ar- $\mathrm{CH}_{2}$ ), 4.28 (brt, 1H, H-7), 3.80 (s, 3H, O-CH $\left.\mathrm{CH}_{3}\right), 3.45-3.33$ (m, 4H, H-1, H-11), 2.69 (qd, $J=7.1,4.1 \mathrm{~Hz}, 1 \mathrm{H}, \mathrm{H}-6), 2.59$ (d, $J=2.8 \mathrm{~Hz}, 1 \mathrm{H}, \mathrm{OH}), 2.57-2.53$ (m, 1H, H-10), $2.52-2.40$ (m, 2H, H-4), $1.67-1.56$ (m, 4H, H-2, H-3), 1.59 (d, $\left.J=1.2 \mathrm{~Hz}, 3 \mathrm{H}, 8-\mathrm{CH}_{3}\right), 1.04$ (d, $J=7.1 \mathrm{~Hz}, 3 \mathrm{H}$, 6- $\left.\mathrm{CH}_{3}\right), 0.93\left(\mathrm{~d}, J=6.7 \mathrm{~Hz}, 3 \mathrm{H}, 10-\mathrm{CH}_{3}\right), 0.88$ (s, 9H, TBS), 0.03 (2xs, 6H, TBS) ppm; ${ }^{13} \mathbf{C}$ NMR $\left(125 \mathrm{MHz}, \mathrm{CDCl}_{3}\right) \delta=215.4(\mathrm{C}-5), 159.3\left(\mathrm{C}_{\text {arom. }} \mathrm{O}\right), 134.0\left(\mathrm{C}_{\text {arom }}\right), 130.8(\mathrm{C}-9), 129.6(\mathrm{C}-8), 129.4$ $\left(\mathrm{C}_{\text {arom. }} \mathrm{H}\right), 113.9\left(\mathrm{C}_{\text {arom. }} \mathrm{H}\right), 75.4(\mathrm{C}-7), 72.7\left(\mathrm{Ar}-\mathrm{CH}_{2}\right), 69.9(\mathrm{C}-1), 68.1(\mathrm{C}-11), 55.4\left(\mathrm{O}-\mathrm{CH}_{3}\right), 48.4(\mathrm{C}-$ 6), 41.7 (C-4), 35.4 (C-10), 29.3 (C-2), 26.1 (TBS), 20.5 (C-3), 18.5 (TBS), 17.3 (10- $\mathrm{CH}_{3}$ ), 13.7 (8- 
$\mathrm{CH}_{3}$ ), $10.3\left(6-\mathrm{CH}_{3}\right),-5.2$ (TBS) ppm; HRMS (ESI+) $\mathrm{m} / z$ : calcd for $\mathrm{C}_{28} \mathrm{H}_{48} \mathrm{O}_{5} \mathrm{SiNa}^{+}[\mathrm{M}+\mathrm{Na}]^{+}$: 515.3163, found: 515.3166 .

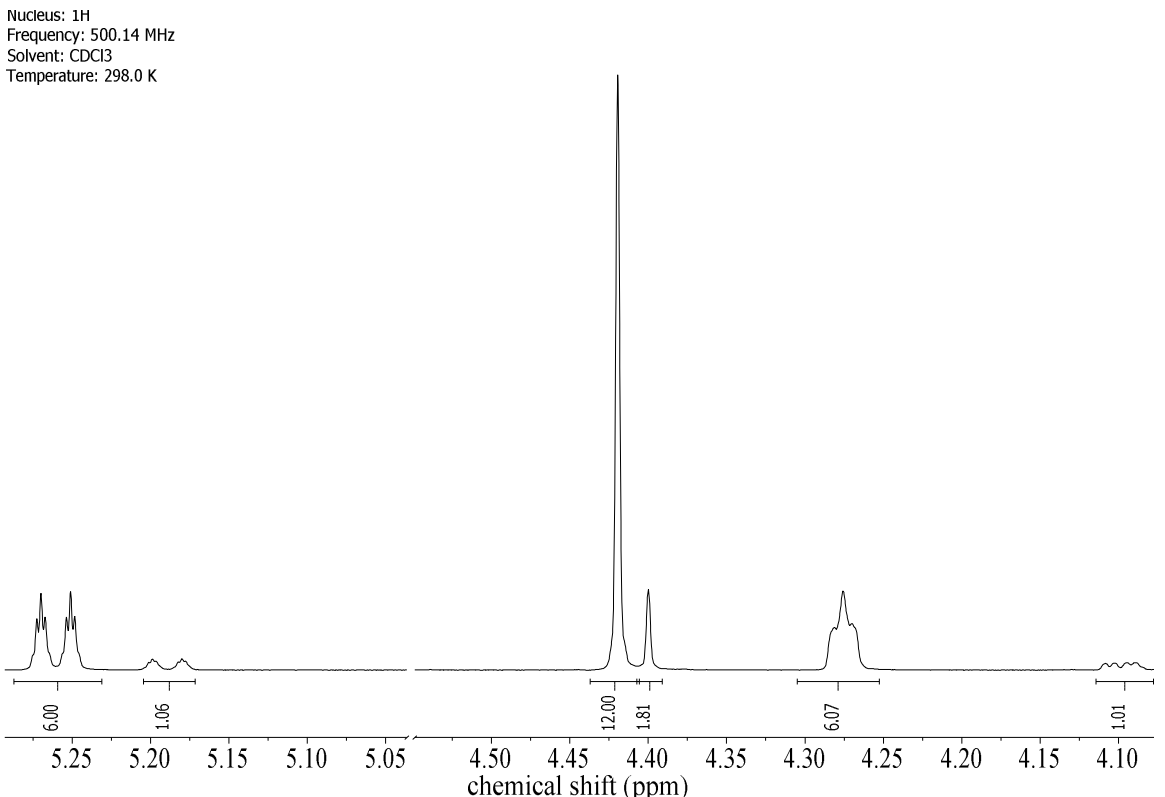

Figure 8. Cutout of the ${ }^{1} \mathrm{H}$ NMR spectrum to show the $d r$ of $6: 1$, resulting out of the aldol reaction to compound 23.

\section{(2S,5S,6S,E)-1-((tert-Butyldimethylsilyl)oxy)-11-((4-methoxybenzyl)oxy)-2,4,6-trimethyl-7-} oxoundec-3-en-5-yl (S)-3,3,3-trifluoro-2-methoxy-2-phenylpropanoate (72)

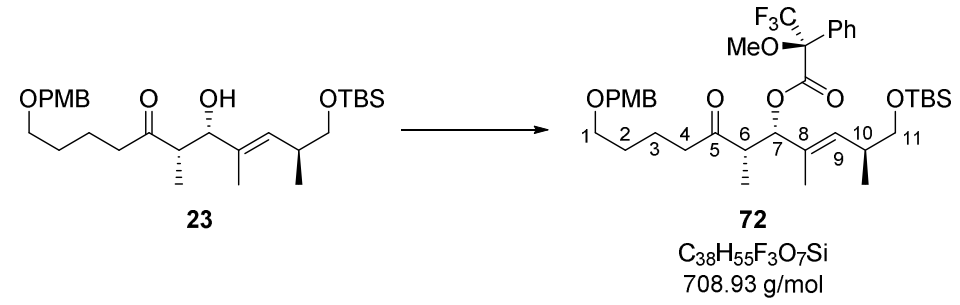

Alcohol 23 (6.00 mg, $12.2 \mu \mathrm{mol}, 1.00$ eq.) was dissolved in pyridine (140 $\mu \mathrm{L})$ and $(-)-(R)-\mathrm{MTPA}-\mathrm{Cl}$ $\left(13.7 \mu \mathrm{L}, 73.1 \mu \mathrm{mol}, 6.00\right.$ eq.) was added at $0{ }^{\circ} \mathrm{C}$. The reaction mixture was stirred $1 \mathrm{~h}$ at $\mathrm{rt}$ followed by the addition of sat. aq. $\mathrm{NH}_{4} \mathrm{Cl}$ solution $(1 \mathrm{~mL})$ and $\mathrm{DCM}(2 \mathrm{~mL})$. After separation of the organic layer, the aqueous phase was extracted with DCM $(3 \times 2 \mathrm{~mL})$, the combined organic phases were dried over $\mathrm{MgSO}_{4}$ and the solvent was removed under reduced pressure. Purification of the crude product was achieved by flash chromatography $(15 \% \mathrm{EtOAc} / \mathrm{CyH})$ to yield the title compound $\mathbf{7 2}$ as a colorless oil $(7.90 \mathrm{mg}, 11.1 \mu \mathrm{mol}, 92 \%, d r=6: 1$ resulting out of the aldol reaction to compound 23) ${ }^{[17]}$

$\mathbf{R}_{f}=0.32(20 \% \mathrm{EtOAc} / \mathrm{CyH}) \cdot[\boldsymbol{\alpha}]_{\boldsymbol{D}}^{\mathbf{2 0}}=-25.3^{\circ}\left(c=1.58\right.$ in $\left.\mathrm{CH}_{2} \mathrm{Cl}_{2}\right) .{ }^{1} \mathbf{H} \mathbf{N M R}\left(700 \mathrm{MHz}, \mathrm{CDCl}_{3}\right) \delta=$ $7.49-7.47$ (m, 2H, $\left.\mathrm{H}_{\text {arom., MTPA }}\right), 7.38-7.37$ (m, 3H, $\left.\mathrm{H}_{\text {arom., MTPA }}\right), 7.24$ (d, J=8.7 Hz, 2H, $\mathrm{H}_{\text {arom. }}$ ), 6.87 $\left(\mathrm{d}, J=8.7 \mathrm{~Hz}, 2 \mathrm{H}, \mathrm{H}_{\text {arom. }}\right), 5.57$ (d, $\left.J=7.8 \mathrm{~Hz}, 1 \mathrm{H}, \mathrm{H}-7\right), 5.31$ (d, $\left.J=9.5 \mathrm{~Hz}, 1 \mathrm{H}, \mathrm{H}-9\right), 4.41(\mathrm{~s}, 2 \mathrm{H}$, 
Ar- $\left.\mathrm{CH}_{2}\right), 3.80$ (s, 3H, O-CH $), 3.51$ (s, 3H, O-CH $\mathrm{CH}_{3, \mathrm{MTPA}}$ ), $3.43-3.40$ (m, 3H, H-1, H-11), 3.35 (dd, $J=$ 9.7, 7.1 Hz, 1H, H-11'), $2.90-2.86$ (m, 1H, H-6), $2.54-2.49$ (m, 1H, H-10), $2.42-2.37$ (m, 2H, H4), 1.65 (d, $\left.J=1.3 \mathrm{~Hz}, 3 \mathrm{H}, 8-\mathrm{CH}_{3}\right), 1.60-1.52$ (m, 4H, H-2, H-3), 0.99 (d, $J=7.0 \mathrm{~Hz}, 3 \mathrm{H}, 6-\mathrm{CH}_{3}$ ), $0.89\left(\mathrm{~d}, J=6.7 \mathrm{~Hz}, 3 \mathrm{H}, 10-\mathrm{CH}_{3}\right), 0.87$ (s, 9H, TBS), 0.02 (2xs, 6H, TBS) ppm; ${ }^{13} \mathbf{C}$ NMR (175 MHz, $\left.\mathrm{CDCl}_{3}\right) \delta=210.5(\mathrm{C}-5), 165.7\left(\mathrm{C}=\mathrm{O}_{\mathrm{MTPA}}\right), 159.3\left(\mathrm{C}_{\text {arom. }} \mathrm{O}\right), 134.2(\mathrm{C}-9), 132.6\left(\mathrm{C}_{\text {arom.MTPA }}\right), 131.1(\mathrm{C}-$ 8), $130.8\left(\mathrm{C}_{\text {arom. }}\right), 129.7\left(\mathrm{C}_{\text {arom. }} \mathrm{H}_{\text {MTPA }}\right), 129.4\left(\mathrm{C}_{\text {arom. }} \mathrm{H}\right), 128.4\left(\mathrm{C}_{\text {arom. }} \mathrm{H}_{\text {MTPA }}\right), 127.6\left(\mathrm{C}_{\text {arom. }} \mathrm{H}_{\text {MTPA }}\right), 123.5$ $\left(\mathrm{q}, J=283.7 \mathrm{~Hz}, \mathrm{CF}_{3}\right), 113.9\left(\mathrm{C}_{\text {arom. }} \mathrm{H}\right), 81.3(\mathrm{C}-7), 72.7\left(\mathrm{Ar}^{\left.-\mathrm{CH}_{2}\right)}, 69.8(\mathrm{C}-1), 67.6(\mathrm{C}-11), 55.4(\mathrm{O}-\right.$ $\mathrm{CH}_{3}$ ), 48.6 (C-6), 41.8 (C-4), 35.5 (C-10), 29.3 (C-2), 26.1 (TBS), 20.3 (C-3), 18.5 (TBS), 17.0 (10$\left.\mathrm{CH}_{3}\right), 13.4\left(8-\mathrm{CH}_{3}\right), 12.6\left(6-\mathrm{CH}_{3}\right),-5.2$ (TBS), -5.3 (TBS) ppm; HRMS (ESI+) $\mathrm{m} / z$ : calcd for $\mathrm{C}_{38} \mathrm{H}_{55} \mathrm{~F}_{3} \mathrm{O}_{7} \mathrm{SiNa}[\mathrm{M}+\mathrm{Na}]^{+}:$731.3561, found: 731.3567 , calcd for $\mathrm{C}_{38} \mathrm{H}_{55} \mathrm{~F}_{3} \mathrm{O}_{7} \mathrm{SiNH}_{4}^{+}\left[\mathrm{M}+\mathrm{NH}_{4}\right]^{+}$: 726.4007, found: 726.4013 .

\section{(2S,5S,6S,E)-1-((tert-Butyldimethylsilyl)oxy)-11-((4-methoxybenzyl)oxy)-2,4,6-trimethyl-7-} oxoundec-3-en-5-yl (R)-3,3,3-trifluoro-2-methoxy-2-phenylpropanoate (73)

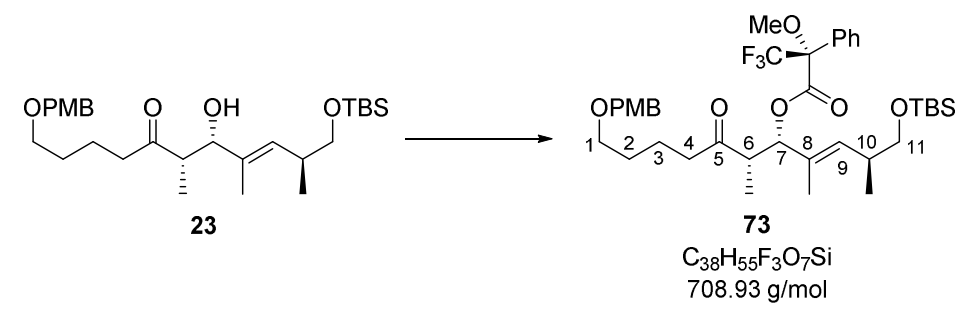

Alcohol 23 (5.70 mg, $11.6 \mu \mathrm{mol}, 1.00$ eq.) was dissolved in pyridine (140 $\mu \mathrm{L})$ and (+)-(S)-MTPA-Cl $\left(13.0 \mu \mathrm{L}, 69.4 \mu \mathrm{mol}, 6.00\right.$ eq.) was added at $0{ }^{\circ} \mathrm{C}$. The reaction mixture was stirred $2 \mathrm{~h}$ at $\mathrm{rt}$ followed by the addition of sat. aq. $\mathrm{NH}_{4} \mathrm{Cl}$ solution $(1 \mathrm{~mL})$ and $\mathrm{DCM}(2 \mathrm{~mL})$. After separation of the organic layer, the aqueous phase was extracted with DCM $(3 \times 2 \mathrm{~mL})$, the combined organic phases were dried over $\mathrm{MgSO}_{4}$ and the solvent was removed under reduced pressure. Purification of the crude product was achieved by flash chromatography $(15 \% \mathrm{EtOAc} / \mathrm{CyH})$ to yield the title compound $\mathbf{7 3}$ as a colorless oil $(6.70 \mathrm{mg}, 9.45 \mu \mathrm{mol}, 82 \%, d r=6: 1$ resulting out of the aldol reaction to compound 23). ${ }^{[17]}$

$\mathbf{R}_{\boldsymbol{f}}=0.31(20 \%$ EtOAc/CyH $) \cdot[\boldsymbol{\alpha}]_{\boldsymbol{D}}^{\mathbf{2 0}}=+8.0^{\circ}\left(c=1.12\right.$ in $\left.\mathrm{CH}_{2} \mathrm{Cl}_{2}\right) .{ }^{\mathbf{1}} \mathbf{H} \mathbf{N M R}\left(700 \mathrm{MHz}, \mathrm{CDCl}_{3}\right) \delta=$ $7.49-7.46$ (m, 2H, $\left.\mathrm{H}_{\text {arom., MTPA }}\right), 7.39-7.33$ (m, 3H, $\left.\mathrm{H}_{\text {arom., мTPA }}\right), 7.24$ (d, J=8.7 Hz, 2H, $\mathrm{H}_{\text {arom. }}$ ), 6.87 (d, $\left.J=8.7 \mathrm{~Hz}, 2 \mathrm{H}, \mathrm{H}_{\text {arom }}\right), 5.53$ (d, $\left.J=6.8 \mathrm{~Hz}, 1 \mathrm{H}, \mathrm{H}-7\right), 5.14$ (d, $\left.J=9.5 \mathrm{~Hz}, 1 \mathrm{H}, \mathrm{H}-9\right), 4.41$ (s, 2H, $\left.\mathrm{Ar}-\mathrm{CH}_{2}\right), 3.80\left(\mathrm{~s}, 3 \mathrm{H}, \mathrm{O}-\mathrm{CH}_{3}\right), 3.52\left(\mathrm{~s}, 3 \mathrm{H}, \mathrm{O}-\mathrm{CH}_{3, \mathrm{MTPA}}\right), 3.42$ (t, $\left.J=6.2 \mathrm{~Hz}, 2 \mathrm{H}, \mathrm{H}-1\right), 3.37$ (dd, $J=$ 9.7, $6.1 \mathrm{~Hz}, 1 \mathrm{H}, \mathrm{H}-11$ ), 3.31 (dd, $J=9.7,7.2 \mathrm{~Hz}, 1 \mathrm{H}, \mathrm{H}-11$ '), $2.88-2.84$ (m, 1H, H-6), $2.50-2.40$ (m, 3H, H-4, H-10), $1.62-1.54$ (m, 4H, H-2, H-3), 1.54 (d, $\left.J=1.2 \mathrm{~Hz}, 3 \mathrm{H}, 8-\mathrm{CH}_{3}\right), 1.09$ (d, $J=$ $\left.7.0 \mathrm{~Hz}, 3 \mathrm{H}, 6-\mathrm{CH}_{3}\right), 0.87$ (s, 9H, TBS), $0.83\left(\mathrm{~d}, J=6.7 \mathrm{~Hz}, 3 \mathrm{H}, 10-\mathrm{CH}_{3}\right), 0.02$ (2xs, 6H), TBS ppm; ${ }^{13} \mathrm{C}$ NMR $\left(175 \mathrm{MHz}, \mathrm{CDCl}_{3}\right) \delta=210.6(\mathrm{C}-5), 165.6\left(\mathrm{C}=\mathrm{O}_{\mathrm{MTPA}}\right), 159.3\left(\mathrm{C}_{\text {arom. }} \mathrm{O}\right), 133.2(\mathrm{C}-9), 132.4$ $\left(\mathrm{C}_{\text {arom.,MTPA }}\right), 130.8(\mathrm{C}-8), 130.8\left(\mathrm{C}_{\text {arom. }}\right), 129.7\left(\mathrm{C}_{\text {arom. }} \mathrm{H}_{\mathrm{MTPA}}\right), 129.4\left(\mathrm{C}_{\text {arom. }} \mathrm{H}\right), 128.5\left(\mathrm{C}_{\text {arom. }} \mathrm{H}_{\mathrm{MTPA}}\right)$, 
$127.6\left(\mathrm{C}_{\text {arom. }} \mathrm{H}_{\mathrm{MTPA}}\right), 123.5\left(\mathrm{q}, J=283.7 \mathrm{~Hz}, \mathrm{CF}_{3}\right), 113.9\left(\mathrm{C}_{\text {arom. }} \mathrm{H}\right), 80.9(\mathrm{C}-7), 72.7\left(\mathrm{Ar}^{-} \mathrm{CH}_{2}\right), 69.8(\mathrm{C}-$ 1), 67.6 (C-11), 55.4 (O-CH ), 48.3 (C-6), 41.7 (C-4), 35.4 (C-10), 29.3 (C-2), 26.1 (TBS), 20.4 (C-3), 18.5 (TBS), $16.9\left(10-\mathrm{CH}_{3}\right), 13.4\left(8-\mathrm{CH}_{3}\right), 12.2\left(6-\mathrm{CH}_{3}\right),-5.2$ (TBS), -5.3 (TBS) ppm; HRMS (ESI+) $m / z$ : calcd for $\mathrm{C}_{38} \mathrm{H}_{55} \mathrm{~F}_{3} \mathrm{O}_{7} \mathrm{SiNa}^{+}[\mathrm{M}+\mathrm{Na}]^{+}:$731.3561, found: 731.3558 .

\section{Mosher Ester Analysis for Compound 23}

Determination of the absolute configuration was accomplished using the Mosher ester analysis. For this purpose, the two Mosher ester derivatives $\mathbf{7 2}$ and $\mathbf{7 3}$ have been synthesized and the respective NMR data have been compared as shown in Table 2. The resulting difference between the ${ }^{1} \mathrm{H}$ NMR data of the $(S)$ - and $(R)$-MTPA ester led to the determination of the absolute configuration as shown below. ${ }^{[17]}$

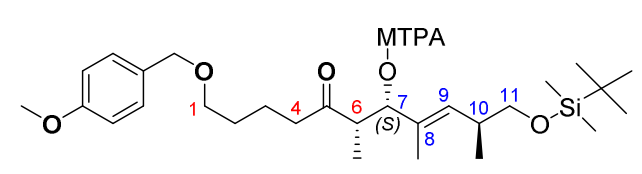

Table 2. Significant ${ }^{1} \mathrm{H}$ NMR signals of $\mathbf{7 2}$ and $\mathbf{7 3}$ and their use in the Mosher ester analysis.

$\begin{array}{cccc}\text { position } & \begin{array}{c}\delta_{H}[\mathrm{ppm}] \\ (\mathbf{7 2})(S)\end{array} & \begin{array}{c}\delta_{H}[\mathrm{ppm}] \\ (\mathbf{7 3})(R)\end{array} & \Delta \delta^{S-R} \\ 1 & 3.41 & 3.52 & -0.11 \\ 6-\mathrm{CH}_{3} & 0.99 & 1.09 & -0.10 \\ 4 & 2.40 & 2.45 & -0.05 \\ 7 & 5.57 & 5.53 & +0.04 \\ 11 & 3.41 & 3.37 & +0.04 \\ 11^{`} & 3.35 & 3.31 & +0.04 \\ 10 & 2.52 & 2.47 & +0.05 \\ 10-\mathrm{CH}_{3} & 0.89 & 0.83 & +0.06 \\ 8-\mathrm{CH}_{3} & 1.65 & 1.54 & +0.11 \\ 9 & 5.31 & 5.14 & +0.17\end{array}$

(2S,5S,6R,7S,E)-1-((tert-Butyldimethylsilyl)oxy)-11-((4-methoxybenzyl)oxy)-2,4,6trimethylundec-3-ene-5,7-diol (74) ${ }^{[29]}$

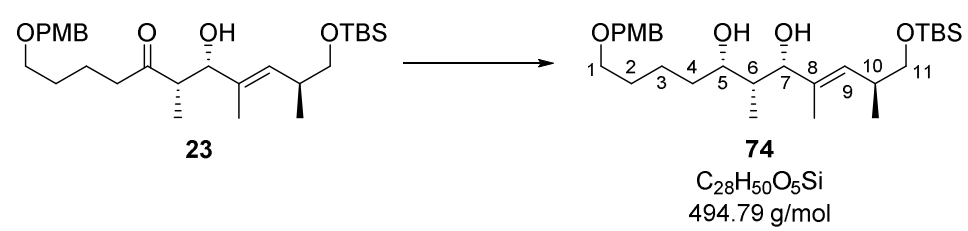

Alcohol 23 (31.7 mg, $64.3 \mu \mathrm{mol}, 1.00$ eq.) was dissolved in THF/methanol (4:1, $0.8 \mathrm{~mL}$ ) and cooled to $-78^{\circ} \mathrm{C}$. $\mathrm{Et}_{2} \mathrm{BOMe}(70.8 \mu \mathrm{L}, 70.8 \mu \mathrm{mol}, 1.10$ eq. $1 \mathrm{M}$ in THF) was added slowly and the mixture was stirred $15 \mathrm{~min}$ at $-78^{\circ} \mathrm{C}$ followed by the addition of $\mathrm{NaBH}_{4}(2.68 \mathrm{mg}, 70.8 \mu \mathrm{mol}, 1.10$ eq.) and further 
stirring for $4 \mathrm{~h}$ at $-78{ }^{\circ} \mathrm{C}$. The reaction mixture was allowed to warm to $0{ }^{\circ} \mathrm{C}$ and quenched with aq. $\mathrm{NaOH}(0.6 \mathrm{~mL}, 3 \mathrm{M})$ and $\mathrm{H}_{2} \mathrm{O}_{2}(0.9 \mathrm{~mL}, 35 \%)$. After stirring $4 \mathrm{~h}$ at $0{ }^{\circ} \mathrm{C}$, water $(1.5 \mathrm{~mL})$ and $\mathrm{Et}_{2} \mathrm{O}$ $(4 \mathrm{~mL})$ were added, the phases separated, the aqueous phase was extracted with $\mathrm{Et}_{2} \mathrm{O}(3 \times 5 \mathrm{~mL})$, the combined organic phases were washed with water $(10 \mathrm{~mL})$, dried over $\mathrm{MgSO}_{4}$ and the solvent was removed under reduced pressure. Purification of the crude product was achieved by flash chromatography $(30 \% \mathrm{EtOAc} / \mathrm{CyH})$ to yield the title compound $\mathbf{7 4}$ as a colorless oil $(31.8 \mathrm{mg}$, $64.3 \mu \mathrm{mol}$, quant.). Furthermore, the separation of the diastereomeric mixture ( $d r=6: 1$, resulting out of the aldol reaction to compound 23) was possible, using the described flash chromatography, and only the major diastereomer was used for following reactions.

$\mathbf{R}_{\boldsymbol{f}}=0.31(40 \% \mathrm{EtOAc} / \mathrm{CyH}) .[\boldsymbol{\alpha}]_{\boldsymbol{D}}^{\mathbf{2 0}}=+2.3^{\circ}\left(c=1.28\right.$ in $\left.\mathrm{CH}_{2} \mathrm{Cl}_{2}\right) .{ }^{1} \mathbf{H} \mathbf{N M R}\left(700 \mathrm{MHz}, \mathrm{CDCl}_{3}\right) \delta=$ 7.26 (d, $\left.J=8.7 \mathrm{~Hz}, 2 \mathrm{H}, \mathrm{H}_{\text {arom. }}\right), 6.87$ (d, $J=8.7 \mathrm{~Hz}, 2 \mathrm{H}, \mathrm{H}_{\text {arom. }}$ ), 5.24 (dq, $J=9.4,1.3 \mathrm{~Hz}, 1 \mathrm{H}, \mathrm{H}-9$ ), 4.43 (s, 2H, Ar-CH $), 4.18$ (brd, $J=2.3 \mathrm{~Hz}, 1 \mathrm{H}, \mathrm{H}-7), 3.85-3.83$ (m, 1H, H-5), 3.80 (s, 3H, O-CH ${ }_{3}$ ), $3.47-3.44$ (m, 3H, H-1, H-11), 3.39 (dd, $J=9.7,7.2 \mathrm{~Hz}, 1 \mathrm{H}, \mathrm{H}-11$ '), 2.67 (s, 1H, 5-OH), $2.63-2.55$ (m, 1H, H-10), 2.39 (s, 1H, 7-OH), $1.67-1.61$ (m, 3H, H-2, H-6), 1.58 (d, J = 1.3 Hz, 3H, 8- $\mathrm{CH}_{3}$ ), $1.56-1.35$ (m, 4H, H-3, H-4), 0.96 (d, $\left.J=6.7 \mathrm{~Hz}, 3 \mathrm{H}, 10-\mathrm{CH}_{3}\right), 0.89$ (s, 9H, TBS), 0.80 (d, $J=$ $\left.7.1 \mathrm{~Hz}, 3 \mathrm{H}, 6-\mathrm{CH}_{3}\right), 0.04$ (2xs, 6H, TBS) ppm; ${ }^{13} \mathbf{C} \mathbf{~ N M R}\left(175 \mathrm{MHz}, \mathrm{CDCl}_{3}\right) \delta=159.3\left(\mathrm{C}_{\text {arom. }} \mathrm{O}\right)$, $135.9(\mathrm{C}-8), 130.8\left(\mathrm{C}_{\text {arom. }}\right), 129.4\left(\mathrm{C}_{\text {arom. }} \mathrm{H}\right), 127.8(\mathrm{C}-9), 113.9\left(\mathrm{C}_{\text {arom. }} \mathrm{H}\right), 80.7$ (C-7), $76.2(\mathrm{C}-5), 72.7$ (Ar- $\left.\mathrm{CH}_{2}\right), 70.1$ (C-1), 68.1 (C-11), $55.4\left(\mathrm{O}-\mathrm{CH}_{3}\right), 39.0$ (C-6), 35.4 (C-4), 35.3 (C-10), 29.8 (C-2), 26.1 (TBS), 23.1 (C-3), 18.5 (TBS), $17.5\left(10-\mathrm{CH}_{3}\right), 14.3\left(8-\mathrm{CH}_{3}\right), 4.8\left(6-\mathrm{CH}_{3}\right),-5.2$ (TBS) ppm; HRMS (ESI+) $m / z$ : calcd for $\mathrm{C}_{28} \mathrm{H}_{50} \mathrm{O}_{5} \mathrm{SiH}^{+}[\mathrm{M}+\mathrm{H}]^{+}: 495.3500$, found: 495.3503 .

\section{tert-Butyl(((S,E)-4-((4S,5R,6S)-6-(4-((4-methoxybenzyl)oxy)butyl)-2,2,5-trimethyl-1,3-dioxan-4- yl)-2-methylpent-3-en-1-yl)oxy)dimethylsilane (75) ${ }^{[18-21]}$}

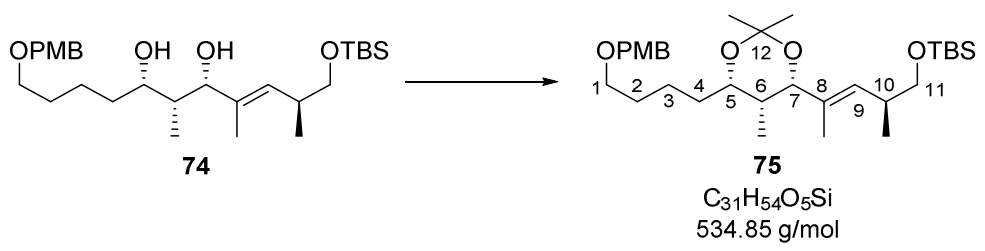

2,2-Dimethoxypropane ( $0.5 \mathrm{~mL})$ was added to diol 74 (9.50 $\mathrm{mg}, 19.2 \mu \mathrm{mol}, 1.00$ eq.) followed by the addition of CSA $(4.47 \mu \mathrm{L}, 0.19 \mu \mathrm{mol}, 0.01$ eq. $43 \mathrm{mM}$ in DCM). The reaction mixture was stirred $3 \mathrm{~h}$ at $\mathrm{rt}$ and quenched with the addition of $\mathrm{NEt}_{3}(7 \mu \mathrm{L})$. All volatiles were removed under reduced pressure. Purification of the crude product was achieved by flash chromatography $(15 \% \mathrm{EtOAc} / \mathrm{CyH})$ to yield the title compound 75 as a colorless oil (10.2 mg, $19.1 \mu \mathrm{mol}$, quant.).

$\mathbf{R}_{\boldsymbol{f}}=0.45(20 \%$ EtOAc/CyH $) \cdot[\boldsymbol{\alpha}]_{\boldsymbol{D}}^{\mathbf{2 0}}=-2.7^{\circ}\left(c=1.46\right.$ in $\left.\mathrm{CH}_{2} \mathrm{Cl}_{2}\right) .{ }^{\mathbf{1}} \mathbf{H} \mathbf{N M R}\left(700 \mathrm{MHz}, \mathrm{CDCl}_{3}\right) \delta=$ 7.27 (d, $\left.J=8.7 \mathrm{~Hz}, 2 \mathrm{H}, \mathrm{H}_{\text {arom. }}\right), 6.87$ (d, $\left.J=8.7 \mathrm{~Hz}, 2 \mathrm{H}, \mathrm{H}_{\text {arom. }}\right), 5.24$ (dq, $\left.J=9.3,1.0 \mathrm{~Hz}, 1 \mathrm{H}, \mathrm{H}-9\right)$, 4.43 (s, 2H, Ar- $\mathrm{CH}_{2}$ ), 4.19 (brs, 1H, H-7), $3.89-3.87$ (m, 1H, H-5), 3.80 (s, 3H, O-CH CH $_{3}, 3.47-3.44$ 
(m, 3H, H-1, H-11), 3.36 (dd, J = 9.7, 7.5 Hz, 1H, H-11'), 2.62 - 2.56 (m, 1H, H-10), 1.65 - 1.61 (m, $2 \mathrm{H}, \mathrm{H}-2), 1.55$ (d, $\left.J=1.0 \mathrm{~Hz}, 3 \mathrm{H}, 8-\mathrm{CH}_{3}\right), 1.53-1.43$ (m, 3H, H-3, H-4, H-6), 1.42 (s, 3H, 12- $\mathrm{CH}_{3}$ ), 1.42 (s, 3H, 12- $\left.\mathrm{CH}_{3}\right), 1.39-1.32$ (m, 2H, H-3', H-4'), 0.97 (d, J=6.7 Hz, 3H, 10-CH $), 0.89$ (s, 9H, TBS), 0.67 (d, $\left.J=6.9 \mathrm{~Hz}, 3 \mathrm{H}, 6-\mathrm{CH}_{3}\right), 0.04$ (2xs, 6H, TBS) ppm; ${ }^{13} \mathbf{C} \mathbf{N M R}\left(175 \mathrm{MHz}, \mathrm{CDCl}_{3}\right) \delta=$ $159.3\left(\mathrm{C}_{\text {arom. }} \mathrm{O}\right), 132.9(\mathrm{C}-8), 130.9\left(\mathrm{C}_{\text {arom. }}\right), 129.4\left(\mathrm{C}_{\text {arom. }} \mathrm{H}\right), 127.0(\mathrm{C}-9), 113.9\left(\mathrm{C}_{\text {arom. }} \mathrm{H}\right), 98.9(\mathrm{C}-12)$, 75.9 (C-7), 73.2 (C-5), $72.7\left(\mathrm{Ar}-\mathrm{CH}_{2}\right), 70.1$ (C-1), $68.3(\mathrm{C}-11), 55.4\left(\mathrm{O}-\mathrm{CH}_{3}\right), 35.3(\mathrm{C}-10), 33.5(\mathrm{C}-6)$, 33.0 (C-4), 30.2 (12- $\left.\mathrm{CH}_{3}\right), 29.9$ (C-2), 26.1 (TBS), 22.3 (C-3), 19.8 (12- $\left.\mathrm{CH}_{3}\right), 18.5$ (TBS), 17.5 (10$\left.\mathrm{CH}_{3}\right), 14.0\left(8-\mathrm{CH}_{3}\right), 5.1\left(6-\mathrm{CH}_{3}\right),-5.1$ (TBS), -5.2 (TBS) ppm; HRMS (ESI+) m/z: calcd for $\mathrm{C}_{31} \mathrm{H}_{54} \mathrm{O}_{5} \mathrm{SiNH}_{4}^{+}\left[\mathrm{M}+\mathrm{NH}_{4}\right]^{+}: 552.4079$, found: 552.4077 .

\section{Acetonide Analysis for Compound 74}

The determination of the correct relative stereochemistry of diol $\mathbf{7 4}$ (stereocenter at position 5 and 7) was accomplished by the accepted model for the analysis of the corresponding acetonide $\mathbf{7 5}$. Thus, the ${ }^{13} \mathrm{C}$ NMR was used and analyzed showing different chemical shifts for the two methyl groups of the acetonide, which indicate a chair conformation, resulting out of the syn-1,3-diol acetonide. In the ${ }^{1} \mathrm{H}$ NMR, a qdd was identified for H-6, although the signal is in the multiplet region of $1.53-1.43 \mathrm{ppm}$. The coupling constant of $6.9 \mathrm{~Hz}$ results from the coupling with the 6- $\mathrm{CH}_{3}$ group and the two coupling constants of $2.3 \mathrm{~Hz}$ indicate a cis coupling to H-5 and H-7. The cis-hydrogens were also confirmed with NOESY correlations as shown in Figure 9. ${ }^{[18-21]}$

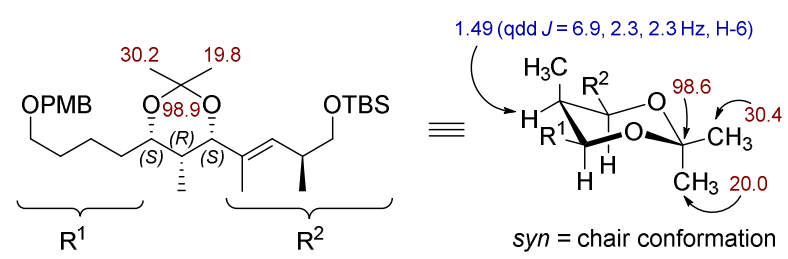

Figure 9. Relevant ${ }^{13} \mathrm{C}$ NMR signals (brown) and the ${ }^{1} \mathrm{H}$ NMR signal for H-6 used for the determination of the 1,3-syn-diol acetonide and the relative configuration of $6-\mathrm{CH}_{3}$. 


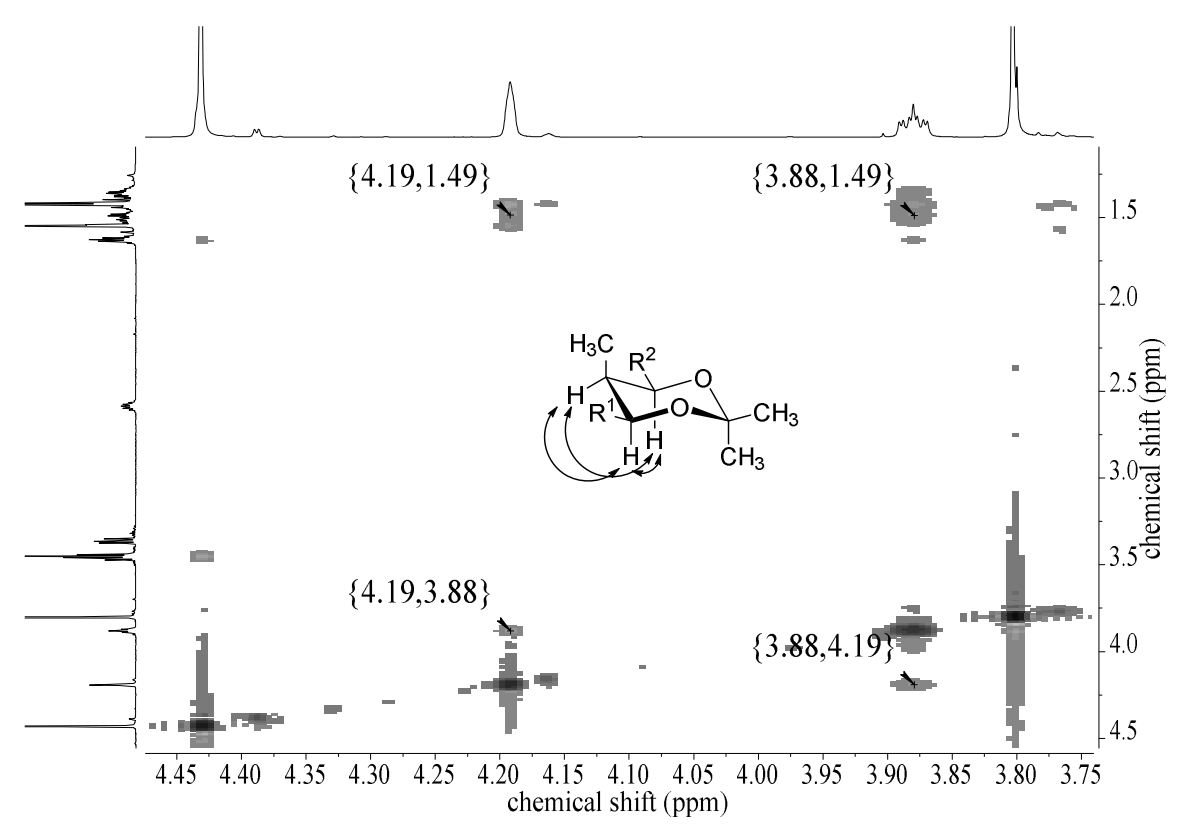

Figure 10. Selected NOESY signals of compound 75, which were used for the determination of the relative configuration of 6- $\mathrm{CH}_{3}$.

\section{4-((4S,5R,6S)-6-((S,E)-5-((tert-Butyldimethylsilyl)oxy)-4-methylpent-2-en-2-yl)-2,2,5-trimethyl-}

\section{1,3-dioxan-4-yl)butan-1-ol (3)}

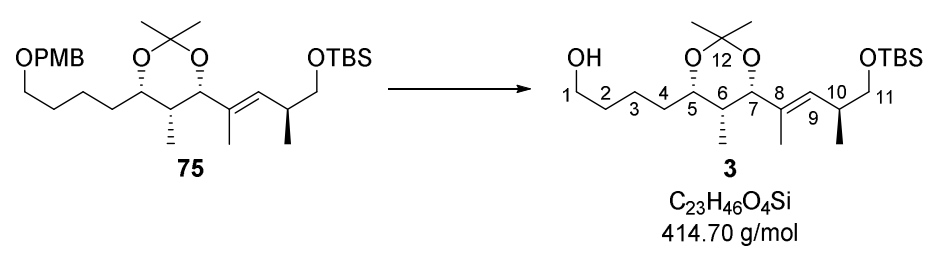

Acetonide 75 (9.60 mg, $18.0 \mu \mathrm{mol}, 1.00$ eq.) was dissolved in DCM $(0.7 \mathrm{~mL})$ and pH7 buffer $(70 \mu \mathrm{L})$ was added. After adding DDQ ( $8.15 \mathrm{mg}, 35.9 \mu \mathrm{mol}, 2.00$ eq.), the reaction mixture was stirred $90 \mathrm{~min}$ at rt. DCM $(4 \mathrm{~mL})$ and water $(3 \mathrm{~mL})$ were added, the phases separated, the aqueous phase was extracted with DCM $(3 \times 4 \mathrm{~mL})$, the combined organic phases were dried over $\mathrm{MgSO}_{4}$ and the solvent was removed under reduced pressure. Purification of the crude product was achieved by flash chromatography $(20 \% \mathrm{EtOAc} / \mathrm{CyH})$ to yield the title compound $\mathbf{3}$ as a colorless oil $(3.70 \mathrm{mg}$, $8.92 \mu \mathrm{mol}, 50 \%)$.

$\mathbf{R}_{f}=0.21(20 \% \mathrm{EtOAc} / \mathrm{CyH}) \cdot[\boldsymbol{\alpha}]_{\boldsymbol{D}}^{\mathbf{2 0}}=-2.1^{\circ}\left(c=0.48\right.$ in $\left.\mathrm{CH}_{2} \mathrm{Cl}_{2}\right) .{ }^{\mathbf{1}} \mathbf{H} \mathbf{N M R}\left(700 \mathrm{MHz}, \mathrm{CDCl}_{3}\right) \delta=$ 5.25 (dq, $J=9.3,1.0 \mathrm{~Hz}, 1 \mathrm{H}, \mathrm{H}-9), 4.20$ (brs, $1 \mathrm{H}, \mathrm{H}-7), 3.91-3.89$ (m, 1H, H-5), 3.67 (t, $J=6.5 \mathrm{~Hz}$, 2H, H-1), 3.46 (dd, $J=9.7,6.0 \mathrm{~Hz}, 1 \mathrm{H}, \mathrm{H}-11$ ), 3.36 (dd, $J=9.7,7.5 \mathrm{~Hz}, 1 \mathrm{H}, \mathrm{H}-11^{\prime}$ ), $2.62-2.57$ (m, 1H, H-10), $1.64-1.58$ (m, 3H, H-2), 1.55 (d, $J=1.0 \mathrm{~Hz}, 3 \mathrm{H}, 8-\mathrm{CH}_{3}$ ), $1.51-1.45$ (m, 3H, H-3, H-4, H-6), 1.43 (s, 3H, 12- $\mathrm{CH}_{3}$ ), 1.42 (s, 3H, 12- $\mathrm{CH}_{3}$ ), $1.40-1.58$ (m, 2H, H-3', H-4'), 0.97 (d, J=6.7 Hz, $\left.3 \mathrm{H}, 10-\mathrm{CH}_{3}\right), 0.68\left(\mathrm{~d}, J=6.9 \mathrm{~Hz}, 3 \mathrm{H}, 6-\mathrm{CH}_{3}\right), 0.04$ (2xs, 6H, TBS) ppm; ${ }^{13} \mathbf{C}$ NMR $(175 \mathrm{MHz}$, $\left.\mathrm{CDCl}_{3}\right) \delta=132.8(\mathrm{C}-8), 127.1$ (C-9), 98.9 (C-12), 75.9 (C-7), 73.3 (C-5), 68.3 (C-11), 63.0 (C-1), 35.3 (C-10), 33.7 (C-6), 33.0 (C-4), 32.8 (C-2), 30.2 (12- $\left.\mathrm{CH}_{3}\right), 26.1$ (TBS), 22.1 (C-3), 19.8 (12- $\left.\mathrm{CH}_{3}\right), 18.5$ 
(TBS), $17.5\left(10-\mathrm{CH}_{3}\right), 14.0\left(8-\mathrm{CH}_{3}\right), 5.1\left(6-\mathrm{CH}_{3}\right),-5.1$ (TBS), -5.2 (TBS) ppm; HRMS (ESI+) $m / z$ : calcd for $\mathrm{C}_{23} \mathrm{H}_{46} \mathrm{O}_{4} \mathrm{SiNH}_{4}^{+}\left[\mathrm{M}+\mathrm{NH}_{4}\right]^{+}: 432.3504$, found: 432.3513 .

$(2 S, 5 S, 6 R, 7 S, E)-2,4,6$-Trimethylundec-3-ene-1,5,7,11-tetraol (40)

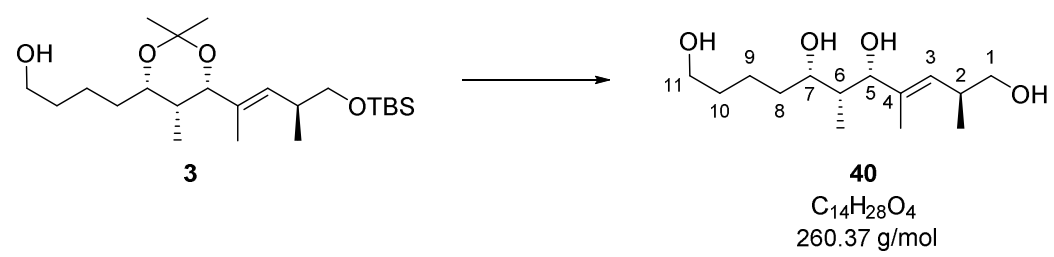

To a solution of 3 (14.0 mg, $33.8 \mu \mathrm{mol}, 1.00$ eq.) in $\mathrm{MeOH}(0.40 \mathrm{~mL})$ was added $\mathrm{HCl}(1.0 \mathrm{M}$ in water, $67.5 \mu \mathrm{L}, 67.5 \mu \mathrm{mol}, 2.00$ eq.) at room temperature. The reaction solution was stirred for $1.5 \mathrm{~h}$ at $\mathrm{rt}$ before addition of water $(5.0 \mathrm{~mL})$ and DCM $(5.0 \mathrm{~mL})$. The phases were separated and the aqueous layer was extracted with DCM $(3 \times 1.0 \mathrm{~mL})$ and ethyl acetate $(5 \times 2.0 \mathrm{~mL})$. The combined phases were dried over $\mathrm{Na}_{2} \mathrm{SO}_{4}$ and concentrated in vacuo to yield title compound $\mathbf{4 0}$ as a colorless resin $(2.80 \mathrm{mg}$, $10.8 \mu \mathrm{mol}, 32 \%)$.

$\boldsymbol{R}_{f}=0.1$ (ethyl acetate). ${ }^{1} \mathbf{H}$ NMR $\left(700 \mathrm{MHz}, \mathrm{MeOD}-d_{4}\right): \delta=5.28$ (ddq, $\left.J=9.3,1.1,0.9 \mathrm{~Hz}, 1 \mathrm{H}, \mathrm{H}-3\right)$, 4.02 (d, $J=6.9 \mathrm{~Hz}, 1 \mathrm{H}, \mathrm{H}-5), 3.66-3.60$ (m, 1H, H-7), 3.57 (dd, $J=6.6 \mathrm{~Hz}, 2 \mathrm{H}, \mathrm{H}-11$ ), 3.43 (dd, $J=$ 10.5, 6.7 Hz, 1H, H-1a), 3.38 (dd, $J=10.5,6.9 \mathrm{~Hz}, 1 \mathrm{H}, \mathrm{H}-1 \mathrm{~b}$ ), 2.64 (dddq, $J=9.3,7.0,6.9,6.7 \mathrm{~Hz}$, 1H, H-2), 1.65 (ddq, $J=6.9,6.8,2.6 \mathrm{~Hz}, 1 \mathrm{H}, \mathrm{H}-6), 1.62$ (d, $J=1.1 \mathrm{~Hz}, 3 \mathrm{H}, 4-\mathrm{Me}), 1.61-1.52$ (m, 3H, H-10), $1.51-1.43$ (m, 2H, H-8), $1.42-1.33$ (m, 1H, H-9), 0.99 (d, $J=6.8 \mathrm{~Hz}, 3 \mathrm{H}, 2-\mathrm{Me}$ ), 0.92 (d, $J=$ $6.9 \mathrm{~Hz}, 3 \mathrm{H}, 6-\mathrm{Me}) \mathrm{ppm} ;{ }^{13} \mathrm{C}$ NMR $\left(175 \mathrm{MHz}, \mathrm{MeOD}-d_{4}\right): \delta=137.7$ (C-4), 130.8 (C-3), 80.9 (C-5), 73.9 (C-7), 68.2 (C-1), 62.9 (C-11), 41.0 (C-6), 36.3 (C-2), 36.2 (C-8), 33.6 (C-10), 23.5 (C-9), 17.5 (2-Me), 12.6 (4-Me), 7.8 (6-Me) ppm; HRMS (ESI+): $m / z$ : calcd for $\mathrm{C}_{14} \mathrm{H}_{29} \mathrm{O}_{4}{ }^{+}[\mathrm{M}+\mathrm{H}]^{+}: 261.2060$, found: 261.2059 .

\section{Synthesis of fragment 4 (C26 to C36)}

Ethyl (E)-5-((tert-Butyldimethylsilyl)oxy)pent-2-enoate (25)

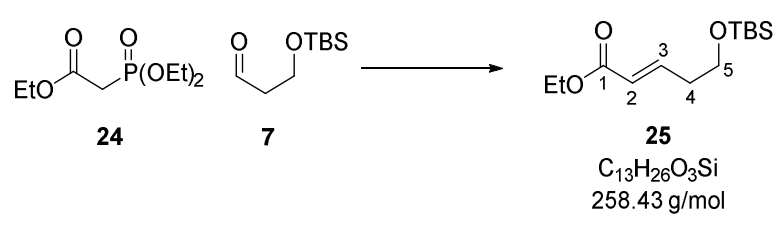

A solution of triethyl phosphonoacetate $(\mathbf{2 4}, 143 \mathrm{mg}, 637 \mu \mathrm{mol}, 1.20$ eq.) in THF (3.5 mL) was cooled to $0{ }^{\circ} \mathrm{C}$ and $\mathrm{NaH}(25.5 \mathrm{mg}, 637 \mu \mathrm{mol}, 1.20$ eq. $60 \%$ dispersion in mineral oil) was added over the period of $5 \mathrm{~min}$. The reaction mixture was stirred $30 \mathrm{~min}$ at $\mathrm{rt}$ before cooling again to $0{ }^{\circ} \mathrm{C}$. Aldehyde 7 (100 mg, $531 \mu \mathrm{mol}, 1.00$ eq. in $1 \mathrm{~mL}$ THF) was added and stirring was continued on at rt. Aq. sat. 
$\mathrm{NH}_{4} \mathrm{Cl}$ solution $(6 \mathrm{~mL})$ and EtOAc $(10 \mathrm{~mL})$ were added, the phases separated, the aqueous phase was extracted with EtOAc $(3 \times 10 \mathrm{~mL})$, the combined organic phases were washed with water $(10 \mathrm{~mL})$ and brine $(10 \mathrm{~mL})$, dried over $\mathrm{MgSO}_{4}$ and the solvent was removed under reduced pressure. Purification of the crude product was achieved by flash chromatography $(5 \% \mathrm{EtOAc} / \mathrm{CyH})$ to yield the title compound 25 as a colorless oil (132 mg, $510 \mu \mathrm{mol}, 96 \%$, only the $E$ isomer was obtained, $E / Z>20: 1$ ). $\mathbf{R}_{f}=0.20\left(5 \%\right.$ EtOAc/CyH). ${ }^{1} \mathbf{H}$ NMR $\left(500 \mathrm{MHz}, \mathrm{CDCl}_{3}\right) \delta=6.96(\mathrm{dt}, J=15.7,7.1 \mathrm{~Hz}, 1 \mathrm{H}, \mathrm{H}-3)$, $5.86(\mathrm{dt}, J=15.7,1.5 \mathrm{~Hz}, 1 \mathrm{H}, \mathrm{H}-2), 4.19$ (q, $\left.J=7.1 \mathrm{~Hz}, 2 \mathrm{H}, \mathrm{OCH}_{2} \mathrm{CH}_{3}\right), 3.72$ (t, $\left.J=6.5 \mathrm{~Hz}, 2 \mathrm{H}, \mathrm{H}-5\right)$, $2.41(\mathrm{dtd}, J=7.1,6.5,1.5 \mathrm{~Hz}, 2 \mathrm{H}, \mathrm{H}-4), 1.28$ (t, $J=7.1 \mathrm{~Hz}, 3 \mathrm{H}, \mathrm{OCH}_{2} \mathrm{CH}_{3}$ ), 0.89 (s, 9H, TBS), 0.05 (s, 6H, TBS) ppm; ${ }^{13} \mathbf{C}$ NMR (125 MHz, $\left.\mathrm{CDCl}_{3}\right) \delta=166.6$ (C-1), 146.0 (C-3), 123.1 (C-2), 61.7 (C5), $60.3\left(\mathrm{OCH}_{2} \mathrm{CH}_{3}\right), 35.8$ (C-4), 26.0 (TBS), 18.4 (TBS), $14.4\left(\mathrm{OCH}_{2} \mathrm{CH}_{3}\right),-5.2$ (TBS) ppm; MS (ESI+) $m / z$ : calcd for $\mathrm{C}_{13} \mathrm{H}_{26} \mathrm{O}_{3} \mathrm{SiH}^{+}[\mathrm{M}+\mathrm{H}]^{+}: 259.173$, found: 259.172 . The large coupling constant of $15.7 \mathrm{~Hz}$ between $\mathrm{H}-2$ and $\mathrm{H}-3$ indicated a trans-coupling confirming the $E$ double bond. The spectroscopic data were in agreement with those previously reported. ${ }^{[3]}$ 


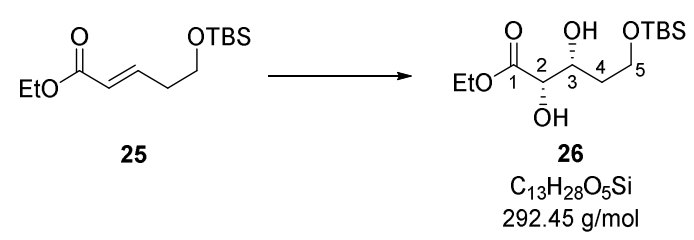

A solution of ester 25 (365 mg, $1.41 \mathrm{mmol}, 1.00$ eq.) in 1:1 tert-butanol/water ( $3.3 \mathrm{~mL}$ ) was added to a $0{ }^{\circ} \mathrm{C}$ cooled solution of methanesulfonamide $(135 \mathrm{mg}, 1.41 \mathrm{mmol}, 1.00 \mathrm{eq}$.) and $\mathrm{AD}-\mathrm{mix}-\beta$ (1.97 g, $1.40 \mathrm{~g}$ per mmol starting material) in 1:1 tert-butanol/water $(1.4 \mathrm{~mL})$. The mixture was stirred for $48 \mathrm{~h}$ at rt, $\mathrm{Na}_{2} \mathrm{SO}_{3}$ (1.78 g, $14.1 \mathrm{mmol}, 10.0$ eq.) was added and stirring was continued for $3 \mathrm{~h}$ at rt. EtOAc $(30 \mathrm{~mL})$ and water $(30 \mathrm{~mL})$ were added the phases separated, the aqueous phase was extracted with EtOAc $(3 \times 30 \mathrm{~mL})$, the combined organic phases were washed with brine $(30 \mathrm{~mL})$, dried over $\mathrm{MgSO}_{4}$ and the solvent was removed under reduced pressure. Purification of the crude product was achieved by flash chromatography (35\% EtOAc/CyH) to yield the title compound $\mathbf{2 6}$ as a colorless oil $(330 \mathrm{mg}$, $1.13 \mathrm{mmol}, 80 \%)$.

$\mathbf{R}_{\boldsymbol{f}}=0.34(40 \% \mathrm{EtOAc} / \mathrm{CyH}) \cdot[\boldsymbol{\alpha}]_{\boldsymbol{D}}^{25}=-2.2^{\circ}\left(c=0.90\right.$ in $\left.\mathrm{CHCl}_{3}\right) .{ }^{1} \mathbf{H} \mathbf{N M R}\left(400 \mathrm{MHz}, \mathrm{CDCl}_{3}\right) \delta=$ 4.29 (q, $J=7.1 \mathrm{~Hz}, 2 \mathrm{H}, \mathrm{OCH}_{2} \mathrm{CH}_{3}$ ), $4.20-4.17$ (brm, 1H, H-3), 4.06 (brs, 1H, H-2), $3.94-3.82$ (m, 2H, H-5), $3.20-3.14$ (m, 2H, 2-OH, 3-OH), $2.00-1.91$ (m, 1H, H-4), $1.77-1.71$ (m, 1H, H-4'), 1.32 (t, $\left.J=7.1 \mathrm{~Hz}, 3 \mathrm{H}, \mathrm{OCH}_{2} \mathrm{CH}_{3}\right), 0.90$ (s, 9H, TBS), 0.08 (s, 6H, TBS) ppm; ${ }^{13} \mathbf{C}$ NMR $(175 \mathrm{MHz}$, $\left.\mathrm{CDCl}_{3}\right) \delta=173.3(\mathrm{C}-1), 73.8(\mathrm{C}-2), 72.3(\mathrm{C}-3), 62.0\left(\mathrm{O}_{\underline{C H}}{ }_{2} \mathrm{CH}_{3}\right), 61.8$ (C-5), 35.4 (C-4), 26.0 (TBS), 18.3 (TBS), $14.3\left(\mathrm{OCH}_{2} \underline{\mathrm{CH}}_{3}\right)$, -5.4 (TBS) ppm; HRMS (ESI+) $m / z$ : calcd for $\mathrm{C}_{13} \mathrm{H}_{28} \mathrm{O}_{5} \mathrm{SiH}^{+}[\mathrm{M}+\mathrm{H}]^{+}$: 293.1779, found: 293.1778 . Due to the similarity of the rotation values (literature: $[\boldsymbol{\alpha}]_{\boldsymbol{D}}^{25}=-3.6^{\circ}(c=$ 0.90 in $\mathrm{CHCl}_{3}$ ) and the literature known procedure to product 27, the ee was not checked again and the literature provided ee of $92 \%$ was assumed. The spectroscopic data were in agreement with those previously reported. ${ }^{[3]}$

Ethyl-(4S,5R)-5-(2-((tert-butyldimethylsilyl)oxy)ethyl)-2,2-dimethyl-1,3-dioxolane-4-carboxylate (76)

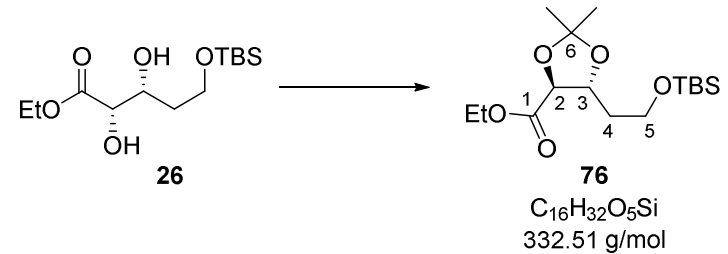

Diol 26 (60.0 mg, $205 \mu \mathrm{mol}, 1.00$ eq.) was dissolved in acetone (1 mL) and 2,2-dimethoxypropane (151 $\mu \mathrm{L}, 1.23 \mathrm{mmol}, 6.00$ eq.) and $p \mathrm{TsOH}(200 \mu \mathrm{L}, 2.40 \mu \mathrm{mol}, 0.01$ eq. $12 \mathrm{mM}$ solution in acetone) were added. The reaction mixture was stirred on at $\mathrm{rt}$ followed by the addition of solid $\mathrm{NaHCO}_{3}$ $(10 \mathrm{mg})$ and further stirring for $15 \mathrm{~min}$ at $\mathrm{rt}$. After filtration of the mixture through a short pad of 
silica, all volatiles were removed under reduced pressure. Purification of the crude product was achieved by flash chromatography $(15 \% \mathrm{EtOAc} / \mathrm{CyH})$ to yield the title compound $\mathbf{7 6}$ as a colorless oil (53.7 mg, $162 \mu \mathrm{mol}, 79 \%)$.

$\mathbf{R}_{\boldsymbol{f}}=0.59(40 \% \mathrm{EtOAc} / \mathrm{CyH}) .[\boldsymbol{\alpha}]_{\boldsymbol{D}}^{\mathbf{2 5}}=+11.8^{\circ}\left(c=1.36\right.$ in $\left.\mathrm{CHCl}_{3}\right) .{ }^{1} \mathbf{H} \mathbf{N M R}\left(700 \mathrm{MHz}, \mathrm{CD}_{2} \mathrm{Cl}_{2}\right) \delta=$ $4.24(\mathrm{td}, J=7.7,3.7 \mathrm{~Hz}, 1 \mathrm{H}, \mathrm{H}-3), 4.19$ (q, $\left.J=7.1 \mathrm{~Hz}, 2 \mathrm{H}, \mathrm{OCH}_{2} \mathrm{CH}_{3}\right), 4.16$ (d, $\left.J=7.7 \mathrm{~Hz}, 1 \mathrm{H}, \mathrm{H}-2\right)$, $3.79-3.72(\mathrm{~m}, 2 \mathrm{H}, \mathrm{H}-5), 1.42\left(\mathrm{~s}, 3 \mathrm{H}, 6-\mathrm{CH}_{3}\right), 1.39\left(\mathrm{~s}, 3 \mathrm{H}, 6-\mathrm{CH}_{3}\right), 1.27(\mathrm{t}, J=7.1 \mathrm{~Hz}, 3 \mathrm{H}$, $\left.\mathrm{OCH}_{2} \mathrm{CH}_{3}\right), 0.89$ (s, 9H, TBS), 0.05 (2xs, 6H, TBS) ppm; ${ }^{13} \mathbf{C}$ NMR $\left(175 \mathrm{MHz}, \mathrm{CD}_{2} \mathrm{Cl}_{2}\right) \delta=171.2(\mathrm{C}-$ 1), 111.1 (C-6), 79.6 (C-2), $76.6(\mathrm{C}-3), 61.7\left(\mathrm{OCH}_{2} \mathrm{CH}_{3}\right), 60.0$ (C-5), $37.2(\mathrm{C}-4), 27.5\left(6-\mathrm{CH}_{3}\right), 26.2$ (TBS), $26.0\left(6-\mathrm{CH}_{3}\right), 18.7$ (TBS), $14.5\left(\mathrm{OCH}_{2} \underline{\mathrm{CH}}_{3}\right),-5.2$ (TBS) ppm; HRMS (ESI+) $\mathrm{m} / \mathrm{z}$ : calcd for $\mathrm{C}_{16} \mathrm{H}_{32} \mathrm{O}_{5} \mathrm{SiH}^{+}[\mathrm{M}+\mathrm{H}]^{+}: 333.2092$, found: 333.2093. The spectroscopic data were in agreement with those previously reported. ${ }^{[3]}$

((4R,5R)-5-(2-((tert-Butyldimethylsilyl)oxy)ethyl)-2,2-dimethyl-1,3-dioxolan-4-yl)methanol (77)

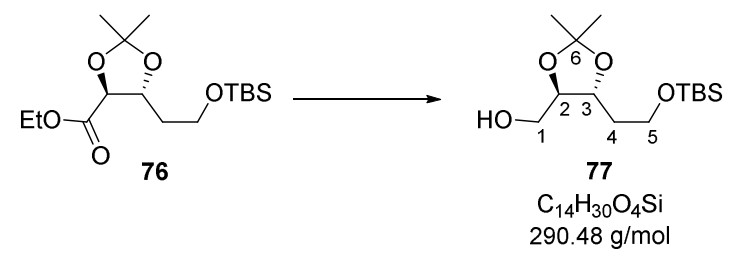

Ester 76 (270 mg, $812 \mu \mathrm{mol}, 1.00$ eq.) was dissolved in THF ( $3.5 \mathrm{~mL})$, the solution was cooled to $-78^{\circ} \mathrm{C}$ and DIBAL-H (2.03 mL, $2.03 \mathrm{mmol}, 2.50$ eq. $1 \mathrm{M}$ in THF) was added slowly. After stirring $1 \mathrm{~h}$ at $-78^{\circ} \mathrm{C}$ and $30 \mathrm{~min}$ at $\mathrm{rt}$ the reaction solution was poured into a sat. aq. Rochelle salt solution $(15 \mathrm{~mL})$. The mixture was stirred until clearly two phases appeared $(2 \mathrm{~h})$. The phases were separated, the aqueous layer was extracted with $\mathrm{Et}_{2} \mathrm{O}(3 \times 30 \mathrm{~mL})$, the combined organic phases were dried over $\mathrm{MgSO}_{4}$ and the solvent was removed under reduced pressure. Purification of the crude product was achieved by flash chromatography $(40 \% \mathrm{EtOAc} / \mathrm{CyH})$ to yield the title compound $\mathbf{7 7}$ as a colorless oil (225 mg, $775 \mu \mathrm{mol}, 96 \%$ ).

$\mathbf{R}_{f}=0.24(30 \% \mathrm{EtOAc} / \mathrm{CyH}) \cdot[\boldsymbol{\alpha}]_{\boldsymbol{D}}^{\mathbf{2 5}}=+17.8^{\circ}\left(c=0.90\right.$ in $\left.\mathrm{CH}_{2} \mathrm{Cl}_{2}\right) .{ }^{\mathbf{1}} \mathbf{H} \mathbf{N M R}\left(500 \mathrm{MHz}, \mathrm{CD}_{2} \mathrm{Cl}_{2}\right) \delta=$ $4.00-3.95$ (m, 1H, H-3), $3.80-3.70$ (m, 4H, H-1, H-2, H-5), $3.62-3.57$ (m, 1H, H-1'), 1.96 (t, $J=$ $6.3 \mathrm{~Hz}, 1 \mathrm{H}, \mathrm{OH}), 1.18-1.76$ (m, 2H, H-4), 1.38 (s, 3H, 6- $\left.\mathrm{CH}_{3}\right), 1.36$ (s, 3H, 6- $\left.\mathrm{CH}_{3}\right), 0.89$ (s, 9H, TBS), 0.06 (2xs, 6H, TBS) ppm; ${ }^{13} \mathbf{C}$ NMR (125 MHz, $\left.\mathrm{CD}_{2} \mathrm{Cl}_{2}\right) \delta=108.9$ (C-6), 82.0 (C-2), 75.0 (C3), 62.6 (C-1), 60.5 (C-5), 36.8 (C-4), 27.7 (6- $\left.\mathrm{CH}_{3}\right), 27.3\left(6-\mathrm{CH}_{3}\right), 26.2$ (TBS), 18.7 (TBS), -5.1 (TBS) ppm; HRMS (ESI+) $m / z$ : calcd for $\mathrm{C}_{14} \mathrm{H}_{30} \mathrm{O}_{4} \mathrm{SiH}^{+}[\mathrm{M}+\mathrm{H}]^{+}:$291.1986, found: 291.1984. 


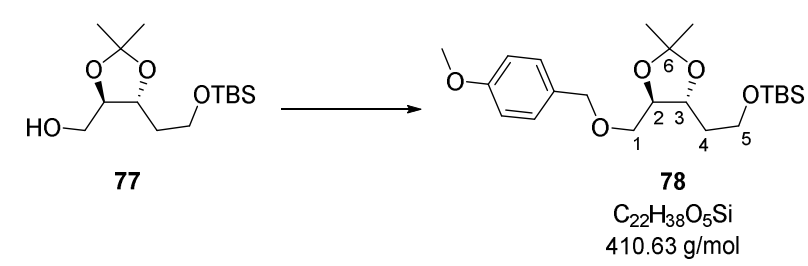

Alcohol 77 (225 mg, $775 \mu \mathrm{mol}, 1.00$ eq.) was dissolved in DMF $(1.5 \mathrm{~mL})$, cooled to $0{ }^{\circ} \mathrm{C}$ and 4-methoxybenzyl chloride ( $115 \mu \mathrm{L}, 852 \mu \mathrm{mol}, 1.10$ eq.) was added. $\mathrm{NaH}$ (34.1 mg, $852 \mu \mathrm{mol}, 1.10 \mathrm{eq}$. $60 \%$ dispersion in mineral oil) was added over the period of $5 \mathrm{~min}$ and the reaction mixture was stirred for $2 \mathrm{~h}$ at $0{ }^{\circ} \mathrm{C}$ and $2 \mathrm{~h}$ at $\mathrm{rt}$. DCM $(15 \mathrm{~mL})$ and water $(10 \mathrm{~mL})$ were added at $0{ }^{\circ} \mathrm{C}$, the phases separated, the aqueous phase extracted with DCM $(3 \times 15 \mathrm{~mL})$, the combined organic phases were dried over $\mathrm{MgSO}_{4}$ and the solvent was removed under reduced pressure. Purification of the crude product was achieved by flash chromatography $(25 \% \mathrm{EtOAc} / \mathrm{CyH})$ to yield the title compound $\mathbf{7 8}$ as a colorless oil (276 mg, $672 \mu \mathrm{mol}, 87 \%)$.

$\mathbf{R}_{\boldsymbol{f}}=0.45(30 \% \mathrm{EtOAc} / \mathrm{CyH}) \cdot[\boldsymbol{\alpha}]_{\boldsymbol{D}}^{\mathbf{2 0}}=+10.7^{\circ}\left(c=1.22\right.$ in $\left.\mathrm{CH}_{2} \mathrm{Cl}_{2}\right) .{ }^{1} \mathbf{H} \mathbf{N M R}\left(700 \mathrm{MHz}, \mathrm{CDCl}_{3}\right) \delta=$ 7.26 (d, $J=8.6 \mathrm{~Hz}, 2 \mathrm{H}, \mathrm{H}_{\text {arom. }}$ ), 6.87 (d, $J=8.6 \mathrm{~Hz}, 2 \mathrm{H}, \mathrm{H}_{\text {arom. }}$ ), $4.57-4.49$ (m, 2H, Ar- $\mathrm{CH}_{2}$ ), 3.92 (td, $J=8.3,3.6 \mathrm{~Hz}, 1 \mathrm{H}, \mathrm{H}-3), 3.87$ (dt, $J=8.3,4.8 \mathrm{~Hz}, 1 \mathrm{H}, \mathrm{H}-2), 3.80$ (s, $\left.3 \mathrm{H}, \mathrm{O}_{-} \mathrm{CH}_{3}\right), 3.77-3.74$ (m, $1 \mathrm{H}$, H-5), $3.73-3.69$ (m, 1H, H-5'), 3.54 (d, J=4.8 Hz, 2H, H-1), $1.83-1.79$ (m, 1H, H-4), 1.77 - 1.72 (m, 1H, H-4'), 1.40 (s, 3H, 6- $\mathrm{CH}_{3}$ ), 1.38 (s, 3H, 6- $\mathrm{CH}_{3}$ ), 0.88 (s, 9H, TBS), 0.04 (2xs, 6H, TBS) ppm; ${ }^{13} \mathbf{C ~ N M R}\left(175 \mathrm{MHz}, \mathrm{CDCl}_{3}\right) \delta=159.3\left(\mathrm{C}_{\text {arom. }} \mathrm{O}\right), 130.3\left(\mathrm{C}_{\text {arom. }}\right), 129.4\left(\mathrm{C}_{\text {arom. }} \mathrm{H}\right), 113.9\left(\mathrm{C}_{\text {arom. }} \mathrm{H}\right)$, 108.9 (C-6), 80.2 (C-2), 75.4 (C-3), $73.3\left(\mathrm{Ar}^{-\mathrm{CH}_{2}}\right), 70.4$ (C-1), $60.0(\mathrm{C}-5), 55.4\left(\mathrm{O}-\mathrm{CH}_{3}\right), 36.5$ (C-4), $27.5\left(6-\mathrm{CH}_{3}\right), 27.1\left(6-\mathrm{CH}_{3}\right), 26.1$ (TBS), 18.4 (TBS), -5.2 (TBS) ppm; HRMS (ESI+) $m / z$ : calcd for $\mathrm{C}_{22} \mathrm{H}_{38} \mathrm{O}_{5} \mathrm{SiNH}_{4}^{+}\left[\mathrm{M}+\mathrm{NH}_{4}\right]^{+}: 428.2827$, found: 428.2834 .

\section{2-((4R,5R)-5-(((4-Methoxybenzyl)oxy)methyl)-2,2-dimethyl-1,3-dioxolan-4-yl)ethan-1-ol (27)}

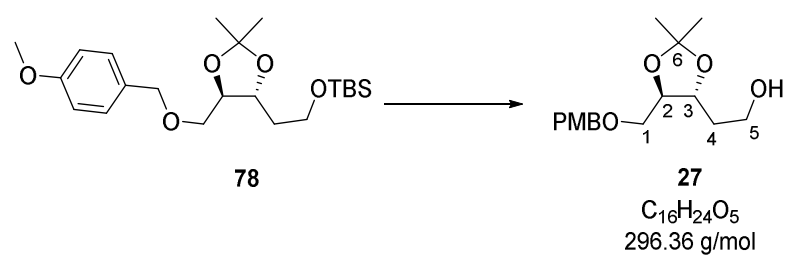

Acetonide 78 (270 mg, $658 \mu \mathrm{mol}, 1.00$ eq.) was dissolved in THF $(12 \mathrm{~mL})$ and cooled to $0{ }^{\circ} \mathrm{C}$. TBAF $\left(986 \mu \mathrm{L}, 986 \mu \mathrm{mol}, 1.50\right.$ eq. $1 \mathrm{M}$ in THF) was added and after stirring for $20 \mathrm{~min}$ at $0{ }^{\circ} \mathrm{C}$, the reaction mixture was stirred further $3 \mathrm{~h}$ at $\mathrm{rt} \mathrm{Et}_{2} \mathrm{O}(15 \mathrm{~mL})$ and water $(15 \mathrm{~mL})$ were added the phases separated, the aqueous phase was extracted with $\mathrm{Et}_{2} \mathrm{O}(3 \times 15 \mathrm{~mL})$, the combined organic phases were dried over $\mathrm{MgSO}_{4}$ and the solvent was removed under reduced pressure. Purification of the crude product was 
achieved by flash chromatography $(60 \% \mathrm{EtOAc} / \mathrm{CyH})$ to yield the title compound $\mathbf{2 7}$ as a colorless oil (171 mg, $577 \mu \mathrm{mol}, 88 \%$ ).

$\mathbf{R}_{f}=0.29(60 \% \mathrm{EtOAc} / \mathrm{CyH}) \cdot[\boldsymbol{\alpha}]_{\boldsymbol{D}}^{\mathbf{2 0}}=+5.4^{\circ}\left(c=0.74\right.$ in $\left.\mathrm{CH}_{2} \mathrm{Cl}_{2}\right) .{ }^{\mathbf{1}} \mathbf{H} \mathbf{N M R}\left(700 \mathrm{MHz}, \mathrm{CDCl}_{3}\right) \delta=$ $7.25\left(\mathrm{~d}, J=8.7 \mathrm{~Hz}, 2 \mathrm{H}, \mathrm{H}_{\text {arom. }}\right), 6.88\left(\mathrm{~d}, J=8.7 \mathrm{~Hz}, 2 \mathrm{H}, \mathrm{H}_{\text {arom. }}\right), 4.53-4.49$ (m, 2H, Ar- $\left.\mathrm{CH}_{2}\right), 3.97$ (td, $J=8.1,4.0 \mathrm{~Hz}, 1 \mathrm{H}, \mathrm{H}-3), 3.91(\mathrm{dt}, J=8.2,5.1 \mathrm{~Hz}, 1 \mathrm{H}, \mathrm{H}-2), 3.81\left(\mathrm{~s}, 3 \mathrm{H}, \mathrm{O}-\mathrm{CH}_{3}\right), 3.78(\mathrm{t}, J=5.6 \mathrm{~Hz}$, 1H, H-5), 3.60 (dd, $J=10.1,5.3 \mathrm{~Hz}, 1 \mathrm{H}, \mathrm{H}-1$ ), 3.52 (dd, $J=10.1,5.1 \mathrm{~Hz}, 1 \mathrm{H}, \mathrm{H}-1$ '), 2.37 (brs, 1H, $\mathrm{OH}), 1.89-1.80(\mathrm{~m}, 2 \mathrm{H}, \mathrm{H}-4), 1.41$ (s, 3H, 6-CH $), 1.39$ (s, 3H, 6- $\left.\mathrm{CH}_{3}\right) \mathrm{ppm} ;{ }^{13} \mathbf{C}$ NMR $(175 \mathrm{MHz}$, $\left.\mathrm{CDCl}_{3}\right) \delta=159.5\left(\mathrm{C}_{\text {arom. }} \mathrm{O}\right), 129.9\left(\mathrm{C}_{\text {arom. }}\right), 129.5\left(\mathrm{C}_{\text {arom. }} \mathrm{H}\right), 114.0\left(\mathrm{C}_{\text {arom. }} \mathrm{H}\right), 109.2(\mathrm{C}-6), 79.8(\mathrm{C}-2)$, 78.4 (C-3), $73.5\left(\mathrm{Ar}-\mathrm{CH}_{2}\right), 70.3(\mathrm{C}-1), 60.9(\mathrm{C}-5), 55.4\left(\mathrm{O}-\mathrm{CH}_{3}\right), 35.5(\mathrm{C}-4), 27.3\left(6-\mathrm{CH}_{3}\right), 27.1$ (6$\mathrm{CH}_{3}$ ) ppm; HRMS (ESI) $m / z$ : calcd for $\mathrm{C}_{16} \mathrm{H}_{24} \mathrm{O}_{5} \mathrm{H}^{+}[\mathrm{M}+\mathrm{H}]^{+}: 297.1697$, found: 297.1696.

2-((4R,5R)-5-(((4-Methoxybenzyl)oxy)methyl)-2,2-dimethyl-1,3-dioxolan-4-yl)acetaldehyde (79)

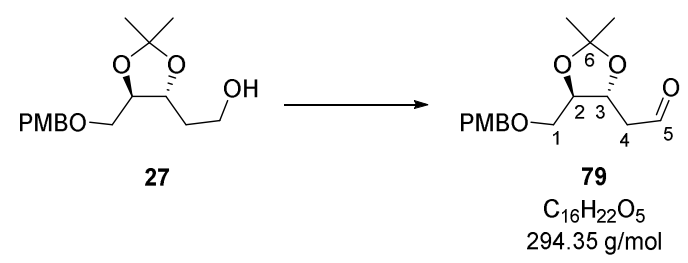

DCM $(0.5 \mathrm{~mL})$ was added to sulfur trioxide pyridine complex (182 mg, $1.44 \mathrm{mmol}, 3.00$ eq.) followed by the addition of $N, N$-diisopropylethylamine $(260 \mu \mathrm{L}, 1.53 \mathrm{mmol}, 4.00$ eq. $)$ and DMSO $(271 \mu \mathrm{L}$, $3.81 \mathrm{mmol}, 10.0$ eq.). The reaction mixture was cooled to $0{ }^{\circ} \mathrm{C}$ and alcohol $27(113 \mathrm{mg}, 381 \mu \mathrm{mol}$, 1.00 eq. in $2 \mathrm{~mL} \mathrm{DCM}$ ) was added. After stirring for $30 \mathrm{~min}$ at $0^{\circ} \mathrm{C}$, sat. aq. $\mathrm{NaHCO}_{3}$ solution $(10 \mathrm{~mL})$ was added and the aqueous phase was extracted with DCM $(3 \times 15 \mathrm{~mL})$. The combined organic layers were washed with $\mathrm{CuSO}_{4}, \mathrm{NH}_{4} \mathrm{Cl}$ and brine solution (each aq. sat. $2 \times 30 \mathrm{~mL}$ ). The organic phase was dried over $\mathrm{MgSO}_{4}$ and the solvent was removed under reduced pressure to yield the title compound 79 as a colorless oil (105 mg, $358 \mu \mathrm{mol}, 94 \%)$.

$\mathbf{R}_{\boldsymbol{f}}=0.50(60 \% \mathrm{EtOAc} / \mathrm{CyH}) \cdot[\boldsymbol{\alpha}]_{\boldsymbol{D}}^{\mathbf{2 0}}=+1.3^{\circ}\left(c=1.50\right.$ in $\left.\mathrm{CH}_{2} \mathrm{Cl}_{2}\right) .{ }^{1} \mathbf{H} \mathbf{N M R}\left(700 \mathrm{MHz}, \mathrm{CDCl}_{3}\right) \delta=$ 9.79 (t, $J=2.0 \mathrm{~Hz}, 1 \mathrm{H}, \mathrm{H}-5), 7.24$ (d, $J=8.7 \mathrm{~Hz}, 2 \mathrm{H}, \mathrm{H}_{\text {arom. }}$ ), 6.88 (d, $J=8.7 \mathrm{~Hz}, 2 \mathrm{H}, \mathrm{H}_{\text {arom. }}$ ), 4.49 (s, 2H, Ar- $\mathrm{CH}_{2}$ ), 4.29 (td, $\left.J=7.7,4.5 \mathrm{~Hz}, 1 \mathrm{H}, \mathrm{H}-3\right), 3.87$ (dt, $\left.J=8.2,5.3 \mathrm{~Hz}, 1 \mathrm{H}, \mathrm{H}-2\right), 3.81$ (s, 3H, O$\mathrm{CH}_{3}$ ), $3.64(\mathrm{dd}, J=10.0,5.1 \mathrm{~Hz}, 1 \mathrm{H}, \mathrm{H}-1), 3.53$ (dd, $J=10.0,5.5 \mathrm{~Hz}, 1 \mathrm{H}, \mathrm{H}-1$ '), $2.73-2.66$ (m, 2H, $\mathrm{H}-4), 1.41$ (s, 6H, 2x6-CH $)$ ppm; ${ }^{13} \mathbf{C}$ NMR $\left(175 \mathrm{MHz}, \mathrm{CDCl}_{3}\right) \delta=200.1$ (C-5), $159.5\left(\mathrm{C}_{\text {arom. }} \mathrm{O}\right)$,

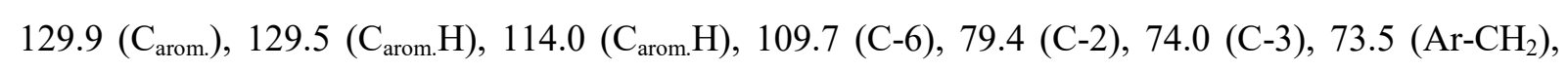
$69.9(\mathrm{C}-1), 55.4\left(\mathrm{O}-\mathrm{CH}_{3}\right), 47.0(\mathrm{C}-4), 27.2\left(6-\mathrm{CH}_{3}\right), 27.0\left(6-\mathrm{CH}_{3}\right) \mathrm{ppm}$; HRMS (ESI+) $\mathrm{m} / z$ : calcd for $\mathrm{C}_{16} \mathrm{H}_{22} \mathrm{O}_{5} \mathrm{NH}_{4}^{+}\left[\mathrm{M}+\mathrm{NH}_{4}\right]^{+}: 312.1805$, found: 312.1802 . The spectroscopic data were in agreement with those previously reported. ${ }^{[30]}$ 


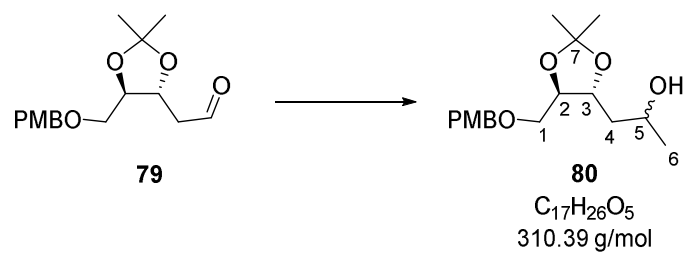

Aldehyde $79\left(100 \mathrm{mg}, 340 \mu \mathrm{mol}, 1.00\right.$ eq.) was dissolved in $\mathrm{Et}_{2} \mathrm{O}(5 \mathrm{~mL})$, cooled to $0{ }^{\circ} \mathrm{C}$ and $\mathrm{MeMgBr}$. (136 $\mu \mathrm{L}, 408 \mu \mathrm{mol}, 1.20$ eq. $3 \mathrm{M}^{\text {in }} \mathrm{Et}_{2} \mathrm{O}$ ) was added. After stirring for 20 min at $0{ }^{\circ} \mathrm{C}_{2} \mathrm{Et}_{2} \mathrm{O}$ $(10 \mathrm{~mL})$ and aq. sat. $\mathrm{NH}_{4} \mathrm{Cl}(15 \mathrm{~mL})$ were added, the phases separated, the aqueous phase was extracted with $\mathrm{Et}_{2} \mathrm{O}(3 \times 15 \mathrm{~mL})$, the combined organic phases were dried over $\mathrm{MgSO}_{4}$ and the solvent was removed under reduced pressure. Purification of the crude product was achieved by flash chromatography $(60 \% \mathrm{EtOAc} / \mathrm{CyH})$ to yield the title compound $\mathbf{8 0}$ as a colorless oil $(95.6 \mathrm{mg}$, $308 \mu \mathrm{mol}, 91 \%, d r=1.5: 1$ but the stereogenic center at C-5 will be removed in the next step).

$\mathbf{R}_{\boldsymbol{f}}=0.33(60 \%$ EtOAc/CyH $) \cdot[\boldsymbol{\alpha}]_{\boldsymbol{D}}^{\mathbf{2 0}}=+5.4^{\circ}\left(c=1.06\right.$ in $\left.\mathrm{CH}_{2} \mathrm{Cl}_{2}\right) .{ }^{\mathbf{1}} \mathbf{H} \mathbf{N M R}\left(700 \mathrm{MHz}, \mathrm{CDCl}_{3}\right) \delta=$ $7.26-7.24$ (d, 2H, $\mathrm{H}_{\text {arom. }}$ ), $6.89-6.87$ (d, 2H, $\mathrm{H}_{\text {arom }}$ ), $4.54-4.48$ (s, 2H, Ar- $\left.\mathrm{CH}_{2}\right), 4.06-3.83$ (m, $3 \mathrm{H}$, H-2, H-3, H-5), 3.81 (s, 3H, O-CH $), 3.60-3.57$ (m, 1H, H-1), 3.53 - 3.50 (m, 1H, H-1'), $1.74-1.61$ (m, 2H, H-4), $1.42-1.39$ (m, 6H, 2x7-CH $), 1.21 / 1.19(2 \mathrm{xd}, J=6.3 \mathrm{~Hz}, 3 \mathrm{H}, \mathrm{H}-6) \mathrm{ppm} ;{ }^{13} \mathbf{C} \mathbf{~ N M R}$ $\left(175 \mathrm{MHz}, \mathrm{CDCl}_{3}\right) \delta=159.5 / 159.5\left(\mathrm{C}_{\text {arom. }} \mathrm{O}\right), 130.0 / 130.0\left(\mathrm{C}_{\text {arom. }}\right), 129.5 / 129.5\left(\mathrm{C}_{\text {arom. }} \mathrm{H}\right), 114.0 / 114.0$ $\left(\mathrm{C}_{\text {arom. }} \mathrm{H}\right), 109.6 / 109.1$ (C-7), 80.4/79.6 (C-2), 78.8/76.6 (C-3), 73.4/73.4 ( $\mathrm{Ar}^{\left.-\mathrm{CH}_{2}\right)}$, 70.3/69.9 (C-1), 67.4/65.3 (C-5), $55.4\left(\mathrm{O}-\mathrm{CH}_{3}\right), 42.0 / 41.3(\mathrm{C}-4), 27.4 / 27.3\left(7-\mathrm{CH}_{3}\right), 27.1 / 27.1\left(7-\mathrm{CH}_{3}\right), 23.8 / 23.4(\mathrm{C}-6)$ ppm; HRMS (ESI+) $m / z$ : calcd for $\mathrm{C}_{17} \mathrm{H}_{26} \mathrm{O}_{5} \mathrm{H}^{+}[\mathrm{M}+\mathrm{H}]^{+}: 311.1853$, found: 311.1852 .

1-((4R,5R)-5-(((4-Methoxybenzyl)oxy)methyl)-2,2-dimethyl-1,3-dioxolan-4-yl)propan-2-one (28)

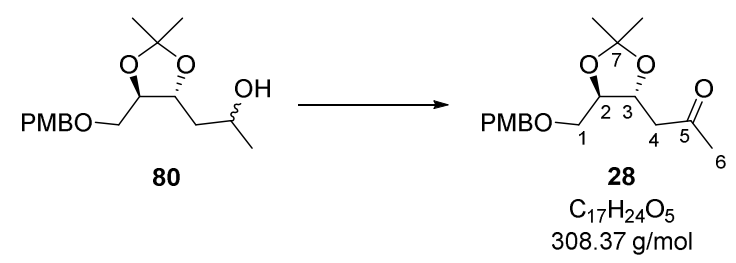

DCM (1.5 mL) was added to sulfur trioxide pyridine complex (147 mg, $924 \mu \mathrm{mol}, 3.00$ eq.) followed by the addition of $N, N$-diisopropylethylamine $(210 \mu \mathrm{L}, 1.23 \mathrm{mmol}, 4.00$ eq. $)$ and DMSO $(219 \mu \mathrm{L}$, $3.08 \mathrm{mmol}, 10.0$ eq.). The reaction mixture was cooled to $0{ }^{\circ} \mathrm{C}$ and alcohol 80 (95.6 mg, $308 \mu \mathrm{mol}$, 1.00 eq., in $1.5 \mathrm{~mL} \mathrm{DCM}$ ) was added. After stirring for $30 \mathrm{~min}$ at $0{ }^{\circ} \mathrm{C}$, the reaction mixture was diluted with DCM $(10 \mathrm{~mL})$ and water $(10 \mathrm{~mL})$, the phases were separated, the aqueous phase was extracted with DCM $(3 \times 15 \mathrm{~mL})$, the combined organic phases were dried over $\mathrm{MgSO}_{4}$ and the solvent was removed under reduced pressure. Purification of the crude product was achieved by flash 
chromatography $(40 \% \mathrm{EtOAc} / \mathrm{CyH})$ to yield the title compound $\mathbf{2 8}$ as a colorless oil $(79.6 \mathrm{mg}$, $258 \mu \mathrm{mol}, 84 \%)$.

$\mathbf{R}_{\boldsymbol{f}}=0.23(40 \% \mathrm{EtOAc} / \mathrm{CyH}) \cdot[\boldsymbol{\alpha}]_{\boldsymbol{D}}^{\mathbf{2 0}}=+4.5^{\circ}\left(c=0.66\right.$ in $\left.\mathrm{CH}_{2} \mathrm{Cl}_{2}\right) .{ }^{\mathbf{1}} \mathbf{H} \mathbf{N M R}\left(500 \mathrm{MHz}, \mathrm{CDCl}_{3}\right) \delta=$ 7.24 (d, $\left.J=8.7 \mathrm{~Hz}, 2 \mathrm{H}, \mathrm{H}_{\text {arom. }}\right), 6.87$ (d, $J=8.7 \mathrm{~Hz}, 2 \mathrm{H}, \mathrm{H}_{\text {arom. }}$ ), 4.49 (d, $\left.J=3.3 \mathrm{~Hz}, 2 \mathrm{H}, \mathrm{Ar}_{-} \mathrm{CH}_{2}\right), 4.24$ (td, $J=8.2,4.1 \mathrm{~Hz}, 1 \mathrm{H}, \mathrm{H}-3), 3.83$ (dt, $J=8.2,5.3 \mathrm{~Hz}, 1 \mathrm{H}, \mathrm{H}-2), 3.80$ (s, 2H, O-CH $\left.{ }_{3}\right), 3.61$ (dd, $J=$ 10.0, $5.4 \mathrm{~Hz}, 1 \mathrm{H}, \mathrm{H}-1), 3.52$ (dd, $J=10.0,5.1 \mathrm{~Hz}, 1 \mathrm{H}, \mathrm{H}-1$ '), 2.73 (dd, $J=16.2,7.9 \mathrm{~Hz}, 1 \mathrm{H}, \mathrm{H}-4$ ), $2.66(\mathrm{dd}, J=16.2,4.1 \mathrm{~Hz}, 1 \mathrm{H}, \mathrm{H}-4$ ') 2.18 (s, 3H, H-6), 1.39 (s, 6H, 2x7-CH $)$ ppm; ${ }^{13} \mathbf{C}$ NMR $\left(125 \mathrm{MHz}, \mathrm{CDCl}_{3}\right) \delta=206.3(\mathrm{C}-5), 159.4\left(\mathrm{C}_{\text {arom. }} \mathrm{O}\right), 130.1\left(\mathrm{C}_{\text {arom }}\right), 129.5\left(\mathrm{C}_{\text {arom. }} \mathrm{H}\right), 113.9\left(\mathrm{C}_{\text {arom. }} \mathrm{H}\right)$,

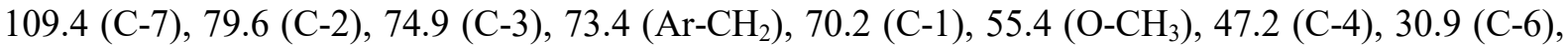
$27.3\left(7-\mathrm{CH}_{3}\right), 27.1\left(7-\mathrm{CH}_{3}\right)$ ppm; HRMS (ESI+) $\mathrm{m} / z$ : calcd for $\mathrm{C}_{17} \mathrm{H}_{24} \mathrm{O}_{5} \mathrm{Na}^{+}[\mathrm{M}+\mathrm{Na}]^{+}: 331.1516$, found: 331.1513 .

(4S,5S)-4-Hydroxy-1-((4R,5R)-5-(((4-methoxybenzyl)oxy)methyl)-2,2-dimethyl-1,3-dioxolan-4yl)-5-methylnonane-2,8-dione (4)

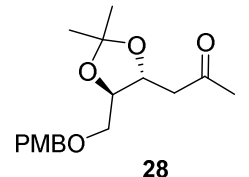

28

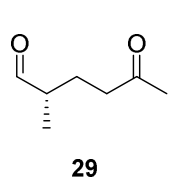

29

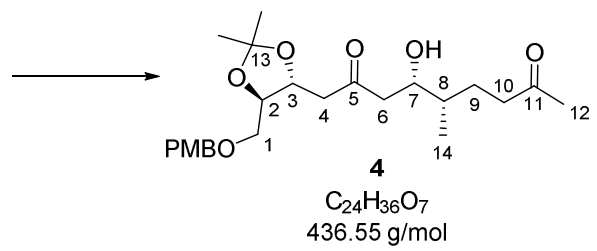

(+)-[Ipc $]_{2} \mathrm{BCl}(80.1 \mathrm{mg}, 250 \mu \mathrm{mol}, 1.10$ eq. $)$ was dissolved in DCM $(1 \mathrm{~mL})$. After cooling to $-78{ }^{\circ} \mathrm{C}$, DIPEA (77.2 $\mu \mathrm{L}, 454 \mu \mathrm{mol}, 2.00$ eq.) and ketone 28 (70.0 mg, $227 \mu \mathrm{mol}, 1.00$ eq. stored over $3 \AA \mathrm{MS}$ in $2 \mathrm{~mL} \mathrm{DCM}$ ) were added dropwise. The reaction mixture was stirred $2.5 \mathrm{~h}$ at $-78^{\circ} \mathrm{C}$ and aldehyde 29 (58.2 mg, $454 \mu \mathrm{mol}, 2.00$ eq. stored $14 \mathrm{~h}$ over $3 \AA \mathrm{MS}$ in $2 \mathrm{~mL}$ DCM) was added dropwise. After stirring $1 \mathrm{~h}$ at $-78^{\circ} \mathrm{C}$, the reaction vessel was placed in a deep freezer $\left(-15^{\circ} \mathrm{C}\right)$ overnight. Methanol (2.8 mL), pH 7 buffer $(1.6 \mathrm{~mL})$ and $\mathrm{H}_{2} \mathrm{O}_{2}(1.6 \mathrm{~mL}, 35 \%)$ were added to quench the reaction. After stirring $1 \mathrm{~h}$ at $\mathrm{rt}$, water $(8 \mathrm{~mL})$ was added, the phases were separated, the aqueous layer was extracted with DCM $(3 \times 10 \mathrm{~mL})$, the combined organic phases were dried over $\mathrm{Na}_{2} \mathrm{SO}_{4}$ and the solvent was removed under reduced pressure. Purification of the crude product was achieved by flash chromatography $(50-80 \% \mathrm{EtOAc} / \mathrm{CyH})$ to yield the title compound 4 (7.80 mg, $17.9 \mu \mathrm{mol}, 8 \%$ (26\% brsm), $d r>4: 1)$.

$\mathbf{R}_{\boldsymbol{f}}=0.35\left(80 \%\right.$ EtOAc/hexane). $[\boldsymbol{\alpha}]_{\boldsymbol{D}}^{\mathbf{2 0}}=-8.7^{\circ}\left(c=0.92\right.$ in $\left.\mathrm{CH}_{2} \mathrm{Cl}_{2}\right) .{ }^{\mathbf{1}} \mathbf{H} \mathbf{N M R}\left(500 \mathrm{MHz}, \mathrm{CDCl}_{3}\right) \delta=$ 7.24 (d, $J=8.7 \mathrm{~Hz}, 2 \mathrm{H}, \mathrm{H}_{\text {arom. }}$ ), 6.87 (d, $J=8.7 \mathrm{~Hz}, 2 \mathrm{H}, \mathrm{H}_{\text {arom. }}$ ), 4.49 (d, $J=3.0 \mathrm{~Hz}, 2 \mathrm{H}, \mathrm{Ar}-\mathrm{CH}_{2}$ ), 4.26 - 4.22 (m, 1H, H-3), 3.98 - 3.93 (m, 1H, H-7), 3.85 - 3.80 (m, 1H, H-2), 3.80 (s, 3H, O-CH $), 3.63$ 3.50 (m, 2H, H-1), $2.79-2.68$ (m, 2H, H-4), $2.64-2.54$ (m, 2H, H-6), 2.53 - 2.44 (m, 2H, H-10), 2.15 (s, 3H, H-12), 1.81 - 1.74 (m, 1H, H-9), 1.52 - 1.46 (m, 1H, H-8), 1.42 - 1.40 (m, 1H, H-9'), $1.39\left(\mathrm{~s}, 6 \mathrm{H}, 2 \mathrm{x} 13-\mathrm{CH}_{3}\right), 0.88(\mathrm{~d}, J=6.7 \mathrm{~Hz}, 3 \mathrm{H}, \mathrm{H}-12) \mathrm{ppm} ;{ }^{13} \mathbf{C} \mathbf{N M R}\left(125 \mathrm{MHz}, \mathrm{CDCl}_{3}\right) \delta=209.7$ (C-5), 209.3 (C-11), $159.4\left(\mathrm{C}_{\text {arom }}\right), 130.0\left(\mathrm{C}_{\text {arom. }}\right), 129.5\left(\mathrm{C}_{\text {arom. }} \mathrm{H}\right), 114.0\left(\mathrm{C}_{\text {arom. }} \mathrm{H}\right), 109.6(\mathrm{C}-13), 79.4$ 


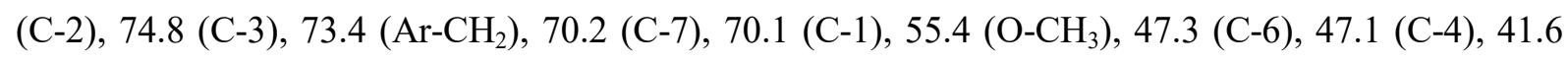
(C-10), 37.5 (C-8), 30.1 (C-12), $27.3\left(13-\mathrm{CH}_{3}\right), 27.1\left(13-\mathrm{CH}_{3}\right), 26.6$ (C-9), 14.4 (C-14) ppm; HRMS (ESI+) $m / z$ : calcd for $\mathrm{C}_{24} \mathrm{H}_{36} \mathrm{O}_{7} \mathrm{NH}_{4}{ }^{+}\left[\mathrm{M}+\mathrm{NH}_{4}\right]^{+}: 454.2799$, found: 454.2796 .

(4S,5S)-1-((4R,5R)-5-(((4-methoxybenzyl)oxy)methyl)-2,2-dimethyl-1,3-dioxolan-4-yl)-5-methyl2,8-dioxononan-4-yl (S)-3,3,3-trifluoro-2-methoxy-2-phenylpropanoate (81)

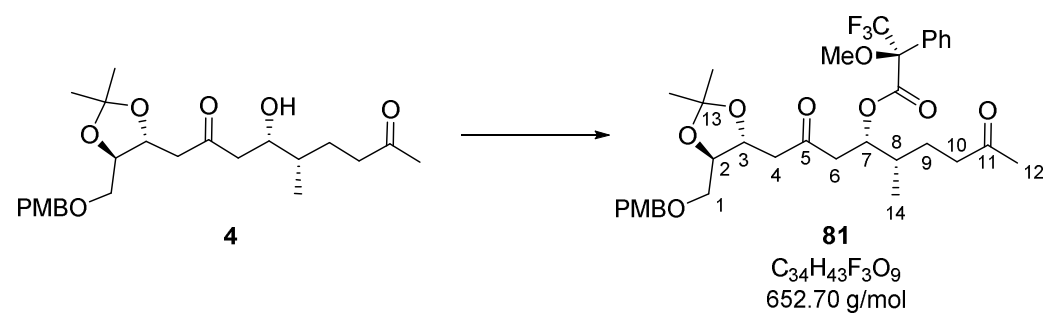

Alcohol 4 (2.90 mg, $6.64 \mu \mathrm{mol}, 1.00$ eq.) was dissolved in pyridine $(200 \mu \mathrm{L})$ and $(-)-(R)-\mathrm{MTPA}-\mathrm{Cl}$ (7.46 $\mu \mathrm{L}, 39.9 \mu \mathrm{mol}, 6.00$ eq.) was added at $0{ }^{\circ} \mathrm{C}$. The reaction mixture was stirred $2 \mathrm{~h}$ at $\mathrm{rt}$ followed by the addition of sat. aq. $\mathrm{NH}_{4} \mathrm{Cl}$ solution $(2 \mathrm{~mL})$ and $\mathrm{DCM}(2 \mathrm{~mL})$. After separation of the organic layer, the aqueous phase was extracted with DCM $(3 \times 2 \mathrm{~mL})$, the combined organic phases were dried over $\mathrm{MgSO}_{4}$ and the solvent was removed under reduced pressure. Purification of the crude product was achieved by flash chromatography (50\% EtOAc/hexane) to yield the title compound $\mathbf{8 1}$ as a colorless oil (4.30 mg, $6.59 \mu \mathrm{mol}, 99 \%)$

$\mathbf{R}_{\boldsymbol{f}}=0.46(50 \%$ EtOAc/hexane $) .[\boldsymbol{\alpha}]_{\boldsymbol{D}}^{\mathbf{2 0}}=-4.7^{\circ}\left(c=0.86\right.$ in $\left.\mathrm{CH}_{2} \mathrm{Cl}_{2}\right) .{ }^{1} \mathbf{H} \mathbf{N M R}\left(700 \mathrm{MHz}, \mathrm{CDCl}_{3}\right) \delta=$ $7.58-7.51\left(\mathrm{~m}, 2 \mathrm{H}, \mathrm{H}_{\text {arom., MTPA }}\right), 7.44-7.36\left(\mathrm{~m}, 3 \mathrm{H}, \mathrm{H}_{\text {arom., MTPA }}\right), 7.24\left(\mathrm{~d}, J=8.7 \mathrm{~Hz}, 2 \mathrm{H}, \mathrm{H}_{\text {arom }}\right), 6.87$ (d, $\left.J=8.7 \mathrm{~Hz}, 2 \mathrm{H}, \mathrm{H}_{\text {arom. }}\right), 5.56-5.53(\mathrm{~m}, 1 \mathrm{H}, \mathrm{H}-7), 4.49$ (d, $\left.J=5.1 \mathrm{~Hz}, 2 \mathrm{H}, \mathrm{Ar}_{-} \mathrm{CH}_{2}\right), 4.14-4.12$ (m, $1 \mathrm{H}, \mathrm{H}-3), 3.81-3.80$ (m, 1H, H-2), 3.80 (s, 3H, O-CH 3 ), 3.59 (dd, $J=10.0,5.3 \mathrm{~Hz}, 1 \mathrm{H}, \mathrm{H}-1), 3.51$ 3.46 (m, 3H, H-1', O-CH ${ }_{3, \mathrm{MTPA}}$ ), 2.84 (dd, $\left.J=17.5,7.7 \mathrm{~Hz}, 1 \mathrm{H}, \mathrm{H}-6\right), 2.65$ (dd, $J=17.5,4.7 \mathrm{~Hz}, 1 \mathrm{H}$, H-6'), 2.62 (d, $J=6.4$ Hz, 1H, H-4), 2.49 (t, J=7.6 Hz, 2H, H-10), 2.13 (s, 3H, H-12), $1.79-1.76$ (m, 1H, H-8), $1.66-1.61$ (m, 1H, H-9), $1.44-1.39$ (m, 1H, H-6'), 1.37 (s, 3H, 13-CH ), 1.35 (s, 3H, 13$\left.\mathrm{CH}_{3}\right), 0.89(\mathrm{~d}, J=6.9 \mathrm{~Hz}, 3 \mathrm{H}, \mathrm{H}-14) \mathrm{ppm} .{ }^{13} \mathbf{C} \mathbf{N M R}\left(175 \mathrm{MHz}, \mathrm{CDCl}_{3}\right) \delta=208.3(\mathrm{C}-11), 204.5(\mathrm{C}-$ 5), $166.1\left(\mathrm{C}=\mathrm{O}_{\mathrm{MTPA}}\right), 159.4\left(\mathrm{C}_{\text {arom. }}\right), 132.1\left(\mathrm{C}_{\text {arom. }, \mathrm{MTPA}}\right), 130.0\left(\mathrm{C}_{\text {arom. }}\right), 129.7\left(\mathrm{C}_{\text {arom. }} \mathrm{H}_{\mathrm{MTPA}}\right), 129.5$ $\left(\mathrm{C}_{\text {arom. }} \mathrm{H}\right), 128.8\left(\mathrm{C}_{\text {arom. }} \mathrm{H}_{\mathrm{MTPA}}\right), 128.6\left(\mathrm{C}_{\text {arom. }} \mathrm{H}_{\mathrm{MTPA}}\right), 127.8\left(\mathrm{C}_{\text {arom.,MTPA }}\right), 114.0\left(\mathrm{C}_{\text {arom. }} \mathrm{H}\right), 109.5(\mathrm{C}-13)$,

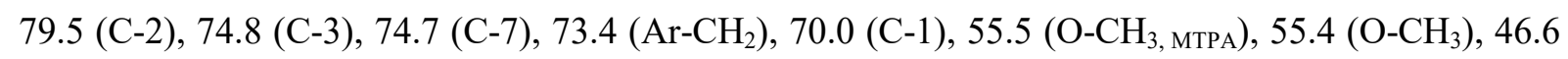
(C-4), 44.4 (C-6), 41.2 (C-10), 35.8 (C-8), 30.1 (C-12), 27.3 (13- $\left.\mathrm{CH}_{3}\right), 27.0$ (13- $\left.\mathrm{CH}_{3}\right), 26.5$ (C-9), 14.6 (C-14) ppm. HRMS (ESI) $m / z$ : calcd for $\mathrm{C}_{34} \mathrm{H}_{43} \mathrm{~F}_{3} \mathrm{O}_{9} \mathrm{NH}_{4}^{+}\left[\mathrm{M}+\mathrm{NH}_{4}\right]^{+}$: 670.3197, found: 670.3199. 


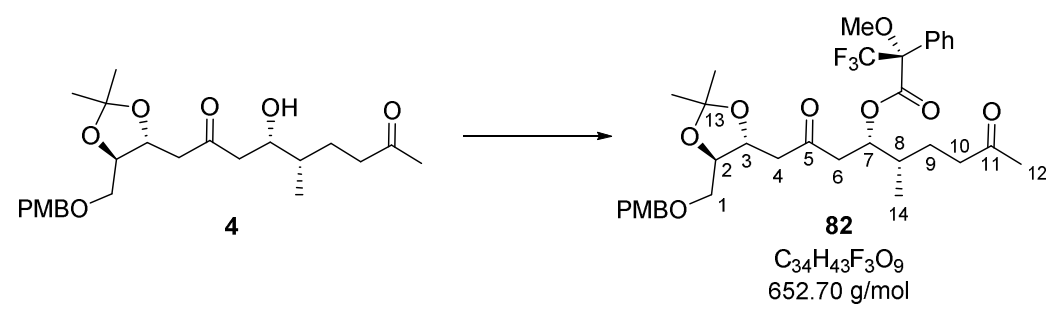

Alcohol 4 (1.70 mg, $3.89 \mu \mathrm{mol}, 1.00$ eq. $)$ was dissolved in pyridine $(200 \mu \mathrm{L})$ and $(+)-(S)-\mathrm{MTPA}-\mathrm{Cl}$ $\left(4.37 \mu \mathrm{L}, 23.4 \mu \mathrm{mol}, 6.00\right.$ eq.) was added at $0{ }^{\circ} \mathrm{C}$. The reaction mixture was stirred $2 \mathrm{~h}$ at $\mathrm{rt}$ followed by the addition of sat. aq. $\mathrm{NH}_{4} \mathrm{Cl}$ solution $(2 \mathrm{~mL})$ and $\mathrm{DCM}(2 \mathrm{~mL})$. After separation of the organic layer, the aqueous phase was extracted with DCM $(3 \times 2 \mathrm{~mL})$, the combined organic phases were dried over $\mathrm{MgSO}_{4}$ and the solvent was removed under reduced pressure. Purification of the crude product was achieved by flash chromatography (50\% EtOAc/hexane) to yield the title compound $\mathbf{8 2}$ as a colorless oil (2.10 mg, $3.22 \mu \mathrm{mol}, 83 \%)$.

$\mathbf{R}_{\boldsymbol{f}} 0.40$ (50\% EtOAc/hexane). $[\boldsymbol{\alpha}]_{\boldsymbol{D}}^{\mathbf{2 0}}=+19.0^{\circ}\left(c=0.42\right.$ in $\left.\mathrm{CH}_{2} \mathrm{Cl}_{2}\right) .{ }^{1} \mathbf{H} \mathbf{N M R}\left(700 \mathrm{MHz}, \mathrm{CDCl}_{3}\right) \delta=$ $7.58-7.49$ (m, 2H, $\left.\mathrm{H}_{\text {arom., MTPA }}\right), 7.42-7.37$ (m, 3H, $\left.\mathrm{H}_{\text {arom., MTPA }}\right), 7.23$ (d, $\left.J=8.7 \mathrm{~Hz}, 2 \mathrm{H}, \mathrm{H}_{\text {arom. }}\right), 6.87$ (d, $J=8.7 \mathrm{~Hz}, 2 \mathrm{H}, \mathrm{H}_{\text {arom }}$ ), $5.55-5.53$ (m, 1H, H-7), 4.48 (d, J=3.7 Hz, 2H, Ar- $\left.\mathrm{CH}_{2}\right), 4.21-4.15$ (m, $1 \mathrm{H}, \mathrm{H}-3), 3.86-3.81$ (m, 1H, H-2), 3.80 (s, 3H, O-CH ${ }_{3}$ ), 3.60 (dd, $\left.J=10.0,5.3 \mathrm{~Hz}, 1 \mathrm{H}, \mathrm{H}-1\right), 3.51$ 3.45 (m, 3H, H-1', O-CH ${ }_{3, \mathrm{MTPA}}$ ), 2.86 (dd, $J=17.6,8.4$ Hz, 1H, H-6), 2.69 - 2.66 (m, 3H, H-4, H-6'), 2.35 (t, $J=7.6 \mathrm{~Hz}, 2 \mathrm{H}, \mathrm{H}-10), 2.08$ (s, 3H, H-12), $1.76-1.69$ (m, 1H, H-8), $1.49-1.44$ (m, 1H, H-9), 1.37 (s, 3H, 13- $\left.\mathrm{CH}_{3}\right), 1.35$ (s, 3H, 13- $\left.\mathrm{CH}_{3}\right), 1.33-1.28$ (m, 1H, H-9'), 0.83 (d, J=6.9 Hz, 3H, H-14) ppm. ${ }^{13} \mathrm{C}$ NMR $\left(176 \mathrm{MHz}, \mathrm{CDCl}_{3}\right) \delta=208.3(\mathrm{C}-11), 204.7(\mathrm{C}-5), 166.1\left(\mathrm{C}=\mathrm{O}_{\mathrm{MTPA}}\right), 159.5\left(\mathrm{C}_{\text {arom. }}\right)$, $132.4\left(\mathrm{C}_{\text {arom.,MTPA }}\right), 130.0\left(\mathrm{C}_{\text {arom }}\right), 129.8\left(\mathrm{C}_{\text {arom. }} \mathrm{H}_{\text {MTPA }}\right), 129.5\left(\mathrm{C}_{\text {arom. }} \mathrm{H}\right), 128.8\left(\mathrm{C}_{\text {arom. }} \mathrm{H}_{\text {MTPA }}\right), 127.8$

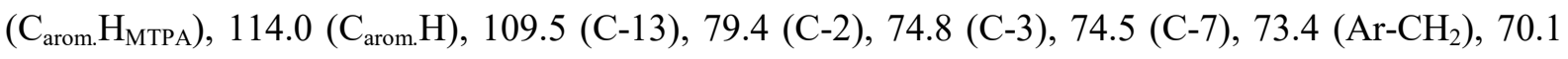
(C-1), $55.7\left(\mathrm{OCH}_{3, \mathrm{MTPA}}\right), 55.4\left(\mathrm{OCH}_{3}\right), 46.6$ (C-4), 44.1 (C-6), 41.3 (C-10), 35.8 (C-8), 30.0 (C-12), $27.3\left(13-\mathrm{CH}_{3}\right), 27.0\left(13-\mathrm{CH}_{3}\right), 26.0$ (C-9), 14.7 (C-14) ppm. HRMS (ESI) m/z: calcd for $\mathrm{C}_{34} \mathrm{H}_{43} \mathrm{~F}_{3} \mathrm{O}_{9} \mathrm{NH}_{4}^{+}\left[\mathrm{M}+\mathrm{NH}_{4}\right]^{+}: 670.3197$, found: 670.3193 .

\section{Mosher Ester Analysis for Compound 4}

Determination of the absolute configuration was accomplished using the Mosher ester analysis. For this purpose, the two Mosher ester derivatives $\mathbf{8 1}$ and $\mathbf{8 2}$ have been synthesized and the respective NMR data have been compared as shown in Table 3. The resulting difference between the ${ }^{1} \mathrm{H}$ NMR data of the $(S)$ - and $(R)$-MTPA ester led to the determination of the absolute configuration as shown below. ${ }^{[17]}$ 


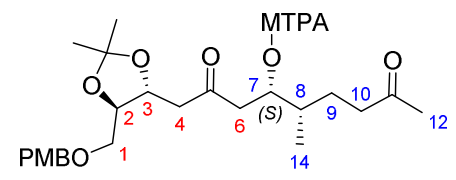

Table 3. Significant ${ }^{1} \mathrm{H}$ NMR signals of $\mathbf{8 1}$ and $\mathbf{8 2}$ and their use in the Mosher ester analysis.

$\begin{array}{llll} & \delta_{H}[\mathrm{ppm}] & \delta_{H}[\mathrm{ppm}] \\ \text { position } & (\mathbf{8 1})(S) & (\mathbf{8 2})(R) & \boldsymbol{\Delta} \boldsymbol{\delta}^{\boldsymbol{S}-\boldsymbol{R}} \\ \text { H-3 } & 4.13 & 4.18 & -0.05 \\ \text { H-4 } & 2.64 & 2.67 & -0.03 \\ \text { H-6 } & 2.65 & 2.68 & -0.03 \\ \text { H-6 } & 2.84 & 2.86 & -0.02 \\ \text { H-2 } & 3.80 & 3.82 & -0.02 \\ \text { H-1 } & 3.58 & 3.60 & -0.02 \\ \text { H-7 } & 5.55 & 5.54 & +0.01 \\ \text { H-12 } & 2.13 & 2.08 & +0.05 \\ \text { H-8 } & 1.77 & 1.72 & +0.05 \\ \text { H-14 } & 0.89 & 0.83 & +0.06 \\ \text { H-10 } & 2.44 & 2.35 & +0.09 \\ \text { H-9 } & 1.46 & 1.30 & +0.16 \\ \text { H-9 } & 1.63 & 1.46 & +0.17\end{array}$

Methyl-D-phenylalaninate hydrochloride $(84)^{[8,9]}$

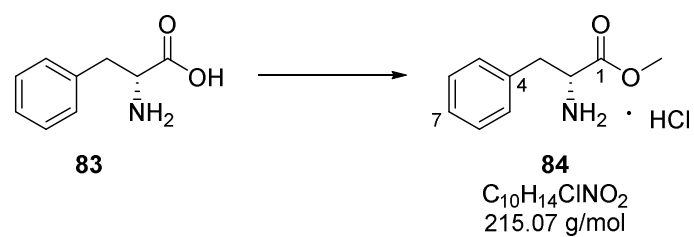

$\mathrm{MeOH}(70 \mathrm{~mL})$ was cooled down to $-5{ }^{\circ} \mathrm{C}$. Thionyl chloride $(14.0 \mathrm{~mL}, 182 \mathrm{mmol}, 3.0$ eq. $)$ was added dropwise within $10 \mathrm{~min}$. D-Phenylalanine $(\mathbf{8 3}, 10.0 \mathrm{~g}, 60.6 \mathrm{mmol})$ was added. The mixture was stirred for $20 \mathrm{~h}$ at room temperature and afterwards concentrated in vacuo. The precipitate was dissolved in $\mathrm{MeOH}(50 \mathrm{~mL})$. The solvent was removed and the procedure was repeated once to remove the excess of $\mathrm{SOCl}_{2}$. The solid was dried to yield hydrochloride 84 (13.1 g, $60.6 \mathrm{mmol}$, quant.) as a colorless solid. 
$\mathbf{R}_{f}=0.02$ (1:1 cyclohexane/ethyl acetate); ${ }^{1} \mathbf{H}$ NMR (500 MHz, MeOD): $\delta=7.42-7.39$ (m, 2H, H-6, $\mathrm{H}-8$ ), 7.36 - 7.33 (m, 1H, H-7), 7.29 - 7.28 (m, 2H, H-9, H-5), 4.36 - 4.33 (m, 1H, H-2), 3.83 (s, 3H, $\left.\mathrm{OCH}_{3}\right), 3.29(\mathrm{dd}, J=14.4,6.1 \mathrm{~Hz}, 1 \mathrm{H}, \mathrm{H}-3 \mathrm{a}), 3.19$ (dd, $\left.J=14.4,7.5 \mathrm{~Hz}, 1 \mathrm{H}, \mathrm{H}-3 \mathrm{~b}\right) \mathrm{ppm} ;{ }^{13} \mathbf{C}$ NMR (125 MHz, MeOD): $\delta=170.5$ (C-1), 135.3 (C-4), 130.4 (C-5, C-9), 130.2 (C-6, C-8), 129.0 (C-7), $55.2(\mathrm{C}-2), 53.6\left(-\mathrm{OCH}_{3}\right), 37.4(\mathrm{C}-3) \mathrm{ppm}$; MS (ESI+) $\mathrm{m} / z$ : for $\mathrm{C}_{10} \mathrm{H}_{14} \mathrm{ClNO}_{2}[\mathrm{M}+\mathrm{H}]^{+}$ 180.102 .

The spectroscopic data were consistent with those previously reported. ${ }^{[8]}$

(R)-3-Benzyl-1-methyl-1,4-diazaspiro[4.4]nonan-2-one $(85)^{[9]}$

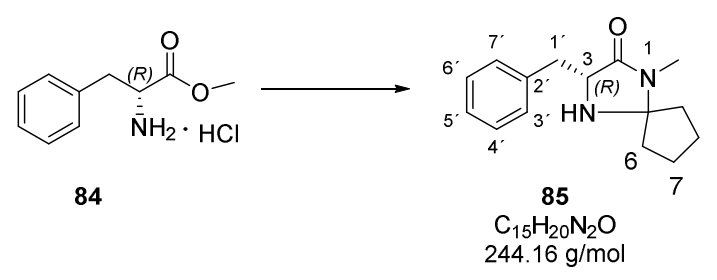

To a solution of ethanolic $\mathrm{MeNH}_{2}$ (33\%weight, $38 \mathrm{~mL}, 400 \mathrm{mmol}, 6.6$ eq.) was added $(R)$-phenylalanine methyl ester hydrochloride $(\mathbf{8 4}, 13.1 \mathrm{~g}, 60.6 \mathrm{mmol})$ and the resulting solution was stirred at room temperature for $20 \mathrm{~h}$. The solvent was removed in vacuo. The residue was suspended in $\mathrm{Et}_{2} \mathrm{O}$ and then, concentrated again. This procedure was repeated two times until $(R)$-phenylalanine- $N$ methyl amide hydrochloride was obtained as a white solid. The solid was treated with aq. sat. $\mathrm{NaHCO}_{3}$ solution $(50 \mathrm{~mL})$ and the aqueous phase was extracted with $\mathrm{CHCl}_{3}(3 \times 50 \mathrm{~mL})$. The combined organic phases were dried over $\mathrm{MgSO}_{4}$ and the solvent was removed under reduced pressure. The residue was dissolved in $\mathrm{MeOH}(120 \mathrm{~mL})$ and cyclopentanone (26.8 mL, $303 \mathrm{mmol}, 5.0$ eq.) and $p$ TSA (116 mg, $0.61 \mathrm{mmol}, 0.01 \mathrm{eq}$.) were added and the reaction mixture was heated to reflux for $18 \mathrm{~h}$. The mixture was cooled down to room temperature and concentrated in vacuo. The residue was taken up in diethyl ether and a solution of $\mathrm{HCl}$ in dioxane $(4 \mathrm{M}, 12.0 \mathrm{~mL})$ was added to precipitate the hydrochloride. The precipitate was recrystallized by solving in a minimum of hot isopropanol. Then, $n$-hexane was added until the cloudiness persisted and then, isopropanol was added until the solution became homogeneous again. After cooling to room temperature, the formed precipitate was filtered off and dried to yield catalyst (7.16 g, $25.6 \mathrm{mmol}, 42 \%$ ) as a colorless solid.

For the organo catalyst reaction, the catalyst was treated with aq. sat. $\mathrm{NaHCO}_{3}$ solution until no further gas evolution was observed. The aqueous phase was extracted with $\mathrm{CHCl}_{3}(3 \times 50 \mathrm{~mL})$ and the solvent was removed in vacuo to yield the acid free catalyst $\mathbf{8 5}$ as colorless oil (6.25 g, 25.6 mmol, quant.).

$\mathbf{R}_{f}=0.10$ (1:1 cyclohexane/ethyl acetate); ${ }^{1} \mathbf{H}$ NMR (500 MHz, $\left.\mathrm{d}_{6}-\mathrm{DMSO}\right): \delta=10.63\left(\mathrm{~s}_{\mathrm{br}}, 1 \mathrm{H}, \mathrm{NH}\right)$, $7.45-7.41$ (m, 2H, H-3', H-7'), 7.35 - 7.33 (m, 2H, H-4', H-6'), 7.28 (t, J= 7.3 Hz, 1H, H-5'), 4.48 
( $\left.\mathrm{s}_{\mathrm{br}}, 1 \mathrm{H}, \mathrm{H}-3\right), 3.28-3.24$ (dd, $J=14.4,4.5 \mathrm{~Hz}, 2 \mathrm{H}, \mathrm{H}-1^{\prime}$ ), 2.80 (s, 3H, N-CH 3 ), $2.17-2.07$ (m, 3H, H-1', H-6, H-9), 1.86 - 1.80 (m, 1H, H-9), 1.76 - 1.69 (m, 4H, H-7, H-8) ppm; ${ }^{13}$ C NMR (125 MHz, $\mathrm{d}_{6}$-DMSO): $\delta=166.8\left(\mathrm{C}_{\mathrm{q}^{-}}\right.$), 136.3 (C-2'), 129.4 (C-3', C-7'), 128.5 (C-4', C-7'), 127.0 (C-5'), 86.2 (C-5), 57.6 (C-3), 33.4 (C-1'), 32.0 (br, C-6, C-9), $25.1\left(\mathrm{~N}-\mathrm{CH}_{3}\right), 23.7$ (C-7/C-8), 23.4 (C-7/C-8) ppm; MS (ESI+) $m / z$ : for $\mathrm{C}_{15} \mathrm{H}_{20} \mathrm{~N}_{2} \mathrm{O}[\mathrm{M}+\mathrm{H}]^{+} 245.165$.

The spectroscopic data were consistent with those previously reported. ${ }^{[9]}$

(S)-2-Methyl-5-oxohexanal (29) ${ }^{[13,14]}$

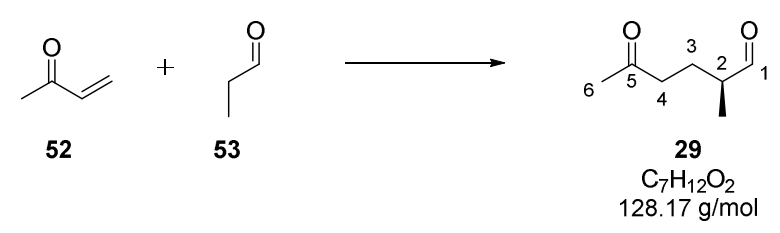

To acid free catalyst 85 (840 mg, 3.44 mmol, 0.2 eq.) and ethyl-3,4,-dihydroxybenzoate (51, $626 \mathrm{mg}$, $3.44 \mathrm{mmol}, 0.2$ eq.) methyl vinyl ketone (52, $4.8 \mathrm{~mL}, 51.6 \mathrm{mmol}, 90 \%, 3.0$ eq.) and propionaldehyde $(\mathbf{5 3}, 1.23 \mathrm{~mL}, 17.2 \mathrm{mmol}, 1.0$ eq.) were added. The mixture was stirred for $16 \mathrm{~h}$ at room temperature. The mixture was purified by silica gel flash column chromatography ( $n$-pentane/diethyl ether 80:20 $\rightarrow$ 70:30) to yield title compound $\mathbf{2 9}(780 \mathrm{mg}, 6.10 \mathrm{mmol}, 35 \%)$ as a yellowish oil.

$\mathbf{R}_{f}=0.20$ (7:3 n-pentane/diethyl ether); $[\alpha]_{\mathbf{D}}{ }^{20}=+13.2\left(c 1.52, \mathrm{CH}_{2} \mathrm{Cl}_{2}\right) ;{ }^{1} \mathbf{H} \mathbf{N M R}\left(500 \mathrm{MHz}, \mathrm{CD}_{2} \mathrm{Cl}_{2}\right)$ : $\delta=9.58(\mathrm{~d}, J=1.7 \mathrm{~Hz}, 1 \mathrm{H}, \mathrm{H}-1), 2.47$ (t, $J=7.7 \mathrm{~Hz}, 2 \mathrm{H}, \mathrm{H}-4), 2.33(6 \mathrm{~d}, J=7.1,1.7 \mathrm{~Hz}, 1 \mathrm{H}, \mathrm{H}-2)$, 2.10 (s, 3H, H-6), $1.96-1.89$ (ddd, $J=14.2,7.7,7.1 \mathrm{~Hz}, 1 \mathrm{H}, \mathrm{H}-3 \mathrm{a}), 1.64-1.57$ (m, 1H, H-3b), 1.08 $\left(\mathrm{d}, J=7.1 \mathrm{~Hz}, 3 \mathrm{H}, 2-\mathrm{CH}_{3}\right) \mathrm{ppm} ;{ }^{13} \mathbf{C} \mathbf{N M R}\left(125 \mathrm{MHz}, \mathrm{CD}_{2} \mathrm{Cl}_{2}\right): \delta=208.2\left(\mathrm{C}_{\mathrm{q}}-5\right), 204.9(\mathrm{C}-1), 46.0$ (C-2), 41.0 (C-4), 30.2 (C-6), $24.6(\mathrm{C}-3), 13.7\left(2-\mathrm{CH}_{3}\right) \mathrm{ppm}$; MS (ESI+) $\mathrm{m} / \mathrm{z}$ : for $\mathrm{C}_{7} \mathrm{H}_{12} \mathrm{O}_{2}[\mathrm{M}+\mathrm{H}]^{+}$ 129.091 .

(2S)-2-Methylhexane-1,5-diol (86) ${ }^{[15]}$

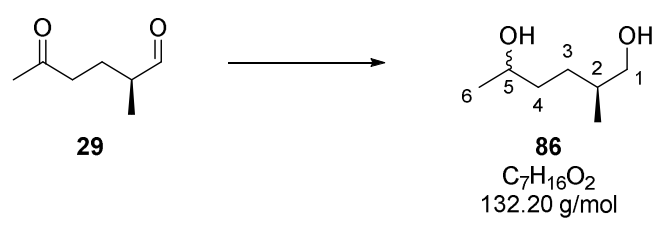

$\mathrm{LiAlH}_{4}$ (4 $\mathrm{M}$ in diethyl ether, $0.4 \mathrm{~mL}, 1.56 \mathrm{mmol}, 2.0$ eq.) was added dropwise to dry diethyl ether $(4 \mathrm{~mL})$ at $0{ }^{\circ} \mathrm{C}$. A solution of $\mathbf{2 9}(100 \mathrm{mg}, 0.78 \mathrm{mmol})$ in dry diethyl ether $(4 \mathrm{~mL})$ was added slowly 
under subsequent cooling. Then, the solution was warmed to room temperature. After complete conversion of the starting material (approx. $15 \mathrm{~min}$ ), the reaction was quenched by very slow addition of $\mathrm{H}_{2} \mathrm{O}(4 \mathrm{~mL})$. Aq. sat. Rochelle salt solution $(2 \mathrm{~mL})$ was added to precipitate the aluminum salts. The phases were separated and the aqueous phase was extracted with diethyl ether $(3 \mathrm{x} 10 \mathrm{~mL})$. The solvent was removed and the crude product $86(120 \mathrm{mg})$, a mixture of diastereomers, was directly used for the next reaction step.

$\mathbf{R}_{f}=0.00$ (7:3 n-pentane/diethyl ether); ${ }^{1} \mathbf{H}$ NMR (700 MHz, $\left.\mathrm{CD}_{2} \mathrm{Cl}_{2}\right): \delta=3.76-3.72(\mathrm{~m}, 1 \mathrm{H}, \mathrm{H}-6)$, $3.47-3.40$ (m, 2H, H-1), 1.62 - 1.56 (m, 1H, H-2), 1.52 - 1.38 (m, 4H, H-3, H-4), 1.16/1.15 (d, $J=$ $6.3 \mathrm{~Hz}, 3 \mathrm{H}, \mathrm{C}-6), 0.91 / 0.90\left(\mathrm{~d}, J=6.7 \mathrm{~Hz}, 3 \mathrm{H}, 2-\mathrm{CH}_{3}\right)$ ppm; ${ }^{13} \mathbf{C} \mathbf{N M R}\left(175 \mathrm{MHz}, \mathrm{CD}_{2} \mathrm{Cl}_{2}\right): \delta=$ 68.9/68.6 (C-5), 68.5/68.4 (C-1), 37.2/37.0 (C-4), 36.5/36.3 (C-2), 29.7/29.6 (C-3), 24.0/23.9 (C-6), 17.0/16.9 $\left(2-\mathrm{CH}_{3}\right)$ ppm; MS (ESI+) $m / z$ : for $\mathrm{C}_{7} \mathrm{H}_{16} \mathrm{O}_{2}[\mathrm{M}+\mathrm{Na}]^{+} 155.1012$.

(5S)-6-((tert-Butyldimethylsilyl)oxy)-5-methylhexan-2-ol (87)

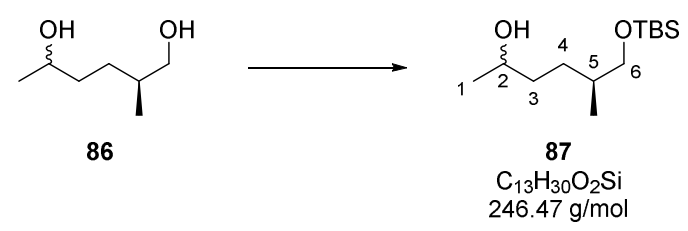

The crude diol 86 (120 mg, max $0.78 \mathrm{mmol})$ was dissolved in dry DCM (3 mL). Imidazole (80 mg, $1.17 \mathrm{mmol}, 1.5$ eq.) was added. Then, a solution of TBSCl (105 mg, $0.70 \mathrm{mmol}, 0.9$ eq.) in dry DCM $(1 \mathrm{~mL})$ was added dropwise at $0{ }^{\circ} \mathrm{C}$. The mixture was stirred for $15 \mathrm{~min}$ at $0{ }^{\circ} \mathrm{C}$. The reaction was quenched by addition of aq. sat. $\mathrm{NH}_{4} \mathrm{Cl}(5 \mathrm{~mL})$. The phases were separated and the aqueous phase was extracted with $\mathrm{Et}_{2} \mathrm{O}$ and DCM $(3 \times 5 \mathrm{~mL})$ successively. The combined organic phases were dried over $\mathrm{MgSO}_{4}$ and the solvent was removed under reduced pressure. The crude product was purified by silica gel flash column chromatography ( $n$-pentane/diethyl ether 7:3) to yield protected diol 87 (121 mg, $0.49 \mathrm{mmol}, 63 \%$ over two steps) as a mixture of diastereomers as a colorless oil.

$\mathbf{R}_{f}=0.52$ (1:1 cyclohexane/ethyl acetate); ${ }^{1} \mathbf{H}$ NMR $\left(500 \mathrm{MHz}, \mathrm{CD}_{2} \mathrm{Cl}_{2}\right): \delta=3.75-3.70(\mathrm{~m}, 1 \mathrm{H}, \mathrm{H}-2)$, $3.47-3.37$ (m, 2H, H-6), $1.60-1.54$ (m, 1H, H-5), 1.50 - 1.06 (m, 4H, H-3, H-4), 1.15 (2d, $J=$ $6.2 \mathrm{~Hz}, 3 \mathrm{H}, \mathrm{H}-1), 0.89$ (s, 9H, OTBS), 0.87 (2d, $\left.J=6.7 \mathrm{~Hz}, 3 \mathrm{H}, 5-\mathrm{CH}_{3}\right), 0.04$ (s, 6H, OTBS) ppm; ${ }^{13} \mathbf{C}$ NMR (125 MHz, $\mathrm{CD}_{2} \mathrm{Cl}_{2}$ ): $\delta=68.9 / 68.7$ (C-2), 68.8/68.7 (C-6), 37.3/37.4 (C-3), 36.3/36.4 (C-5), 29.7/29.8 (C-4), 26.3 (OTBS), 23.8/23.9 (C-1), 18.8 (OTBS), 17.1 (5- $\left.\mathrm{CH}_{3}\right),-5.1$ (OTBS) ppm; HRMS (ESI+) $m / z$ : $[\mathrm{M}+\mathrm{H}]^{+}$Calcd for $\mathrm{C}_{13} \mathrm{H}_{31} \mathrm{O}_{2} \mathrm{Si}^{+}$247.2088, found 247.2084. 


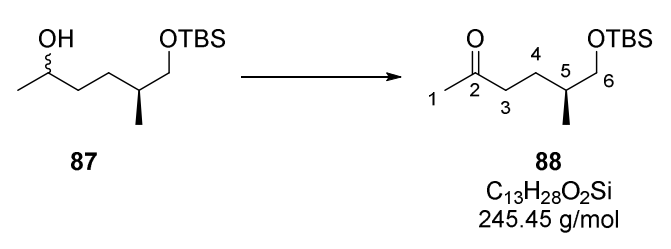

$\mathrm{SO}_{3}$ pyridine complex (234 mg, $1.47 \mathrm{mmol}, 3.0$ eq.) was dried under high vacuum for an hour. DCM $(5 \mathrm{~mL})$ was added. The solution was cooled down to $0{ }^{\circ} \mathrm{C}$. Then, DMSO $(350 \mu \mathrm{L}, 4.9 \mathrm{mmol}, 10$ eq.) and DIPEA ( $333 \mu \mathrm{L}, 1.96 \mathrm{mmol}, 4.0$ eq.) were added. A solution of 87 (120 mg, $0.49 \mathrm{mmol})$ in dry DCM $(1 \mathrm{~mL})$ was added dropwise. The reaction was quenched after $5 \mathrm{~min}$ by addition of aq. sat. $\mathrm{NaHCO}_{3}$ solution $(5 \mathrm{~mL})$. The phases were separated and the aqueous phase was extracted with DCM $(3 \times 5 \mathrm{~mL})$. The combined organic layers were washed with aq. sat. $\mathrm{CuSO}_{4}$ solution $(10 \mathrm{~mL})$, aq. sat. $\mathrm{NH}_{4} \mathrm{Cl}$ solution $(10 \mathrm{~mL})$ and brine $(10 \mathrm{~mL})$ successively, dried over $\mathrm{MgSO}_{4}$ and the solvent was removed under reduced pressure. The crude product was purified by silica gel flash column chromatography ( $n$-pentane/diethyl ether 70:30) to yield title compound $\mathbf{8 8}$ (87 mg, $0.36 \mathrm{mmol}, 73 \%)$ as a colorless oil.

$\mathbf{R}_{f}=0.55$ (2:1 cyclohexane/ethyl acetate); $[\alpha]_{\mathbf{D}}{ }^{20}=-34.5$ (c 1.16, $\left.\mathrm{CHCl}_{3}\right) ;{ }^{1} \mathbf{H}$ NMR $(500 \mathrm{MHz}$, $\mathrm{CD}_{2} \mathrm{Cl}_{2}$ ): $\delta=3.45-3.39$ (m, 2H, H-6), $2.49-2.37$ (m, 2H, H-3), 2.09 (s, 3H, H-1), 1.66 (dddd, $J=$ 13.6, 9.5, 6.2, 5.6 Hz, 1H, H-4a), $1.60-1.51$ (m, 1H, H-5), 1.34 (dddd, $J=13.6 \mathrm{~Hz}, 9.5,7.7,6.0 \mathrm{~Hz}$, $1 \mathrm{H}, \mathrm{H}-4 \mathrm{~b}), 0.89$ (s, 9H, OTBS), 0.86 (d, $\left.J=6.7 \mathrm{~Hz}, 3 \mathrm{H}, 5-\mathrm{CH}_{3}\right), 0.04$ (s, 6H, OTBS) ppm; ${ }^{13} \mathbf{C}$ NMR (125 MHz, $\mathrm{CD}_{2} \mathrm{Cl}_{2}$ ): $\delta=209.3$ (C-2), 68.5 (C-6), 41.9 (C-3), 30.9 (C-5), 35.1 (C-1), 27.9 (C-4), 26.2 (OTBS), 18.8 (OTBS), 16.9 (5- $\mathrm{CH}_{3}$ ), -5.2 (OTBS) ppm; HRMS (ESI+) $\mathrm{m} / z$ : $[\mathrm{M}+\mathrm{H}]^{+} \mathrm{Calcd}$ for $\mathrm{C}_{13} \mathrm{H}_{29} \mathrm{O}_{2} \mathrm{Si}^{+} 245.1931$, found 245.1928 .

The spectroscopic data were consistent with those previously reported. ${ }^{[16]}$

$(3 R, 4 S)-2,4-D i m e t h y l h e x-5-e n-3-01(31)^{[31,32]}$

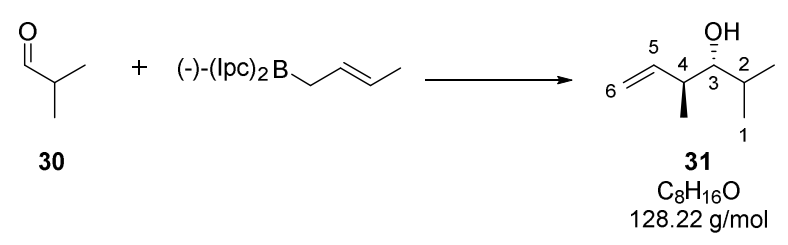

Isobutyraldehyde (30, $1.5 \mathrm{~mL}, 16.4 \mathrm{mmol})$ was dissolved in dry THF $(8 \mathrm{~mL})$. The solution was dried over molecular sieve ( $3 \AA$ ) for $72 \mathrm{~h}$. Dry $\mathrm{KO}^{\mathrm{t}} \mathrm{Bu}(2.4 \mathrm{~g}, 21.3 \mathrm{mmol}, 1.3 \mathrm{eq}$.) was dissolved in THF $(25 \mathrm{~mL})$ and cooled down to $-78{ }^{\circ} \mathrm{C}$. In a separate Schlenk tube, trans-2-butene ( $3 \mathrm{~mL}, 49.2 \mathrm{mmol}$, 
3.0 eq.) was condensed at $-78{ }^{\circ} \mathrm{C}$ and transferred with a transfer canula into the $\mathrm{KO}{ }^{\mathrm{t}} \mathrm{Bu}$ solution. $n$ BuLi (2.5 M in hexanes, $8.5 \mathrm{~mL}, 21.3 \mathrm{mmol}, 1.3$ eq.) was added in a period of $15 \mathrm{~min}$ to result in an orange colored mixture which was stirred for additional $20 \mathrm{~min}$ at $-78{ }^{\circ} \mathrm{C}$. (-)-(Ipc) $)_{2} \mathrm{BOMe}(6.7 \mathrm{~g}$, $21.3 \mathrm{mmol}, 1.3 \mathrm{eq}$.) was added dropwise as a $1 \mathrm{M}$ solution in dry $\mathrm{Et}_{2} \mathrm{O}(21.3 \mathrm{~mL})$ over a period of $20 \mathrm{~min}$ to the orange mixture which becomes colorless again. The mixture was stirred for additional 30 min. $\mathrm{BF}_{3} \mathrm{OEt}_{2}(48 \%, 2.9 \mathrm{~mL}, 23.0 \mathrm{mmol}, 1.4$ eq.) was added dropwise. Instantly after the addition, isobutyraldehyde (30,1.5 mL, $16.4 \mathrm{mmol})$ was added slowly as a solution in dry THF (12 mL) and the mixture was stirred for another $2 \mathrm{~h}$ at $-78^{\circ} \mathrm{C}$. After $1 \mathrm{~h}$, the mixture got solid, so additional dry THF $(20 \mathrm{~mL})$ was added. The mixture was treated with $\mathrm{NaOH}(3 \mathrm{~N}, 20 \mathrm{~mL})$ and $\mathrm{H}_{2} \mathrm{O}_{2}(35 \%, 10 \mathrm{~mL})$, warmed to room temperature and heated to reflux for $1 \mathrm{~h}$. After cooling down to room temperature, the phases were separated and the aqueous phase was extracted with DCM ( $3 \times 25 \mathrm{~mL})$. The combined organic phases were washed with aq. sat. sodium thiosulfate solution, aq. sat. $\mathrm{NH}_{4} \mathrm{Cl}$ solution and brine (each $50 \mathrm{~mL}$ ). The organic phase was dried over $\mathrm{Na}_{2} \mathrm{SO}_{4}$ and the solvent was removed under reduced pressure. The crude product was purified by silica gel flash column chromatography (npentane $\rightarrow n$-pentane/diethyl ether $50: 1 \rightarrow 10: 1)$ to yield volatile $31(1.7 \mathrm{~g}, 13.3 \mathrm{mmol}, \sim 81 \%)$ as a colorless oil with some unidentified impurities.

$\mathbf{R}_{f}=0.40$ (7:3 n-pentane/diethyl ether); $[\alpha]_{\mathbf{D}}{ }^{20}=-144.9\left(c 1.38\right.$, DCM); HRMS (APCI) $m / z:[\mathrm{M}+\mathrm{H}]^{+}$ Calcd for $\mathrm{C}_{8} \mathrm{H}_{7} \mathrm{O}^{+} 129.1274$, found 129.1275 .

Major diastereomer:

${ }^{1} \mathbf{H}$ NMR $\left(700 \mathrm{MHz}, \mathrm{CD}_{2} \mathrm{Cl}_{2}\right): \delta=5.82-5.77(\mathrm{~m}, 1 \mathrm{H}, \mathrm{H}-5), 5.11-5.08(\mathrm{~m}, 2 \mathrm{H}, \mathrm{H}-6), 3.06(\mathrm{td}, J=$ $5.7,4.8 \mathrm{~Hz}, 1 \mathrm{H}, \mathrm{H}-3), 2.33-2.29$ (m, 1H, H-4), $1.75-1.70$ (pd, $J=6.8,5.5 \mathrm{~Hz}, 1 \mathrm{H}, \mathrm{H}-2), 1.42$ (d, $J=$ $4.8 \mathrm{~Hz}, 1 \mathrm{H}, 3-\mathrm{OH}), 1.01$ (d, $\left.J=6.8 \mathrm{~Hz}, 3 \mathrm{H}, 4-\mathrm{CH}_{3}\right), 0.94$ (d, $\left.J=6.8 \mathrm{~Hz}, 3 \mathrm{H}, \mathrm{H}-1 / 2-\mathrm{CH}_{3}\right), 0.90$ (d, $J=$ $\left.6.8 \mathrm{~Hz}, 3 \mathrm{H}, \mathrm{H}-1 / 2-\mathrm{CH}_{3}\right) \mathrm{ppm} ;{ }^{13} \mathbf{C}$ NMR $\left(175 \mathrm{MHz}, \mathrm{CD}_{2} \mathrm{Cl}_{2}\right): \delta=141.4(\mathrm{C}-5), 116.1(\mathrm{C}-6), 79.9(\mathrm{C}-3)$, $42.0(\mathrm{C}-4), 31.0(\mathrm{C}-2), 20.2\left(\mathrm{C}-1 / 2-\mathrm{CH}_{3}\right), 17.4\left(4-\mathrm{CH}_{3}\right), 16.8\left(\mathrm{C}-1 / 2-\mathrm{CH}_{3}\right) \mathrm{ppm}$.

Minor diastereomer:

${ }^{1} \mathbf{H}$ NMR $\left(700 \mathrm{MHz}, \mathrm{CD}_{2} \mathrm{Cl}_{2}\right): \delta=5.82-5.77(\mathrm{~m}, 1 \mathrm{H}, \mathrm{H}-5), 5.06-5.03(\mathrm{~m}, 2 \mathrm{H}, \mathrm{H}-6), 3.14$ (td, $J=$ 5.7, $4.8 \mathrm{~Hz}, 1 \mathrm{H}, \mathrm{H}-3), 2.35-2.32$ (m, 1H, H-4), $1.64-1.60$ (m, 1H, H-2), 1.42 (d, J=4.8 Hz, 1H, 3$\mathrm{OH}), 1.01\left(\mathrm{~d}, J=6.8 \mathrm{~Hz}, 3 \mathrm{H}, 4-\mathrm{CH}_{3}\right), 0.94\left(\mathrm{~d}, J=6.8 \mathrm{~Hz}, 3 \mathrm{H}, \mathrm{H}-1 / 2-\mathrm{CH}_{3}\right), 0.90(\mathrm{~d}, J=6.8 \mathrm{~Hz}, 3 \mathrm{H}$, $\left.\mathrm{H}-1 / 2-\mathrm{CH}_{3}\right)$ ppm; ${ }^{13} \mathbf{C}$ NMR $\left(175 \mathrm{MHz}, \mathrm{CD}_{2} \mathrm{Cl}_{2}\right.$ ): $\delta=142.7$ (C-5), 114.7 (C-6), 78.0 (C-3), 41.3 (C-4), $34.1(\mathrm{C}-2), 20.0\left(\mathrm{C}-1 / 2-\mathrm{CH}_{3}\right), 14.4\left(\mathrm{C}-1 / 2-\mathrm{CH}_{3}\right), 14.1\left(4-\mathrm{CH}_{3}\right) \mathrm{ppm}$.

The diastereomeric ratio was determined by ${ }^{1} \mathrm{H}$ NMR analysis to be $d r=14: 1$ (anti:syn). The signals of H-3 (3.14 ppm (minor) and 3.06 ppm (major)) are separated. The enantiomeric excess could not be determined at this step since the compound could not be detected by UV light detector of the HPLC. The absolute stereochemistry and the enantiomeric excess of the main product was determined in the next step by Mosher ester analysis. 


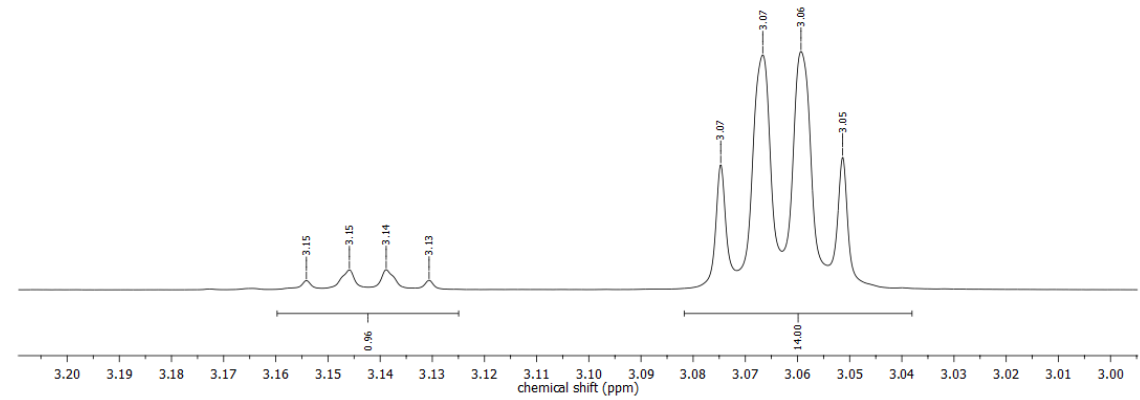

Figure 11. Selected ${ }^{1} \mathrm{H}$ NMR signals of compound $\mathbf{3 1}$ to determine the $d r$ to be 14:1.

$(3 R, 4 S)$-2,4-Dimethylhex-5-en-3-yl-(S)-3,3,3-trifluoro-2-methoxy-2-phenylpropanoate $(89)^{[17]}$

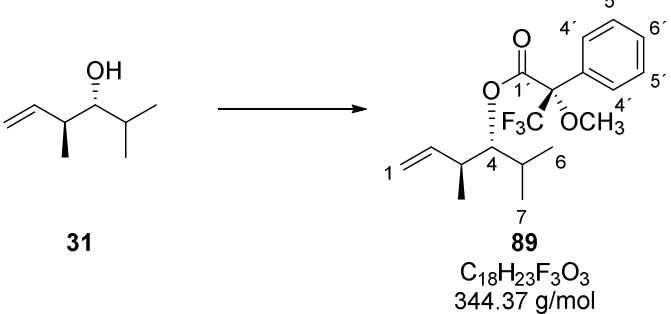

Alcohol 31 (4 mg, $31 \mu \mathrm{mol})$ was dissolved in dry DCM $(0.5 \mathrm{~mL})$. Then, dry pyridine $(7.5 \mu \mathrm{L}$, $93 \mu \mathrm{mol}, 3.0$ eq.) and $R$-MTPA-Cl $(12 \mu \mathrm{L}, 62 \mu \mathrm{mol}, 2.0$ eq. $)$ were added at room temperature. The solution was stirred for $2 \mathrm{~h}$. The reaction was quenched by addition of $\mathrm{H}_{2} \mathrm{O}(1 \mathrm{~mL})$, the phases were separated and the aqueous phase was extracted with DCM $(3 \times 1 \mathrm{~mL})$. The combined organic phases were dried over $\mathrm{MgSO}_{4}$ and the solvent was removed. The crude product was purified by silica gel flash column chromatography ( $n$-pentane $\rightarrow n$-pentane/diethyl ether 9:1) to give $S$-Mosher ester 89 (4.8 $\mathrm{mg}, 14 \mu \mathrm{mol}, 45 \%)$ as a colorless oil.

$\mathbf{R}_{f}=0.64$ (n-pentane/diethyl ether 7:3); $[\boldsymbol{\alpha}]_{\mathbf{D}}{ }^{20}=-225.8\left(c \quad 0.62, \mathrm{CH}_{2} \mathrm{Cl}_{2}\right) ;{ }^{1} \mathbf{H}-\mathbf{N M R}(700 \mathrm{MHz}$, $\left.\mathrm{CD}_{2} \mathrm{Cl}_{2}\right): \delta=7.58-7.53(\mathrm{~m}, 2 \mathrm{H}, \mathrm{Ph}), 7.43-7.38(\mathrm{~m}, 3 \mathrm{H}, \mathrm{Ph}), 5.66(\mathrm{ddd}, J=17.2,10.3,8.7 \mathrm{~Hz}, 1 \mathrm{H}$, $\mathrm{H}-2), 4.99-4.92$ (m, 2H, H-1), 4.90 (t, $J=6.0 \mathrm{~Hz}, 1 \mathrm{H}, \mathrm{H}-4), 3.51-3.49$ (m, 3H, $\left.\mathrm{OCH}_{3}\right), 2.55-2.50$ (m, 1H, H-3), $2.00-1.95$ (m, 1H, H-5), 0.96 (d, $\left.J=6.9 \mathrm{~Hz}, 3 \mathrm{H}, 3-\mathrm{CH}_{3}\right), 0.93$ (d, $J=6.8 \mathrm{~Hz}, 3 \mathrm{H}, \mathrm{H}-$ 6/H-7), 0.90 (d, $J=6.8 \mathrm{~Hz}, 3 \mathrm{H}, \mathrm{H}-6 / \mathrm{H}-7) \mathrm{ppm} ;{ }^{13} \mathbf{C}$ NMR (125 MHz, $\left.\mathrm{CD}_{2} \mathrm{Cl}_{2}\right): \delta=166.8\left(\mathrm{C}-1^{\prime}\right), 139.8$ (C-2), $132.5\left(\mathrm{C}_{\mathrm{q}}-3^{\prime}\right), 130.1$ (C-5'/C-6'), 128.8 (C-5'/C-6'), 128.5 (C-4') 128.0 (q, C-2'), 127.4 (C- 
5'/C-6'), 116.2 (C 1), 85.1 (C-4), $55.9\left(\mathrm{OCH}_{3}\right), 40.7$ (C-3), 30.3 (C-5), 19.7 (C-6/C-7), 17.9 (3-CH 3 ), 17.6 (C-6/C-7) ppm; HRMS (ESI+) $\mathrm{m} / z$ : [M + Na $]^{+}$Calcd for $\mathrm{C}_{18} \mathrm{H}_{23} \mathrm{~F}_{3} \mathrm{O}_{3}{ }^{+} 367.1492$, found 367.1432. $(3 R, 4 S)$-2,4-Dimethylhex-5-en-3-yl-( $R)-3,3,3$-trifluoro-2-methoxy-2-phenylpropanoate $(90)^{[17]}$

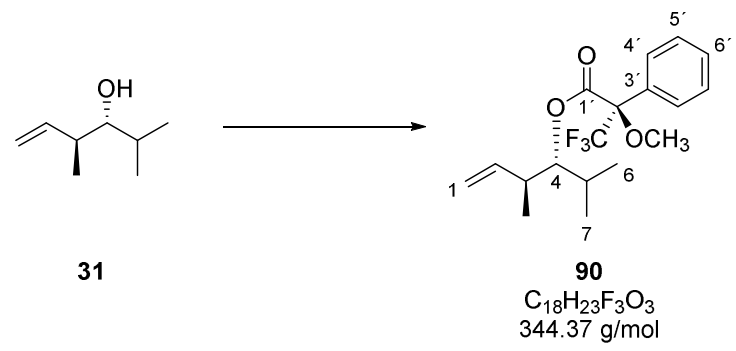

Alcohol 31 (4 mg, $31 \mu \mathrm{mol})$ was dissolved in dry DCM $(0.5 \mathrm{~mL})$. Then, dry pyridine $(7.5 \mu \mathrm{L}$, $93 \mu \mathrm{mol}, 3.0$ eq.) and $S$-MTPA-Cl $(12 \mu \mathrm{L}, 62 \mu \mathrm{mol}, 2.0$ eq.) were added at room temperature. The solution was stirred for $2.5 \mathrm{~h}$. Because no full conversion was observed, additional S-MTPA-Cl (12 $\mu \mathrm{L}, 62 \mu \mathrm{mol}, 2.0$ eq) was added and after $5.5 \mathrm{~h}$, again $S$-MTPA-Cl (12 $\mu \mathrm{L}, 62 \mu \mathrm{mol}, 2.0 \mathrm{eq})$ and pyridine $(7.5 \mu \mathrm{L}, 93 \mu \mathrm{mol}, 3.0$ eq.) were given into the reaction mixture and stirred overnight. The reaction was quenched by addition of $\mathrm{H}_{2} \mathrm{O}(1 \mathrm{~mL})$, the phases were separated and the aqueous phase was extracted with DCM $(3 \times 0.5 \mathrm{~mL})$. The combined organic phases were dried over $\mathrm{MgSO}_{4}$ and the solvent was removed. The crude product was purified by silica gel flash column chromatography $(n-$ pentane $\rightarrow n$-pentane/diethyl ether 9:1) to give $R$-Mosher ester 90 (6 mg, $17 \mu \mathrm{mol}, 56 \%)$ as a colorless oil.

$\mathbf{R}_{f}=0.63$ (n-pentane/diethyl ether 7:3); $[\alpha]_{\mathbf{D}}{ }^{20}=+333.3\left(c\right.$ 1.17, $\left.\mathrm{CH}_{2} \mathrm{Cl}_{2}\right) ;{ }^{1} \mathbf{H}-\mathbf{N M R}(500 \mathrm{MHz}$, $\mathrm{CD}_{2} \mathrm{Cl}_{2}$ ): $\delta=7.58-7.52\left(\mathrm{~m}, 2 \mathrm{H}, \mathrm{H}-4^{\prime}\right), 7.44-7.38$ (m, 3H, H-5'/H-6'), 5.75 (ddd, $J=17.2,10.3$, $8.6 \mathrm{~Hz}, 1 \mathrm{H}, \mathrm{H}-2), 5.07-5.01$ (m, 2H, H-1), 4.91 (t, $J=6.0 \mathrm{~Hz}, 1 \mathrm{H}, \mathrm{H}-4), 3.53-3.49$ (m, 3H, $\left.\mathrm{OCH}_{3}\right)$, $2.61-2.54$ (m, 1H, H-3), $1.99-1.90$ (m, 1H, H-5), 1.02 (d, $\left.J=7.0 \mathrm{~Hz}, 3 \mathrm{H}, 3-\mathrm{CH}_{3}\right), 0.87$ (d, $J=$ $6.8 \mathrm{~Hz}, 3 \mathrm{H}, \mathrm{H}-6 / \mathrm{H}-7), 0.81(\mathrm{~d}, J=6.8 \mathrm{~Hz}, 3 \mathrm{H}, \mathrm{H}-6 / \mathrm{H}-7) \mathrm{ppm} ;{ }^{13} \mathbf{C} \mathbf{N M R}\left(125 \mathrm{MHz}, \mathrm{CD}_{2} \mathrm{Cl}_{2}\right): \delta=$ 166.8 (C-1'), 140.2 (C-2), 132.6 (C-3'), 130.1 (C-5'/C-6'), 128.8 (C-5'/C-6'), 128.4 (C-4'), 128.0 (q, C-2'), 116.3 (C-1), 85.2 (C-4), $55.9\left(\mathrm{OCH}_{3}\right), 40.8$ (C-3), 30.2 (C-5), 19.7 (C-6/C-7), 18.1 (3-CH $\left.{ }_{3}\right)$, 17.3 (C-6/C-7) ppm; HRMS (APCI) $m / z$ : $[\mathrm{M}+\mathrm{Na}]^{+}$Calcd for $\mathrm{C}_{18} \mathrm{H}_{23} \mathrm{~F}_{3} \mathrm{O}_{3}{ }^{+}$367.1492, found 367.1450 . 


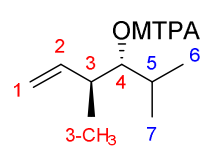

Table 4. Mosher ester analysis.

$\begin{array}{llll} & \delta_{H}[\mathrm{ppm}] & \delta_{H}[\mathrm{ppm}] \\ \text { position } & (\mathbf{8 9})(S) & (\mathbf{9 0})(R) & \boldsymbol{\Delta \delta}^{S-\boldsymbol{R}} \\ \text { H-7 } & 0.90 & 0.81 & +0.09 \\ \mathrm{H}-6 & 0.93 & 0.87 & +0.06 \\ \mathrm{H}-5 & 1.98 & 1.95 & +0.03 \\ \mathrm{H}-4 & 4.90 & 4.91 & -0.01 \\ 3-\mathrm{CH}_{3} & 0.96 & 1.02 & -0.06 \\ \mathrm{H}-3 & 2.52 & 2.58 & -0.06 \\ \mathrm{H}-1 & 4.95 & 5.04 & -0.09 \\ \mathrm{H}-2 & 5.66 & 5.75 & -0.11\end{array}$

For multiplets, the center of the signals was selected to calculate the differences. The enantiomeric excess was determined from the ${ }^{1} \mathrm{H}$ NMR spectra of the Mosher esters $\mathbf{8 9}$ and $\mathbf{9 0}$ to be $e e=88 \%$.

tert-Butyl(((3R,4S)-2,4-dimethylhex-5-en-3-yl)oxy)dimethylsilane (91)

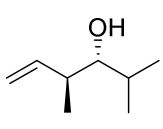

31

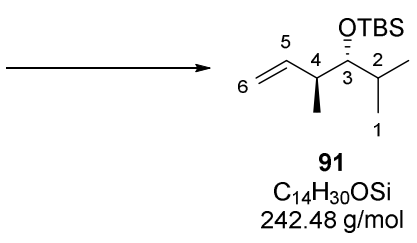

Alcohol 31 (500 mg, $3.9 \mathrm{mmol})$ was dissolved in dry DCM/diethyl ether $(1: 1,25 \mathrm{~mL})$ at $-20^{\circ} \mathrm{C}$. 2,6-Lutidine (1.5 mL, $12.9 \mathrm{mmol}, 3.3$ eq.) and TBSOTf $(1.8 \mathrm{~mL}, 7.8 \mathrm{mmol}, 2.0$ eq.) were added, subsequently. The mixture was stirred for $2.5 \mathrm{~h}$ and allowed to warm to $10^{\circ} \mathrm{C}$ (by no further addition of ice cubes to the cooling bath). The reaction was quenched by addition of aq. $\mathrm{NH}_{4} \mathrm{Cl}$ solution $(20 \mathrm{~mL}, 1 \mathrm{~N})$. The phases were separated and the aqueous phase was extracted with DCM $(3 \times 20 \mathrm{~mL})$. The combined organic phases were washed with water and dried with $\mathrm{MgSO}_{4}$, the solvent was removed off and the crude product was purified by silica gel flash column chromatography ( $n$-pentane 100\%) to yield TBS protected alcohol $91(940 \mathrm{mg})$ as a volatile, colorless oil. Due to the volatile and nonpolar character of this compound, it was not possible to remove unreacted TBSOH. It could be removed after the next step. 
$\mathbf{R}_{f}=0.57\left(100 \% n\right.$-pentane); $[\alpha]_{\mathbf{D}}{ }^{20}=+6.9\left(c 1.44, \mathrm{CH}_{2} \mathrm{Cl}_{2}\right) ;{ }^{1} \mathbf{H}-\mathbf{N M R}\left(700 \mathrm{MHz}, \mathrm{CD}_{2} \mathrm{Cl}_{2}\right): \delta=5.90$ (ddd, $J=17.3,10.4,8.0 \mathrm{~Hz}, 1 \mathrm{H}, \mathrm{H}-5), 4.99-4.93$ (m, 2H, H-6), 3.31 (dd, $J=5.5,3.9 \mathrm{~Hz}, 1 \mathrm{H}, \mathrm{H}-3$ ), $2.39-2.35$ (m, 1H, H-4), 1.74 (pd, $J=6.8,5.5 \mathrm{~Hz}, 1 \mathrm{H}, \mathrm{H}-2), 1.01$ (d, $\left.J=7.0 \mathrm{~Hz}, 3 \mathrm{H}, 4-\mathrm{CH}_{3}\right), 0.91$ (s, 9H, OTBS), 0.88 (d, $J=6.8 \mathrm{~Hz}, 3 \mathrm{H}, 2-\mathrm{CH}_{3}$ ), 0.87 (d, $J=6.8 \mathrm{~Hz}, 3 \mathrm{H}, \mathrm{H}-1$ ), 0.06 (s, 3H, OTBS), 0.05 (s, 3H, OTBS) ppm; ${ }^{13} \mathbf{C}$ NMR $\left(175 \mathrm{MHz}, \mathrm{CD}_{2} \mathrm{Cl}_{2}\right): \delta=142.7$ (C-5), 113.9 (C-6), 81.5 (C-3), 42.7 (C-4), 32.7 (C-2), 26.5 (OTBS), 20.4 (C-1/2- $\left.\mathrm{CH}_{3}\right), 19.0\left(\mathrm{C}-1 / 2-\mathrm{CH}_{3}\right), 18.9$ (OTBS), $18.5\left(4-\mathrm{CH}_{3}\right),-3.4$ (OTBS), -3.5 (OTBS) ppm.

The exact mass could not be determined due to the high volatility of compound $\mathbf{9 1 .}$

\section{(2S,3R)-3-((tert-Butyldimethylsilyl)oxy)-2,4-dimethylpentan-1-ol (32) ${ }^{[33]}$}

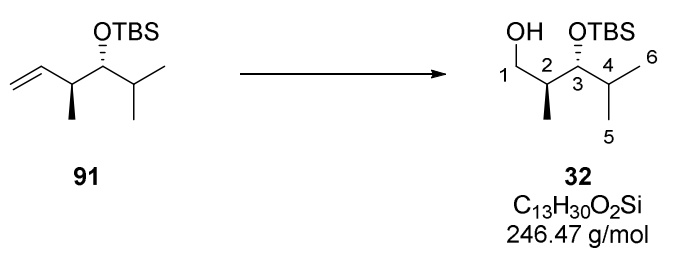

TBS protected alcohol $91(940 \mathrm{mg}$, max. $3.9 \mathrm{mmol})$ was dissolved in dry DCM (55 mL, approx. $15 \mathrm{~mL} / \mathrm{mmol}$ ) and dry $\mathrm{MeOH}(5 \mathrm{~mL})$. The solution was cooled down to $-78{ }^{\circ} \mathrm{C}$. Ozone (approx. $1 \mathrm{~min} / \mathrm{mmol}$ ) was bubbled through the solution. Full conversion was indicated by the blue color of the reaction solution. The reaction flask was purged with $\mathrm{O}_{2}$ and then, $\mathrm{NaBH}_{4}(740 \mathrm{mg}, 19.5 \mathrm{mmol}$, 5.0 eq.) was added. The mixture was stirred at room temperature for $30 \mathrm{~min}$, followed by quenching with water $(20 \mathrm{~mL})$. The phases were separated and the aqueous phase was extracted with DCM $(3 \mathrm{x}$ $15 \mathrm{~mL}$ ). The combined organic phases were dried over $\mathrm{Na}_{2} \mathrm{SO}_{4}$ and the solvent was removed. The crude product was purified by silica gel flash column chromatography ( $n$-pentane/diethyl ether 9:1 $\rightarrow$ 7:3) to yield the primary alcohol 32 (660 mg, $2.67 \mathrm{mmol}, 67 \%$ over three steps) as a colorless oil.

$\mathbf{R}_{f}=0.34$ (7:3 n-pentane/diethyl ether); $[\alpha]_{\mathbf{D}}{ }^{20}=-65.6\left(c 1.22, \mathrm{CH}_{2} \mathrm{Cl}_{2}\right) ;{ }^{1} \mathbf{H} \mathbf{N M R}\left(500 \mathrm{MHz}, \mathrm{CD}_{2} \mathrm{Cl}_{2}\right)$ : $\delta=3.62-3.52(\mathrm{~m}, 2 \mathrm{H}, \mathrm{H}-1), 3.44(\mathrm{dd}, J=4.9 \mathrm{~Hz}, 1 \mathrm{H}, \mathrm{H}-3), 2.32(\mathrm{t}, J=5.8 \mathrm{~Hz}, 1 \mathrm{H},-\mathrm{OH}), 1.87-$ 1.79 (m, 2H, H-2, H-4), 0.96 (d, $\left.J=7.1 \mathrm{~Hz}, 3 \mathrm{H}, 2-\mathrm{CH}_{3}\right), 0.92$ (d, $\left.J=6.9 \mathrm{~Hz}, 3 \mathrm{H}, \mathrm{H}-6\right), 0.92$ (s, 9H, OTBS), 0.91 (d, $J=6.9 \mathrm{~Hz}, 3 \mathrm{H}, \mathrm{H}-5), 0.11$ (s, 3H, OTBS), 0.09 (s, 3H, OTBS) ppm; ${ }^{13} \mathbf{C}$ NMR (125 MHz, $\mathrm{CD}_{2} \mathrm{Cl}_{2}$ ): $\delta=82.4$ (C-3), 66.3 (C-1), 38.0 (C-2), 33.4 (C-4), 26.4 (OTBS), 19.5 (C5/C-6), 18.8 (OTBS), 18.6 (C-5/C-6), 16.6 (2- $\mathrm{CH}_{3}$ ), -3.70 (OTBS), -3.71 (OTBS); HRMS (APCI) $m / z:[\mathrm{M}+\mathrm{H}]^{+}$Calcd for $\mathrm{C}_{13} \mathrm{H}_{31} \mathrm{O}_{2} \mathrm{Si}^{+}$247.2088, found 247.2092. 


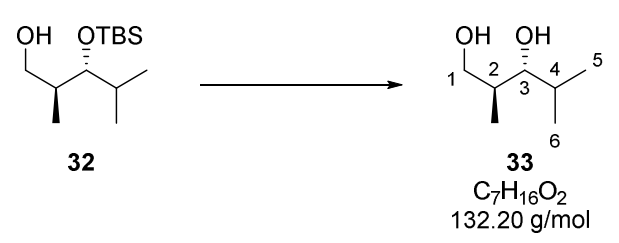

TBS protected alcohol $32(123 \mathrm{mg}, 0.50 \mathrm{mmol})$ was dissolved in DCM/MeOH $(5: 1,2.8 \mathrm{~mL})$. CSA (12 mg, $0.05 \mathrm{mmol}, 0.1$ eq.) was added and the solution was stirred for $20 \mathrm{~h}$ at room temperature. The solvents were removed under reduced pressure. The crude product was purified by silica gel flash column chromatography (diethyl ether) to yield diol $\mathbf{3 3}$ (60 mg, $0.45 \mathrm{mmol}, 91 \%)$ as a colorless solid.

$\mathbf{R}_{f}=0.27$ (diethyl ether); $[\alpha]_{\mathbf{D}}{ }^{20}=-182.7\left(c 1.04, \mathrm{CH}_{2} \mathrm{Cl}_{2}\right) ;{ }^{1} \mathbf{H} \mathbf{N M R}\left(500 \mathrm{MHz}, \mathrm{CD}_{2} \mathrm{Cl}_{2}\right): \delta=3.72-$ 3.58 (m, 2H, H-1), $3.33-3.30$ (m, 1H, H-3), $2.62\left(\mathrm{~s}_{\mathrm{br}}, 1 \mathrm{H}, 1-\mathrm{OH}\right), 2.41\left(\mathrm{~s}_{\mathrm{br}}, 1 \mathrm{H}, 3-\mathrm{OH}\right), 1.86-1.78$ (m, 1H, H-4), $1.83-1.74$ (m, 1H, H-2), 0.95 (d, $J=7.0 \mathrm{~Hz}, 3 \mathrm{H}, \mathrm{H}-5 / \mathrm{H}-6), 0.88$ (d, J=7.0 Hz, 3H, H5/H-6), 0.85 (d, $\left.J=7.0 \mathrm{~Hz}, 3 \mathrm{H}, 2-\mathrm{CH}_{3}\right) \mathrm{ppm} ;{ }^{13} \mathbf{C} \mathbf{N M R}\left(125 \mathrm{MHz}, \mathrm{CD}_{2} \mathrm{Cl}_{2}\right): \delta=82.3(\mathrm{C}-3), 68.5$ (C-1), 37.9 (C-2), 30.9 (C-4), 20.2 (C-5/C-6), 15.4 (C-5/C-6), $14.2\left(2-\mathrm{CH}_{3}\right) \mathrm{ppm}$; HRMS (ESI+) m/z: $[\mathrm{M}+\mathrm{H}]^{+}$Calcd for $\mathrm{C}_{7} \mathrm{H}_{17} \mathrm{O}_{2}^{+}$133.1223, found 133.1222.

\section{(S)-Ir-SEGPHOS Krische catalyst (34)}

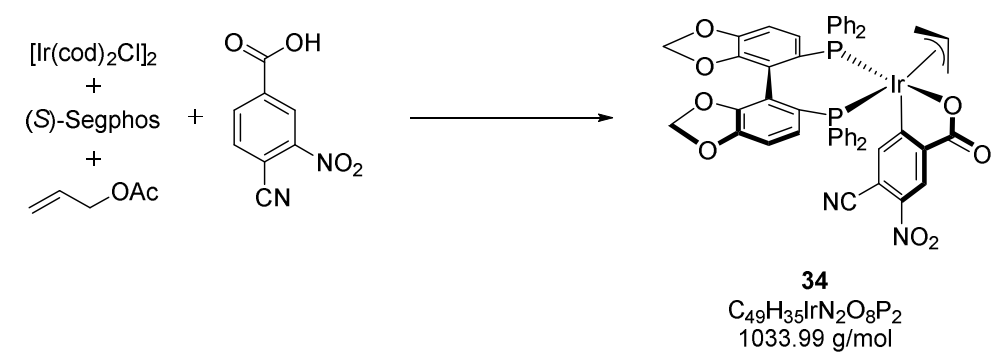

Under argon a mixture of $\left[\operatorname{Ir}(\operatorname{cod})_{2} \mathrm{Cl}\right]_{2}(44 \mathrm{mg}, 65 \mu \mathrm{mol}),(S)$-SEGPHOS $(80 \mathrm{mg}, 130 \mu \mathrm{mol}, 2.0$ eq. $)$, $\mathrm{Cs}_{2} \mathrm{CO}_{3}$ (85 mg, $260 \mu \mathrm{mol}, 4.0$ eq.), 4-cyano-3-nitro-benzoic acid (50 mg, $260 \mu \mathrm{mol}, 4.0$ eq.) and allyl acetate $(35 \mu \mathrm{L}, 330 \mu \mathrm{mol}, 5.0$ eq.) were suspended in THF $(1.3 \mathrm{~mL})$. It was stirred for $30 \mathrm{~min}$ at room temperature and heated for $1.5 \mathrm{~h}$ at $80^{\circ} \mathrm{C}$. After cooling to room temperature, the mixture was filtered and the precipitate was washed with THF $(5 \mathrm{~mL})$. The filtrate was concentrated in vacuo (approx. 2/3 volume) and $n$-hexane was added. The formed precipitate was filtered off and dried in vacuo to yield $(S)$-Ir-SEGPHOS Krische catalyst (34, $59 \mathrm{mg}, 57 \mu \mathrm{mol}, 88 \%$ ) as a yellow solid.

The catalyst $\mathbf{3 4}$ was prepared according to a known procedure. ${ }^{[34]}$ 


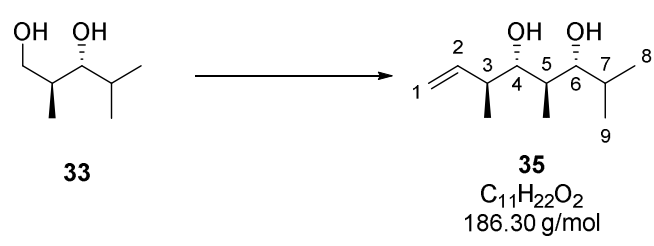

(S)-Ir-SEGPHOS Krische catalyst (34, $21 \mathrm{mg}, 21 \mu \mathrm{mol}, 2.5 \mathrm{~mol} \%)$, diol 33 (108 mg, $0.82 \mathrm{mmol})$ and $\mathrm{K}_{2} \mathrm{CO}_{3}(213 \mathrm{mg}, 1.64 \mathrm{mmol}, 2.0$ eq.) were suspended in THF ( $0.8 \mathrm{~mL}$, approx. $1 \mathrm{~mL} / \mathrm{mmol})$ and $\mathrm{H}_{2} \mathrm{O}$ ( $60 \mu \mathrm{L}, 3.28 \mathrm{mmol}, 4.0$ eq.). Crotyl acetate $(260 \mu \mathrm{L}, 2.05 \mathrm{mmol}, 2.5$ eq.) was added. The mixture was sparged with argon for $2 \mathrm{~min}$ and then, heated to $90{ }^{\circ} \mathrm{C}$ for $2 \mathrm{~d}$. The reaction mixture was cooled down to room temperature and evaporated on silica gel $(63-200 \mu \mathrm{m})$. The resulting residue was subjected to silica gel flash column chromatography (7:3 n-pentane/diethyl ether $\rightarrow 100 \%$ diethyl ether) to yield the crotylation product 35 ( $89 \mathrm{mg}, 0.48 \mathrm{mmol}, 58 \%)$ as a colorless oil.

$\mathbf{R}_{f}=0.56$ (diethyl ether); $[\alpha]_{\mathbf{D}}{ }^{20}=-59.4\left(c 1.01, \mathrm{CH}_{2} \mathrm{Cl}_{2}\right) ;{ }^{1} \mathbf{H} \mathbf{N M R}\left(500 \mathrm{MHz}, \mathrm{CD}_{2} \mathrm{Cl}_{2}\right): \delta=5.71$ (ddd, $J=17.0,10.4,8.7 \mathrm{~Hz}, 1 \mathrm{H}, \mathrm{H}-2), 5.16-5.11(\mathrm{~m}, 2 \mathrm{H}, \mathrm{H}-1), 3.42$ (dt, $J=9.2,2.0 \mathrm{~Hz}, 1 \mathrm{H}, \mathrm{H}-4), 3.31$ (dt, $J=9.0,1.8 \mathrm{~Hz}, 1 \mathrm{H}, \mathrm{H}-6), 2.31-2.23$ (m, 1H, H-3), $1.89-1.87$ (m, 1H, H-5), $1.73-1.66$ (m, 1H, $\mathrm{H}-7$ ), 0.98 (d, $J=6.6 \mathrm{~Hz}, 3 \mathrm{H}, \mathrm{H}-8 / \mathrm{H}-9), 0.95$ (d, $\left.J=6.8 \mathrm{~Hz}, 3 \mathrm{H}, 3-\mathrm{CH}_{3}\right), 0.86$ (d, $J=7.0 \mathrm{~Hz}, 3 \mathrm{H}, 5-$ $\left.\mathrm{CH}_{3}\right), 0.83(\mathrm{~d}, J=6.8 \mathrm{~Hz}, 3 \mathrm{H}, \mathrm{H}-8 / \mathrm{H}-9) \mathrm{ppm} ;{ }^{13} \mathbf{C} \mathbf{N M R}\left(125 \mathrm{MHz}, \mathrm{CD}_{2} \mathrm{Cl}_{2}\right): \delta=142.2(\mathrm{C}-2), 117.0$ (C-1), 83.2 (C-6), 80.4 (C-4), 43.2 (C-3), 35.1 (C-5), 31.8 (C-7), 19.9 (C-8/C-9), 19.4 (C-8/C-9), 16.8 $\left(3-\mathrm{CH}_{3}\right), 4.7\left(5-\mathrm{CH}_{3}\right)$ ppm; HRMS (ESI+) $\mathrm{m} / \mathrm{z}$ : $[\mathrm{M}+\mathrm{H}]^{+}$Calcd for $\mathrm{C}_{11} \mathrm{H}_{23} \mathrm{O}_{2}{ }^{+}$187.1693, found 187.1690 .

The diastereomeric ratio 9:2:1 of the reaction was roughly determined by ${ }^{1} \mathrm{H}$ NMR analysis. The signals of H-2 (5.71, ddd) are partly overlapping. Compound $\mathbf{3 5}$ did not show UV activity making an analysis by HPLC impractically. The syn-configuration of the 1,3 diol was verified with the acetonide.

Nucleus: $1 \mathrm{H}$

Frequency: $499.13 \mathrm{MHz}$

Solvent: $\mathrm{CD} 2 \mathrm{Cl} 2$

Temperature: $298.0 \mathrm{~K}$

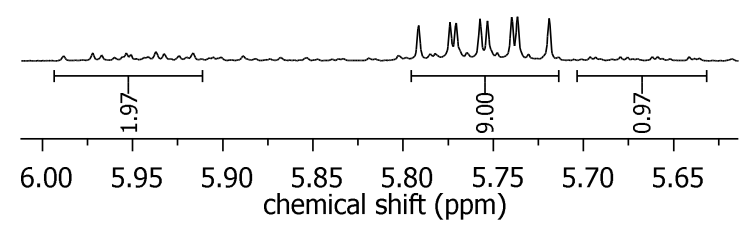

Figure 12. Selected ${ }^{1} \mathrm{H}$ NMR signals of compound 35 to determine the $d r$ to be $\sim 9: 2: 1$. 


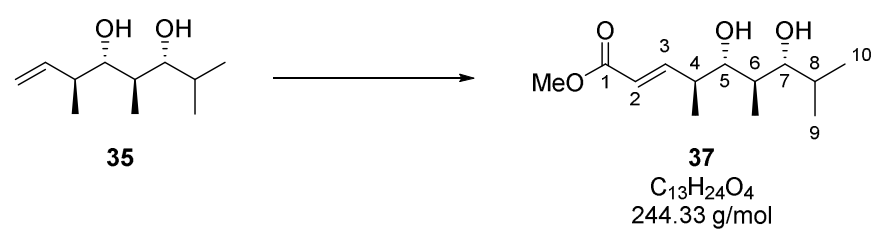

The diol 35 (30 mg, $0.16 \mathrm{mmol})$ and methyl acrylate (36, $73 \mu \mathrm{L}, 0.81 \mathrm{mmol}, 5.0$ eq.) were dissolved in dry DCM $(1 \mathrm{~mL})$. Hoveyda-Grubbs-catalyst $2^{\text {nd }}$ generation $(5 \mathrm{mg}, 8 \mu \mathrm{mol}, 0.05 \mathrm{eq}$.) was added and the mixture was heated to reflux for $19 \mathrm{~h}$. The solvent was removed under reduced pressure. The crude product was purified by silica gel flash column chromatography ( $n$-pentane/diethyl ether 7:3 $\rightarrow 100 \%$ diethyl ether) to yield unsaturated ester $\mathbf{3 7}(38 \mathrm{mg}, 155 \mu \mathrm{mol}, 97 \%)$ as a green oil.

$\mathbf{R}_{f(\text { Silica) }}=0.44$ (diethyl ether), $\mathbf{R}_{\text {f(reversed phase) }}=0.30\left(1: 1 \mathrm{MeCN} / \mathrm{H}_{2} \mathrm{O}\right) ;[\alpha]_{\mathbf{D}}{ }^{20}=-206.5\left(c 0.92, \mathrm{CH}_{2} \mathrm{Cl}_{2}\right)$; ${ }^{1} \mathbf{H}$ NMR $\left(500 \mathrm{MHz}, \mathrm{CD}_{2} \mathrm{Cl}_{2}\right): \delta=6.95(\mathrm{dd}, J=15.7,8.6 \mathrm{~Hz}, 1 \mathrm{H}, \mathrm{H}-3), 5.88(\mathrm{dd}, J=15.7,1.0 \mathrm{~Hz}, 1 \mathrm{H}$, $\mathrm{H}-2), 3.70\left(\mathrm{~s}, 3 \mathrm{H}, \mathrm{OCH}_{3}\right), 3.58$ (dt, $\left.J=8.8,1.3 \mathrm{~Hz}, 1 \mathrm{H}, \mathrm{H}-5\right), 3.33$ (dt, $\left.J=9.1,2.2 \mathrm{~Hz}, 1 \mathrm{H}, \mathrm{H}-7\right), 2.97$ $\left(\mathrm{s}_{\mathrm{br}}, 1 \mathrm{H}, 5-\mathrm{OH}\right), 2.52\left(\mathrm{~s}_{\mathrm{br}}, 1 \mathrm{H}, 7-\mathrm{OH}\right), 2.51-2.43$ (m, 1H, H-4), 1.89 (qt, $J=7.0,2.1 \mathrm{~Hz}, 1 \mathrm{H}, \mathrm{H}-6$ ), $1.74-1.67$ (m, 1H, H-8), 0.99 (t, $\left.J=6.6 \mathrm{~Hz}, 6 \mathrm{H}, 4-\mathrm{CH}_{3}, \mathrm{H}-9\right), 0.88$ (d, $\left.J=7.1 \mathrm{~Hz}, 3 \mathrm{H}, 6-\mathrm{CH}_{3}\right), 0.85$ $(\mathrm{d}, J=6.7 \mathrm{~Hz}, 3 \mathrm{H}, \mathrm{H}-10) \mathrm{ppm} ;{ }^{13} \mathbf{C}$ NMR $\left(125 \mathrm{MHz}, \mathrm{CD}_{2} \mathrm{Cl}_{2}\right): \delta=167.3(\mathrm{C}-1), 152.6(\mathrm{C}-3), 121.9(\mathrm{C}-$ 2), 83.6 (C-7), 80.6 (C-5), $51.8\left(\mathrm{OCH}_{3}\right), 41.3$ (C-4), 35.5 (C-6), 32.1 (C-8), 19.7 (C-9/C-10), 19.3 (C9/C-10), $16.4\left(4-\mathrm{CH}_{3}\right), 4.7\left(6-\mathrm{CH}_{3}\right) \mathrm{ppm}$; HRMS (ESI+) $\mathrm{m} / \mathrm{z}$ : $[\mathrm{M}+\mathrm{H}]^{+}$Calcd for $\mathrm{C}_{13} \mathrm{H}_{25} \mathrm{O}_{4}{ }^{+} 245.1747$, found 245.1750 .

The diastereomeric enantiomer pairs were separated by chiral HPLC at this step. Only the main fraction was isolated due to the lack of baseline separated peaks. Therefore, the $d r$ was roughly determined by analysis by the ${ }^{1} \mathrm{H}$ NMR of compound $\mathbf{3 5}$. The configuration of the diol was ascertained with the acetonide. The separation was carried out on System A (analytical), a DAICEL Chiralpak IC$\mathrm{U}$ column $(<2 \mu \mathrm{m}, 3.0 \times 100 \mathrm{~mm})$ with an isocratic solvent mixture of $n$-hexane and isopropanol (80:20), $261 \mathrm{bar}, 0.85 \mathrm{~mL} / \mathrm{min}$ flow rate, $25^{\circ} \mathrm{C}$ and 6 min total runtime.

Methyl-(4S,5S,6R,7R)-5,7-dihydroxy-4,6,8-trimethylnonanoate $(92)^{[18-21]}$

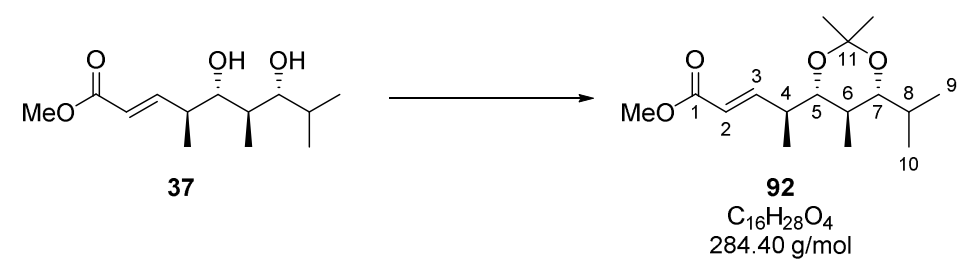

The unprotected diol 37 (10 mg, $41 \mu \mathrm{mol})$ was dissolved in 2,2-dimethoxypropane (0.5 mL). CSA (10 mg, $43 \mu \mathrm{mol})$ was dissolved in dry DCM $(1 \mathrm{~mL})$. The required quantity $(9.5 \mu \mathrm{L}, 95 \mu \mathrm{g}, 4.1 \mu \mathrm{mol}$, 
0.1 eq.) was taken from this standard solution and added to the dissolved diol 37. The mixture was stirred for $4 \mathrm{~h}$ at room temperature. The reaction was quenched by addition of $\mathrm{NEt}_{3}(10 \mu \mathrm{L})$, diluted with DCM $(2 \mathrm{~mL})$ and the solvents were evaporated. The crude product was purified by silica gel flash column chromatography (100\% diethyl ether) to yield acetonide $92(10.8 \mathrm{mg}, 37.9 \mu \mathrm{mol}, 93 \%)$ as a colorless solid.

$\mathbf{R}_{f}=0.74$ (diethyl ether); $[\alpha]_{\mathbf{D}}{ }^{20}=-38.8\left(\mathrm{c} 1.03, \mathrm{CH}_{2} \mathrm{Cl}_{2}\right) ;{ }^{1} \mathbf{H} \mathbf{N M R}\left(500 \mathrm{MHz}, \mathrm{CD}_{2} \mathrm{Cl}_{2}\right): \delta=6.97(\mathrm{dd}$, $J=15.8,7.2 \mathrm{~Hz}, 1 \mathrm{H}, \mathrm{H}-3), 5.82(\mathrm{dd}, J=15.8,1.3 \mathrm{~Hz}, 1 \mathrm{H}, \mathrm{H}-2), 3.69$ (s, 3H, $\left.\mathrm{OCH}_{3}\right), 3.57$ (dd, $J=9.8$, $2.2 \mathrm{~Hz}, 1 \mathrm{H}, \mathrm{H}-5), 3.29$ (dd, $J=9.6,2.1 \mathrm{~Hz}, 1 \mathrm{H}, \mathrm{H}-7), 2.47-2.39$ (m, 1H, H-4), $1.63-1.59$ (m, 2H, H-6, H-8), $1.35\left(\mathrm{~s}, 3 \mathrm{H}, 11-\mathrm{CH}_{3}\right) 1.32\left(\mathrm{~s}, 3 \mathrm{H}, 11-\mathrm{CH}_{3}\right), 0.96$ (d, $\left.J=7.0 \mathrm{~Hz}, 3 \mathrm{H}, 4-\mathrm{CH}_{3}\right), 0.93(\mathrm{~d}, J=$ $6.4 \mathrm{~Hz}, 3 \mathrm{H}, \mathrm{H}-9 / \mathrm{H}-10), 0.82$ (d, $\left.J=6.8 \mathrm{~Hz}, 3 \mathrm{H}, 6-\mathrm{CH}_{3}\right), 0.81$ (d, $\left.J=6.7 \mathrm{~Hz}, 3 \mathrm{H}, \mathrm{H}-9 / \mathrm{H}-10\right) \mathrm{ppm} ;{ }^{13} \mathbf{C}$ NMR (125 MHz, $\mathrm{CD}_{2} \mathrm{Cl}_{2}$ ): $\delta=167.6$ (C-1), 153.4 (C-3), 120.8 (C-2), $99.4\left(\mathrm{C}_{\mathrm{q}}-11\right), 79.8$ (C-7), 77.5 (C-5), $51.7\left(\mathrm{OCH}_{3}\right), 38.4$ (C-4), 31.2 (C-6), 30.2 (11-CH 3$), 29.9$ (C-8), 20.1 (C-9/C-10), 19.8 (11$\left.\mathrm{CH}_{3}\right), 17.7(\mathrm{C}-9 / \mathrm{C}-10), 14.8\left(4-\mathrm{CH}_{3}\right), 4.7\left(6-\mathrm{CH}_{3}\right) \mathrm{ppm}$; HRMS (APCI) $m / z:[\mathrm{M}+\mathrm{H}]^{+}$Calcd for $\mathrm{C}_{16} \mathrm{H}_{29} \mathrm{O}_{4}^{+}$285.2060, found 285.2053.

The configuration between C-5 and C-7 was verified by the ${ }^{13} \mathrm{C}$ NMR spectrum which shows chemical shifts in agreement with a syn configuration, according to the method of Rychnovsky. ${ }^{[18-21]}$

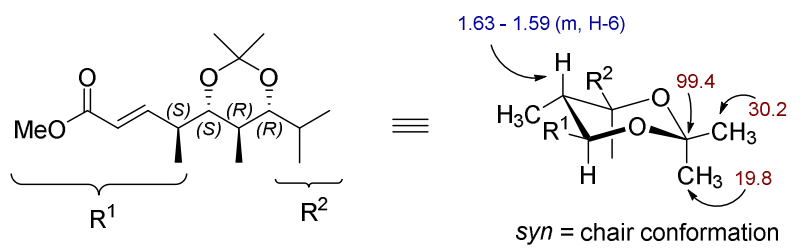

Figure 13. Relevant ${ }^{13} \mathrm{C}$ NMR signals (brown) and the ${ }^{1} \mathrm{H}$ NMR signal for $\mathrm{H}-6$ used for the determination of the 1,3-syn-diol acetonide and the relative configuration of $6-\mathrm{CH}_{3}$. 


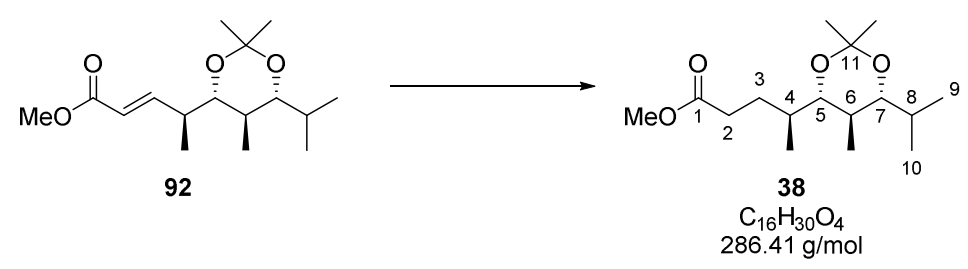

The unsaturated methyl ester 92 (40 mg, $0.14 \mathrm{mmol})$ was dissolved in THF $(0.4 \mathrm{~mL})$. Palladium on charcoal $(10 \% \mathrm{Pd}, 8 \mathrm{mg})$ was added and the solution was saturated with $\mathrm{H}_{2}$. The mixture was stirred for $45 \mathrm{~min}^{1}$ at room temperature. After completion of the reaction, the hydrogen gas was removed by purging with argon. The mixture was filtered through Celite ${ }^{\circledR}$ and the solvent was removed. The crude product was purified by silica gel flash column chromatography (9:1 $n$-pentane/diethyl ether) to yield title compound $\mathbf{3 8}$ (30 $\mathrm{mg}, 0.11 \mathrm{mmol}, 75 \%)$ as a colorless solid.

$\mathbf{R}_{f}=0.56$ (7:3 n-pentane/diethyl ether); $[\boldsymbol{\alpha}]_{\mathbf{D}}{ }^{20}=-40\left(\mathrm{c} 1.00, \mathrm{CH}_{2} \mathrm{Cl}_{2}\right) ;{ }^{1} \mathbf{H} \mathbf{N M R}\left(500 \mathrm{MHz}, \mathrm{CD}_{2} \mathrm{Cl}_{2}\right): \delta$ $=3.63\left(\mathrm{~s}, 3 \mathrm{H}, \mathrm{OCH}_{3}\right), 3.38(\mathrm{dd}, J=9.8,2.2 \mathrm{~Hz}, 1 \mathrm{H}, \mathrm{H}-5), 3.26(\mathrm{dd}, J=9.6,2.1 \mathrm{~Hz}, 1 \mathrm{H}, \mathrm{H}-7), 2.37-$ 2.25 (m, 2H, H-2), 1.99 - 1.92 (m, 1H, H-3a), 1.65 - 1.52 (m, 3H, H-4, H-6, H-8), 1.40 - 1.36 (m, 1H, $\mathrm{H}-3 \mathrm{~b}), 1.36$ (s, 3H, 11- $\mathrm{CH}_{3}$ ), 1.33 (s, 3H, 11- $\left.\mathrm{CH}_{3}\right), 0.92$ (d, $\left.J=6.5 \mathrm{~Hz}, 3 \mathrm{H}, \mathrm{C}-9 / \mathrm{C}-10\right), 0.80$ (d, $J=$ $6.7 \mathrm{~Hz}, 3 \mathrm{H}, \mathrm{C}-9 / \mathrm{C}-10), 0.79$ (d, $\left.J=6.7 \mathrm{~Hz}, 3 \mathrm{H}, 4-\mathrm{CH}_{3}\right), 0.77$ (d, $\left.J=6.8 \mathrm{~Hz}, 3 \mathrm{H}, 6-\mathrm{CH}_{3}\right) \mathrm{ppm} ;{ }^{13} \mathbf{C}$ NMR $\left(125 \mathrm{MHz}, \mathrm{CD}_{2} \mathrm{Cl}_{2}\right): \delta=174.9(\mathrm{C}-1), 99.2$ (C-11), 80.1 (C-7), $78.2(\mathrm{C}-5), 51.8\left(\mathrm{OCH}_{3}\right), 34.4$ (C-4), 32.1 (C-2), 31.2 (C-6), 30.3 (11- $\left.\mathrm{CH}_{3}\right), 29.9$ (C-8), 29.1 (C-3), 20.1 (C-9/C-10), 19.8 (11- $\left.\mathrm{CH}_{3}\right)$, $17.8(\mathrm{C}-9 / \mathrm{C}-10), 14.4\left(4-\mathrm{CH}_{3}\right), 4.8\left(6-\mathrm{CH}_{3}\right) \mathrm{ppm}$; HRMS (ESI+) $\mathrm{m} / z$ : $[\mathrm{M}+\mathrm{H}]^{+}$Calcd for $\mathrm{C}_{16} \mathrm{H}_{31} \mathrm{O}_{4}{ }^{+}$ 287.2217, found 287.2220.

(S)-4-((4S,5R,6R)-6-Isopropyl-2,2,5-trimethyl-1,3-dioxan-4-yl)pentan-1-ol (5)

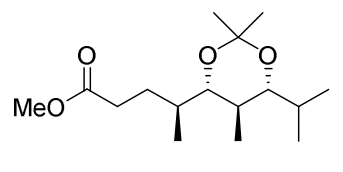

38

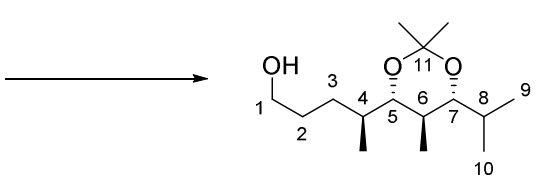

5

$\mathrm{C}_{15} \mathrm{H}_{30} \mathrm{O}_{3}$
$258.40 \mathrm{~g} / \mathrm{mol}$

$\mathrm{LiAlH}_{4}\left(20 \mu \mathrm{L}, 0.02 \mathrm{mmol}, 1.0\right.$ eq. $1 \mathrm{M}$ in $\left.\mathrm{Et}_{2} \mathrm{O}\right)$ was added to dry THF $(0.1 \mathrm{~mL})$ at $0{ }^{\circ} \mathrm{C}$. Methyl ester $38(6 \mathrm{mg}, 0.02 \mathrm{mmol})$ was added dropwise as a solution in THF $(0.2 \mathrm{~mL})$. The mixture was warmed to room temperature and stirred for $15 \mathrm{~min}$. The reaction was quenched by slow addition of

\footnotetext{
${ }^{1}$ The $\mathrm{R}_{\mathrm{f}}$ values of starting material and product are equal which makes observation via TLC challenging. The product spot on TLC will stain later than the starting material with potassium permanganate.
} 
$\mathrm{H}_{2} \mathrm{O}(0.3 \mathrm{~mL})$ and stirred for another hour. The mixture was extracted with diethyl ether $(3 \mathrm{x} 1 \mathrm{~mL})$, the combined organic phases were dried over $\mathrm{Na}_{2} \mathrm{SO}_{4}$ and the solvent was removed. The crude product was purified by silica gel flash column chromatography ( $n$-pentane/diethyl ether 7:3 $\rightarrow$ diethyl ether) to yield primary alcohol 5 (5 mg, $19 \mu \mathrm{mol}, 97 \%)$ as a colorless oil.

$\mathbf{R}_{f}=0.12\left(7: 3 n\right.$-pentane/diethyl ether); $[\alpha]_{\mathbf{D}}{ }^{20}=-45.5\left(\mathrm{c} 0.22, \mathrm{CH}_{2} \mathrm{Cl}_{2}\right) ;{ }^{1} \mathbf{H} \mathbf{N M R}\left(500 \mathrm{MHz}, \mathrm{CD}_{2} \mathrm{Cl}_{2}\right)$ : $\delta=3.58(\mathrm{t}, J=6.4 \mathrm{~Hz}, 2 \mathrm{H}, \mathrm{H}-1), 3.38(\mathrm{dd}, J=9.8,2.1 \mathrm{~Hz}, 1 \mathrm{H}, \mathrm{H}-5), 3.26(\mathrm{dd}, J=9.6,2.2 \mathrm{~Hz}, 1 \mathrm{H}$, H-7), 1.73 - 1.68 (m, 1H, H-3a), 1.66 - 1.60 (m, 2H, H-2a, H-8), 1.62 - 1.57 (m, 1H, H-6), 1.57 $1.52(\mathrm{~m}, 1 \mathrm{H}, \mathrm{H}-4), 1.48-1.44$ (m, 1H, H-2b), 1.36 (s, 3H, 11- $\left.\mathrm{CH}_{3}\right), 1.33$ (s, 3H, 11- $\left.\mathrm{CH}_{3}\right), 1.10-1.03$ (m, 1H, H-3b), 0.92 (d, $J=6.4 \mathrm{~Hz}, 3 \mathrm{H}, \mathrm{H}-9 / \mathrm{H}-10), 0.80$ (d, $J=6.7 \mathrm{~Hz}, 3 \mathrm{H}, \mathrm{H}-9 / \mathrm{H}-10), 0.80$ (d, $J=$ $\left.6.8 \mathrm{~Hz}, 3 \mathrm{H}, 4-\mathrm{CH}_{3}\right), 0.78\left(\mathrm{~d}, J=6.8 \mathrm{~Hz}, 3 \mathrm{H}, 6-\mathrm{CH}_{3}\right) \mathrm{ppm} ;{ }^{13} \mathbf{C} \mathbf{N M R}\left(125 \mathrm{MHz}, \mathrm{CD}_{2} \mathrm{Cl}_{2}\right): \delta=99.2(\mathrm{C}-$ 11), 80.1 (C-7), 78.4 (C-5), 63.6 (C-1), 34.4 (C-4), 31.2 (C-6), 30.4 (11- $\left.\mathrm{CH}_{3}\right), 30.3$ (C-2), 29.9 (C-8), 29.7 (C-3), 20.1 (C-9/C-10), $19.8\left(11-\mathrm{CH}_{3}\right), 17.8(\mathrm{C}-9 / \mathrm{C}-10), 14.7\left(4-\mathrm{CH}_{3}\right), 4.7\left(6-\mathrm{CH}_{3}\right)$ ppm; HRMS (ESI+) $m / z$ : $[\mathrm{M}+\mathrm{H}]^{+}$Calcd for $\mathrm{C}_{15} \mathrm{H}_{31} \mathrm{O}_{3}{ }^{+} 259.2268$, found 259.2267.

$(4 S, 5 S, 6 R, 7 R)-4,6,8-$ Trimethylnonane-1,5,7-triol (41)

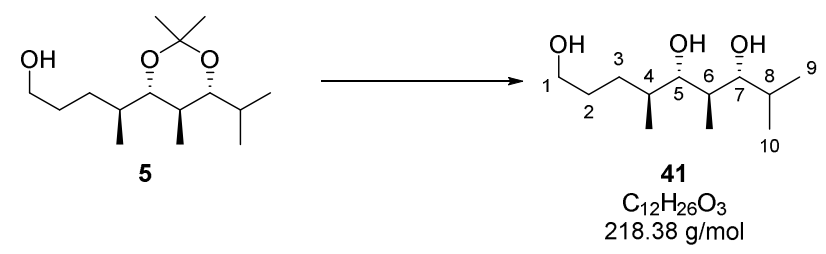

Crude primary alcohol 5 (5 mg, max. $0.02 \mathrm{mmol})$ was dissolved in $\mathrm{MeOH}(0.3 \mathrm{~mL})$. $\mathrm{HCl}(40 \mu \mathrm{L}$, $0.04 \mathrm{mmol}, 2.0$ eq. $1 \mathrm{M}$ in $\mathrm{H}_{2} \mathrm{O}$ ) was added at room temperature and the solution was stirred until TLC indicated full conversion ( $3.5 \mathrm{~h}$ ). After diluting with $\mathrm{H}_{2} \mathrm{O}$ and DCM (each $0.5 \mathrm{~mL}$ ), the phases were separated and the aqueous phase was extracted with DCM $(3 \times 0.5 \mathrm{~mL})$. The combined organic phases were dried over $\mathrm{Na}_{2} \mathrm{SO}_{4}$ and the solvent was evaporated. The crude product was purified by silica gel flash column chromatography (cyclohexane/ethyl acetate $1: 1 \rightarrow$ ethyl acetate) to obtain triol 41 (1.2 $\mathrm{mg}, 5.5 \mu \mathrm{mol}, 29 \%)$ as a colorless oil.

$\mathbf{R}_{f}=0.01$ (diethyl ether); $[\alpha]_{\mathbf{D}}{ }^{20}=-166.7\left(\mathrm{c} 0.24, \mathrm{CH}_{2} \mathrm{Cl}_{2}\right) ;{ }^{1} \mathbf{H}$ NMR (400 MHz, MeOD): $\delta=3.55$ (td, $J=6.6,2.1 \mathrm{~Hz}, 2 \mathrm{H}, \mathrm{H}-1), 3.36$ (dd, $J=8.1,3.4 \mathrm{~Hz}, 1 \mathrm{H}, \mathrm{H}-5), 3.28$ (dd, $J=7.7,4.0 \mathrm{~Hz}, 1 \mathrm{H}, \mathrm{H}-7), 1.87$ - 1.80 (m, 1H, H-6), 1.82 - 1.74 (m, 1H, H-8), $1.74-1.68$ (m, 1H, H-3a), 1.71 - 1.65 (m, 1H, H-2a), $1.65-1.59$ (m, 1H, H-4), $1.49-1.44$ (m, 1H, H-2b), 1.16 - 1.10 (m, 1H, H-3b), 0.95 (d, J=6.6 Hz, $3 \mathrm{H}, \mathrm{H}-9 / \mathrm{H}-10), 0.91$ (d, $\left.J=6.9 \mathrm{~Hz}, 3 \mathrm{H}, 6-\mathrm{CH}_{3}\right), 0.88$ (d, $\left.J=6.8 \mathrm{~Hz}, 3 \mathrm{H}, \mathrm{H}-9 / \mathrm{H}-10\right), 0.87$ (d, $J=$ $\left.6.6 \mathrm{~Hz}, 3 \mathrm{H}, 4-\mathrm{CH}_{3}\right)$ ppm; ${ }^{13} \mathrm{C}$ NMR $(100 \mathrm{MHz}, \mathrm{MeOD}): \delta=82.0$ (C-7), 80.6 (C-5), 63.5 (C-1), 37.2 
(C-6), 36.9 (C-4), 31.9 (C-8), 31.0 (C-2), 29.4 (C-3), 19.8 (C-9/C-10), 18.8 (6- $\left.\mathrm{CH}_{3}\right), 16.3\left(4-\mathrm{CH}_{3}\right), 6.7$ (C-9/C-10) ppm; HRMS (ESI+) $\mathrm{m} / z$ : $[\mathrm{M}+\mathrm{H}]^{+}$Calcd for $\mathrm{C}_{12} \mathrm{H}_{27} \mathrm{O}_{3}{ }^{+} 219.1955$, found 219.1955 . 


\section{Bioinformatic analysis}

\section{Material \& Methods}

Stereochemistry in the polyketide backbone was predicted from KR- (ketoreductase-) domain and ER(enoyl reductase-) domain sequences in the respective module. For KR-domains antiSMASH ${ }^{[37]}$ prediction of the $6 \mathrm{KR}$-domain types (A1, A2, B1, B2, C1, C2) was taken into account and was verified by identifying the catalytic regions the alignment of $\mathrm{KR}$ domain sequences according to Keatinge-Clay ${ }^{[38]}$. Thereby, vancoresmycin KR-domains (var4 - var12) were aligned with erythromycin KR-domains (SACE_0721 - SACE_0724) and pimaricin KR-domains (pimS1 - pimS4). Alignment was performed using MAFFT 7.222 implemented in geneious 9.1.6 using the default settings. Additionally, D- or L-configuration of alcohol and methyl groups was predicted using the webtool for a pHMM based approach (https://akitsche.shinyapps.io/profileHMM_App/) by Kitsche \& Kalesse. ${ }^{[39]}$

Stereocenters introduced by ER-domains were predicted based on the model proposed by Kwan and colleagues. ${ }^{[40]}$ Vancoresmycin ER-domain sequences were aligned with ER-domains from erythromycin (Ery_SACE_0721), megalomycin (megAIII), tylosin (tylG), rapamycin (rapA-C) and geldanamycin (gdmAI-III). Alignment was performed using MAFFT 7.222 implemented in geneious 9.1.6 using the default settings.

\section{Results}

In order to predict stereochemical properties of the vancoresmycin molecule produced by Amycolatopsis sp. DEM30355, predictions from antiSMASH as well as the catalytic regions proposed by Keatinge-Clay were taken into account. In the alignment of vancoresmycin KR-domains with KRdomains of known stereospecificity catalytic regions 1-6 according to Keatinge-Clay corresponded to amino acid positions 96-98, 149, 154, 157, 159 and 161. (Figure 14: KR alignment). Stereocenters introduced by ER-domains were predicted based on the presence of tyrosine $(\mathrm{Y})$ in the catalytic region. Tyrosine at position 44 in our alignment correlates with (2S)-configuration in the growing polyketide chain. (Figure 15: ER alignment) In the vancoresmycin molecule, the only stereocenter introduced by an ER is at var11/module_2 (C32).

Predictions for the incorporated polyketide extender units and the respective reduction state and stereocenters are summed up in Table 5 (Table 5: Vancoresmycin modules and predictions). Thereby, predictions based on the model proposed by Keatinge-Clay ${ }^{[38]}$ were in accordance with the pHMM 
based predictions. The proposed structure for the polyketide backbone is shown in Figure 16 (Figure 16.1, 16.2, 16.3: Vancoresmycin polyketide backbone prediction). 


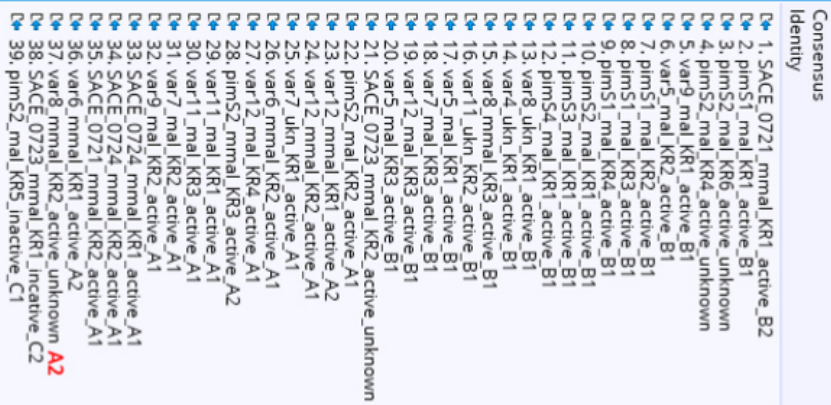

[व口ग ดี $\gg \gg$ ดดดดดดด〈ดดดดดดดดดดดดดดดดดดดดดดดดดด जनिनरयगIन

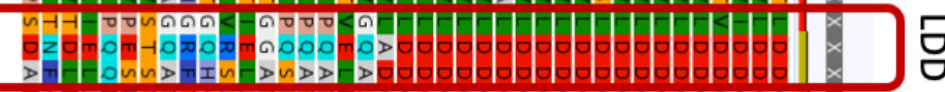
प्रय

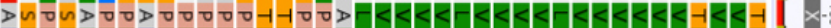

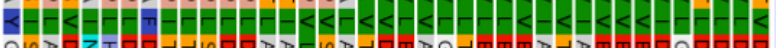
4 - $>$ -

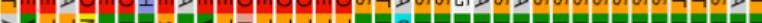

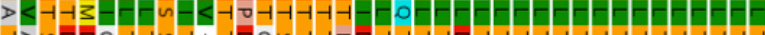

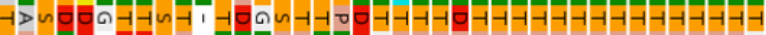
-

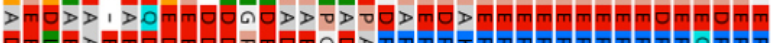

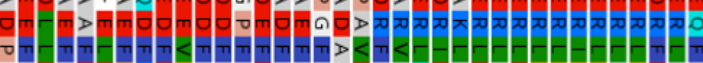
0

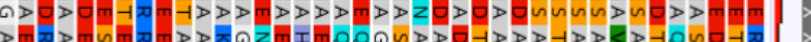

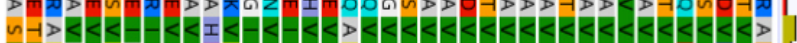

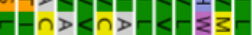

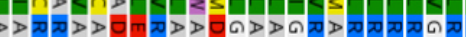

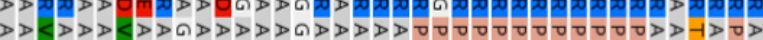

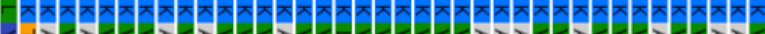

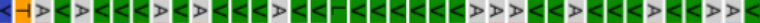

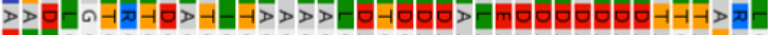

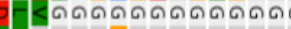

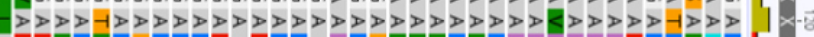
>

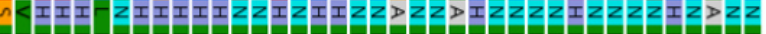

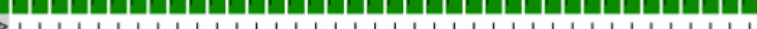

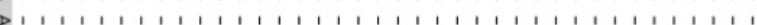

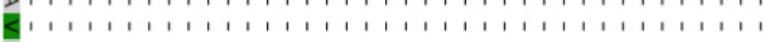

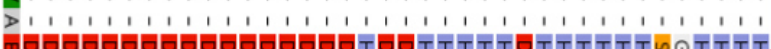

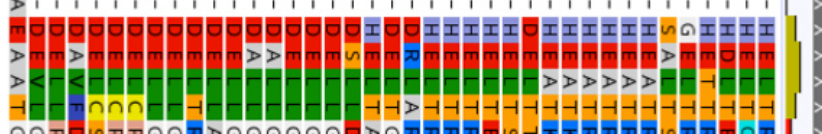

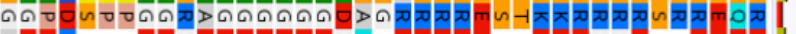

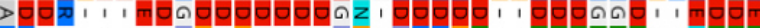
可, $1,1,1,1,1,-1,1,1,1$

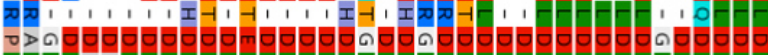

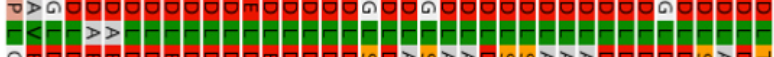

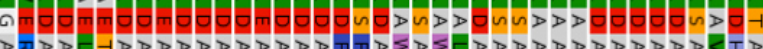

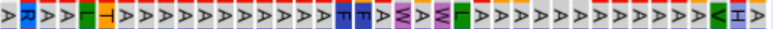
同

$<$

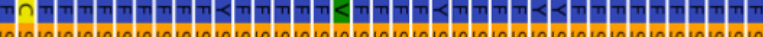

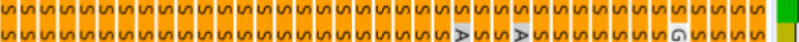

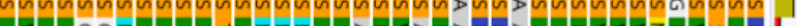

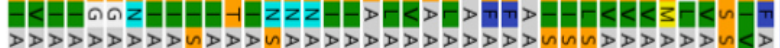

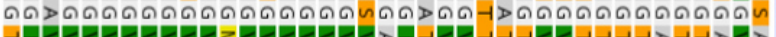

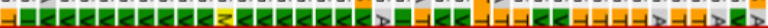

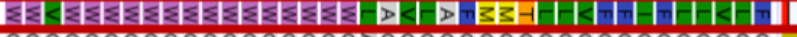

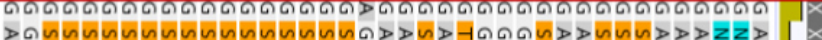

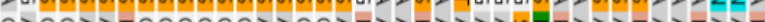

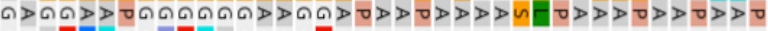

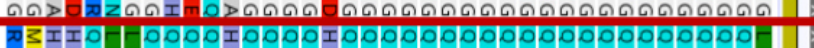
nompans $|-|<|<|<|<|<|<|<|<|<|<|<|<|<|<|<|<|<|<|<|<|<|<|<|<|<|<|<|<|<|<|<|<|<|<|<|<|<|<| \mid x$ । nnzzzzzzzzzzzzzzzzzzzzzzzzzzzzzzzzzzzzzzzzzzzzzz|| |x

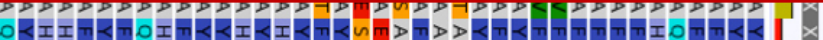

Figure 14. KR alignment. 


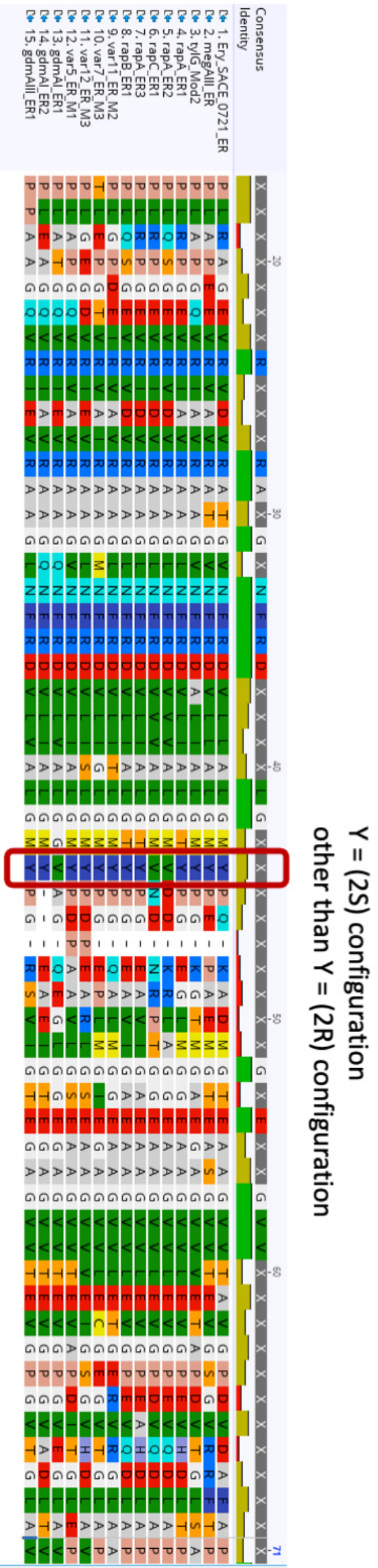

Figure 15. ER alignment. 
Table 5. Vancoresmycin overview modules and predictions: blue $=$ ScoreDiff value unreliable below +15 (D) above -15 (L); grey = KR inactive or ER final "decision" for stereochemistry.

\begin{tabular}{|c|c|c|c|c|c|c|c|c|c|}
\hline gene/module & AT & KR & KR-type & $\mathrm{DH}$ & ER & $\begin{array}{l}\text { ER- } \\
\text { type }\end{array}$ & prediction & $\begin{array}{c}\text { Kitsche \& } \\
\text { Kalesse } \\
(2013) \\
\text { HMM } \\
\text { prediction }\end{array}$ & $\begin{array}{c}\text { ScoreDiff } \\
\text { value } \\
\text { (reliable } \\
\pm 15 \text { ) }\end{array}$ \\
\hline var13/loading & $\begin{array}{c}\text { Isobutyryl- } \\
\text { CoA }\end{array}$ & $\mathrm{x}$ & - & $\mathrm{x}$ & $\mathrm{x}$ & - & & - & - \\
\hline var12/m1 & $\begin{array}{c}\text { Meth-Mal- } \\
\text { CoA }\end{array}$ & $\checkmark$ & $\mathrm{A} 2$ & $\mathrm{x}$ & $\mathrm{x}$ & - & & $\begin{array}{l}\mathrm{OH} \rightarrow \mathrm{L} \\
\mathrm{CH}_{3} \rightarrow \mathrm{D}\end{array}$ & $\begin{array}{c}-85.77 \\
4.83\end{array}$ \\
\hline var12/m2 & $\begin{array}{c}\text { Meth-Mal- } \\
\text { CoA }\end{array}$ & $\checkmark$ & A1 & $\mathrm{X}$ & $\mathrm{x}$ & - & & $\begin{array}{l}\mathrm{OH} \rightarrow \mathrm{L} \\
\mathrm{CH}_{3} \rightarrow \mathrm{D}\end{array}$ & $\begin{array}{l}-74.65 \\
42.65\end{array}$ \\
\hline var $12 / \mathrm{m} 3$ & Mal-CoA & $\checkmark$ & B1 & $\checkmark$ & $\checkmark$ & $\begin{array}{l}\text { Pos } \\
44 \\
Y=S\end{array}$ & & - & - \\
\hline var12/m4 & Mal-CoA & $\checkmark$ & A1 & $\mathrm{x}$ & $\mathrm{x}$ & - & & $\mathrm{OH} \rightarrow \mathrm{L}$ & -73.1 \\
\hline var11/m1 & Mal-CoA & $\checkmark$ & A1 & $\mathrm{x}$ & $\mathrm{X}$ & - & & $\mathrm{OH} \rightarrow \mathrm{L}$ & -69.04 \\
\hline var11/m2 & $\begin{array}{l}\text { Meth-Mal- } \\
\text { COA }\end{array}$ & $\checkmark$ & B1 & $\checkmark$ & $\checkmark$ & $\begin{array}{c}\text { Pos } \\
44 \\
Y=S\end{array}$ & & $\begin{array}{l}\mathrm{OH} \rightarrow \mathrm{D} \\
\mathrm{CH}_{3} \rightarrow \mathrm{D}\end{array}$ & $\begin{array}{l}37.17 \\
27.37\end{array}$ \\
\hline var11/m3 & Mal-COA & $\checkmark$ & A1 & $\mathrm{x}$ & $\mathrm{x}$ & - & & $\mathrm{OH} \rightarrow \mathrm{L}$ & -61.73 \\
\hline $\operatorname{var} 10 / \mathrm{m} 1$ & Mal-CoA & $\mathrm{x}$ & - & $\mathrm{X}$ & $\mathrm{X}$ & - & & - & - \\
\hline var9/m1 & $\mathrm{x}$ & $\checkmark$ & B1 & $\mathrm{x}$ & $x$ & - & & $\mathrm{OH} \rightarrow \mathrm{D}$ & 27.31 \\
\hline var9/m2 & Mal-COA & $\checkmark$ & A1 & $\mathrm{x}$ & $\mathrm{x}$ & - & & $\mathrm{OH} \rightarrow \mathrm{L}$ & -62.25 \\
\hline var8/m1 & $\begin{array}{l}\text { Meth-Mal- } \\
\text { CoA }\end{array}$ & $\checkmark$ & B1 & $\checkmark$ & $\mathrm{x}$ & - & 0 & - & - \\
\hline
\end{tabular}




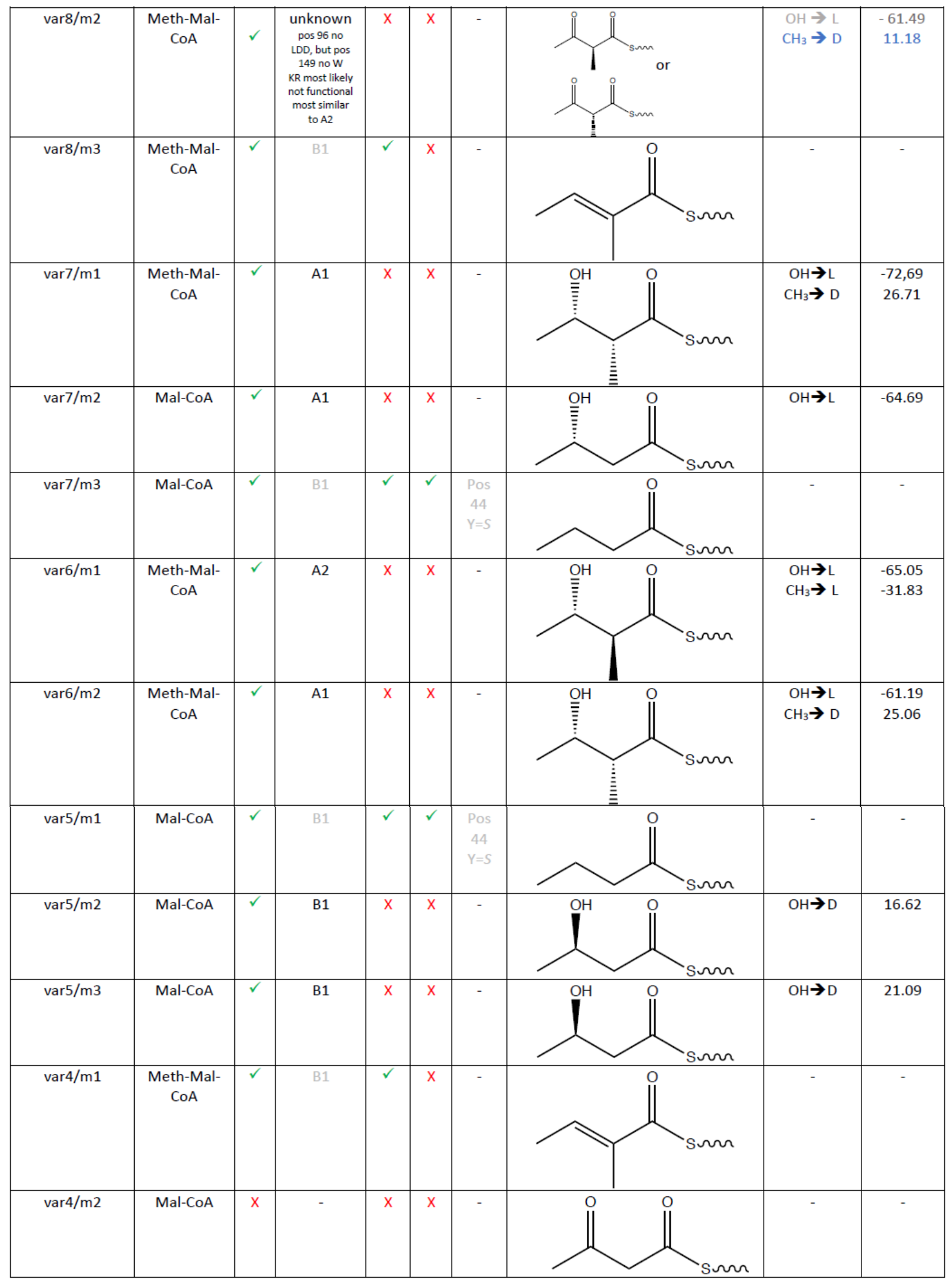




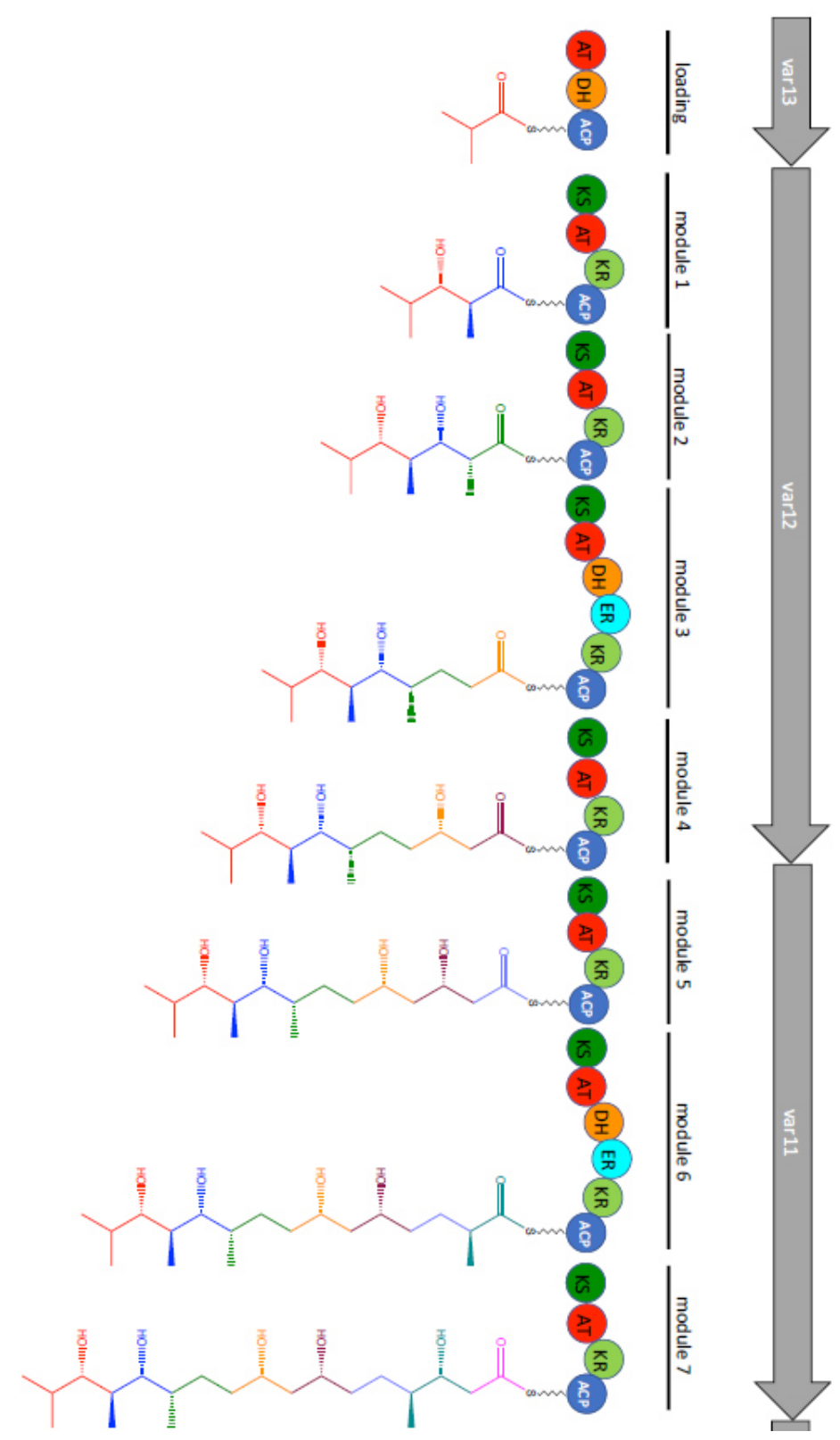

Figure 16.1. Vancoresmycin polyketide backbone prediction (var13-var11). 


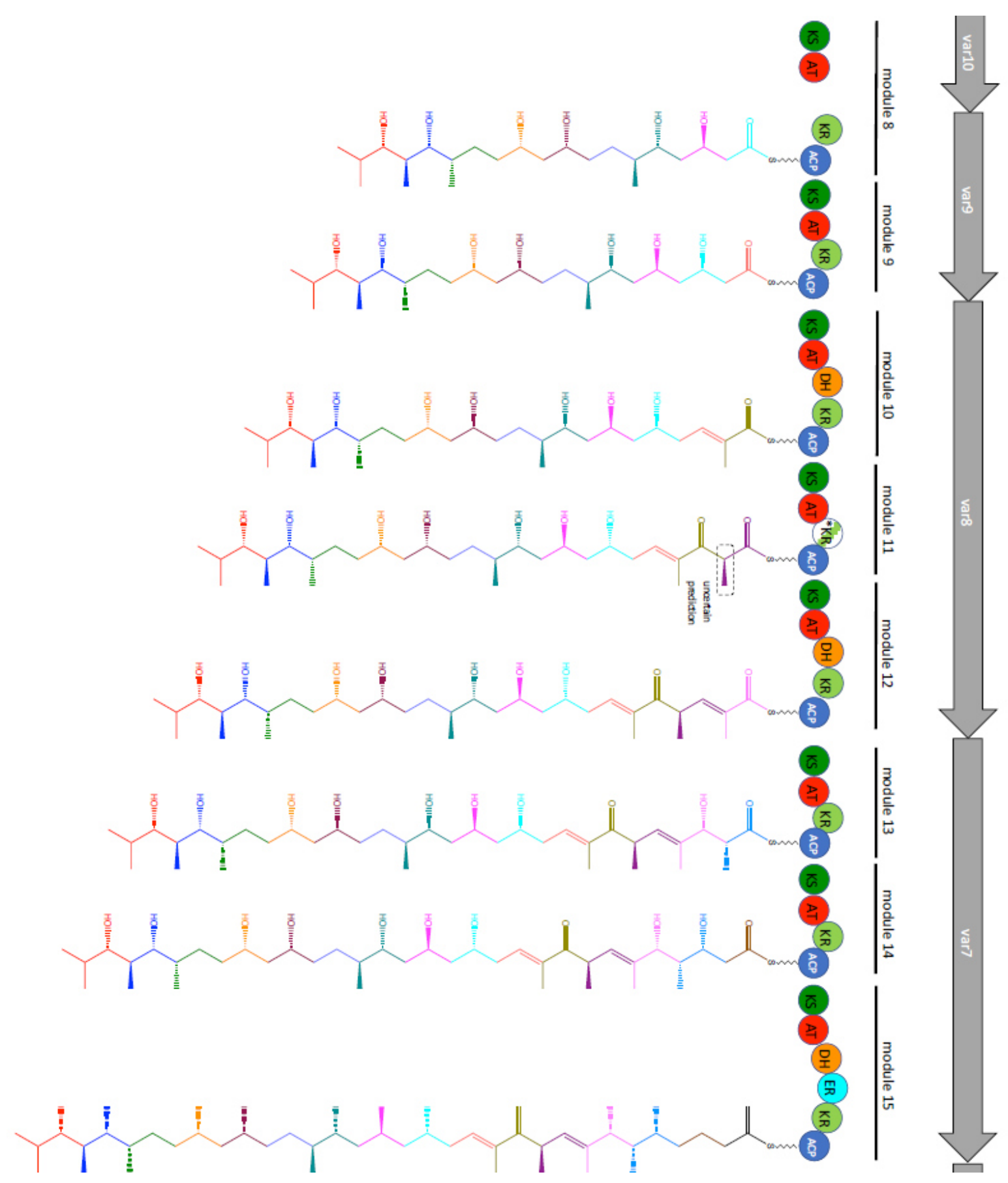

Figure 16.2. Vancoresmycin polyketide backbone prediction (var10-var7). 


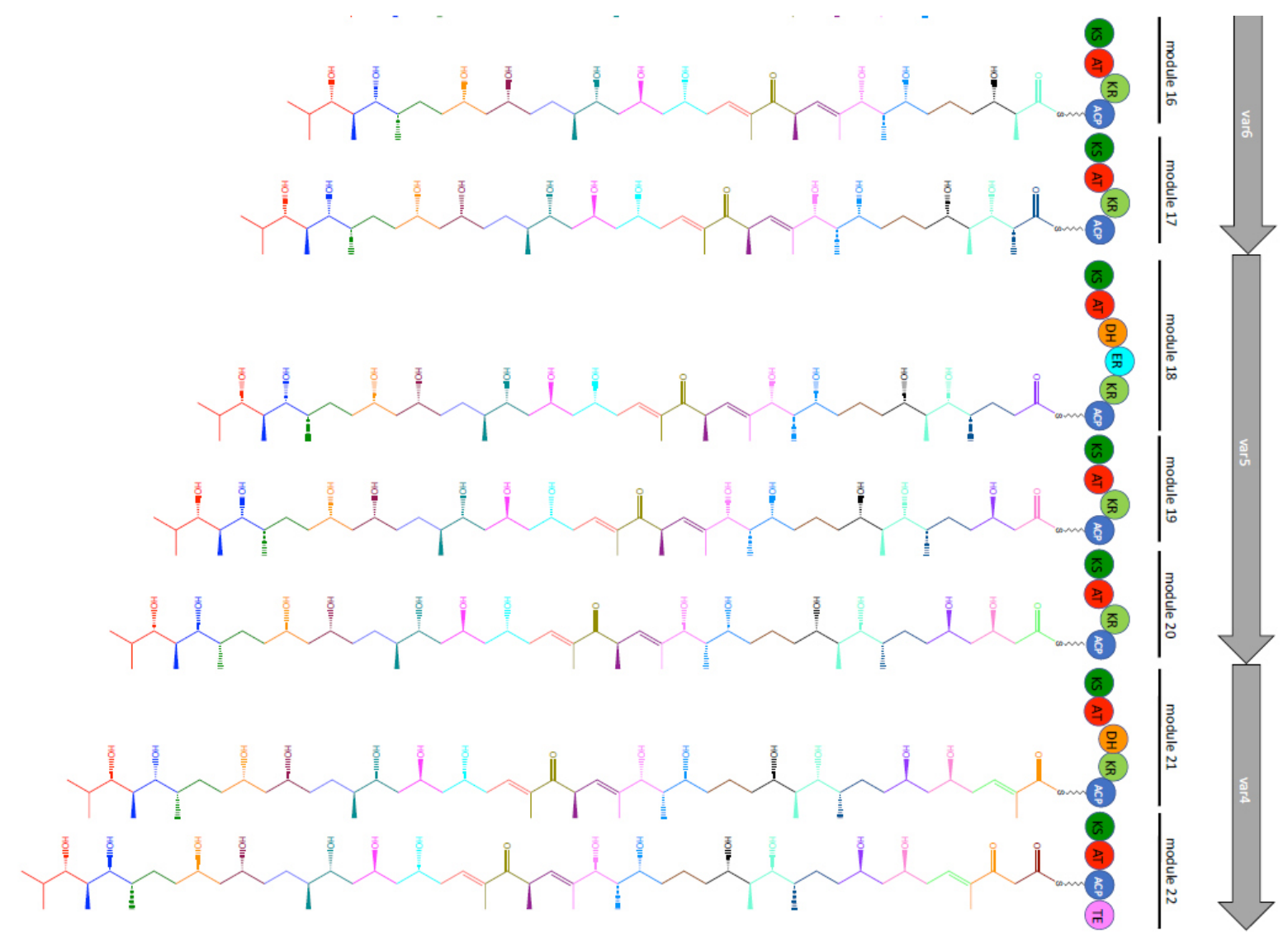

Figure 16.3. Vancoresmycin polyketide backbone prediction (var6-var4). 


\section{References}

[1] Fulmer, G. R.; Miller, A. J.; Sherden, N. H.; Gottlieb, H. E.; Nudelman, A.; Stoltz, B. M.; Bercaw, J. E.; Goldberg, K. I. NMR Chemical Shifts of Trace Impurities. Organometallics 2010, 29, 2176-2179.

[2] Baenziger, M.; McGarrity, J. F. A facile Synthesis of $N$-protected Statine and Analogs via a Lipase-catalyzed Kinetic Resolution. Meul, T. J. Org. Chem, 1993, 58, 4010-4012.

[3] Fernandes, R. A.; Chavan, V. P. A Dötz Benzannulation Route to the Enantioselective Synthesis of (-)- and (+)-Juglomycin A. Tetrahedron Asymmetry 2011, 22, 1312-1319.

[4] Freeman, J. L.; Brimble, M. A.; Furkert, D. P. A chiral Auxiliary-based Synthesis of the C5C17 trans-decalin Framework of Anthracimycin. Org. Chem. Front. 2019, 6, 2954-2963.

[5] Lin, L.; Romano, C.; Mazet, C. Palladium-Catalyzed Long-Range Deconjugative Isomerization of Highly Substituted $\alpha, \beta$-Unsaturated Carbonyl Compounds. J. Am. Chem. Soc. 2016, 138, 10344-10350.

[6] Ghosh, S. K.; Wei, Y.; Gerasyuto, A. I.; Feltenberger, J. B.; Wang, J.; Hsung, R. P. A Cyclic Acetal Tethered Intramolecular Diels-Alder Cycloaddition. Studies Directed toward a Total Synthesis of ( \pm )-Fusidilactone C. Heterocycles 2011, 82, 1379-1409.

[7] Freund, R. R. A.; Gobrecht, P.; Moser, P.; Fischer, D.; Arndt, H. D. Synthesis and Biological Profiling of Parthenolide ether analogs. Org. Biomol. Chem. 2019, 17, 9703-9707.

[8] Muszak, D.; Łabuzek, B.; Brela, M. Z.; Twarda-Clapa, A.; Czub, M.; Musielak, B.; Surmiak, E.; Holak, T. A. The Synthesis and Characterization of Tetramic acid derivatives as Mdm2p53 inhibitors. J. Mol. Struct. 2019, 1189, 161-174.

[9] Ahrendt, K. A.; Borths, C. J.; MacMillan, D. W. C. New Strategies for Organic Catalysis: The First Highly Enantioselective Organocatalytic Diels-Alder Reaction. J. Am. Chem. Soc. 2000, $122,4243-4244$.

[10] Holland, M. C.; Metternich, J. B.; Daniliuc, C.; Schweizer, W. B.; Gilmour, R. Aromatic Interactions in Organocatalyst Design: Augmenting Selectivity Reversal in Iminium Ion Activation. Chem. Eur. J. 2015, 21, 10031-10038.

[11] Straniero, V.; Zanotto, C.; Straniero, L.; Casiraghi, A.; Duga, S.; Radaelli, A.; de Giuli Morghen, C.; Valoti, E. 2,6-Difluorobenzamide Inhibitors of Bacterial Cell Division Protein FtsZ. ChemMedChem 2017, 12, 1303-1318.

[12] Tian, J.; Yi, C.; Fang, H.; Sang, D.; He, Z.; Wang, J.; Gan, Y.; An, Q. Ethyl Acetate as a Cosolvent and Sacrificial Ester in the Aluminum Triiodide Promoted Chemoselective Demethylation of Methyl Vanillate. Tetrahedron Lett. 2017, 58, 3522-3524.

[13] ElMarrouni, A.; Joolakanti, S. R.; Colon, A.; Heras, M.; Arseniyadis, S.; Cossy, J. Two Concise Total Syntheses of (-)-Bitungolide F. Org. Lett. 2010, 12, 4074-4077.

[14] Peelen, T. J.; Chi, Y.; Gellman, S. H. Enantioselective Organocatalytic Michael Additions of Aldehydes to Enones with Imidazolidinones. J. Am. Chem. Soc. 2005, 127, 11598-11599. 
[15] Zhu, G.; Negishi, E.-i. Fully Reagent-Controlled Asymmetric Synthesis of (-)-Spongidepsin via the Zr-Catalyzed Asymmetric Carboalumination of Alkenes (ZACA Reaction). Org. Lett. 2007, 9, 2771-2774.

[16] White, J. D.; Hanselmann, R.; Jackson, R. W.; Porter, W. J.; Ohba, Y.; Tiller, T.; Wang, S. Total Synthesis of Rutamycin B, a Macrolide Antibiotic from Streptomyces aureofaciens. J. Org. Chem. 2001, 66, 5217-5231.

[17] Hoye, T. R.; Jeffrey, C. S.; Shao, F. Mosher Ester Analysis for the Determination of Absolute Configuration of Stereogenic (chiral) Carbinol Carbons. Nat. Protoc. 2007, 2, 2451-2458.

[18] Dias, L. C., de Lucca, E. C. Total Synthesis of (-)-Marinisporolide C. J. Org. Chem. 2017, 82, 3019-3045.

[19] Rychnovsky, S. D.; Rogers, B. N., Yang, G. Analysis of two Carbon-13 NMR Correlations for Determining the Stereochemistry of 1,3-Diol Acetonides. J. Org. Chem. 1993, 58, 3511-3515.

[20] Rychnovsky, S. D.; Rogers, B. N., Richardson, T. I. Configurational Assignment of Polyene Macrolide Antibiotics Using the [13C]Acetonide Analysis. Acc. Chem. Res. 1998, 31, 9-17.

[21] Rychnovsky, S. D.; Skalitzky, D. J. Stereochemistry of Alternating Polyol Chains: 13C NMR Analysis of 1,3-Diol Acetonides. Tetrahedron Lett. 1990, 31, 945-948.

[22] Abe, M.; Sawada, Y.; Uno, S.; Chigasaki, S.; Oku, M.; Sakai, Y.; Miyoshi, H. Role of Acyl Chain Composition of Phosphatidylcholine in Tafazzin-Mediated Remodeling of Cardiolipin in Liposomes. Biochemistry 2017, 56, 6268-6280.

[23] Tanaka-Yanuma, A.; Watanabe, S.; Ogawa, K.; Watanabe, S.; Aoki, N.; Ogura, T.; Usuki, T. Synthesis of the Polyketide moiety of the Jamaicamides. Tetrahedron Lett. 2015, 56, 67776781.

[24] Millán, A.; Grigol Martinez, P. D.; Aggarwal, V. K. Stereocontrolled Synthesis of Polypropionate Fragments based on a Building Block Assembly Strategy using Lithiation Borylation Methodologies. Chem. - A Eur. J. 2018, 24, 730-735.

[25] Kubota, K.; Yamamoto, E.; Ito, H. Copper(I)-Catalyzed Enantioselective Nucleophilic Borylation of Aldehydes: An Efficient Route to Enantiomerically Enriched $\alpha$ Alkoxyorganoboronate Esters. J. Am. Chem. Soc. 2015, 137, 420-424.

[26] Matsui, K.; Zheng, B.; Kusaka, S.; Kuroda, M.; Yoshimoto, K.; Yamada, H.; Yonemitsu, O. Stereoselective Synthesis of the C(1)-C(12) Fragments of Tedanolides - Application of a synSelective Tin(II)-Mediated Aldol Reaction and a Convertible Methoxybenzyl Protecting Group. Eur. J. Org. Chem. 2001, 2001, 3615-3624.

[27] Paterson, I.; Goodman, J. M.; Lister, M. A.; Schumann, R. C.; McClure, C. K.; Norcross, R. D. Enantio- and Diastereoselective Aldol Reactions of Achiral Ethyl and Methyl Ketones with Aldehydes: the Use of Enol Diisopinocampheylborinates. Tetrahedron 1990, 46, 4663-4684.

[28] Brown, H. C.; Singaram B. Improved Procedures for the Synthesis of Diisopinocampheylborane of High Optical Purity. J. Org. Chem. 1984, 49, 945-947. 
[29] Chen, K.-M.; Hardtmann, G. E.; Prasad, K.; Repič, O.; Shapiro, M. J. 1,3- Diastereoselective Reduction of $\beta$-Hydroxyketones Utilizing Alkoxydialkylboranes. Tetrahedron Lett. 1987, 28 , $155-158$.

[30] Wang, B.; Hansen, T. M.; Wang, T.; Wu, D.; Weyer, L.; Ying, L.; Engler, M. M.; Sanville, M.; Leitheiser, C.; Christmann, M.; Lu, Y.; Chen, J.; Zunker, N.; Cink, R. D.; Ahmed, F.; Lee, C.-S.; Forsyth, C. J. Total Synthesis of Phorboxazole A via de Novo Oxazole Formation: Strategy and Component Assembly. J. Am. Chem. Soc. 2011, 133, 1484-1505.

[31] Brown, H. C.; Bhat, K. S. Chiral Synthesis via Organoboranes. 7. Diastereoselective and enantioselective synthesis of erythro- and threo-, beta-methylhomoallyl alcohols via enantiomeric (Z)- and (E)-crotylboranes. J. Am. Chem. Soc. 1986, 108, 5919-5923.

[32] Brown, H. C.; Bhat, K. S. Enantiomeric Z- and E-crotyldiisopinocampheylboranes. Synthesis in high optical purity of all four possible stereoisomers of beta-methylhomoallyl alcohols. $J$. Am. Chem. Soc. 1986, 108, 293-294.

[33] Willand-Charnley, R.; Fisher, T. J.; Johnson, B. M.; Dussault, P. H. Pyridine is an organocatalyst for the reductive ozonolysis of alkenes. Org. Lett. 2012, 14, 2242-2245.

[34] Han, S. B.; Han, H.; Krische, M. J. Diastereo- and Enantioselective anti-Alkoxyallylation Employing Allylic gem-Dicarboxylates as Allyl Donors via Iridium-Catalyzed Transfer Hydrogenation. J. Am. Chem. Soc. 2010, 132, 1760-1761.

[35] Shin, I.; Krische, M. J. Formal Synthesis of Premisakinolide A and C(19)-C(32) of Swinholide A via Site-Selective C-H Allylation and Crotylation of Unprotected Diols. Org. Lett. 2015, 17, 4686-4689.

[36] Fu, F.; Loh, T.-P. An asymmetric synthesis of the polyol fragment of the polyene macrolide antibiotic RK-397. Tetrahedron Lett. 2009, 50, 3530-3533.

[37] Blin, K.; Shaw, S.; Steinke, K.; Villebro, R.; Ziemert, N.; Lee, S. Y.; Medema, M. H.; Weber, T. antiSMASH 5.0: updates to the secondary metabolite genome mining pipeline. Nucleic Acids Res. 2019, 47, W81-W87.

[38] Keatinge-Clay, A. T. A tylosin ketoreductase reveals how chirality is determined in polyketides. Chem. Biol. 2007, 14, 898-908.

[39] Kitsche, A.; Kalesse, M. Configurational Assignment of Secondary Hydroxyl Groups and Methyl Branches in Polyketide Natural Products through Bioinformatic Analysis of the Ketoreductase Domain. ChemBioChem 2013, 14, 851-861.

[40] Kwan, D. H. Sun, Y.; Schulz, F.; Hong, H.; Popovic, B.; Sim-Stark, J. C.C.; Haydock, S. F.; Leadlay, P. F. Prediction and Manipulation of the Stereochemistry of Enoylreduction in Modular Polyketide Synthases. Chem. Biol. 2008, 15, 1231-1240. 


\section{Copies of NMR spectra}

NMR-Spectra for Compound 16b

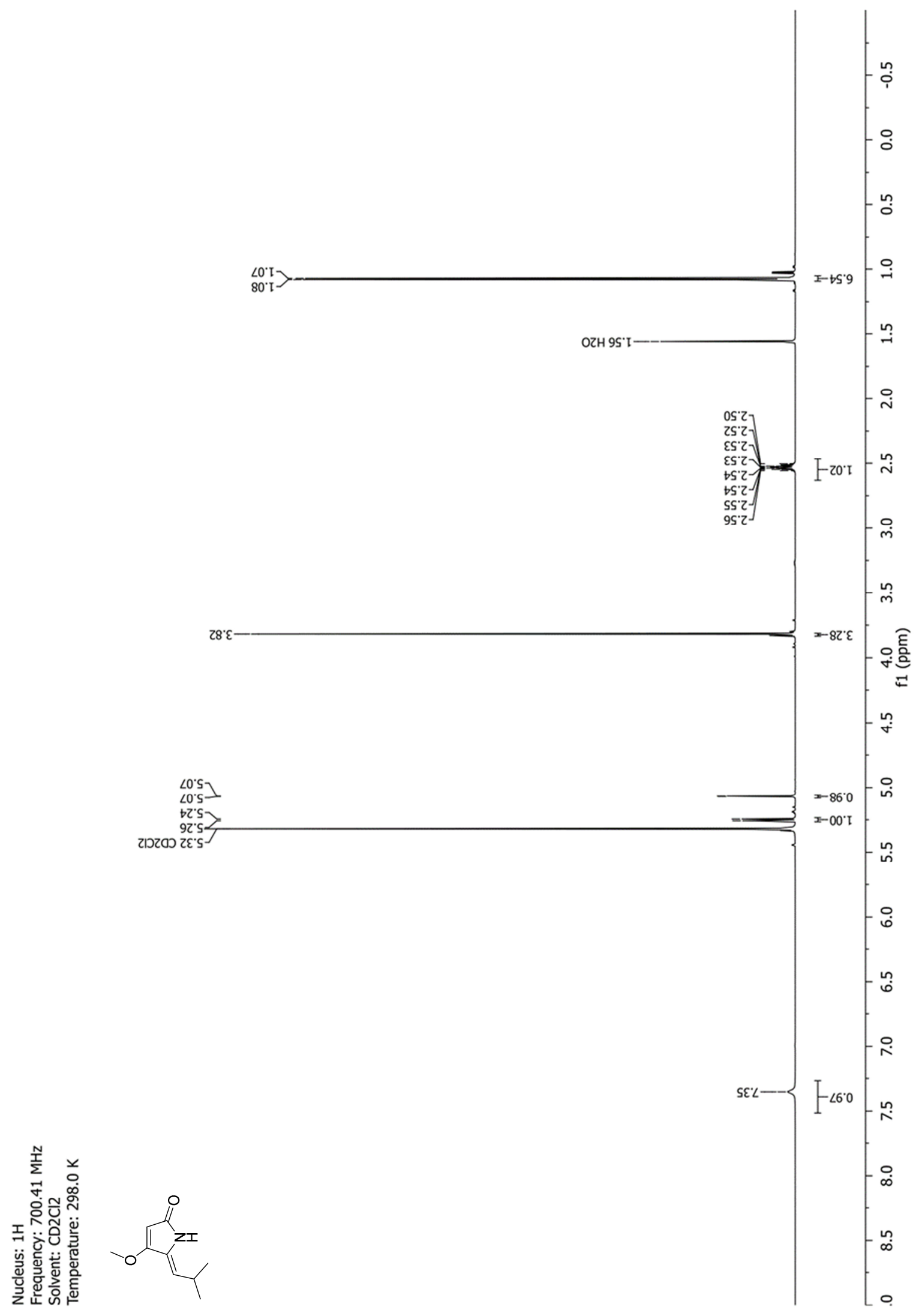




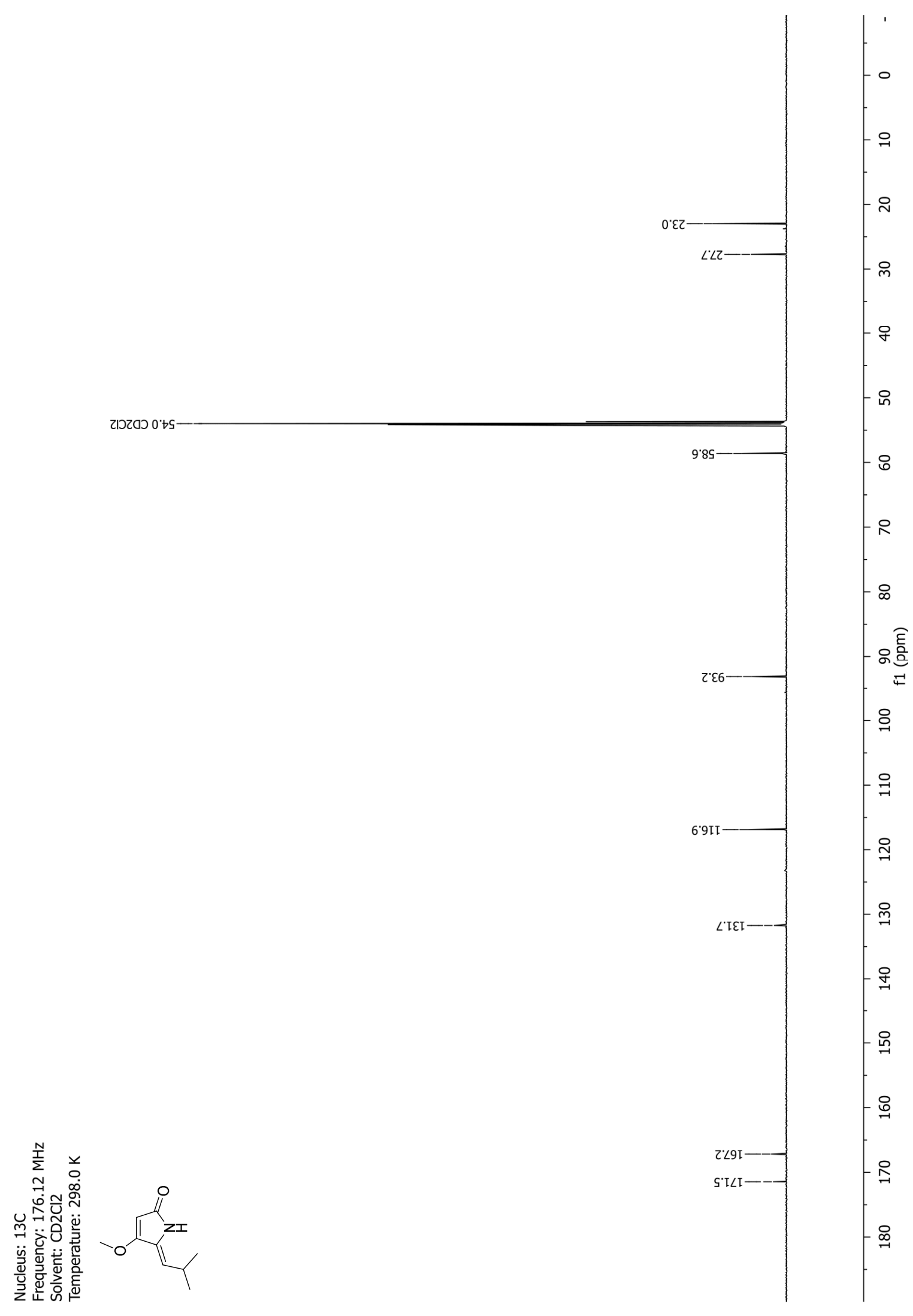


NMR-Spectra for Compound 16c

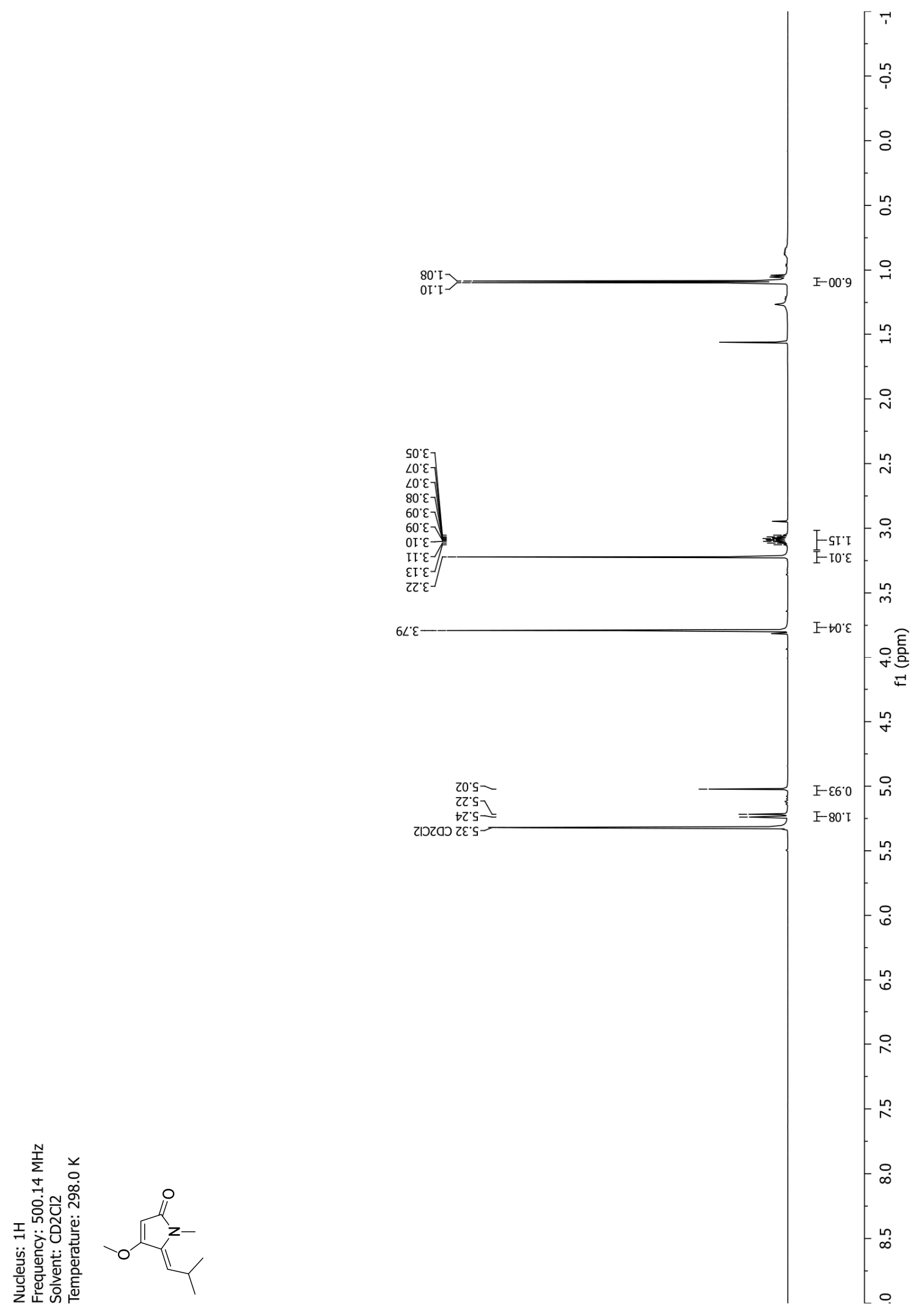




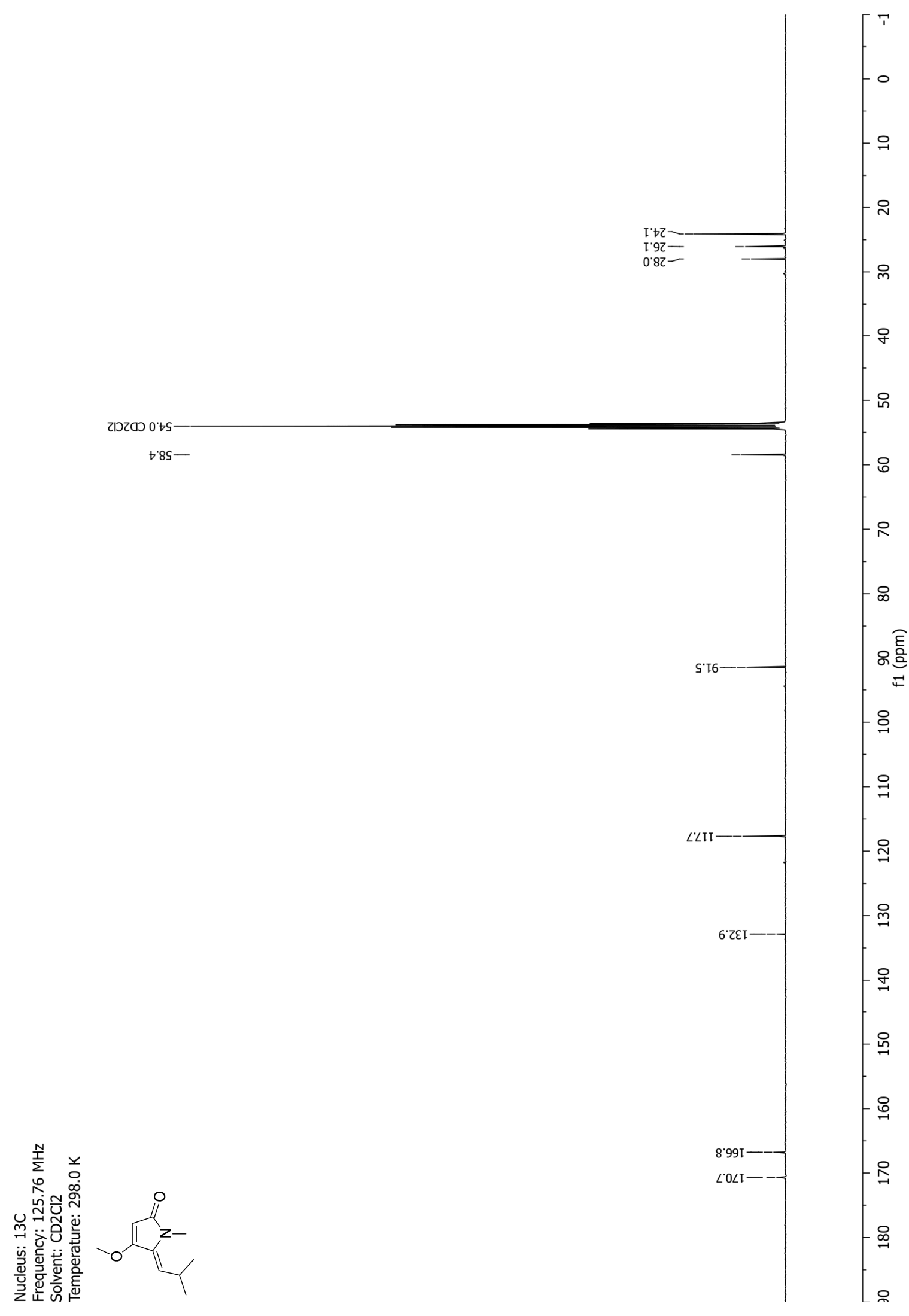


NMR-Spectra for Compound 16

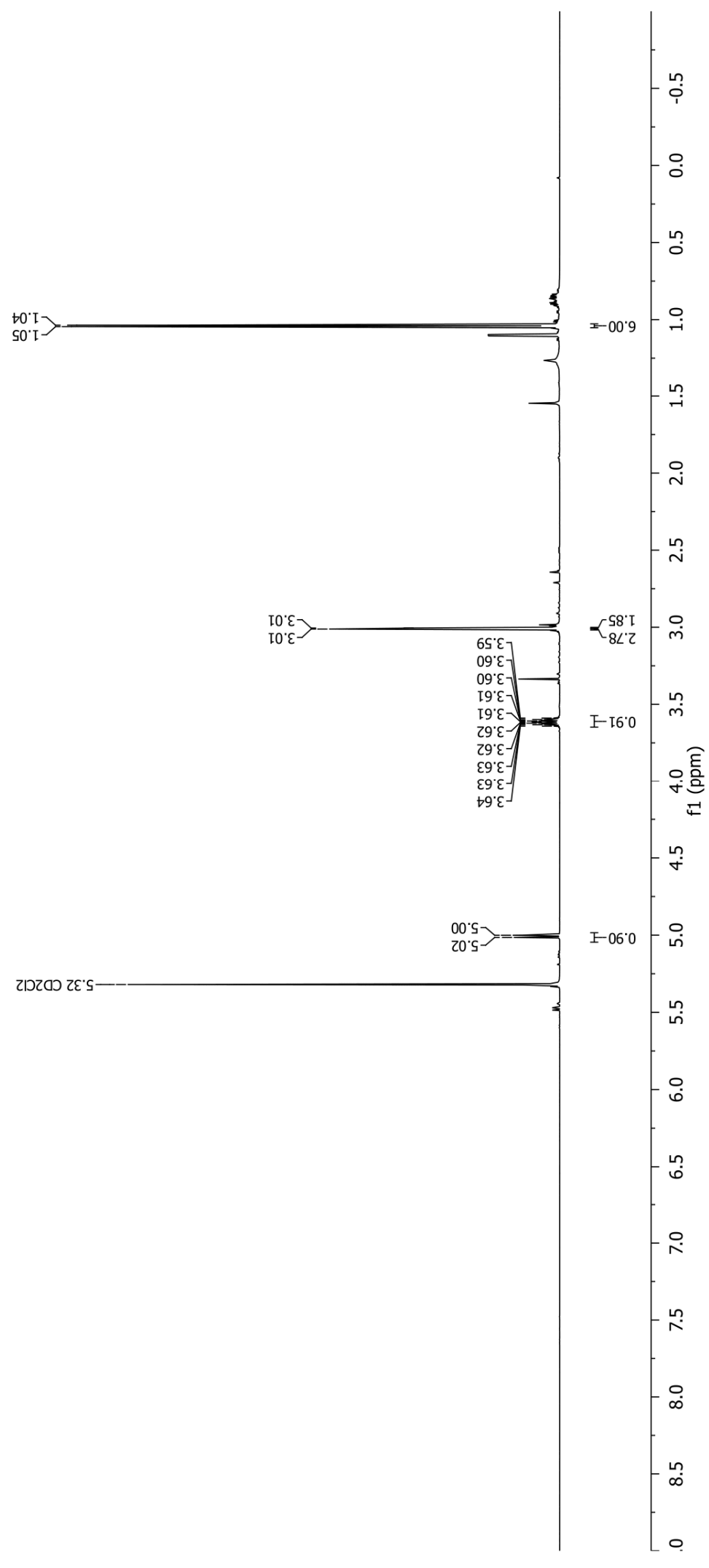




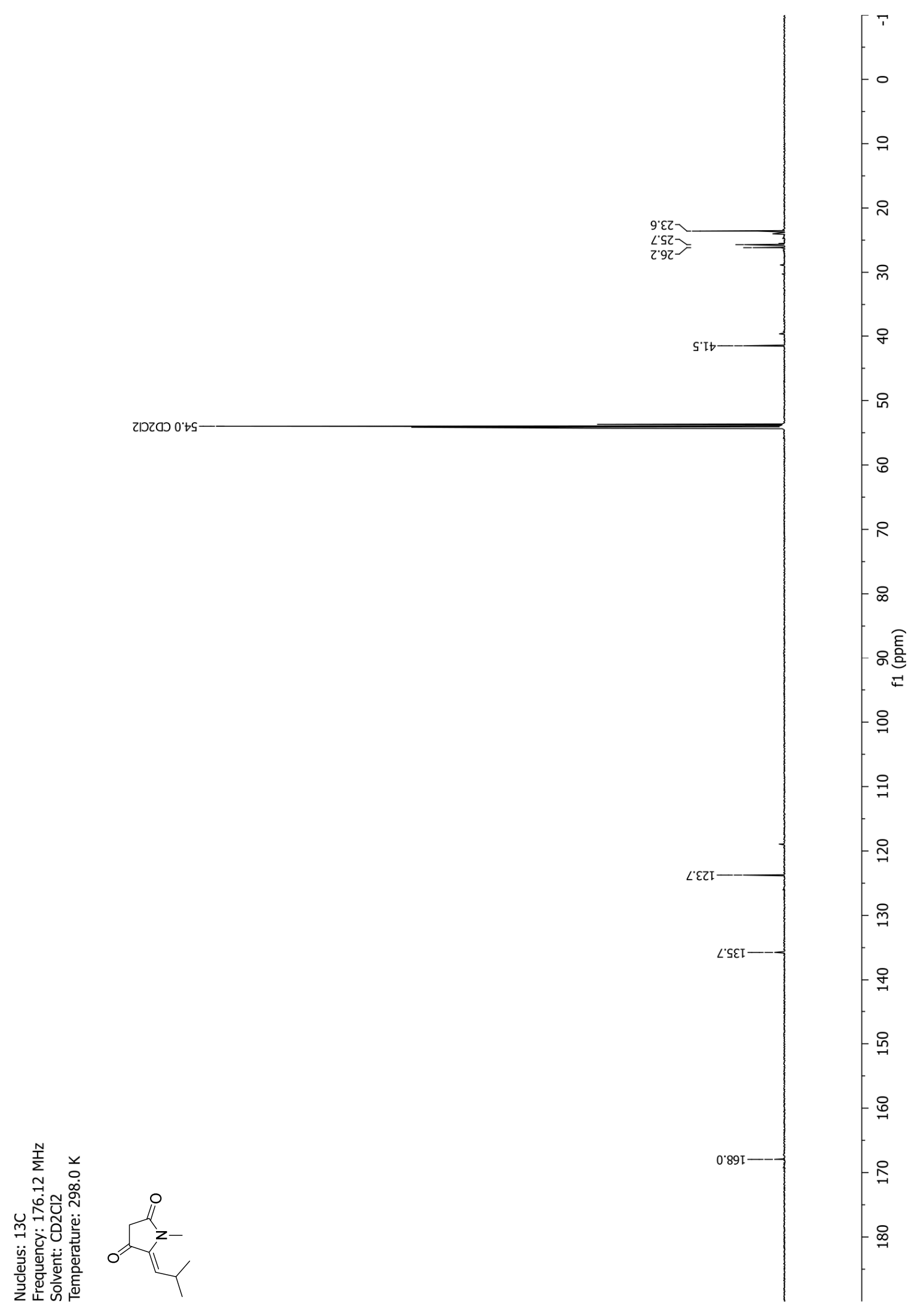


NMR-Spectra for Compound 43

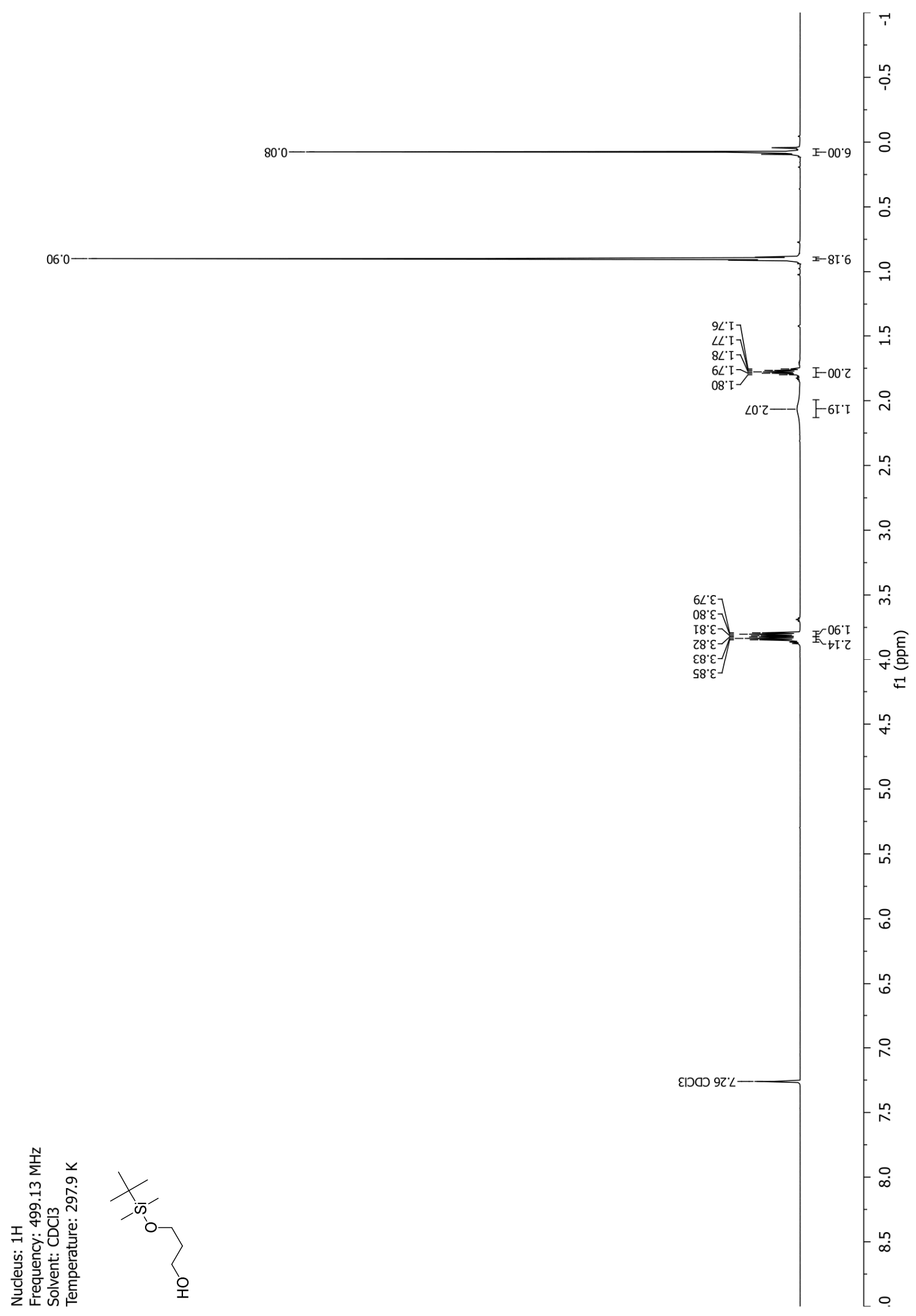




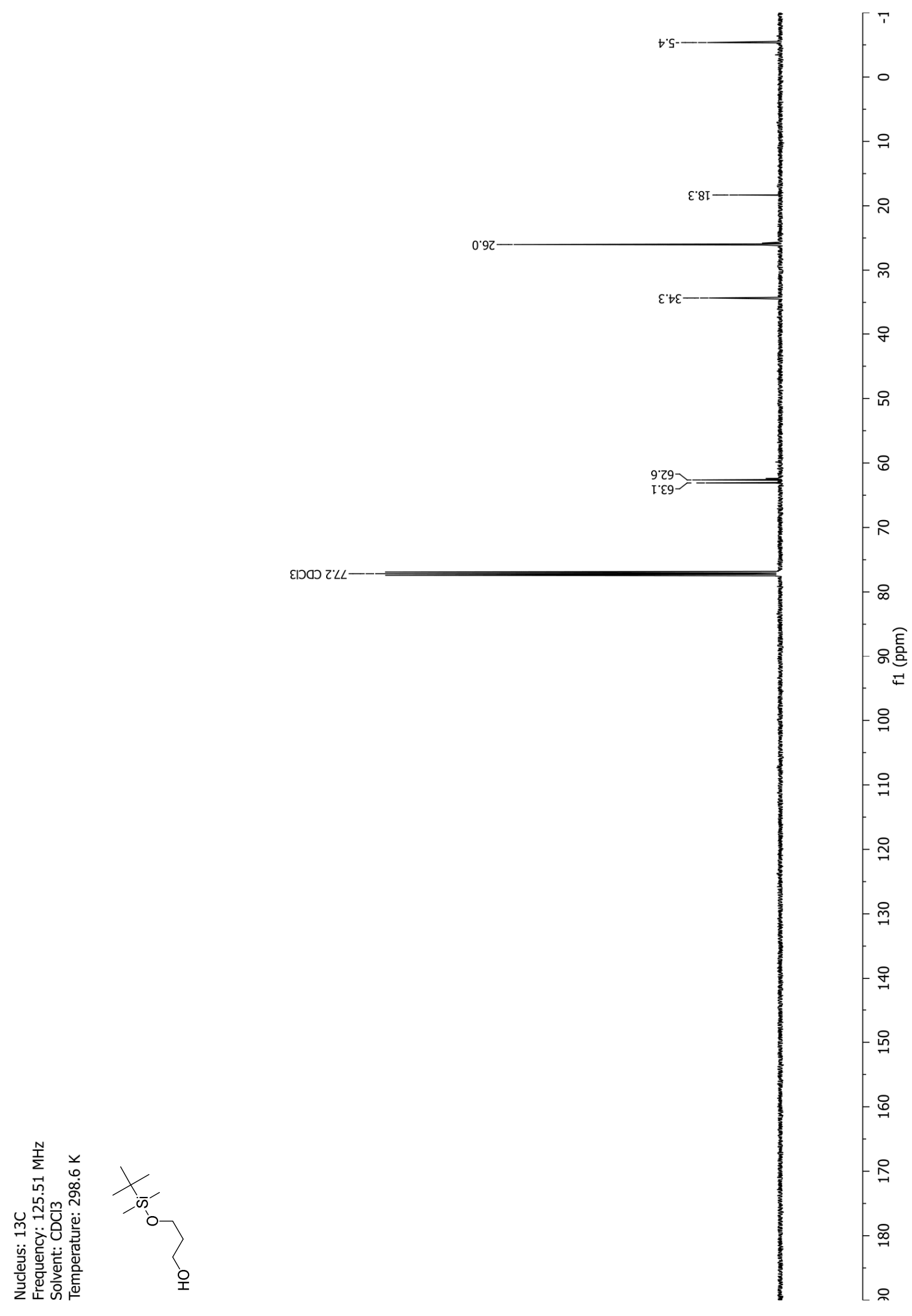


NMR-Spectra for Compound 7

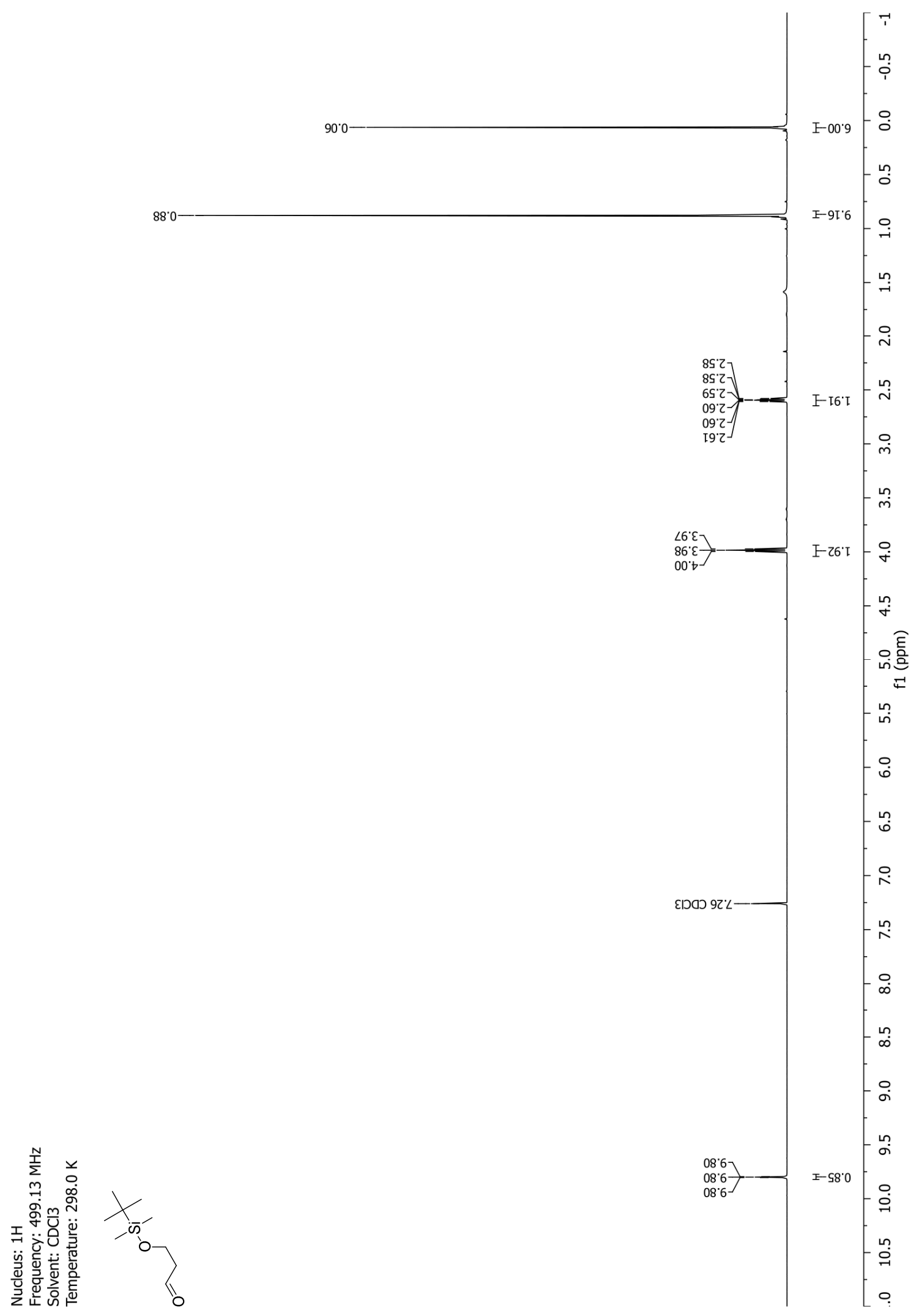




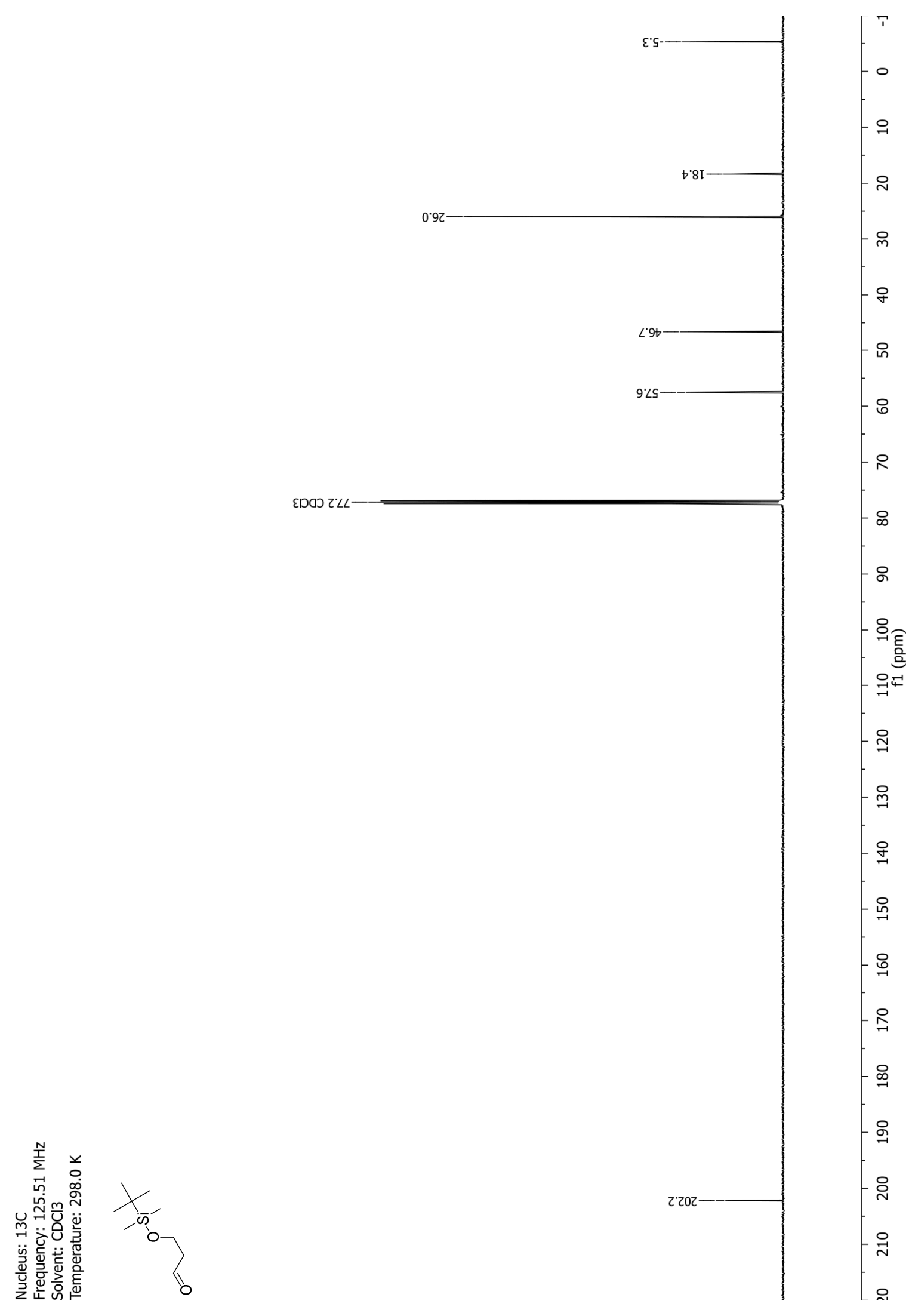


NMR-Spectra for Compound $44 E$

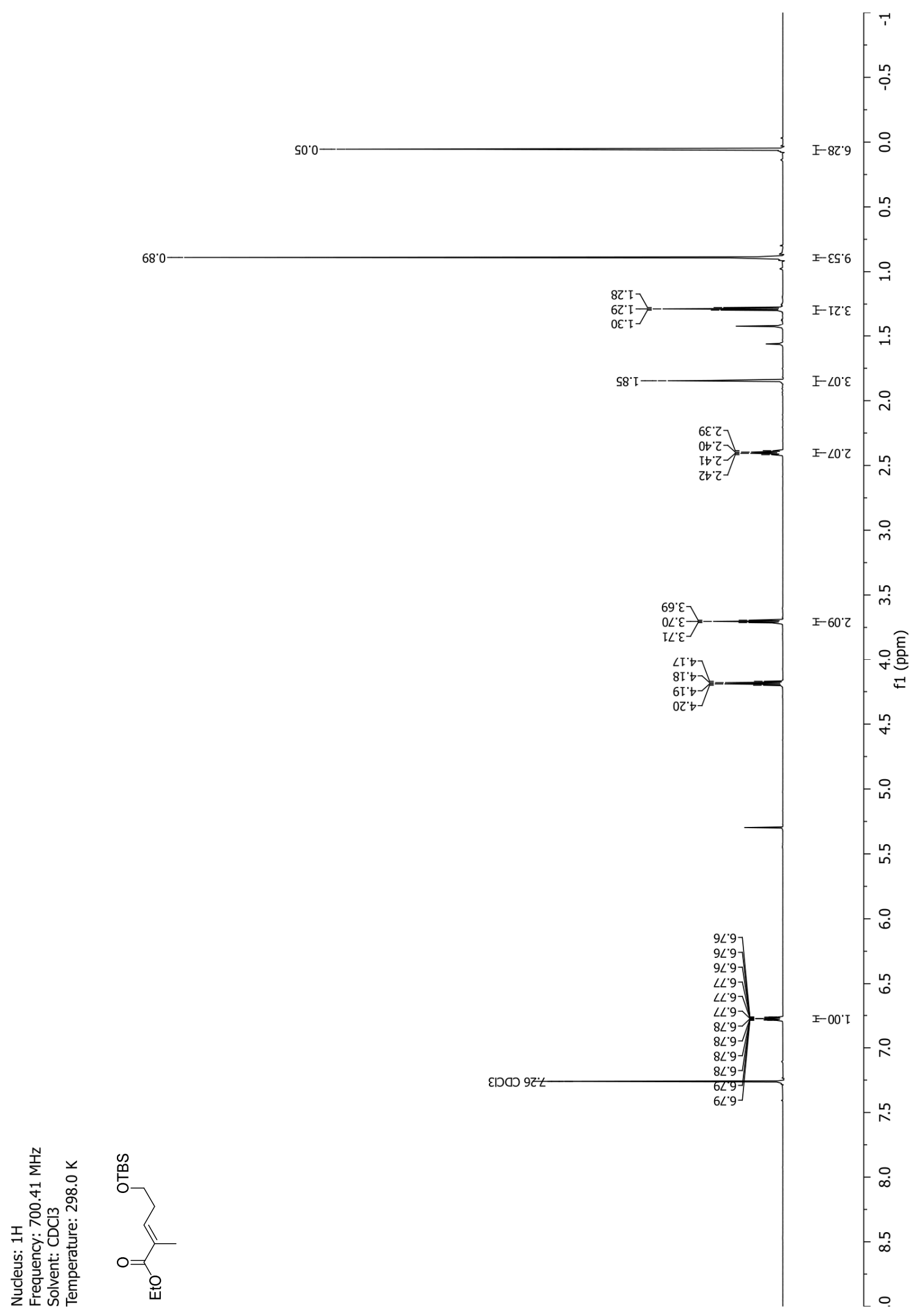




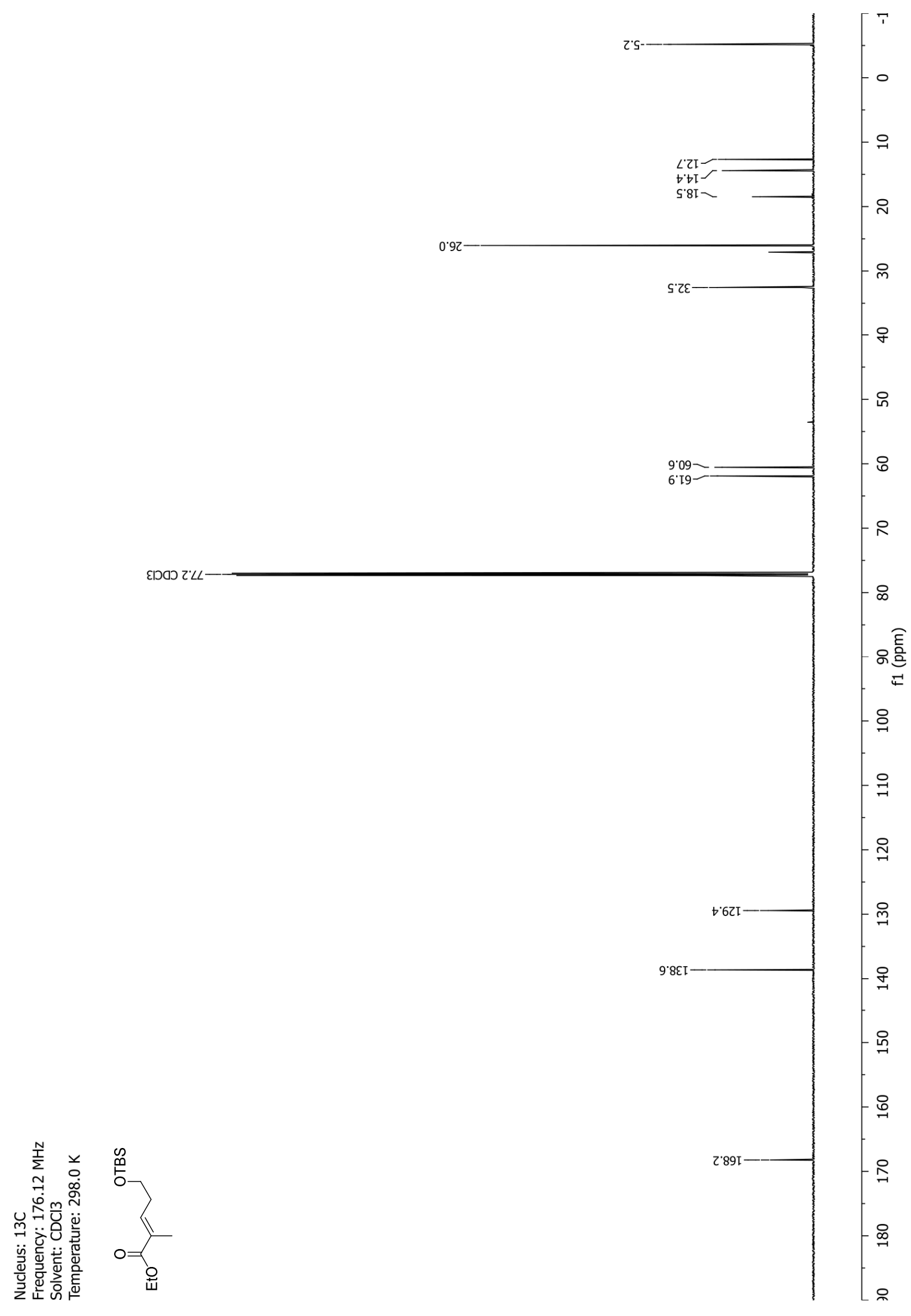


NMR-Spectra for Compound $44 Z$

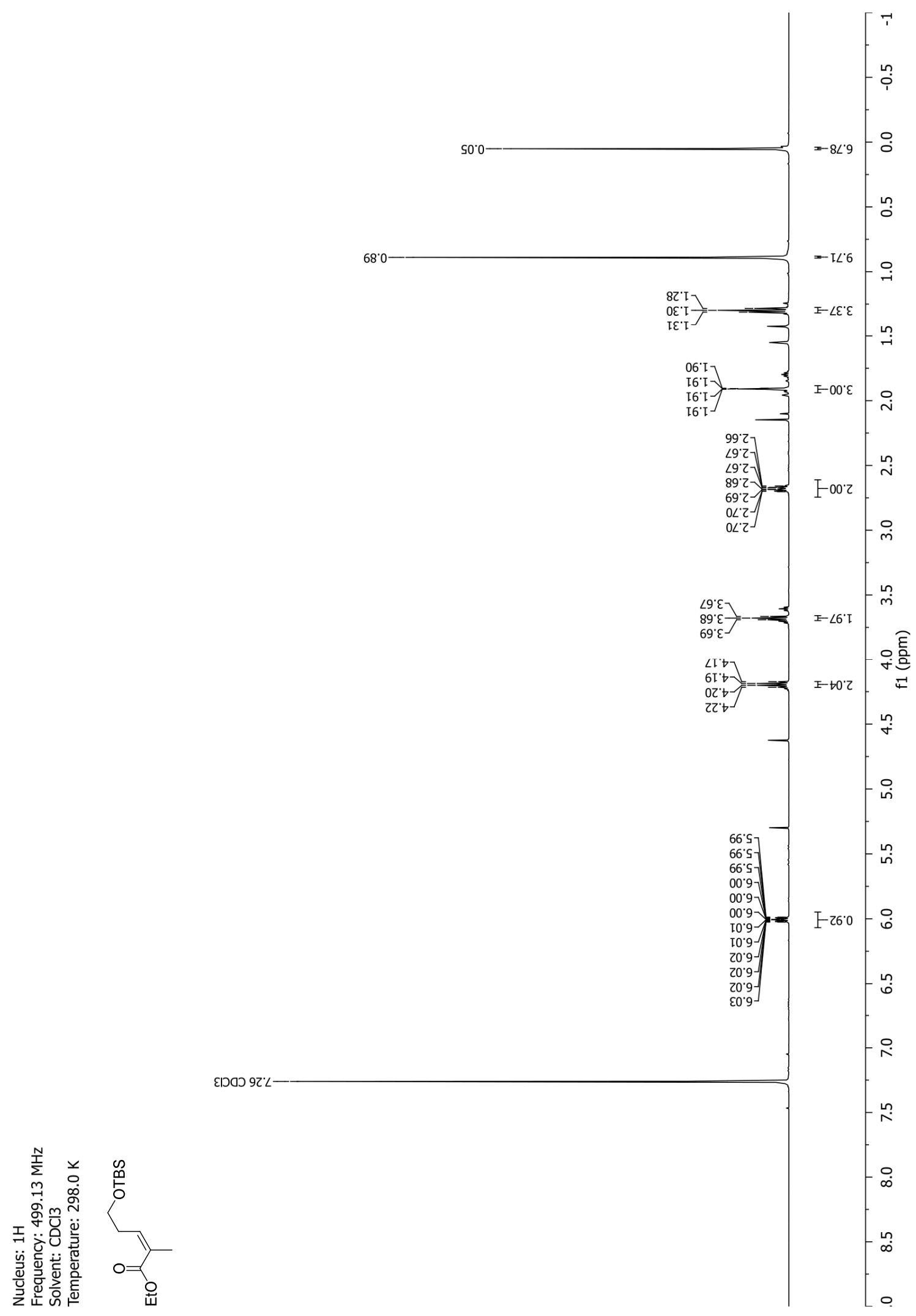




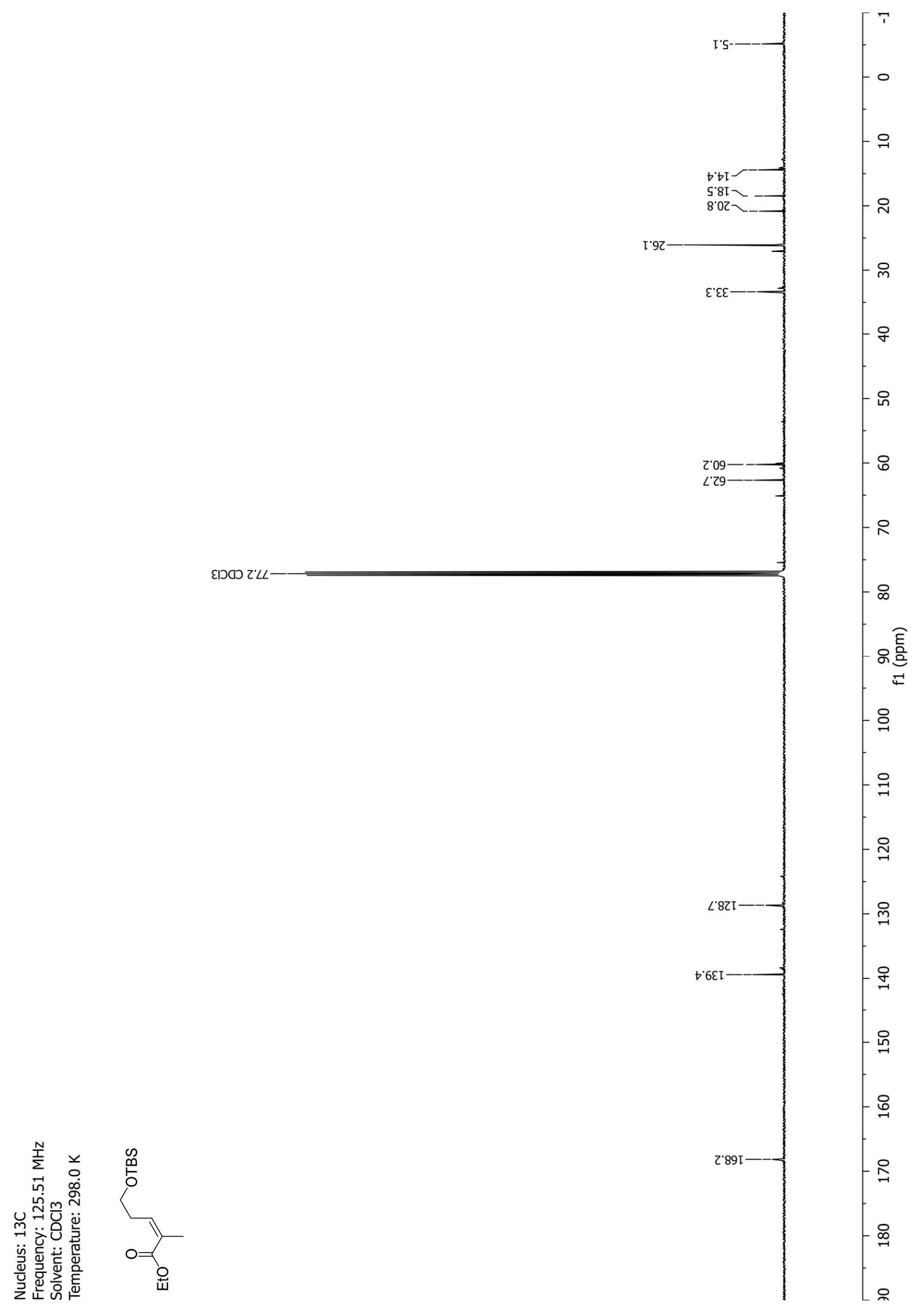


NMR-Spectra for Compound 9

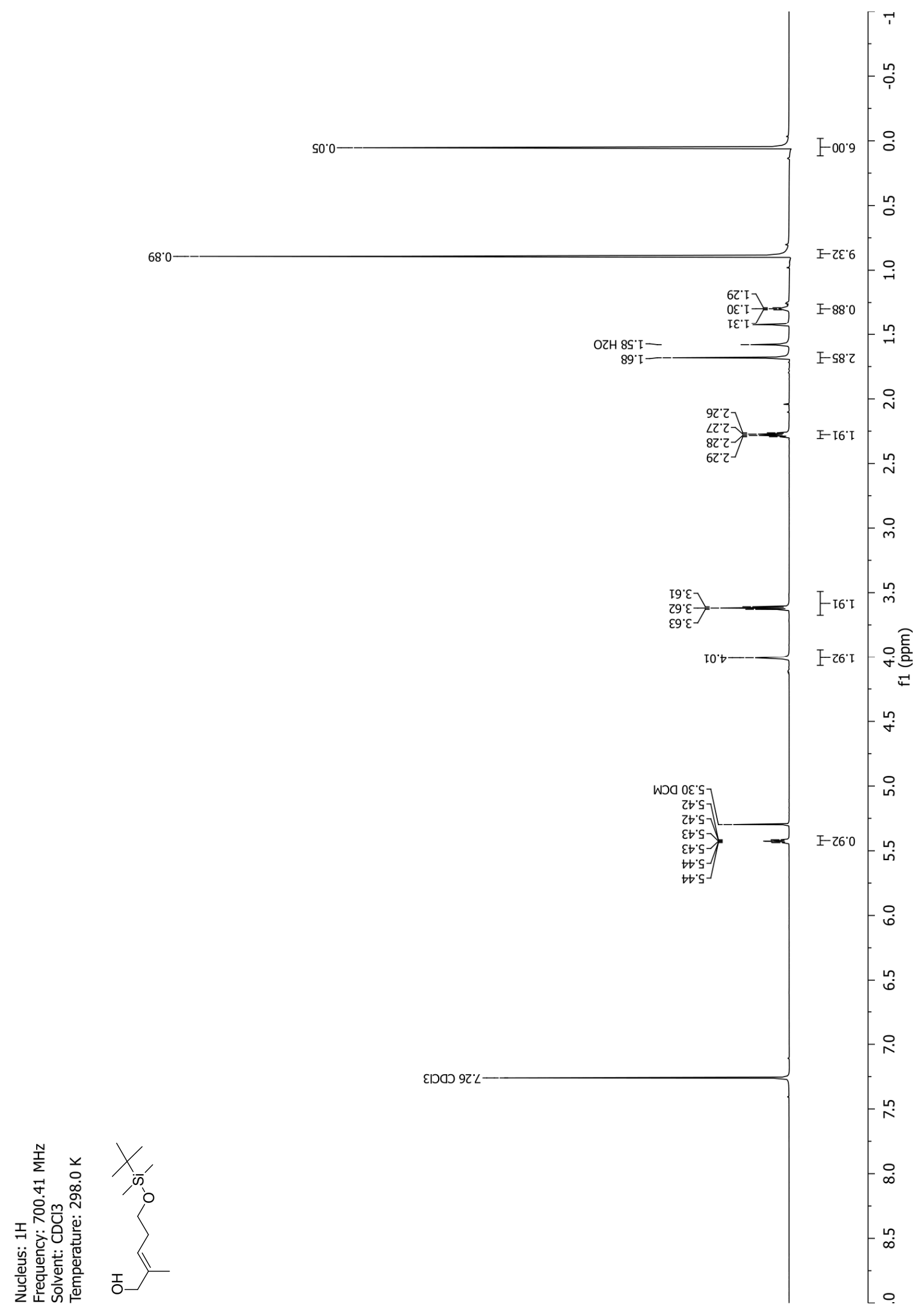




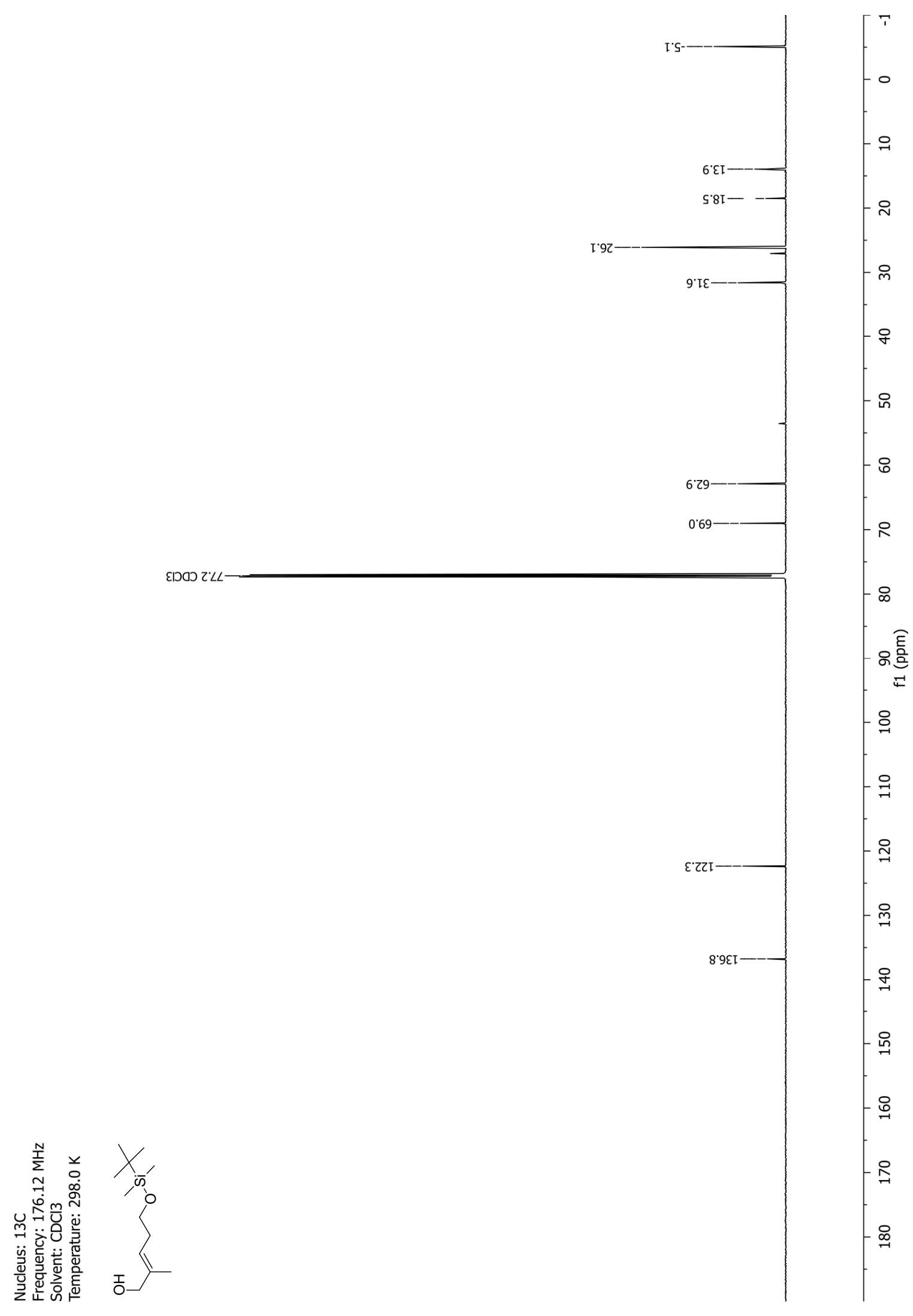


NMR-Spectra for Compound 45

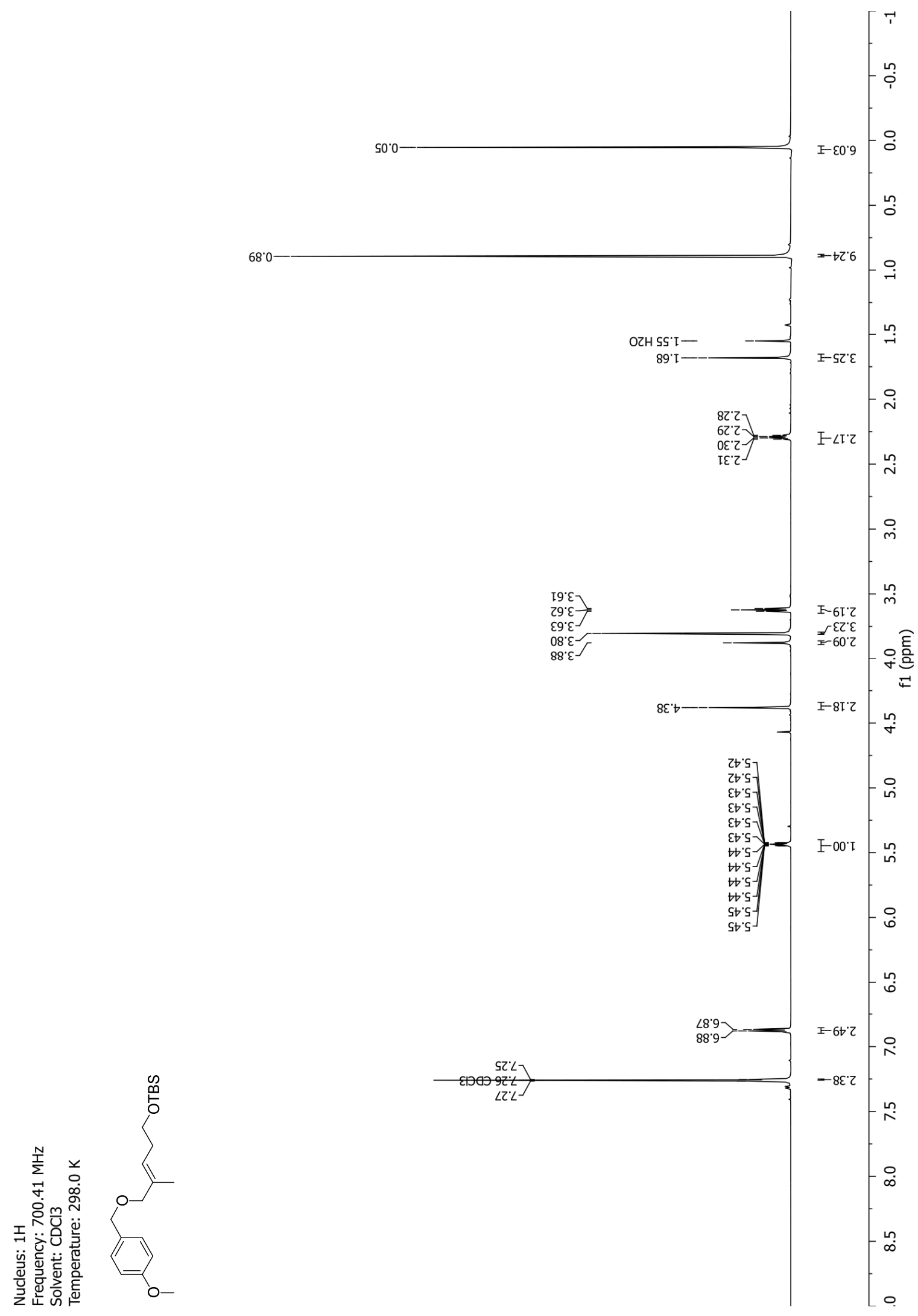




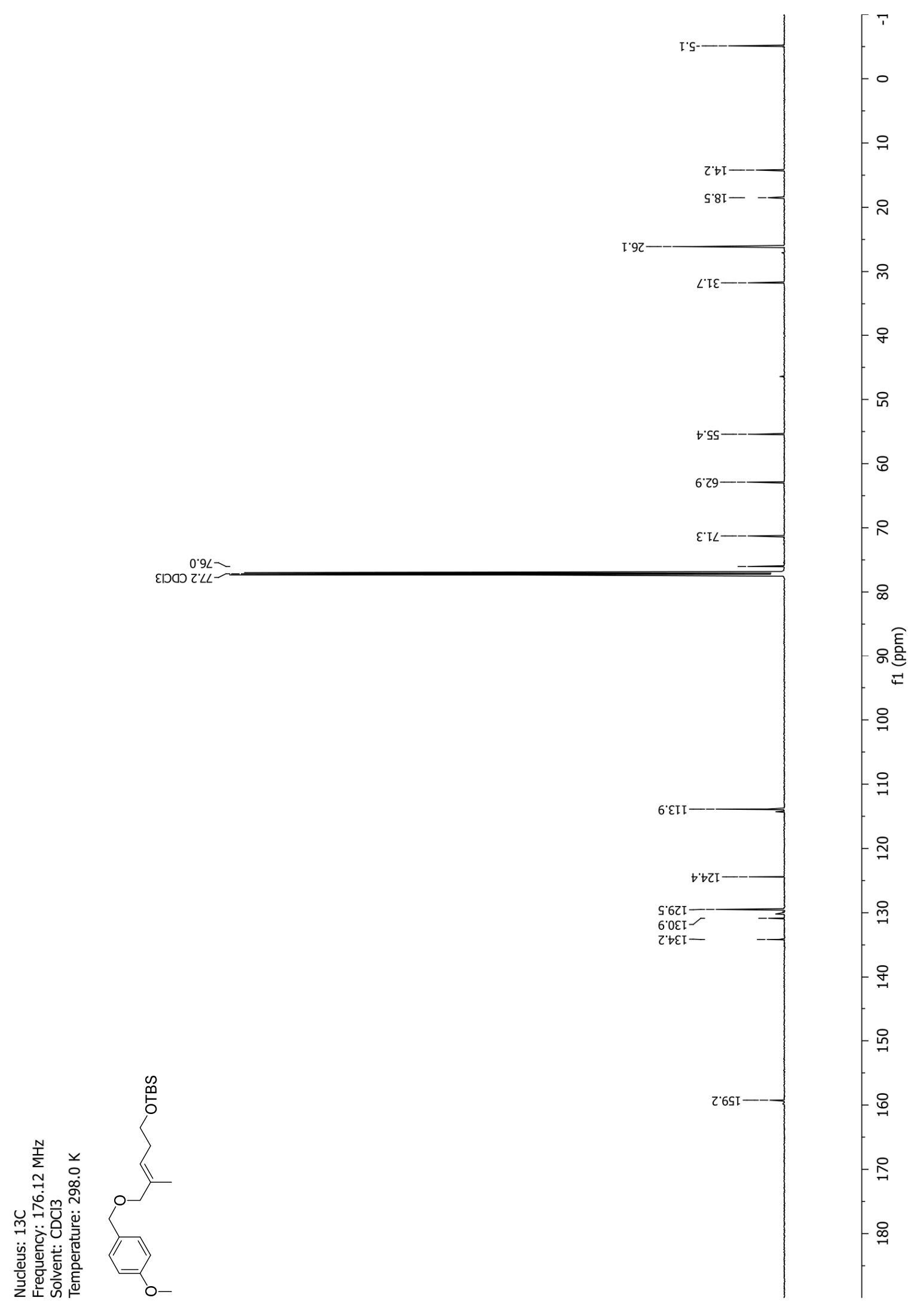


NMR-Spectra for Compound 46

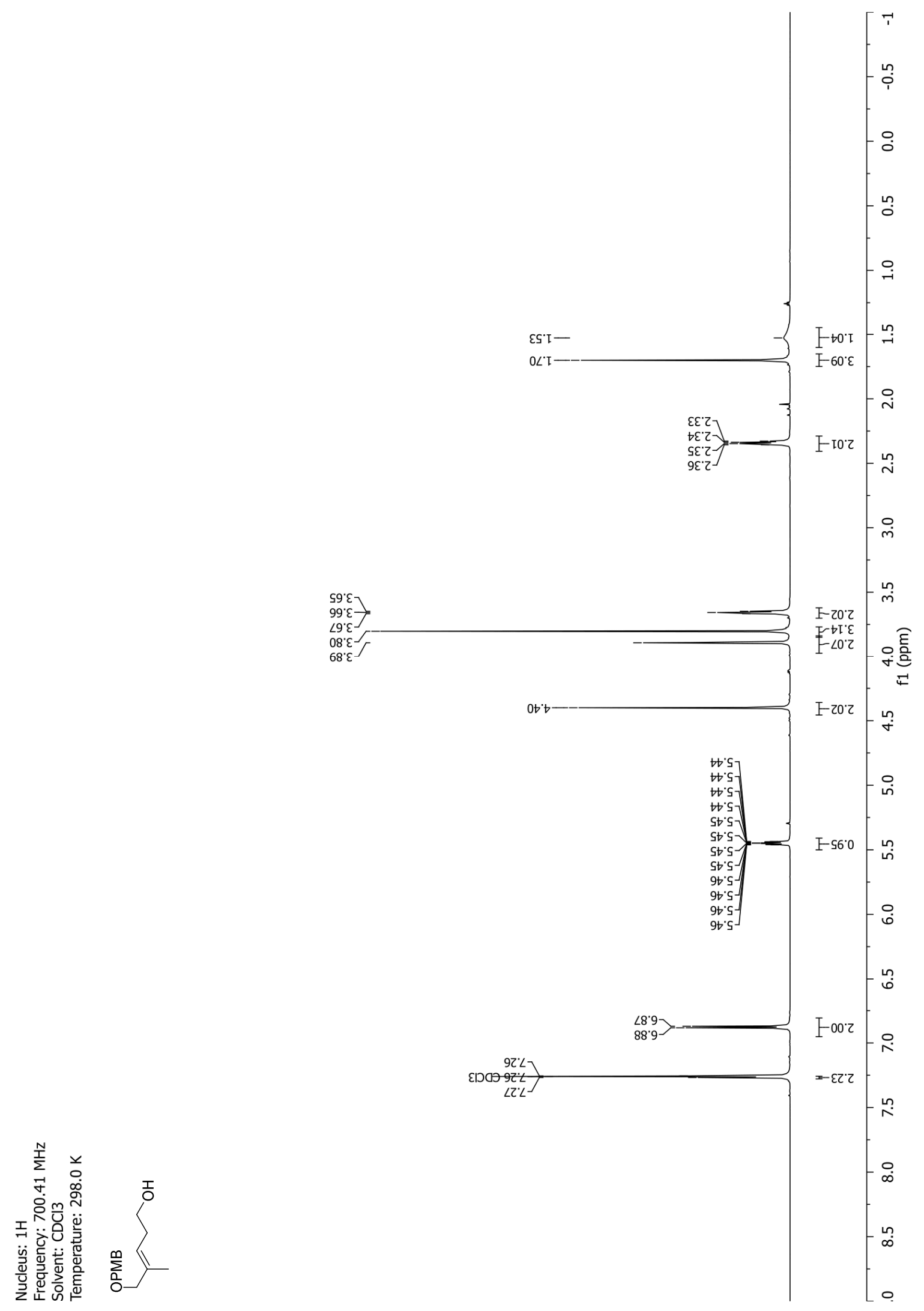




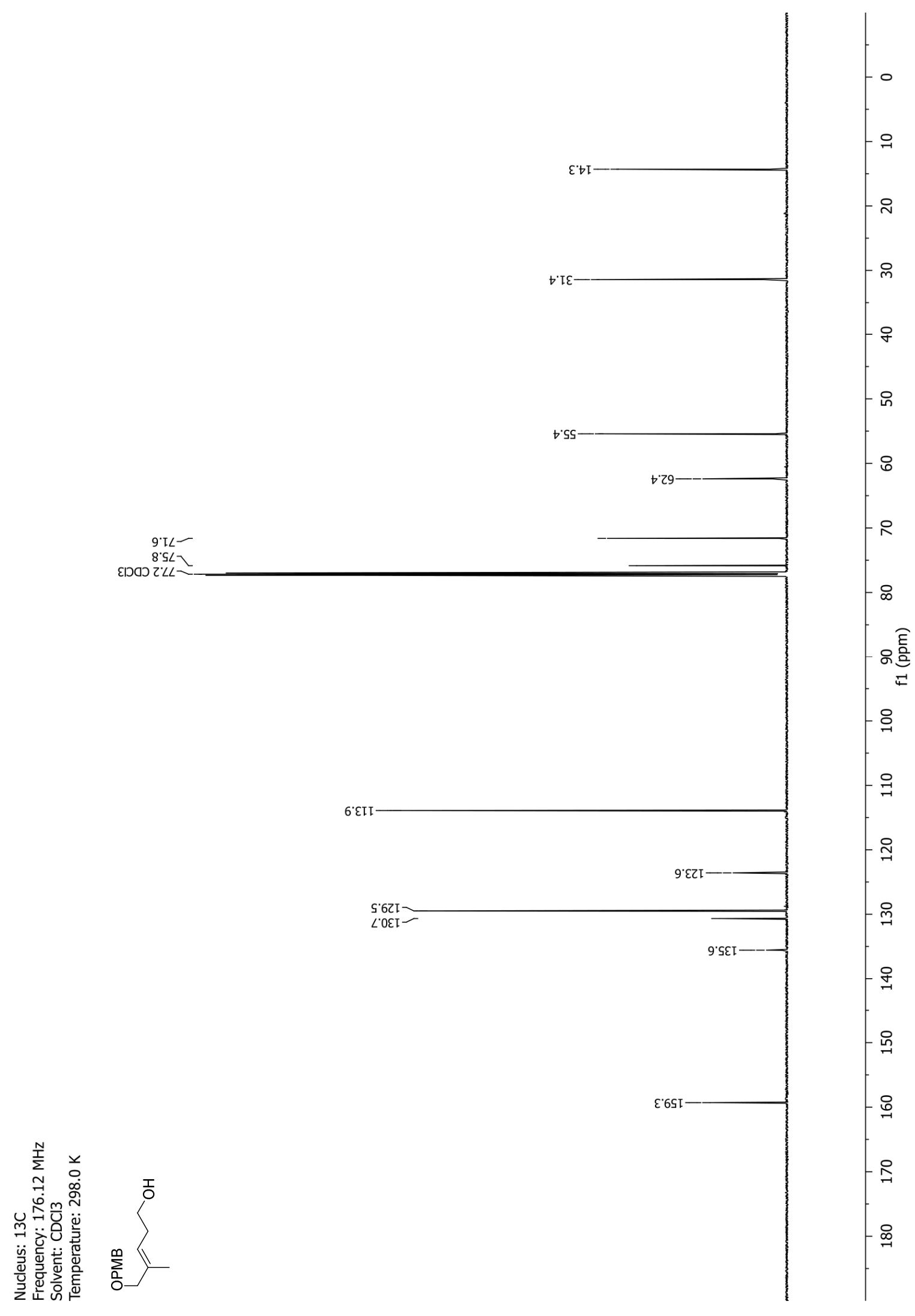


NMR-Spectra for Compound 10

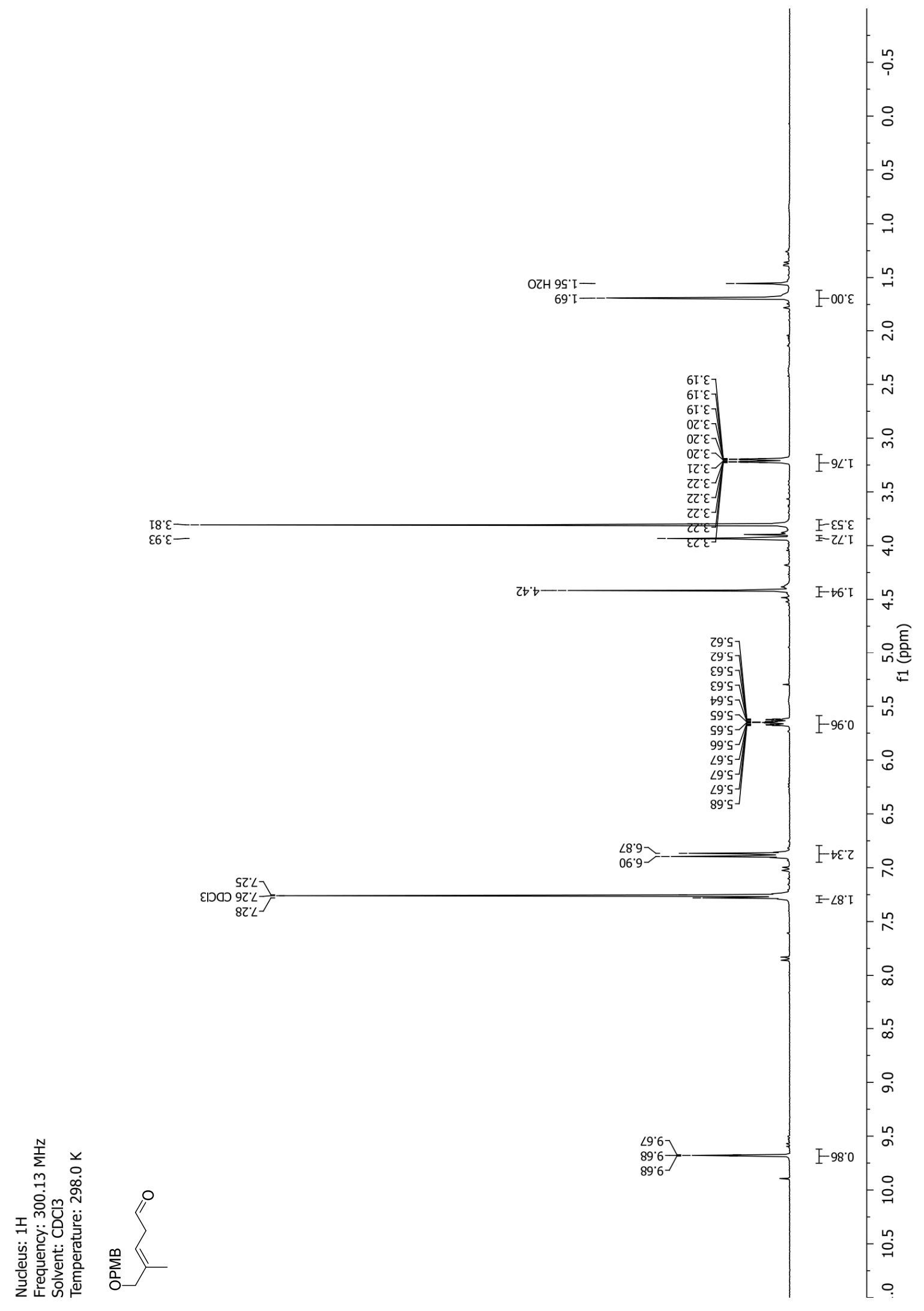




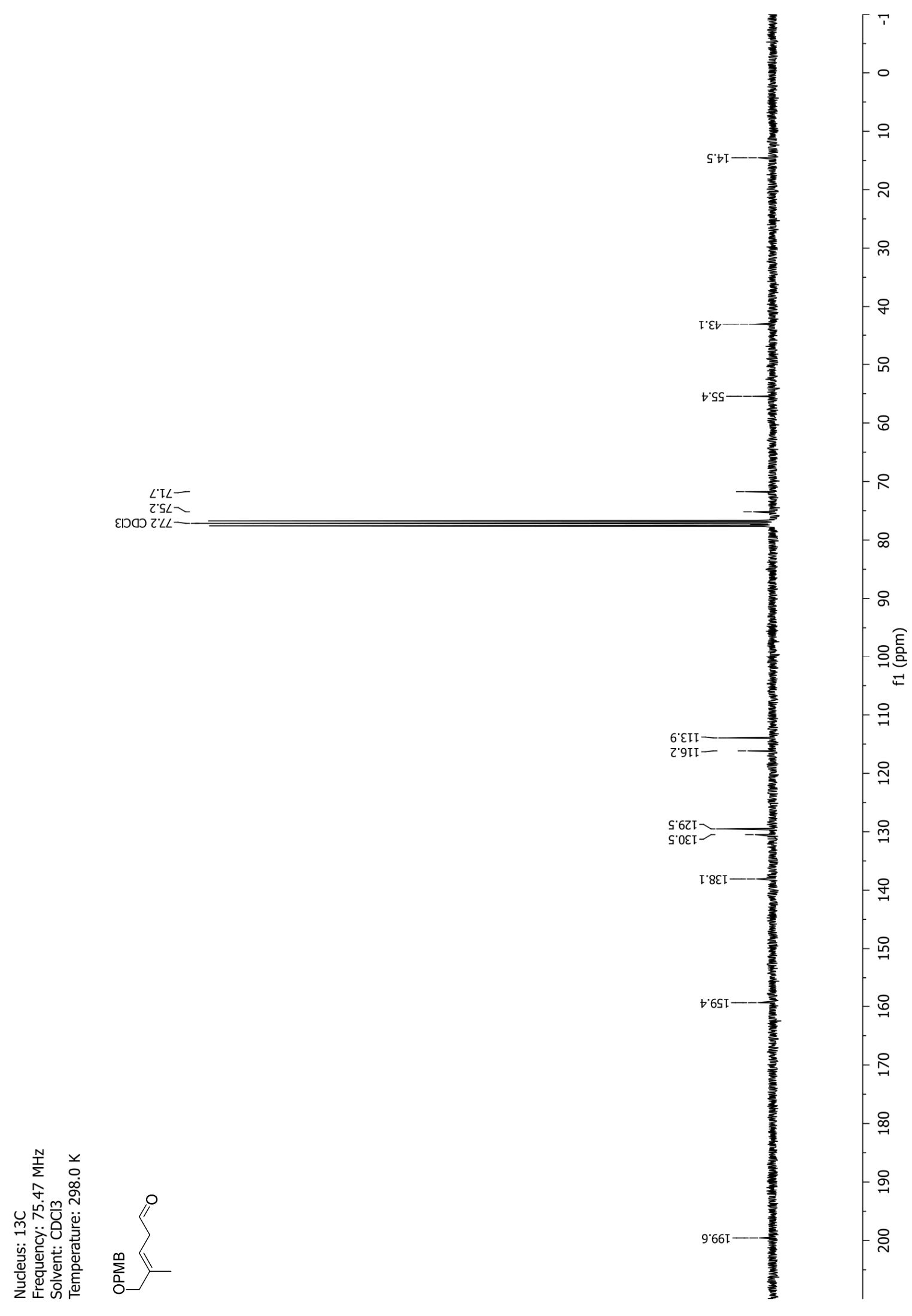


NMR-Spectra for Compound 48

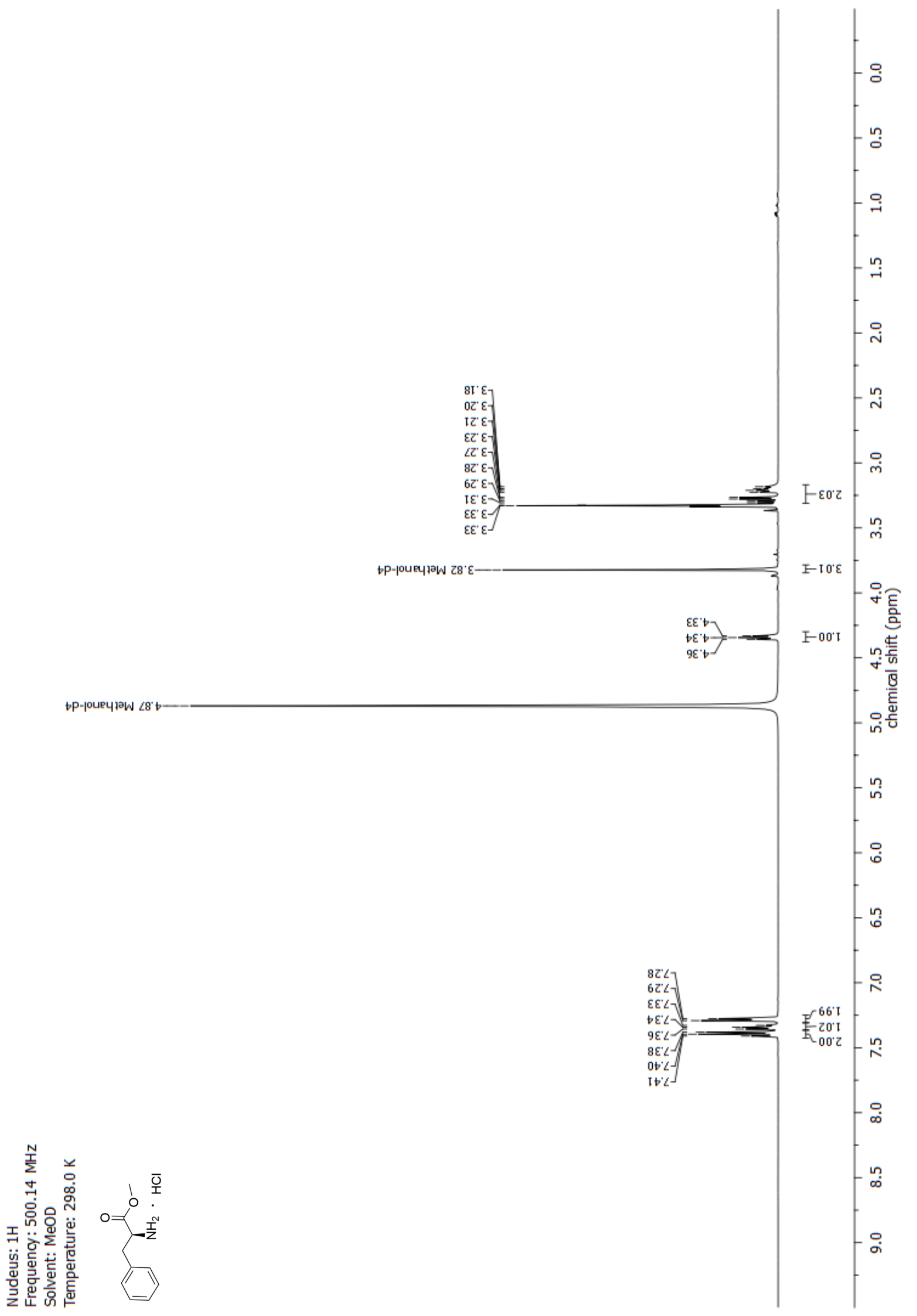




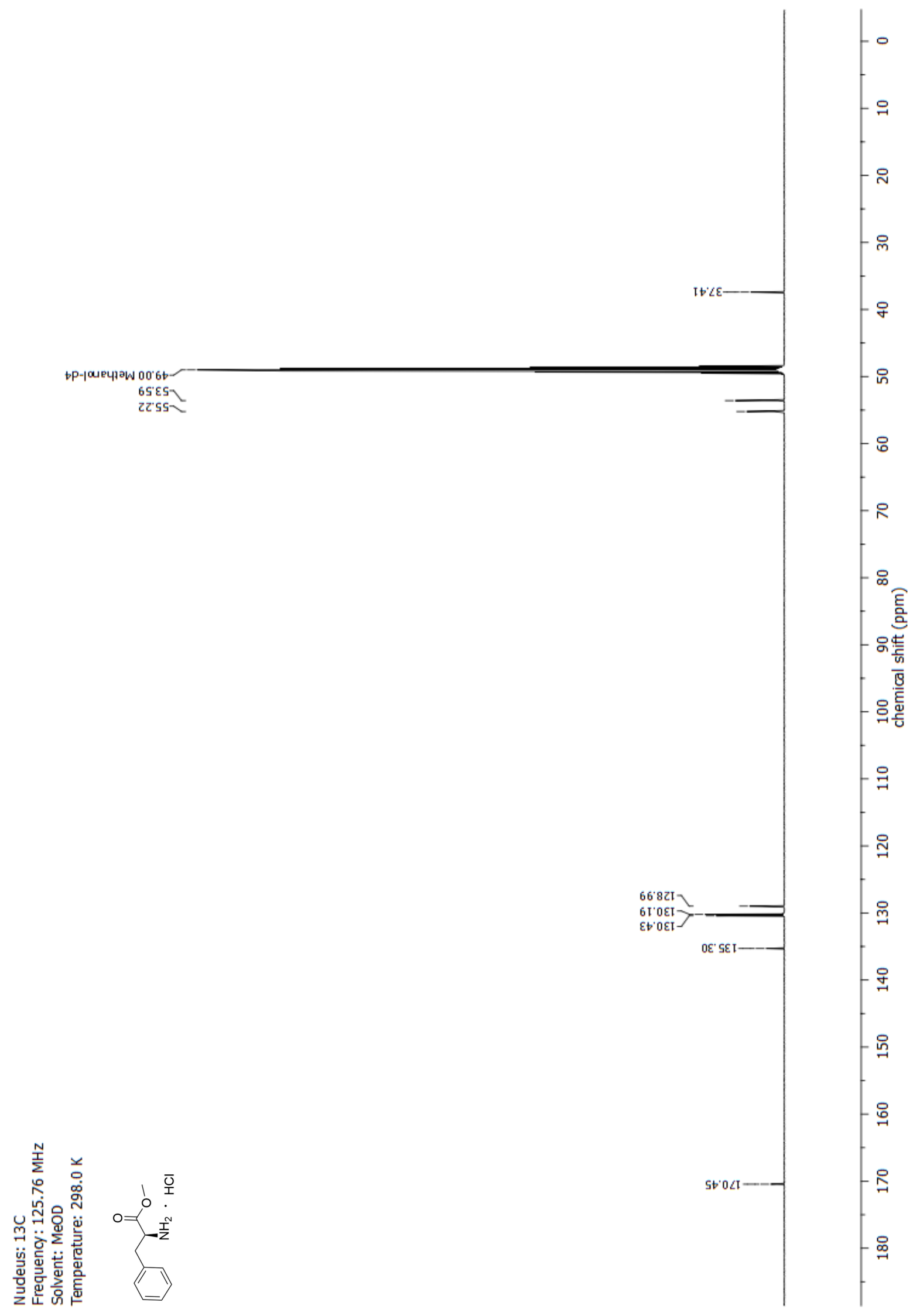


NMR-Spectra for Compound 49

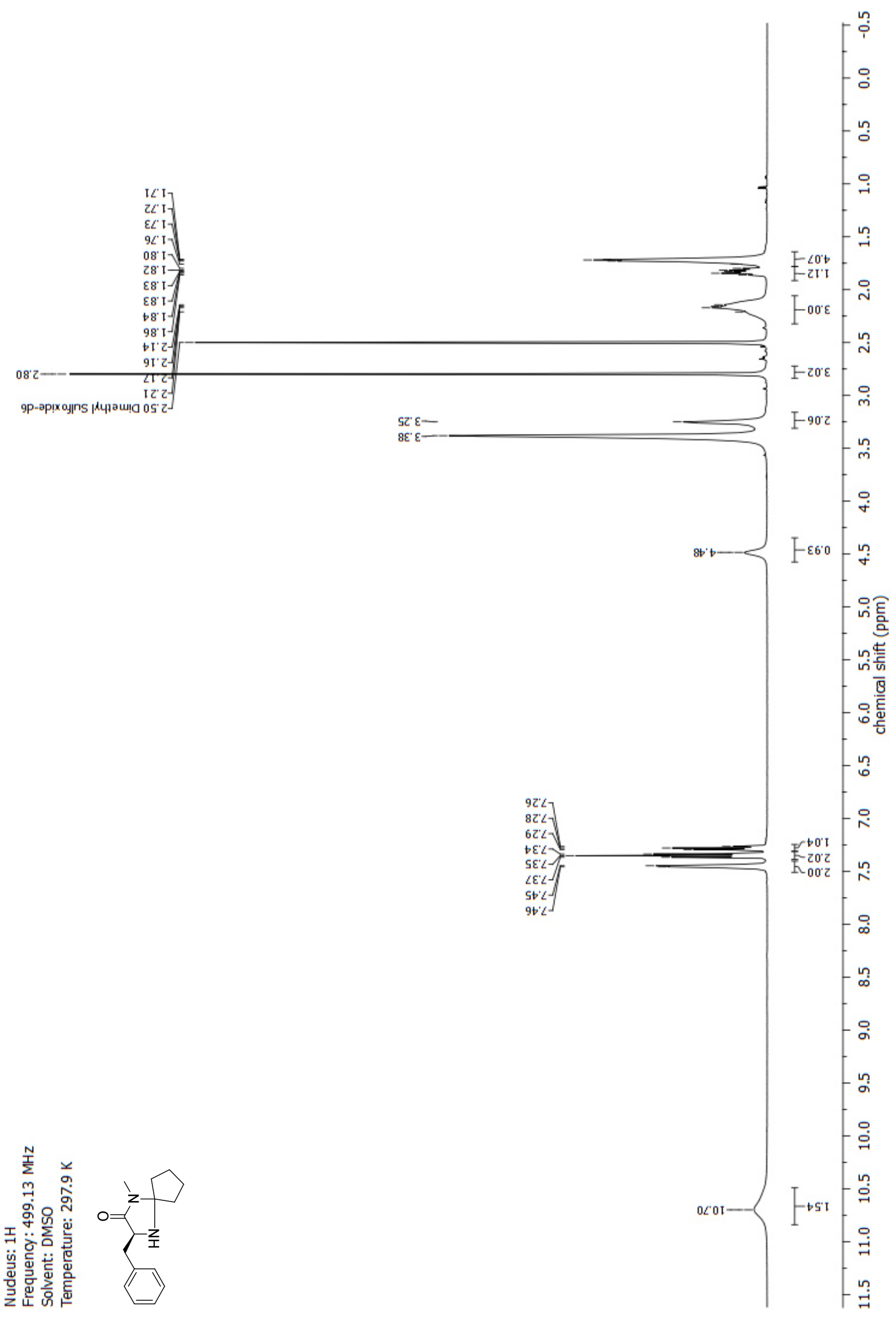




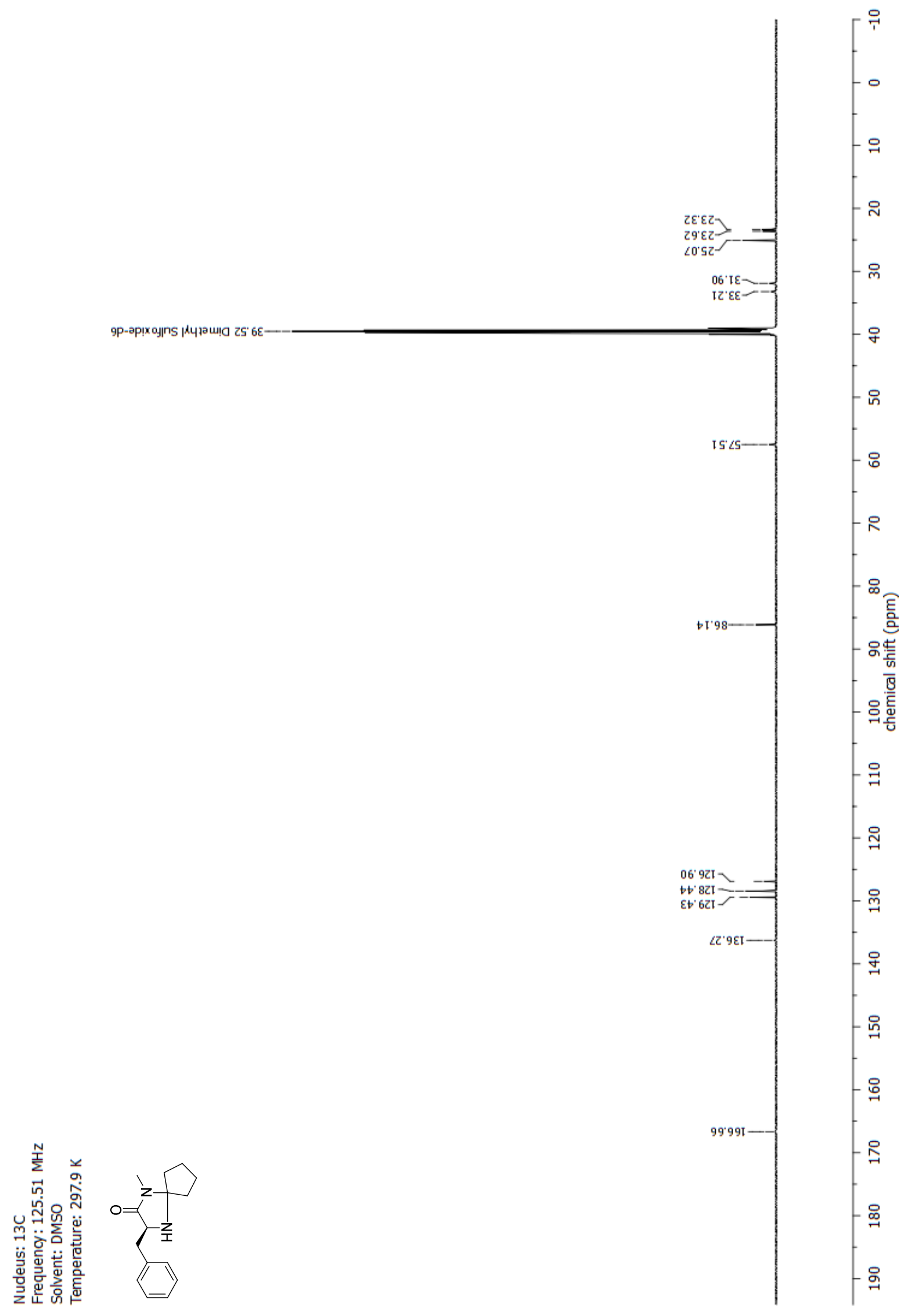


NMR-Spectra for Compound 51

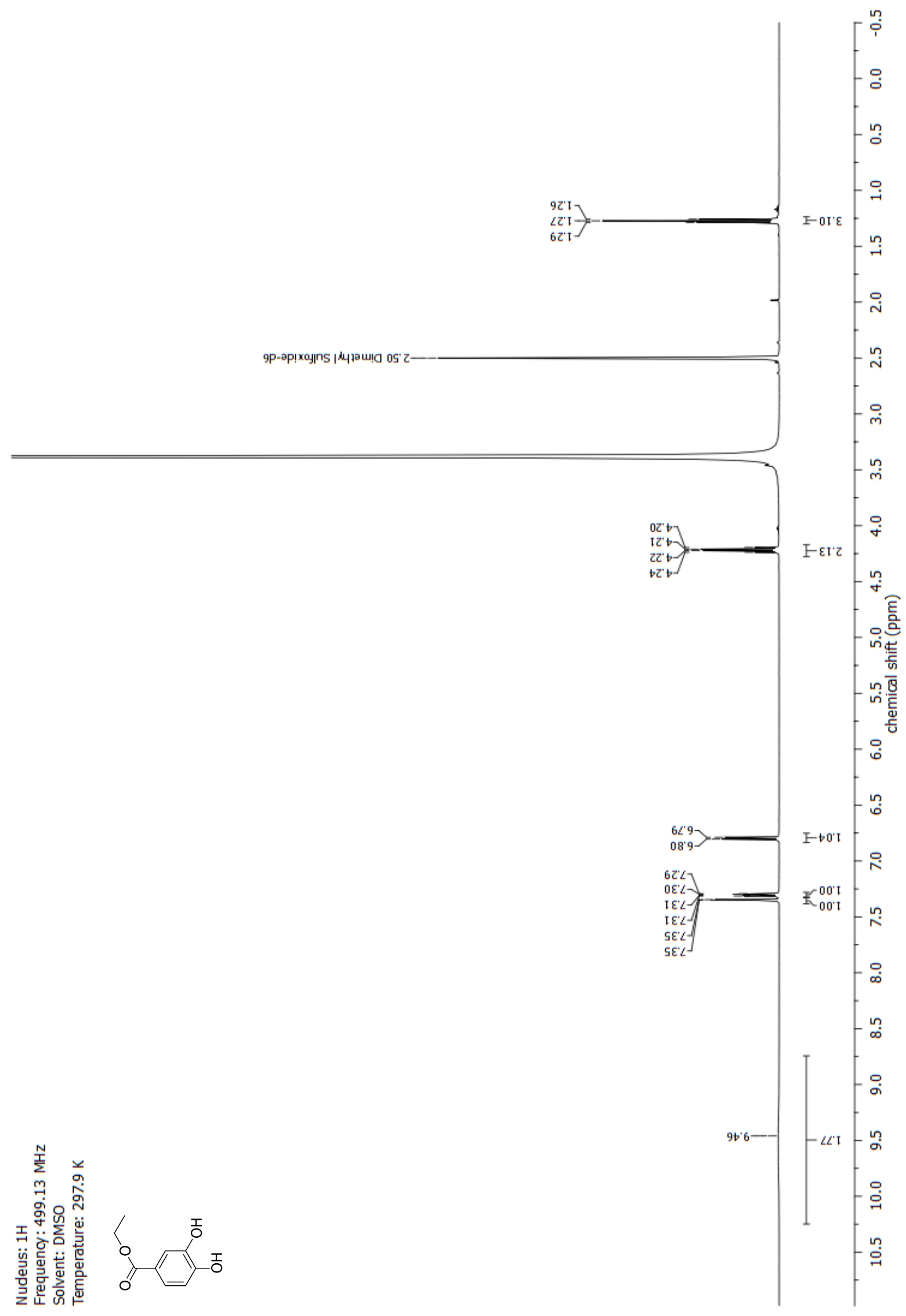




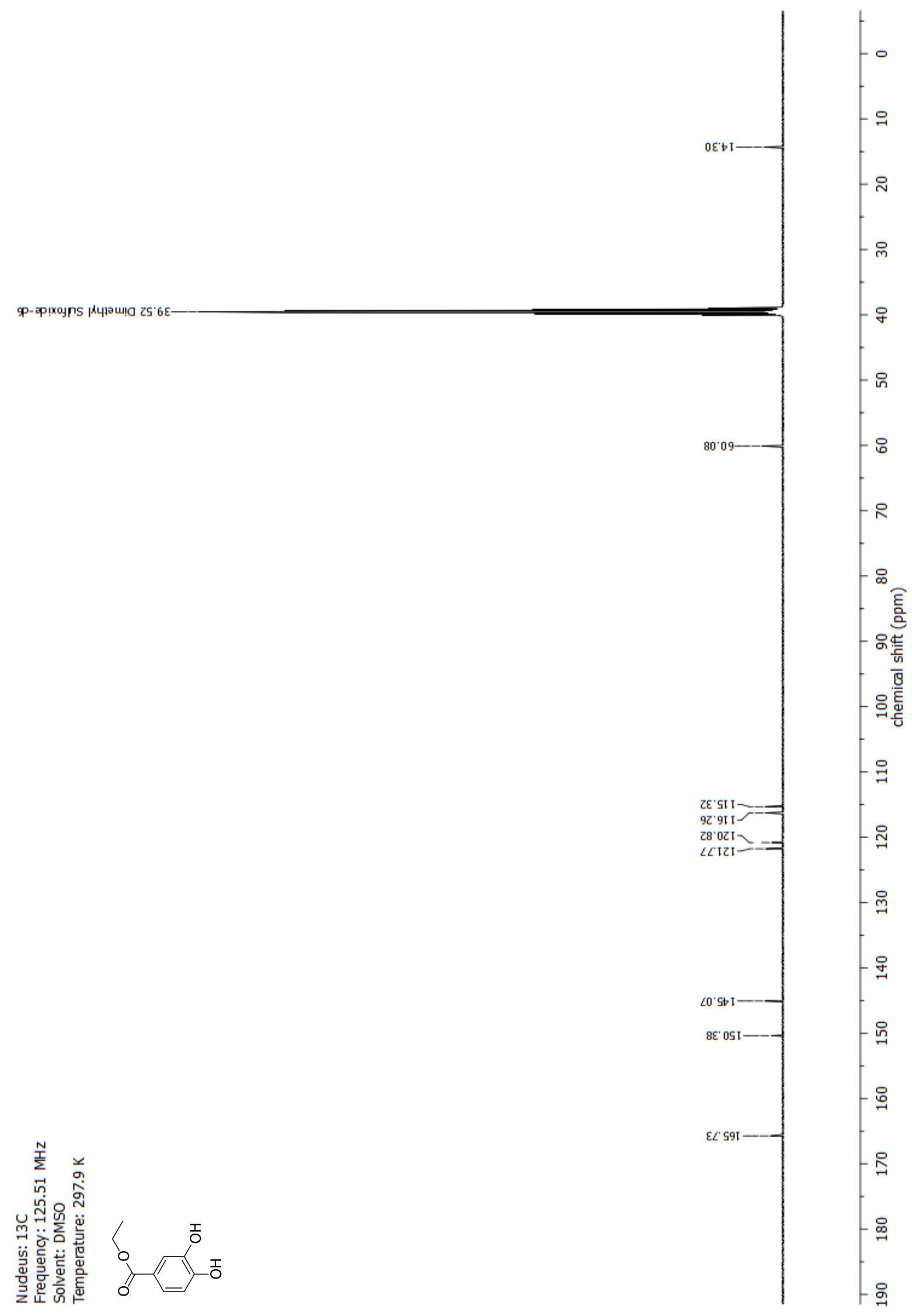


NMR-Spectra for Compound 12

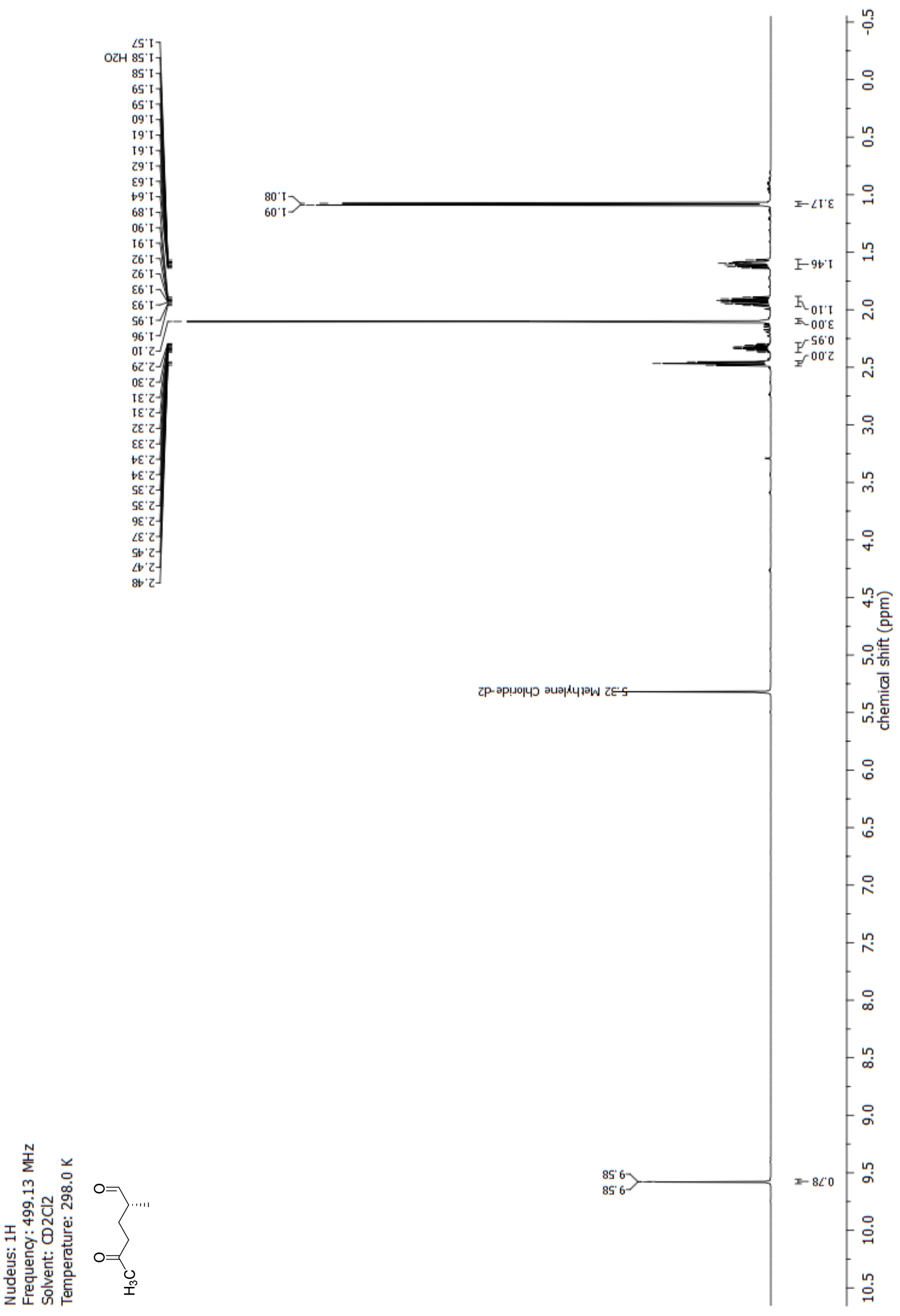




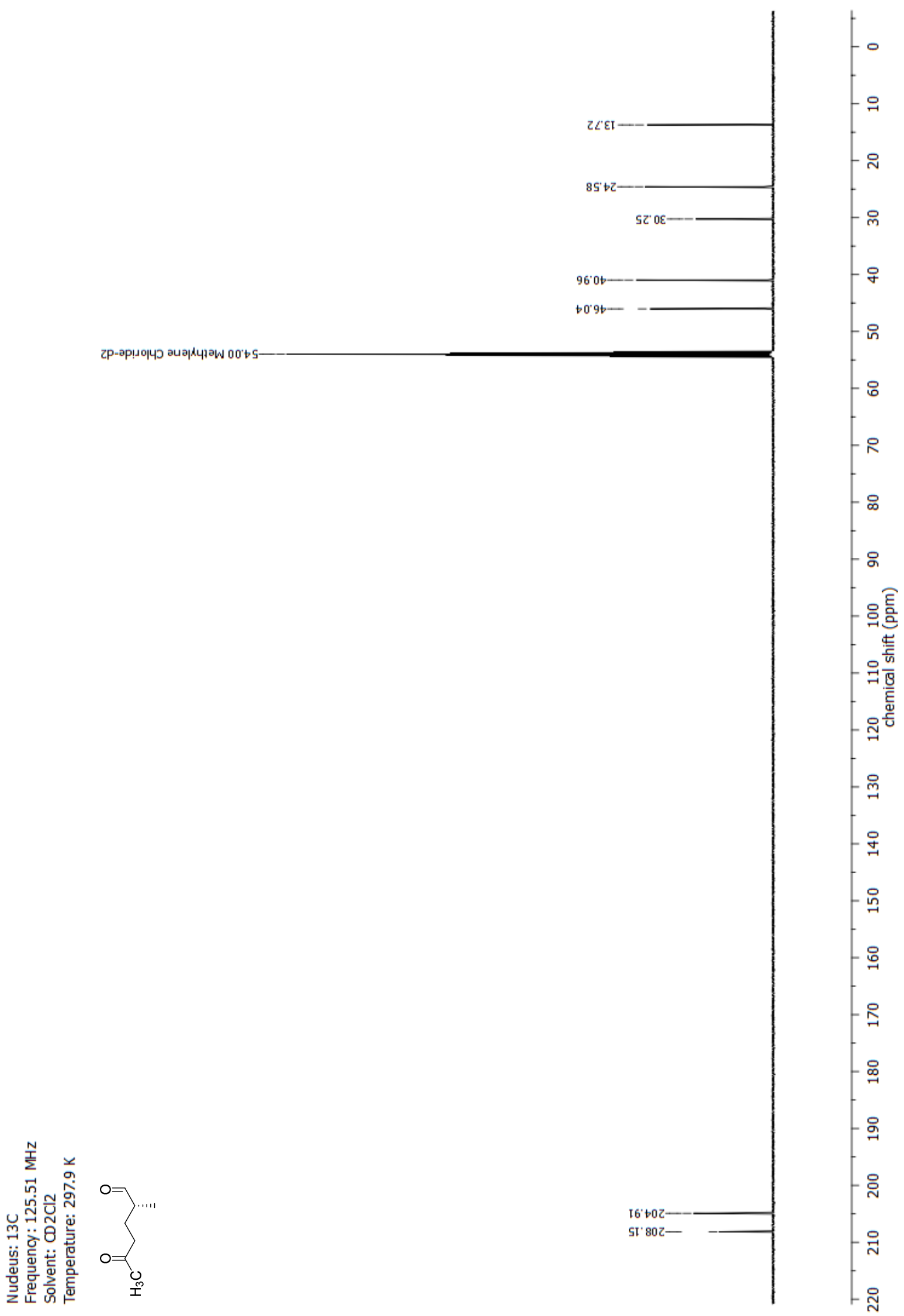


NMR-Spectra for Compound 54

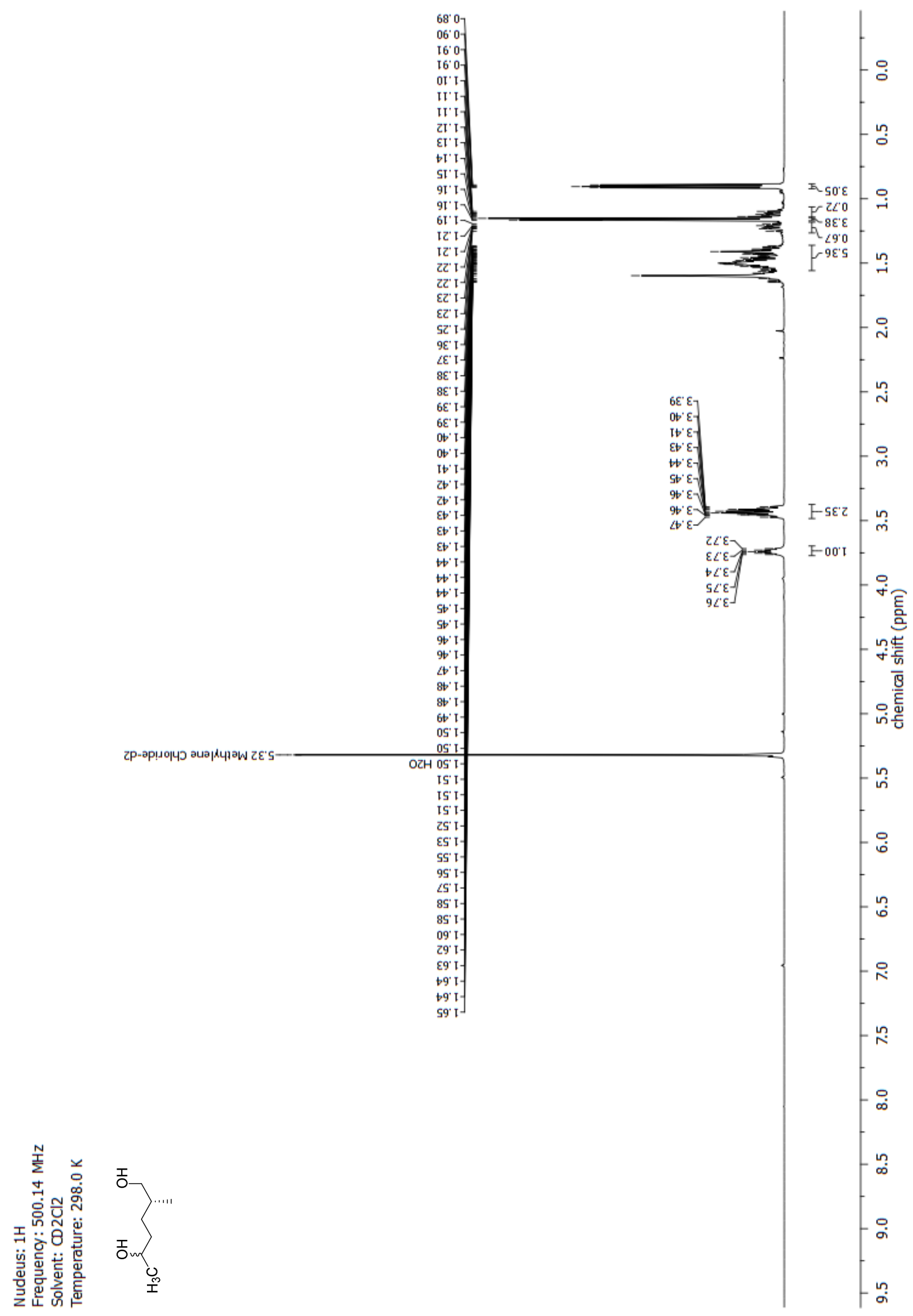




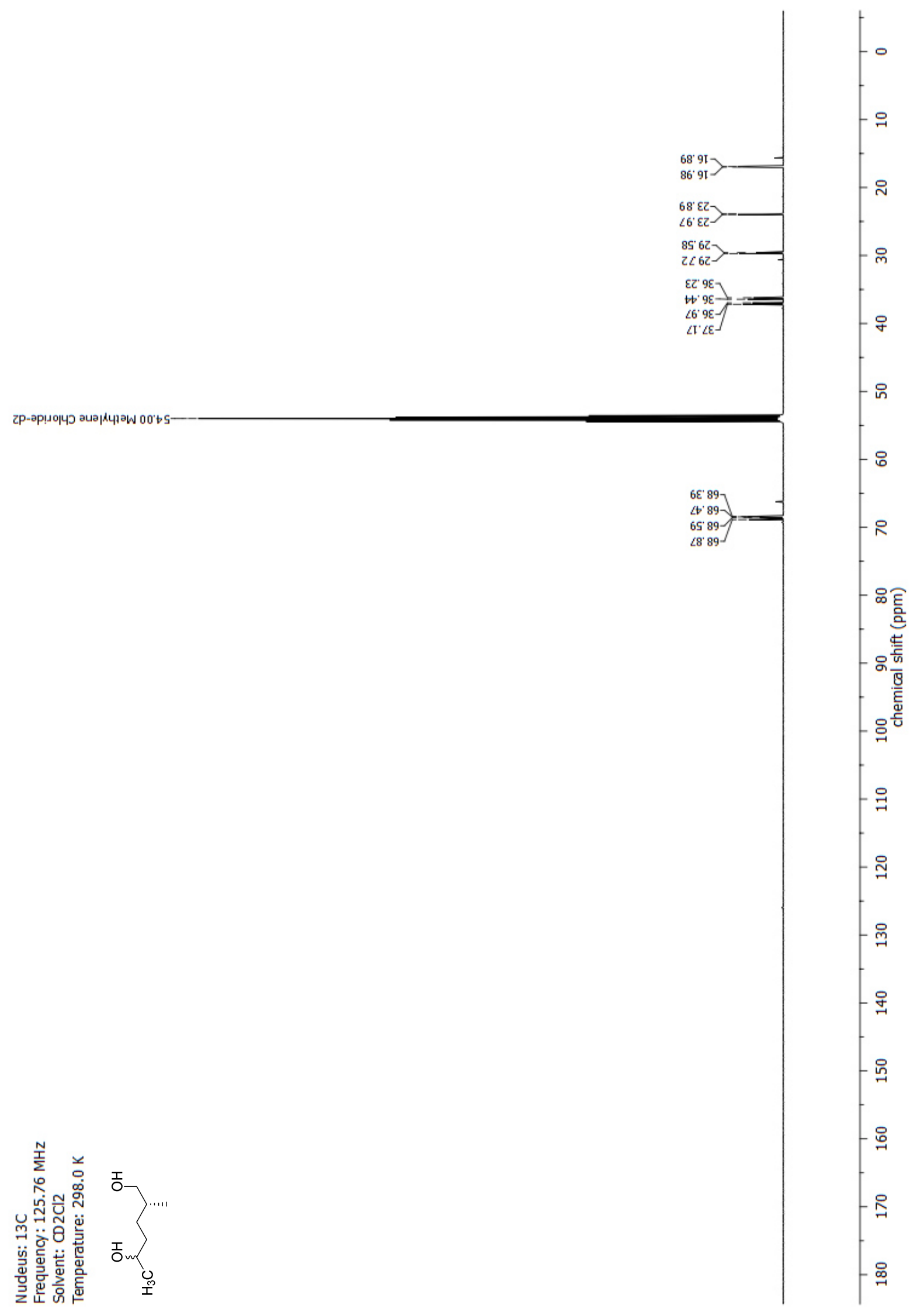


NMR-Spectra for Compound 55

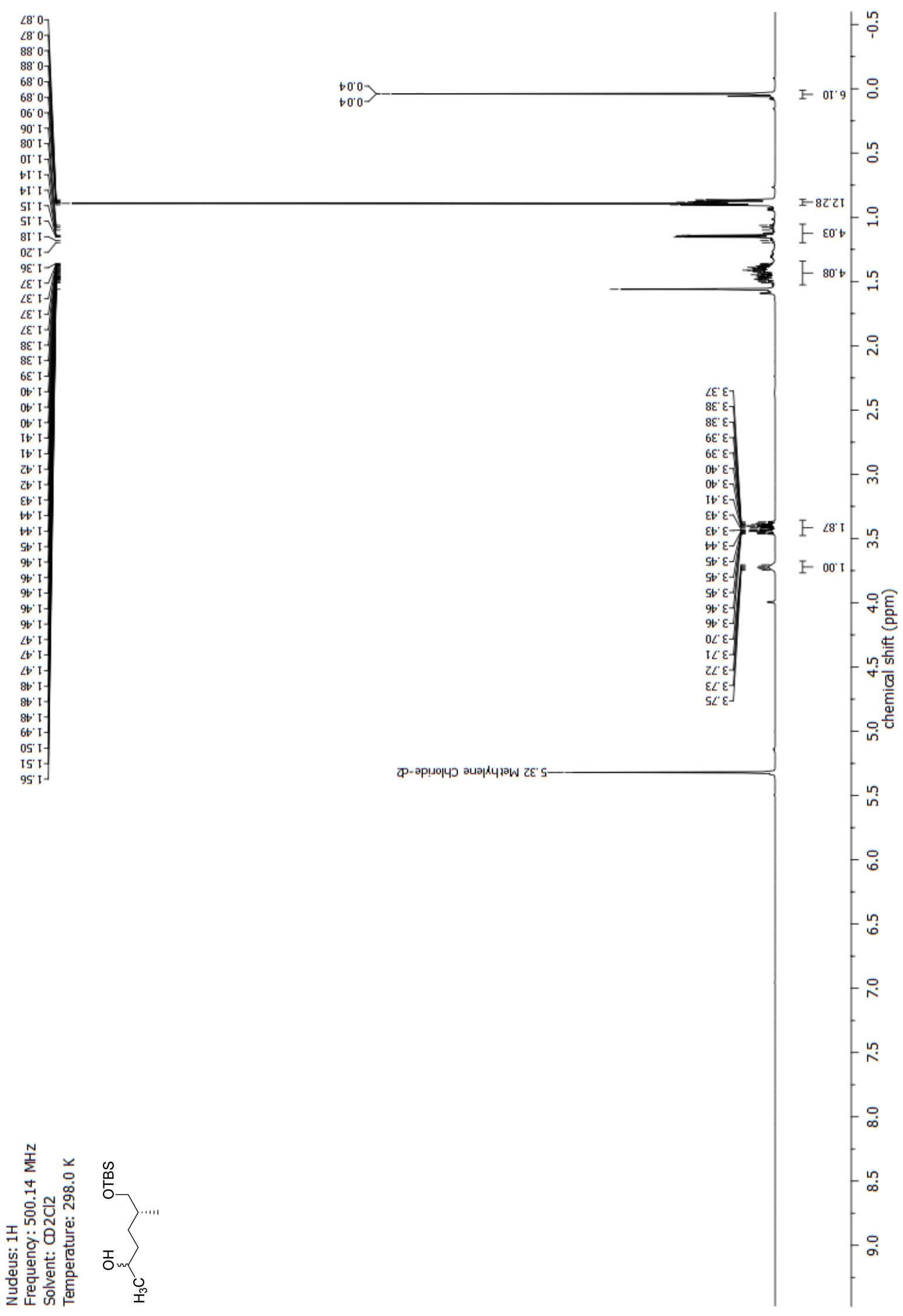




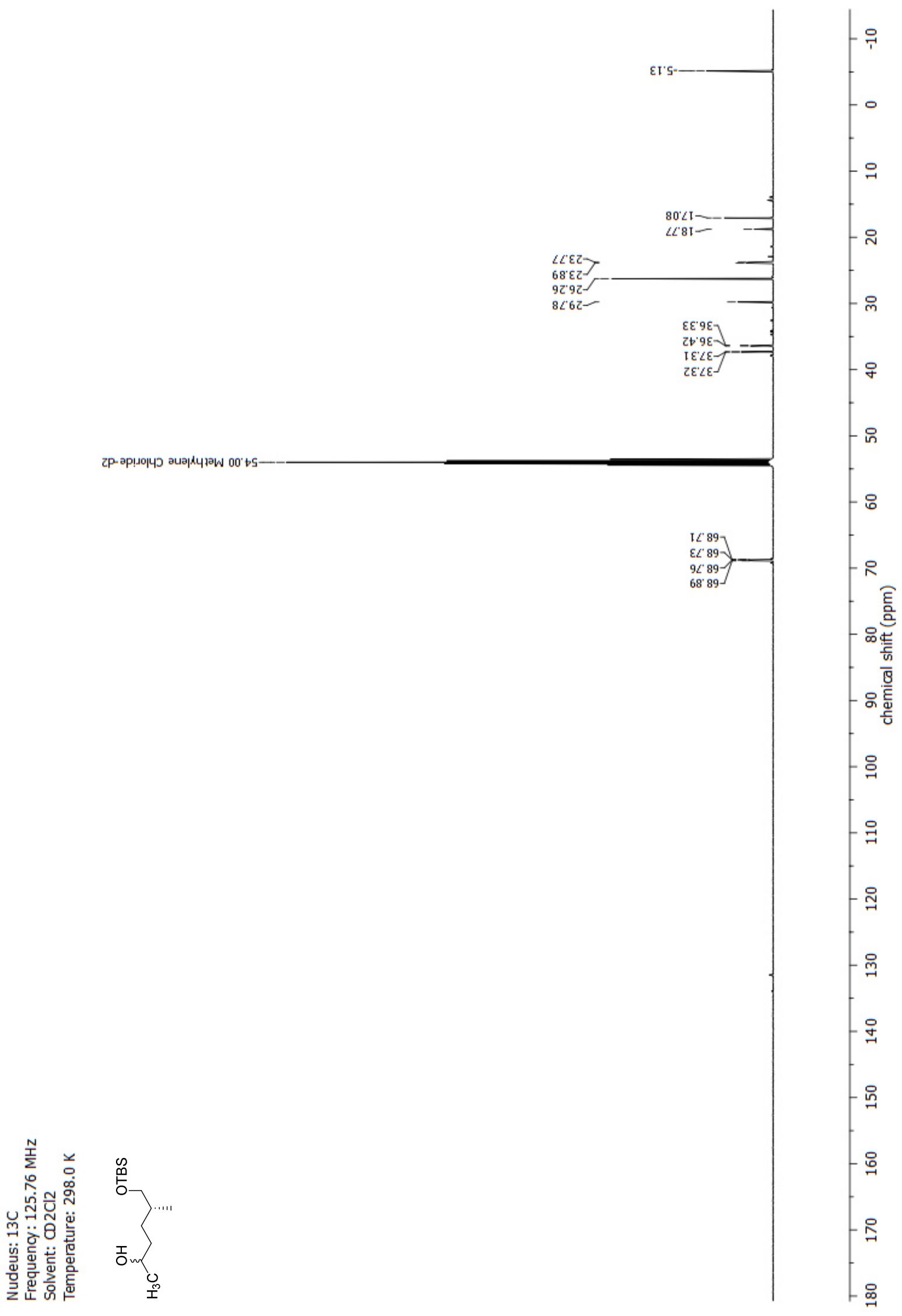


NMR-Spectra for Compound 11

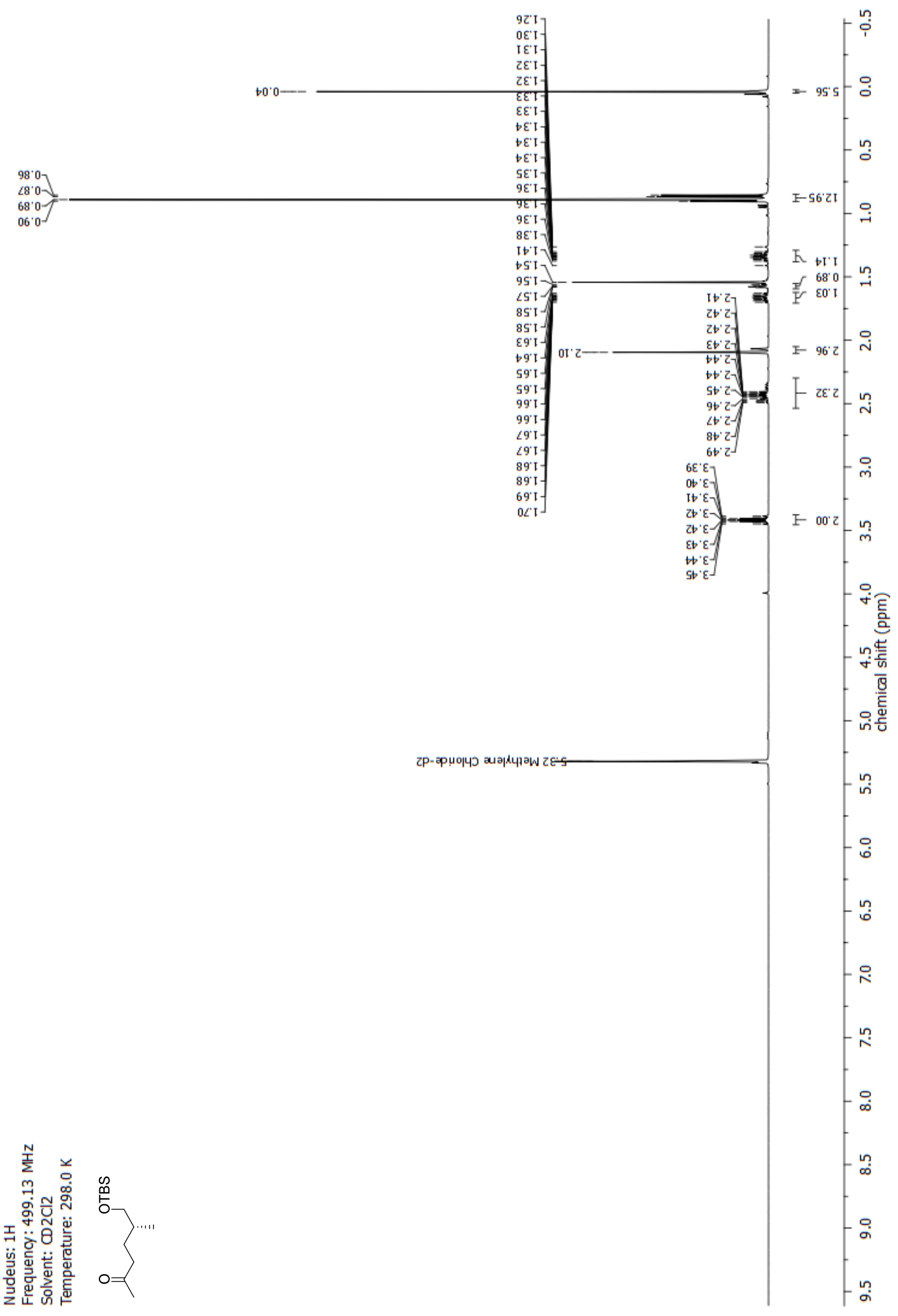




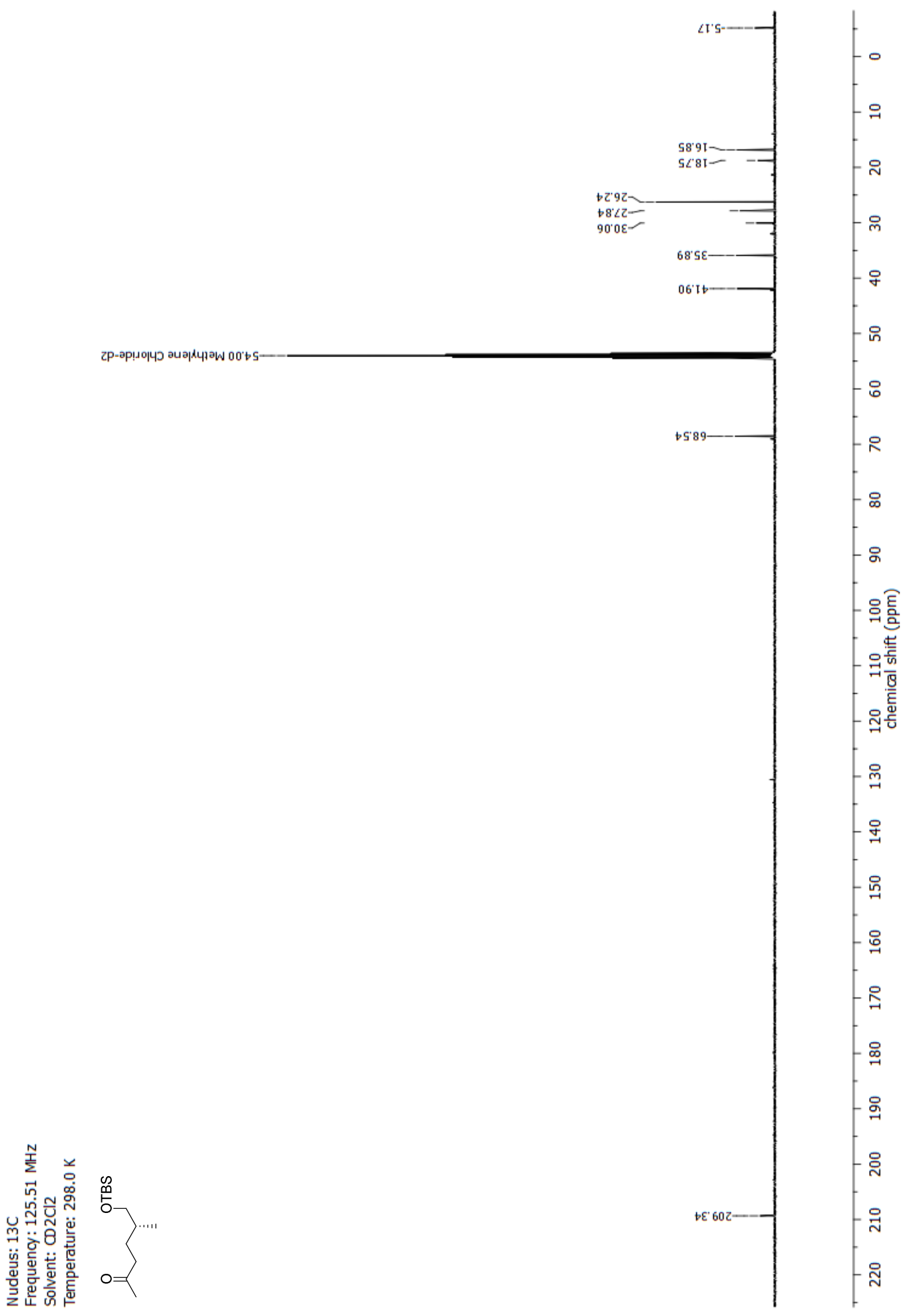


NMR-Spectra for Compound 56

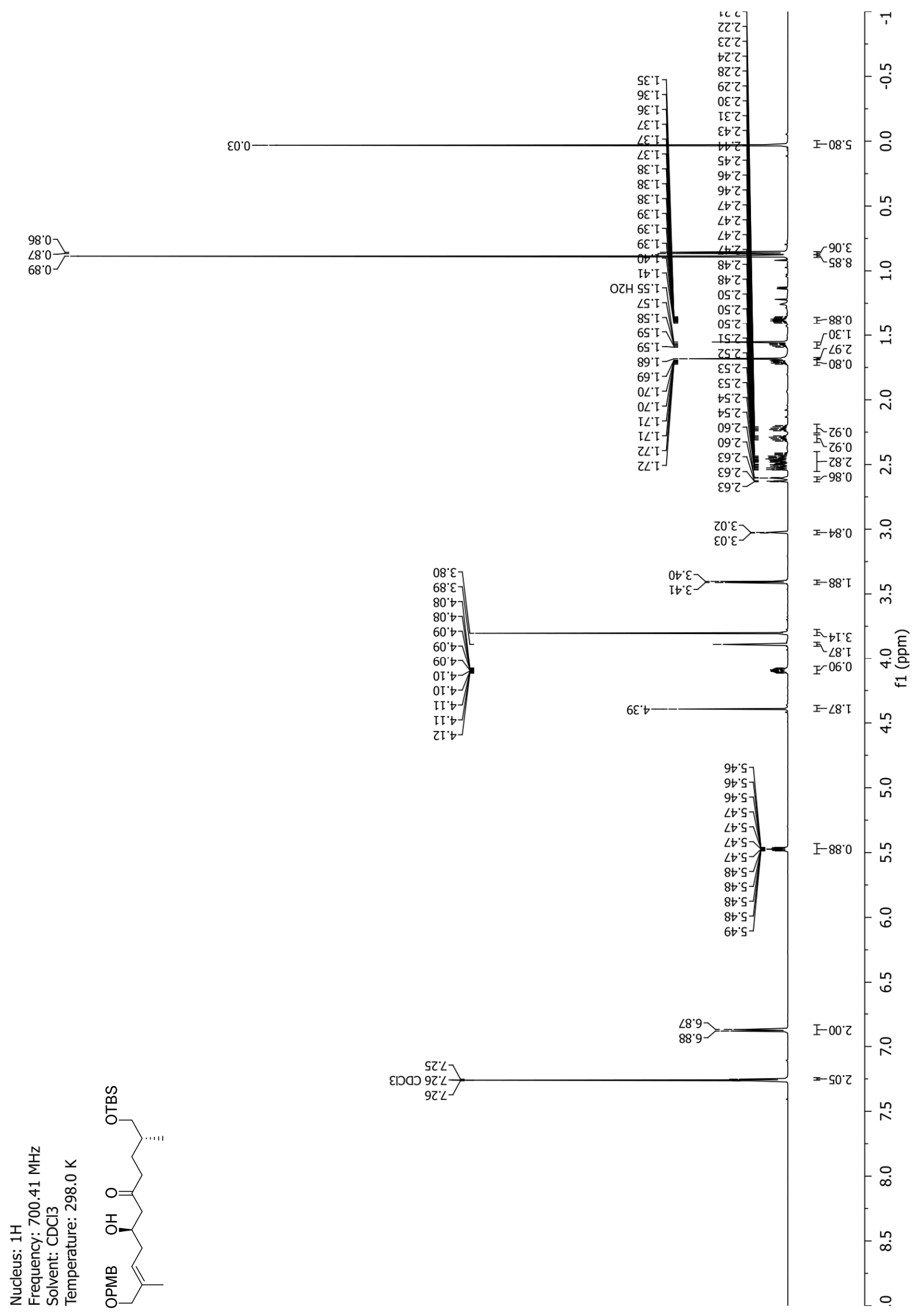




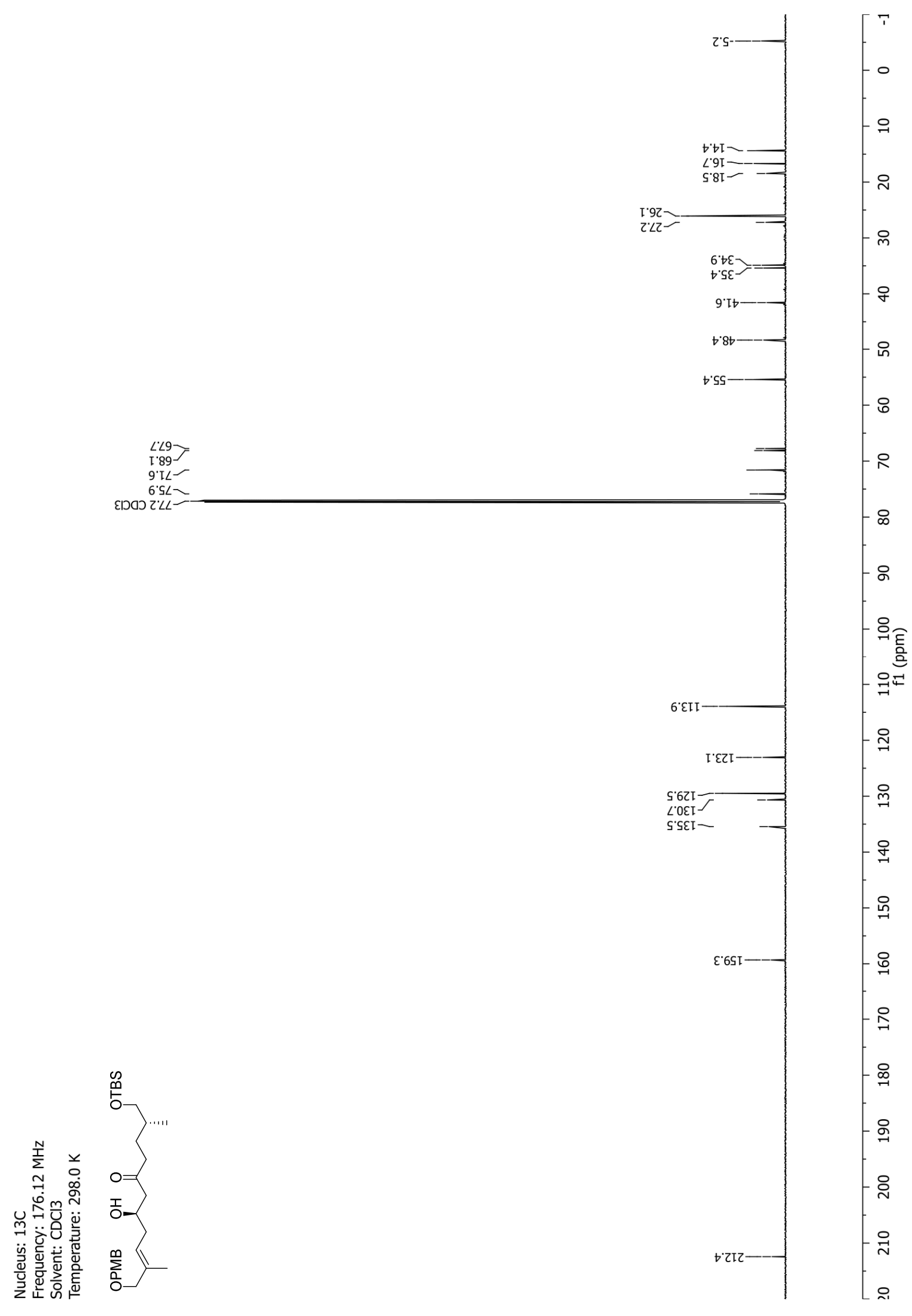




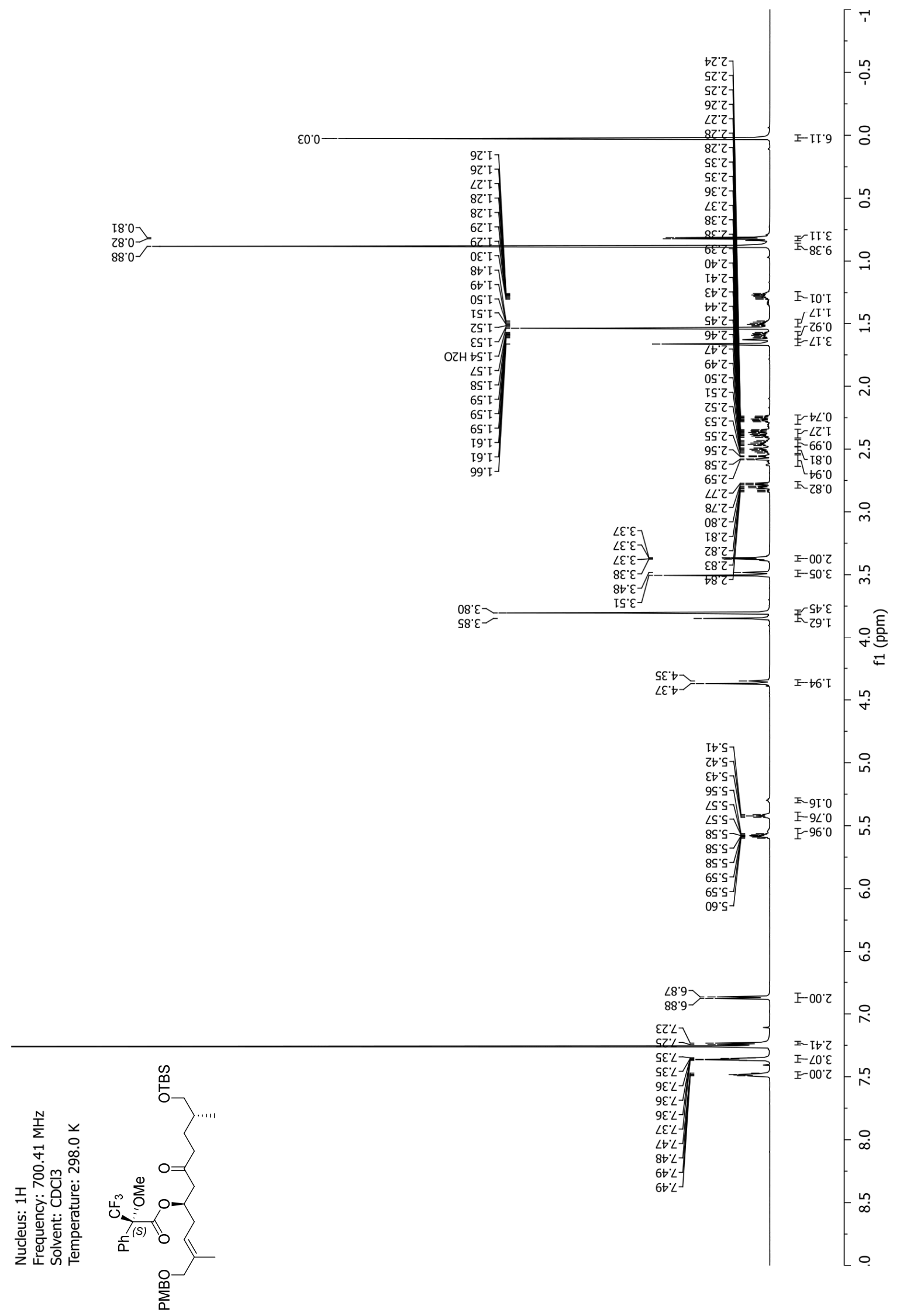




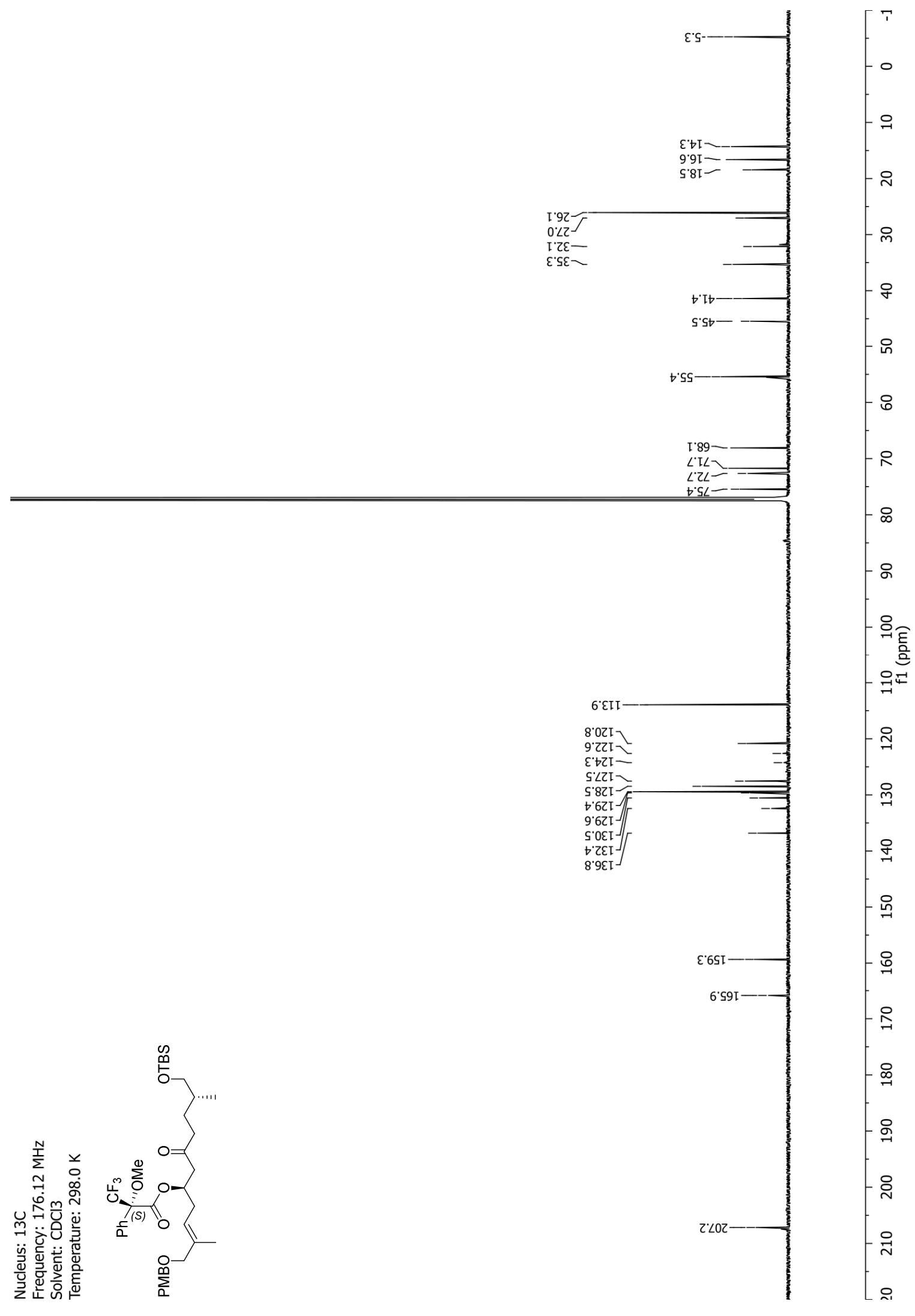


NMR-Spectra for Compound 58

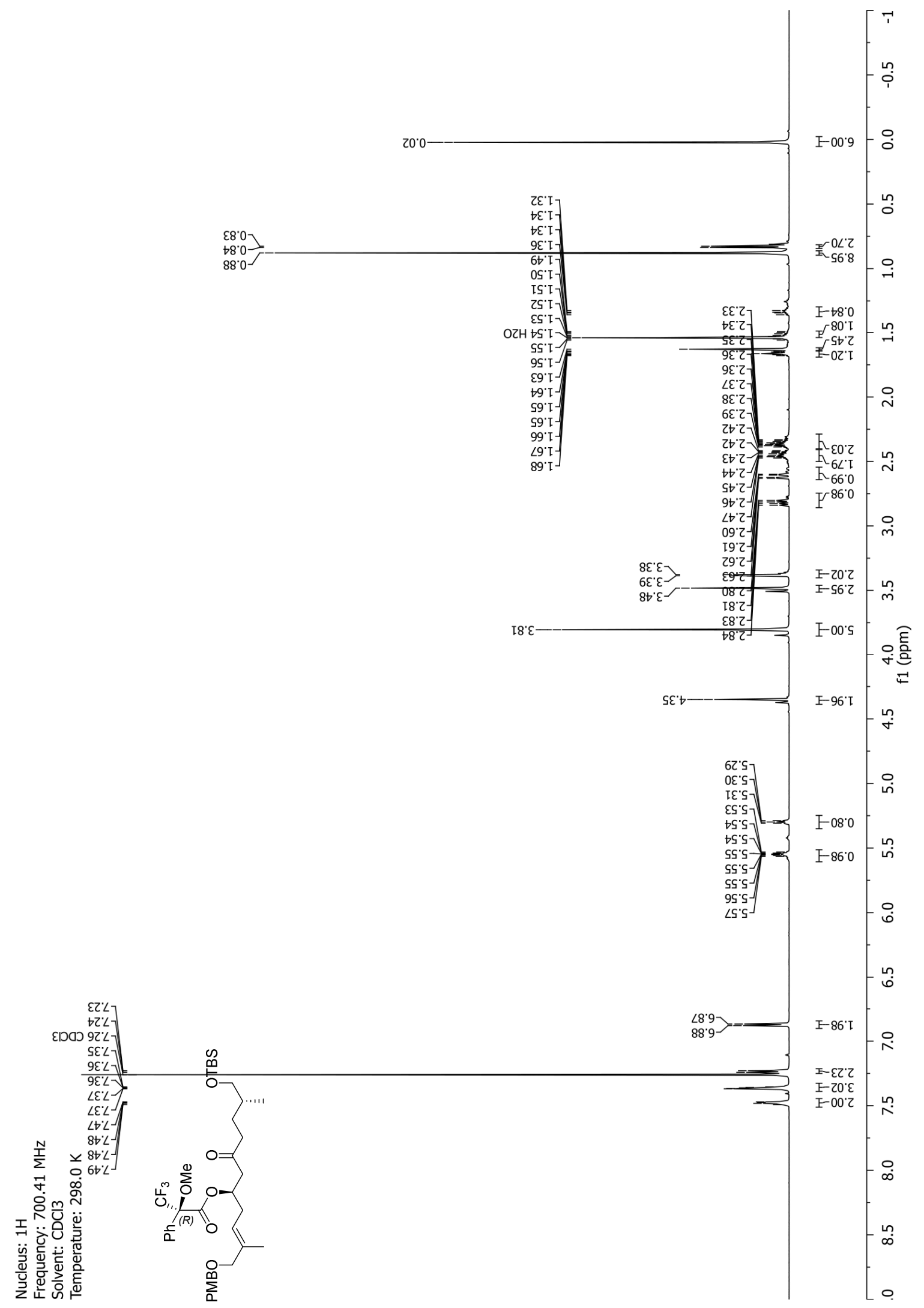




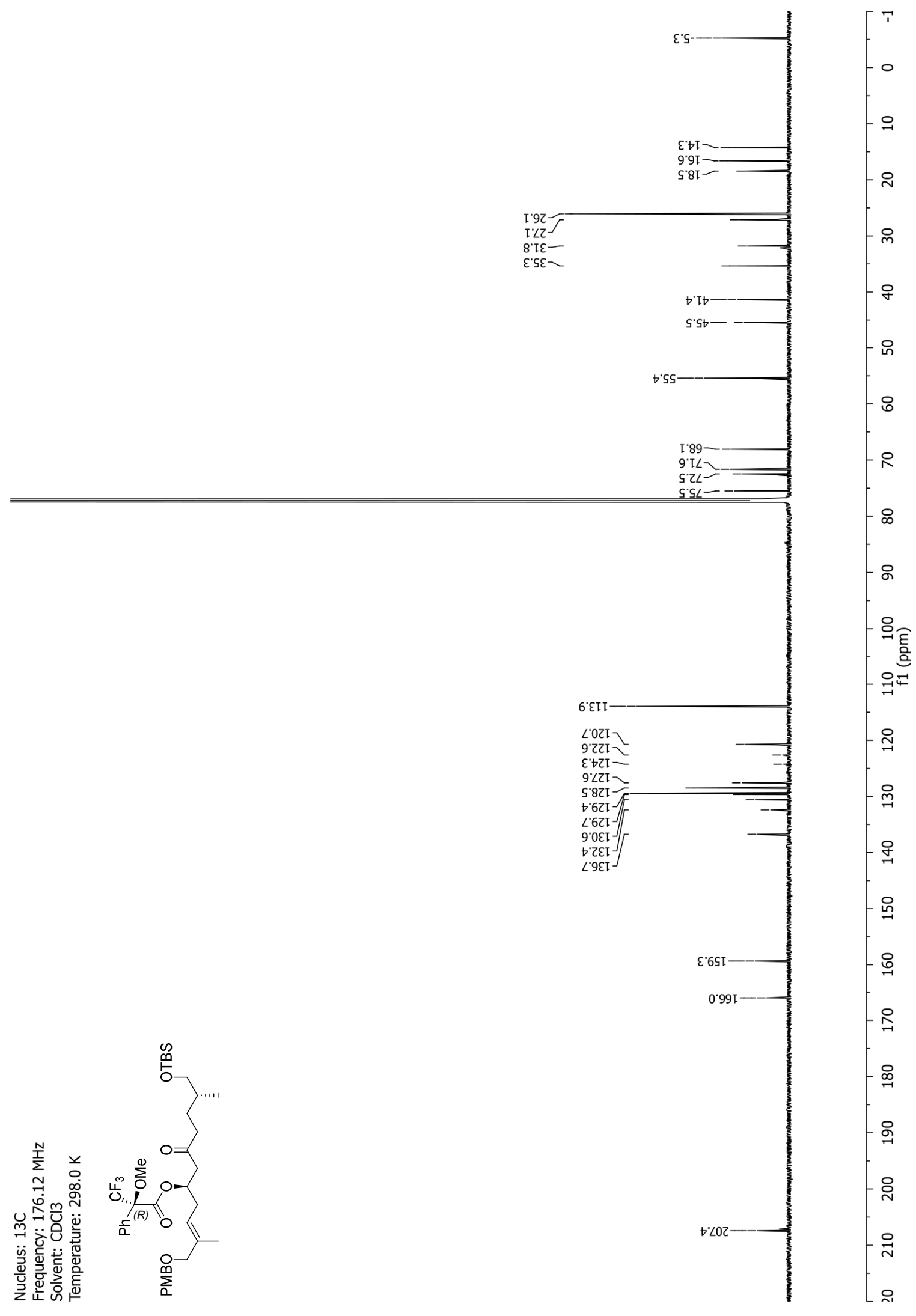


NMR-Spectra for Compound 59

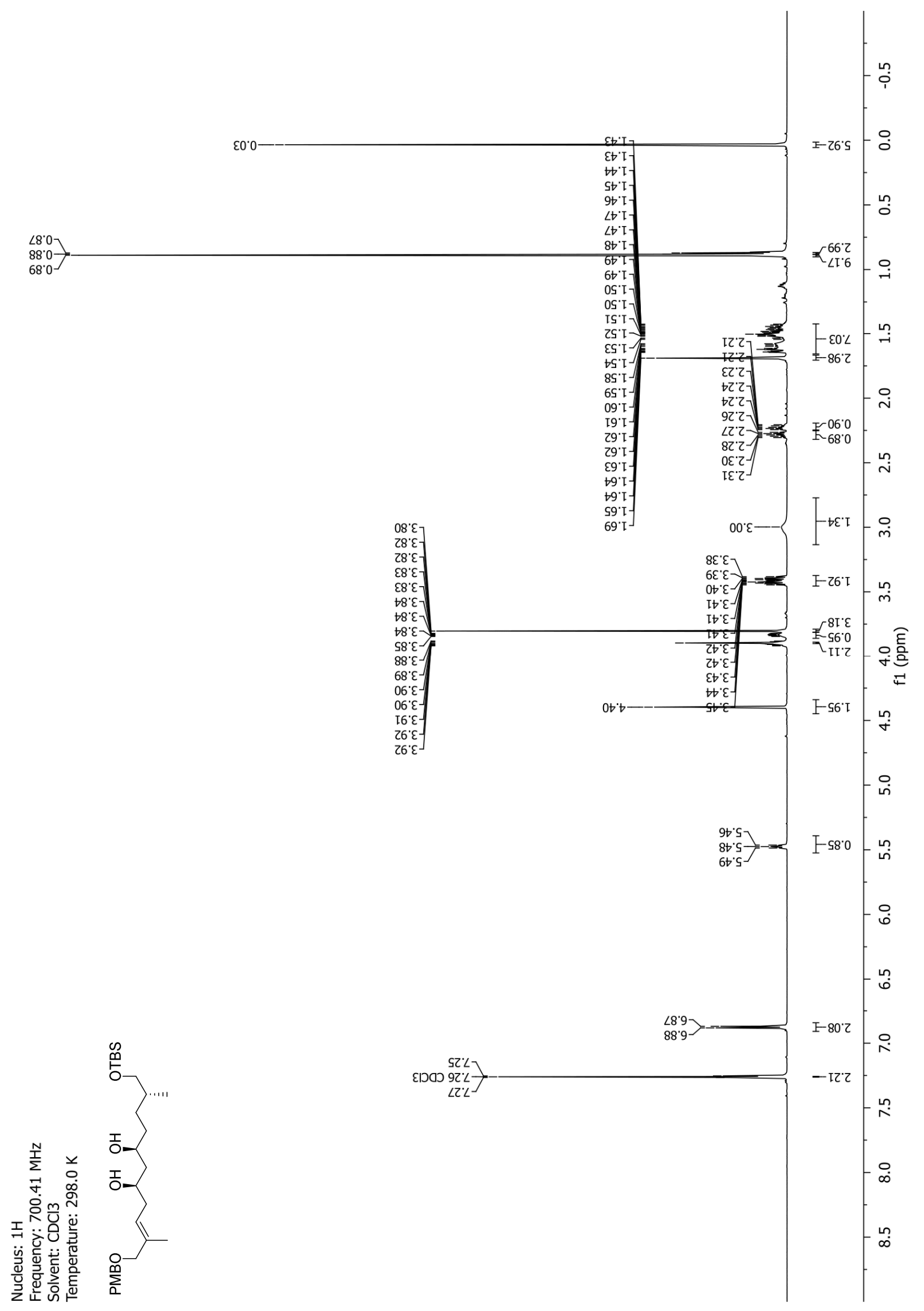




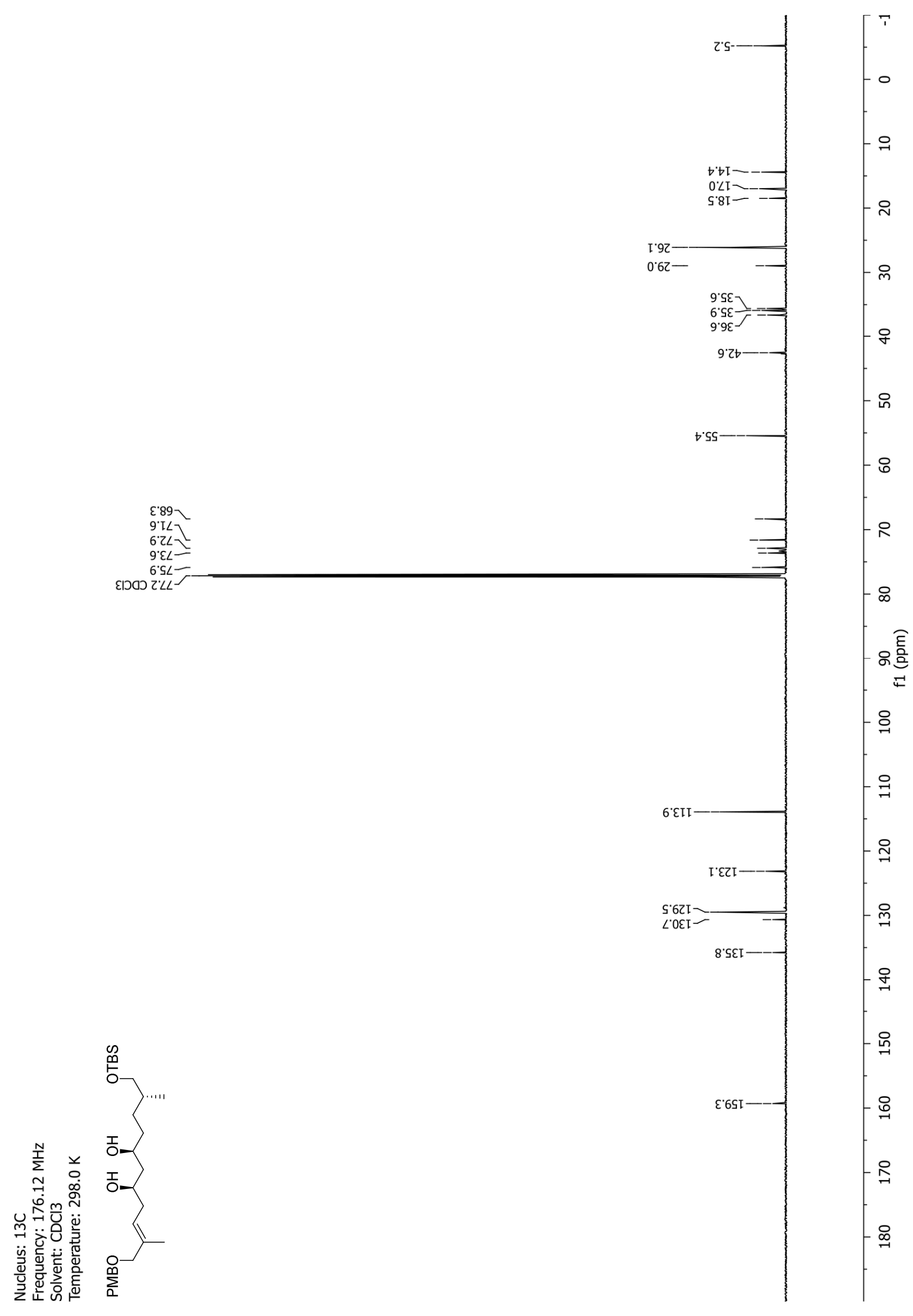




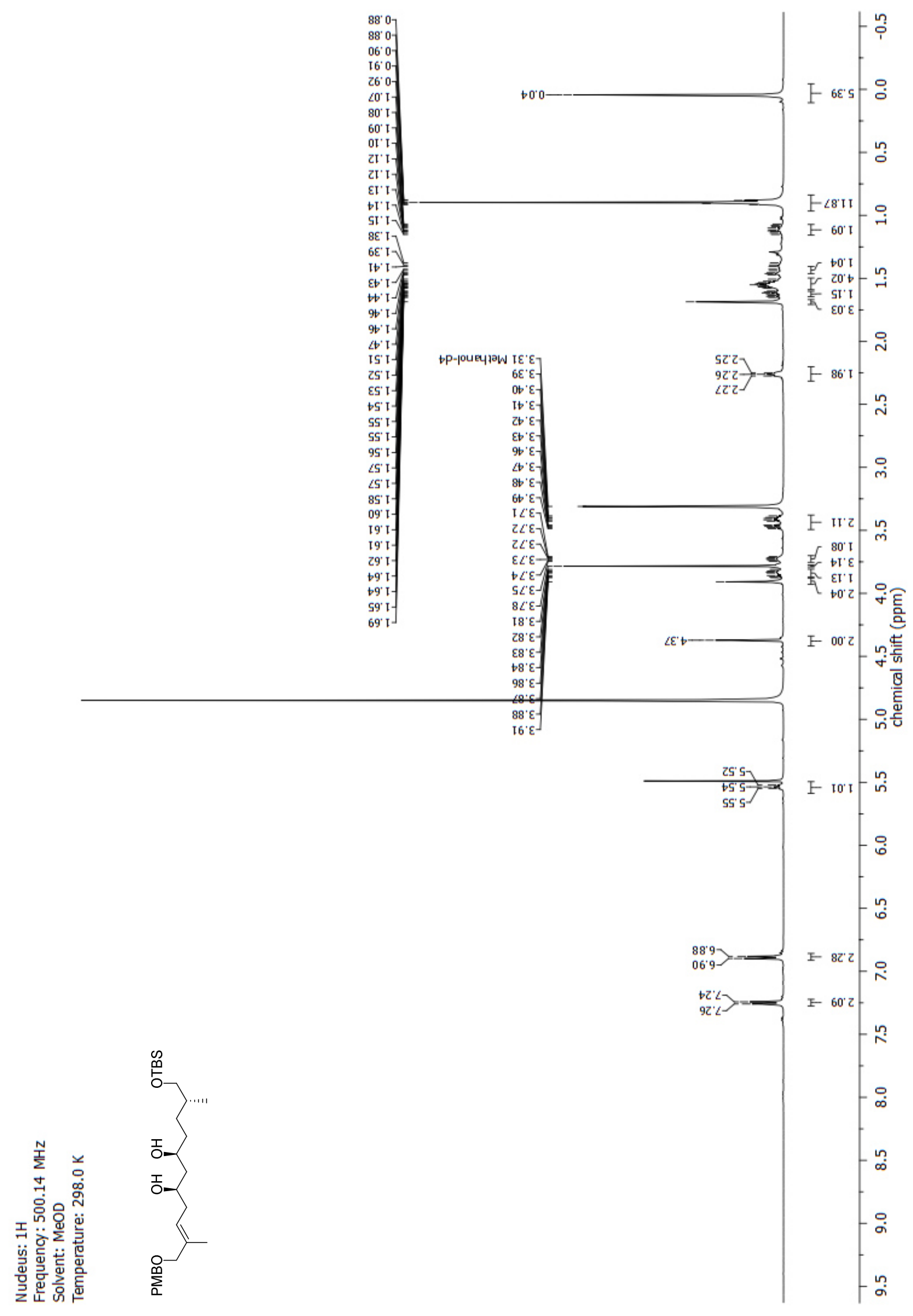




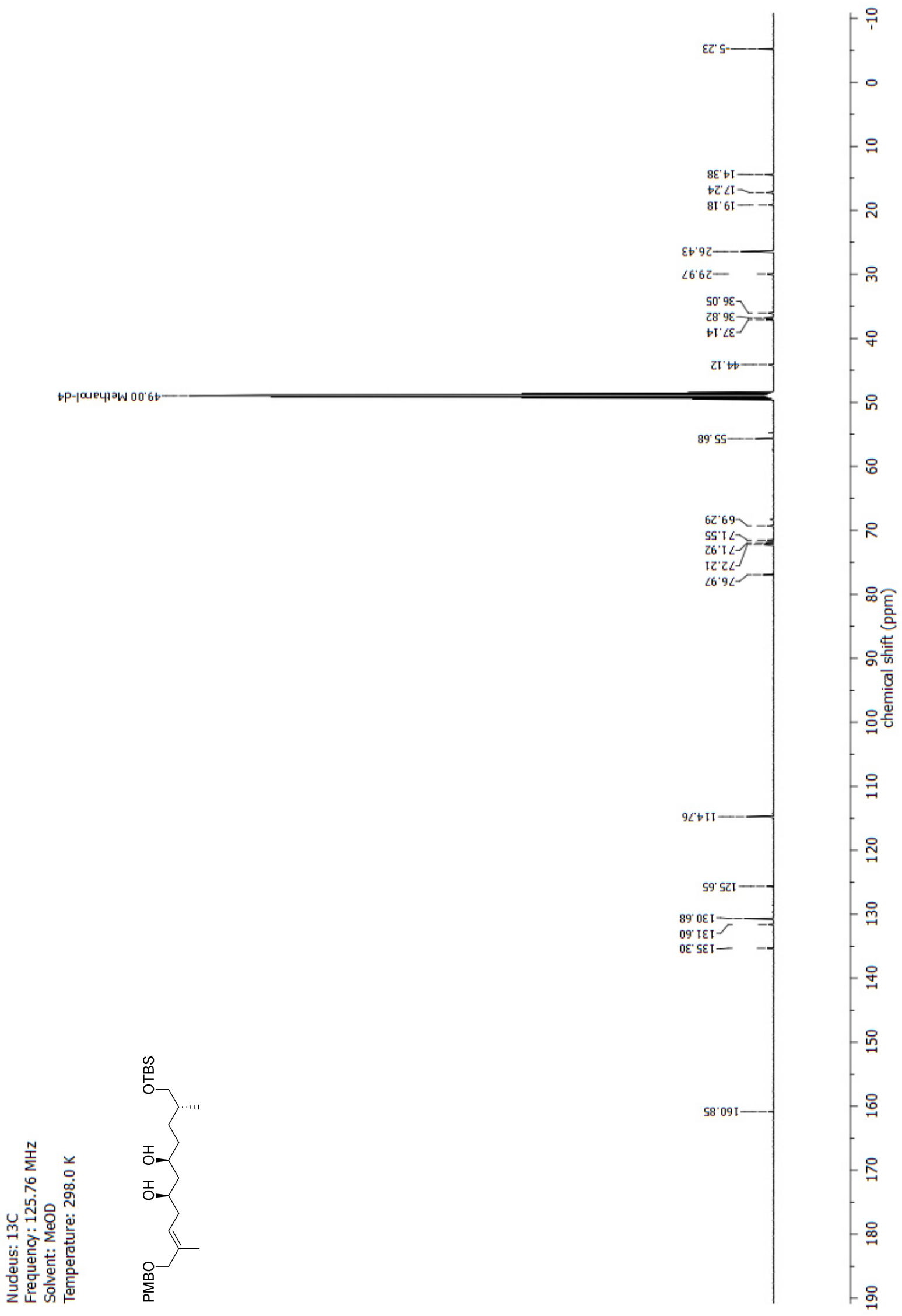


NMR-Spectra for Compound 13

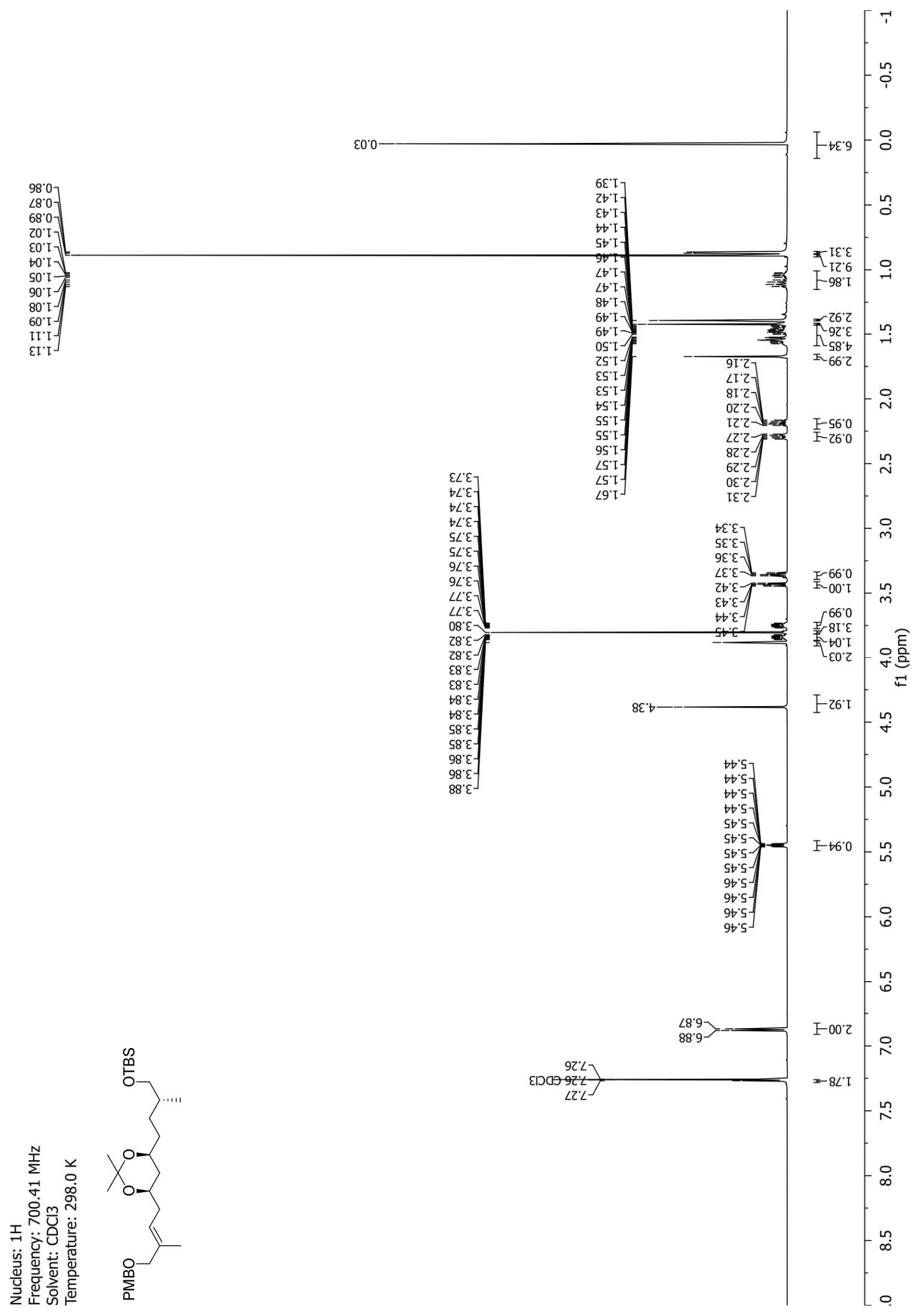




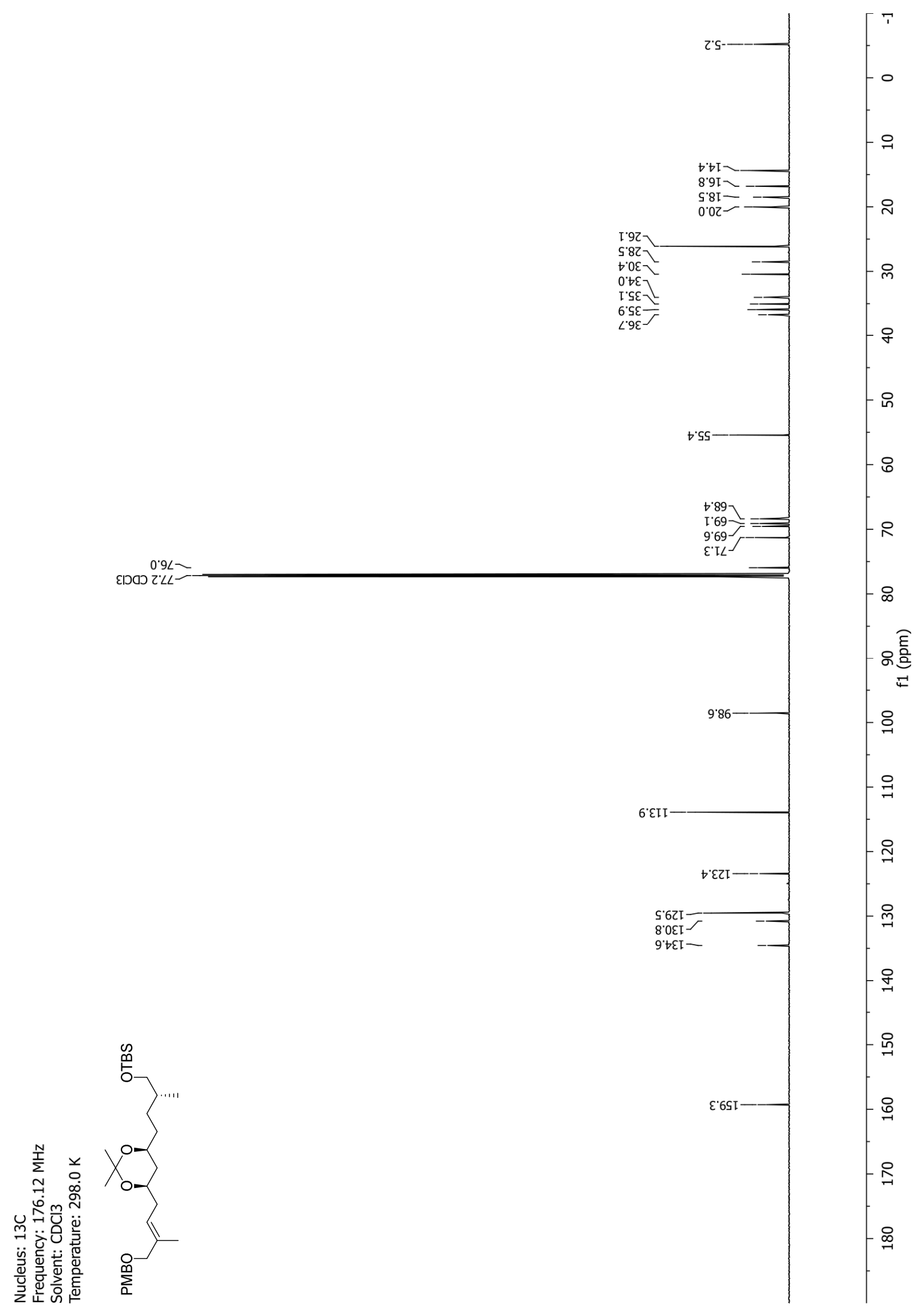




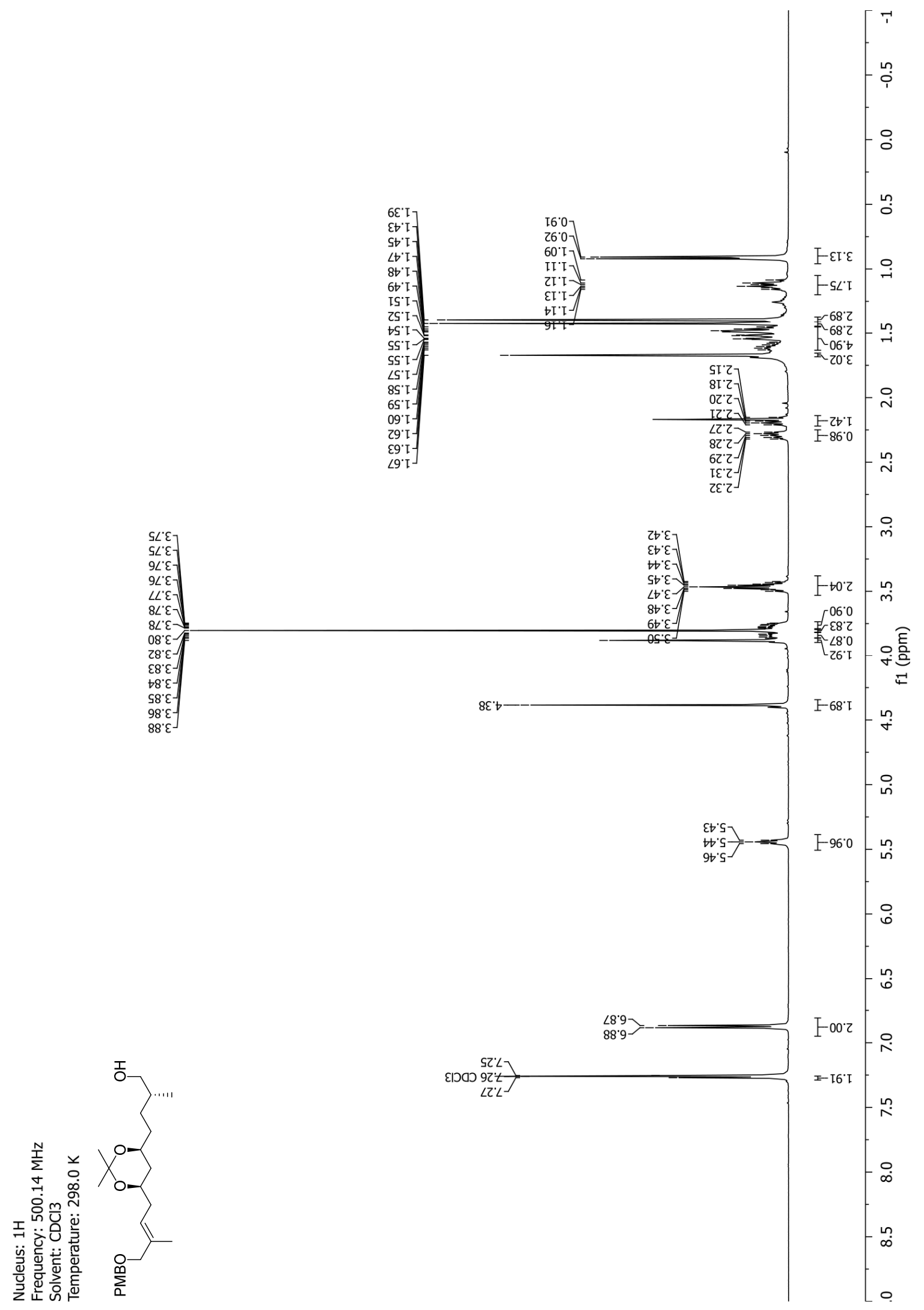




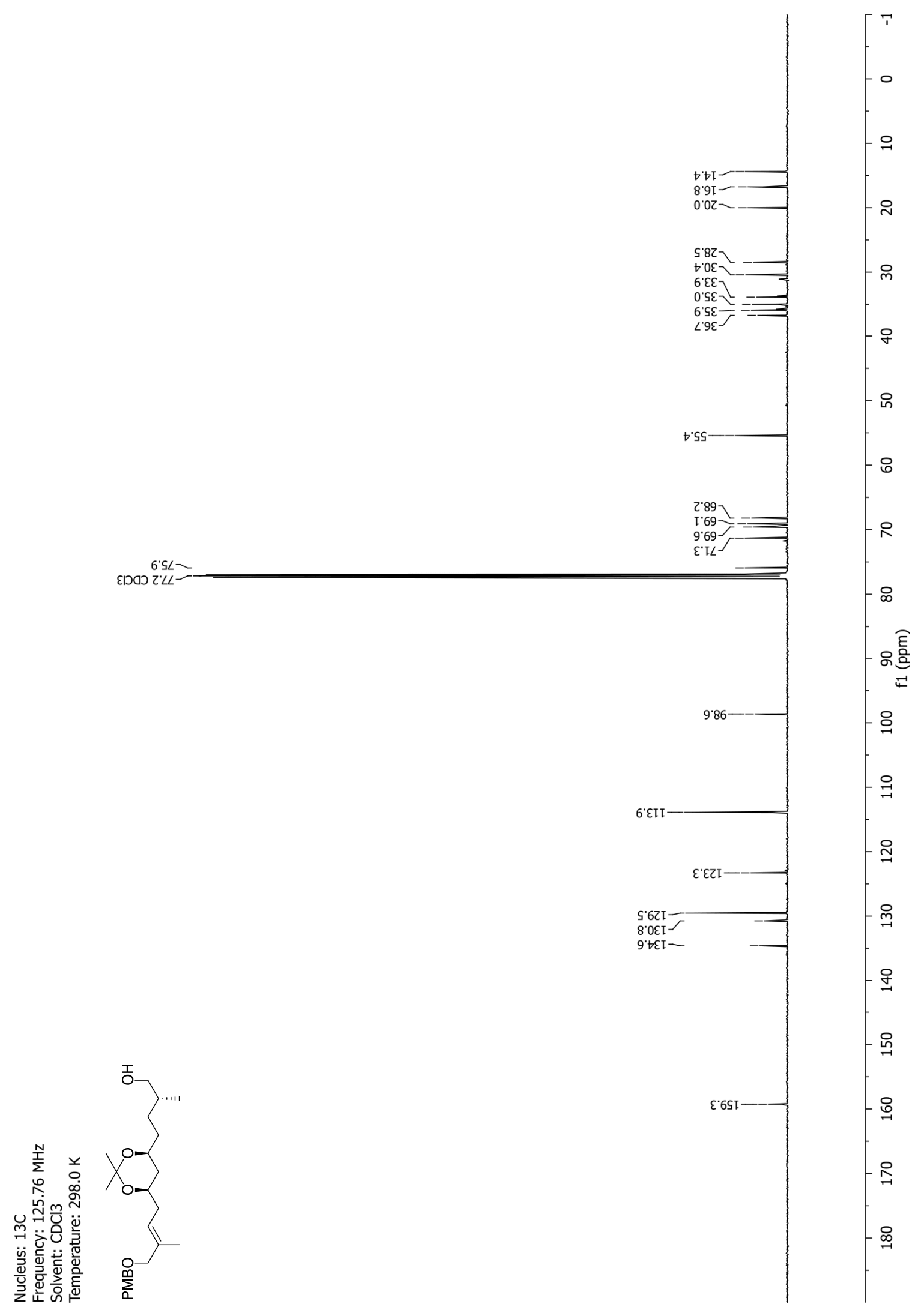




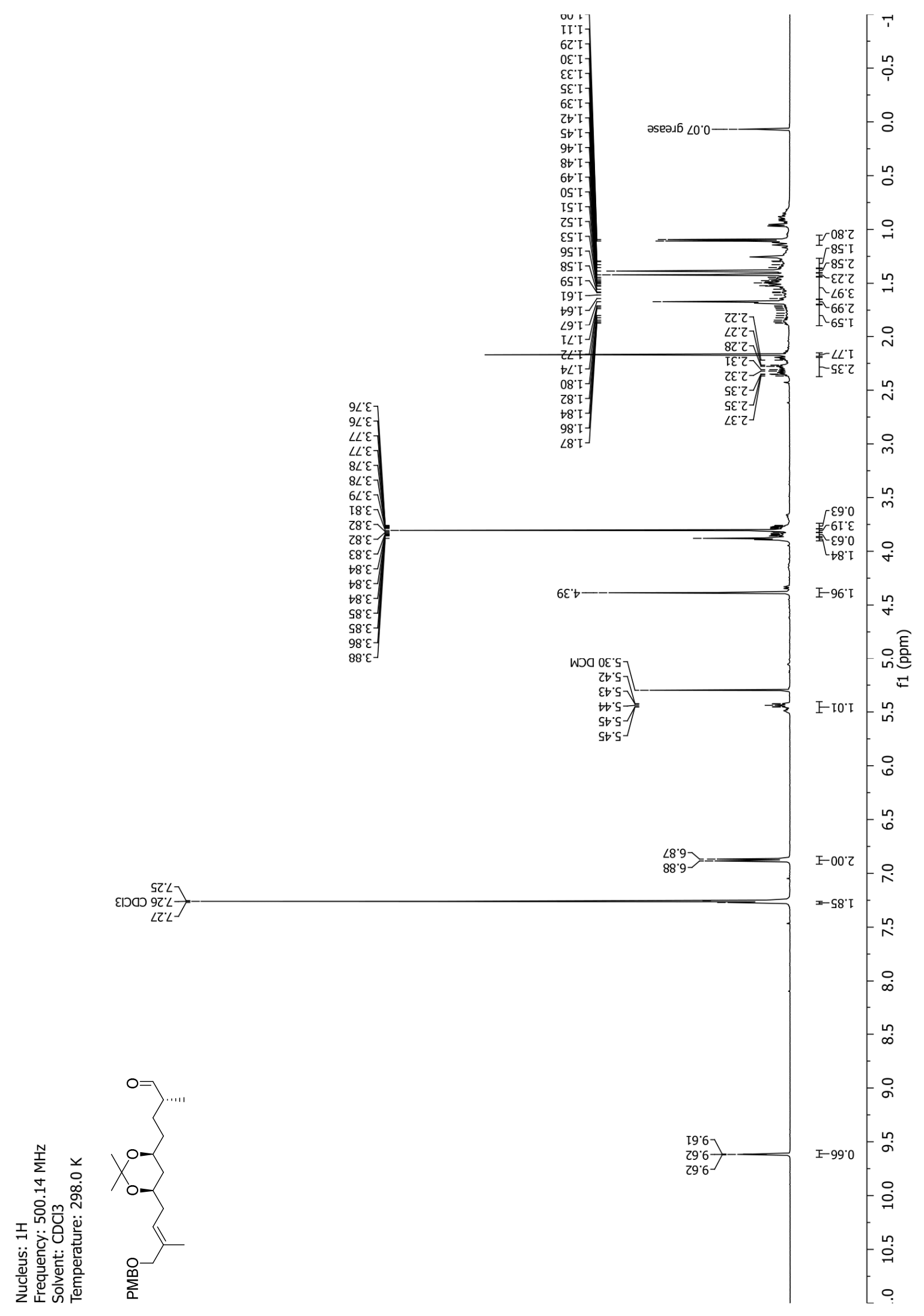




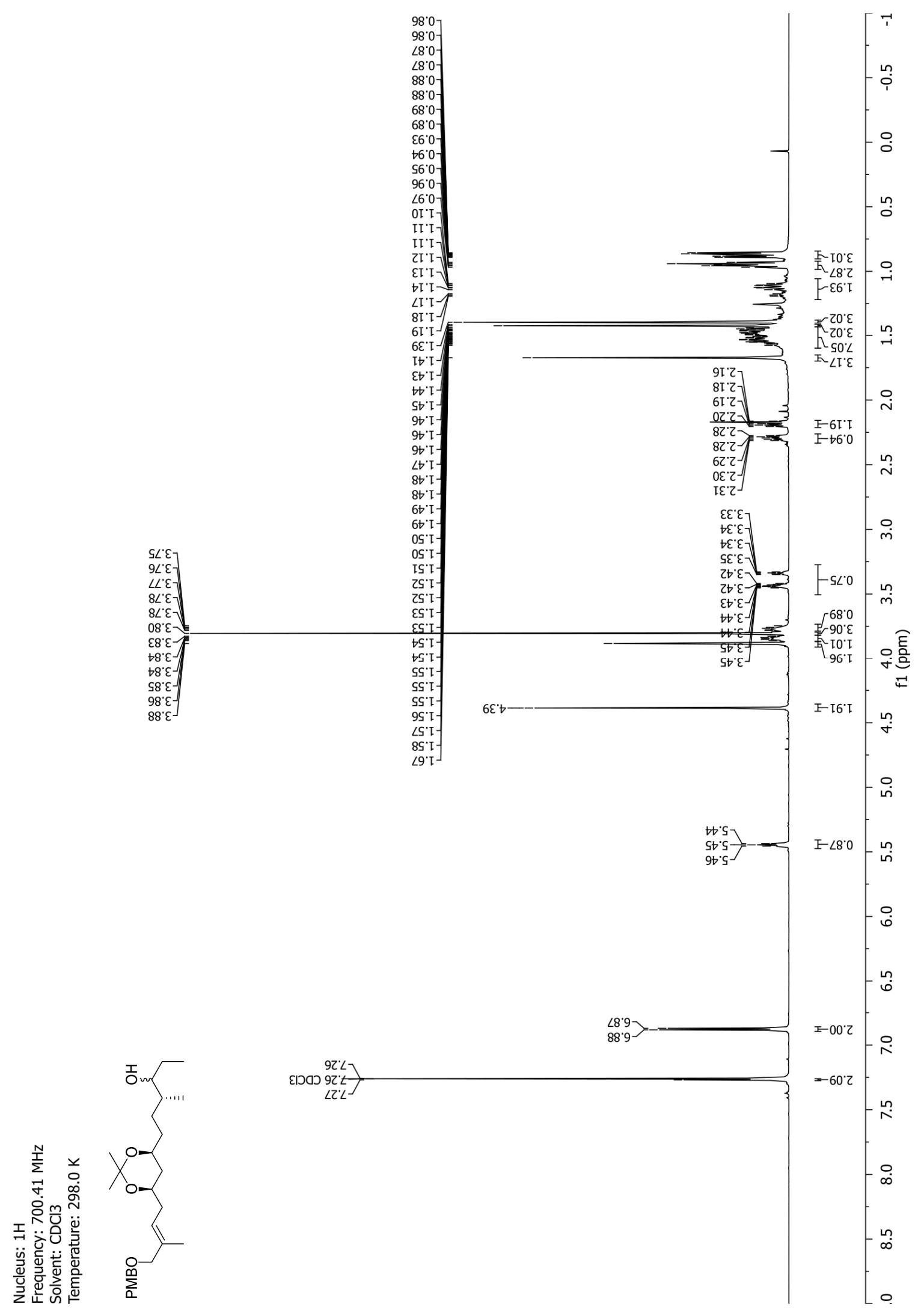




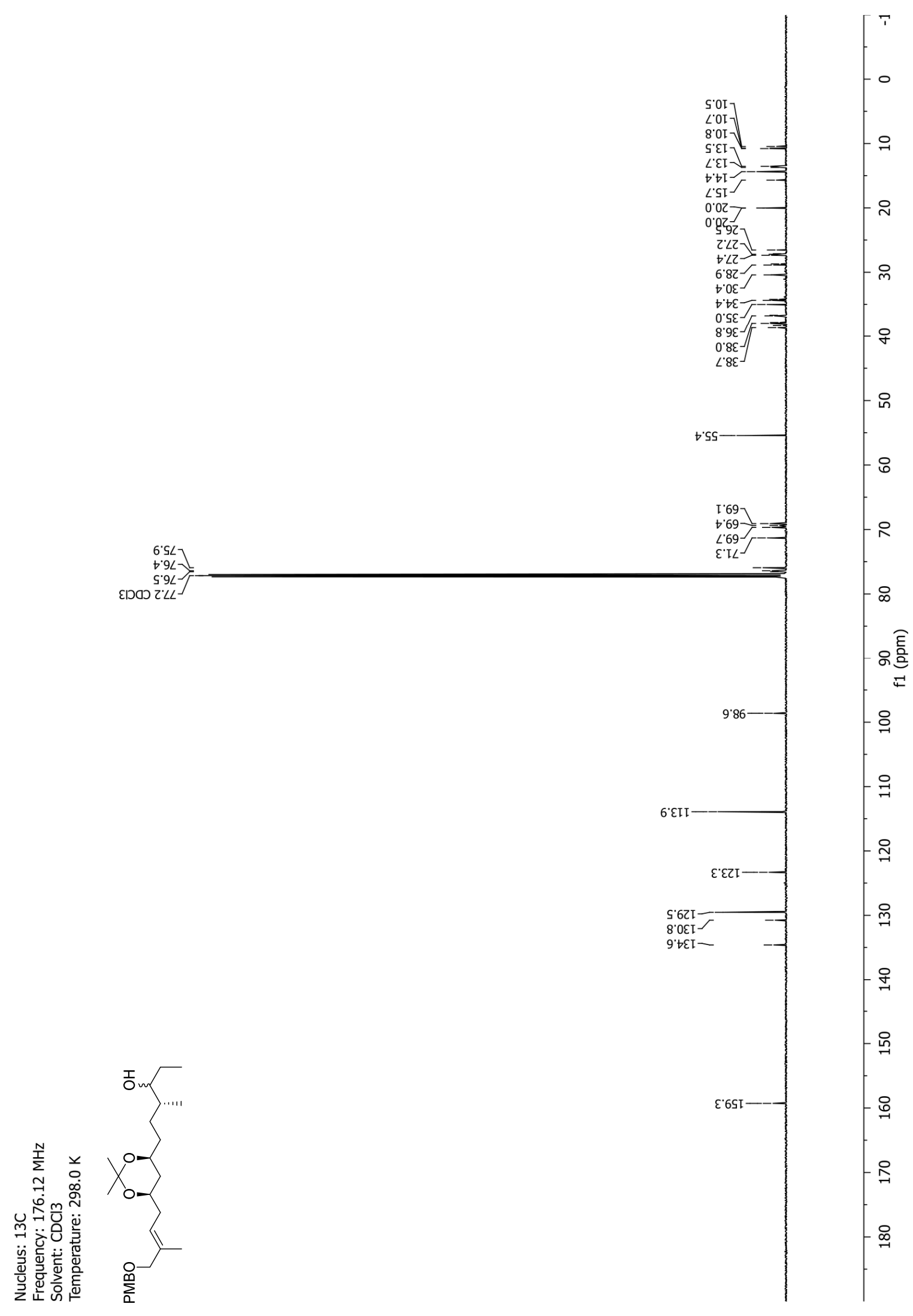




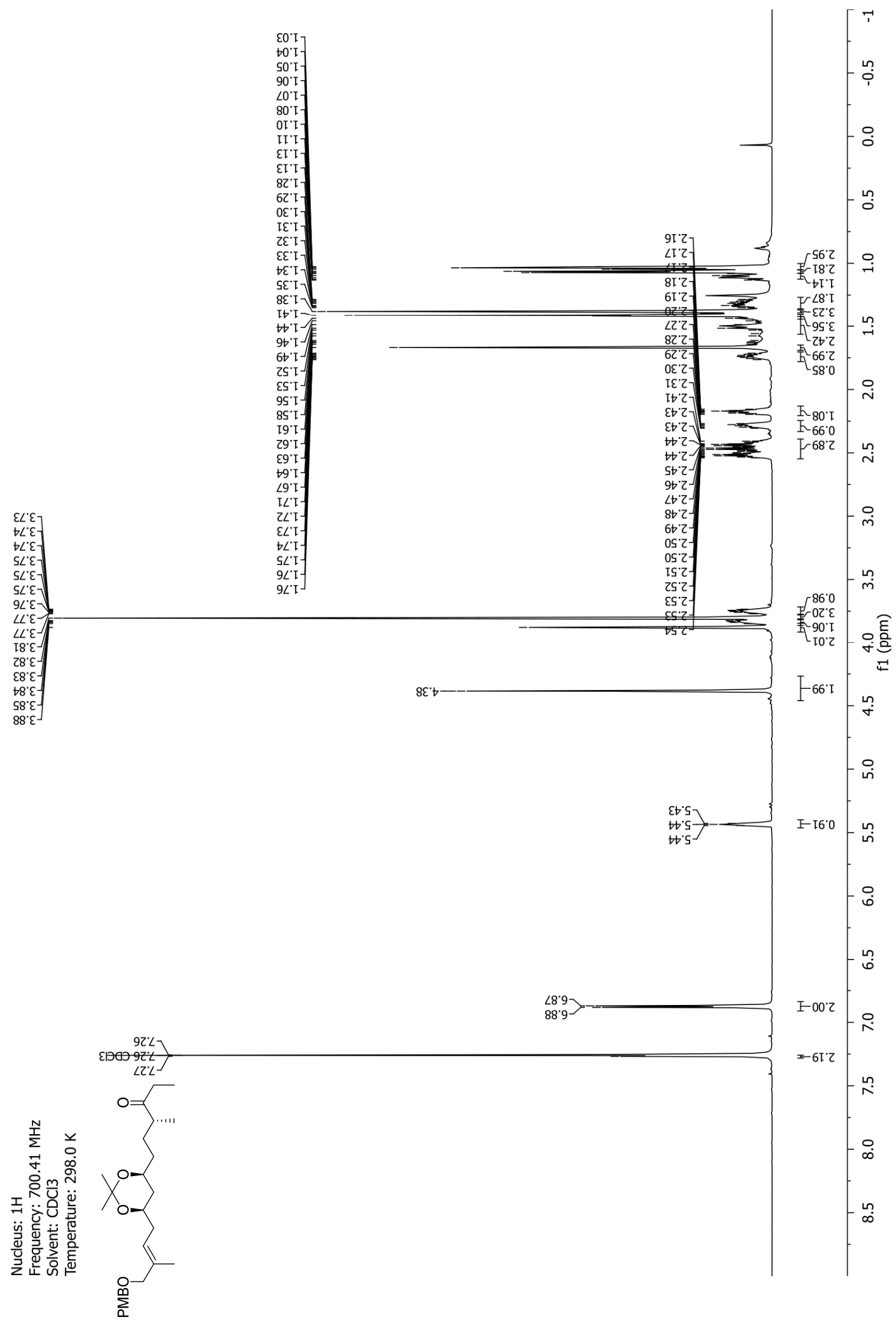




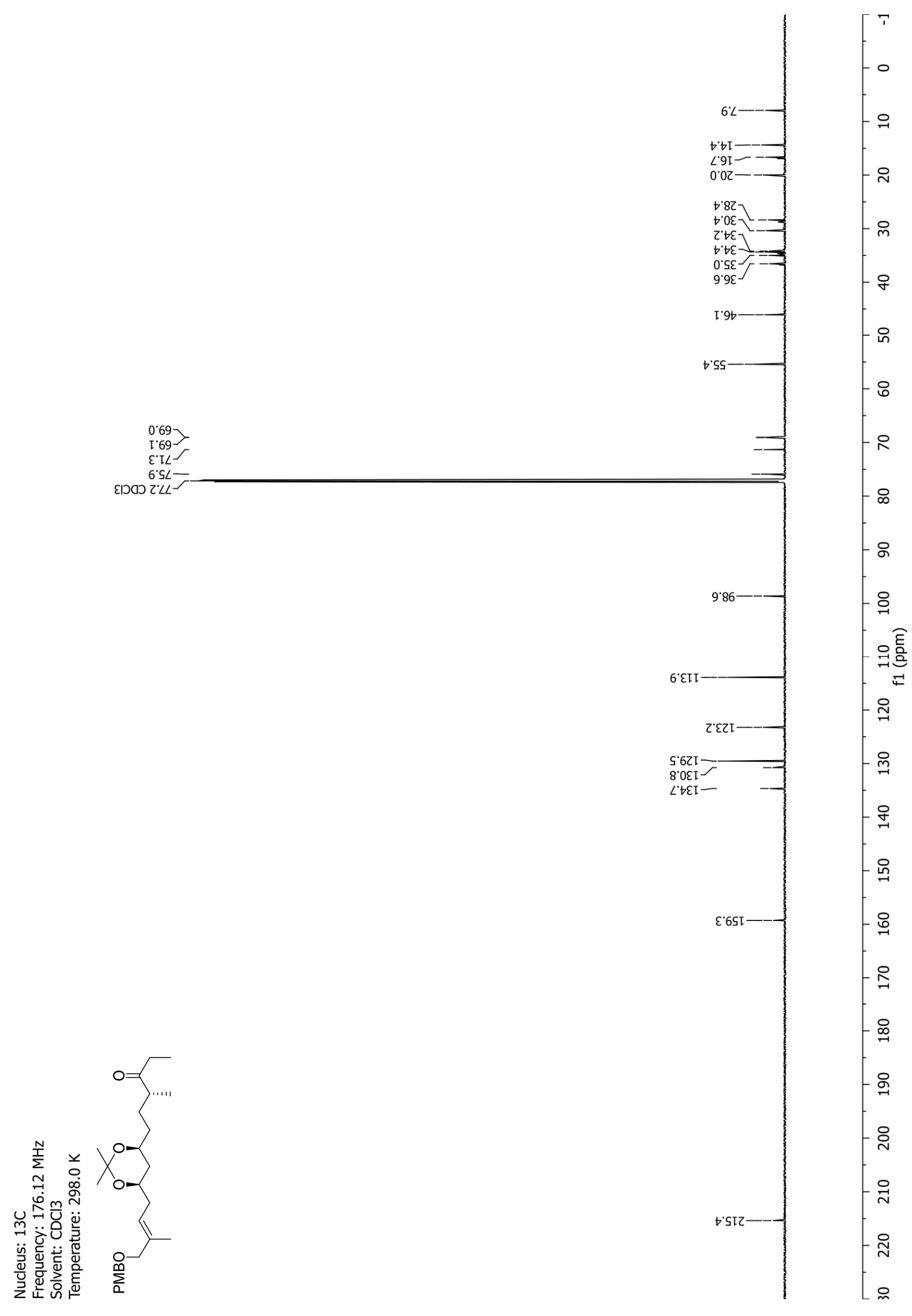


NMR-Spectra for Compound 63

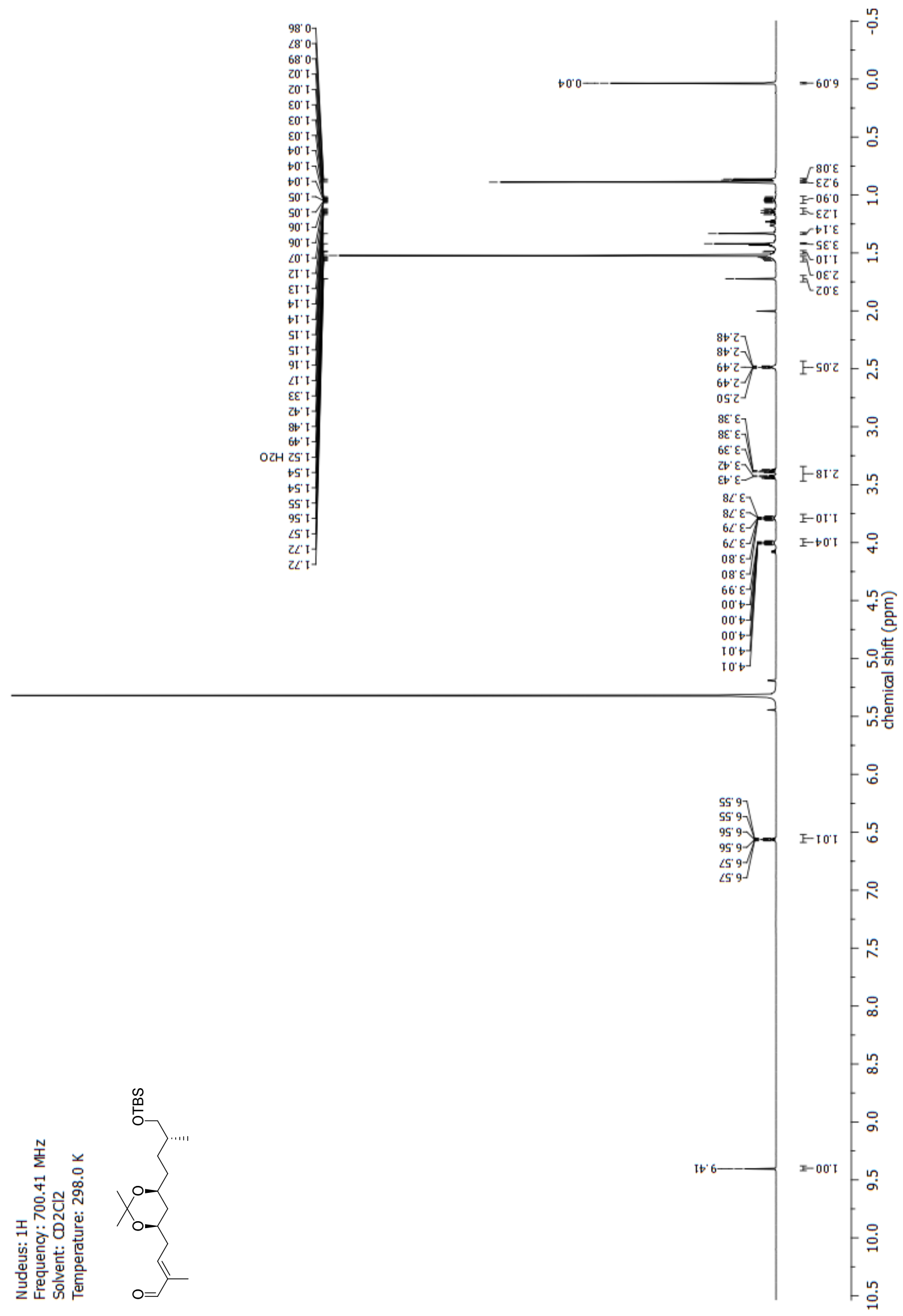




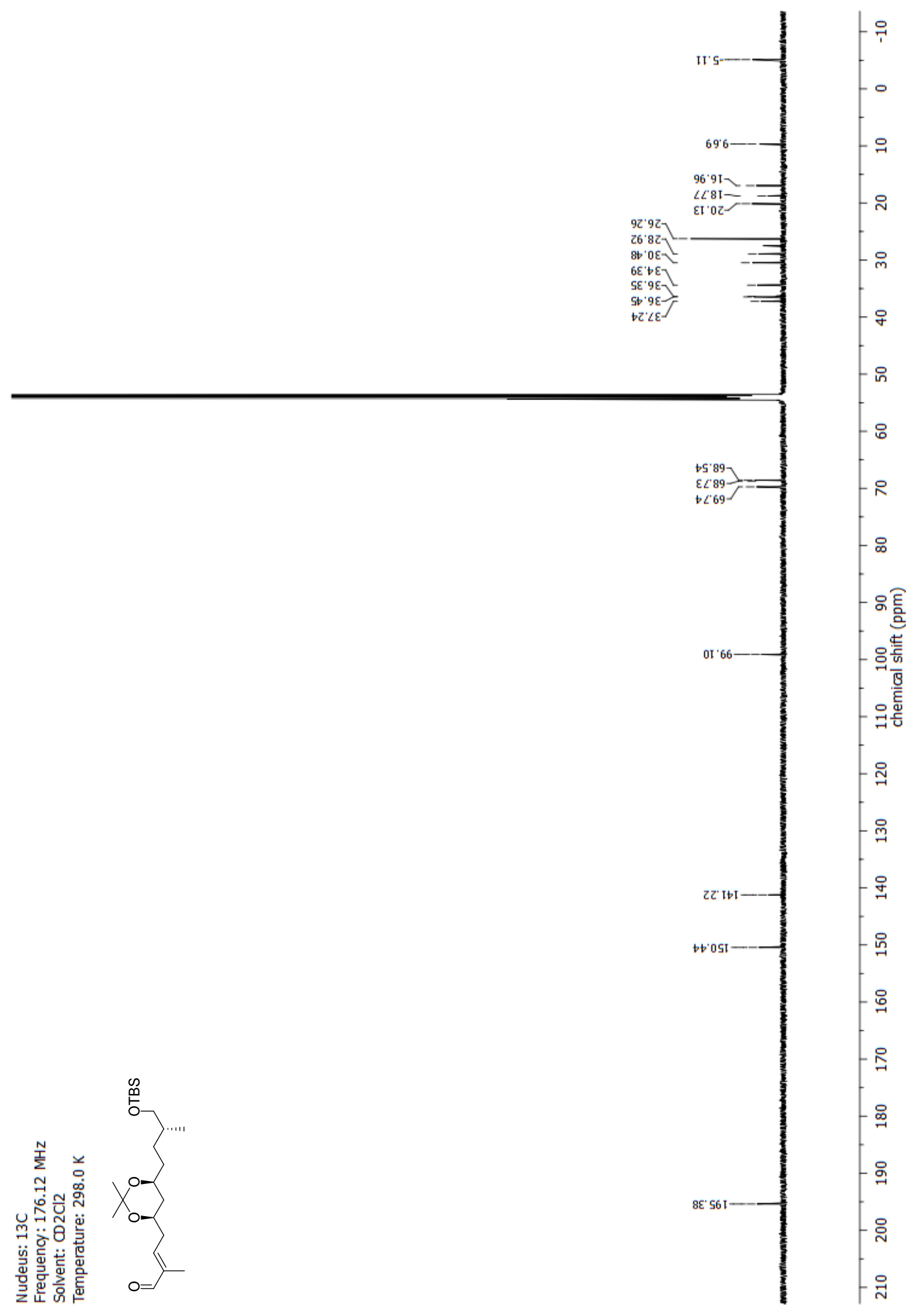


NMR-Spectra for Compound 15

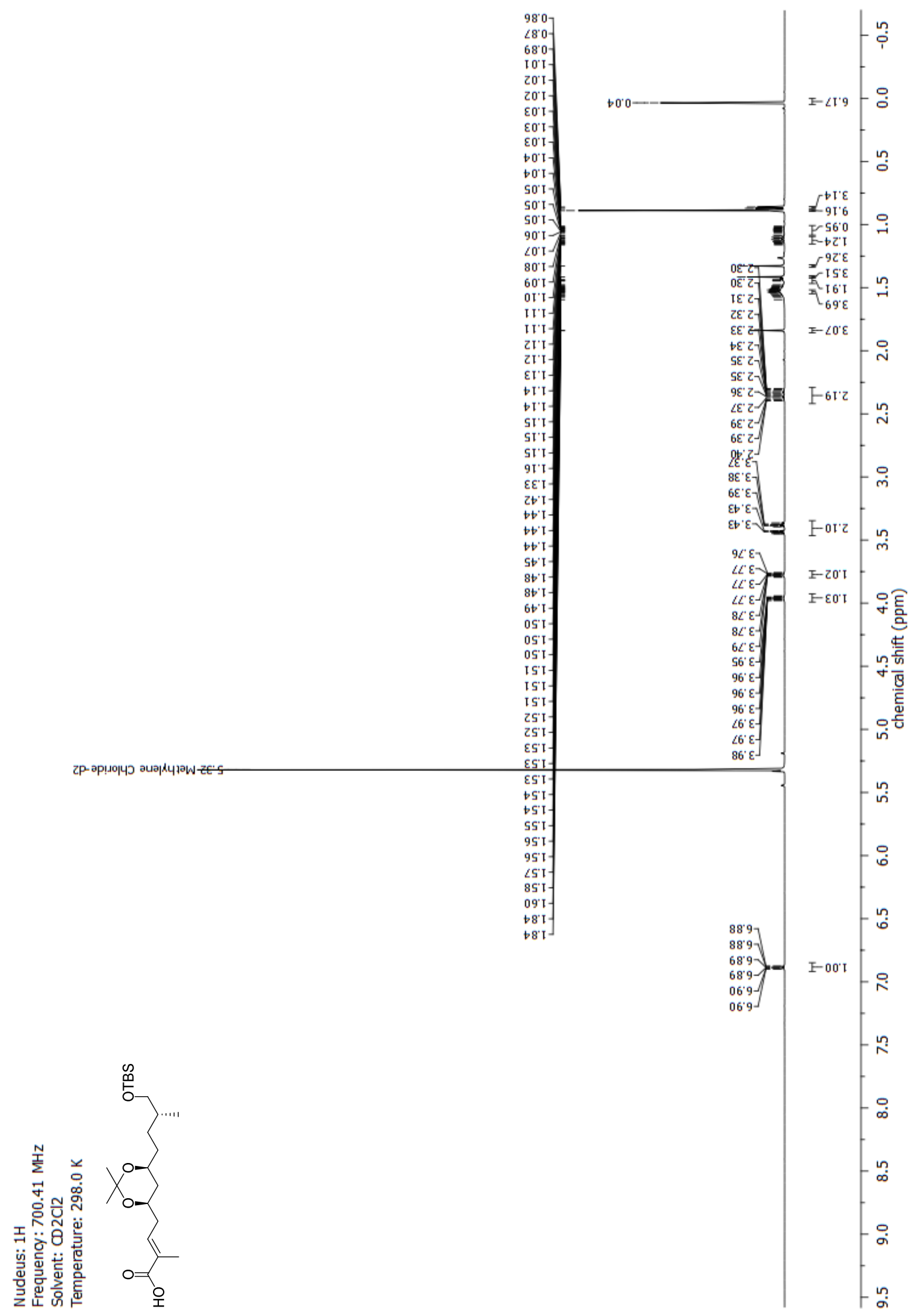




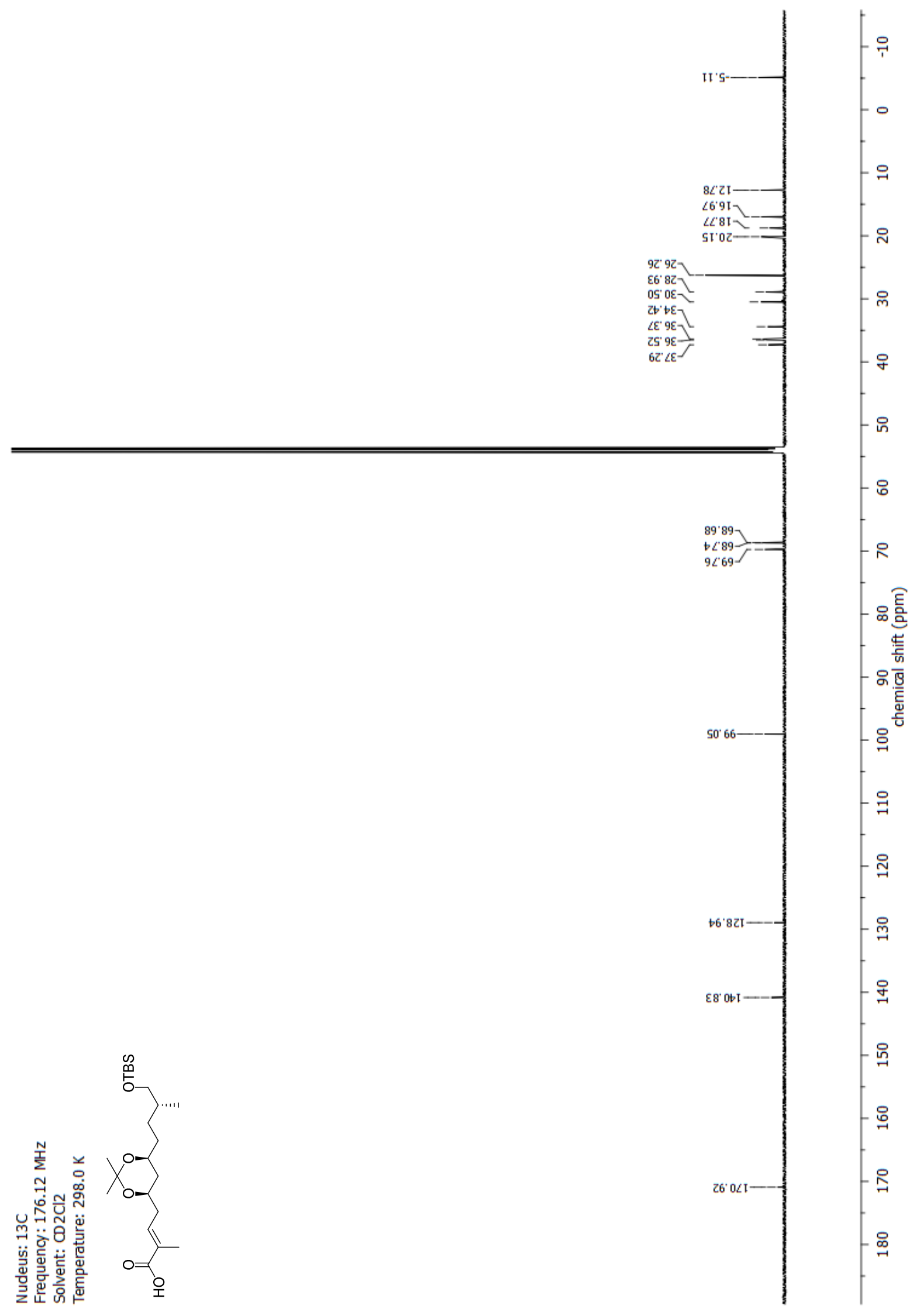


NMR-Spectra for Compound 17

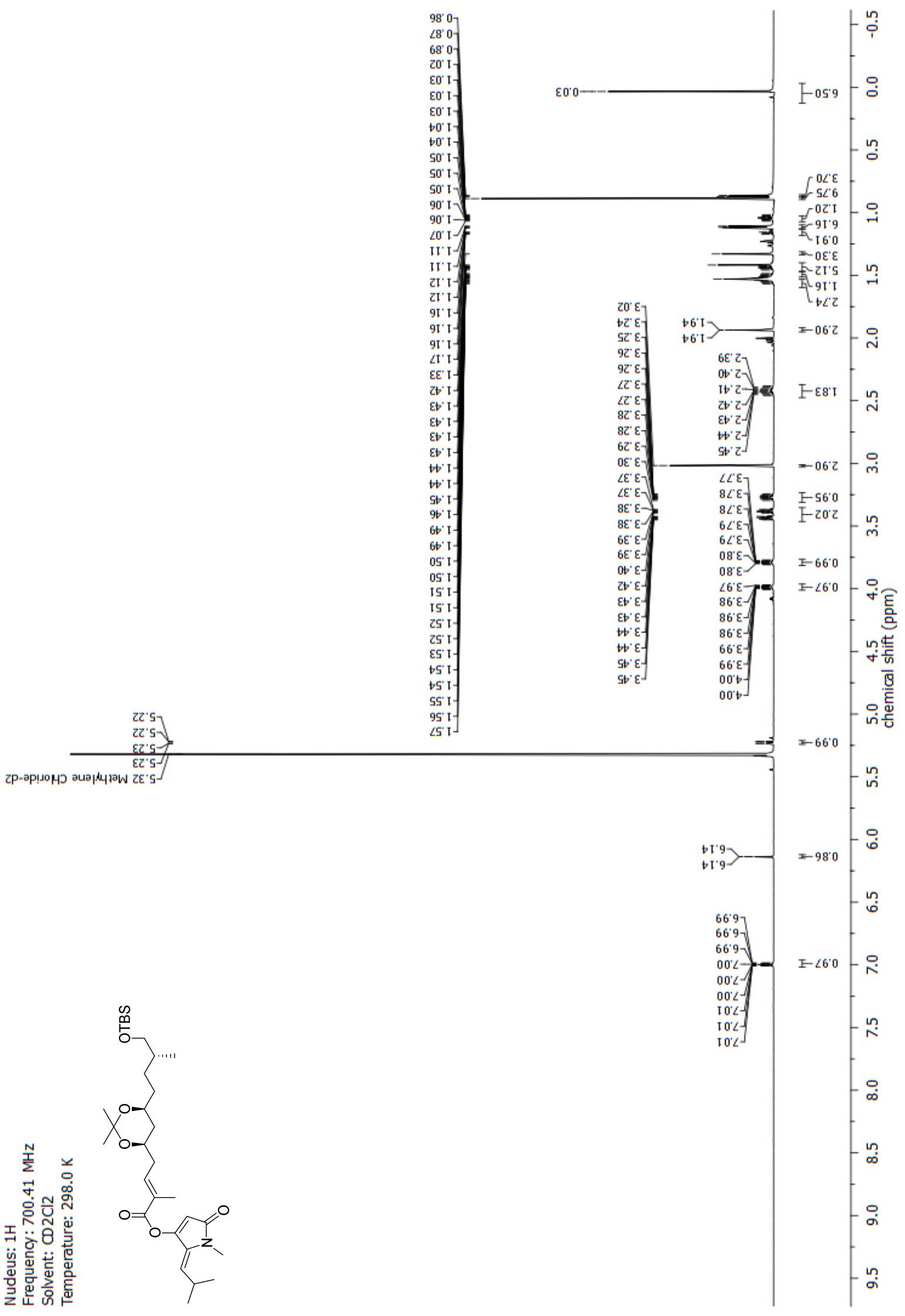




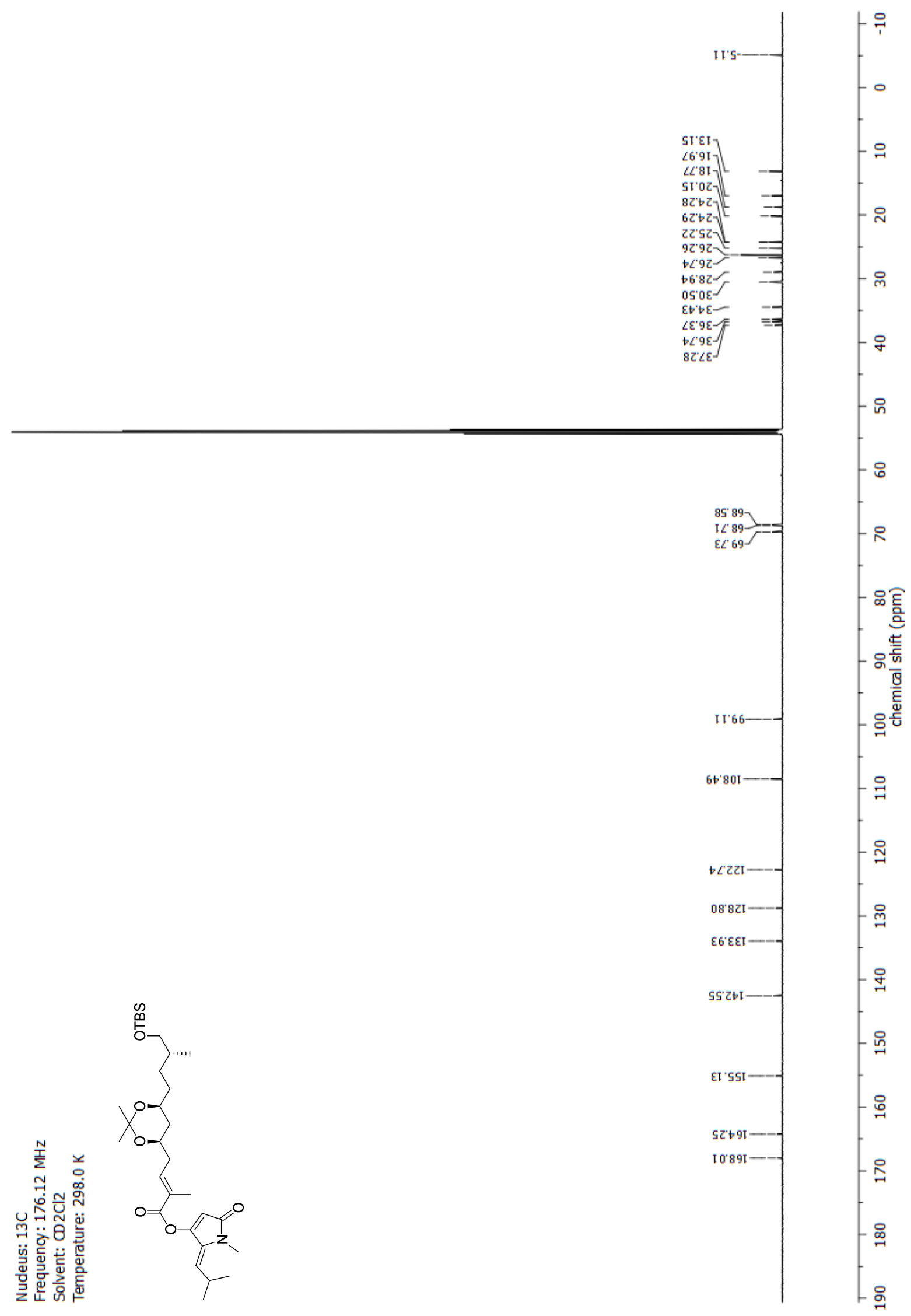


NMR-Spectra for Compound 2

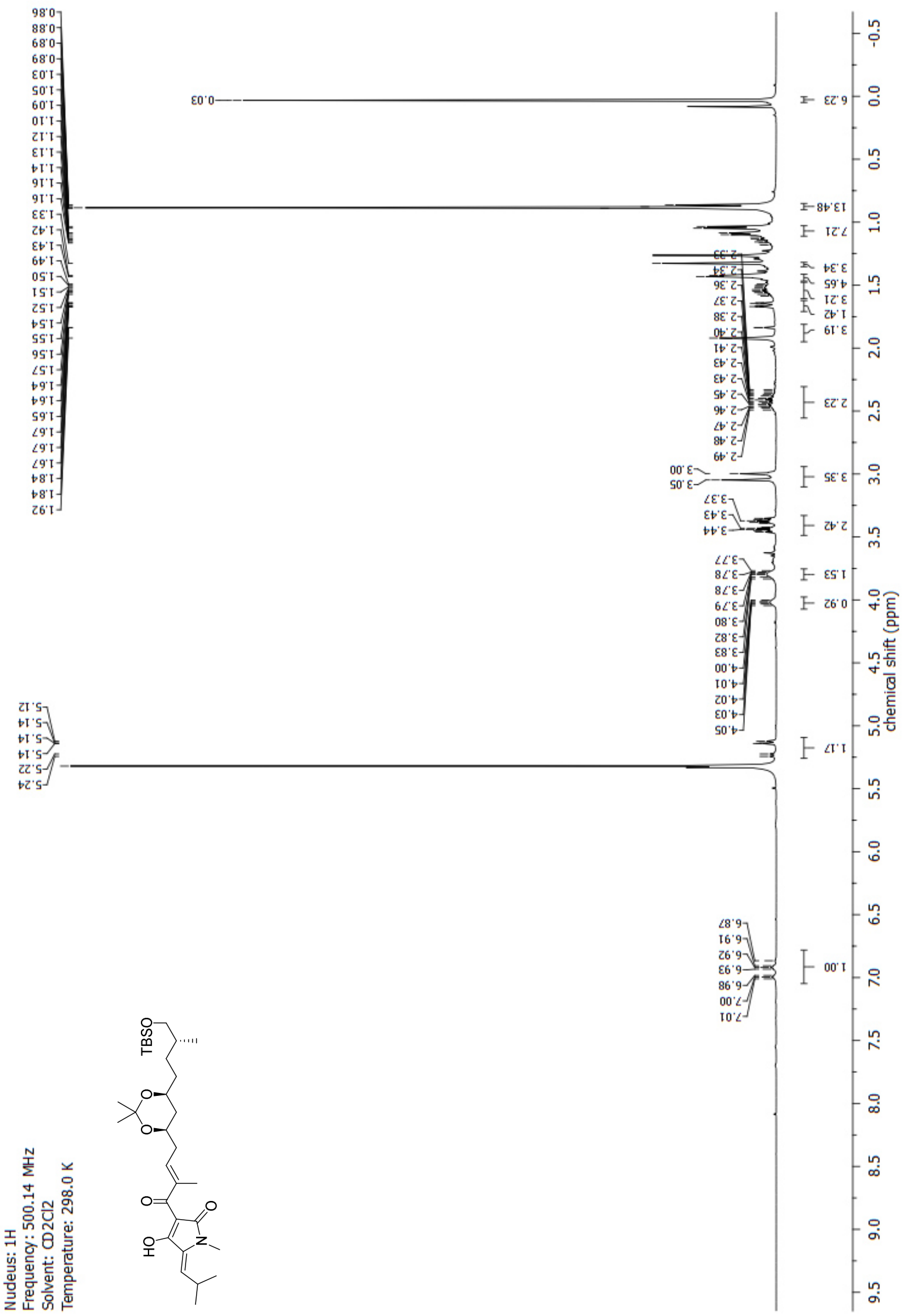




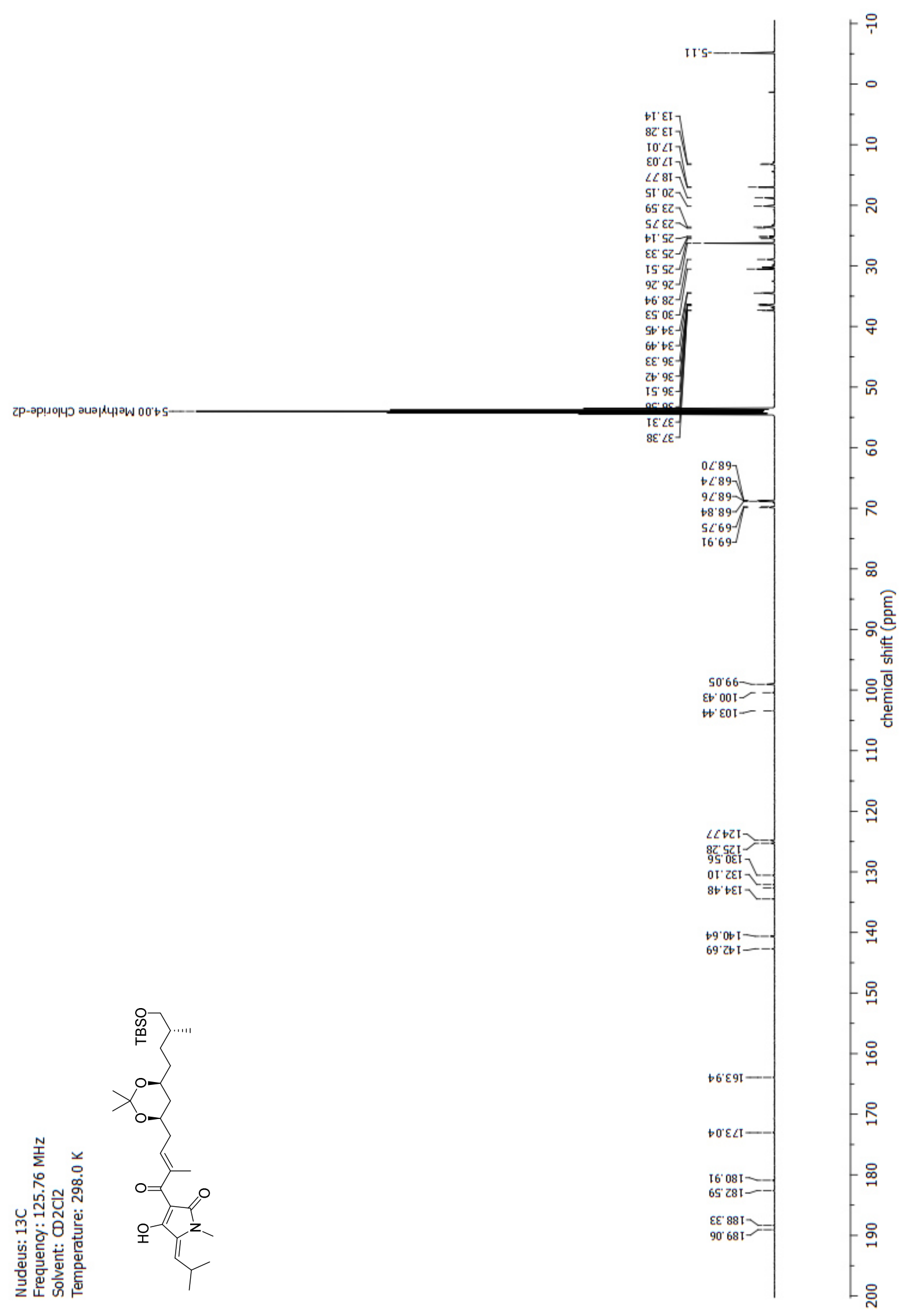


NMR-Spectra for Compound 64

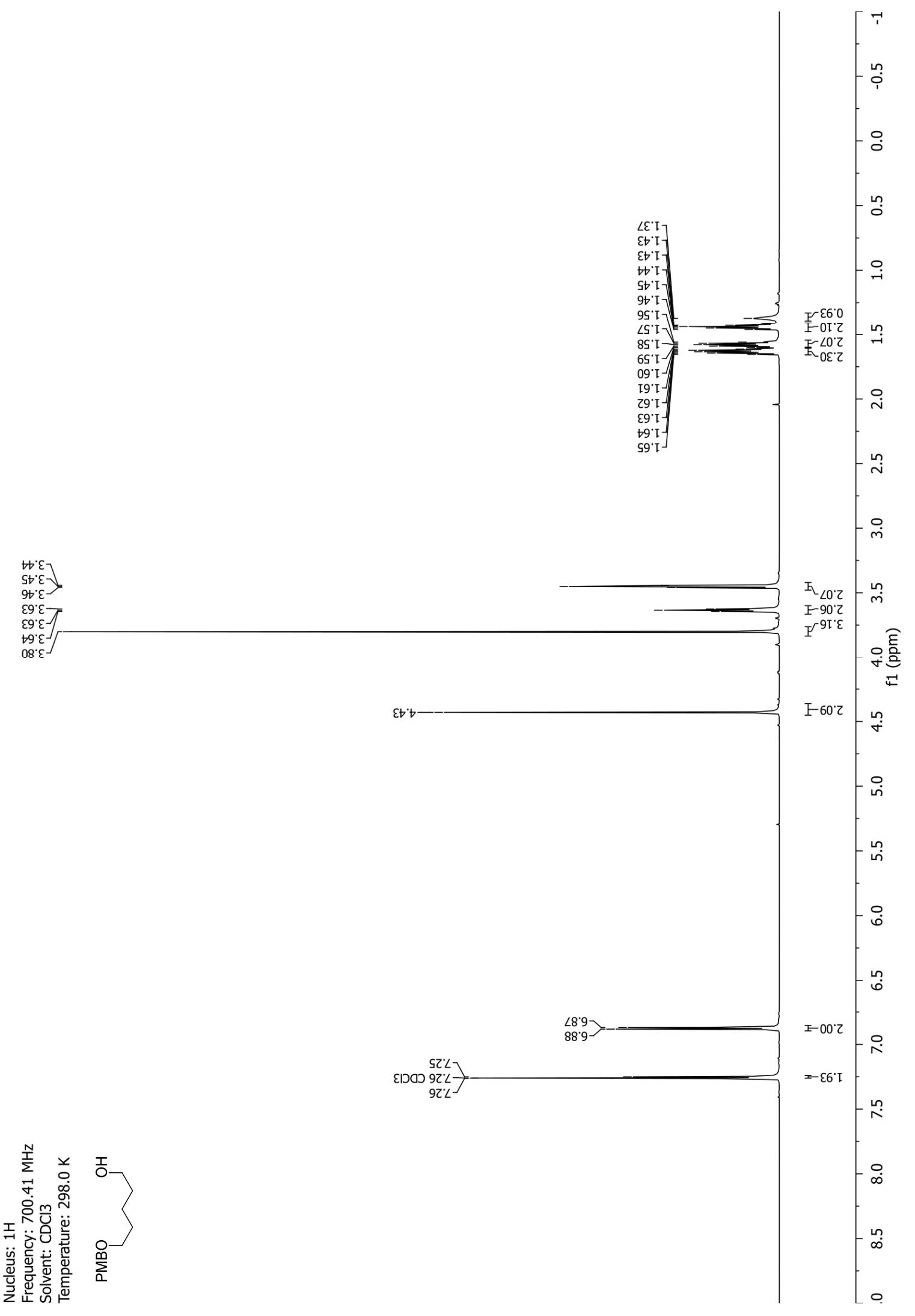




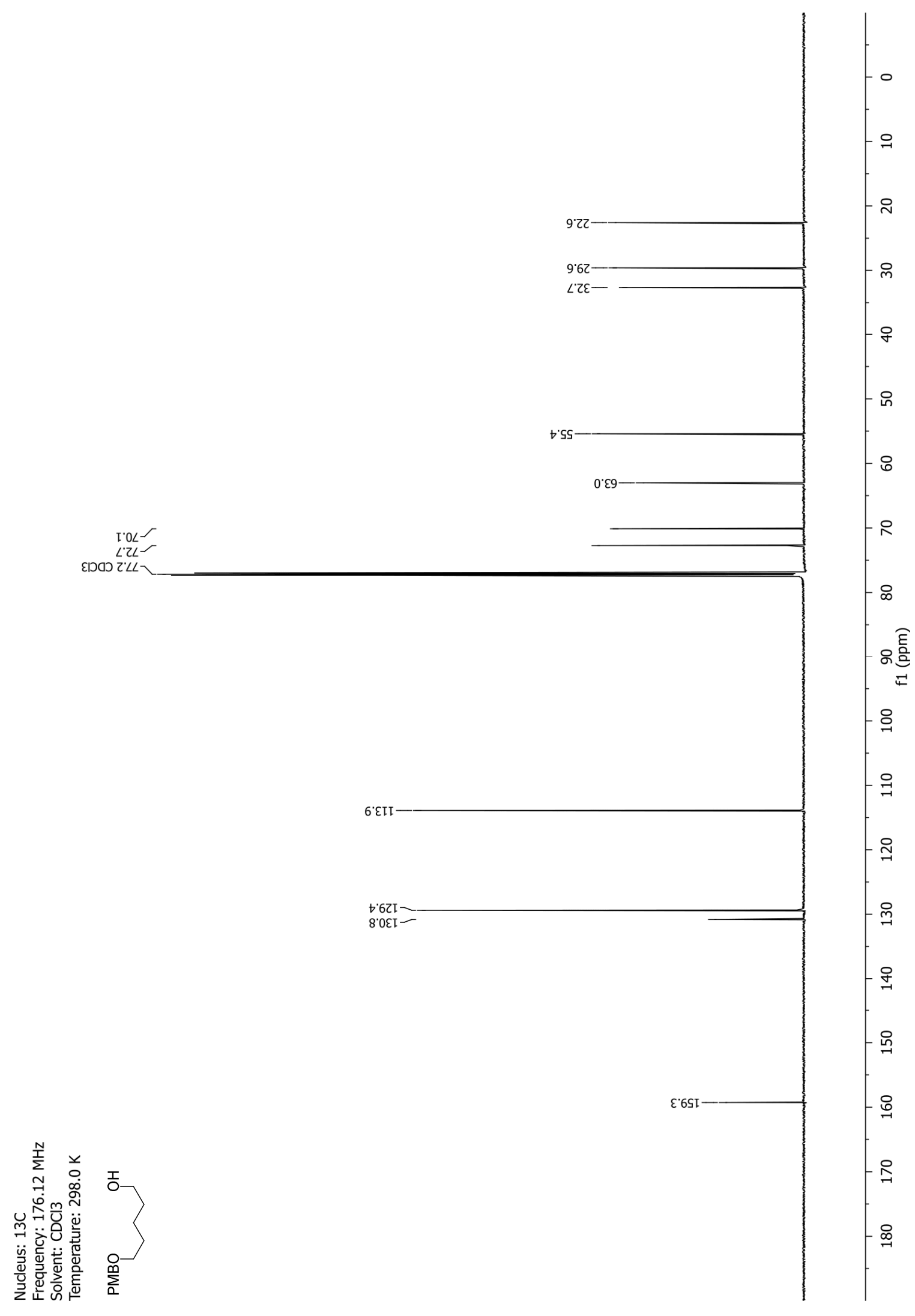


NMR-Spectra for Compound 65

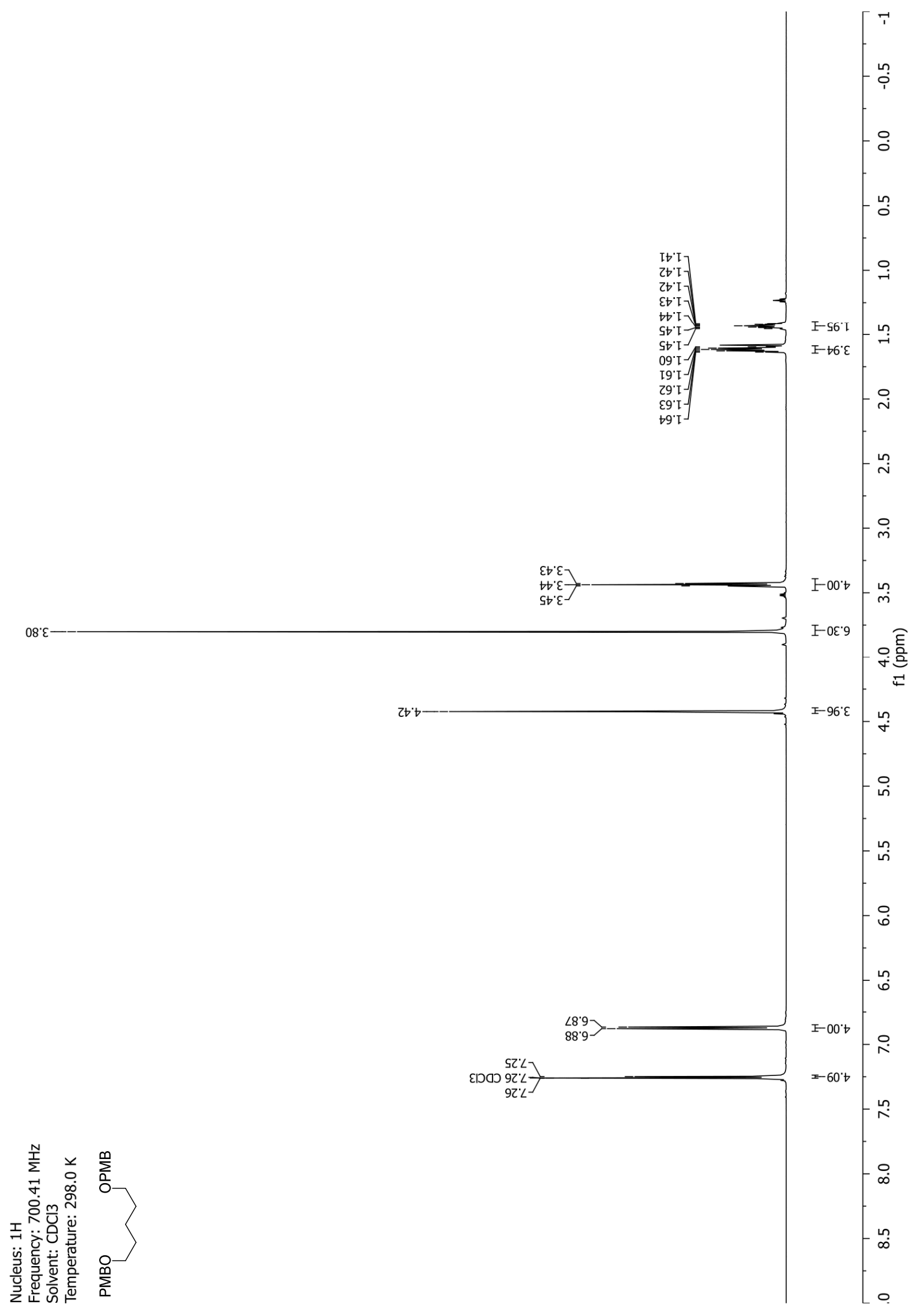




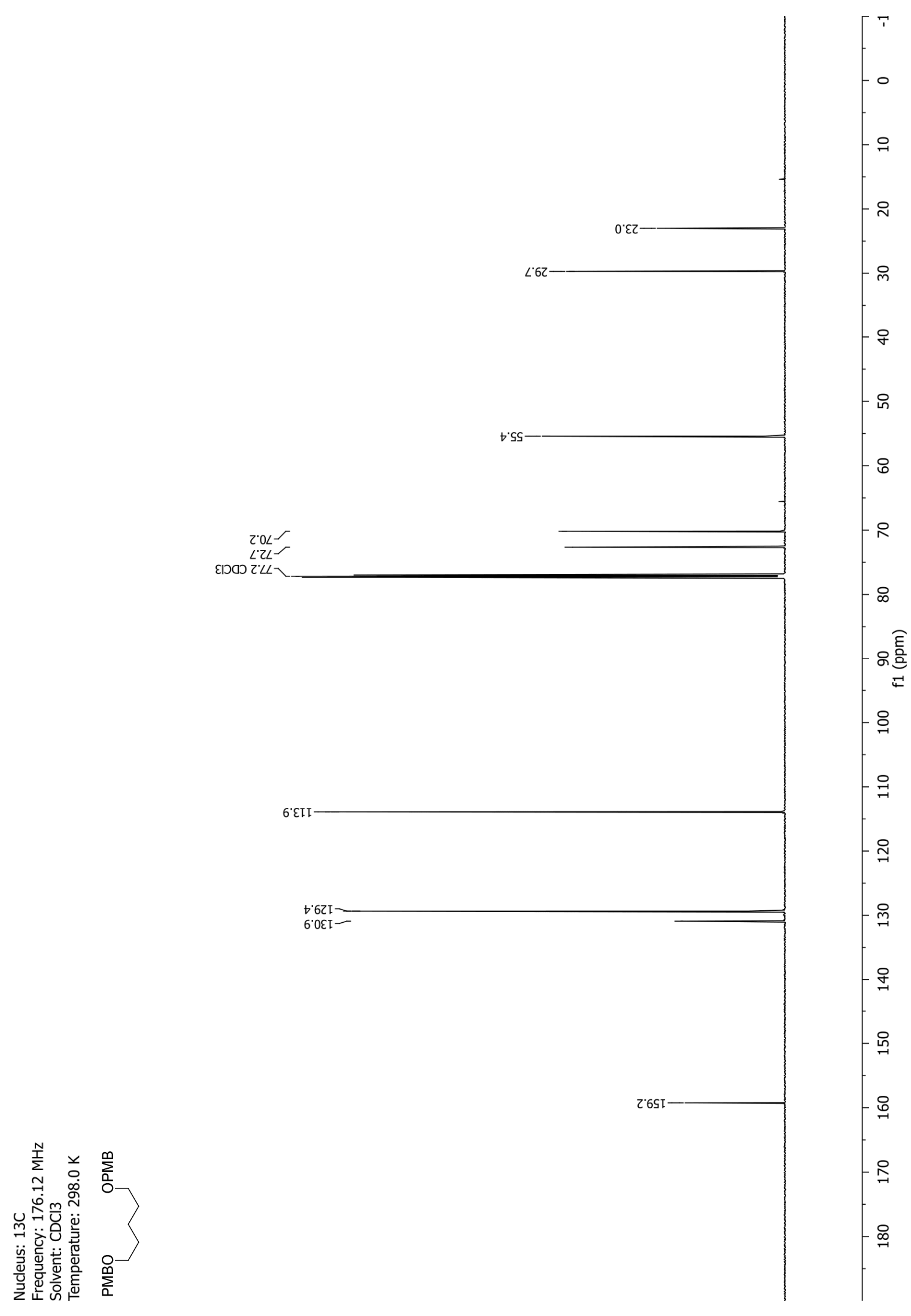




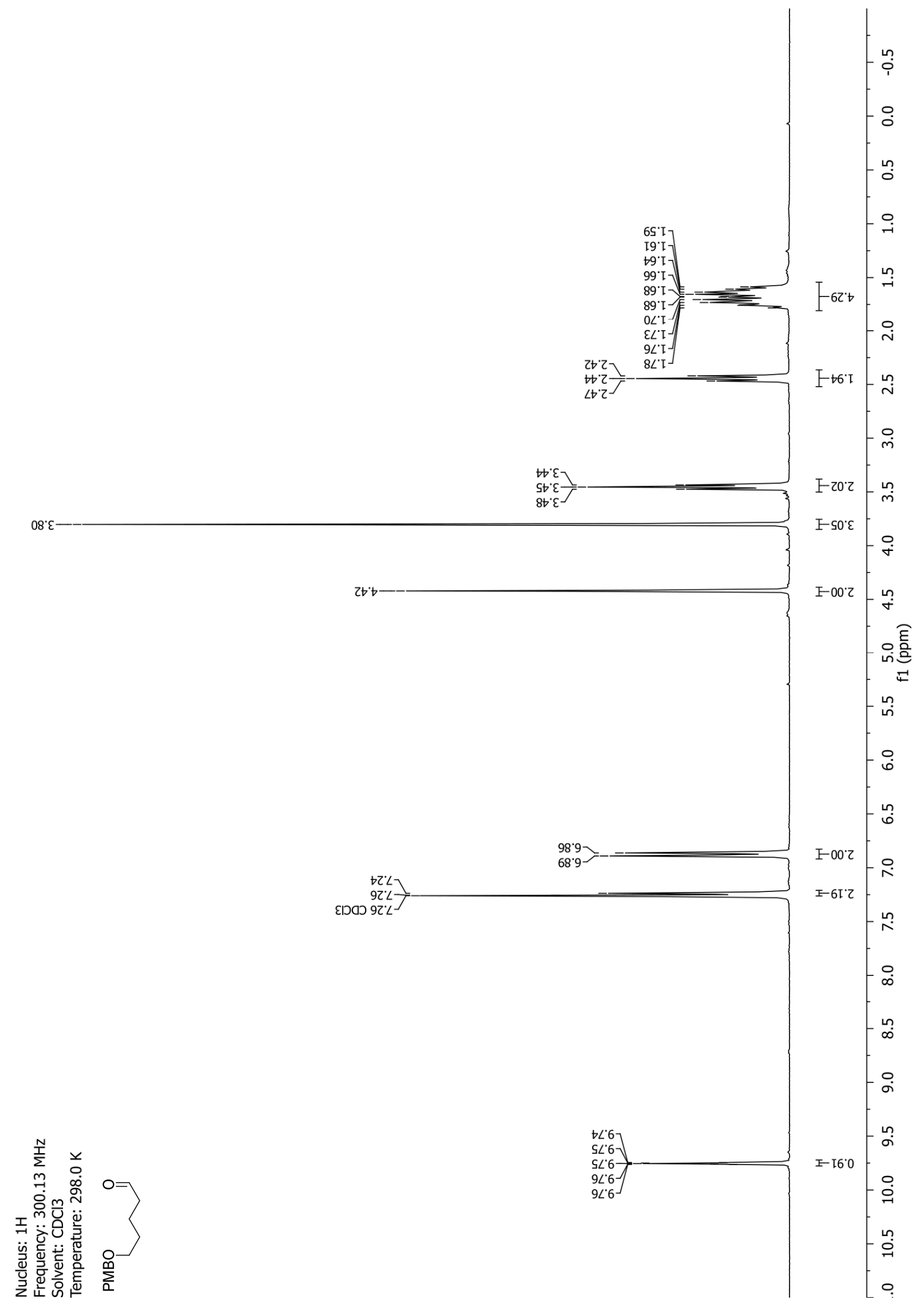




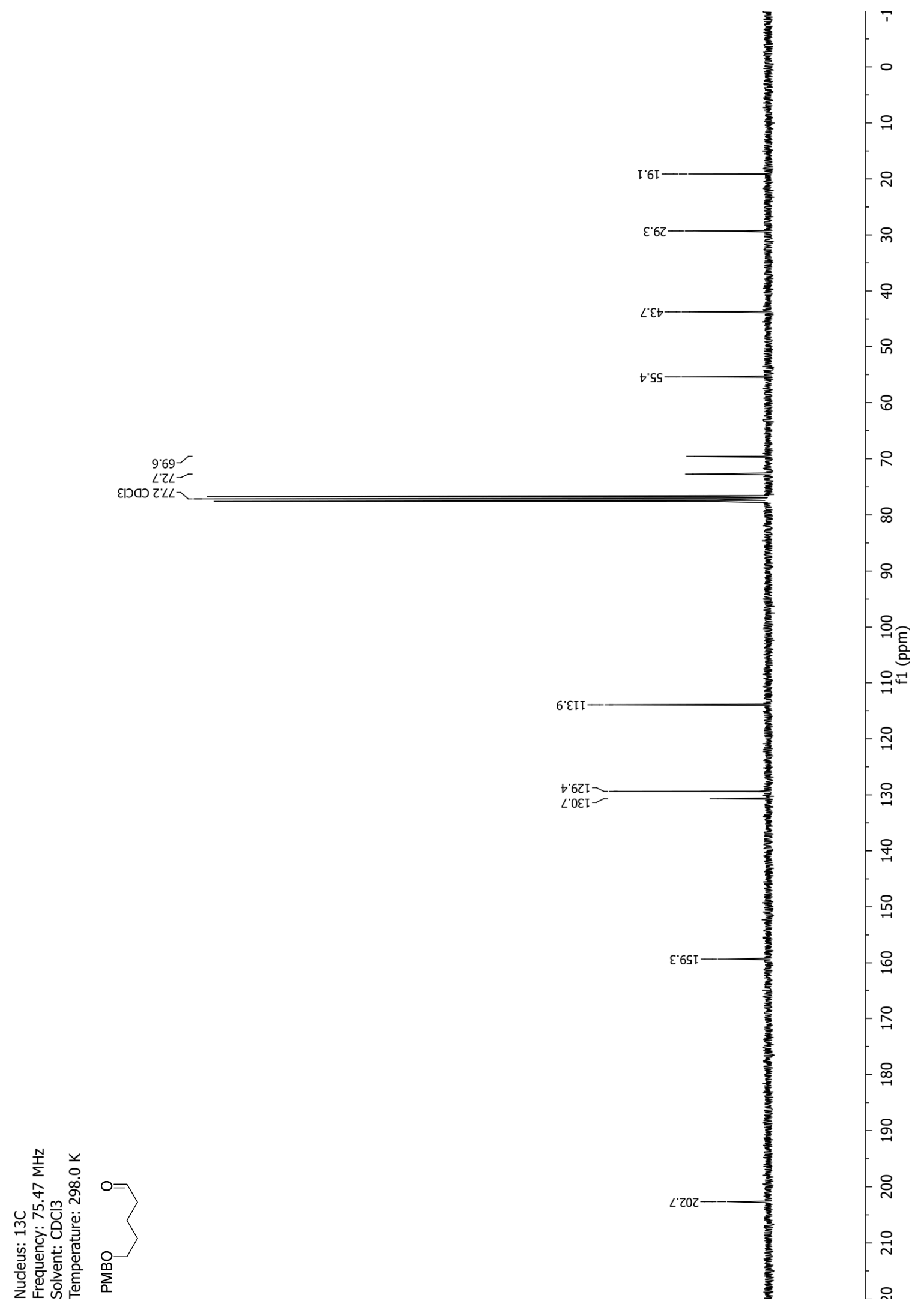




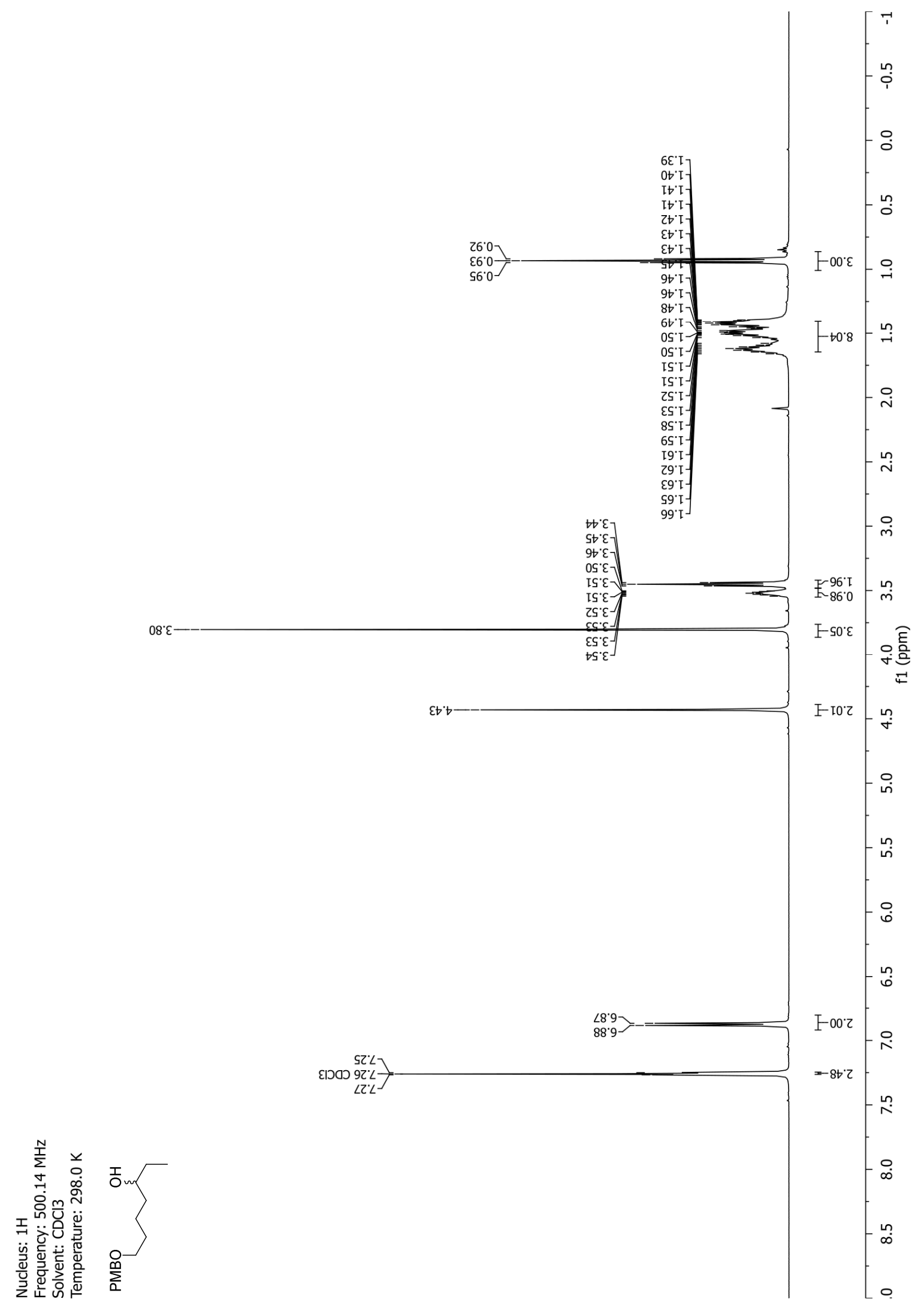




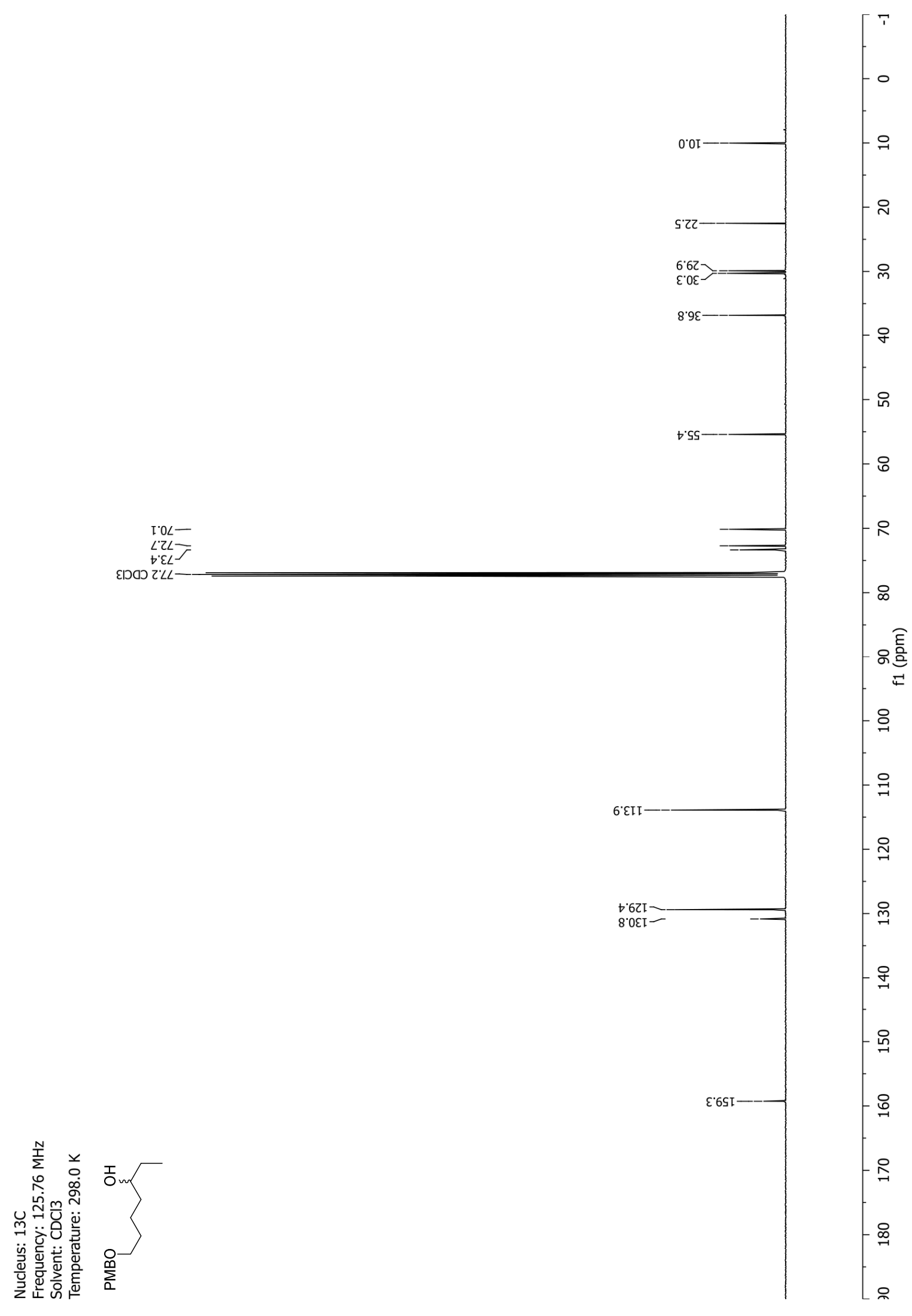


NMR-Spectra for Compound 21

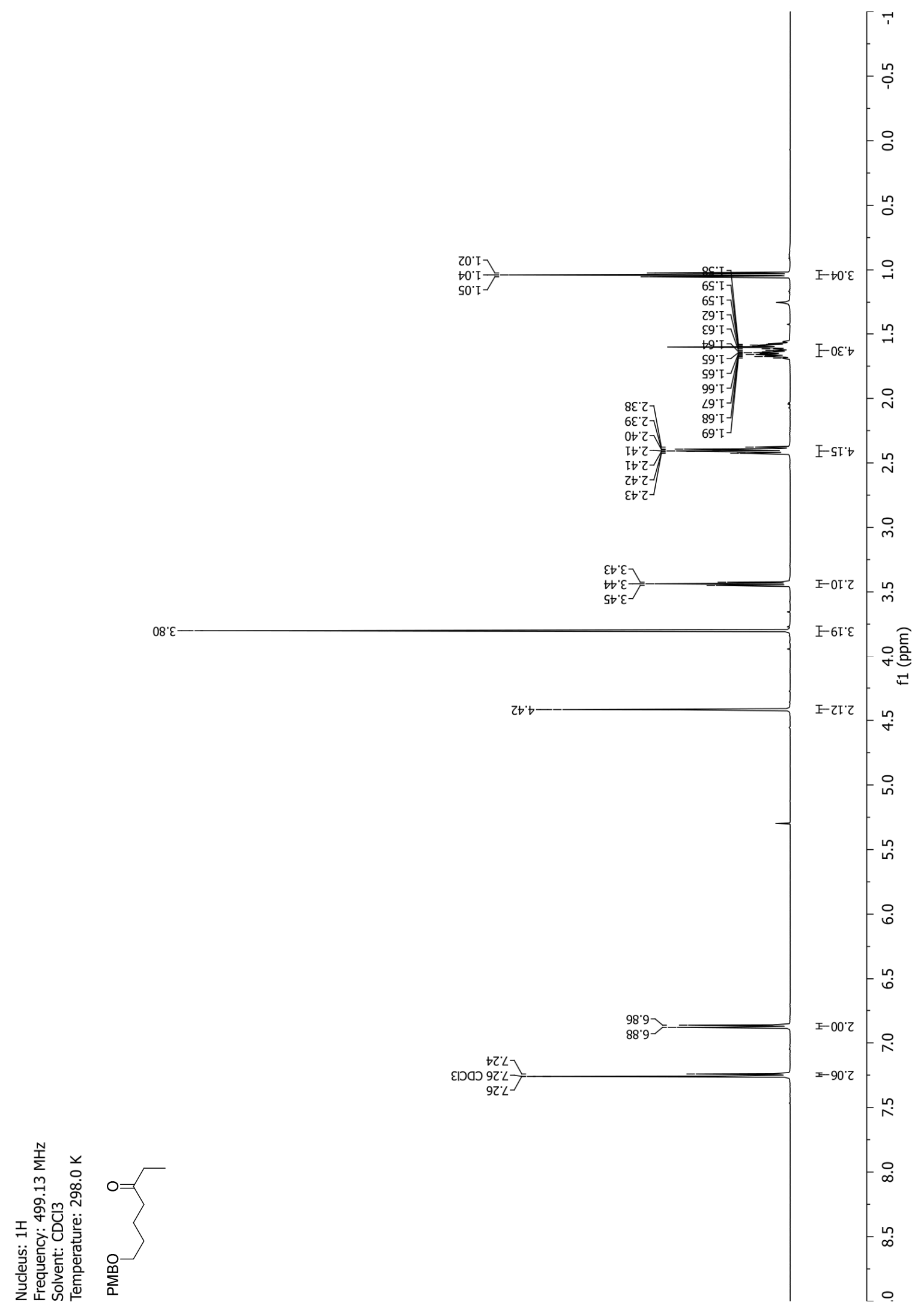




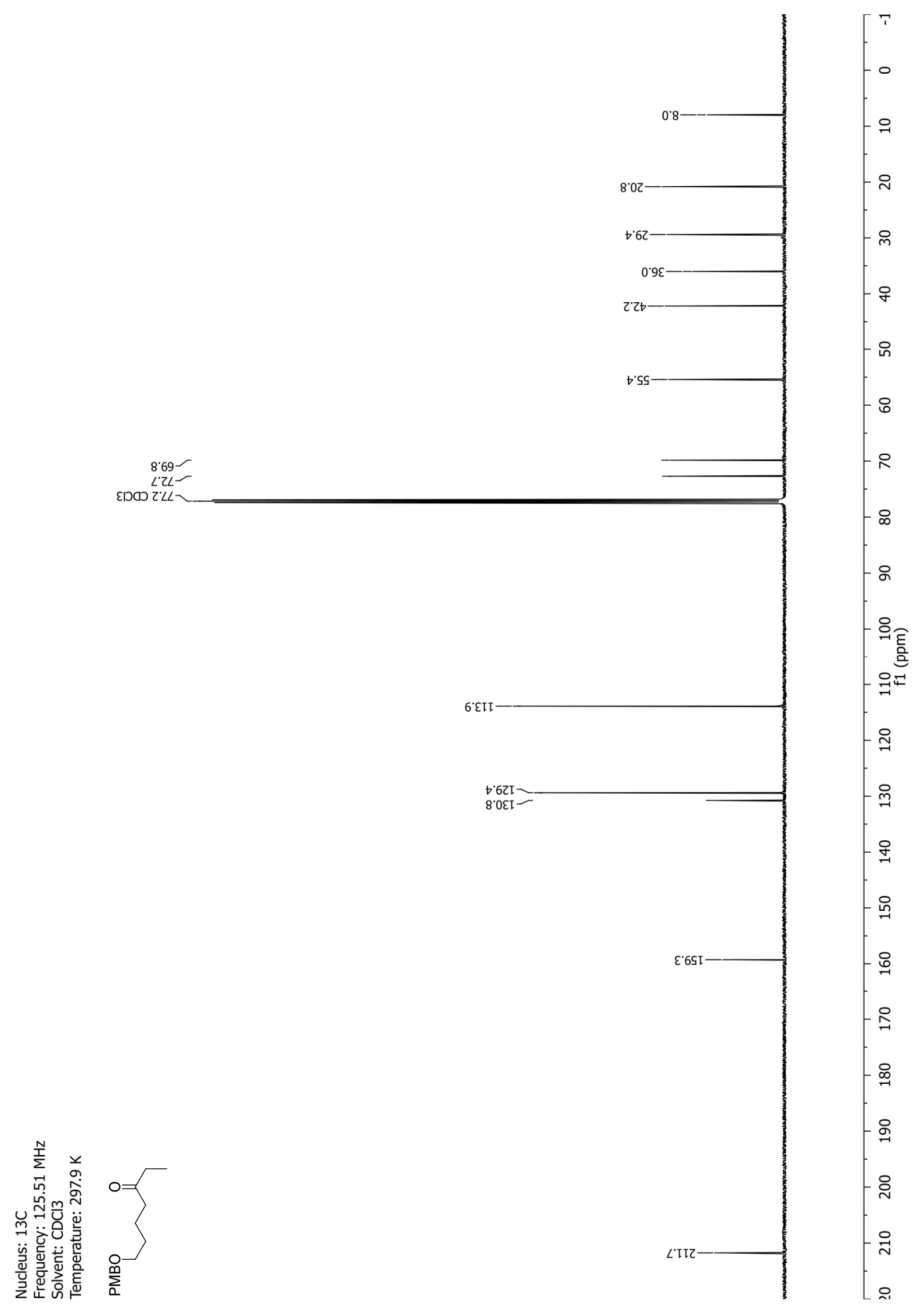


NMR-Spectra for Compound 69

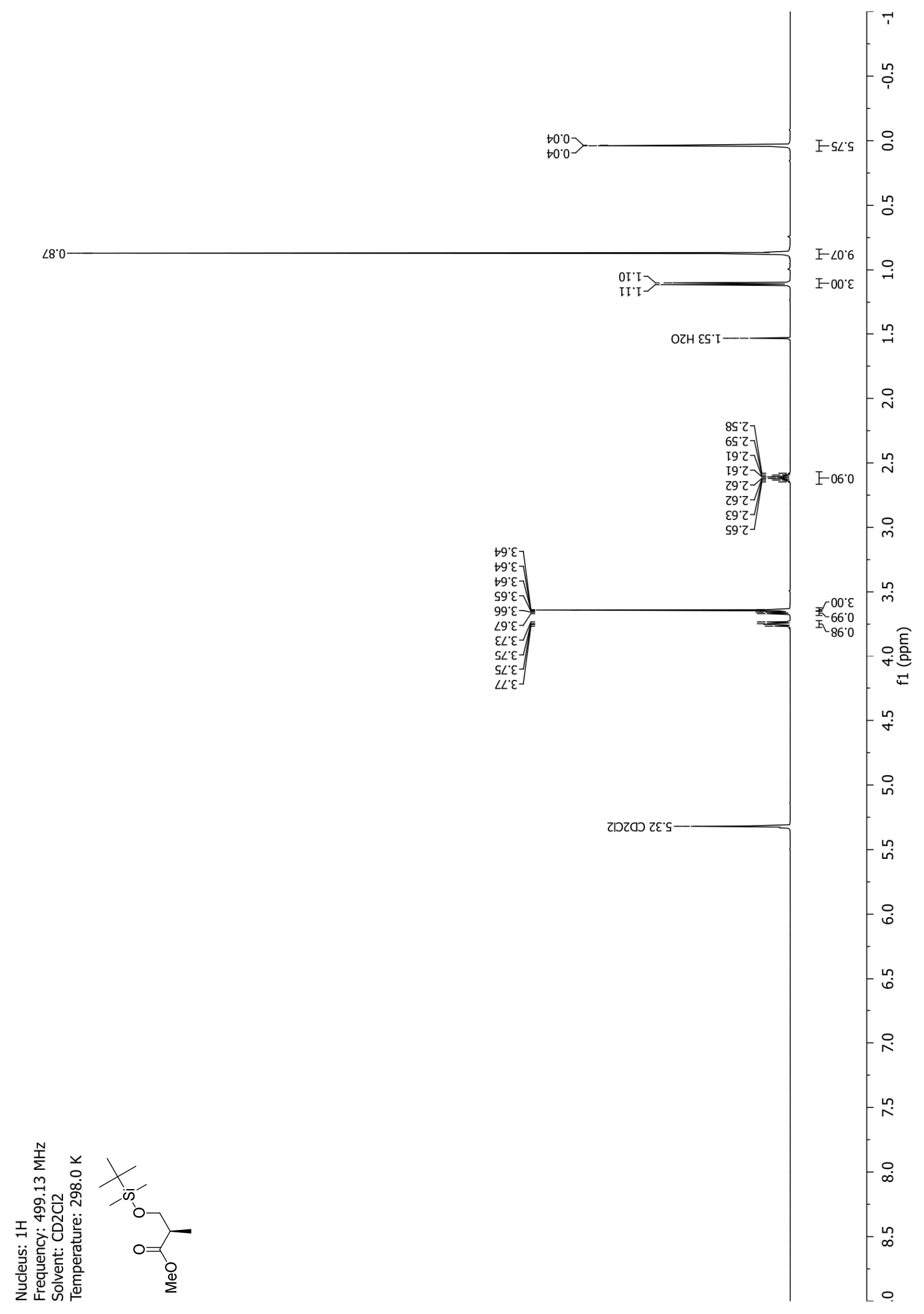




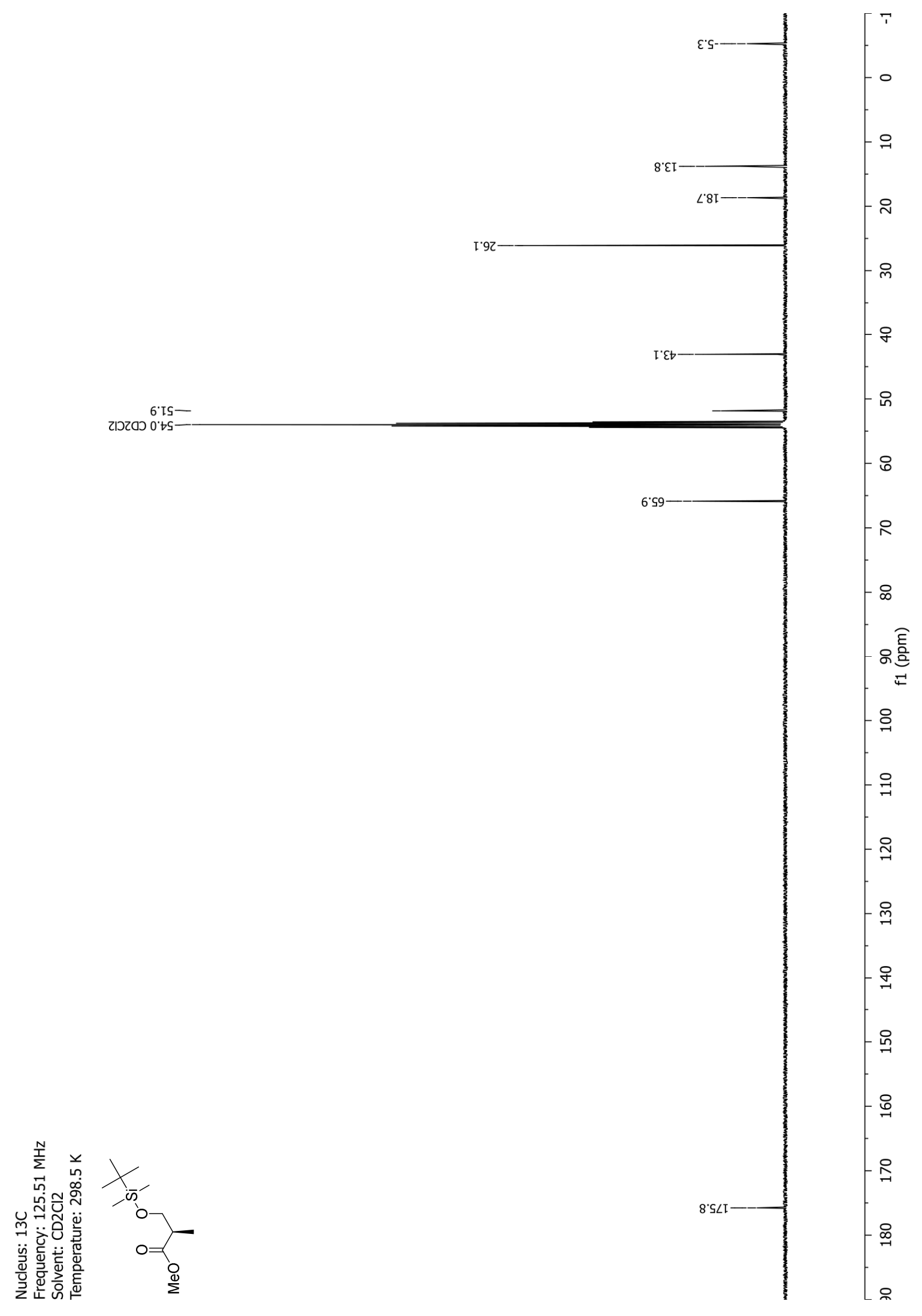


NMR-Spectra for Compound 70

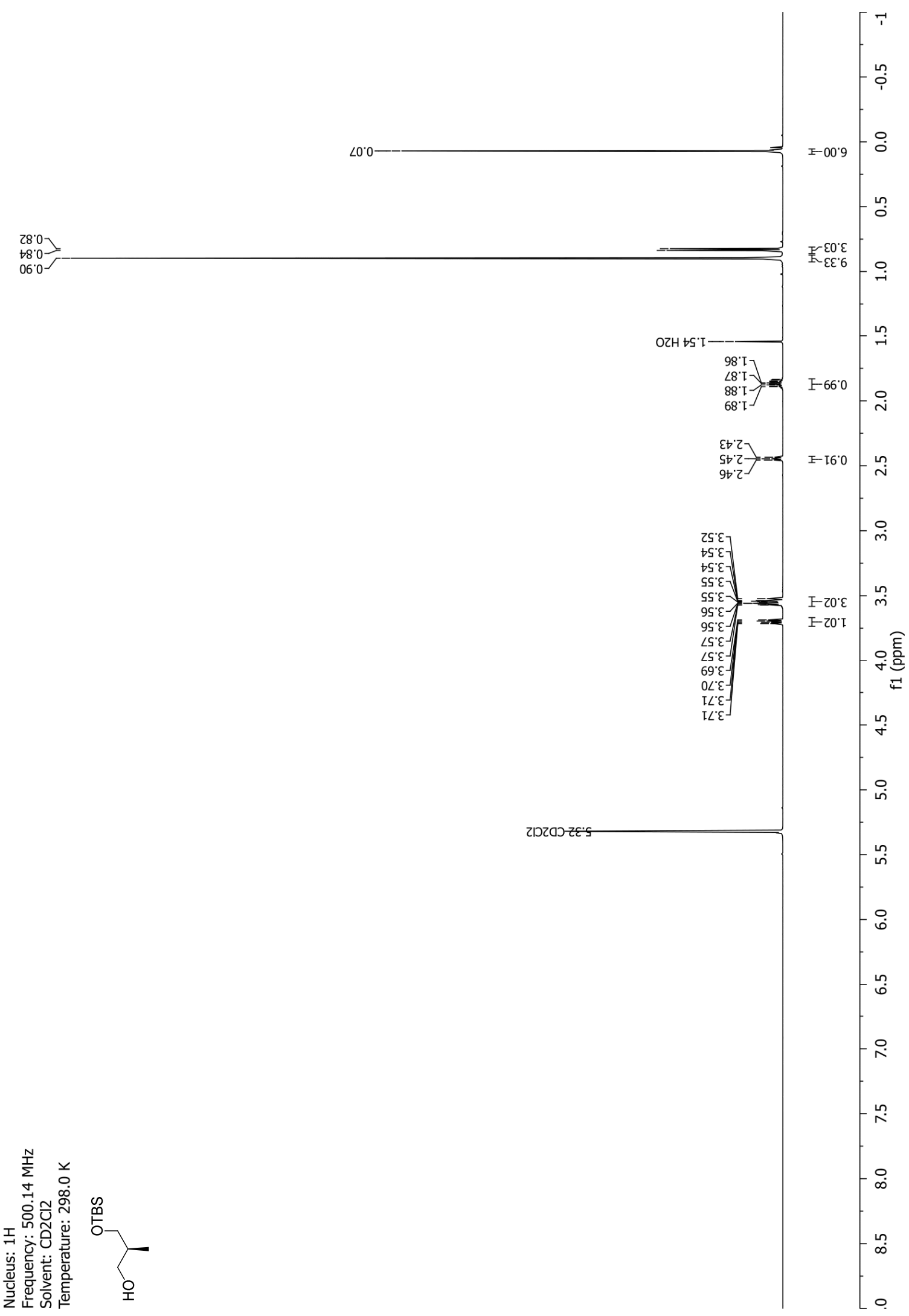




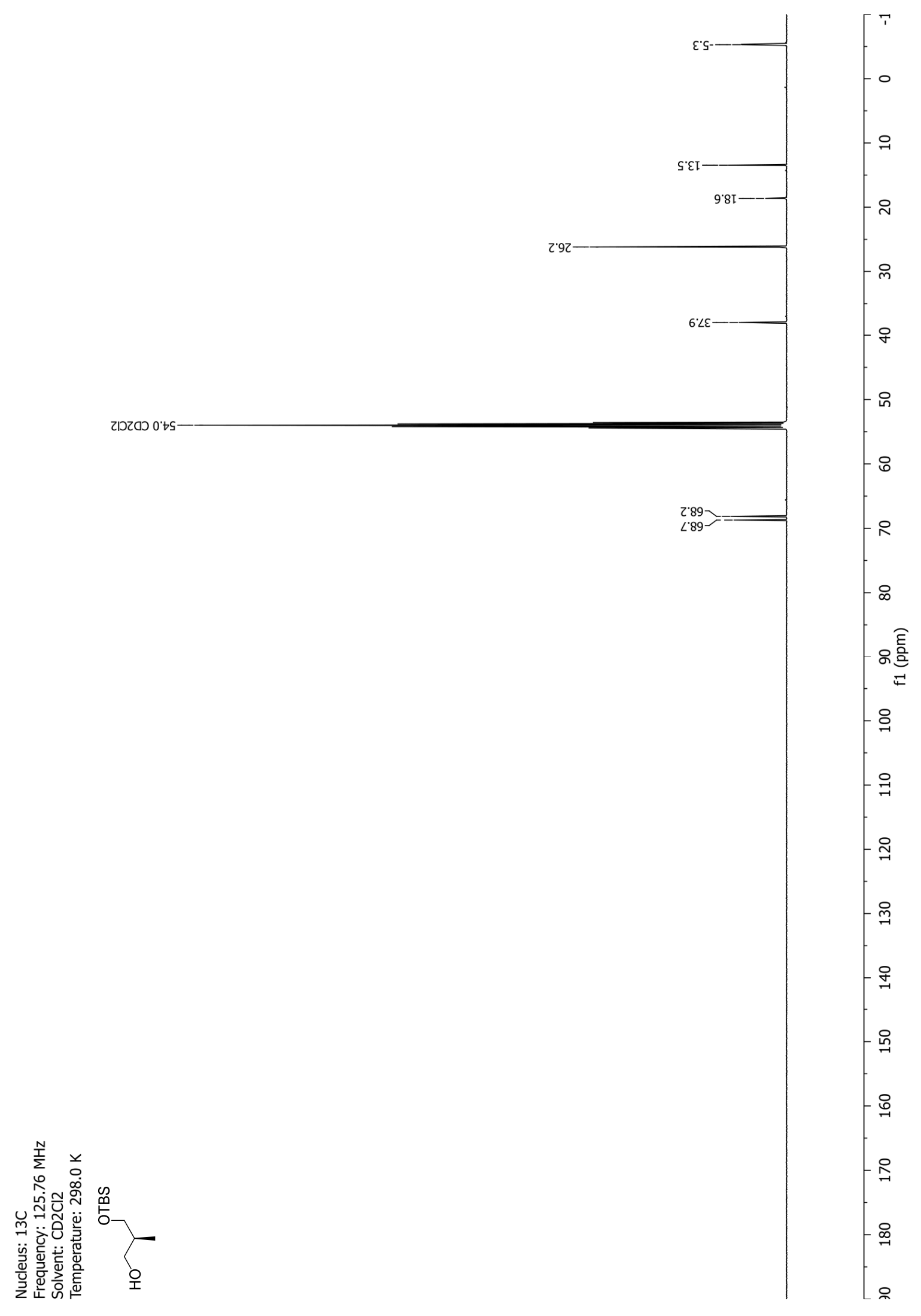


NMR-Spectra for Compound 18

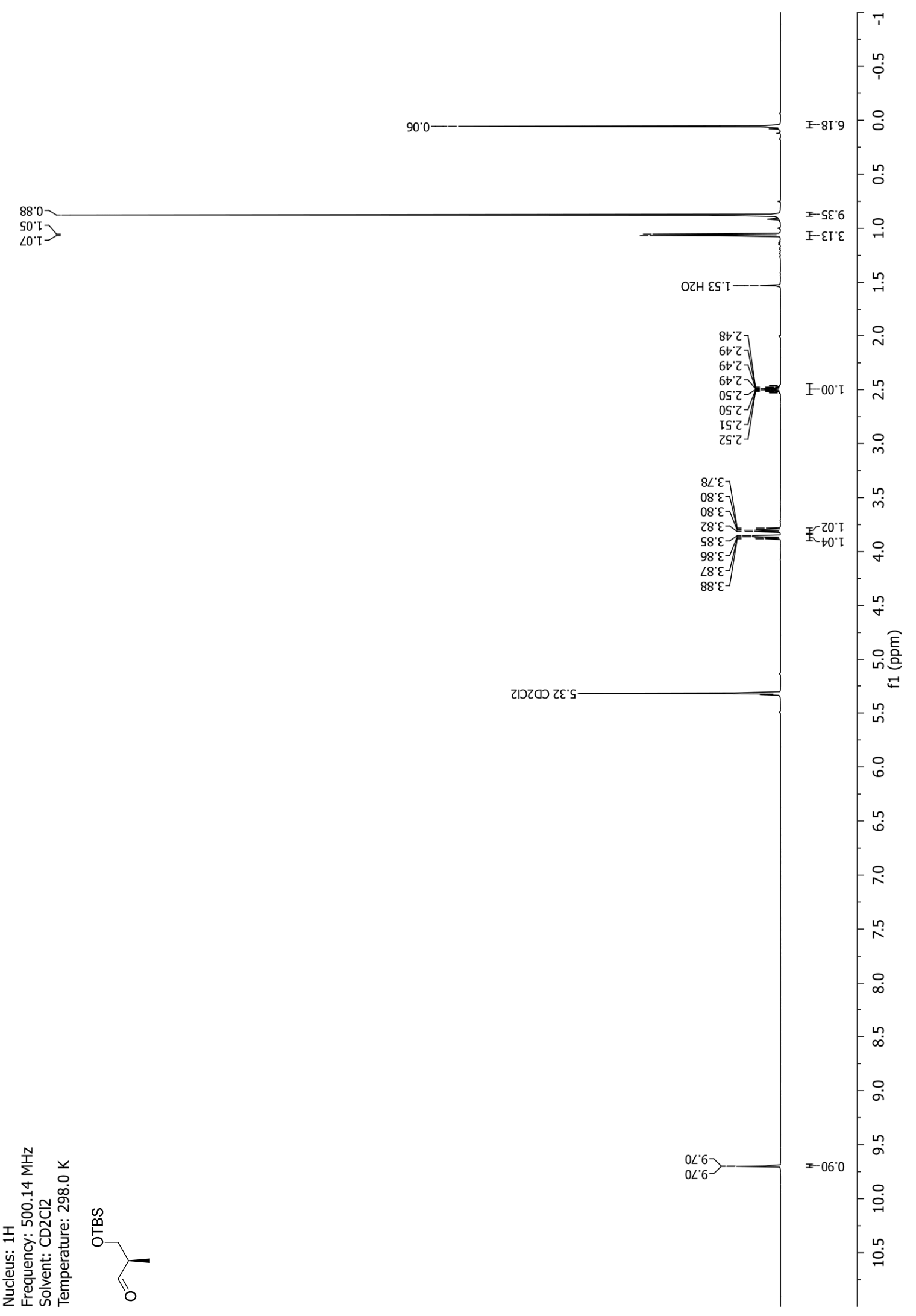




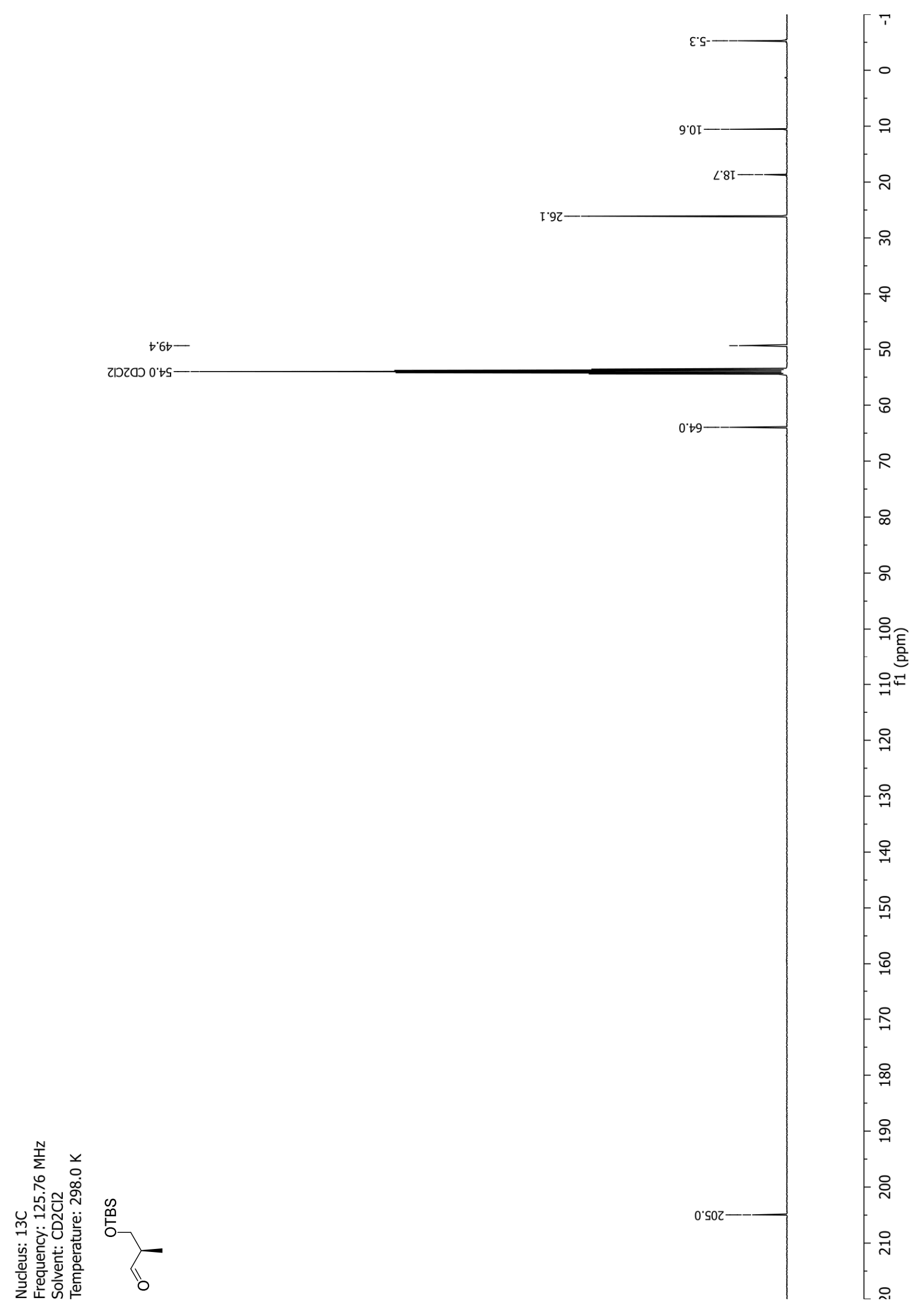




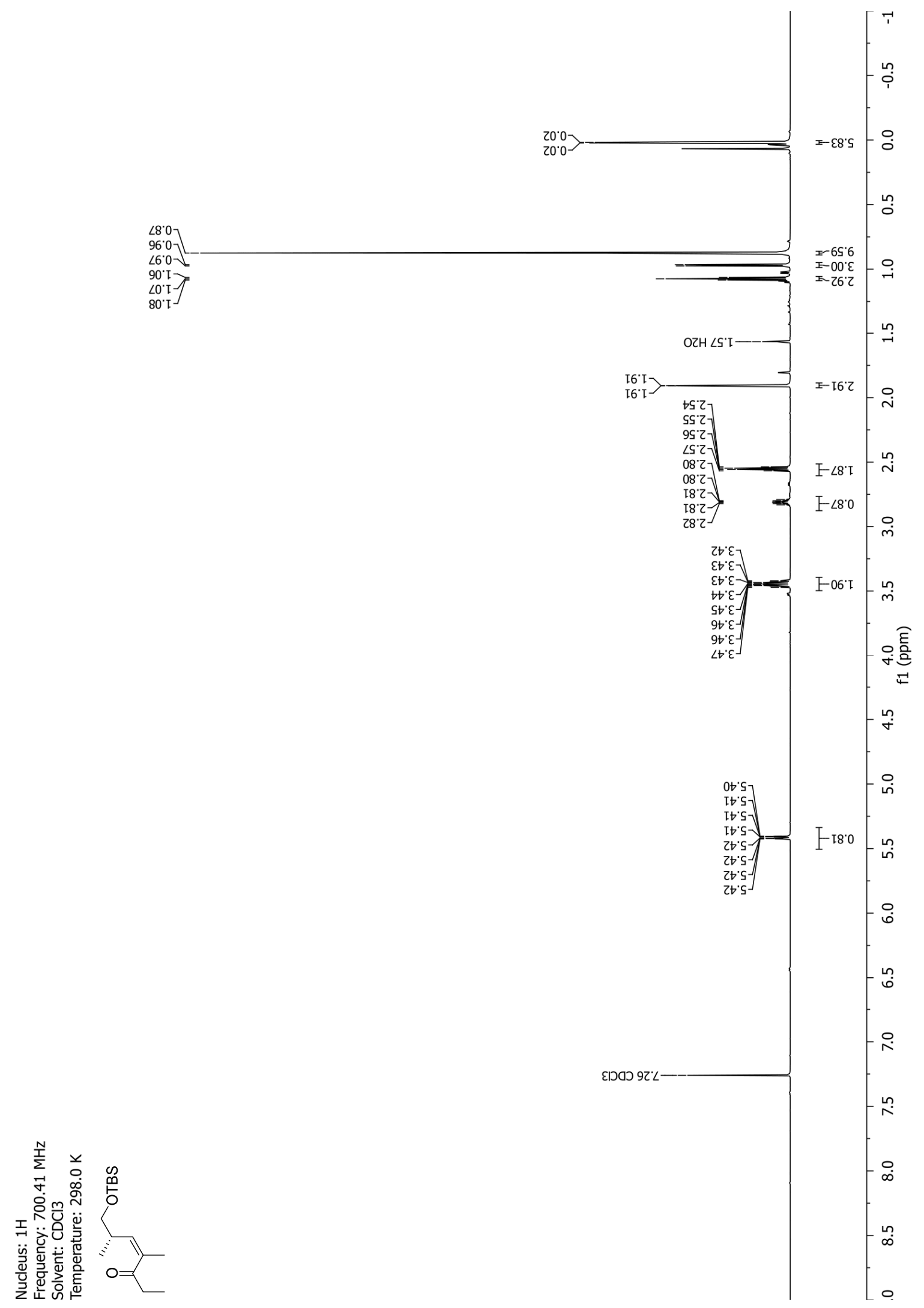




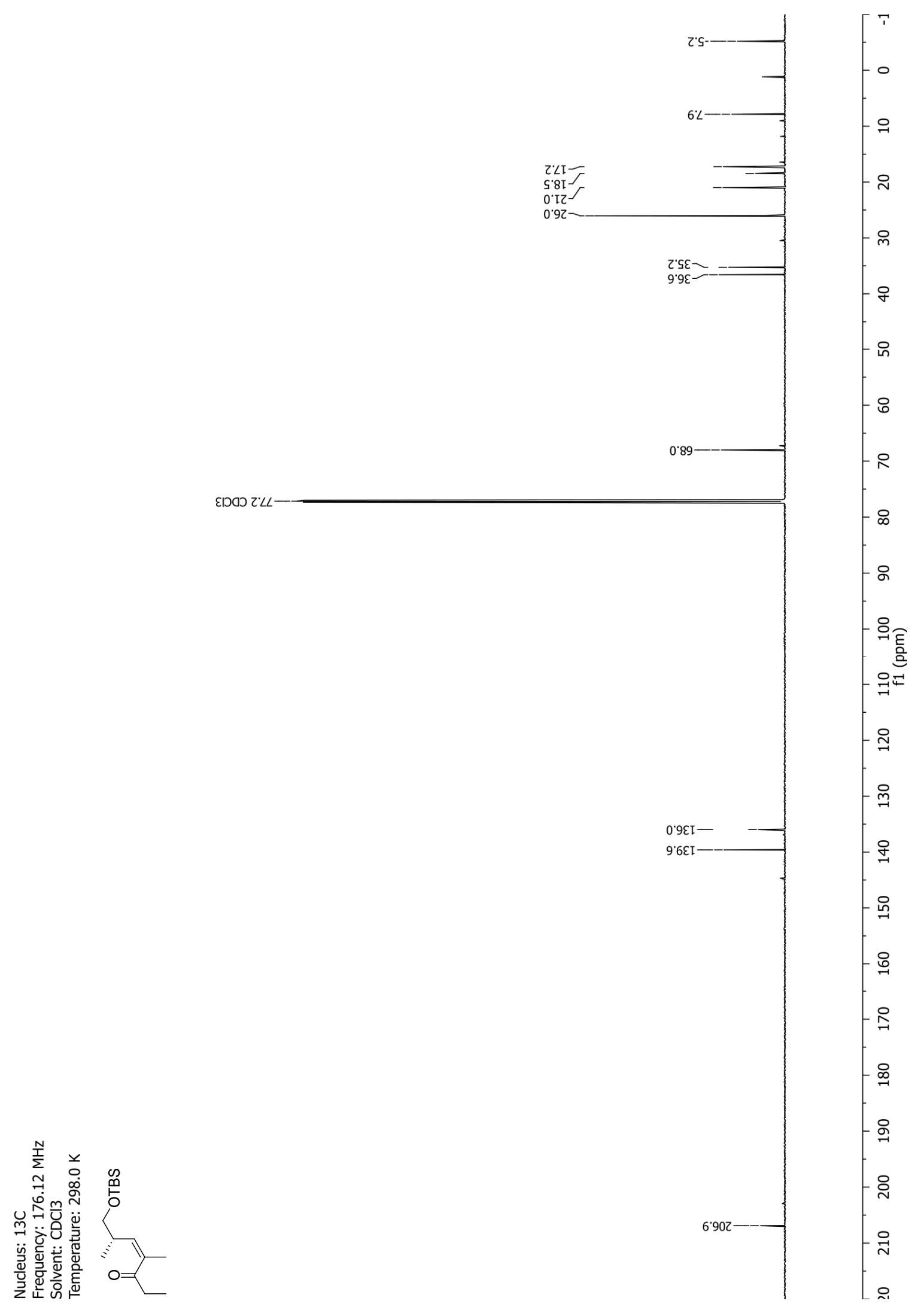


NMR-Spectra for Compound 19

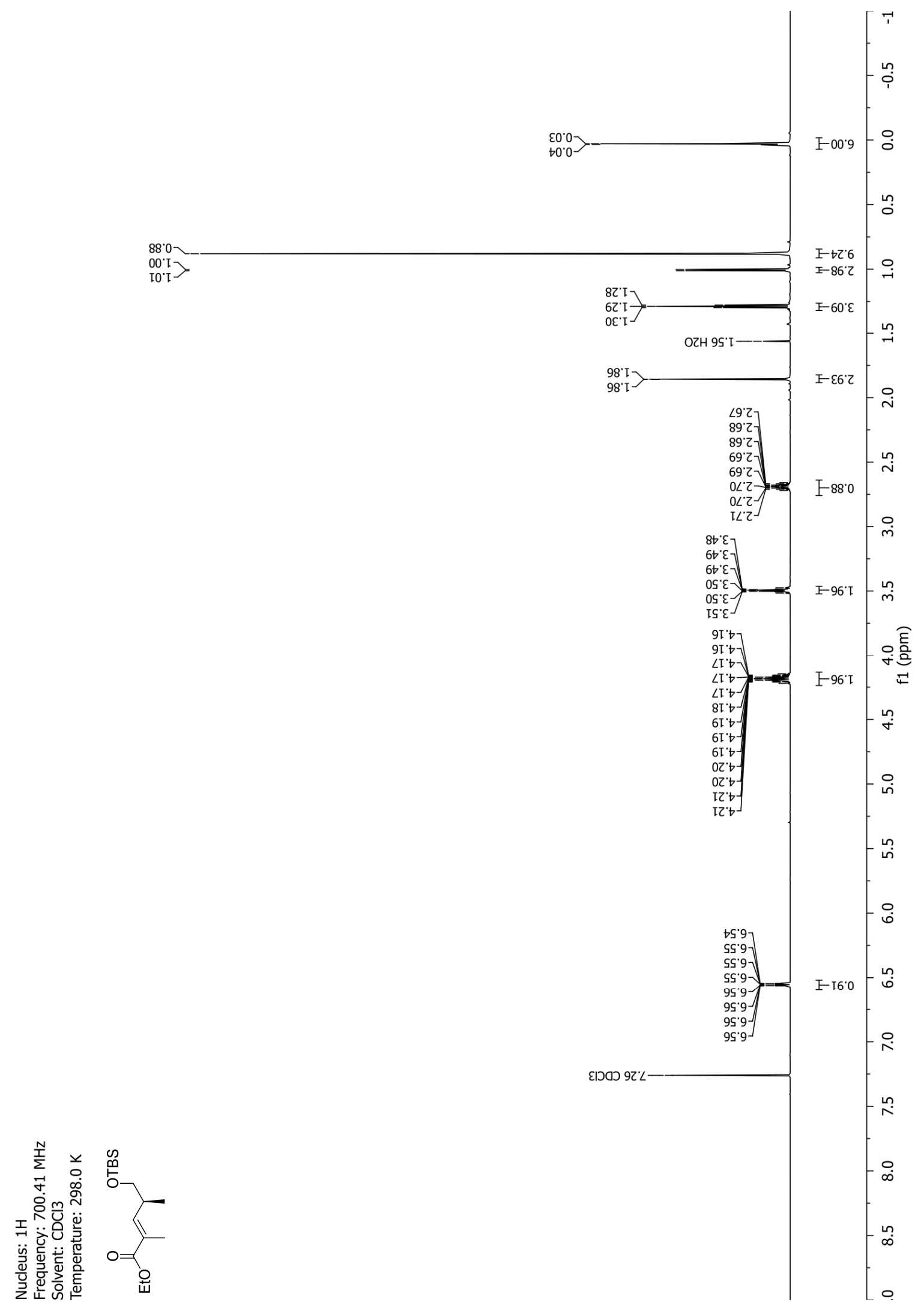




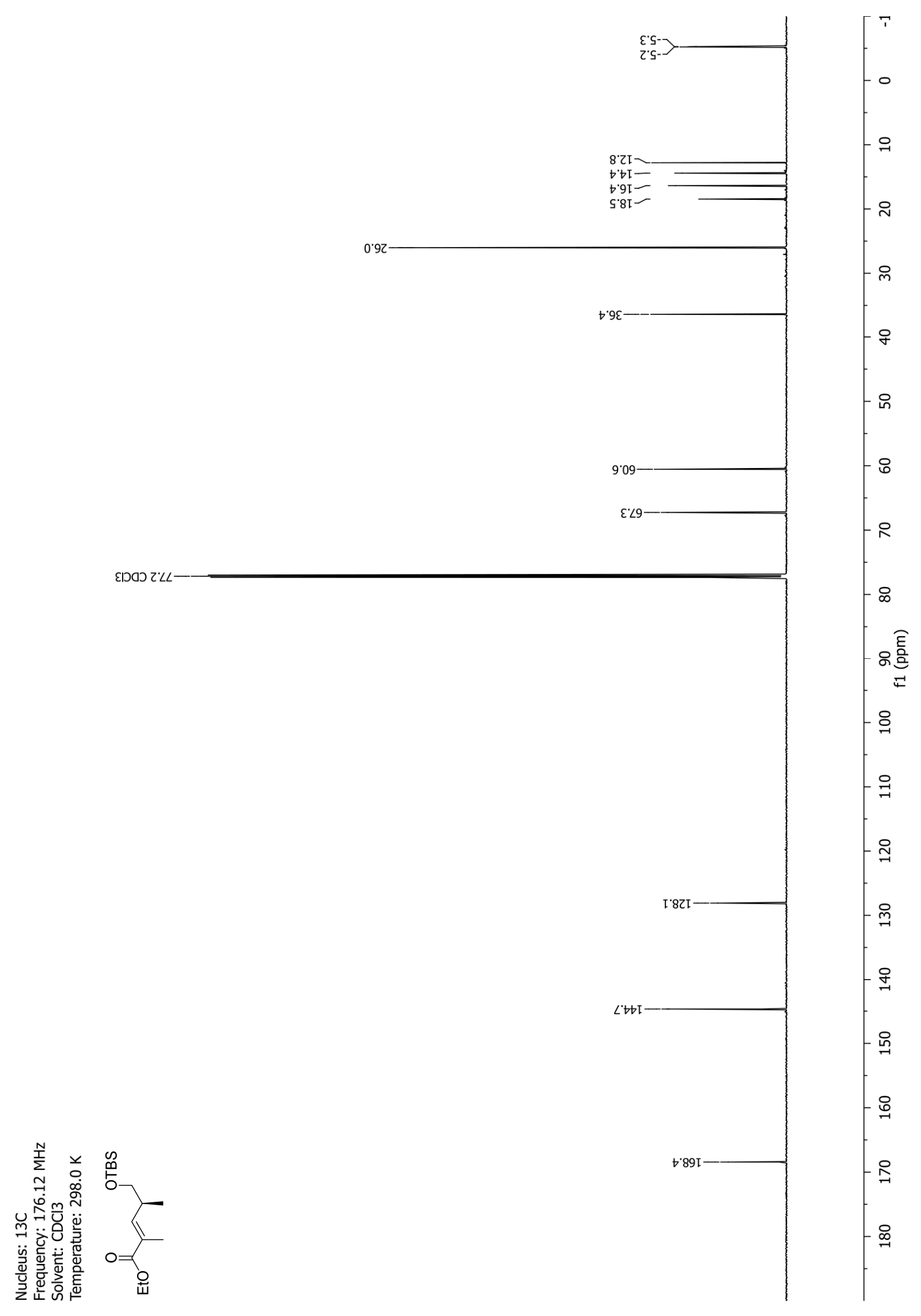




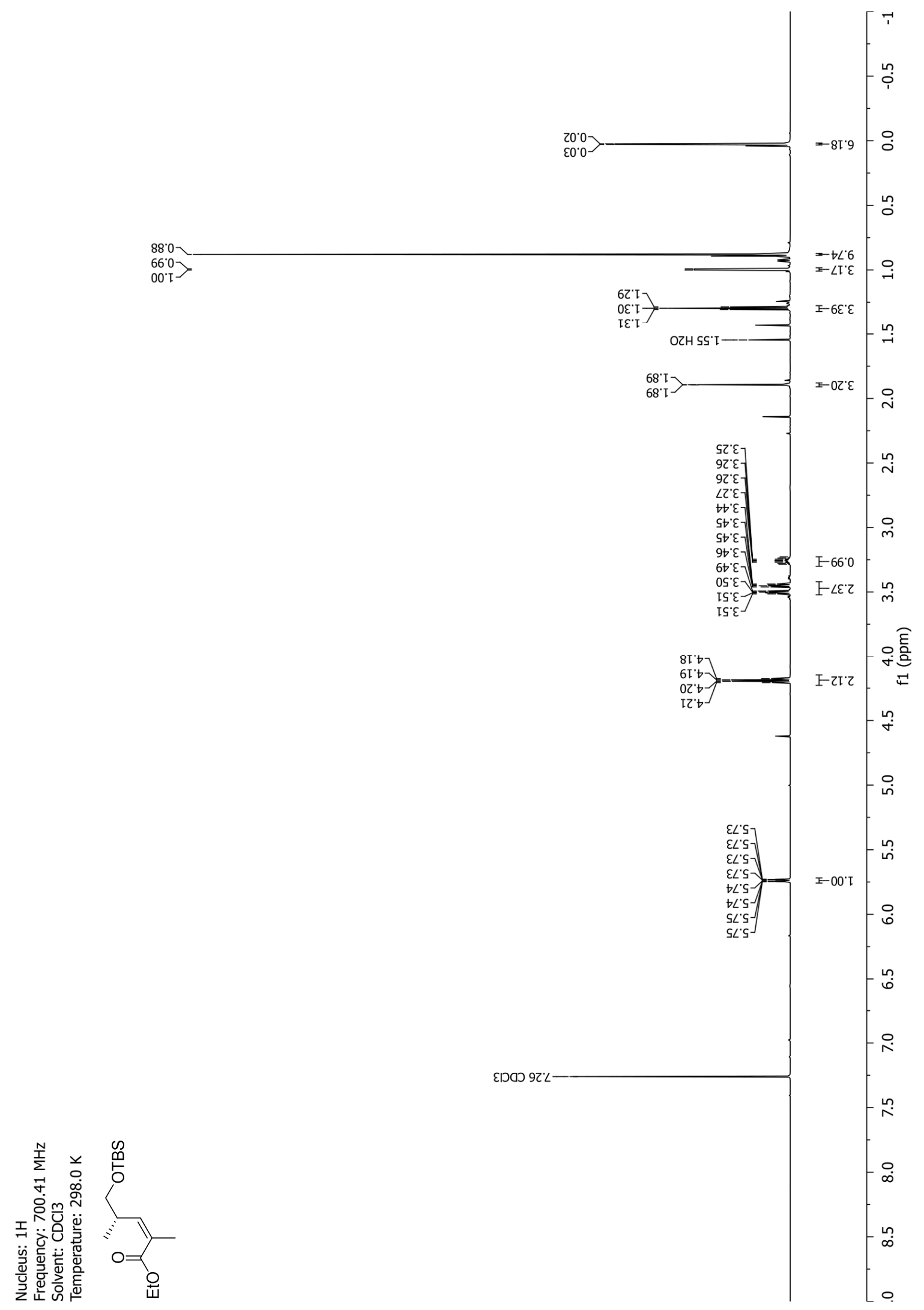




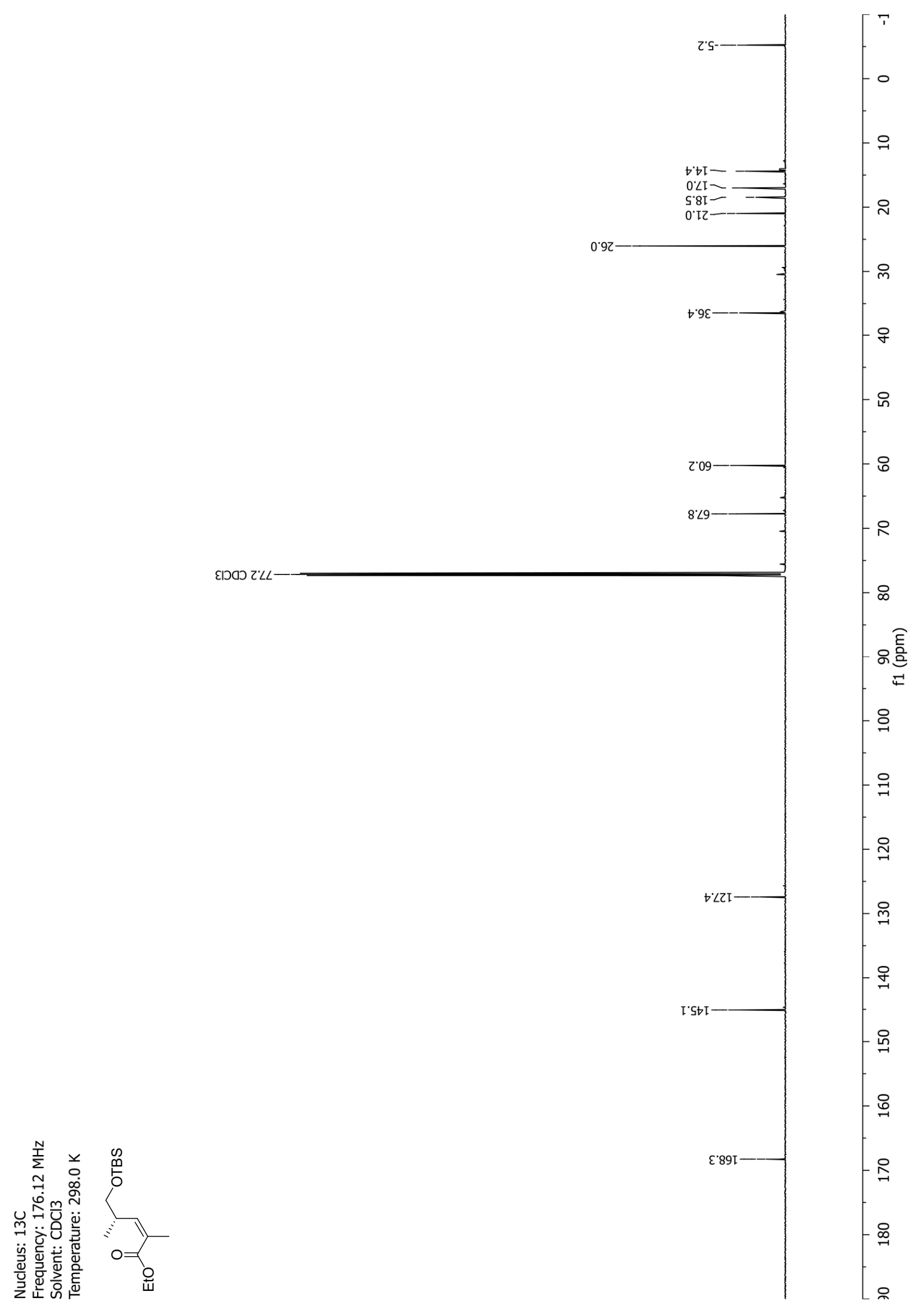


NMR-Spectra for Compound 71

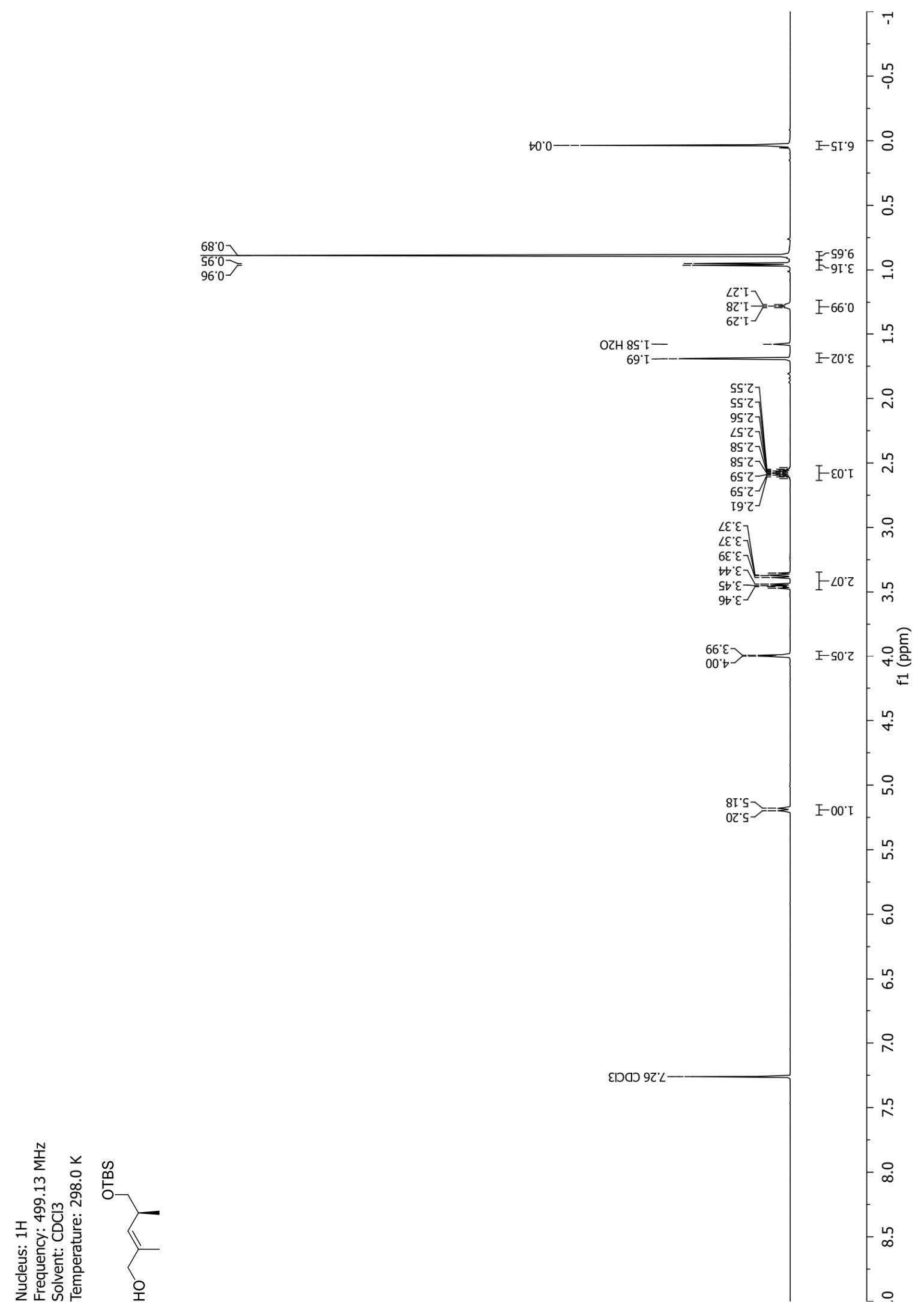




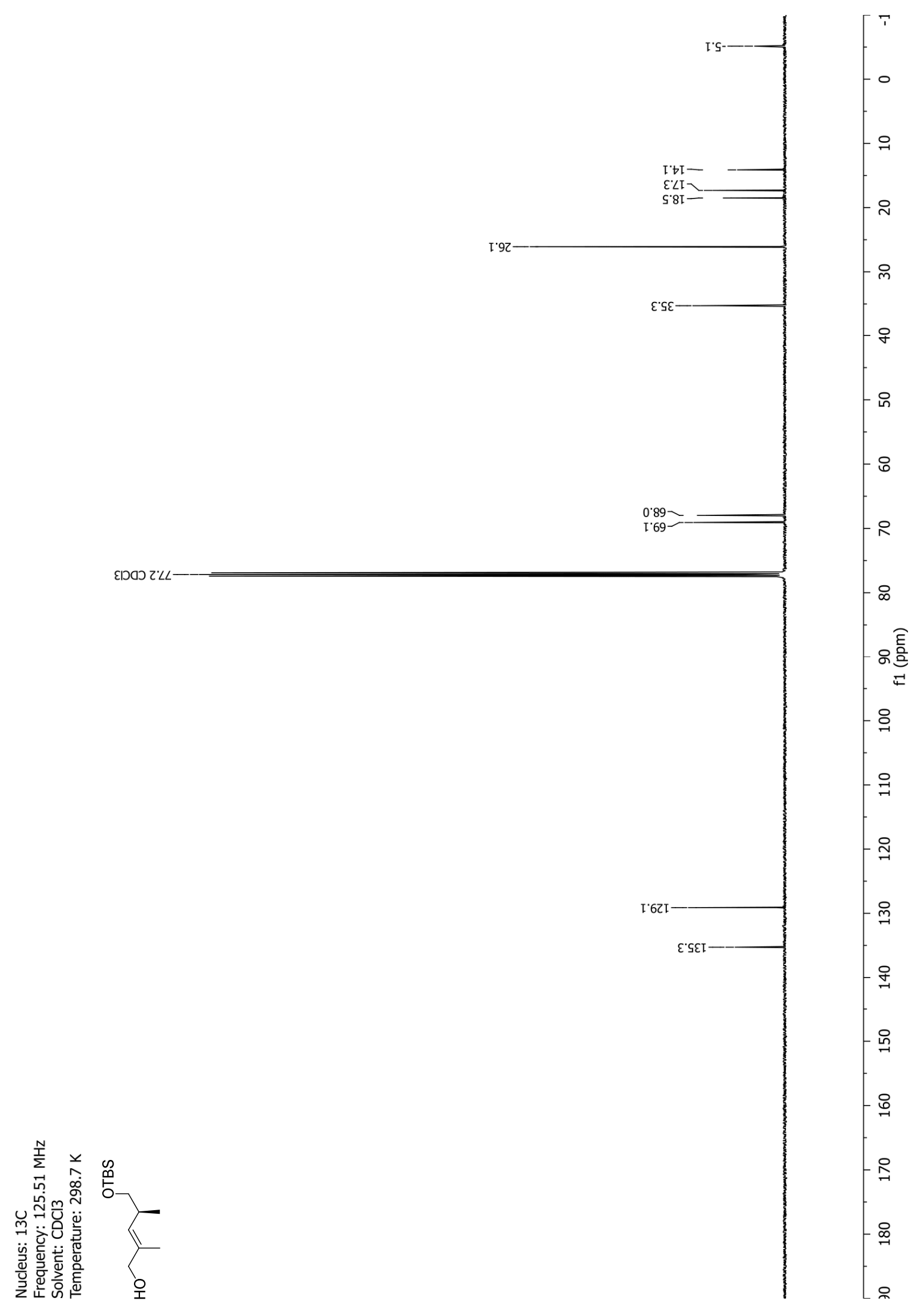


NMR-Spectra for Compound 20

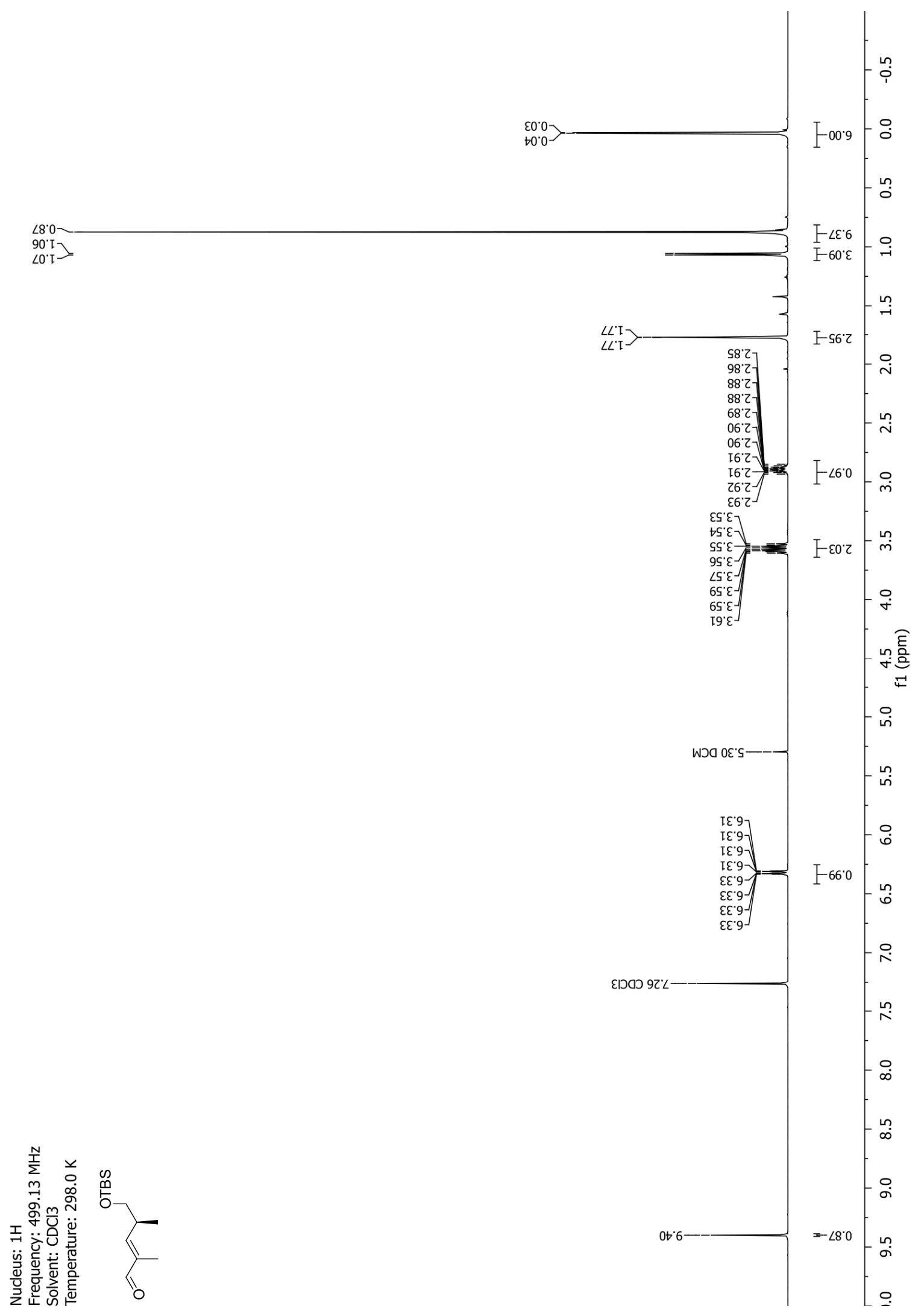




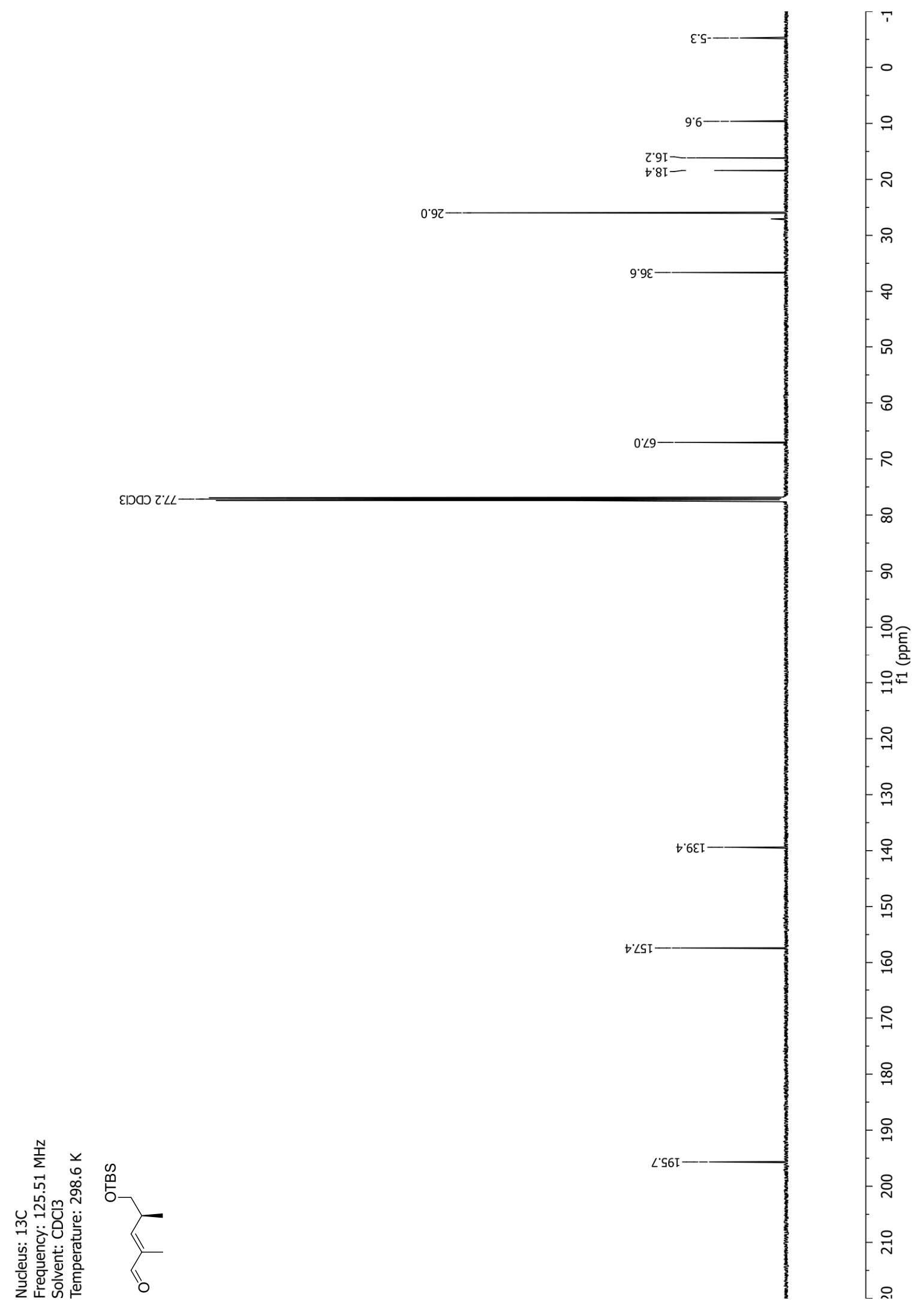


NMR-Spectra for Compound 23

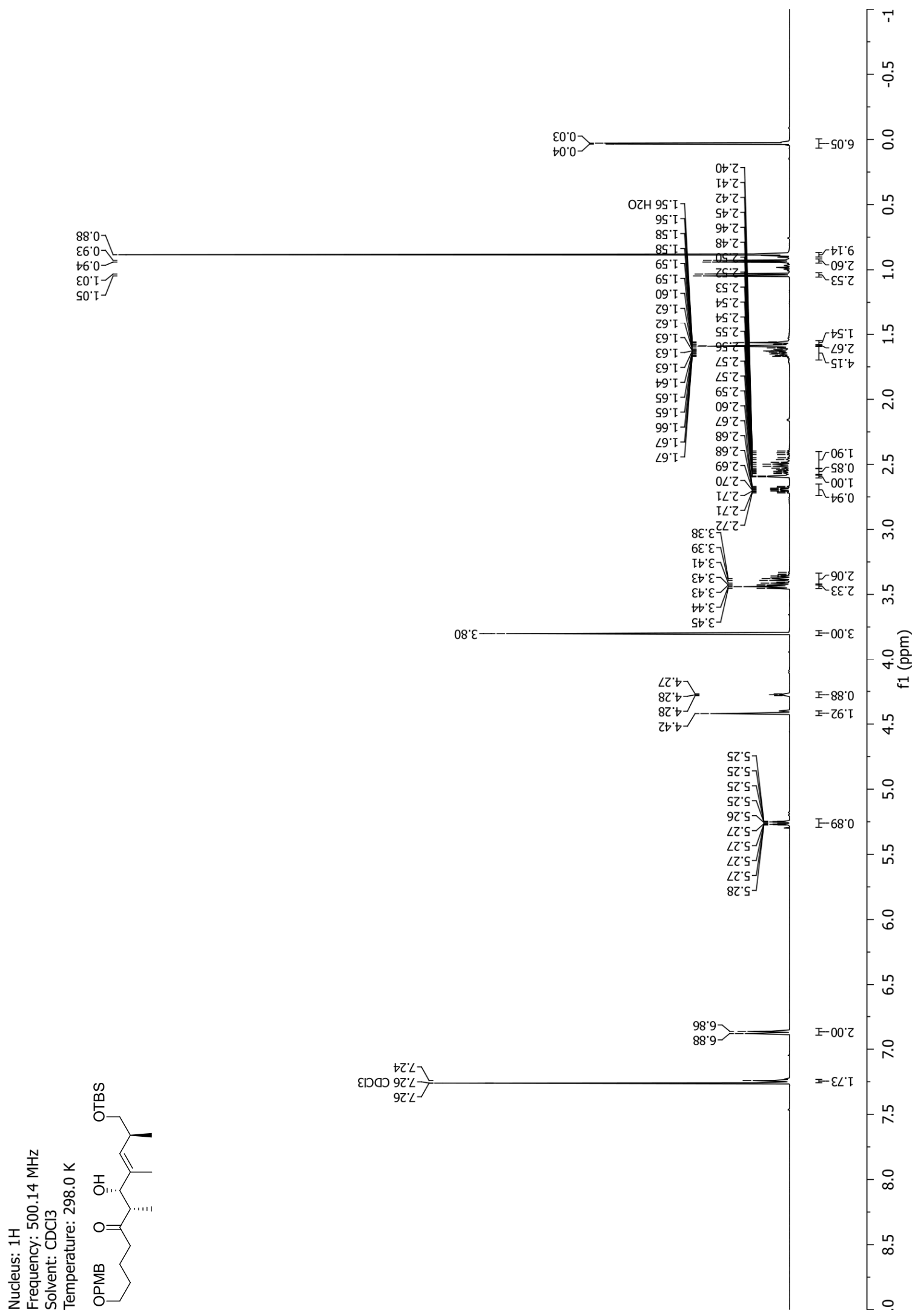




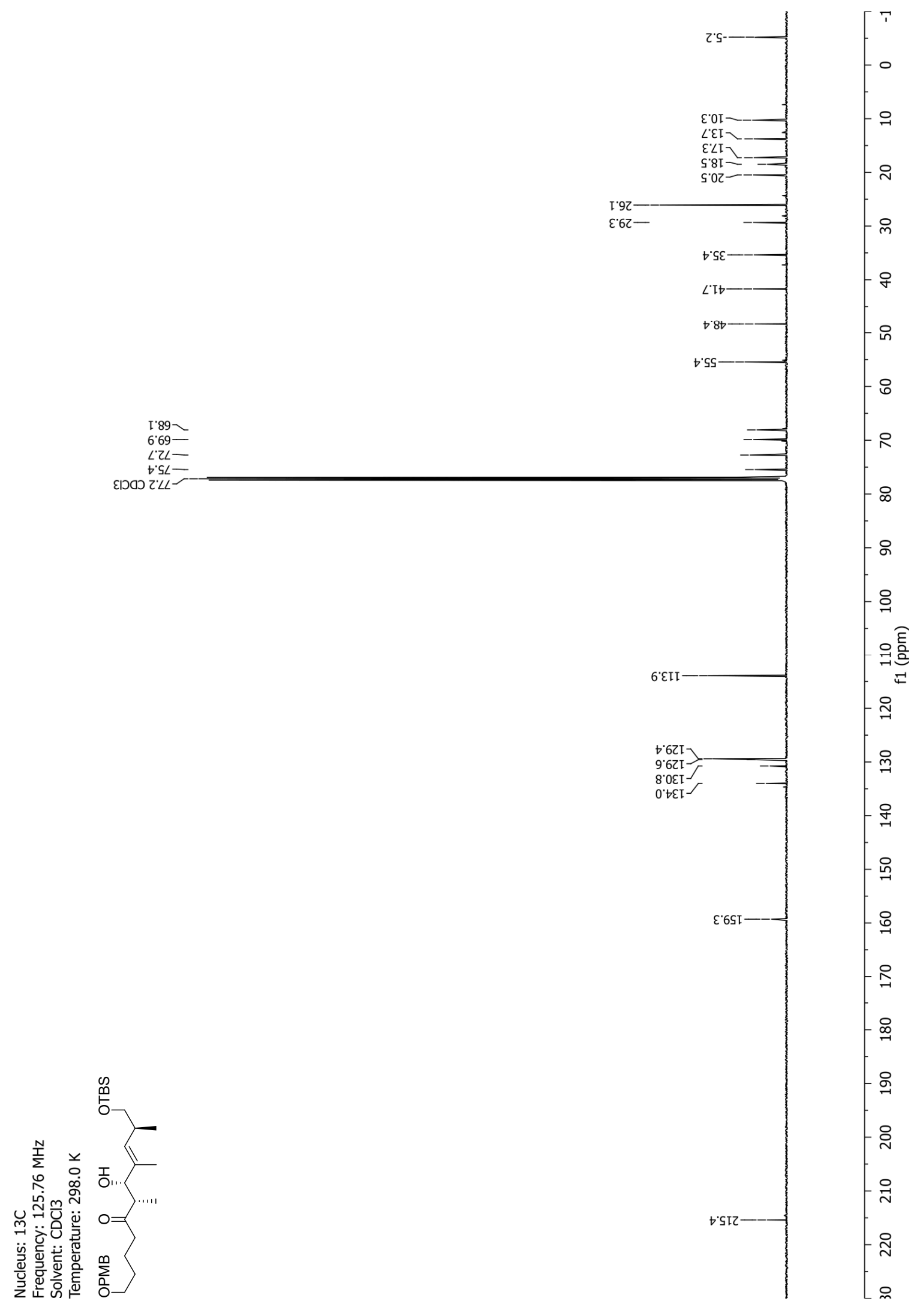




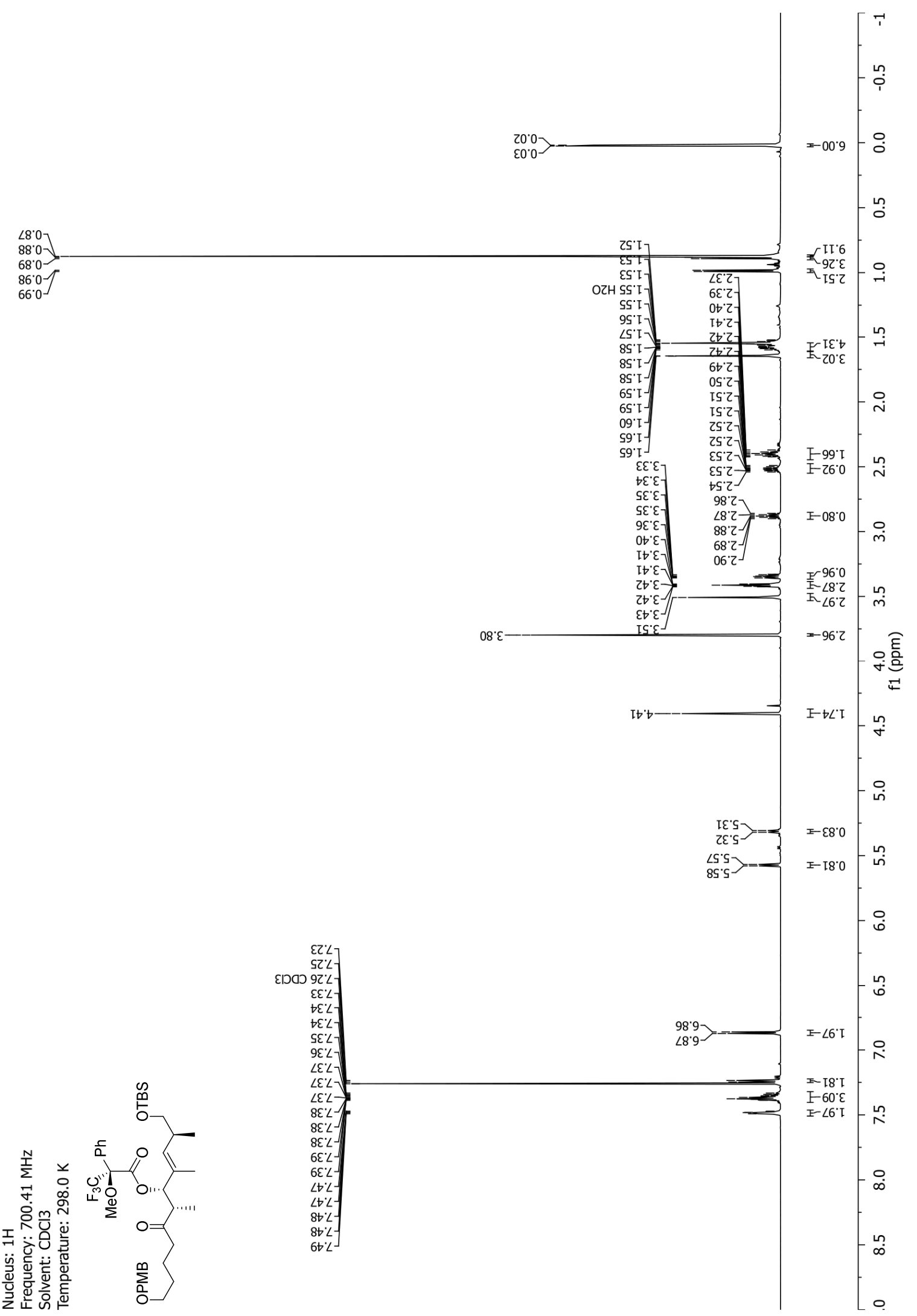




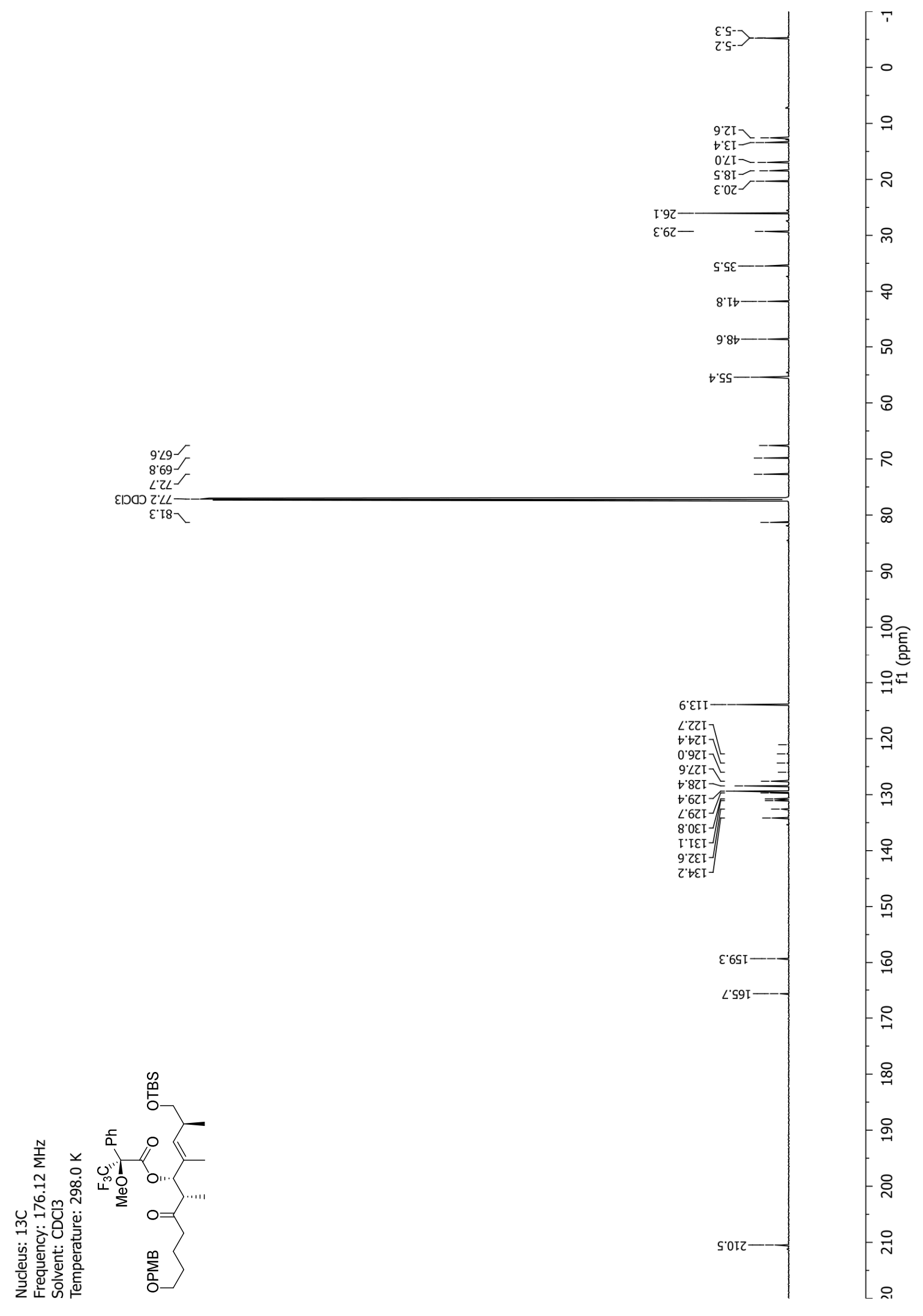


NMR-Spectra for Compound 73

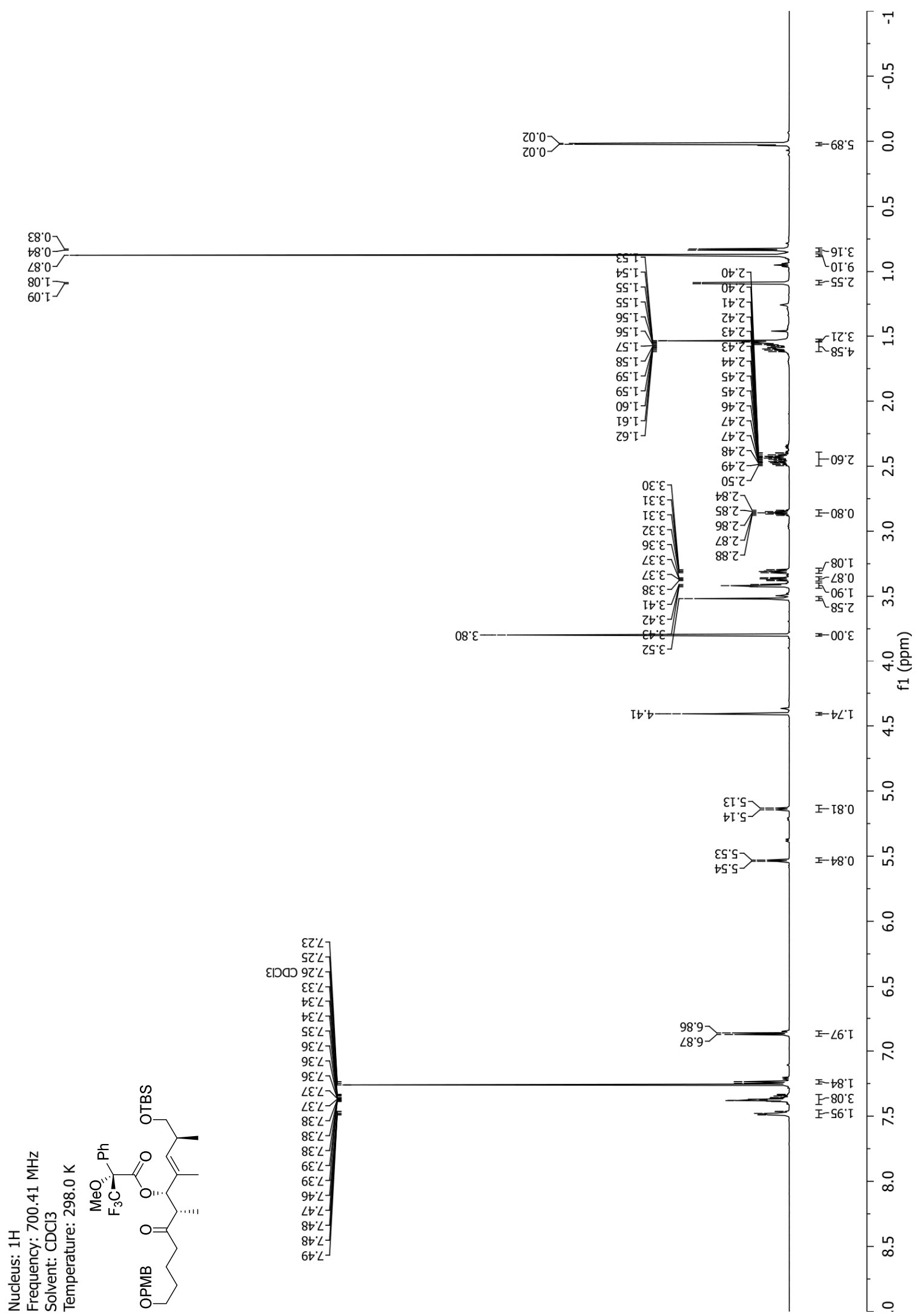




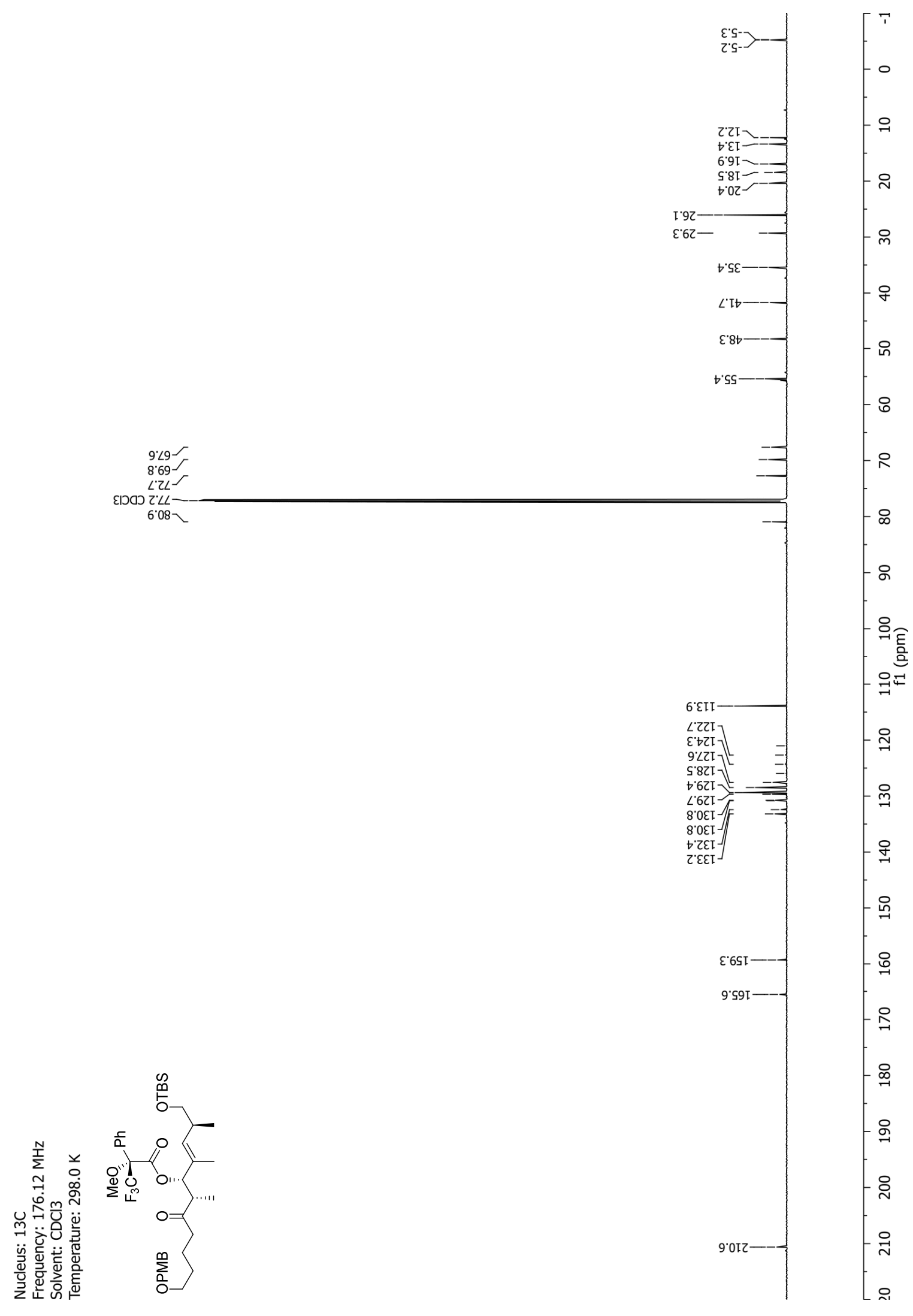




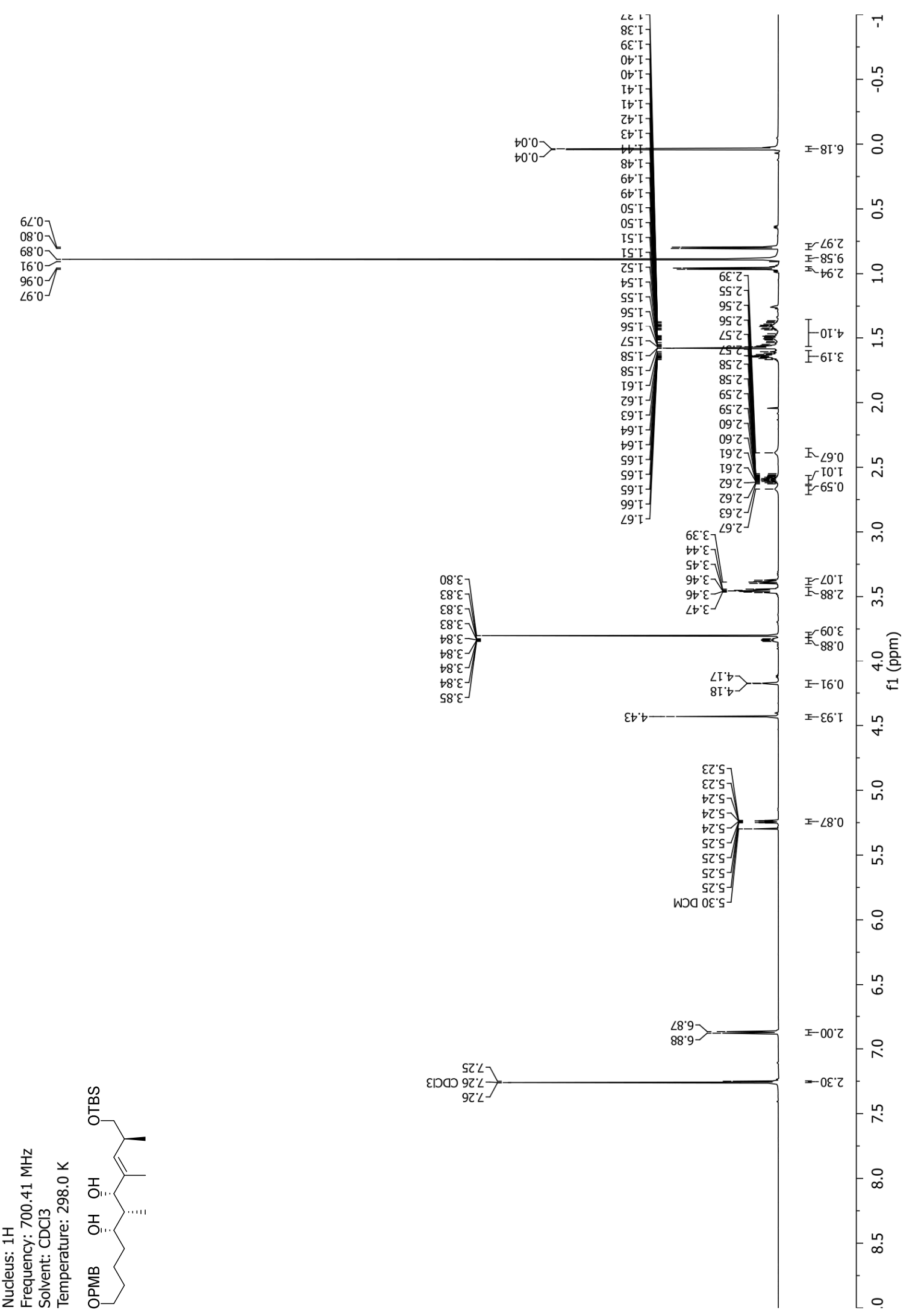




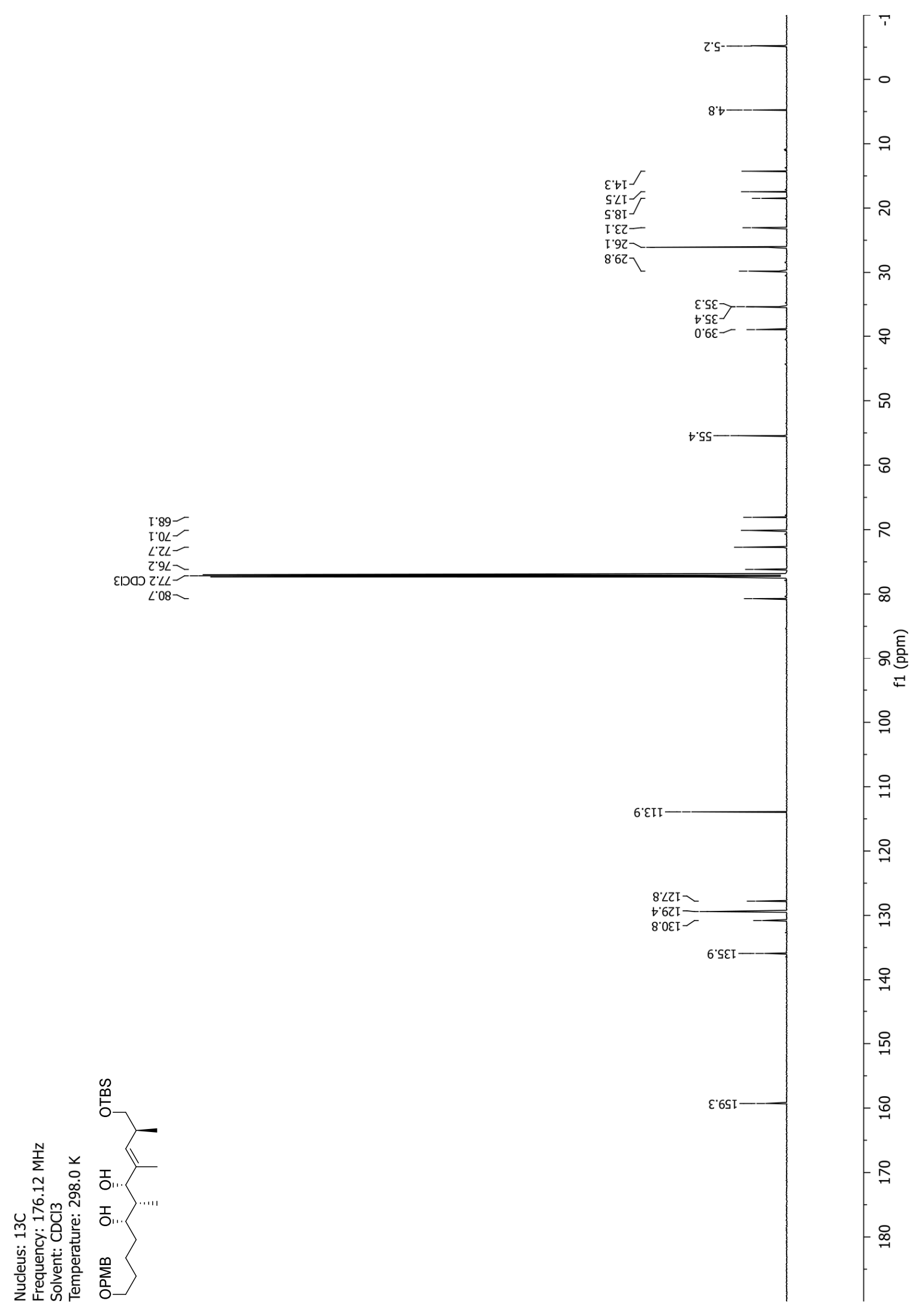




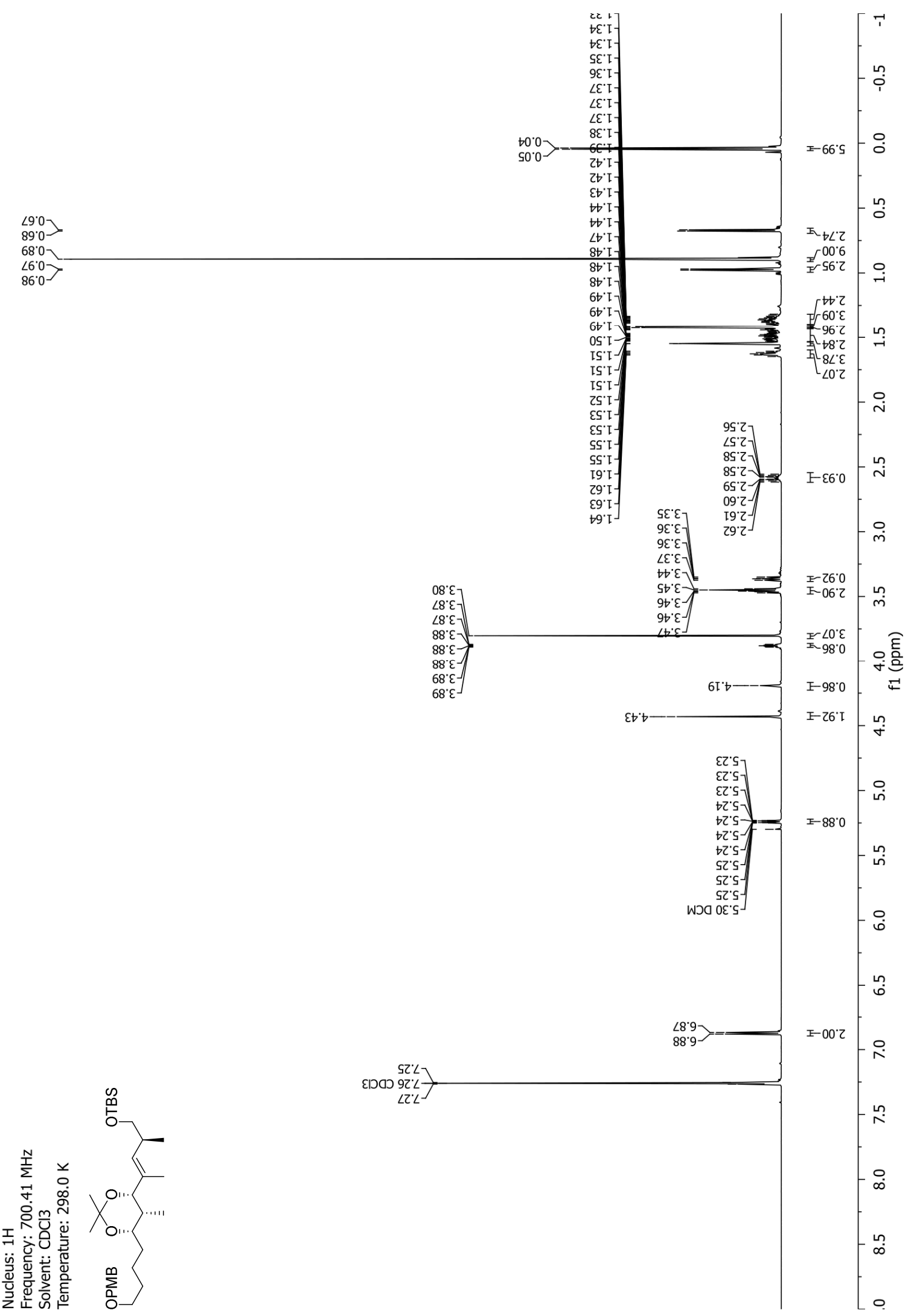




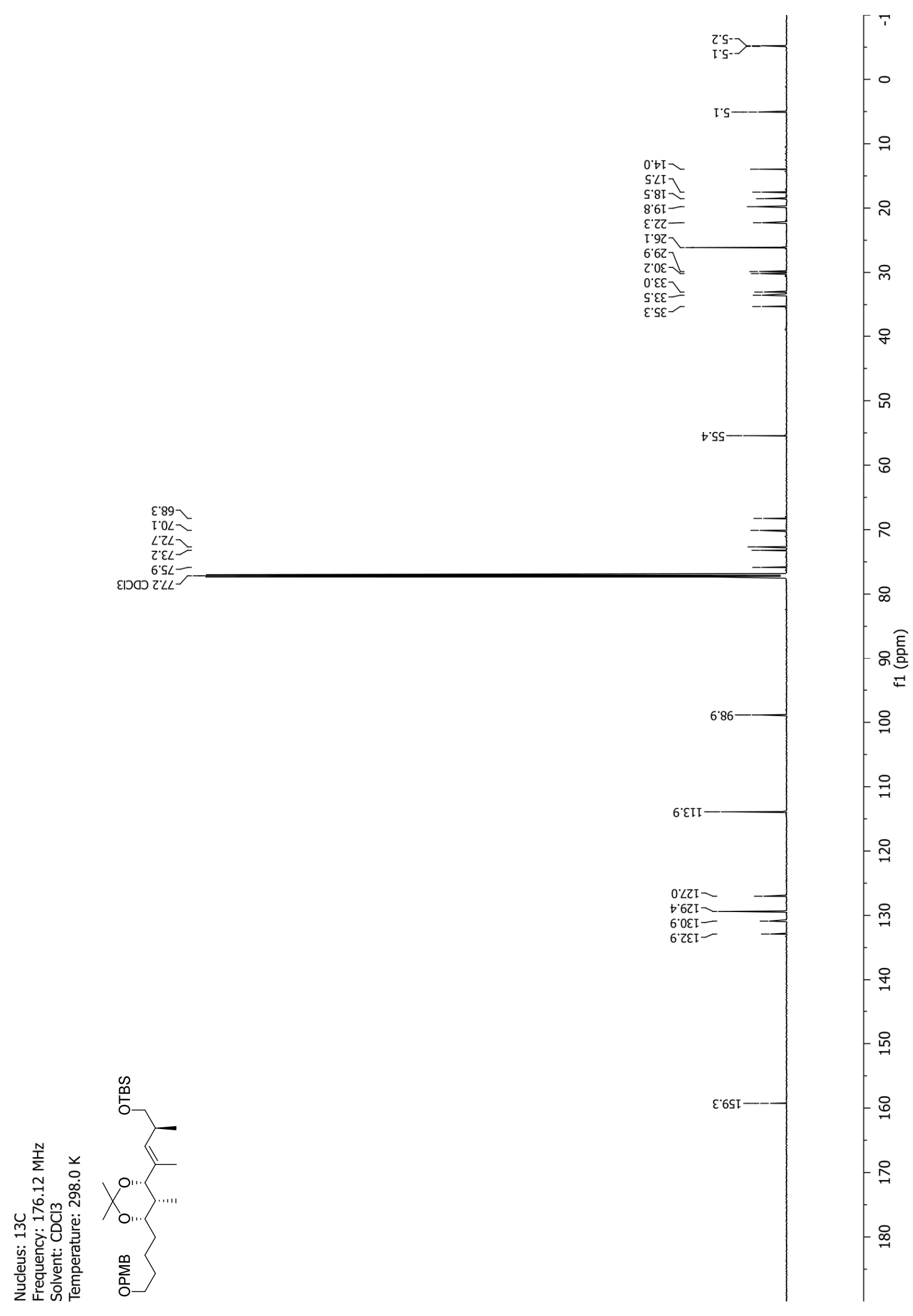


NMR-Spectra for Compound 3

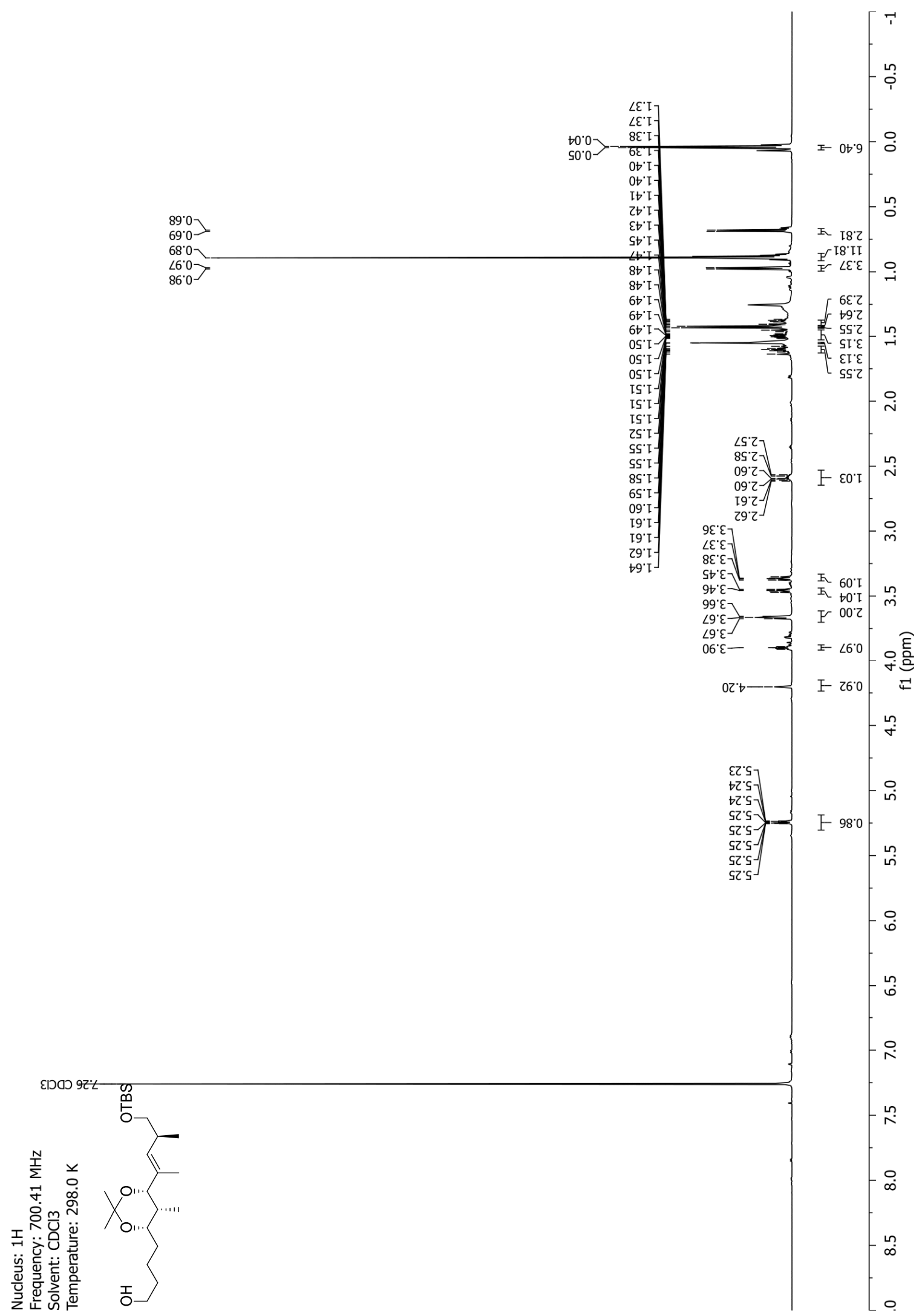




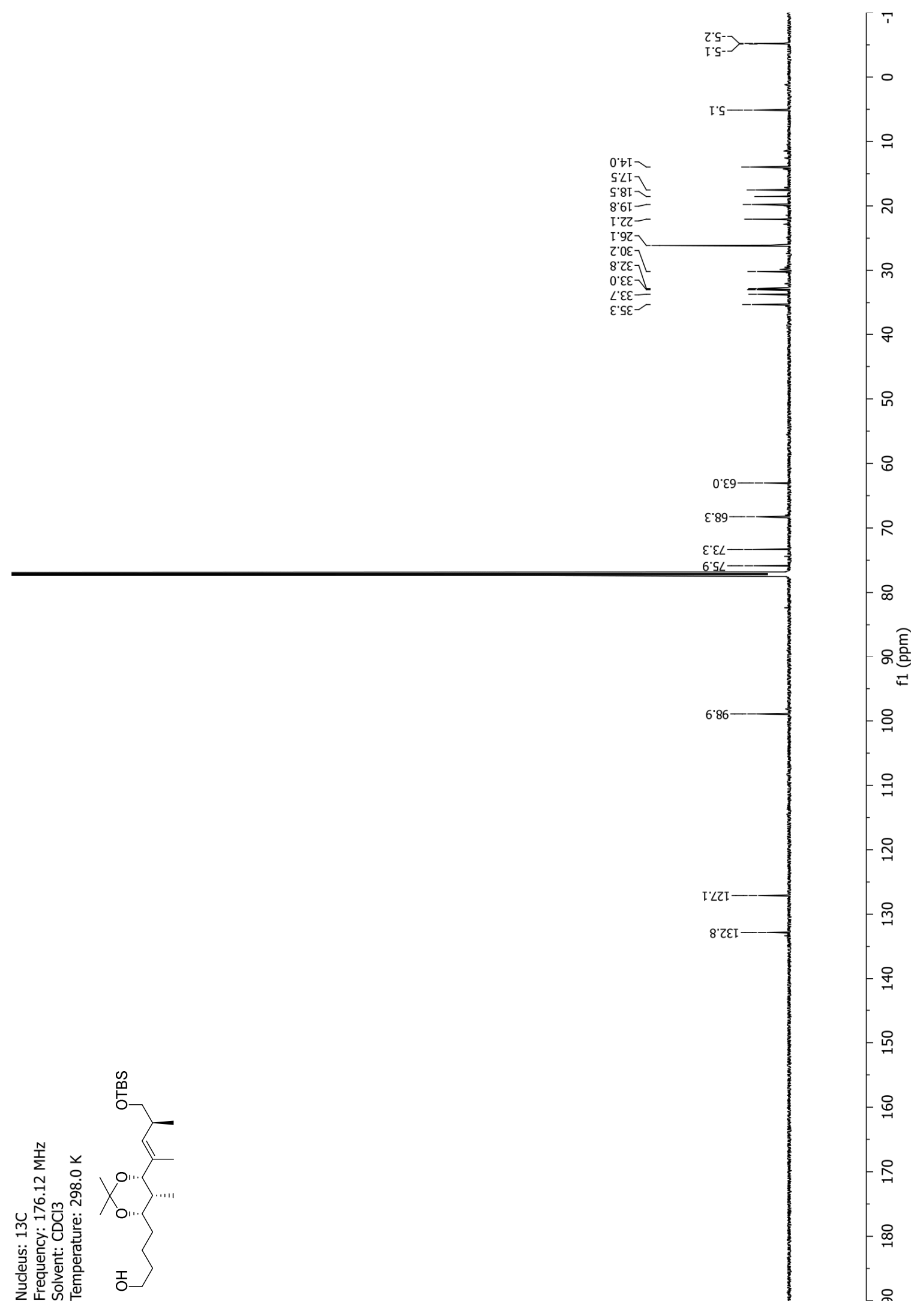


NMR-Spectra for Compound 40

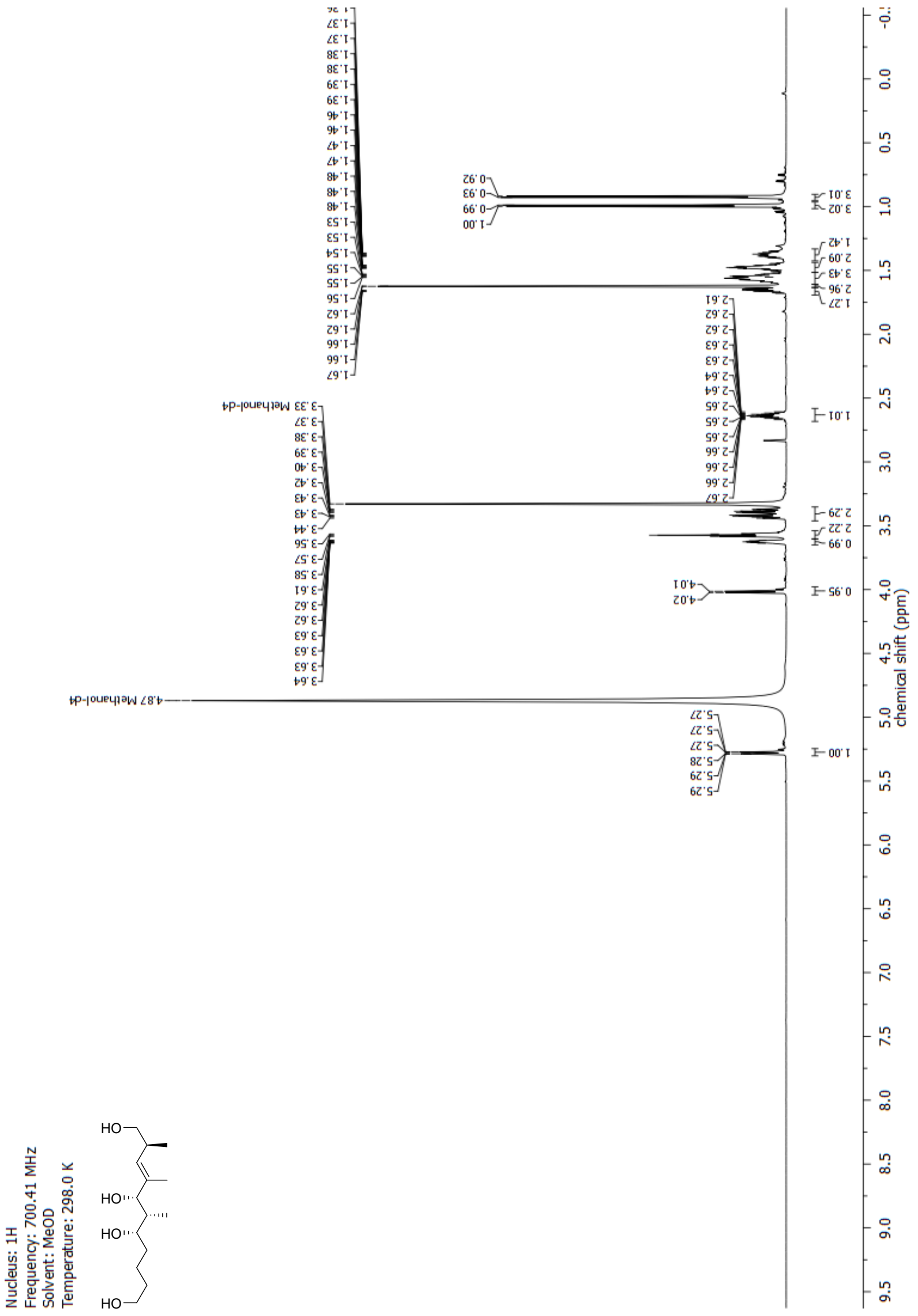




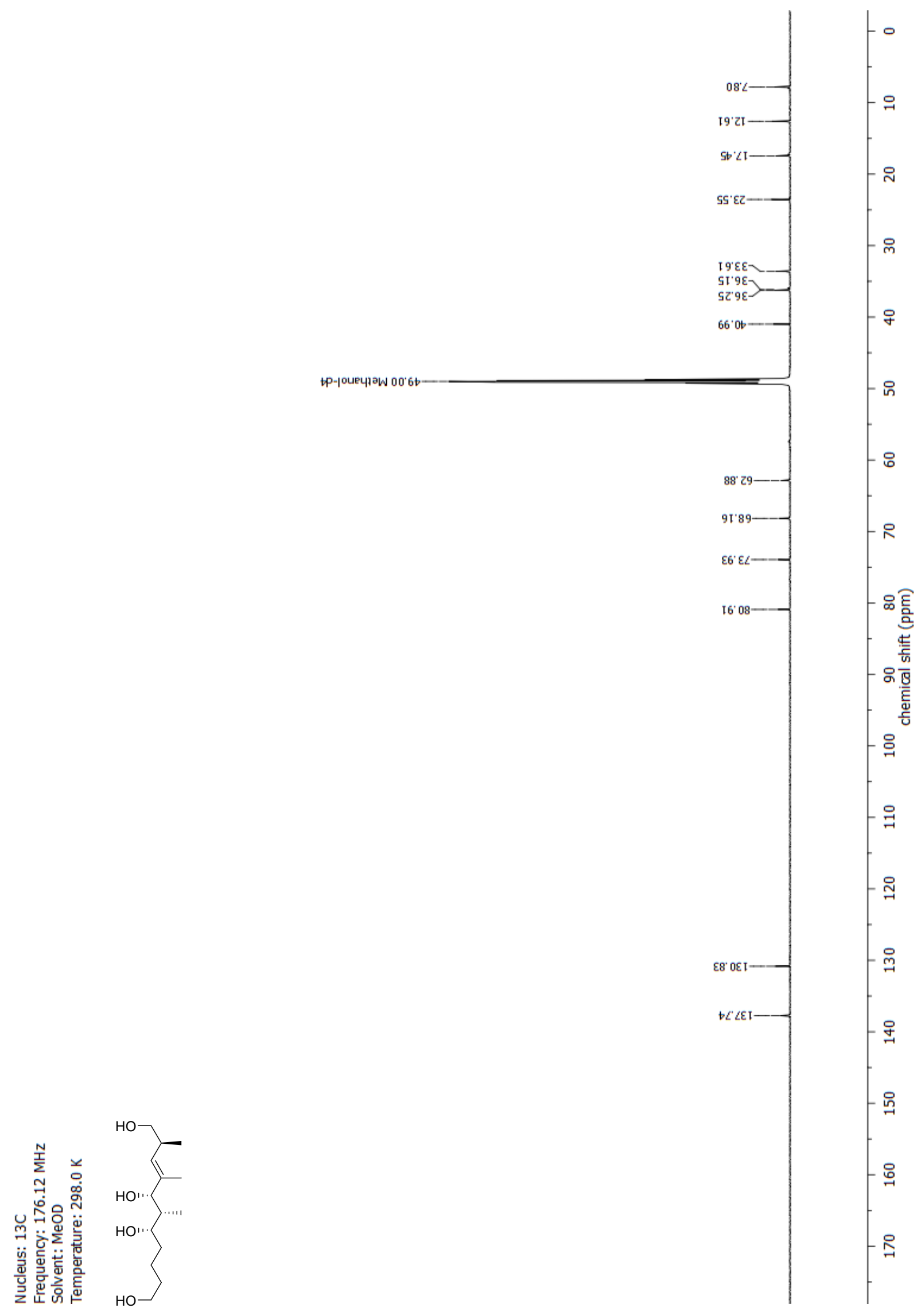


NMR-Spectra for Compound 25

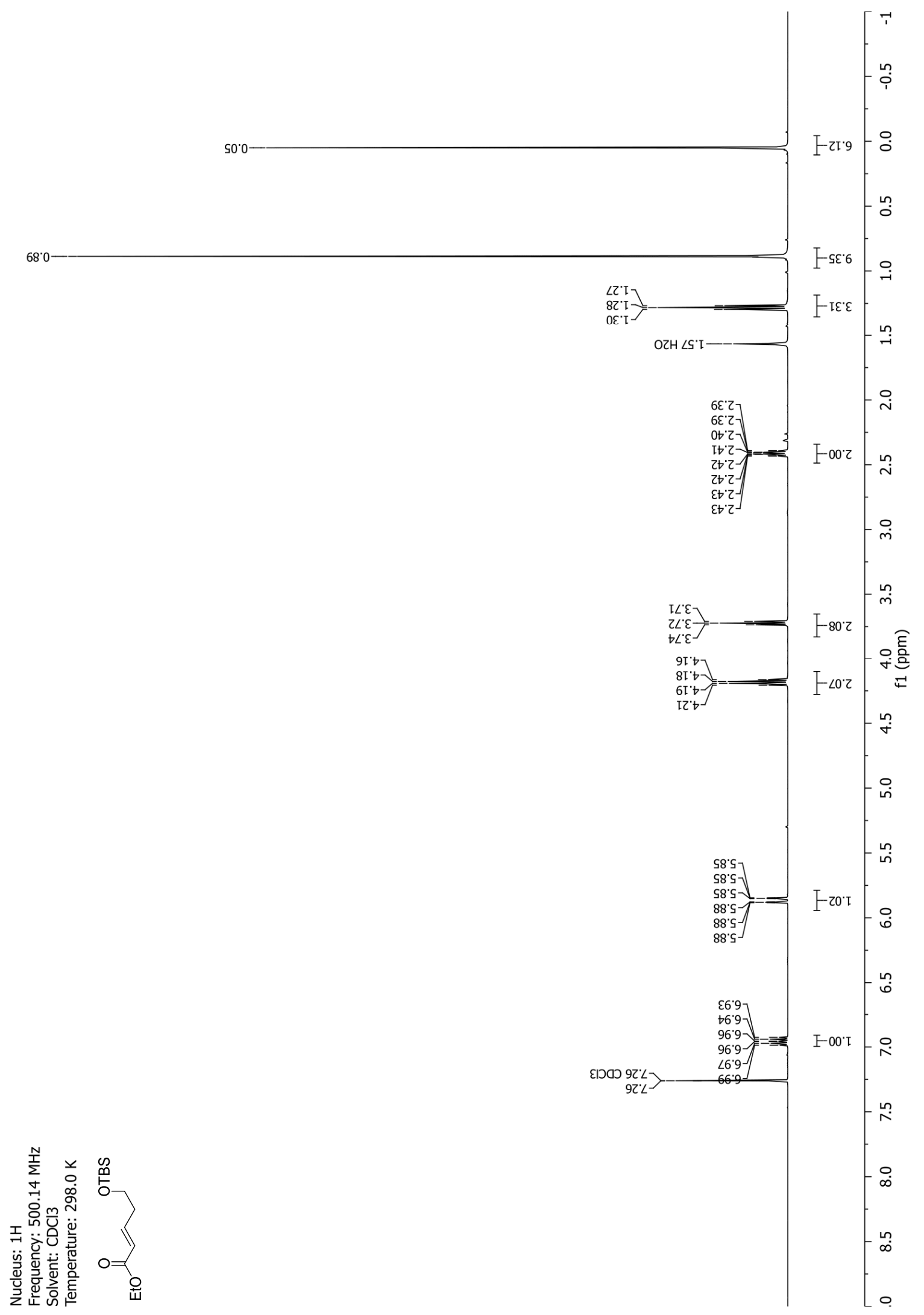




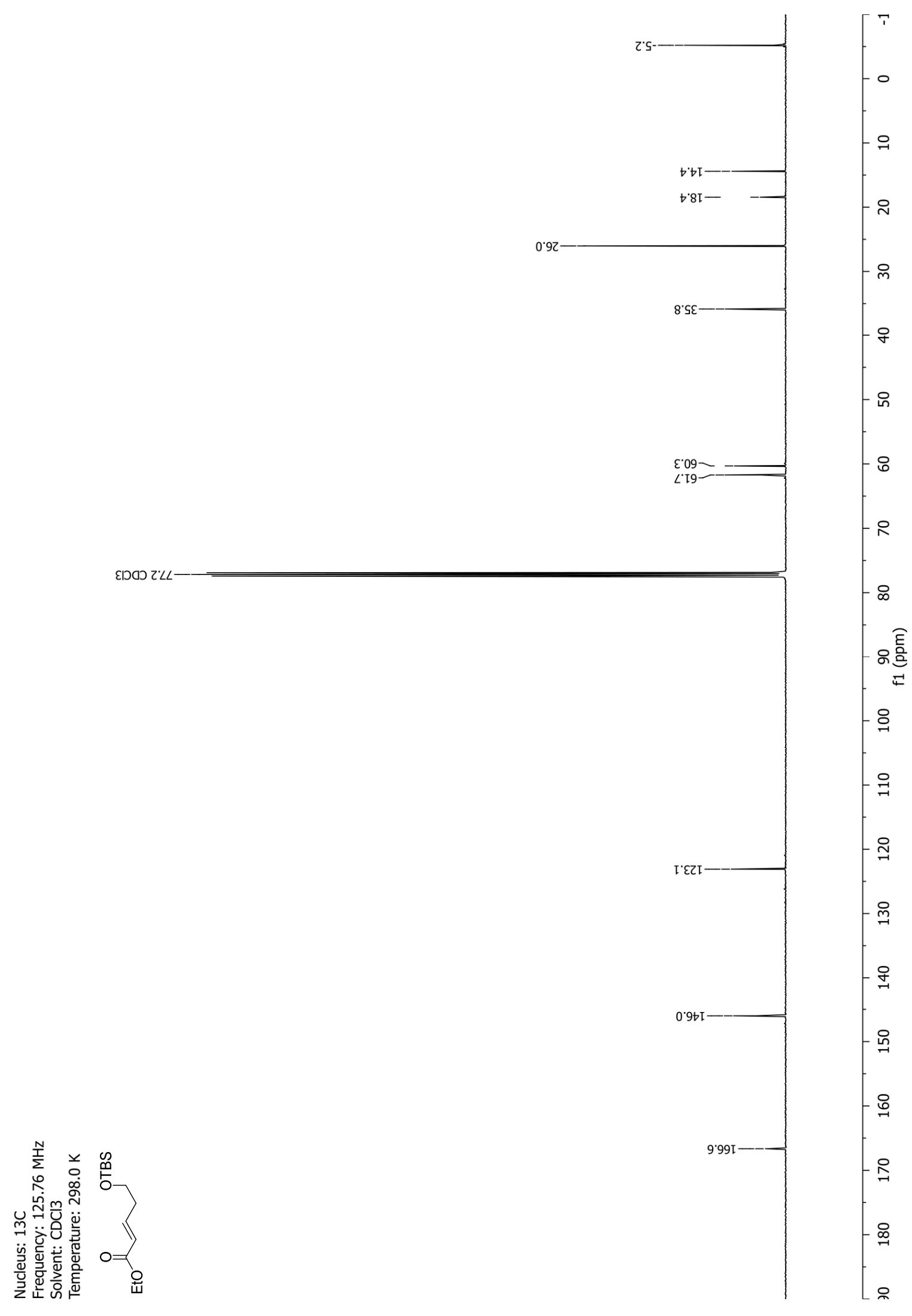


NMR-Spectra for Compound 26

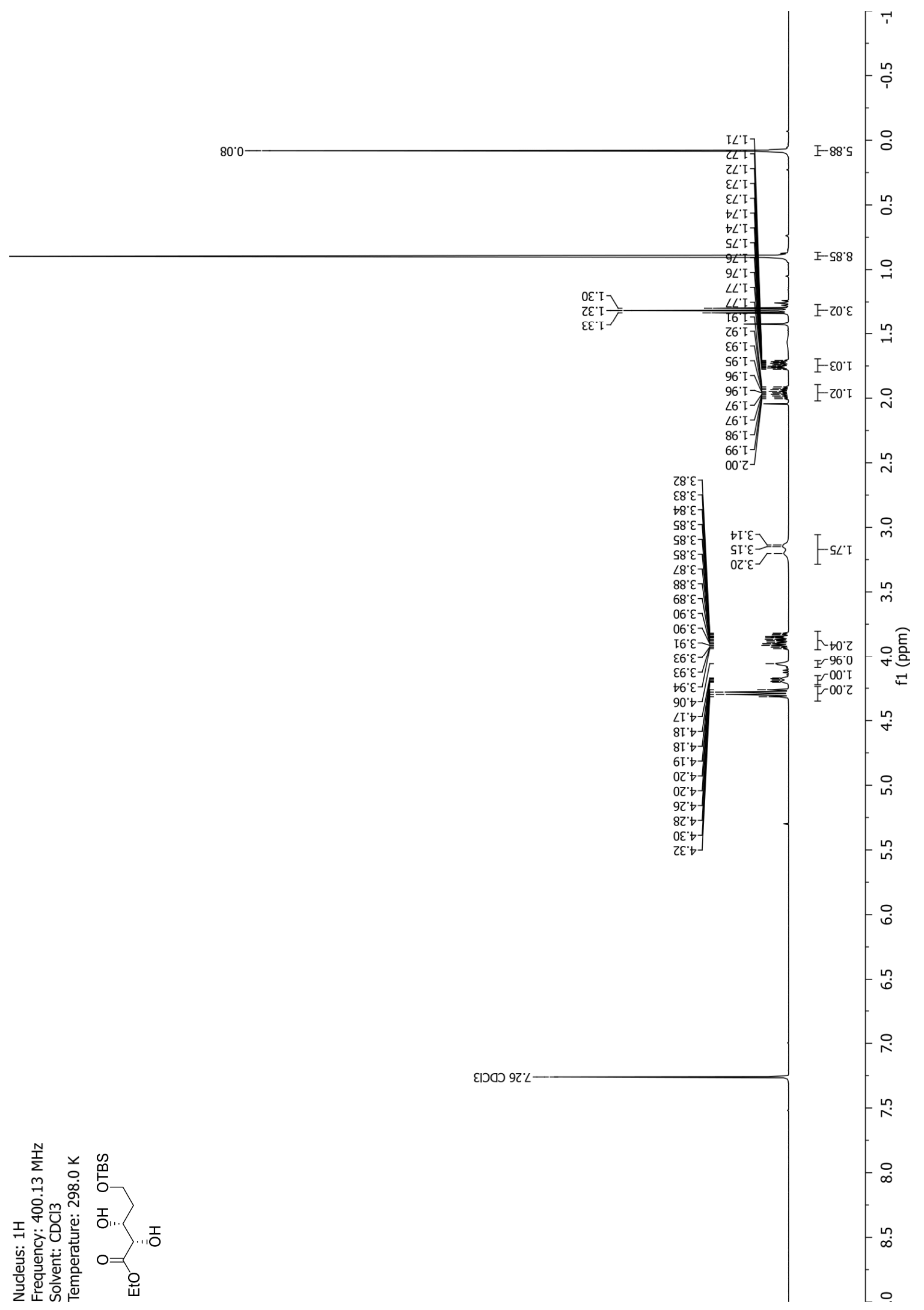




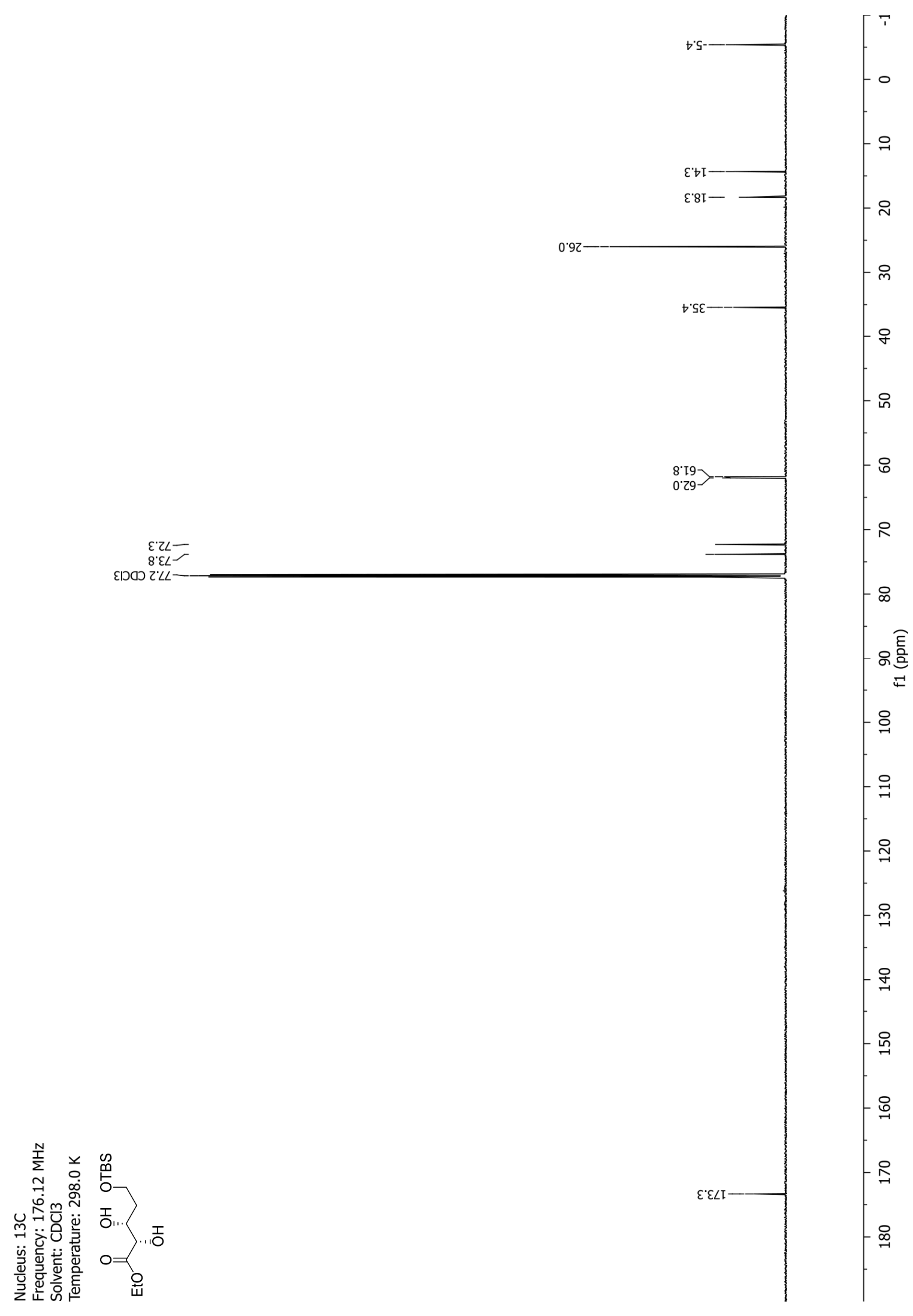


NMR-Spectra for Compound 76

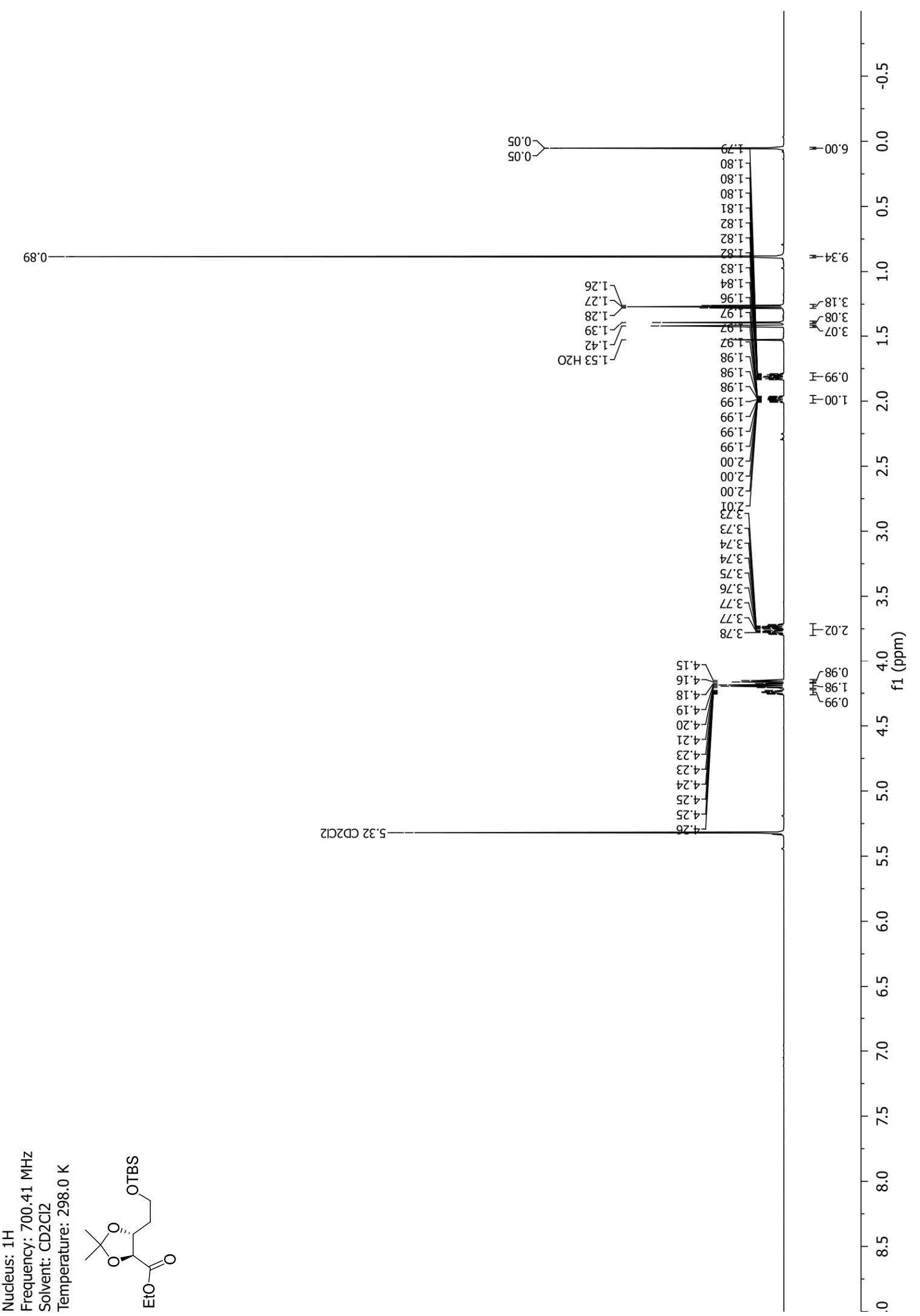




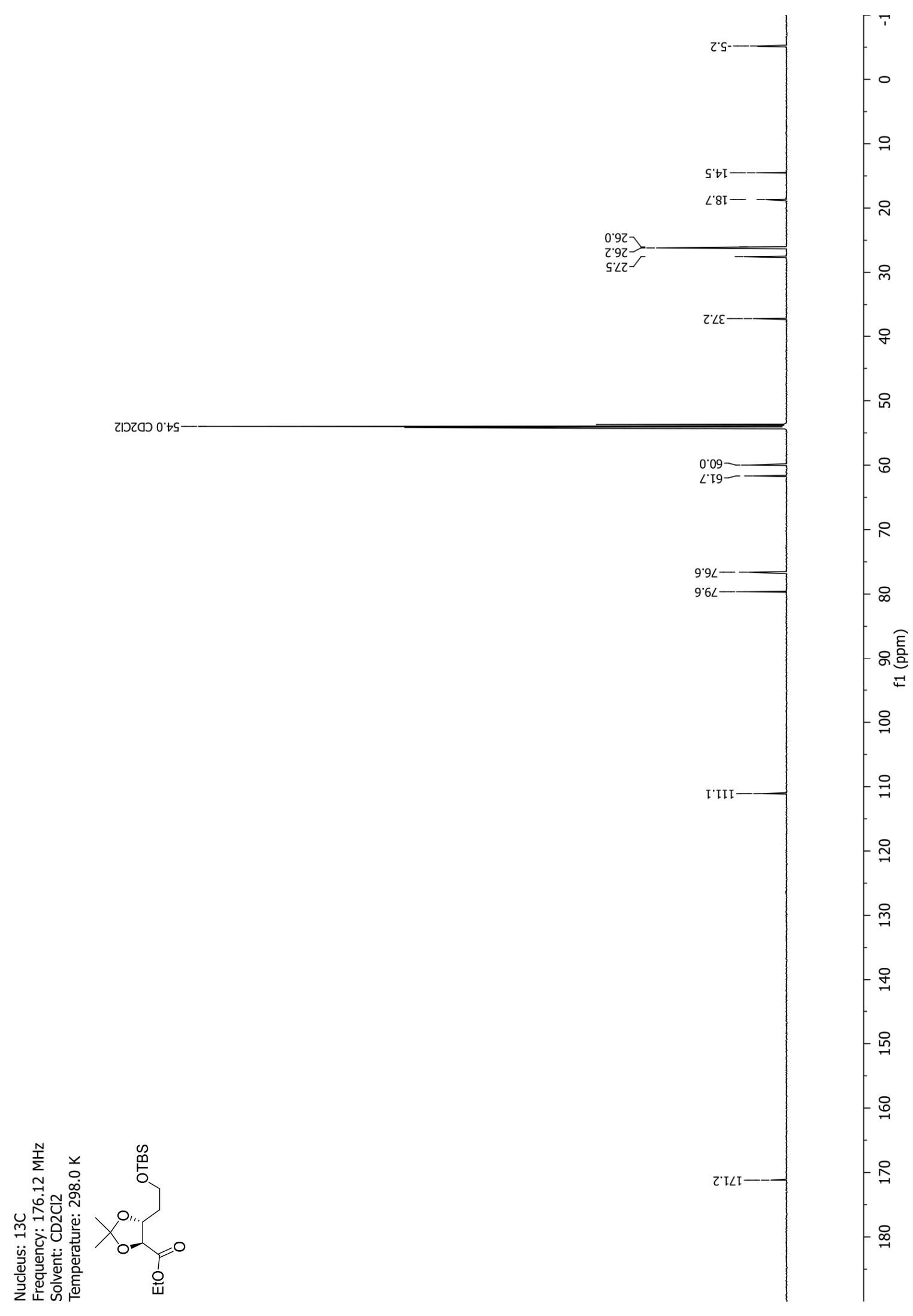


NMR-Spectra for Compound 77

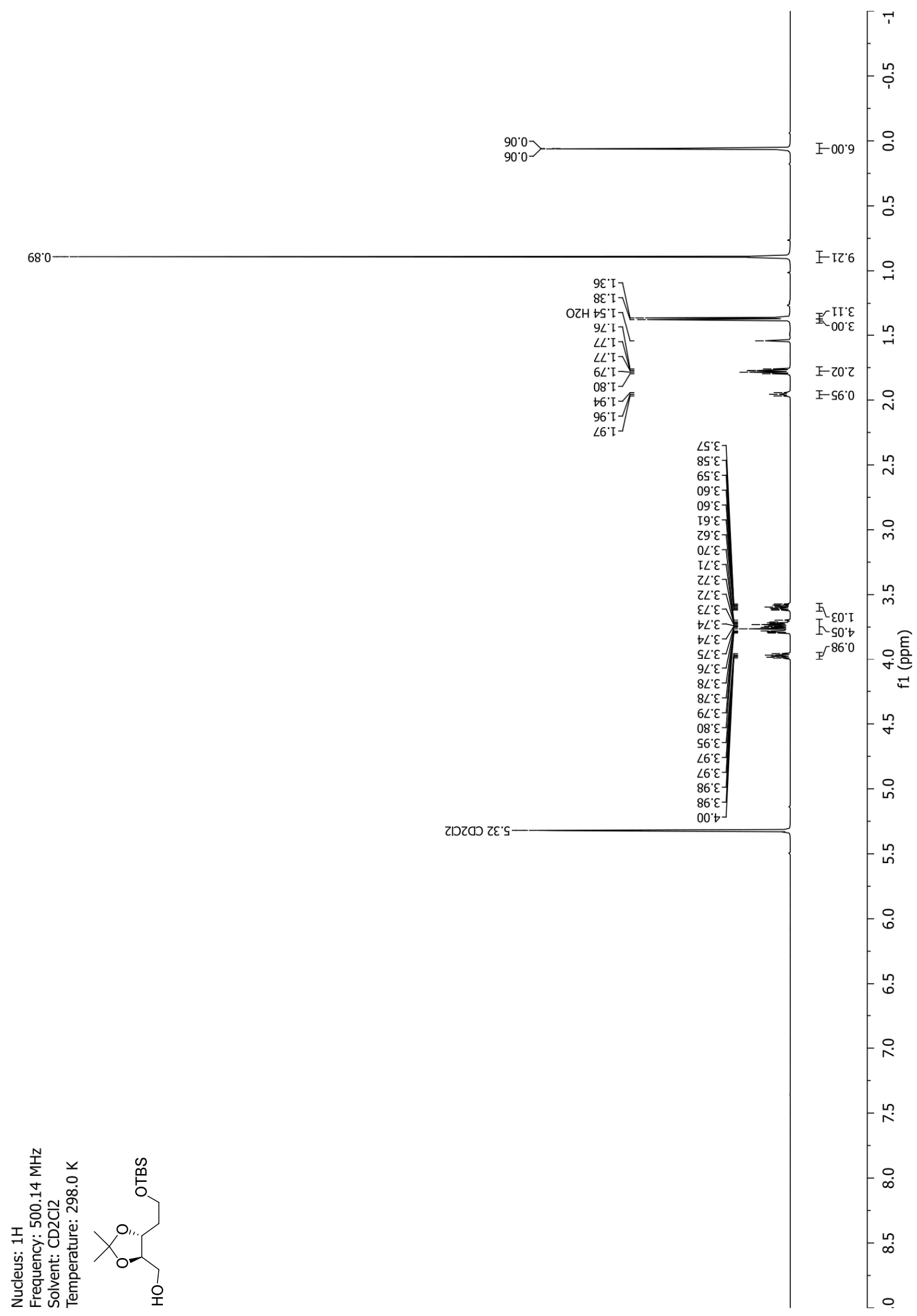




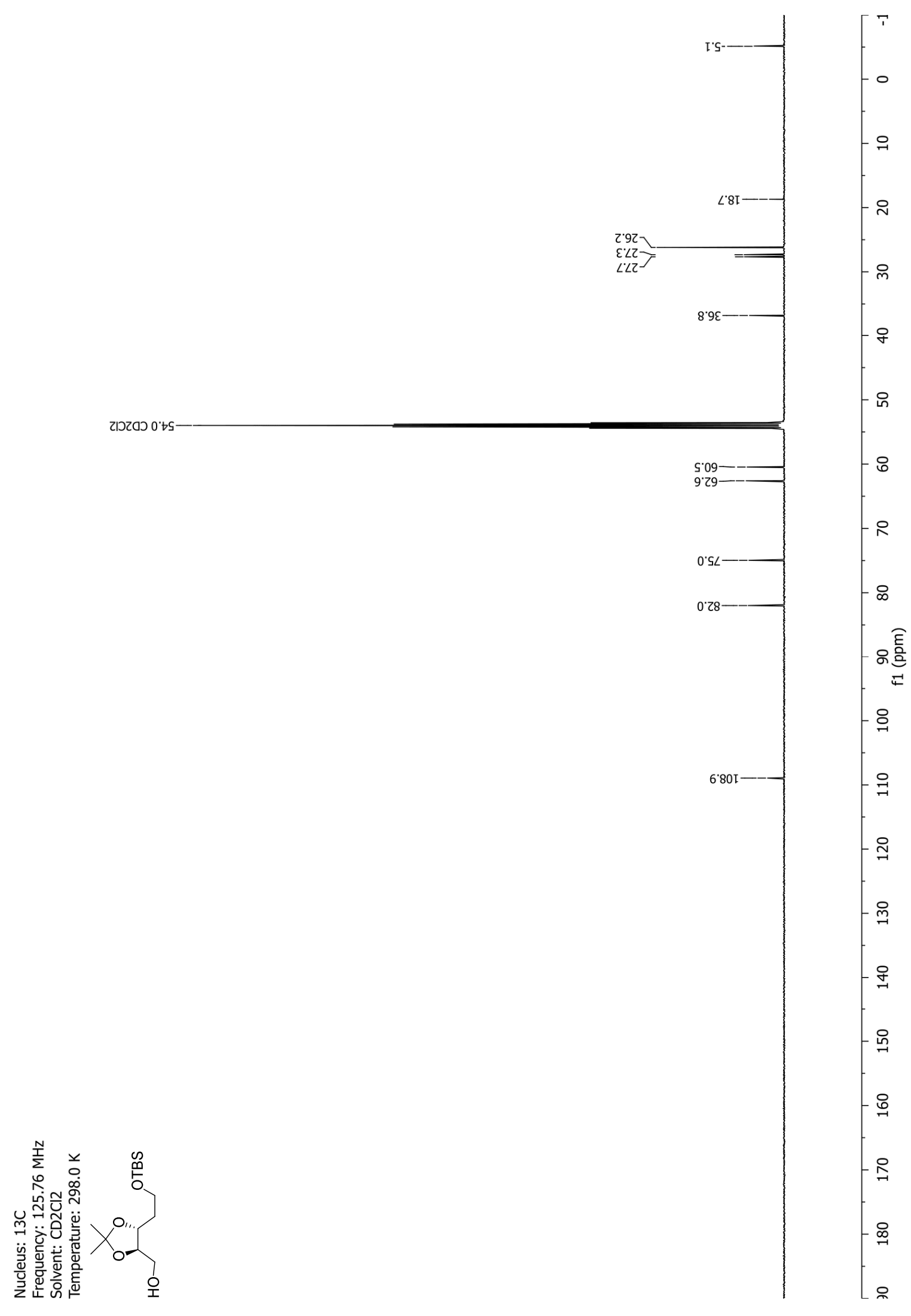


NMR-Spectra for Compound 78

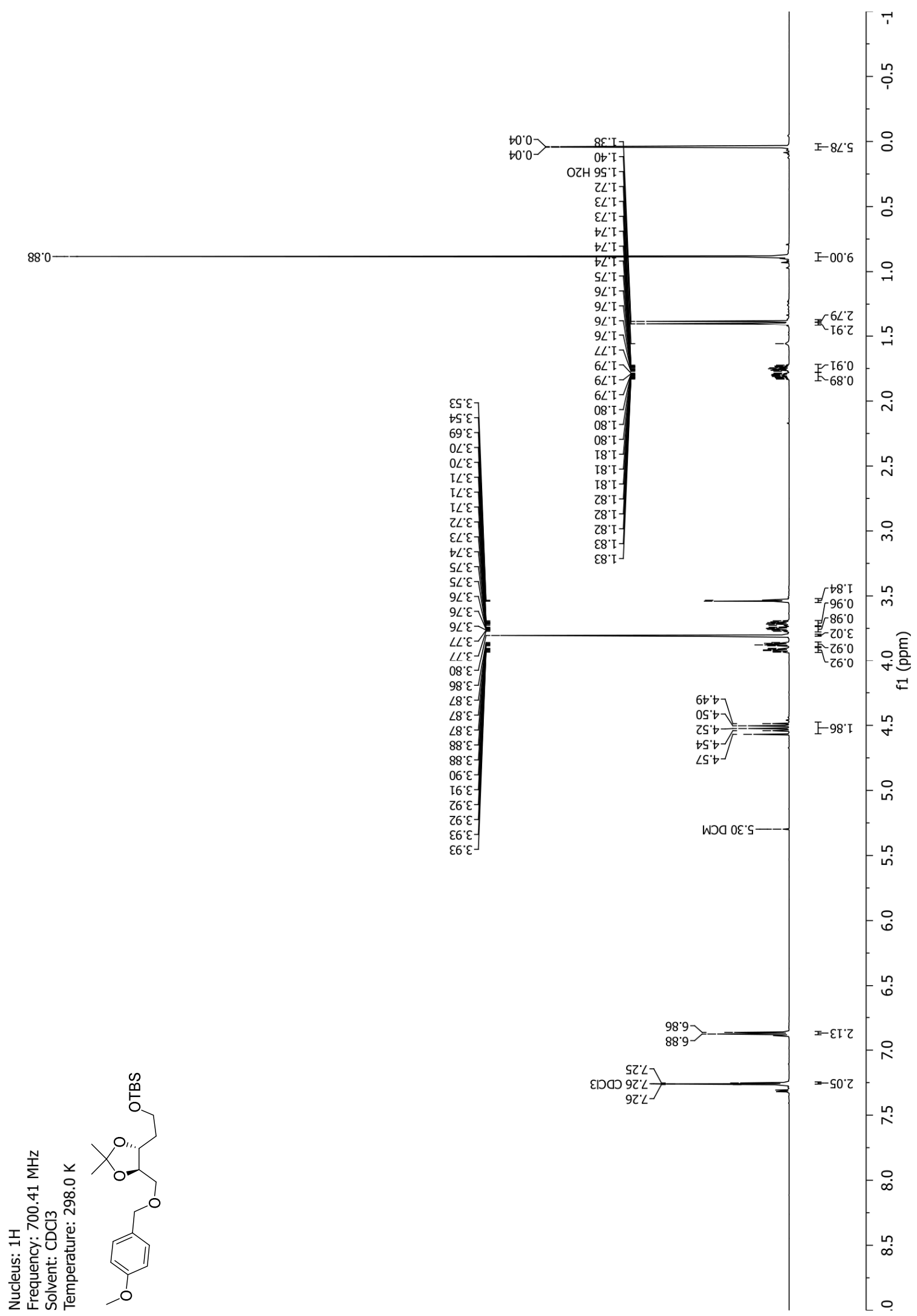




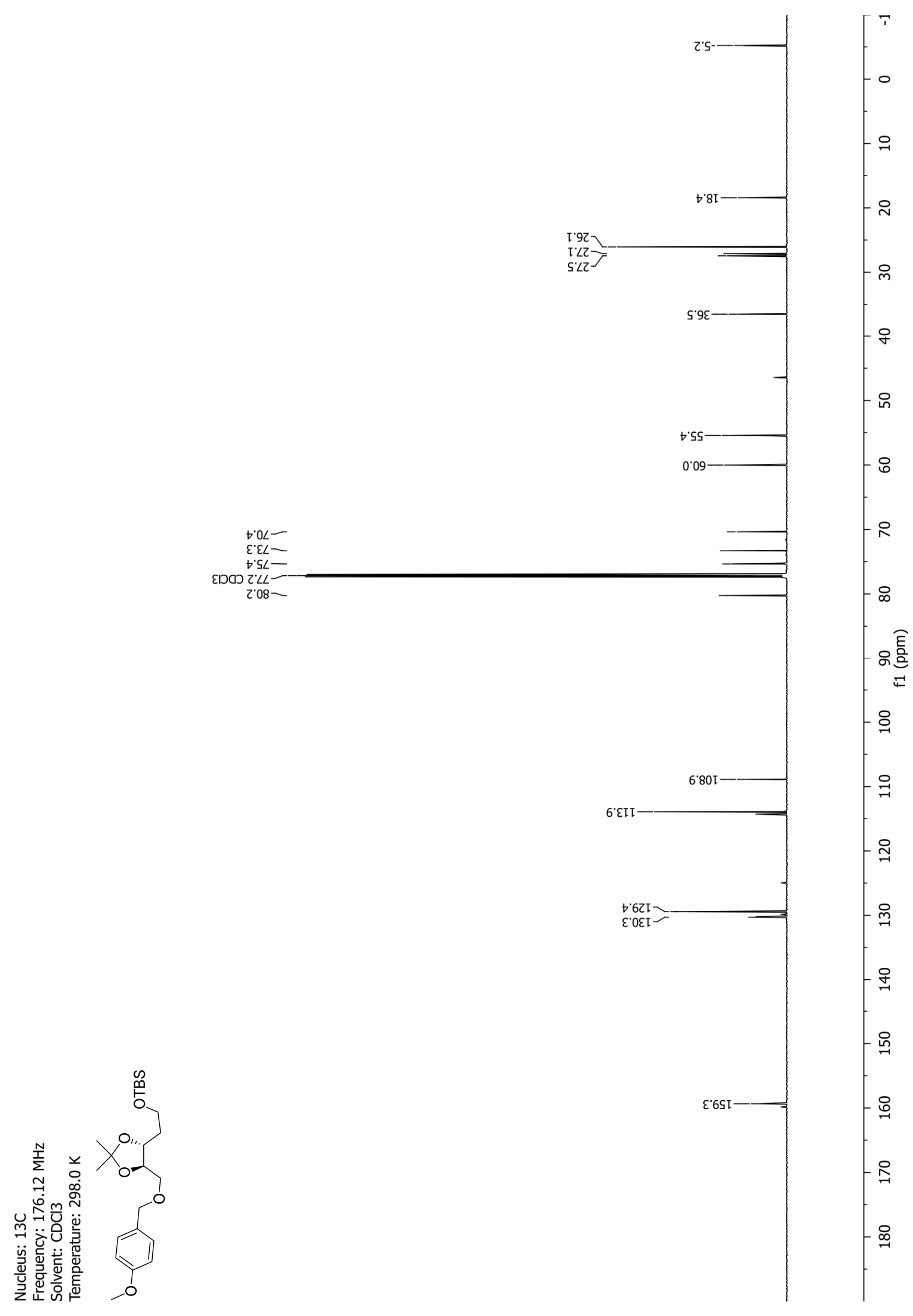


NMR-Spectra for Compound 27

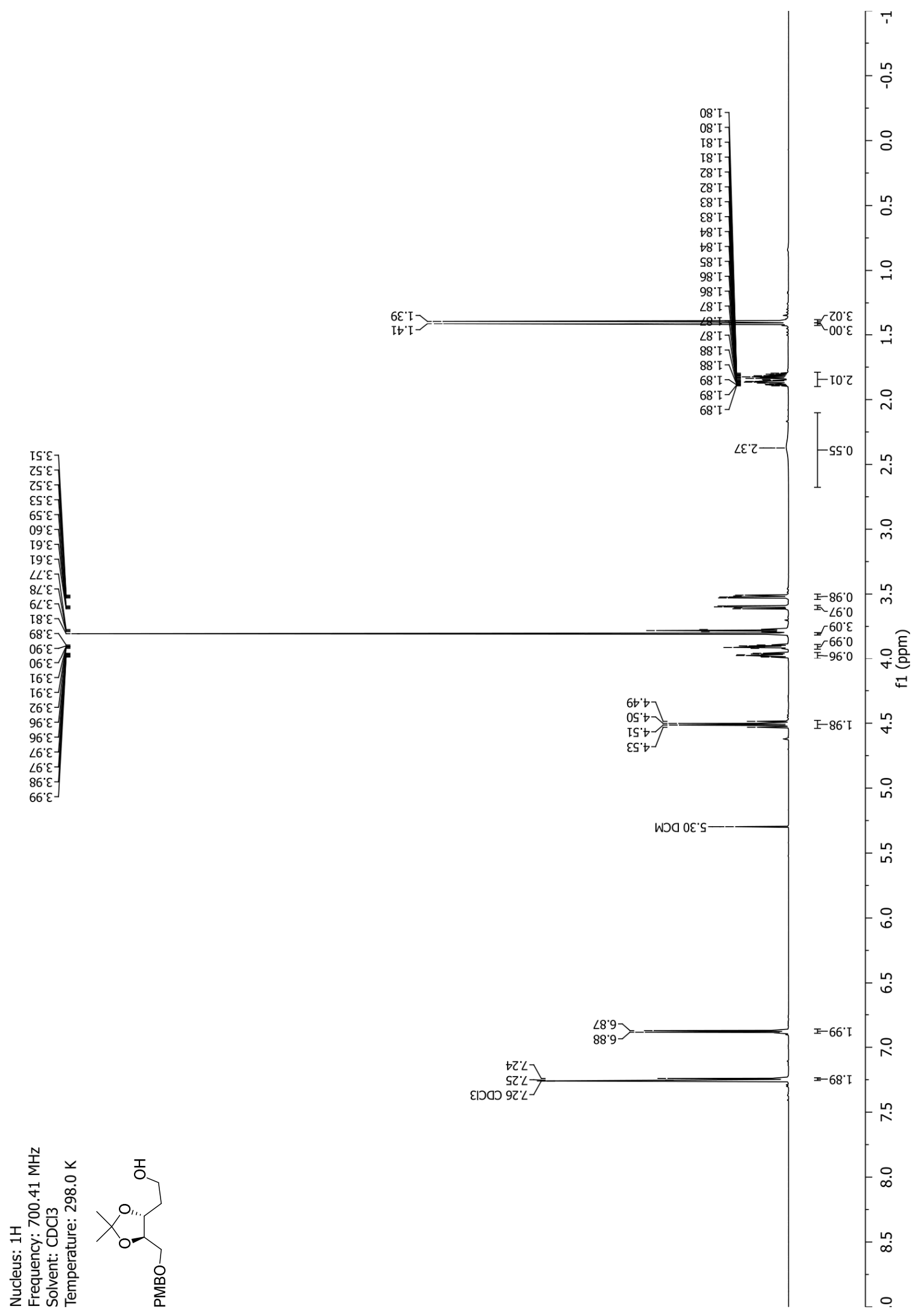




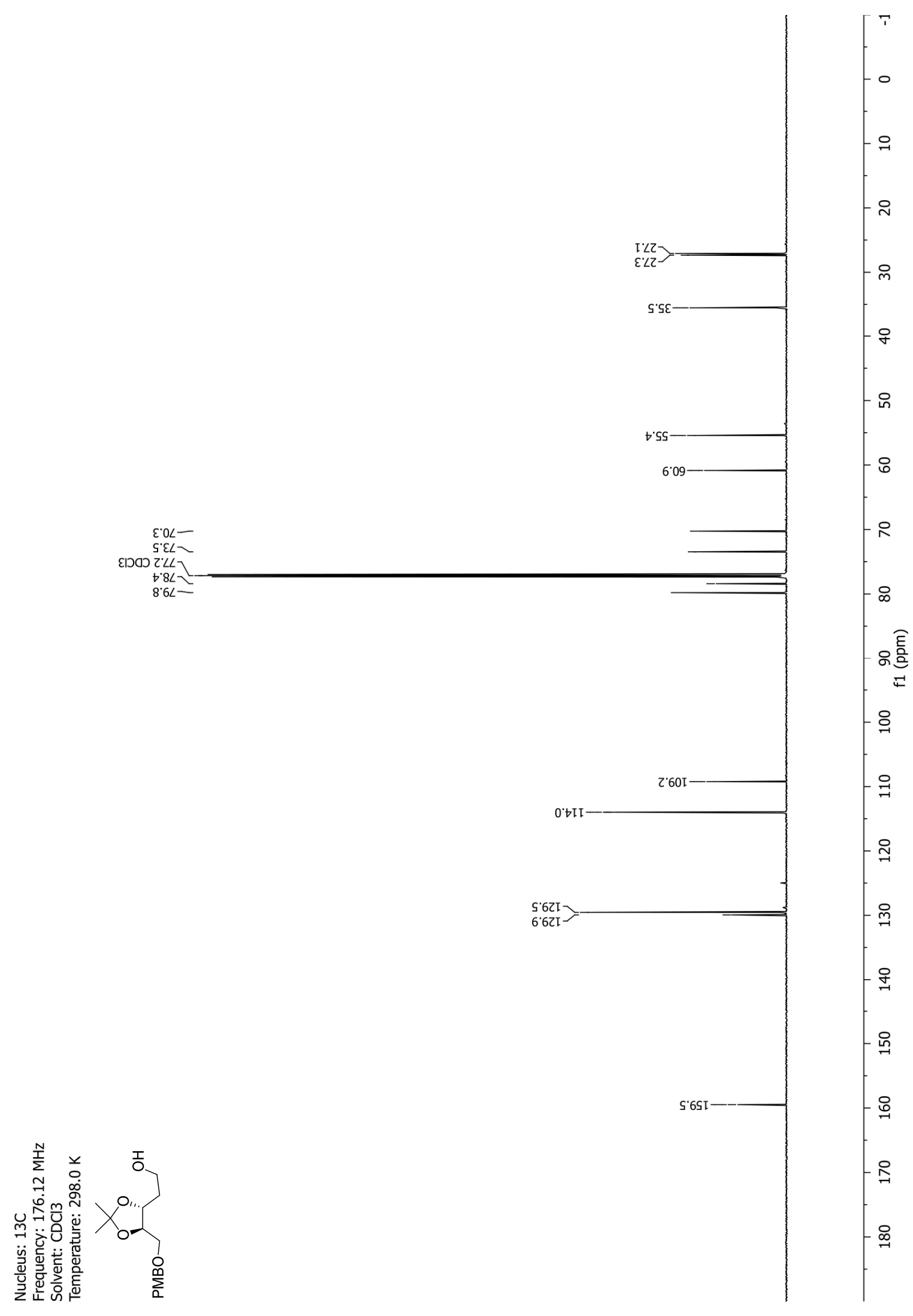


NMR-Spectra for Compound 79

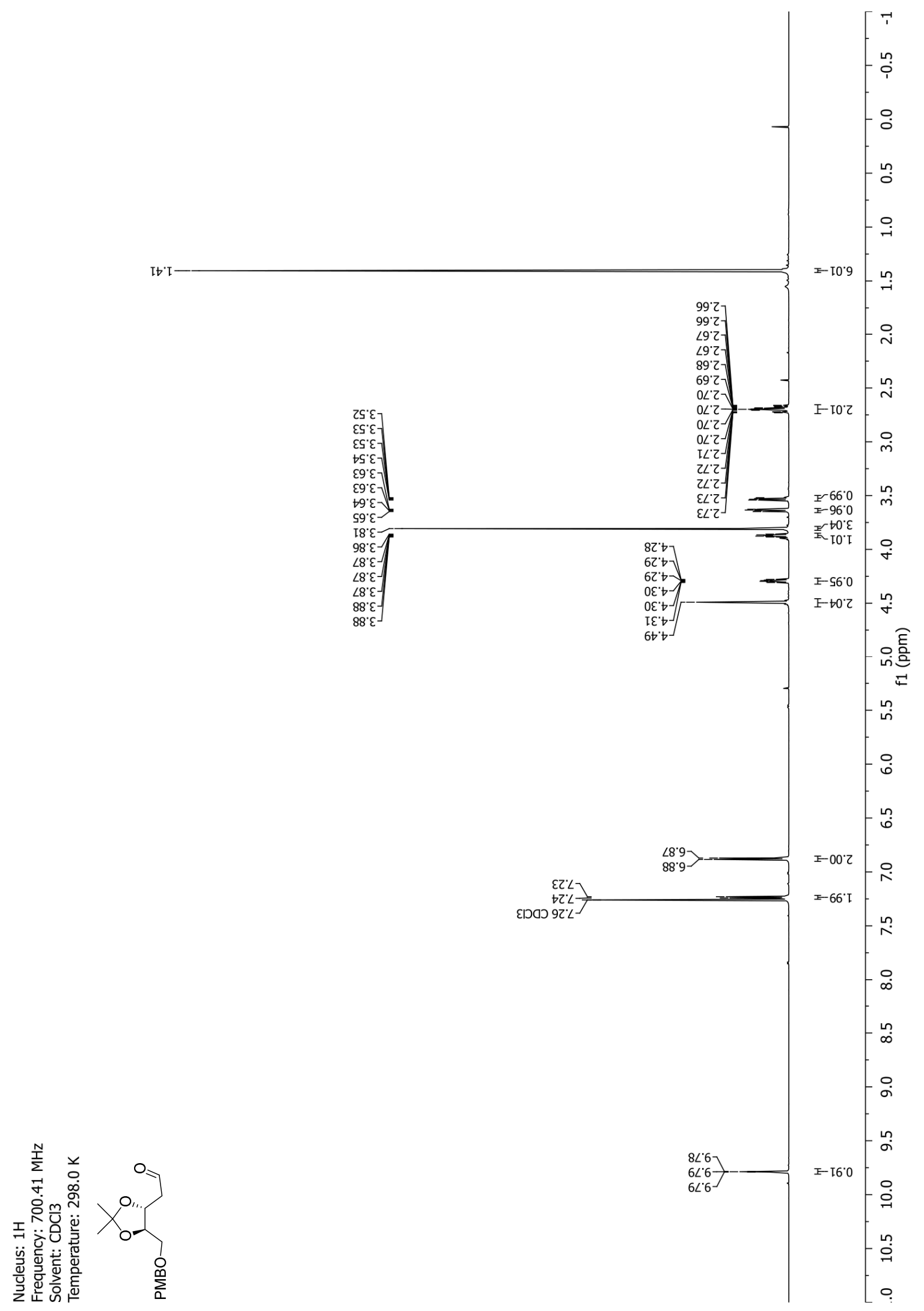




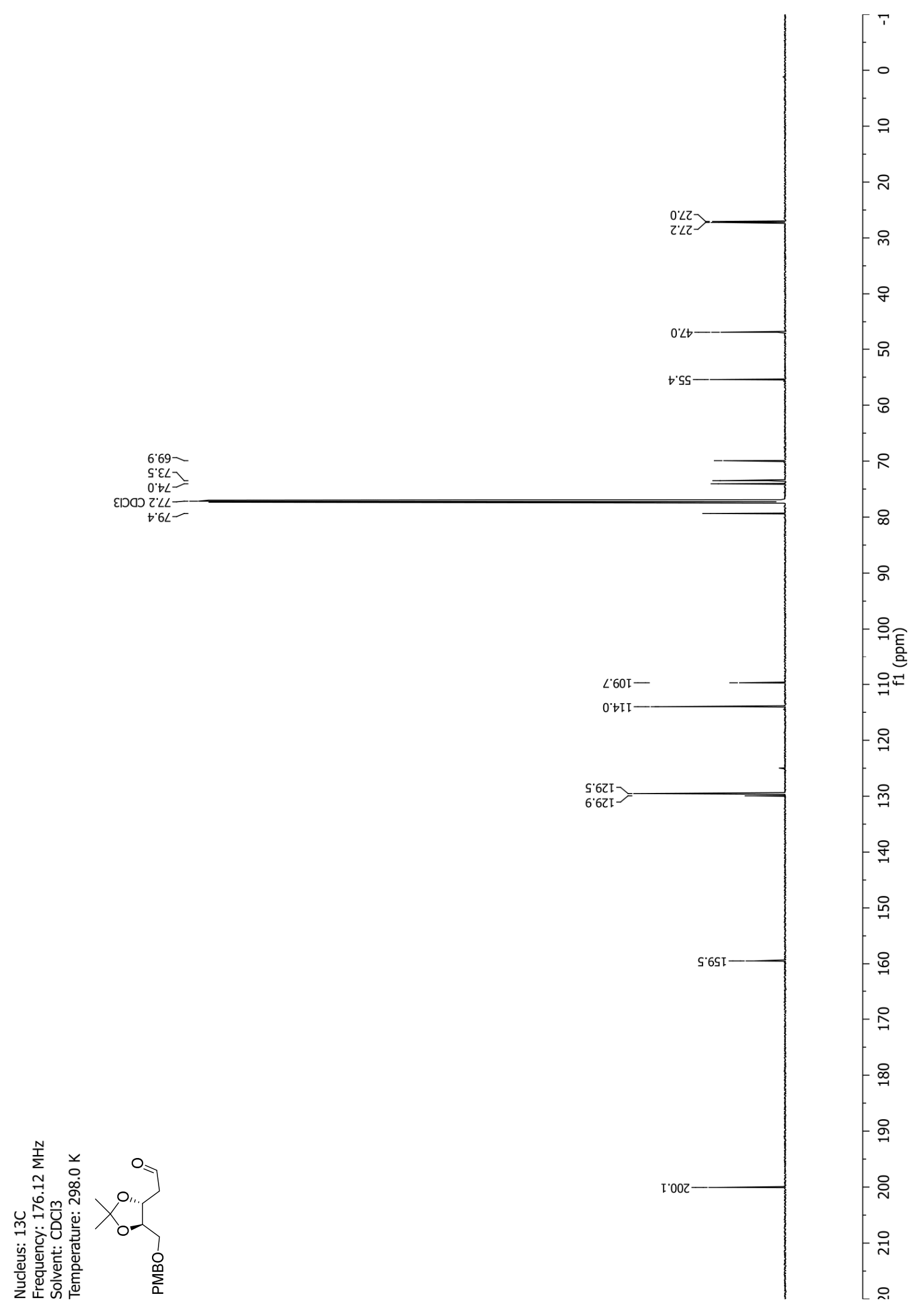




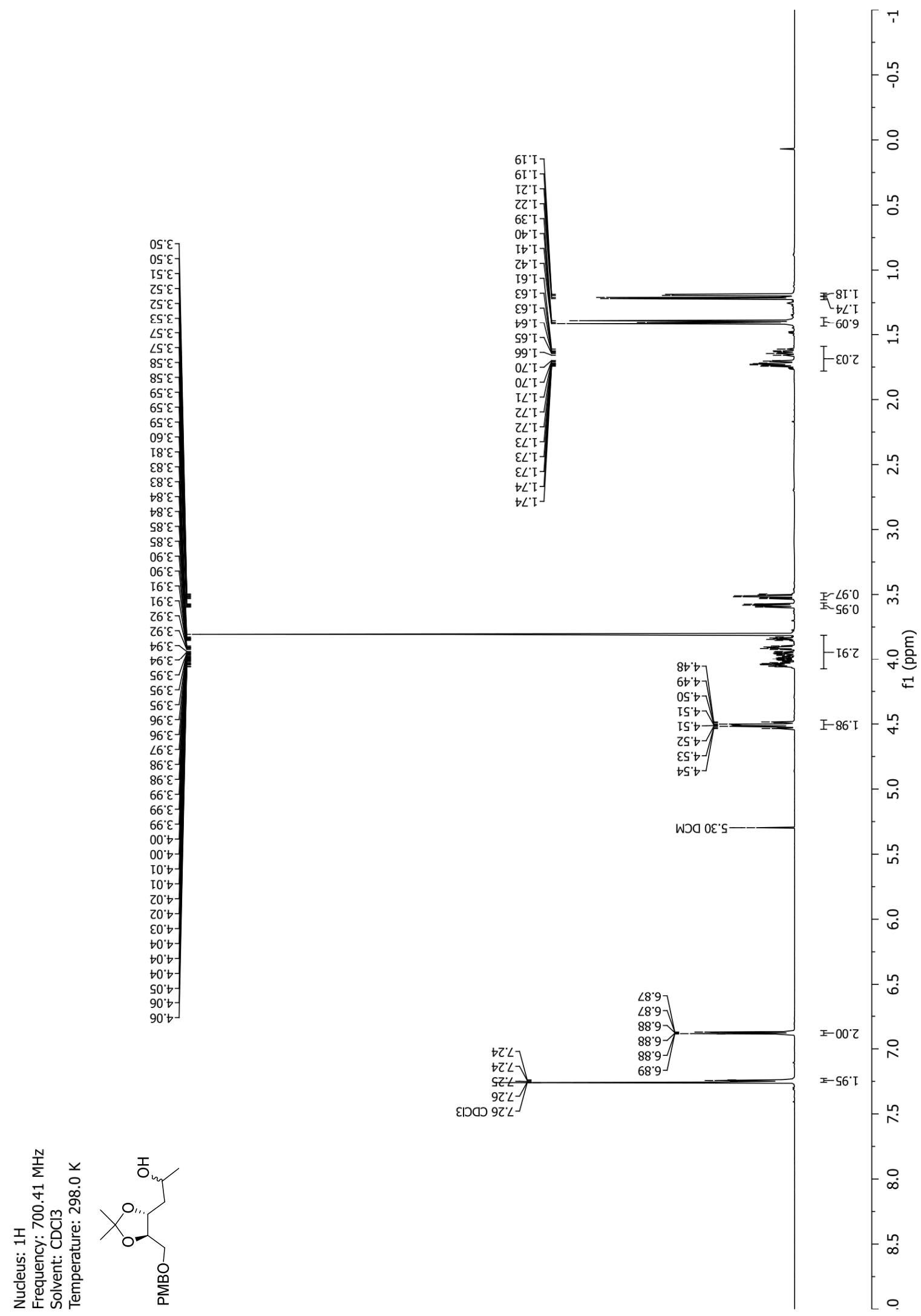




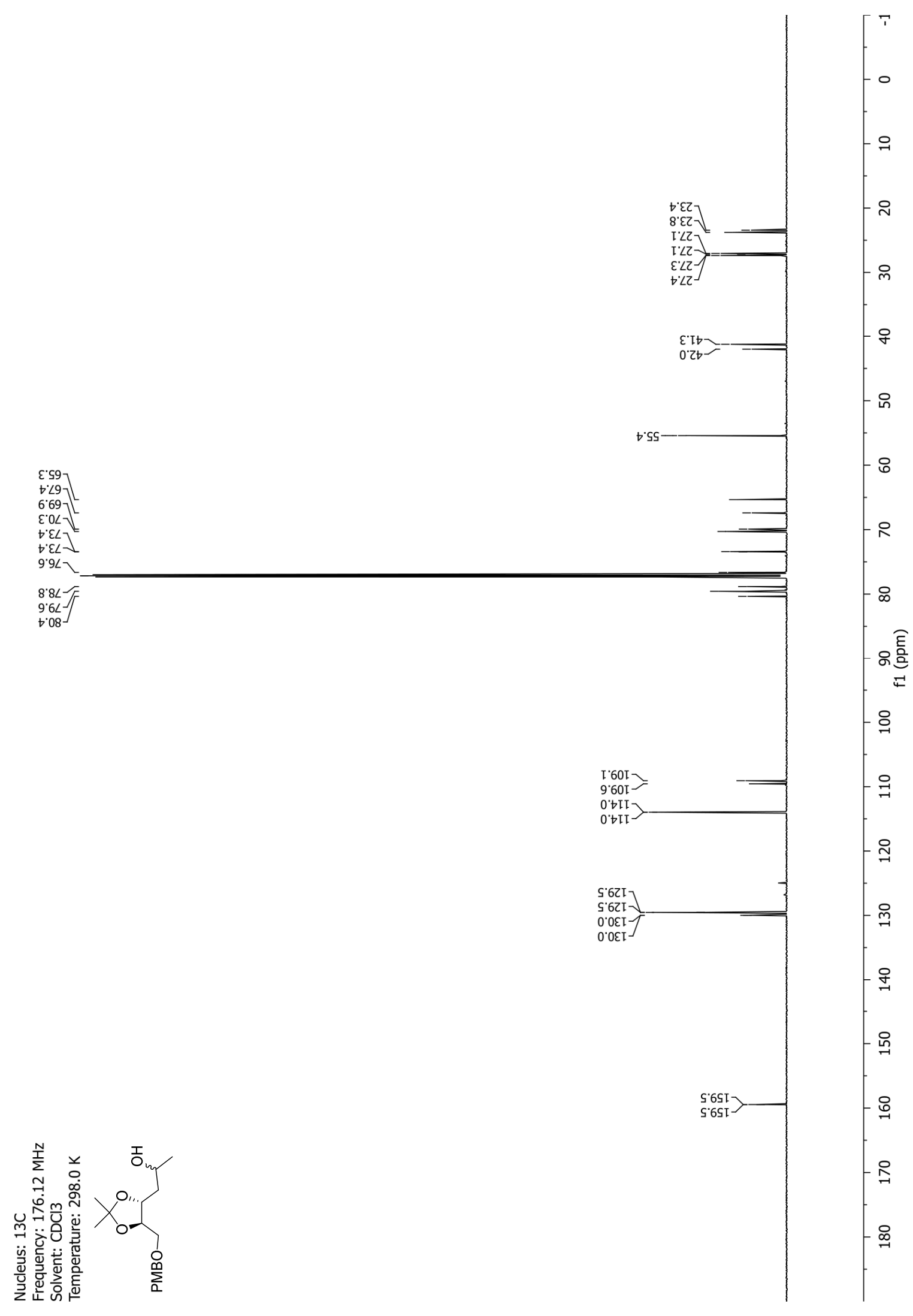


NMR-Spectra for Compound 28

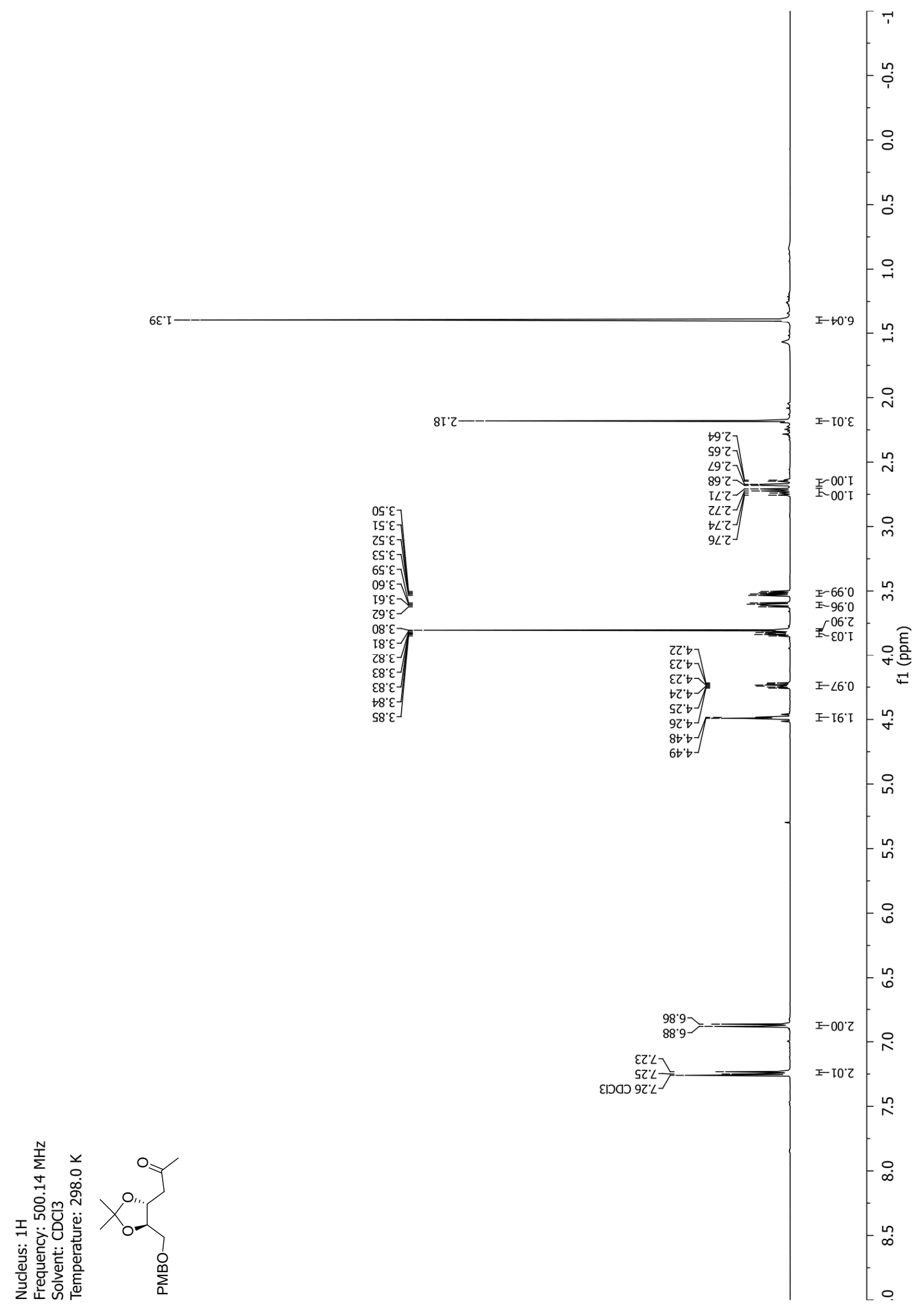




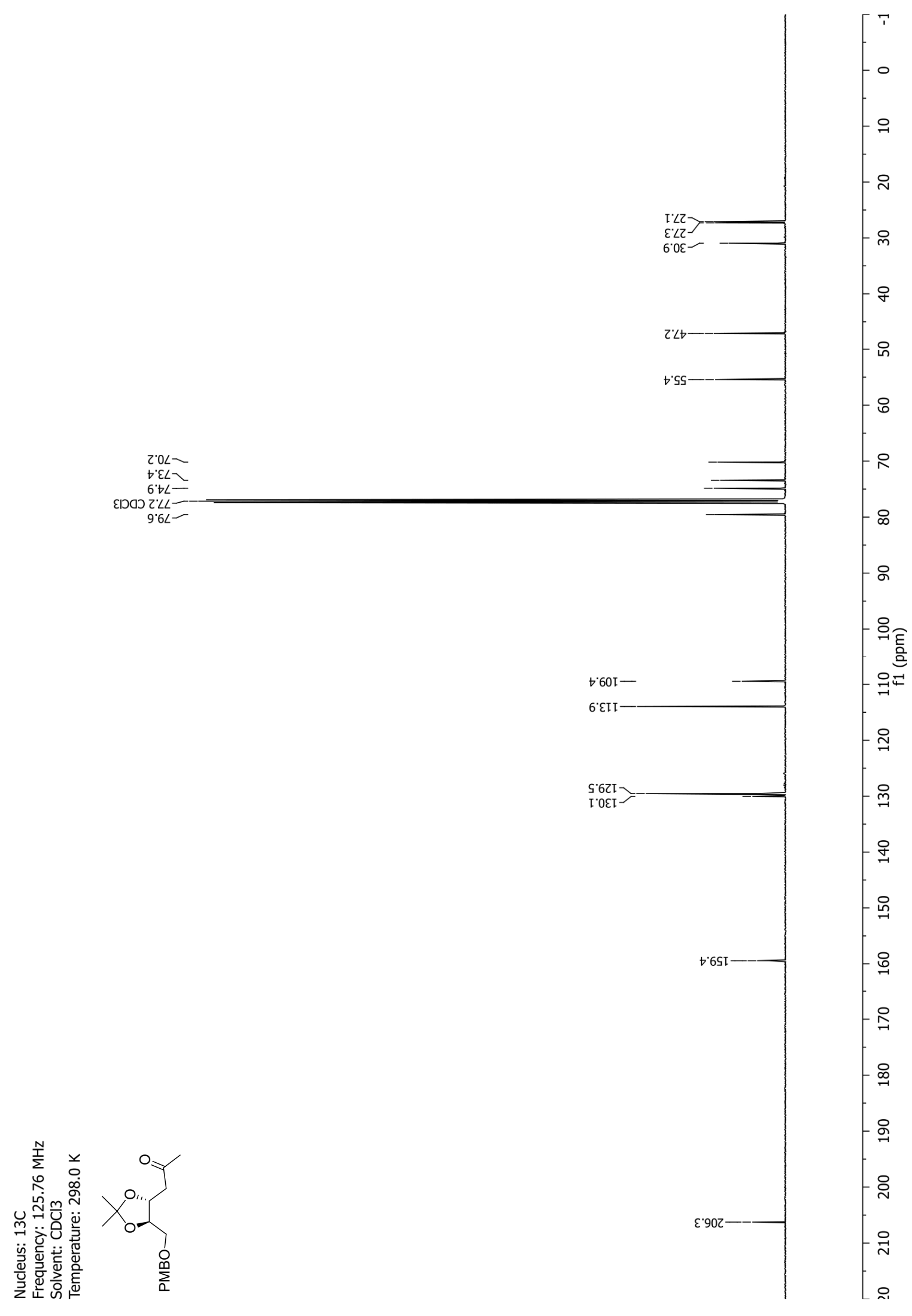


NMR-Spectra for Compound 4

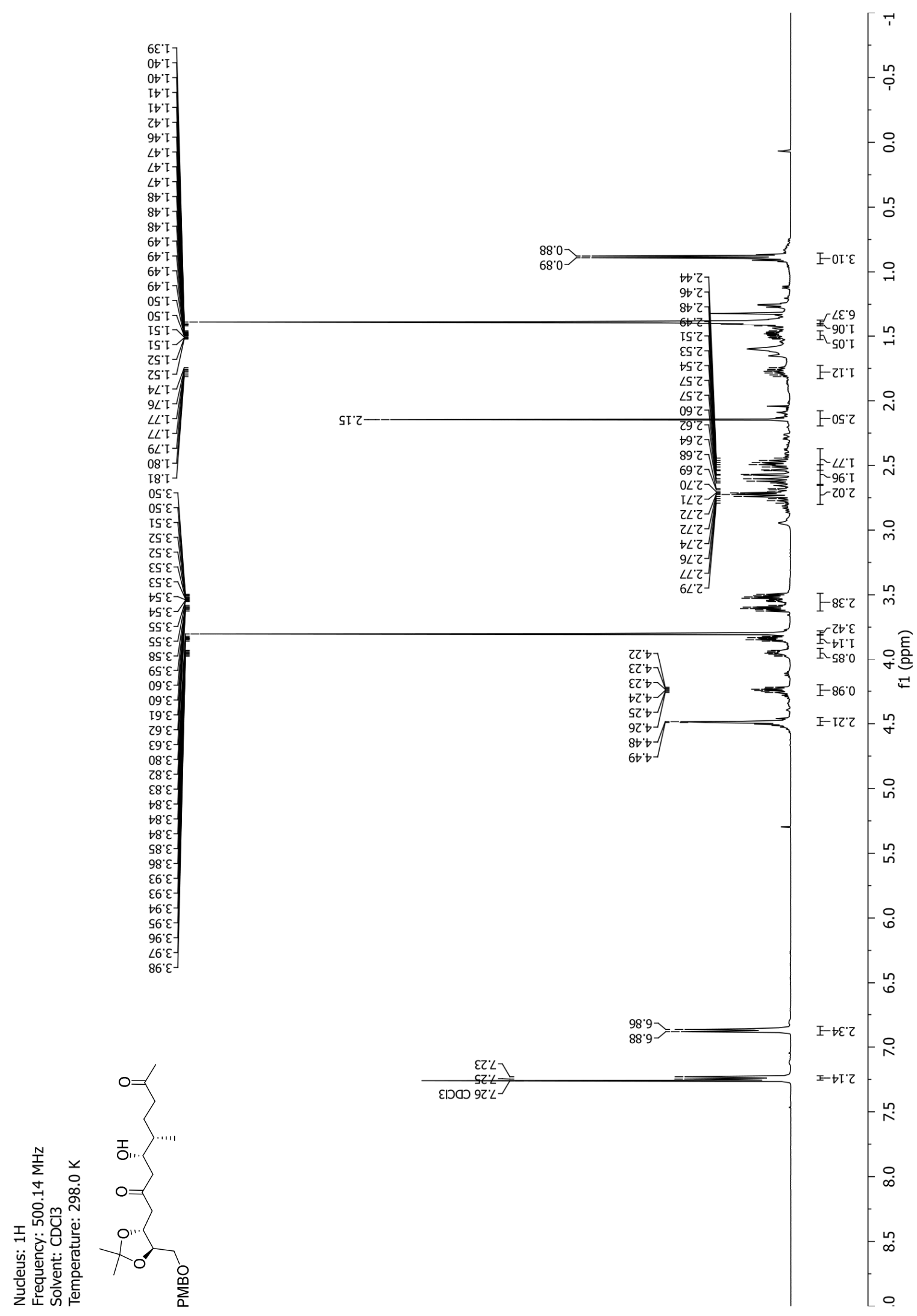




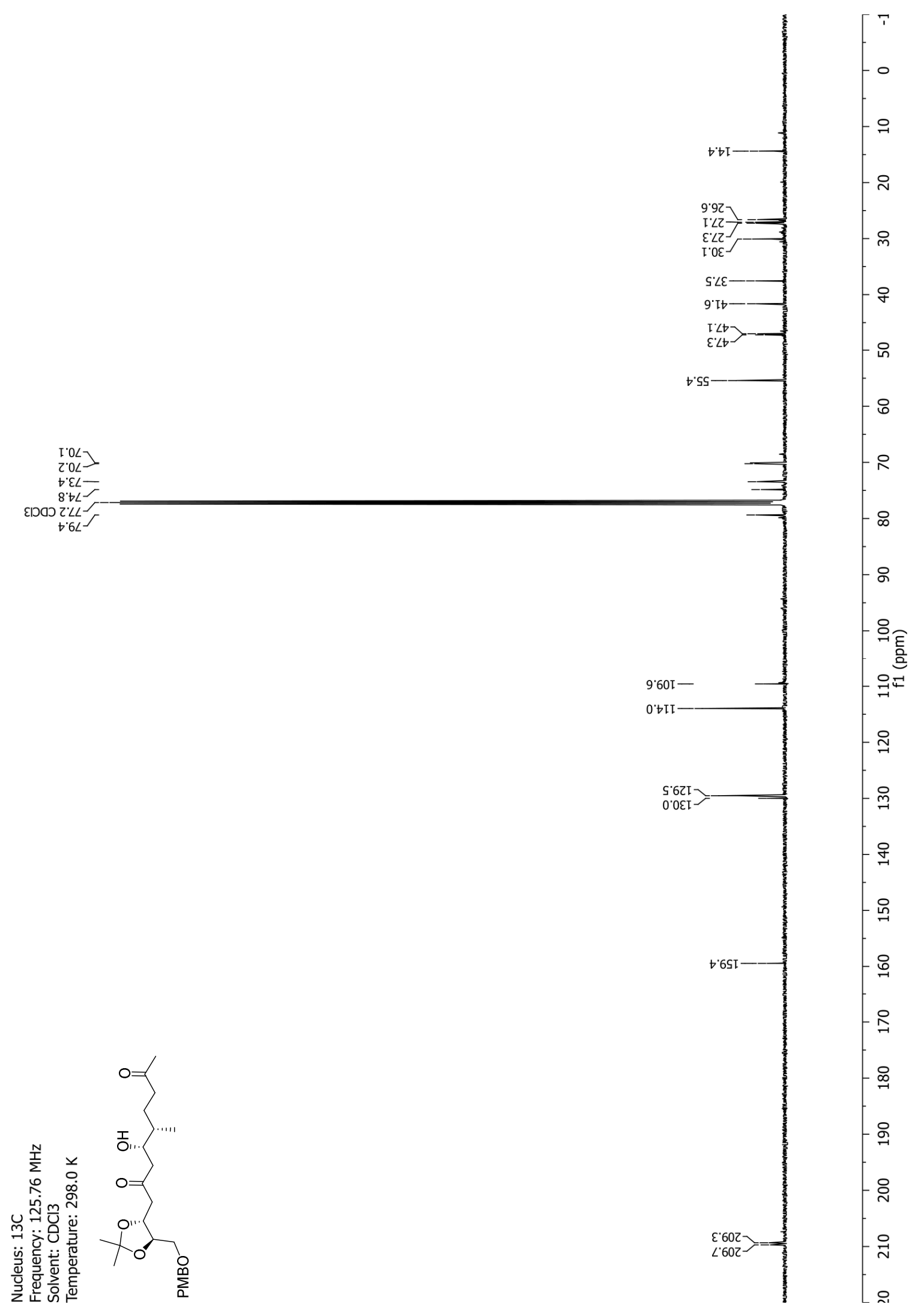




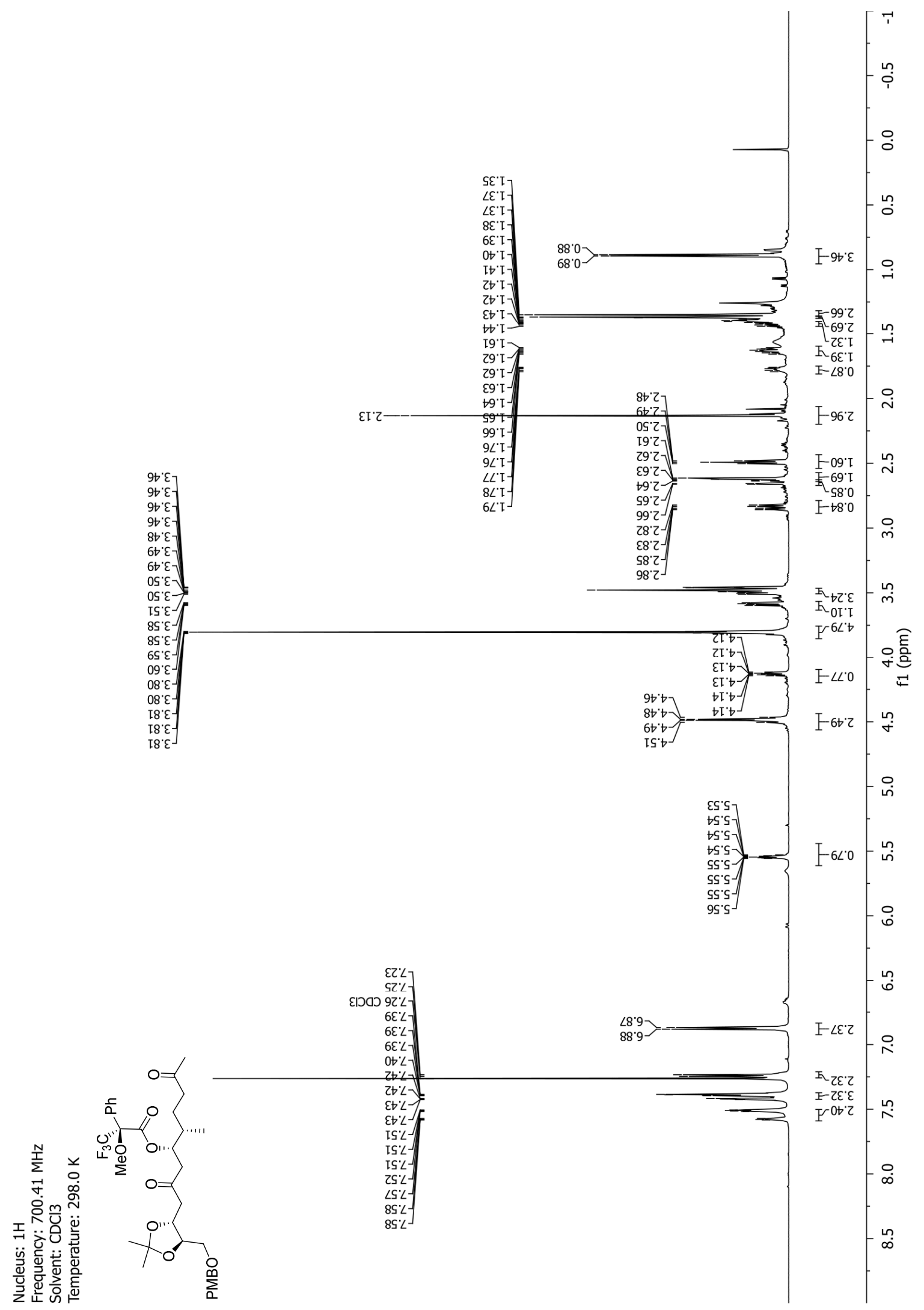




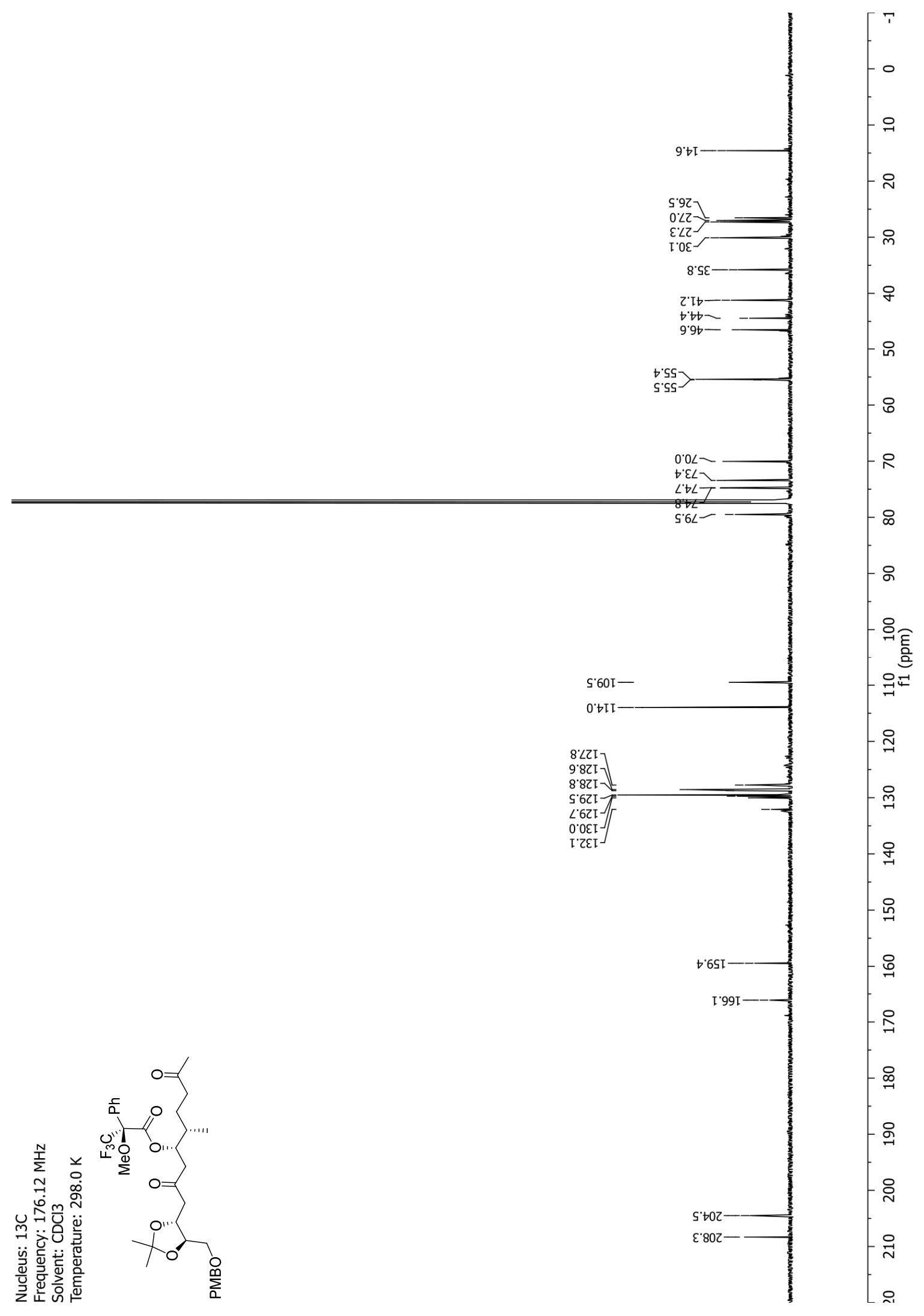



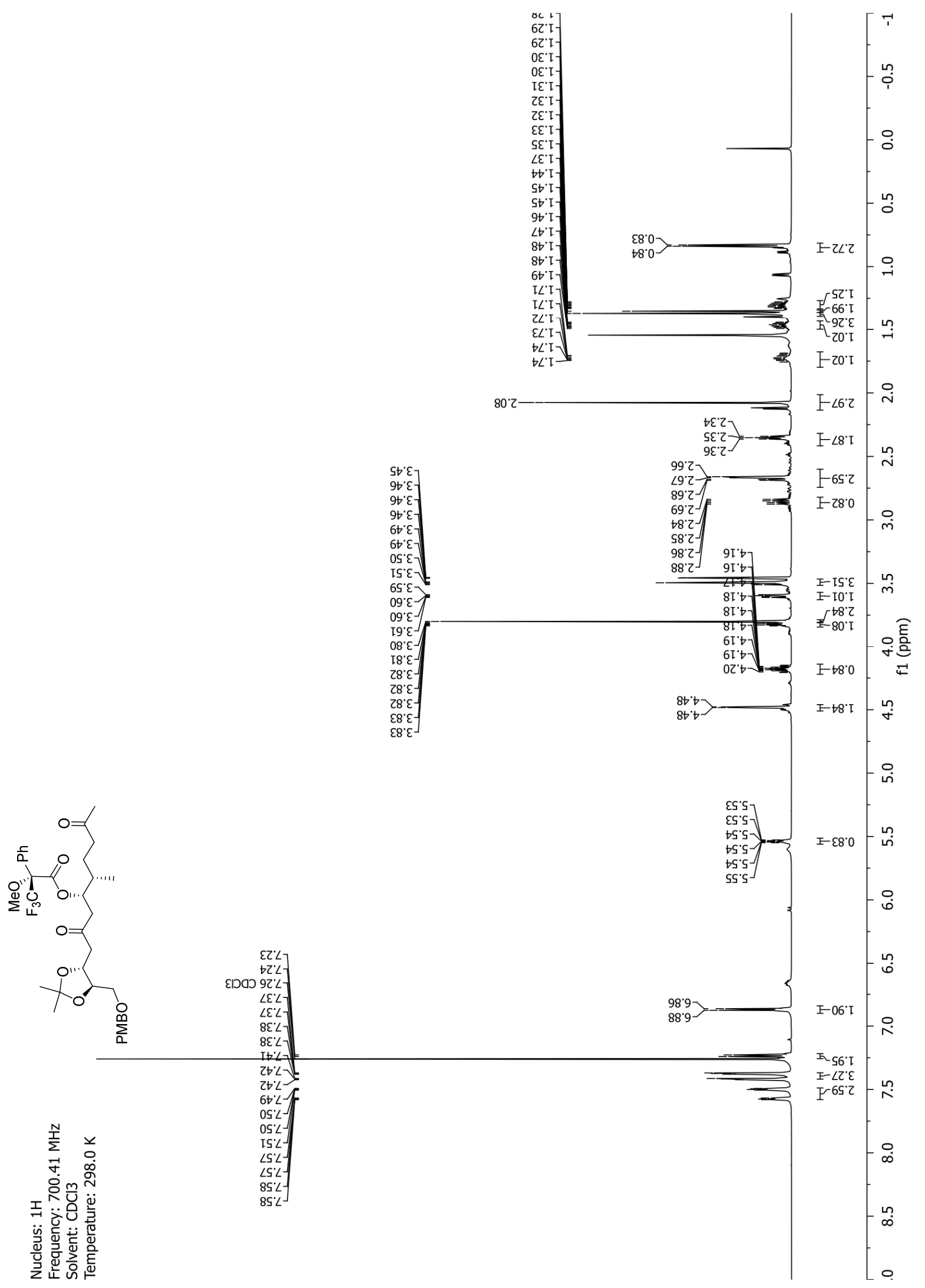


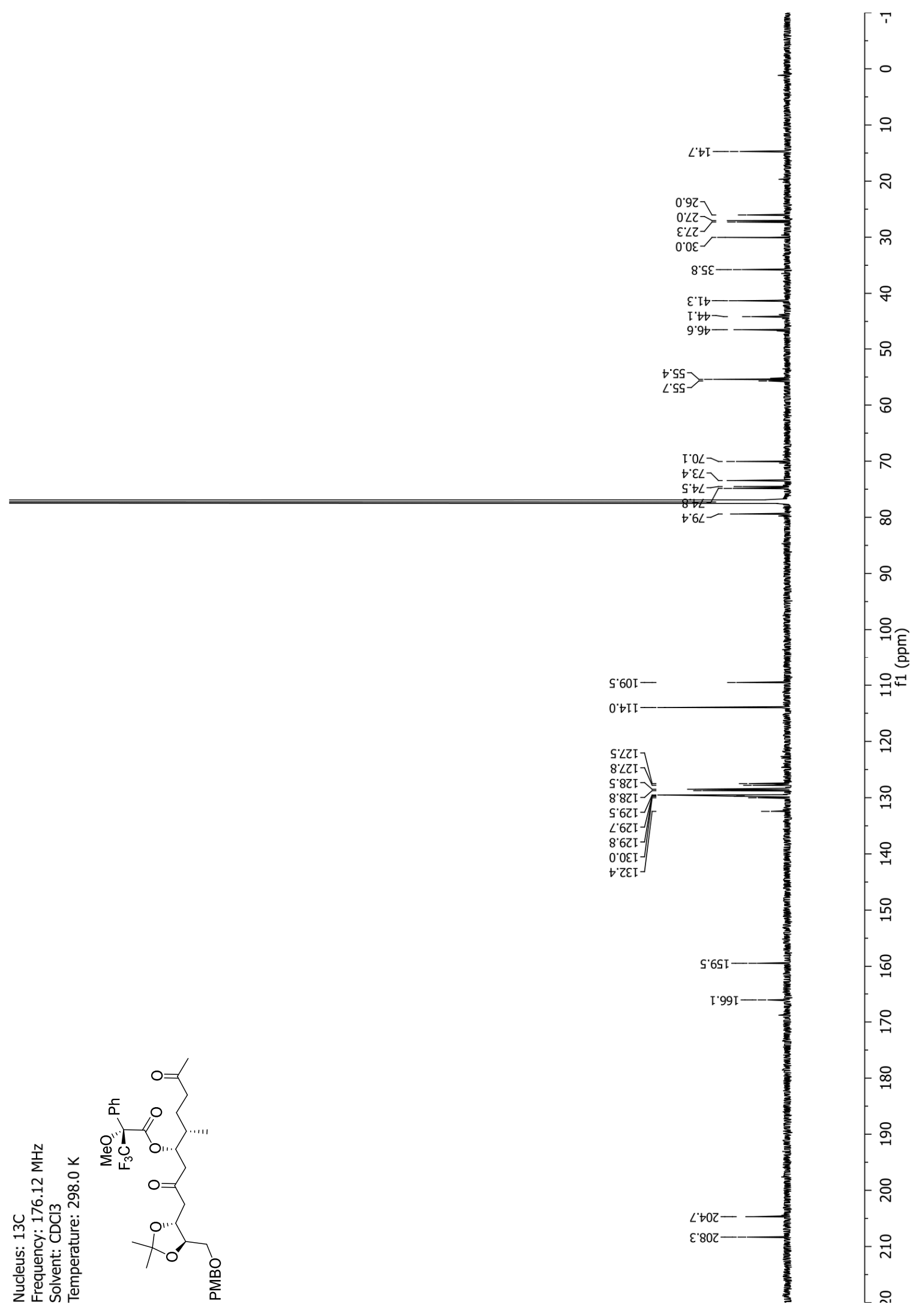


NMR-Spectra for Compound 84

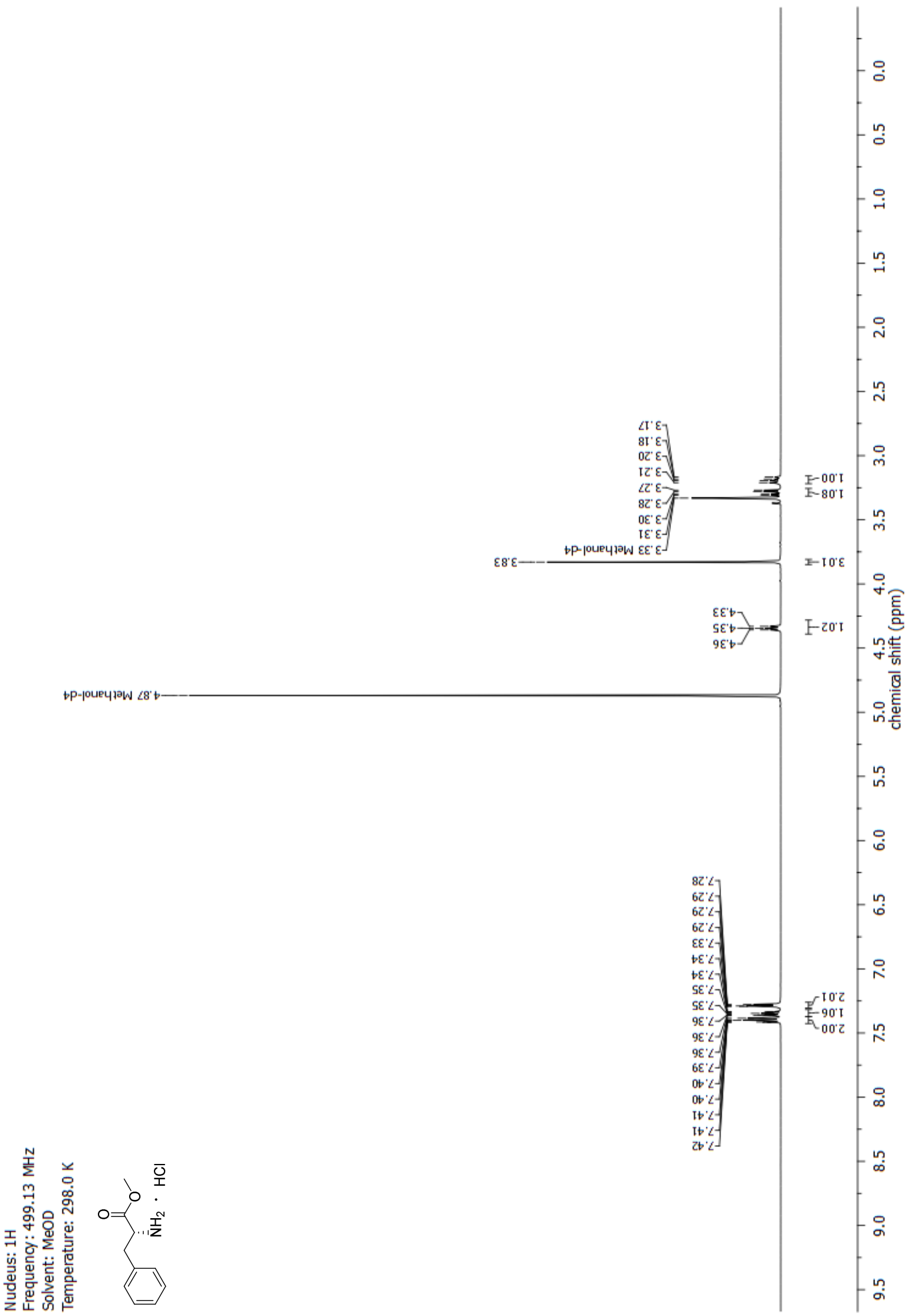




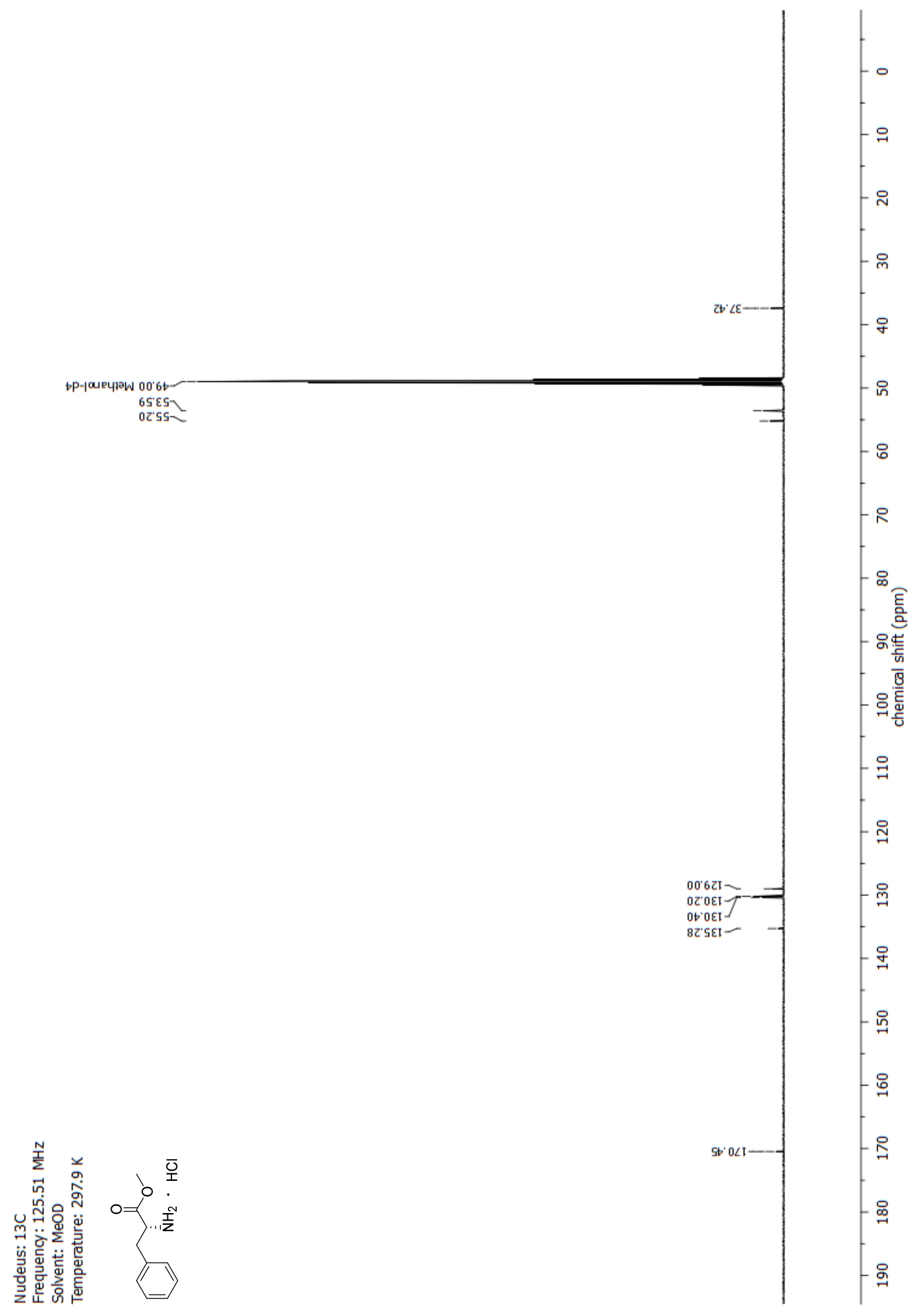


NMR-Spectra for Compound 85

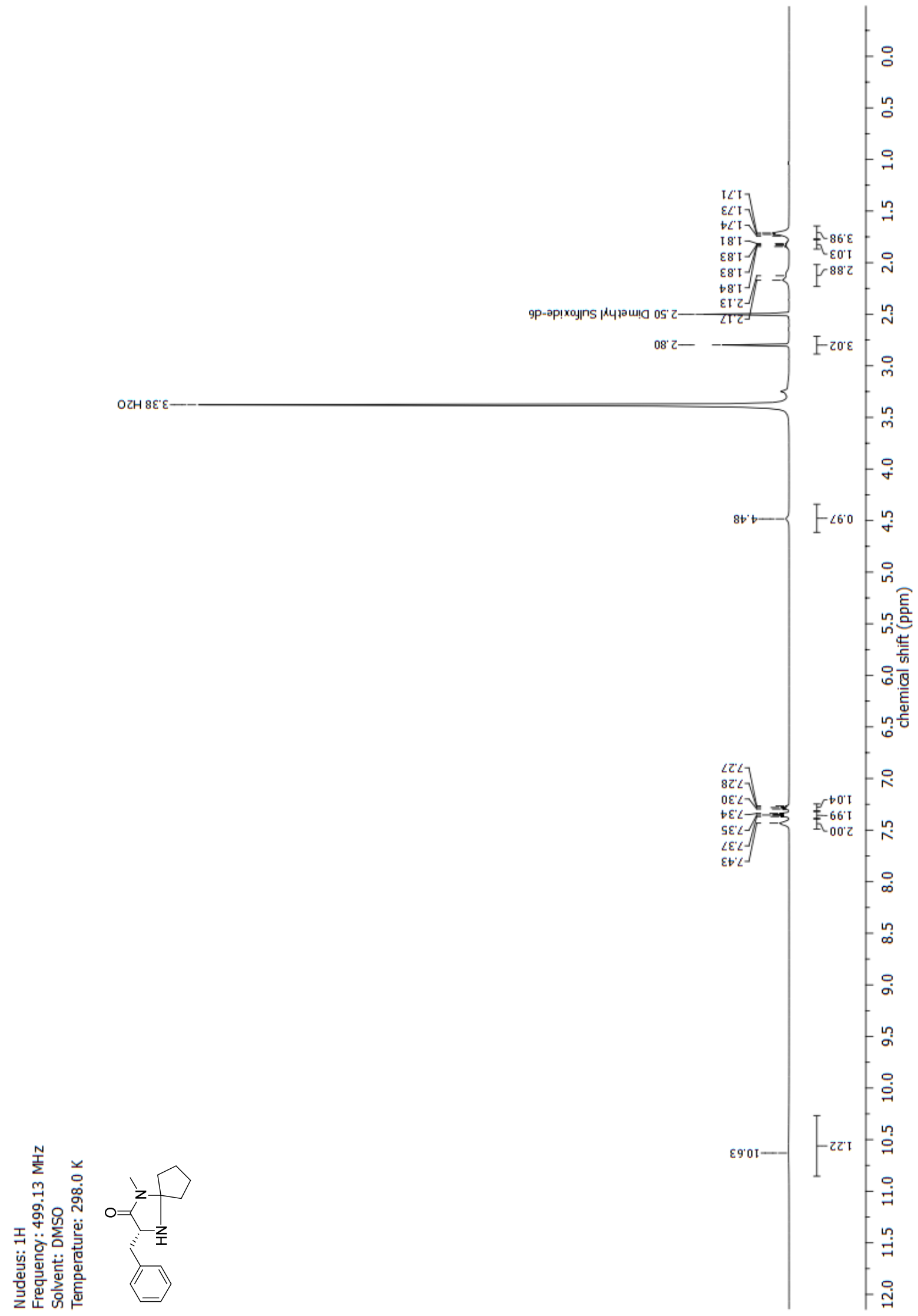




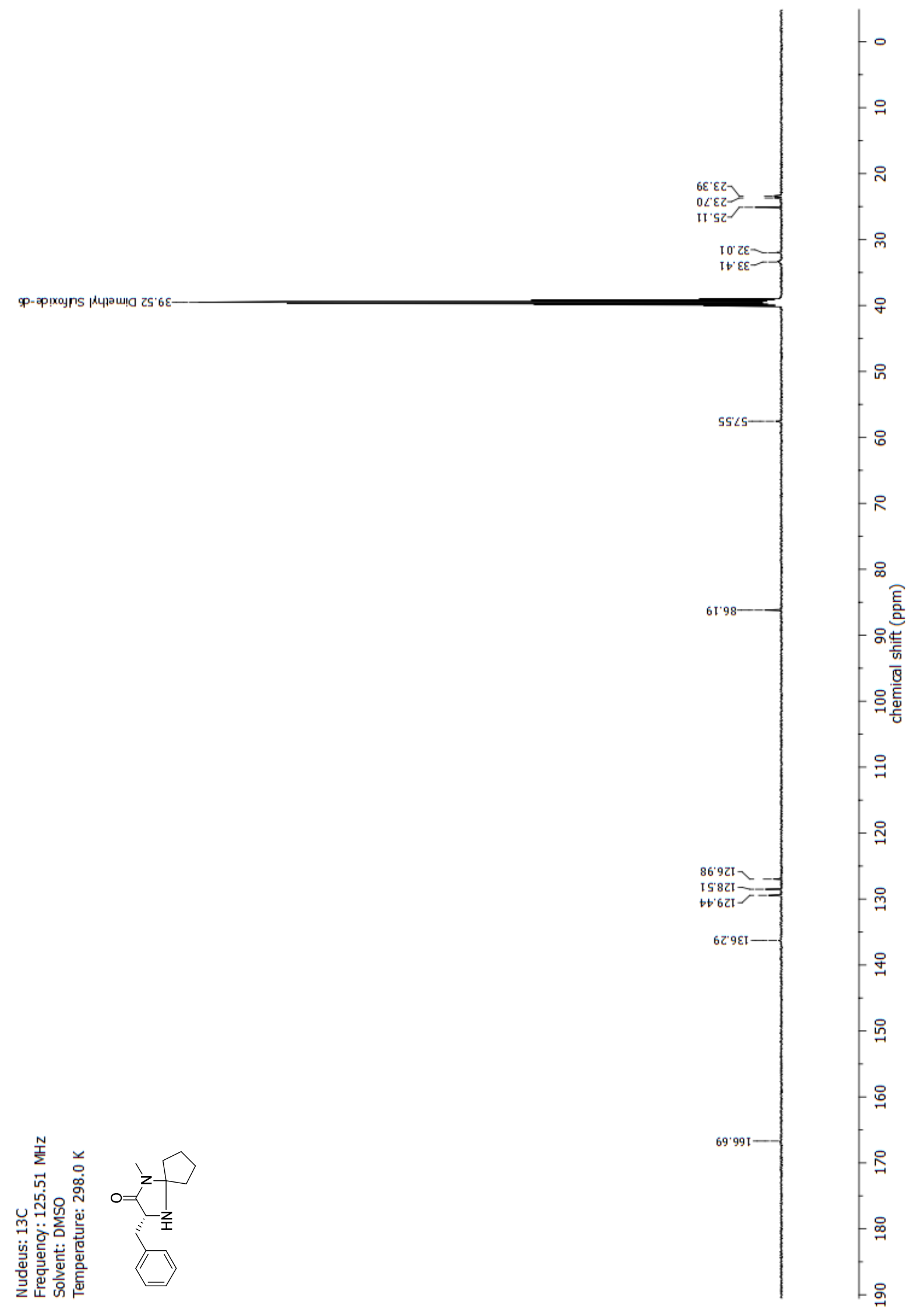


NMR-Spectra for Compound 29

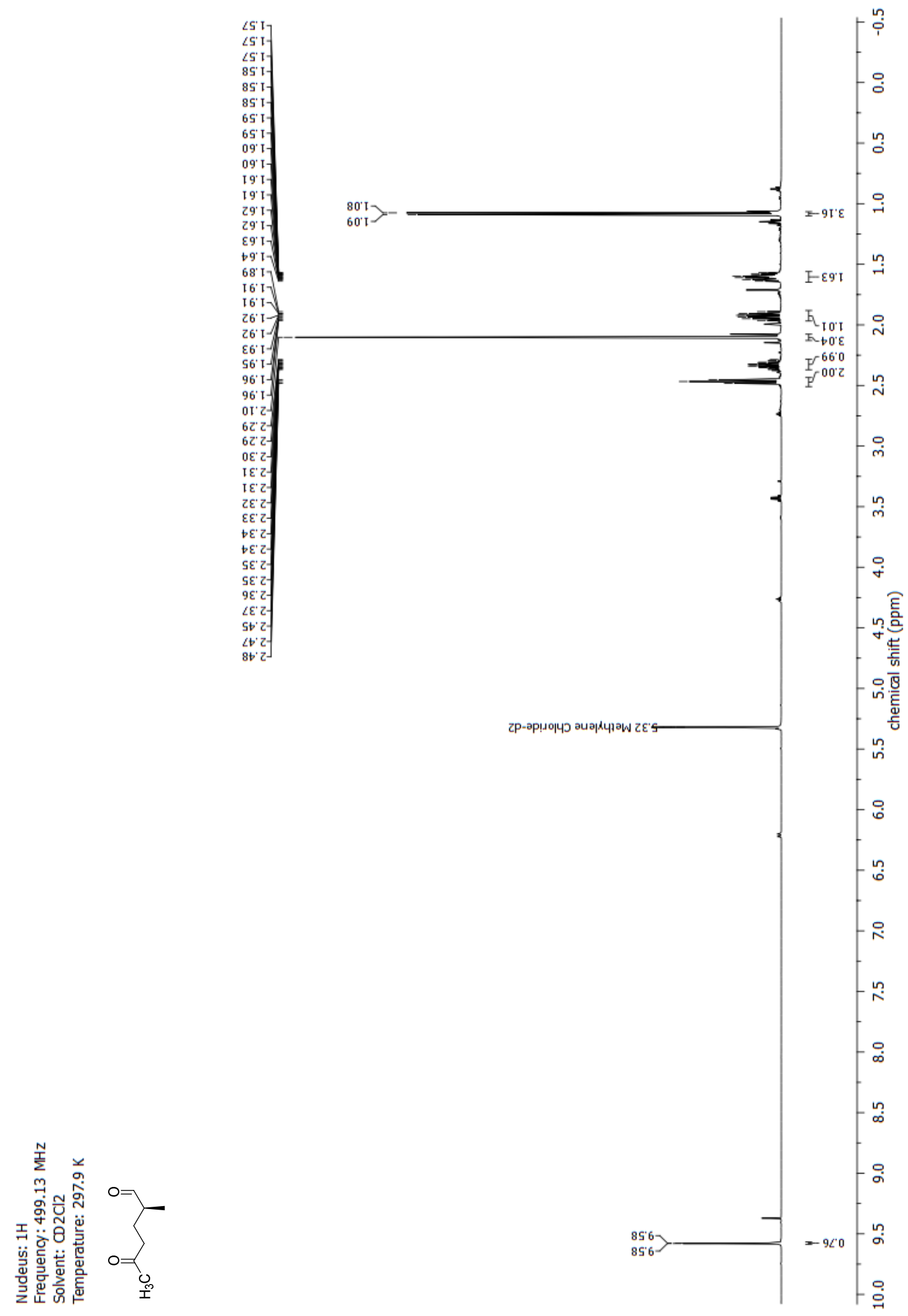




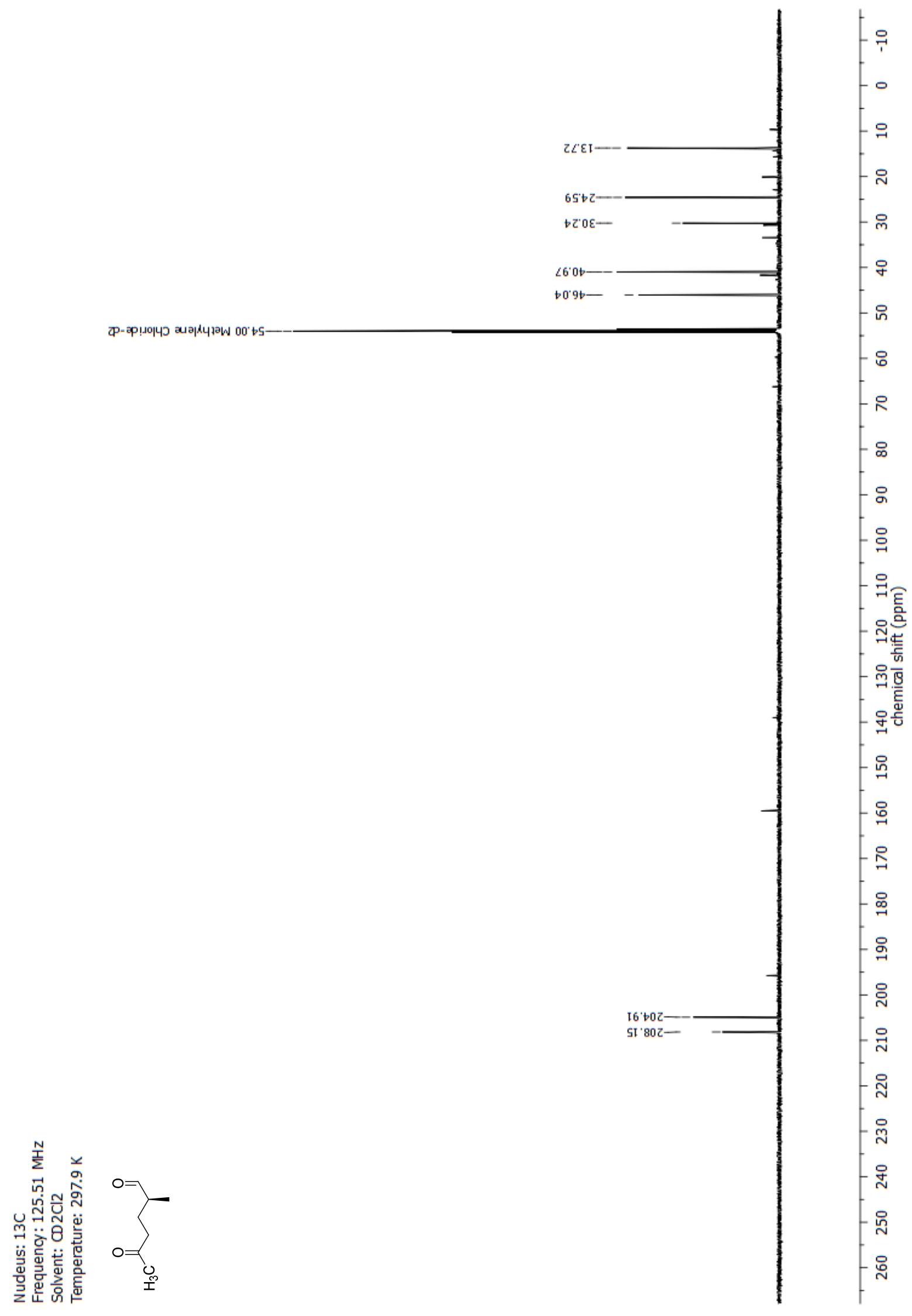


NMR-Spectra for Compound 86

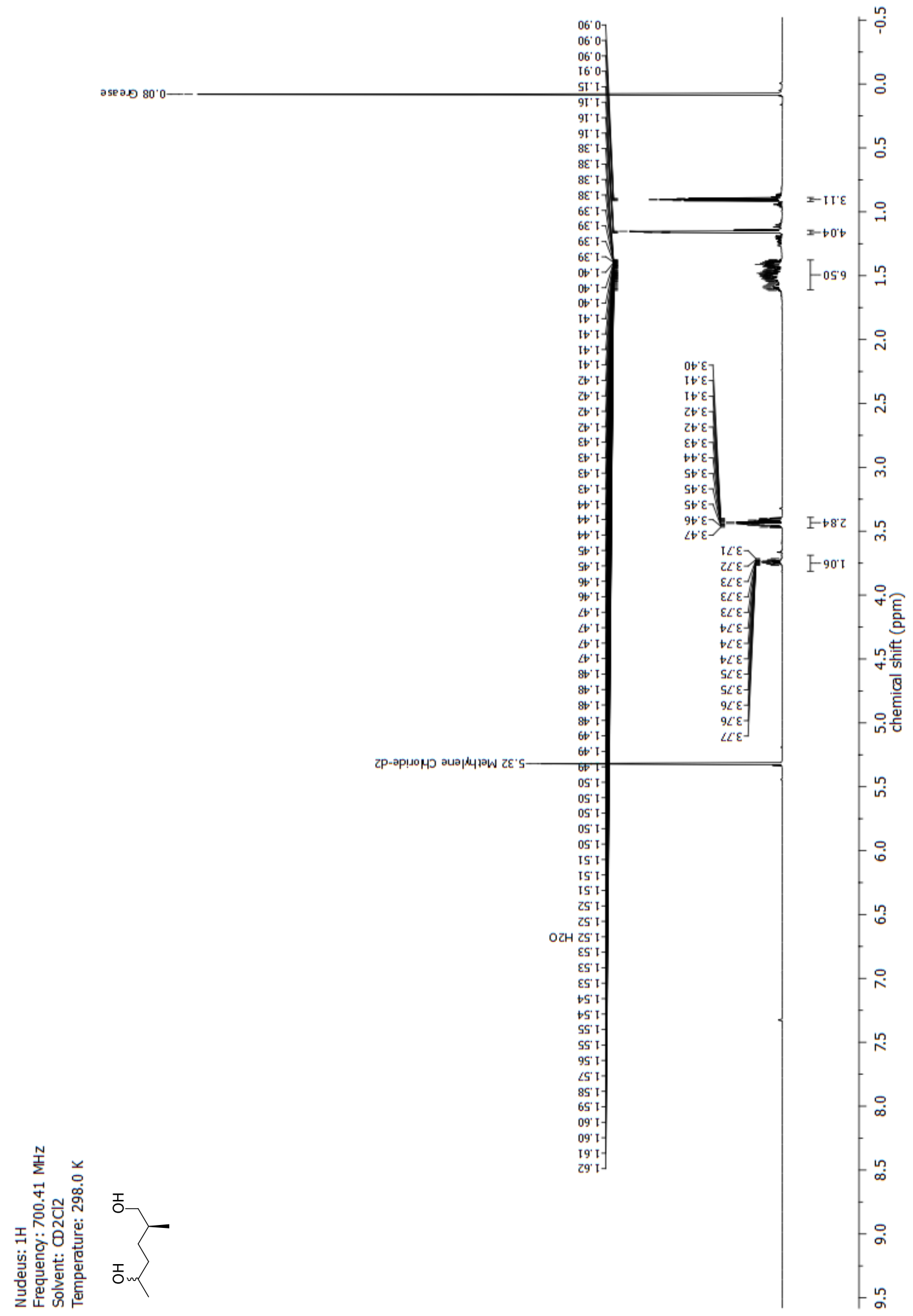




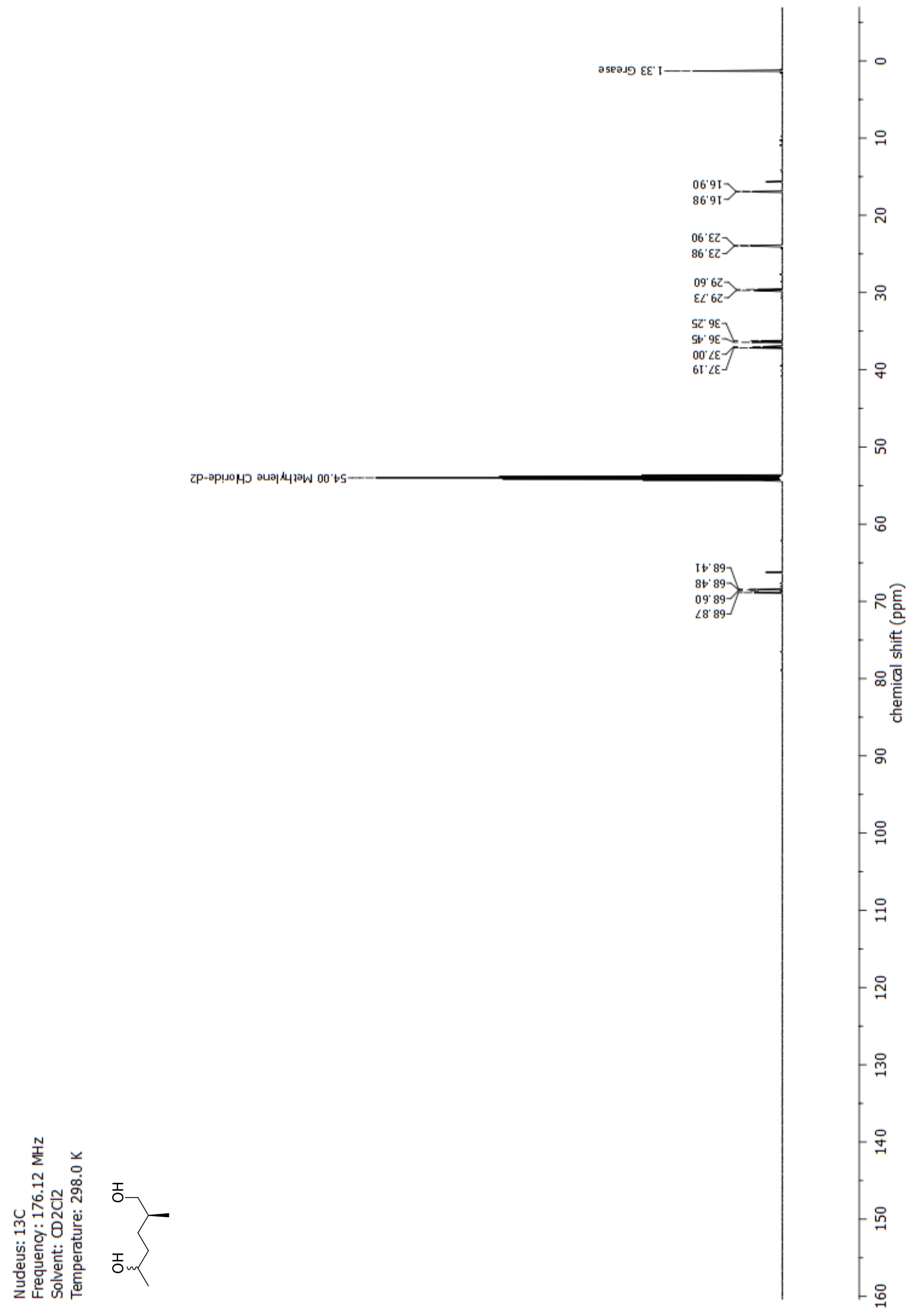


NMR-Spectra for Compound 87

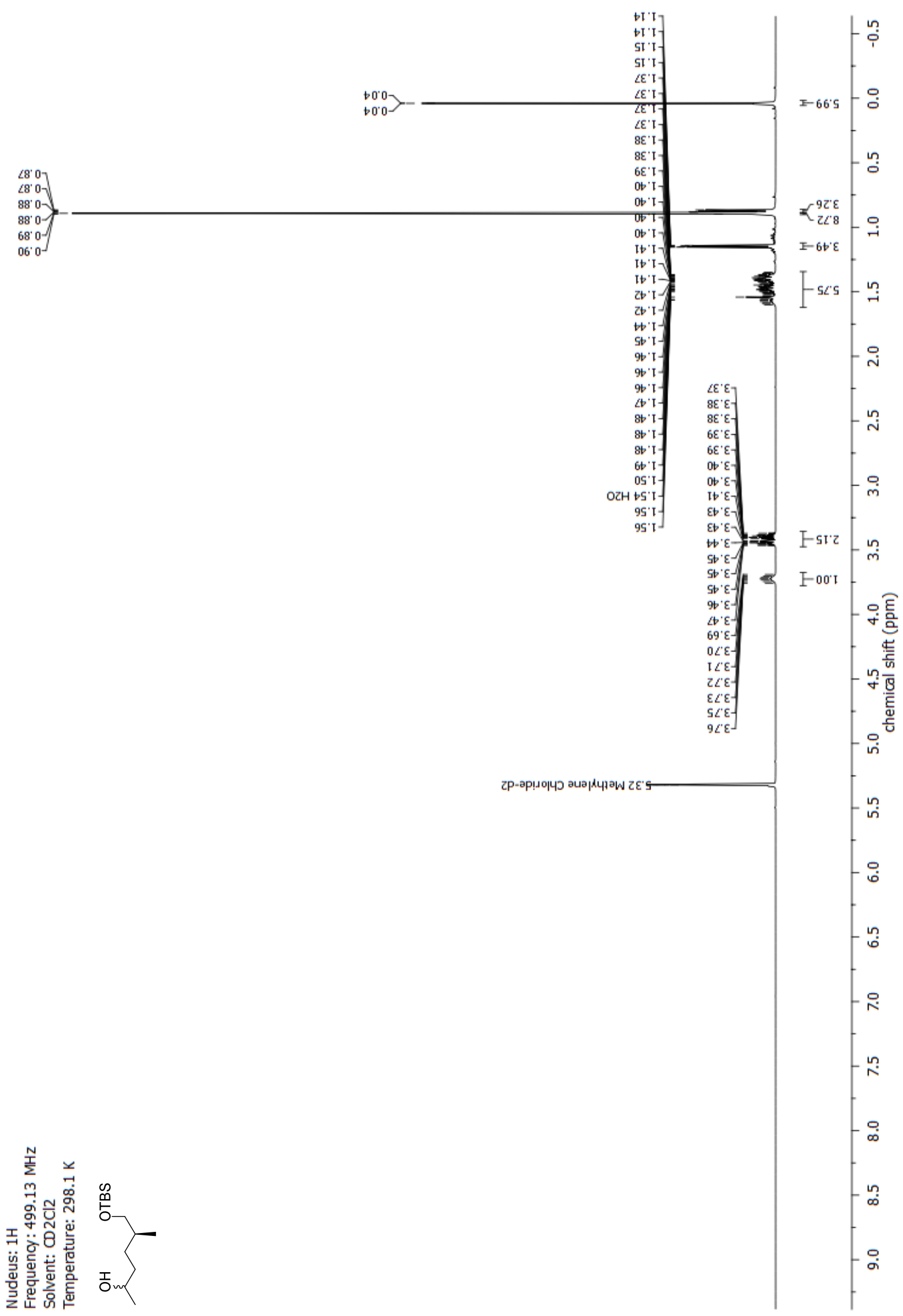




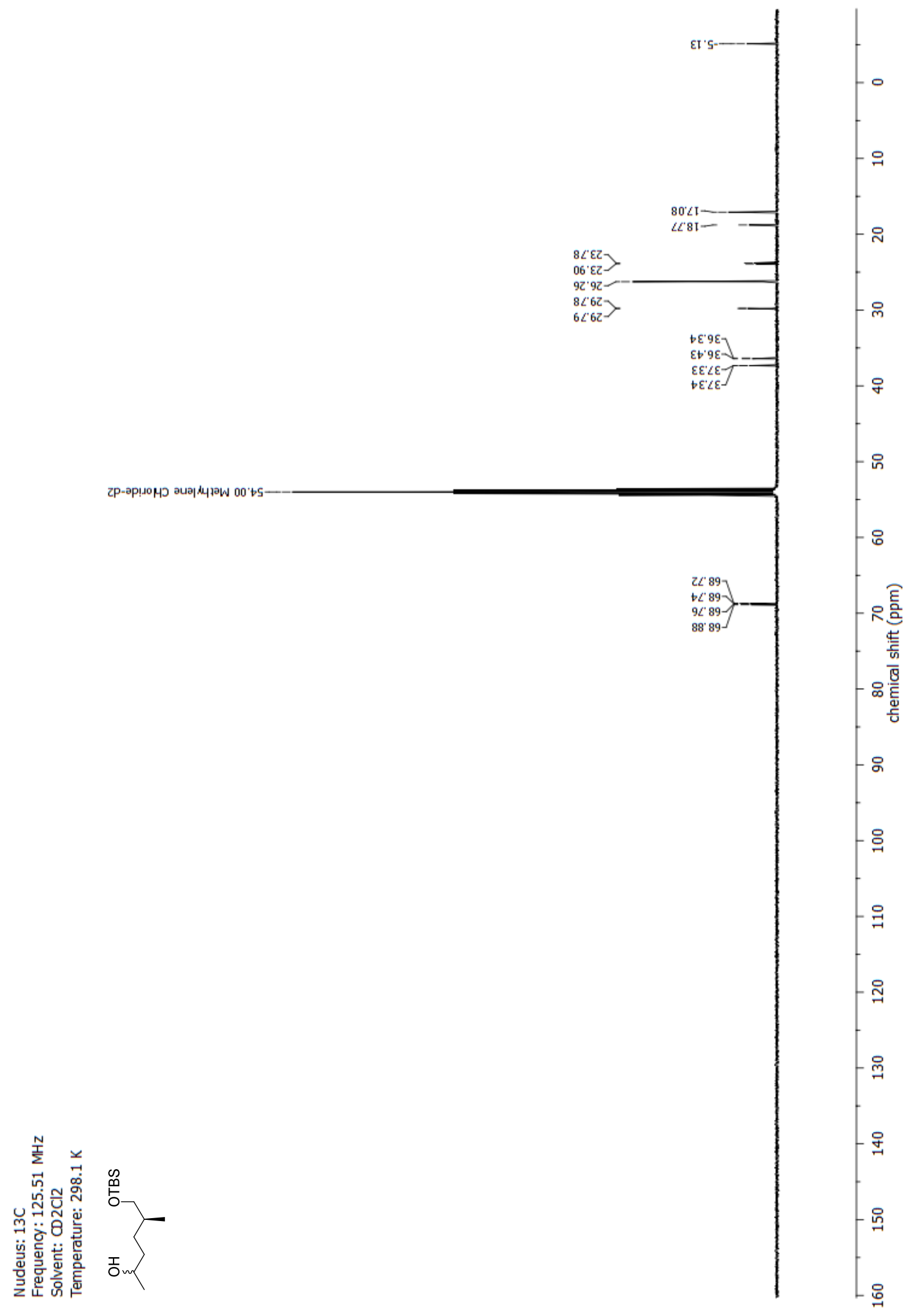


NMR-Spectra for Compound 88

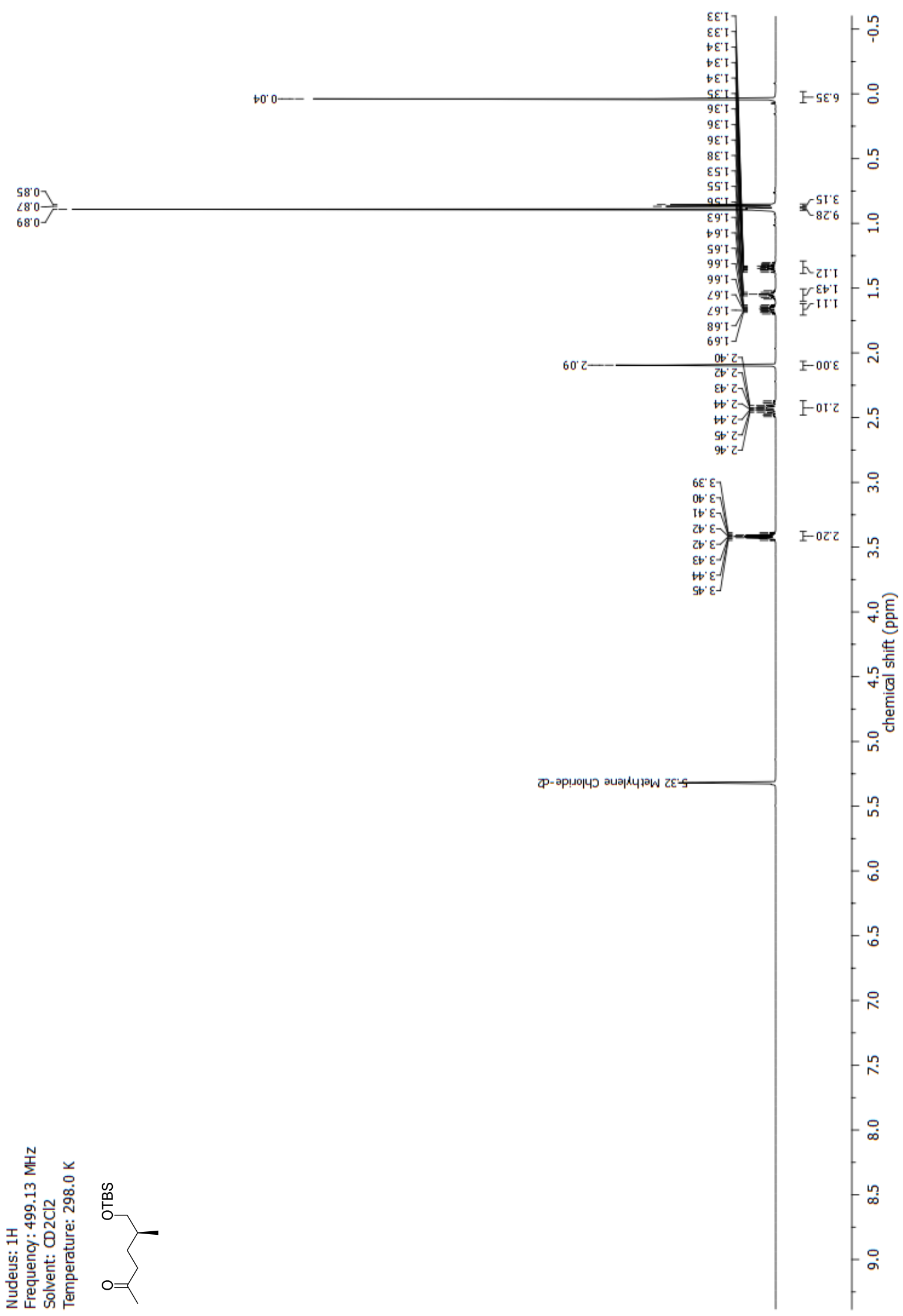




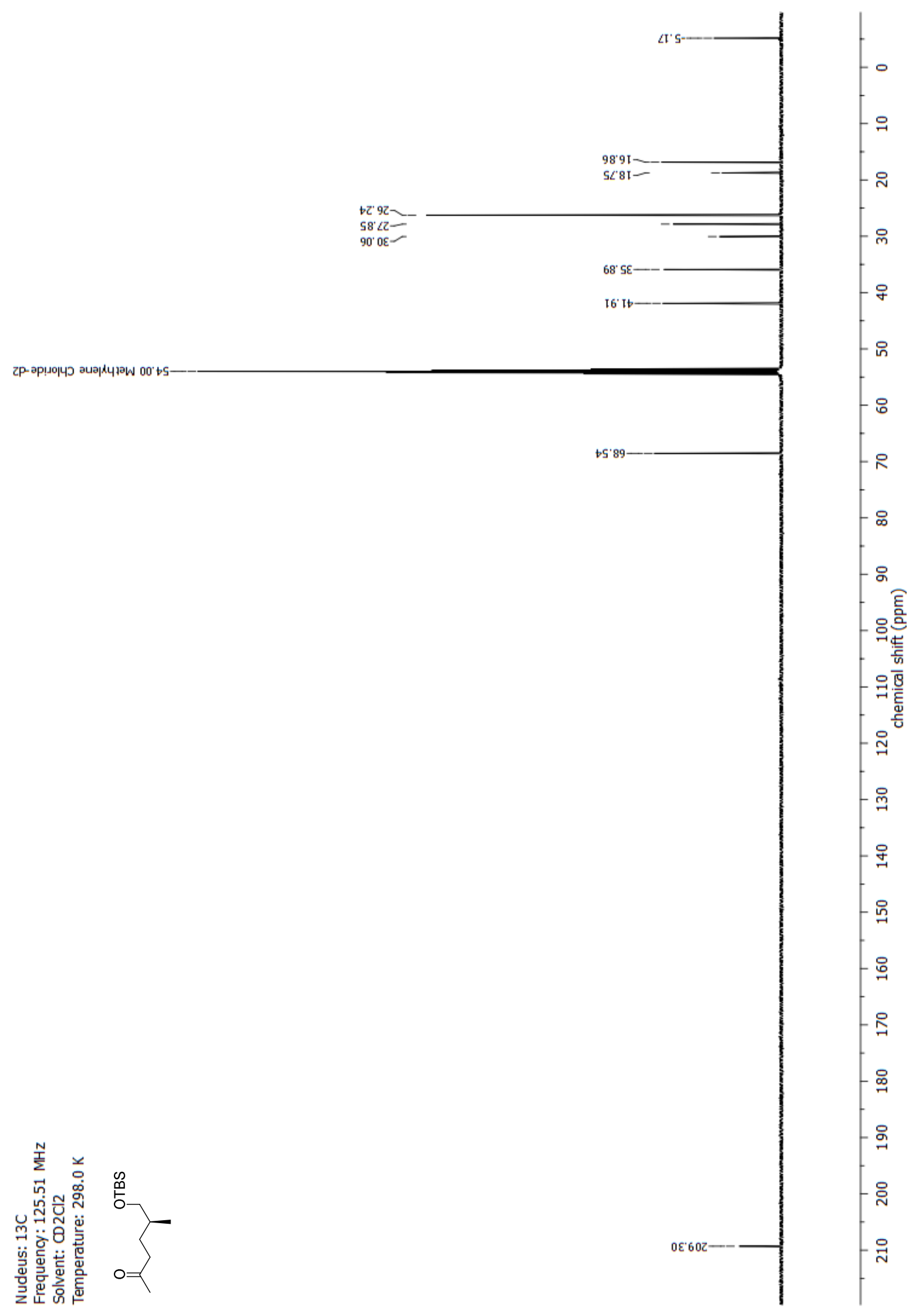


NMR-Spectra for Compound 31

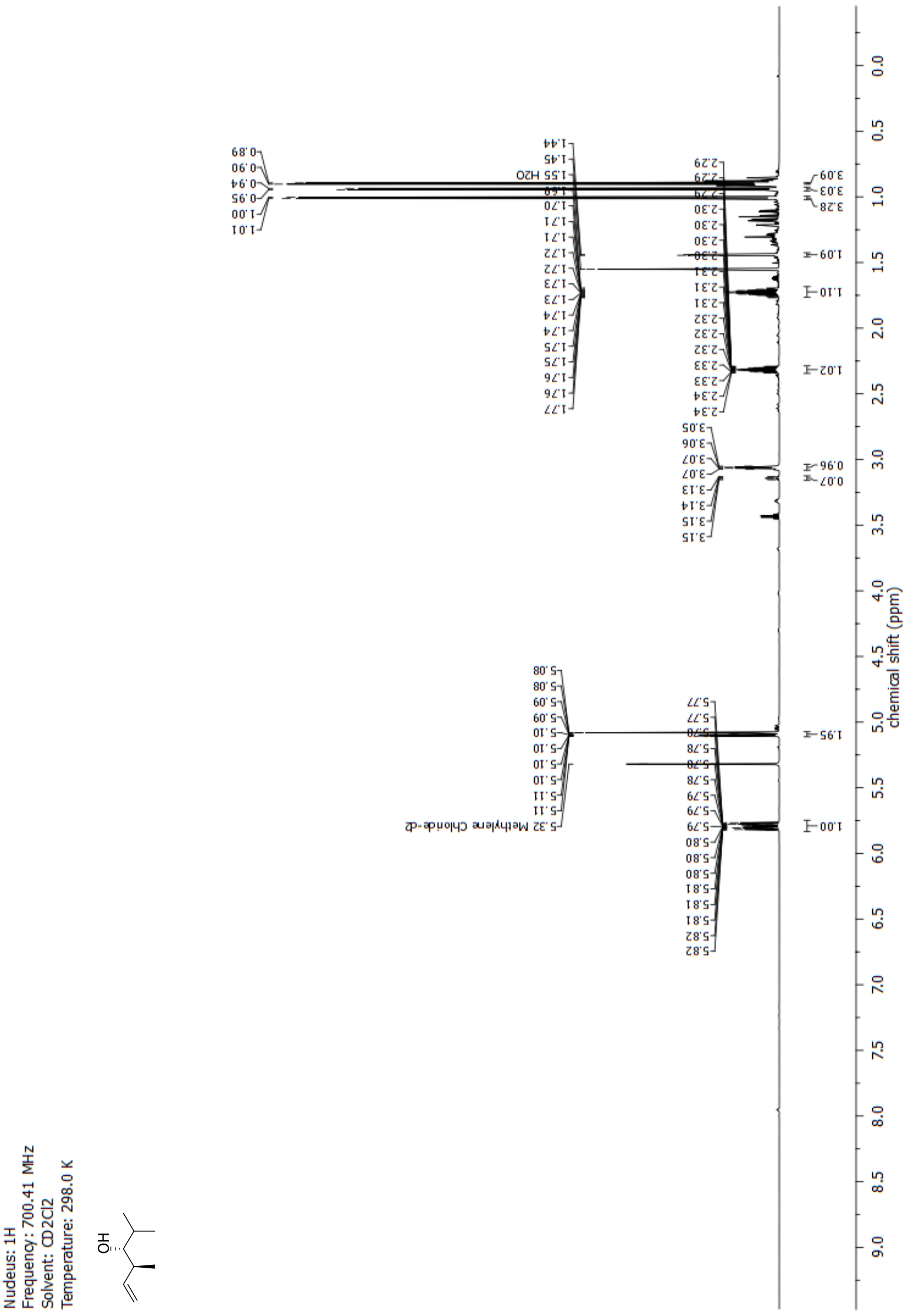




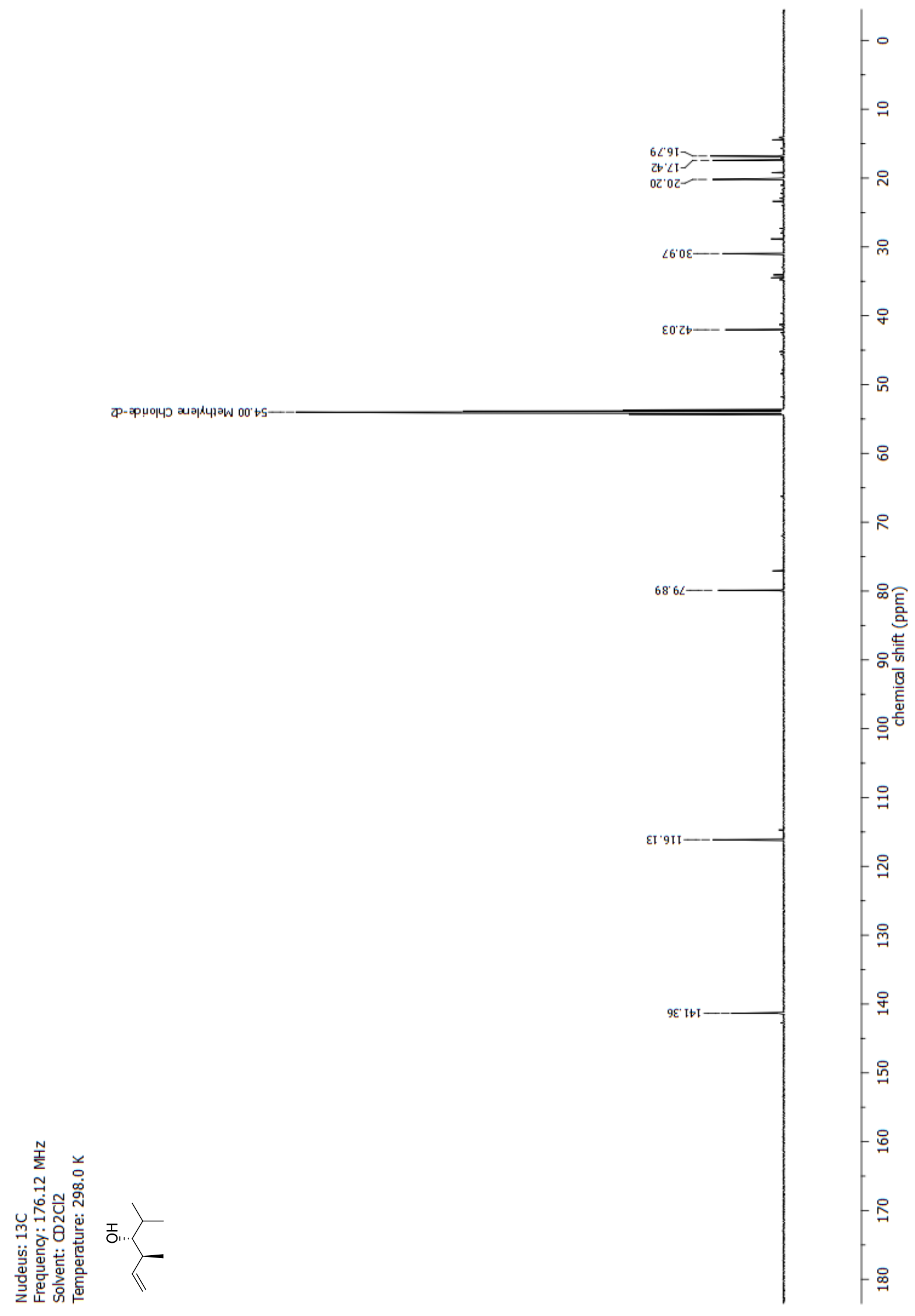


NMR-Spectra for Compound 89

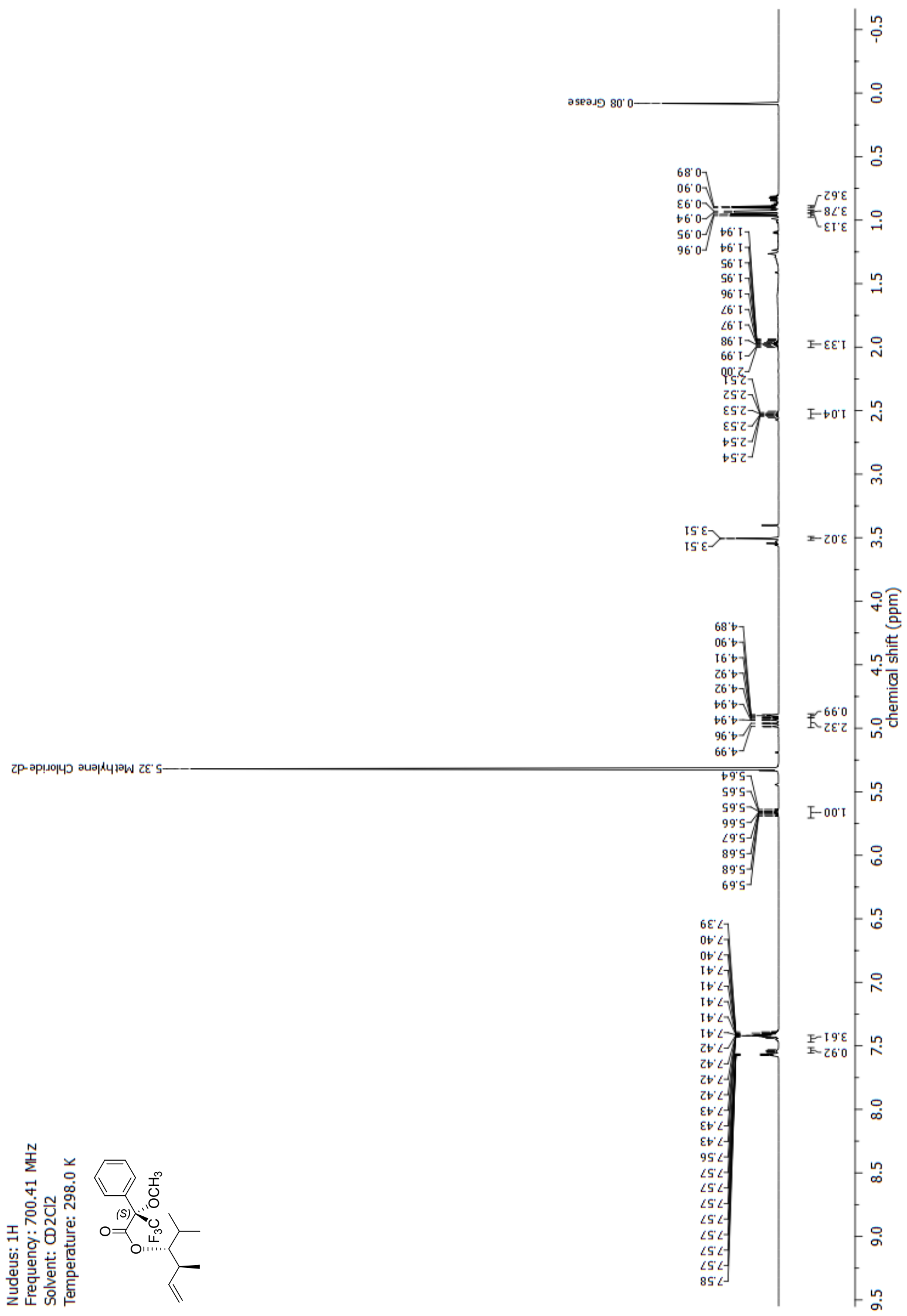




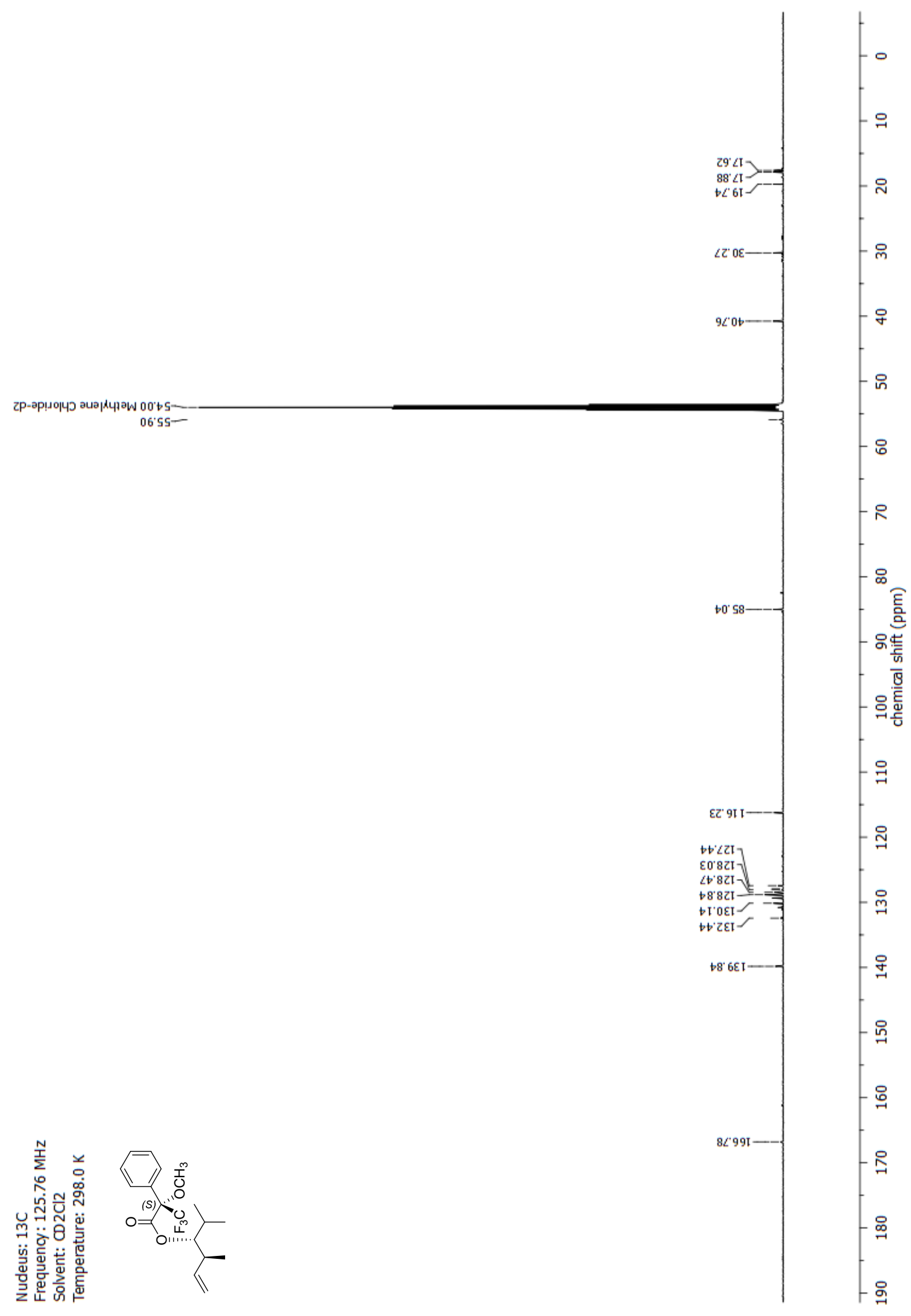




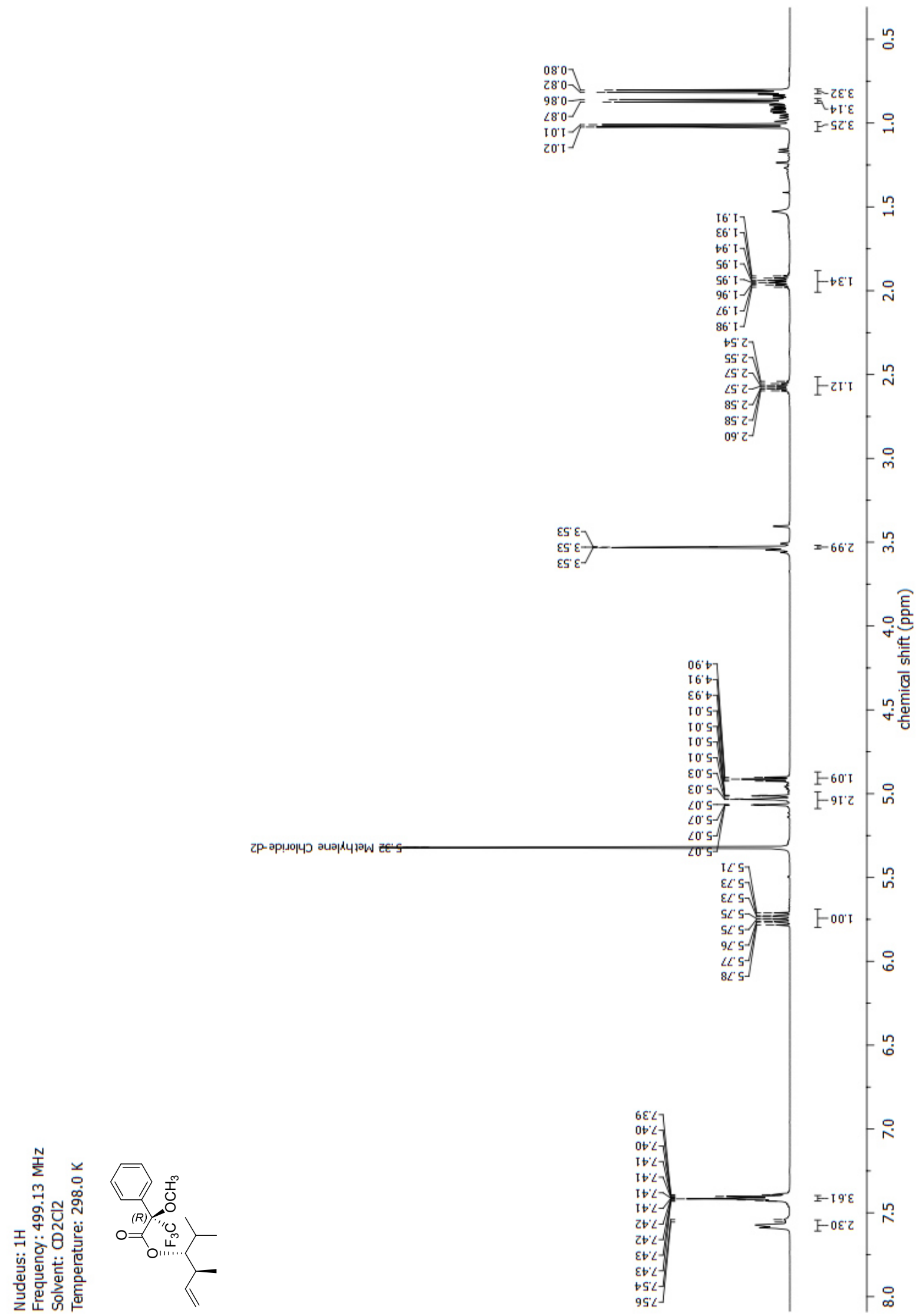




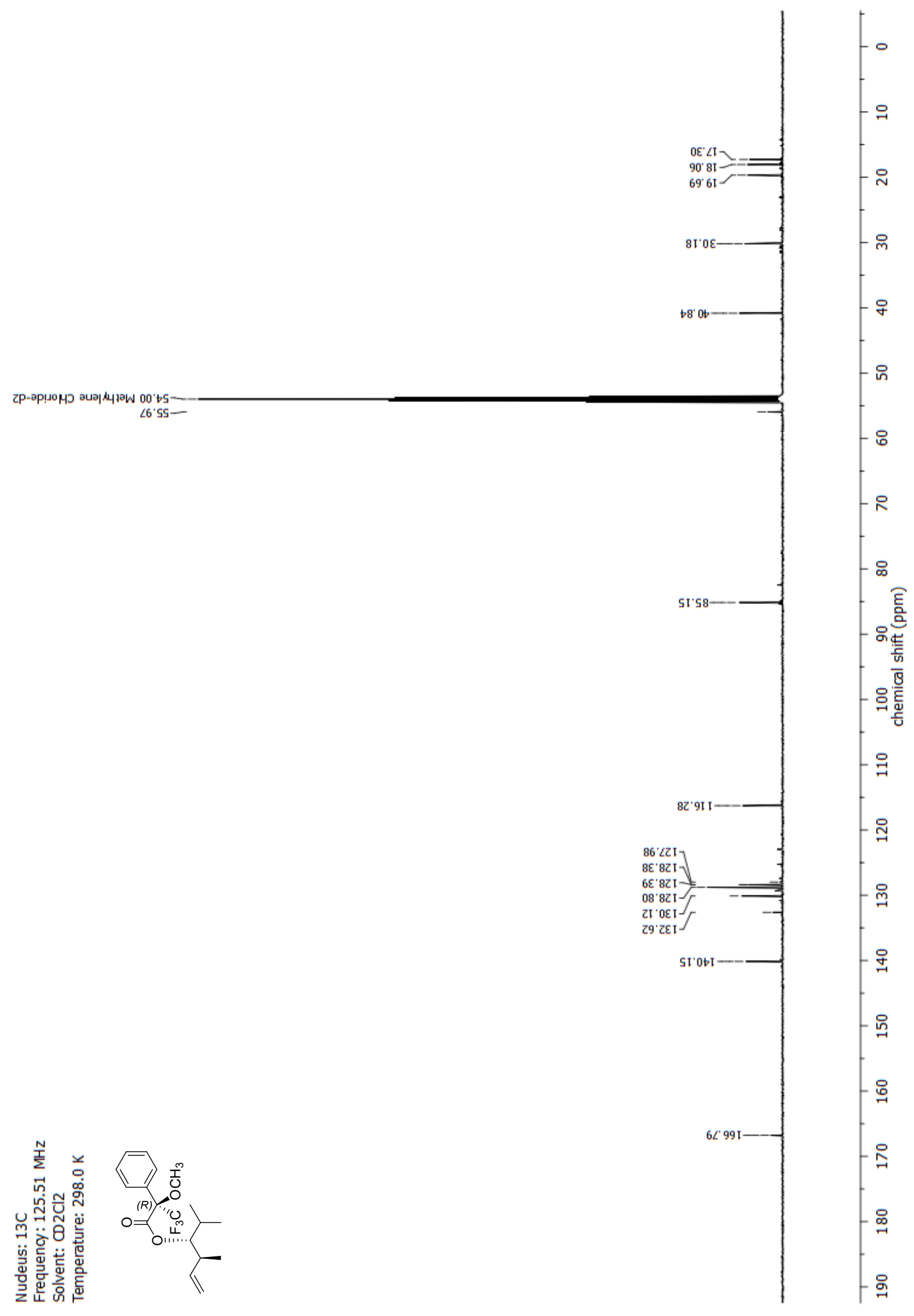




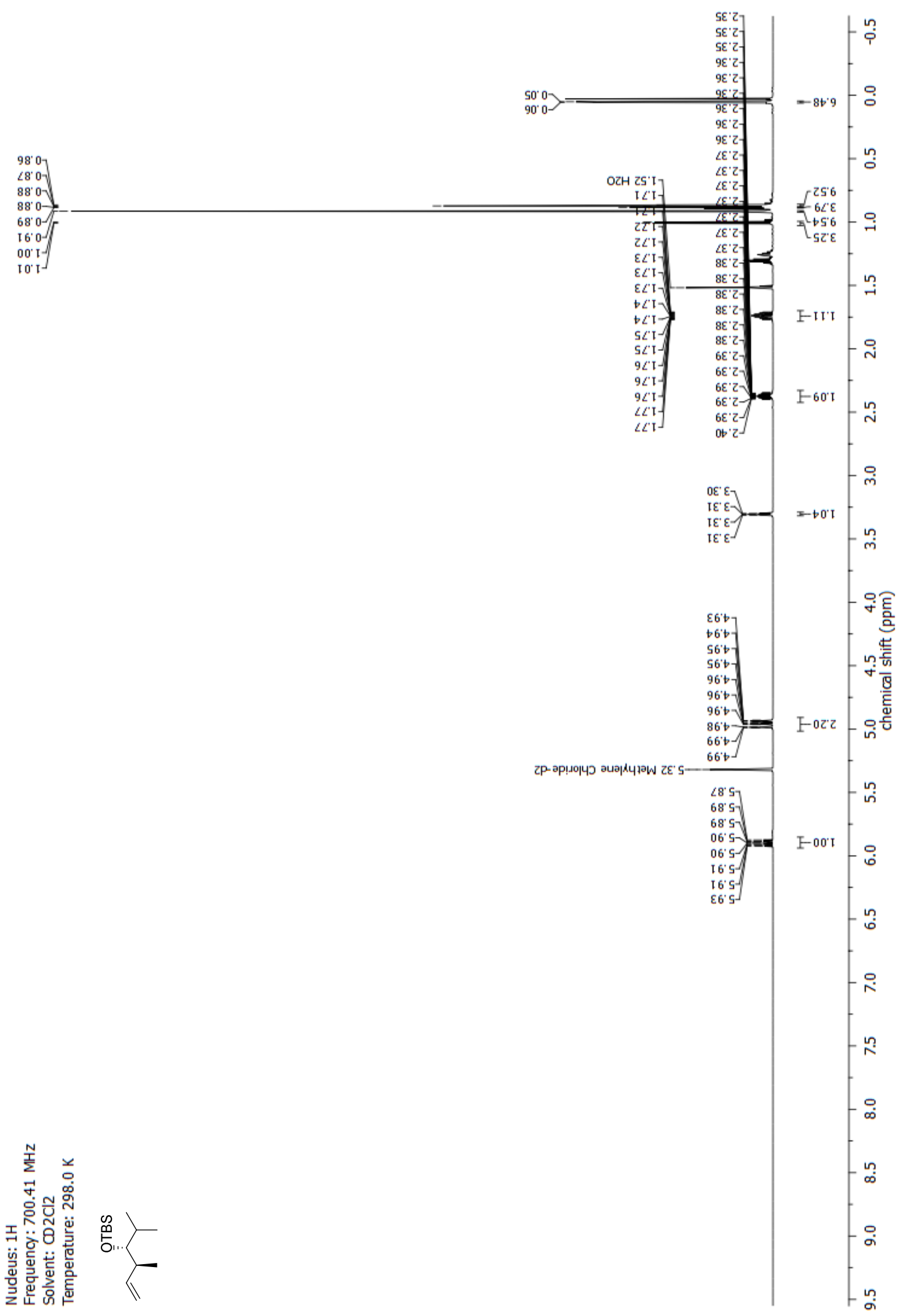




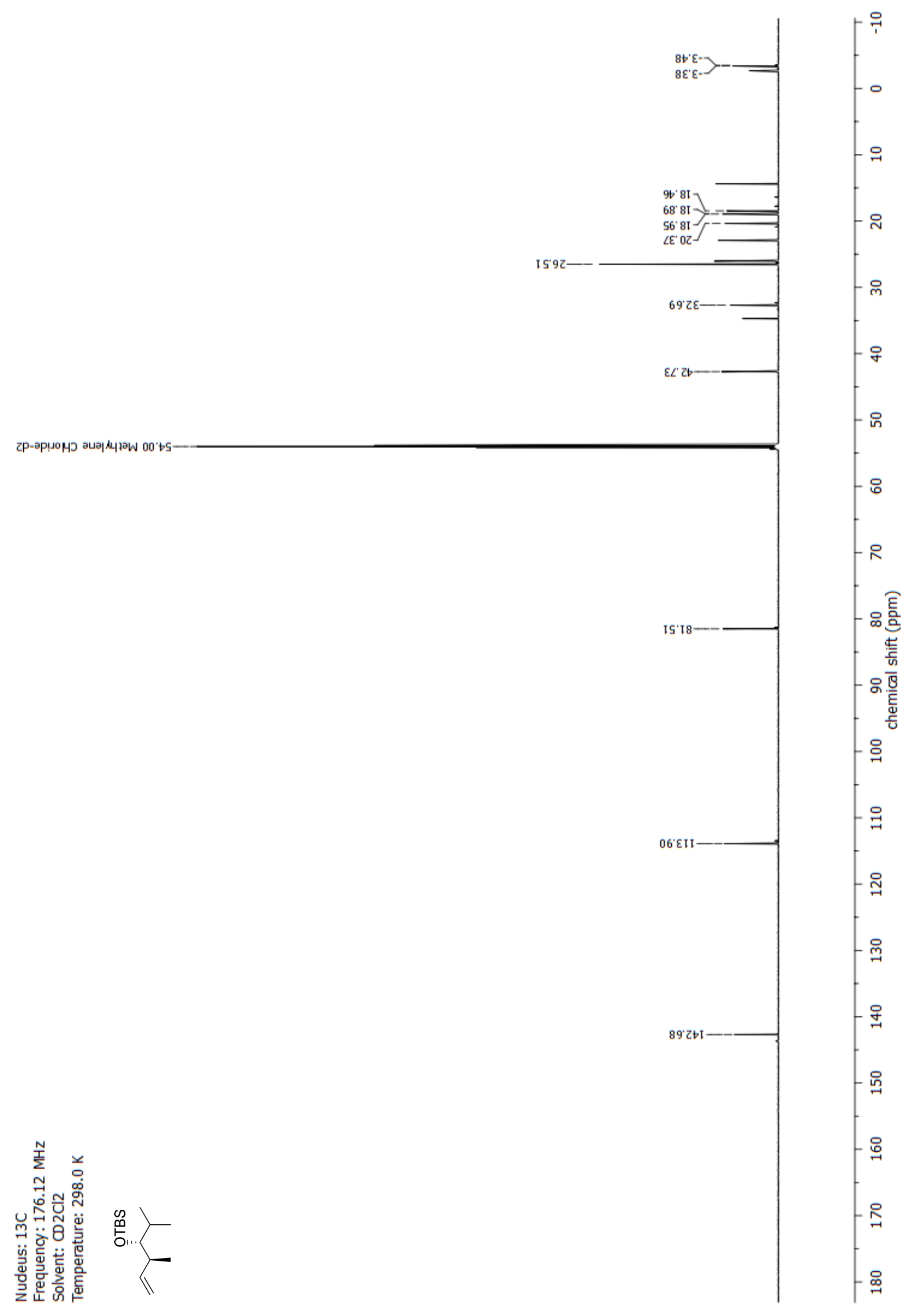


NMR-Spectra for Compound 32

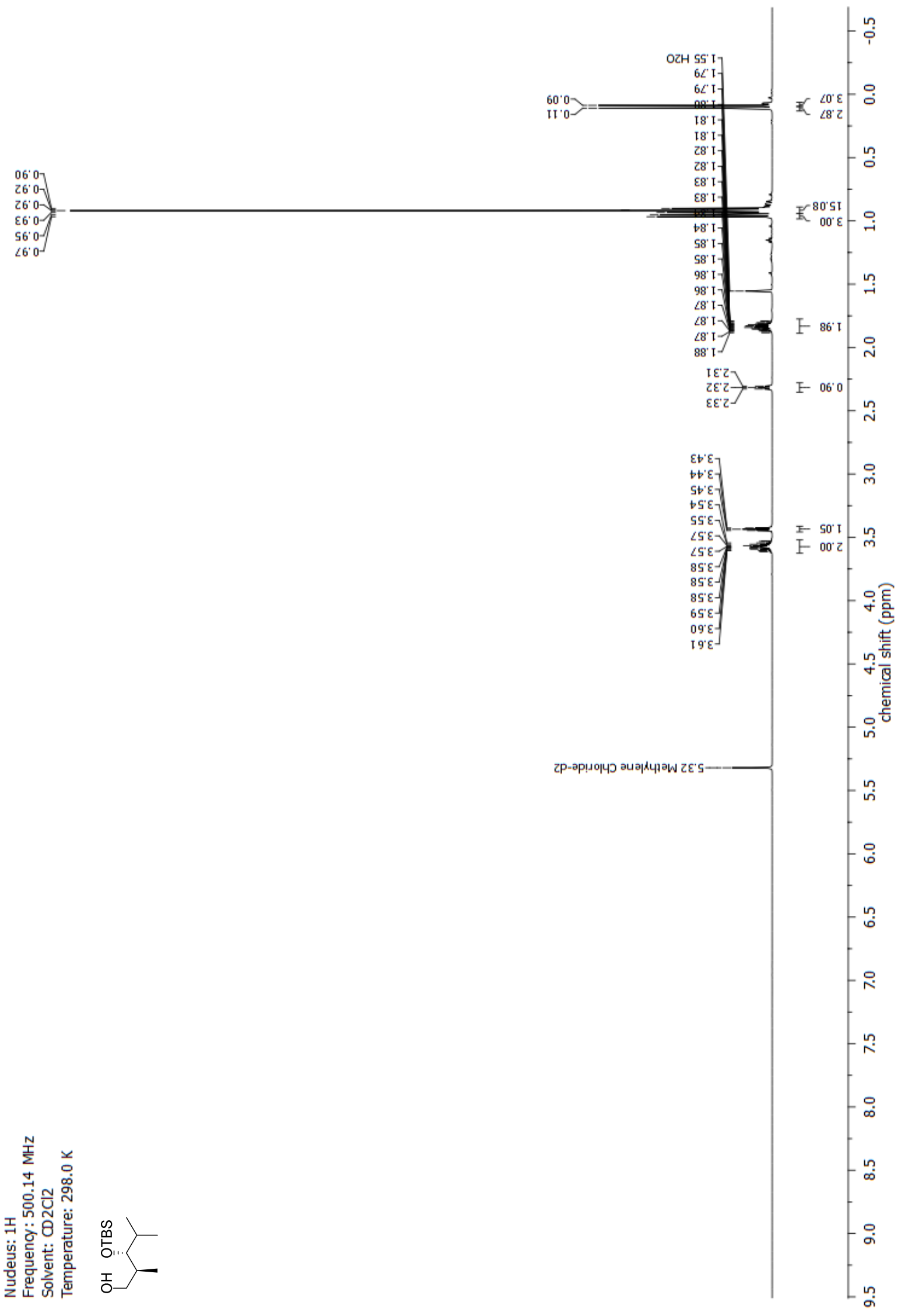




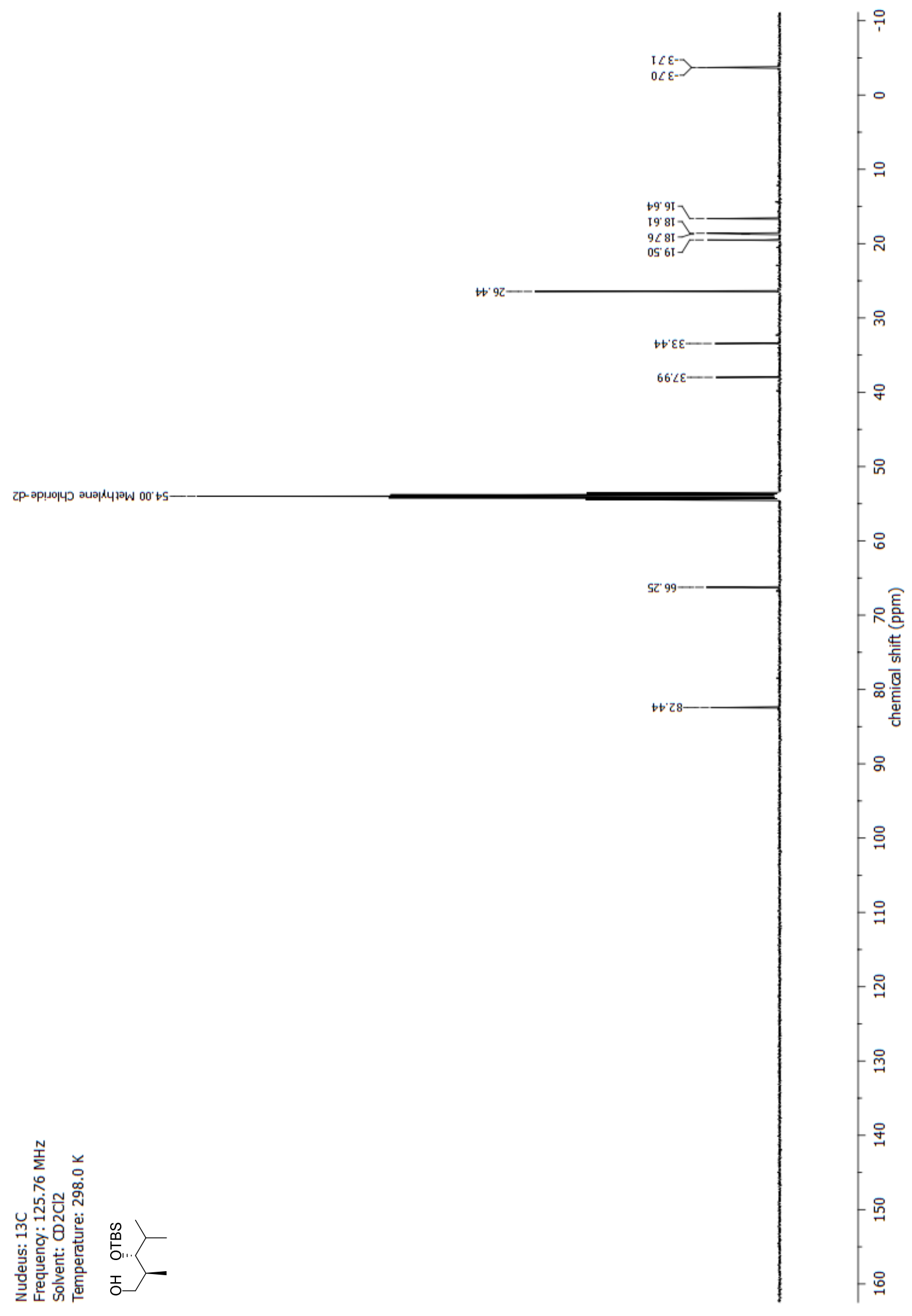


NMR-Spectra for Compound 33

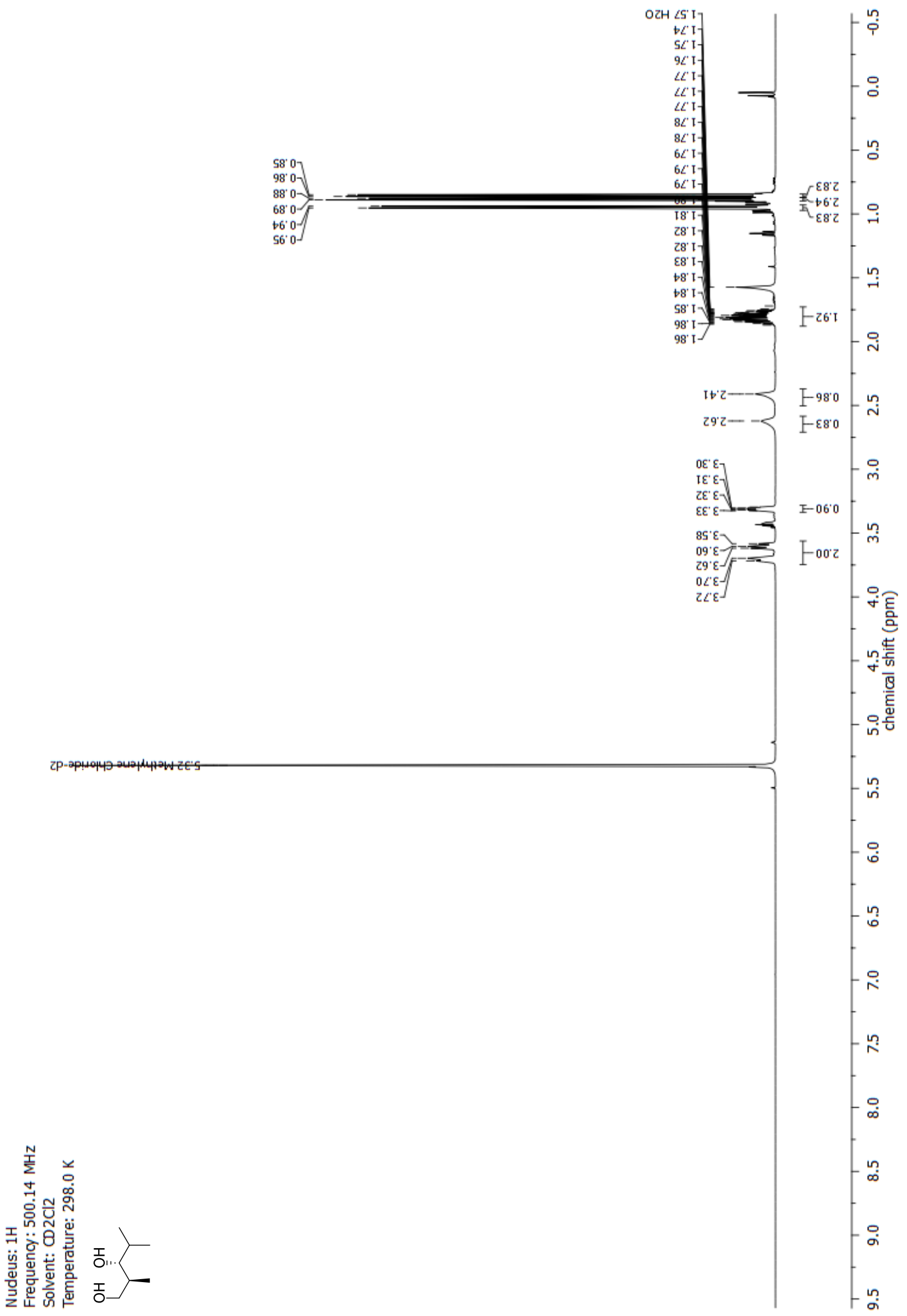




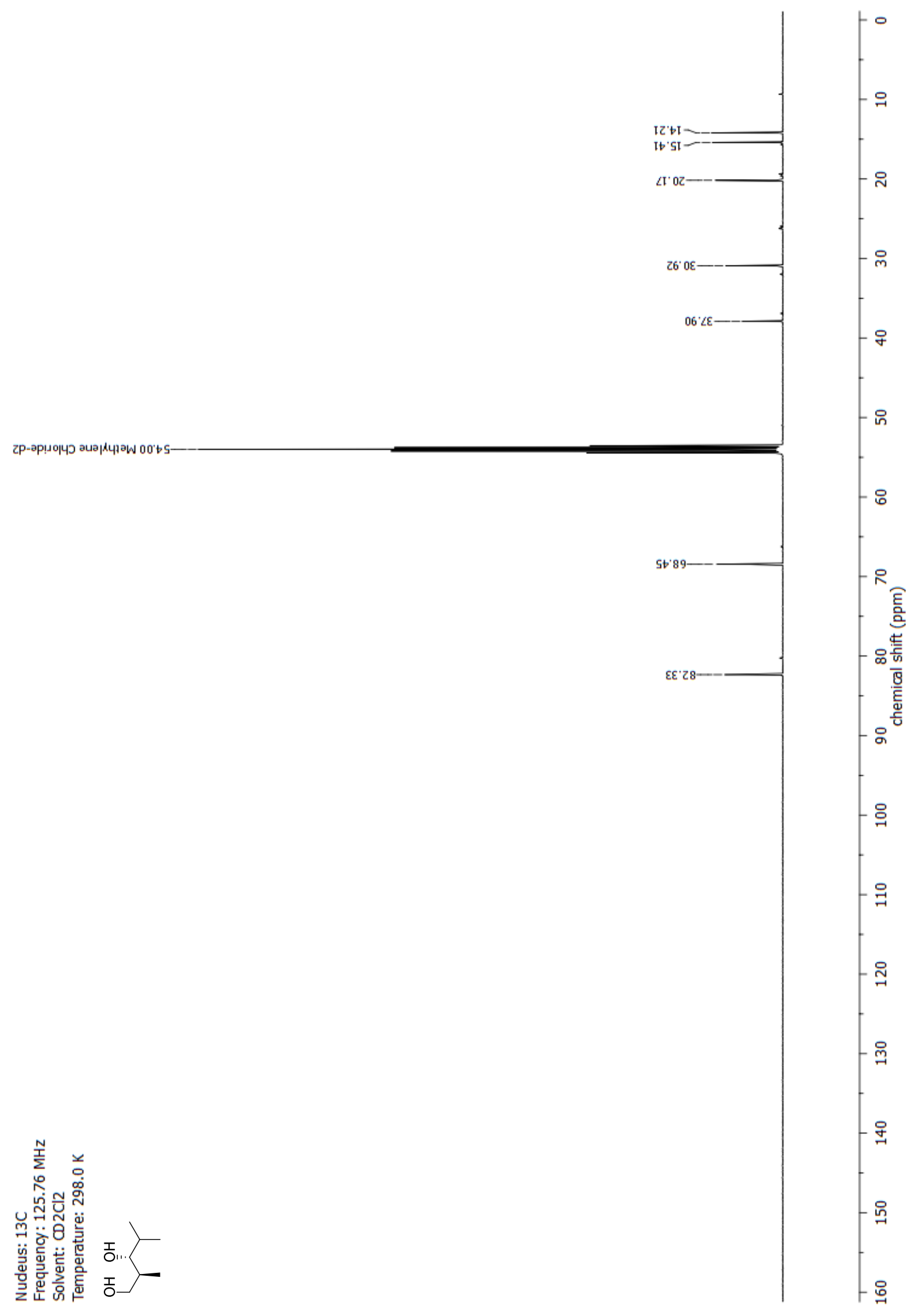


NMR-Spectra for Compound 35

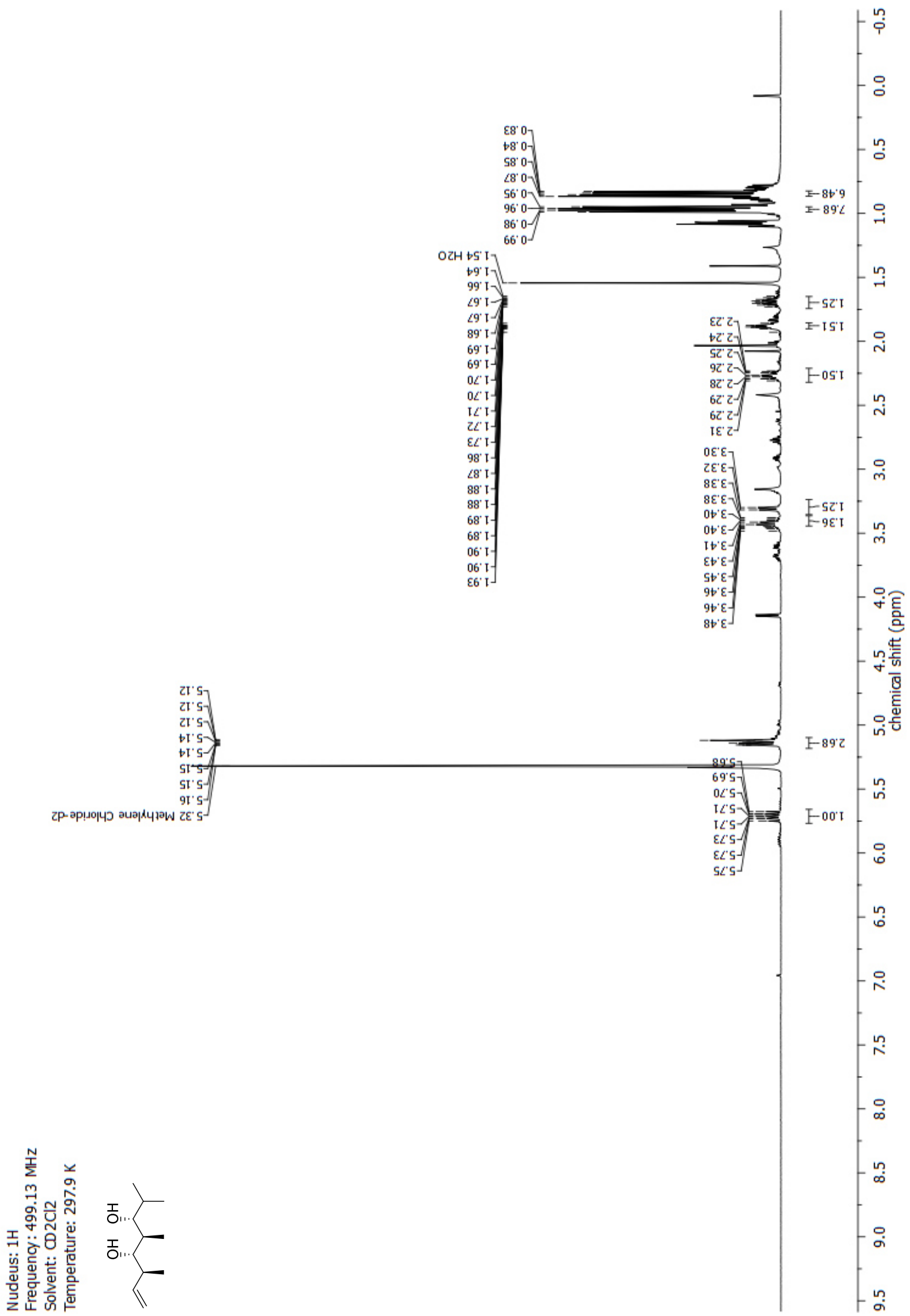




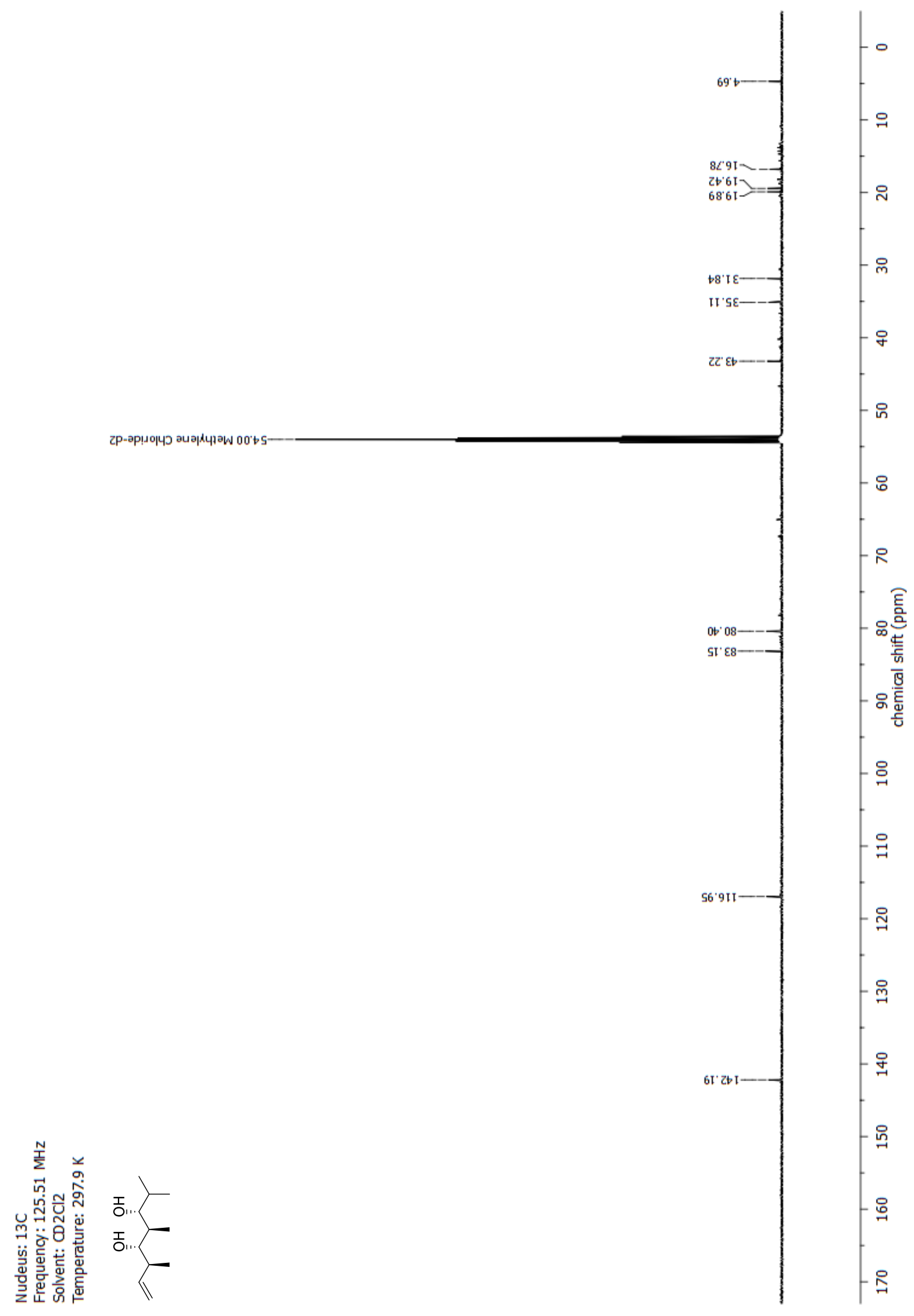


NMR-Spectra for Compound 37

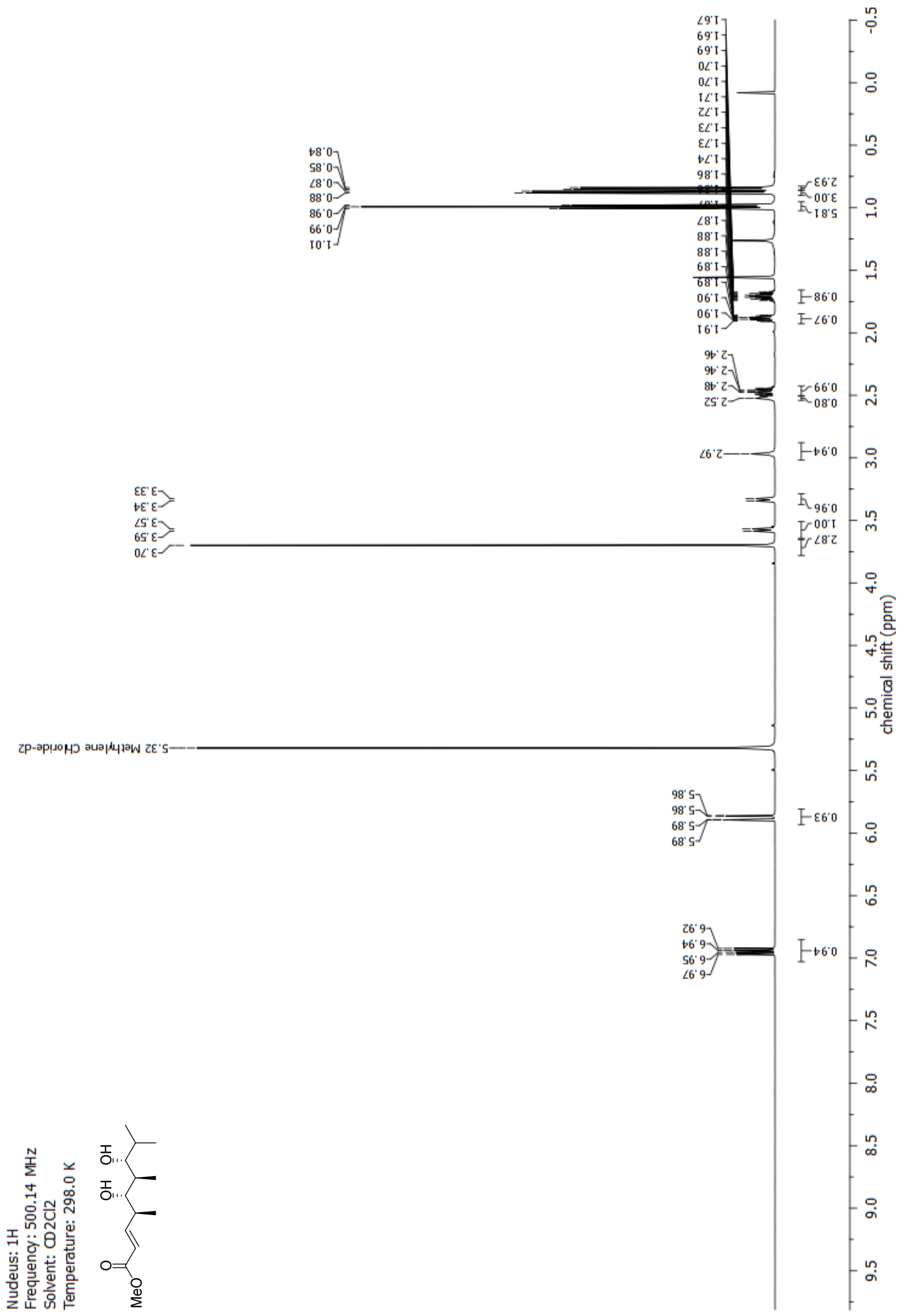




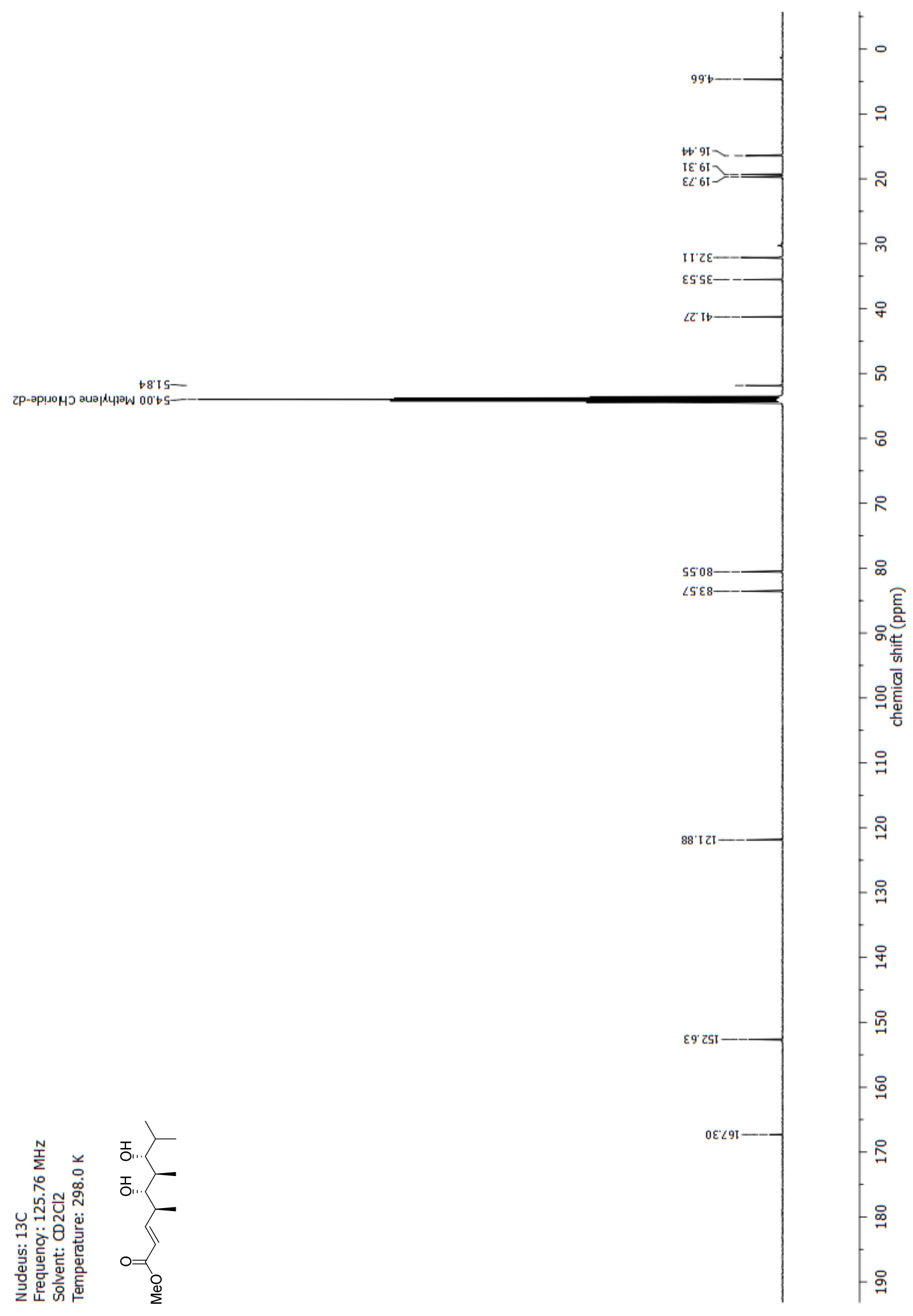




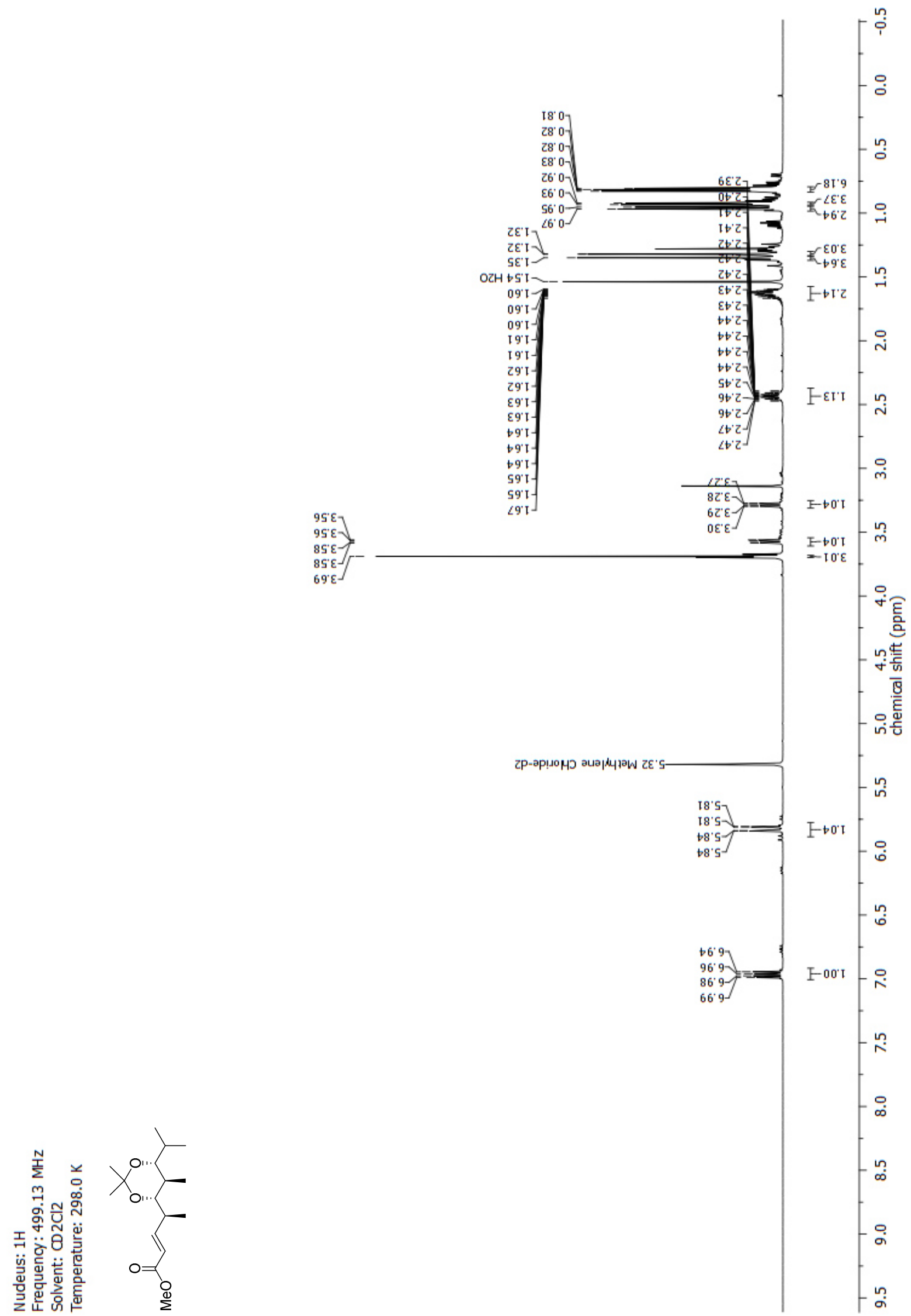




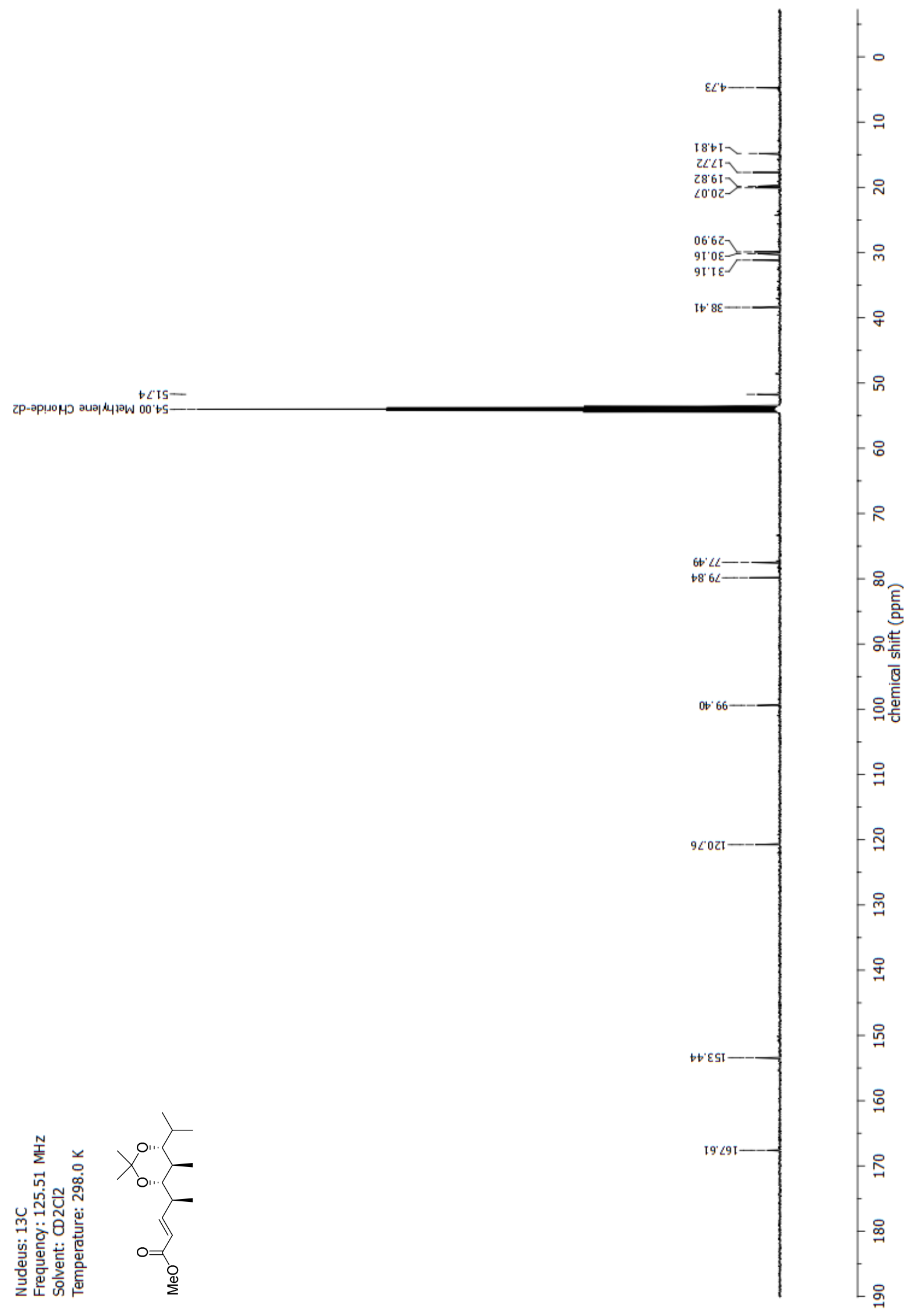


NMR-Spectra for Compound 38

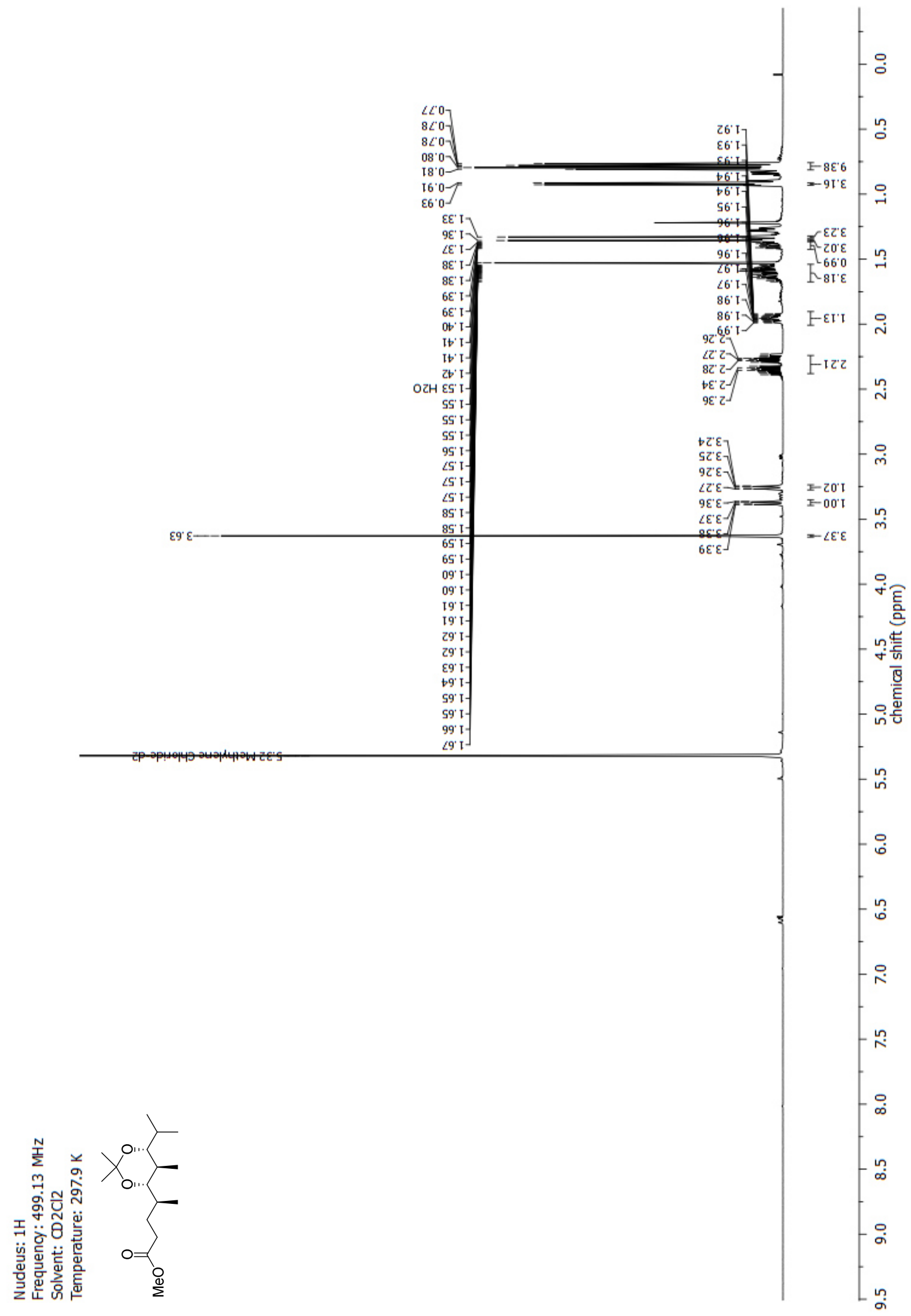




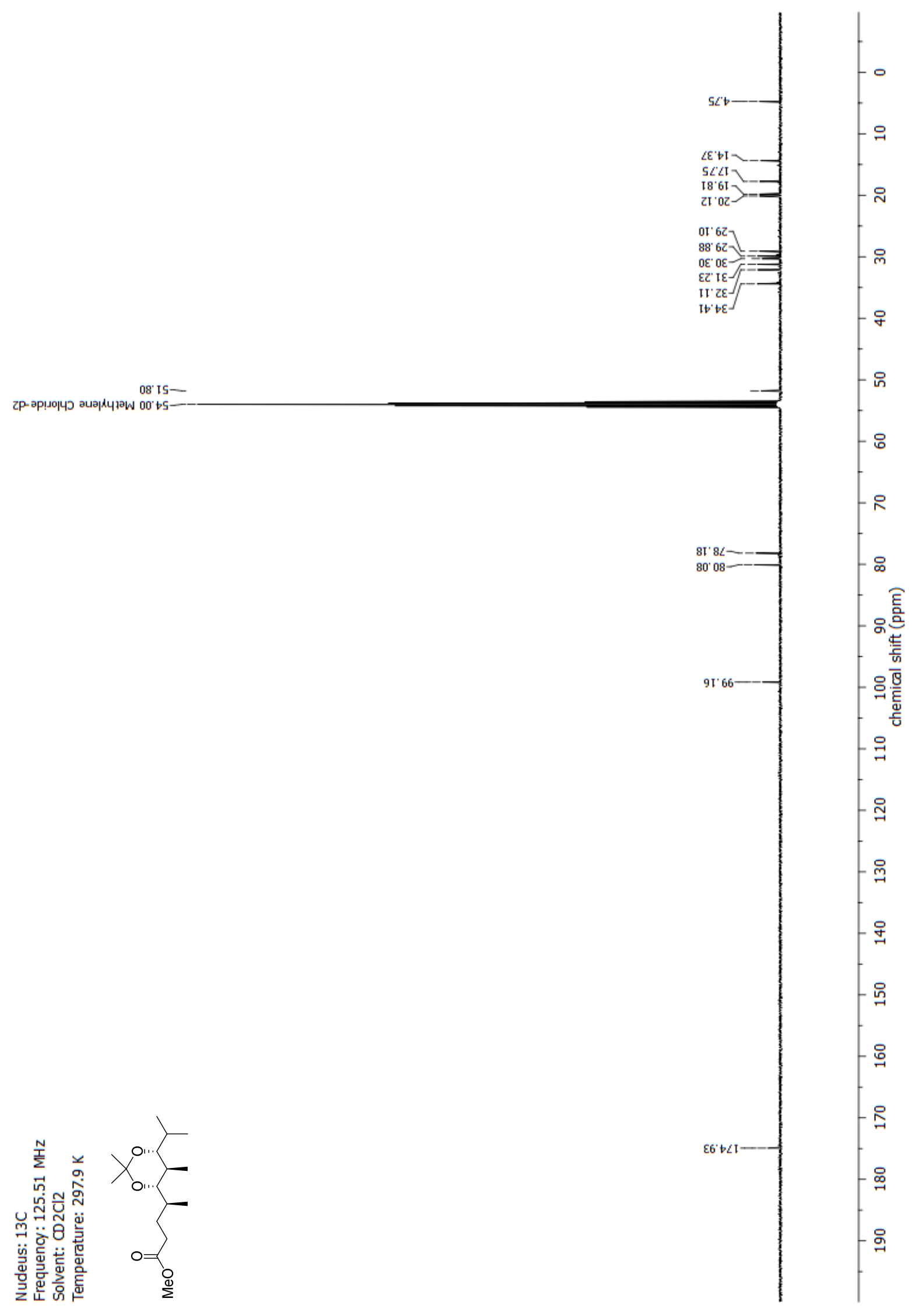


NMR-Spectra for Compound 5

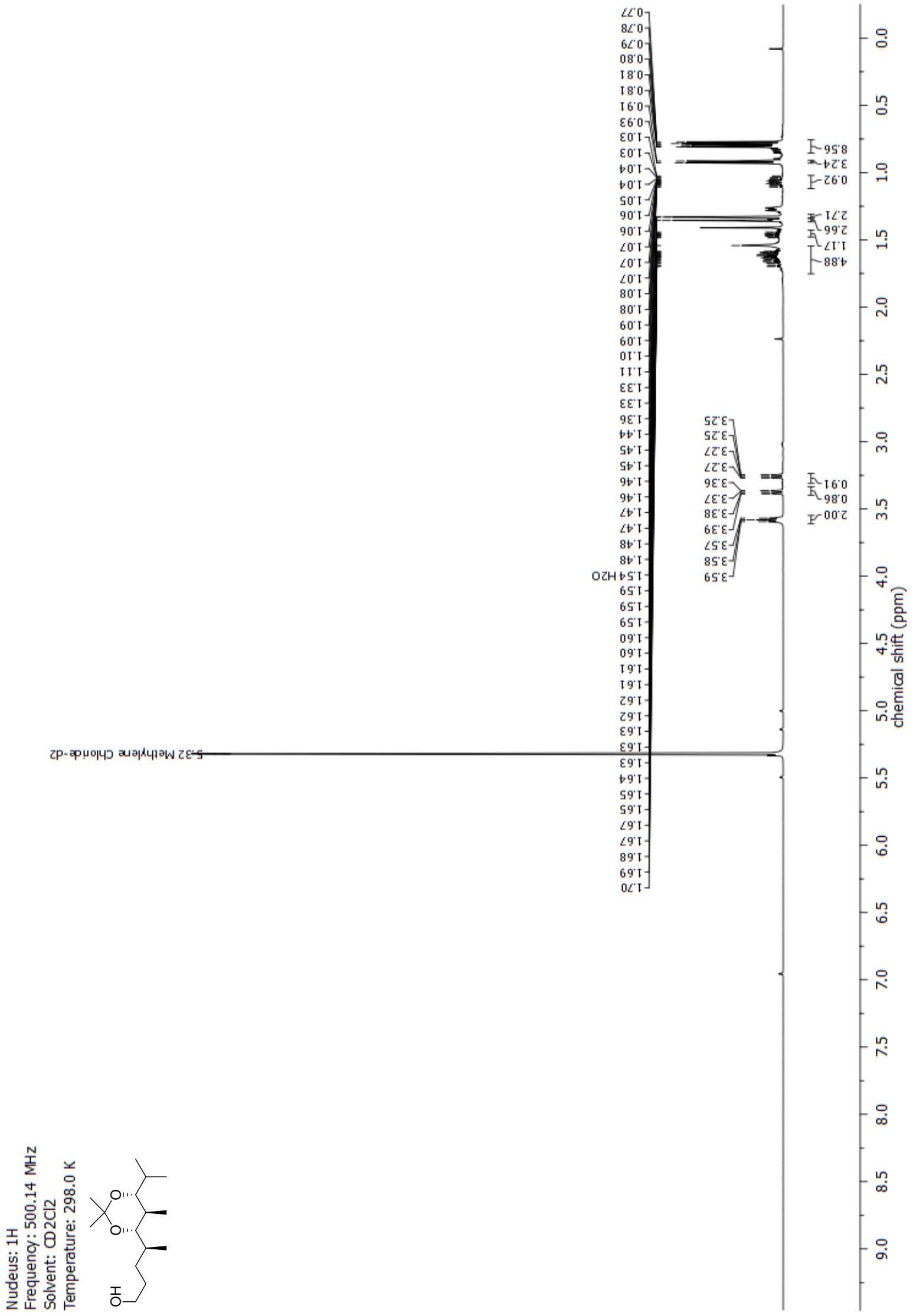




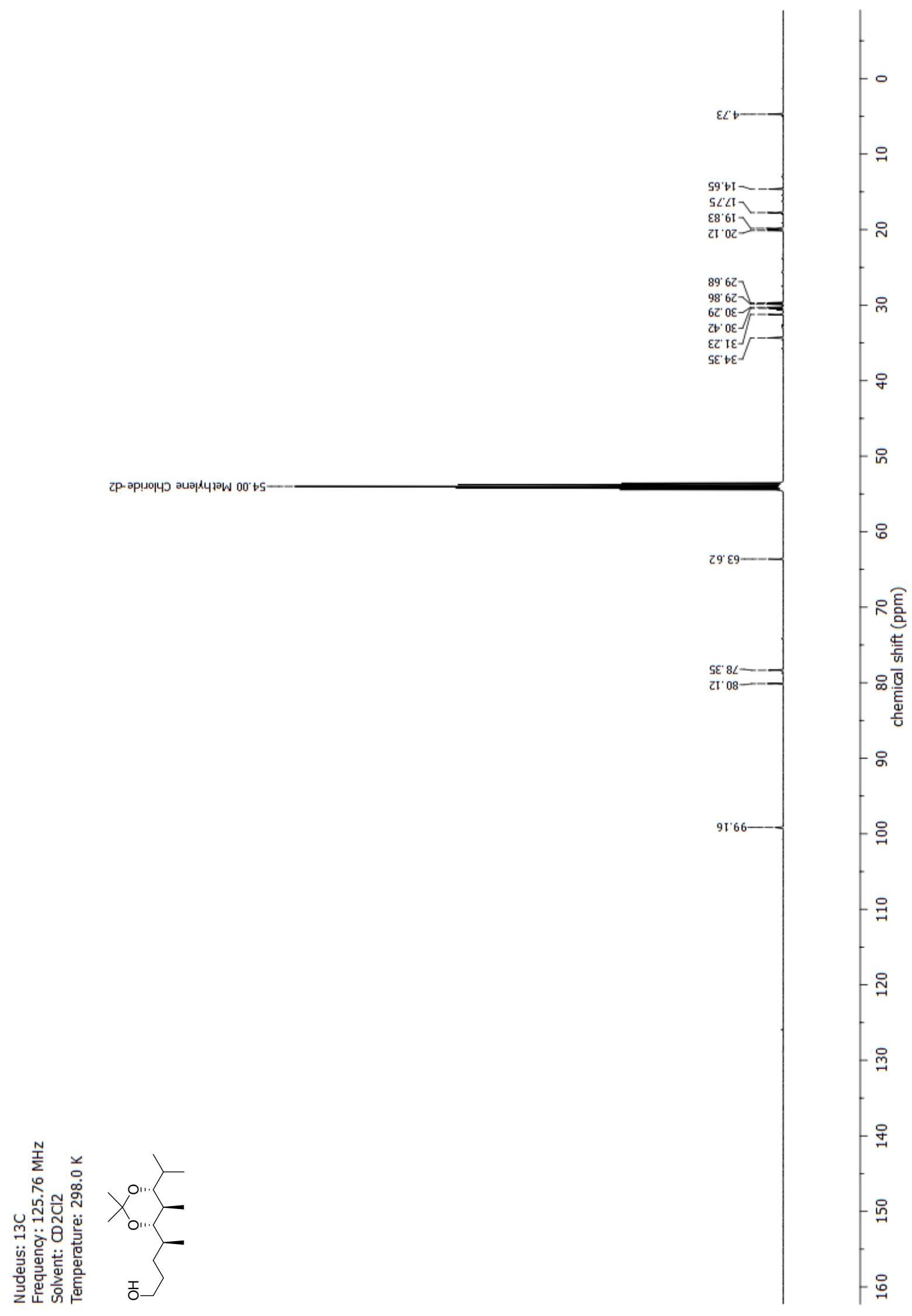


NMR-Spectra for Compound 41

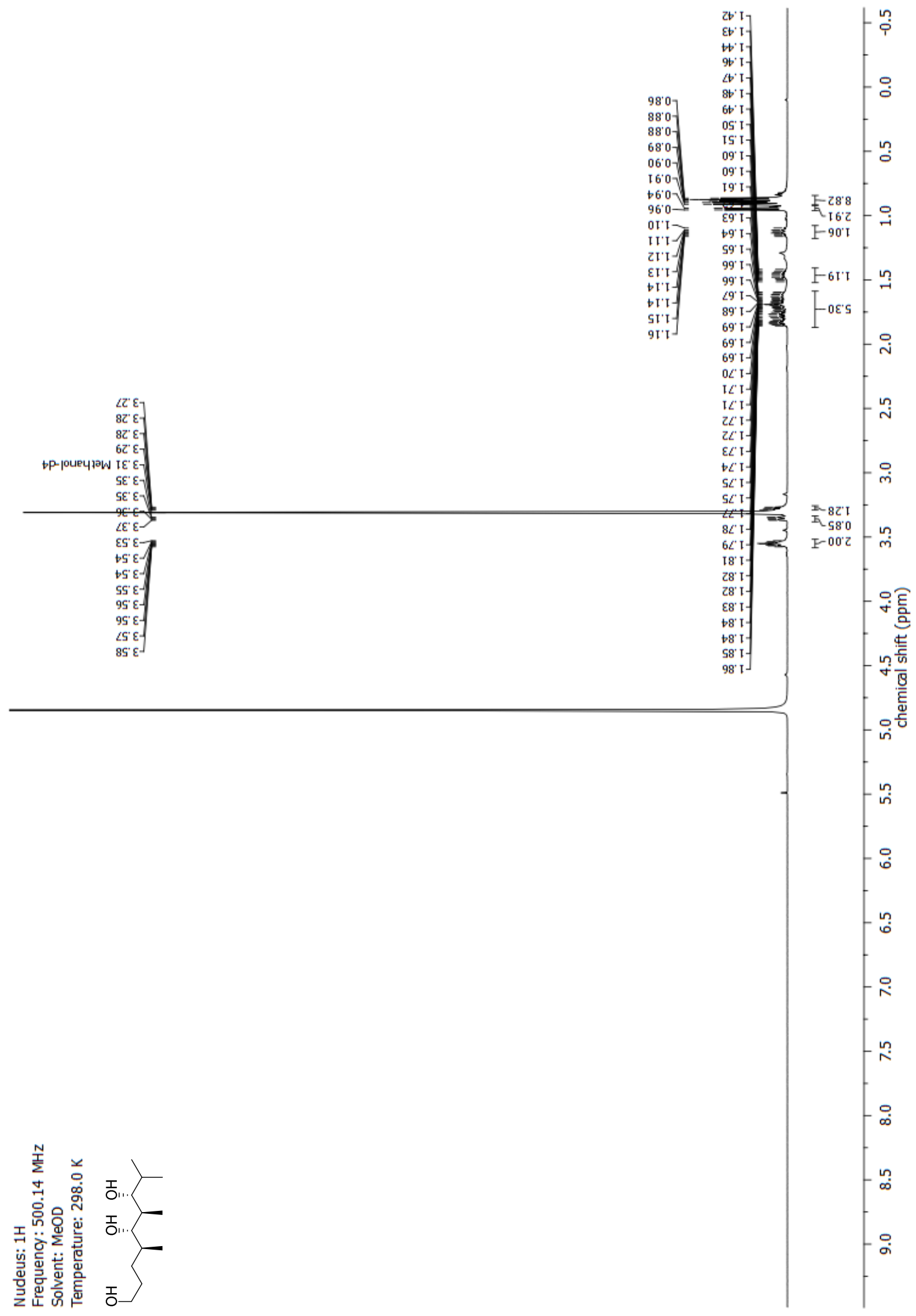




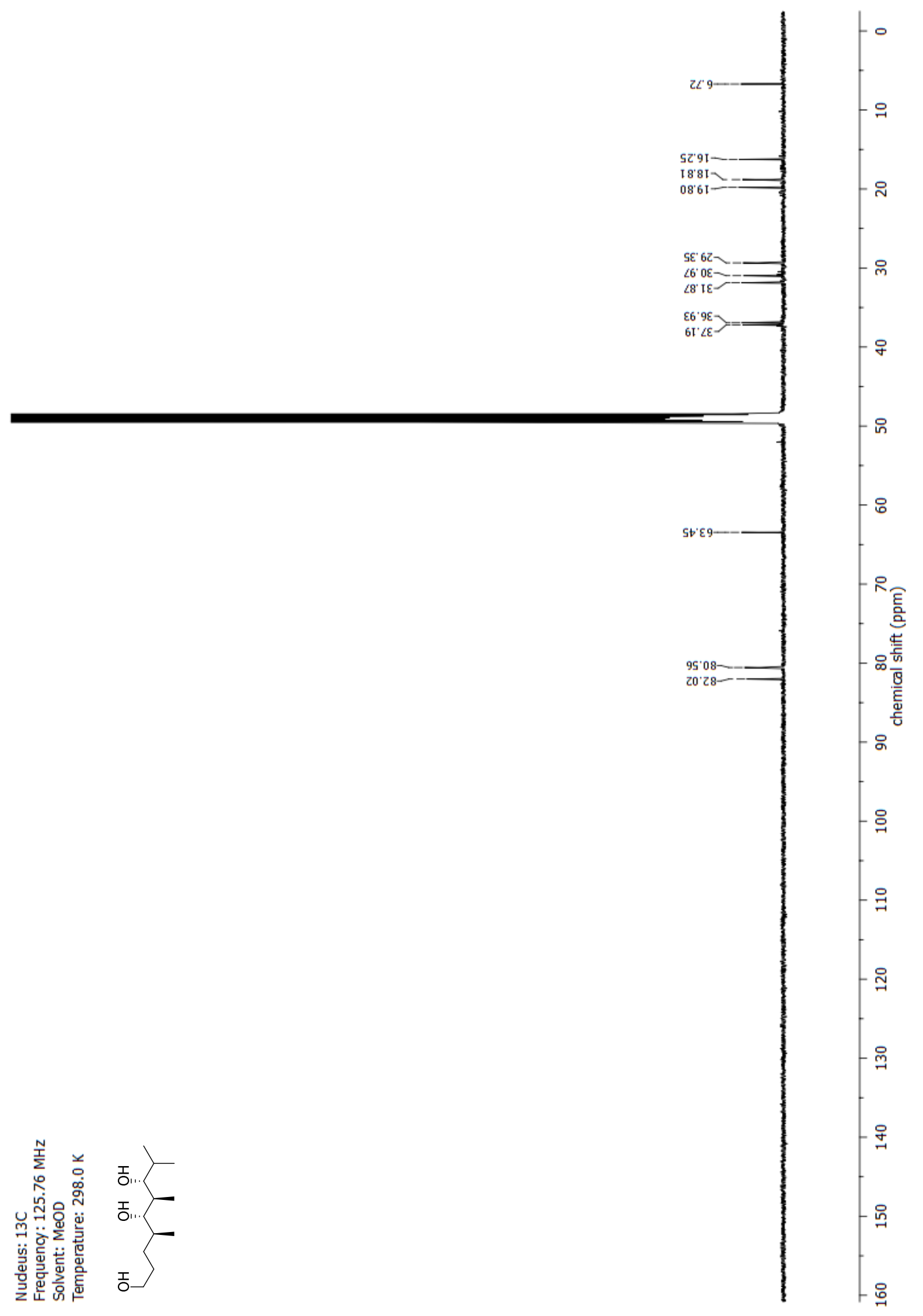

